\title{
FCC-hh: The Hadron Collider
}

\section{Future Circular Collider Conceptual Design Report Volume 3}

A. Abada ${ }^{33}$, M. Abbrescia ${ }^{118,258}$, S.S. AbdusSalam ${ }^{219}$, I. Abdyukhanov ${ }^{17}$, J. Abelleira Fernandez ${ }^{143}$, A. Abramov ${ }^{205}$, M. Aburaia ${ }^{285}$, A.O. Acar ${ }^{239}$, P.R. Adzic ${ }^{288}$, P. Agrawal ${ }^{80}$, J.A. Aguilar-Saavedra ${ }^{47}$, J.J. Aguilera-Verdugo ${ }^{107}$, M. Aiba ${ }^{192}$, I. Aichinger ${ }^{65}$, G. Aielli ${ }^{135,273}$, A. Akay ${ }^{239}$, A. Akhundov ${ }^{46}$, H. Aksakal ${ }^{146}$, J.L. Albacete ${ }^{47}$, S. Albergo ${ }^{121,261}$, A. Alekou ${ }^{313}$, M. Aleksa ${ }^{65}$, R. Aleksan ${ }^{40}$, R.M. Alemany Fernandez ${ }^{65}$, Y. Alexahin ${ }^{71}$, R.G. Alía ${ }^{65}$, S. Alioli ${ }^{127}$, N. Alipour Tehrani65, B.C. Allanach ${ }^{299}$, P.P. Allport ${ }^{291}$, M. Altinll ${ }^{63,113}$, W. Altmannshofer ${ }^{298}$, G. Ambrosio ${ }^{71}$, D. Amorim ${ }^{65}$, O. Amstutz ${ }^{162}$, L. Anderlini ${ }^{124,263}$, A. Andreazza ${ }^{128,267}$, M. Andreini ${ }^{65}$, A. Andriatis ${ }^{168}$, C. Andris ${ }^{166}$, A. Andronic ${ }^{346}$, M. Angelucci ${ }^{116}$, F. Antinori ${ }^{130,268}$, S.A. Antipov ${ }^{65}$, M. Antonelli ${ }^{116}$, M. Antonello ${ }^{128,265}$, P. Antonioli ${ }^{119}$, S. Antusch $^{287}$, F. Anulli ${ }^{134,272}$, L. Apolinário ${ }^{159}$, G. Apollinari ${ }^{71}$, A. Apollonio ${ }^{65}$, D. Appelö ${ }^{302}$, R.B. Appleby ${ }^{303,313}$, A. Apyan ${ }^{71}$, A. Apyan $^{1}$, A. Arbey ${ }^{337}$, A. Arbuzov ${ }^{18}$, G. Arduini ${ }^{65}$, V. Ar1 ${ }^{10}$, S. Arias ${ }^{67,311}$, N. Armesto ${ }^{109}$, R. Arnaldi ${ }^{137,275}$, S.A. Arsenyev ${ }^{65}$, M. Arzeo ${ }^{65}$, S. Asai ${ }^{237}$, E. Aslanides ${ }^{32}$, R.W. Aßmann ${ }^{50}$, D. Astapovych ${ }^{229}$, M. Atanasov ${ }^{65}$, S. Atieh ${ }^{65}$, D. Attié ${ }^{40}$, B. Auchmann ${ }^{65}$,

A. Audurier ${ }^{120,260}$, S. Aull ${ }^{65}$, S. Aumon ${ }^{65}$, S. Aune ${ }^{40}$, F. Avino ${ }^{65}$,

G. Avrillaud ${ }^{84}$, G. Aydın ${ }^{174}$, A. Azatov ${ }^{138,215}$, G. Azuelos ${ }^{242}$, P. Azzi ${ }^{130,268}$,

O. Azzolini ${ }^{117}$, P. Azzurri ${ }^{133,216}$, N. Bacchetta ${ }^{130,268}$, E. Bacchiocchi ${ }^{267}$,

H. Bachacou ${ }^{40}$, Y.W. Baek ${ }^{75}$, V. Baglin ${ }^{65}$, Y. Bai ${ }^{333}$, S. Baird ${ }^{65}$, M.J. Baker ${ }^{335}$, M.J. Baldwin ${ }^{168}$, A.H. Ball ${ }^{65}$, A. Ballarino ${ }^{65}$, S. Banerjee ${ }^{55}$, D.P. Barber ${ }^{50,318}$, D. Barducci ${ }^{138,215}$, P. Barjhoux ${ }^{3}$, D. Barna ${ }^{173}$, G.G. Barnaföldi ${ }^{173}$, M.J. Barnes ${ }^{65}$, A. Barr ${ }^{191}$, J. Barranco García ${ }^{57}$, J. Barreiro Guimarães da Costa ${ }^{98}$, W. Bartmann ${ }^{65}$, V. Baryshevsky ${ }^{96}$, E. Barzi ${ }^{71}$, S.A. Bass ${ }^{54}$, A. Bastianin ${ }^{267}$, B. Baudouy ${ }^{40}$, F. Bauer ${ }^{40}$, M. Bauer ${ }^{55}$, T. Baumgartner ${ }^{233}$, I. BautistaGuzmán $^{16}$, C. Bayındır ${ }^{20,83}$, F. Beaudette ${ }^{33}$, F. Bedeschi ${ }^{133,216}$, M. Béguin ${ }^{65}$, I. Bellafont ${ }^{7}$, L. Bellagamba ${ }^{119,259}$, N. Bellegarde ${ }^{65}$, E. Belli ${ }^{134,209,272}$, E. Bellingeri $^{44}$, F. Bellini ${ }^{65}$, G. Bellomo ${ }^{128,267}$, S. Belomestnykh ${ }^{71}$, G. Bencivenni ${ }^{116}$, M. Benedikt ${ }^{65, a}$, G. Bernardi ${ }^{33}$, J. Bernardi $^{233}$, C. Bernet ${ }^{33,337}$, J.M. Bernhardt ${ }^{3}$, C. Bernini ${ }^{44}$, C. Berriaud ${ }^{40}$, A. Bertarelli ${ }^{65}$, S. Bertolucci ${ }^{119,259}$ ', M.I. Besana ${ }^{192}$, M. Besançon ${ }^{40}$, O. Beznosov ${ }^{318}$, P. Bhat ${ }^{71}$, C. Bhat ${ }^{71}$, M.E. Biagini ${ }^{116}$, J.-L. Biarrotte ${ }^{33}$, A. Bibet Chevalier ${ }^{28}$, E.R. Bielert ${ }^{306}$, M. Biglietti ${ }^{136,274}$, G.M. Bilei ${ }^{132,271}$, B. Bilki ${ }^{307}$, C. Biscari ${ }^{7}$, F. Bishara ${ }^{50,191}$, O.R. Blanco-García ${ }^{116}$, F.R. Blánquez ${ }^{65}$, F. Blekman ${ }^{342}$, A. Blondel ${ }^{305}$,

J. Blümlein ${ }^{50}$, T. Boccali ${ }^{133,216}$, R. Boels ${ }^{85}$, S.A. Bogacz ${ }^{238}$, A. Bogomyagkov ${ }^{24}$, O. Boine-Frankenheim ${ }^{229}$, M.J. Boland ${ }^{323}$, S. Bologna ${ }^{292}$, O. Bolukbasi ${ }^{113}$,

M. Bomben ${ }^{33}$, S. Bondarenko ${ }^{18}$, M. Bonvini ${ }^{134,272}$, E. Boos ${ }^{222}$, B. Bordini ${ }^{65}$,

F. Bordry ${ }^{65}$, G. Borghello ${ }^{65,276}$, L. Borgonovi ${ }^{119,259}$, S. Borowka ${ }^{65}$, D. Bortoletto ${ }^{191}$,

D. Boscherini ${ }^{119,259}$, M. Boscolo ${ }^{116}$, S. Boselli ${ }^{131,270}$, R.R. Bosley ${ }^{291}$, F. Bossu ${ }^{33}$,

C. Botta ${ }^{65}$, L. Bottura ${ }^{65}$, R. Boughezal ${ }^{12}$, D. Boutin ${ }^{40}$, G. Bovone ${ }^{44}$,

I. Božović Jelisavčićc ${ }^{341}$, A. Bozbey ${ }^{239}$, C. Bozzi ${ }^{123,262}$, D. Bozzini ${ }^{65}$,

V. Braccini ${ }^{44}$, S. Braibant-Giacomelli ${ }^{119,259}$, J. Bramante ${ }^{194,201}$, P. BraunMunzinger $^{78}$, J.A. Briffa ${ }^{312}$, D. Britzger ${ }^{170}$, S.J. Brodsky ${ }^{226}$, J.J. Brooke ${ }^{292}$, 
R. Bruce ${ }^{65}$, P. Brückman De Renstrom ${ }^{100}$, E. Bruna ${ }^{137,275}$, O. Brüning ${ }^{65}$, O. Brunner ${ }^{65}$, K. Brunner ${ }^{173}$, P. Bruzzone ${ }^{57}$, X. Buffat ${ }^{65}$, E. Bulyak ${ }^{182}$,

F. Burkart ${ }^{65}$, H. Burkhardt ${ }^{65}$, J.-P. Burnet ${ }^{65}$, F. Butin ${ }^{65}$, D. Buttazzo ${ }^{133,216}$,

A. Butterworth ${ }^{65}$, M. Caccia ${ }^{128,265}$, Y. Cai ${ }^{226}$, B. Caiffi ${ }^{125,264}$, V. Cairo ${ }^{226}$,

O. Cakir $^{10}$, R. Calaga ${ }^{65}$, S. Calatroni ${ }^{65}$, G. Calderini ${ }^{33}$, G. Calderola ${ }^{117}$,

A. Caliskan ${ }^{79}$, D. Calvet ${ }^{31,282}$, M. Calviani ${ }^{65}$, J.M. Camalich ${ }^{103}$, P. Camarri ${ }^{135,273}$,

M. Campanelli ${ }^{284}$, T. Camporesi ${ }^{65}$, A.C. Canbay ${ }^{10}$, A. Canepa ${ }^{71}$, E. Cantergiani ${ }^{84}$,

D. Cantore-Cavalli ${ }^{128,267}$, M. Capeans ${ }^{65}$, R. Cardarelli ${ }^{135,273}$, U. Cardella ${ }^{162}$,

A. Cardini ${ }^{120}$, C.M. Carloni Calame ${ }^{131,270}$, F. Carra ${ }^{65}$, S. Carra ${ }^{128,267}$,

A. Carvalho ${ }^{159}$, S. Casalbuoni ${ }^{147}$, J. Casas ${ }^{7}$, M. Cascella ${ }^{284}$, P. Castelnovo ${ }^{267}$,

G. Castorina ${ }^{134,272}$, G. Catalano ${ }^{267}$, V. Cavasinni ${ }^{133,216}$, E. Cazzato ${ }^{287}$,

E. Cennini ${ }^{65}$, A. Cerri ${ }^{329}$, F. Cerutti ${ }^{65}$, J. Cervantes ${ }^{65}$, I. Chaikovska ${ }^{33}$,

J. Chakrabortty ${ }^{88}$, M. Chala ${ }^{55}$, M. Chamizo-Llatas ${ }^{21}$, H. Chanal ${ }^{31}$, D. Chanal ${ }^{28}$,

S. Chance ${ }^{33}$, A. Chancé ${ }^{40}$, P. Charitos ${ }^{65}$, J. Charles ${ }^{5}$, T.K. Charles ${ }^{316}$,

S. Chattopadhyay ${ }^{187}$, R. Chehab ${ }^{154}$, S.V. Chekanov ${ }^{12}$, N. Chen ${ }^{175}$,

A. Chernoded ${ }^{222}$, V. Chetvertkova ${ }^{78}$, L. Chevalier ${ }^{40}$, G. Chiarelli ${ }^{133,216}$,

G. Chiarello ${ }^{134,209,272}$, M. Chiesa ${ }^{145}$, P. Chiggiato ${ }^{65}$, J.T. Childers ${ }^{12}$,

A. Chmielińska ${ }^{57,65}$, A. Cholakian ${ }^{80,168}$, P. Chomaz ${ }^{40}$, M. Chorowski ${ }^{348}$,

W. Chou ${ }^{98}$, M. Chrzaszcz ${ }^{100}$, E. Chyhyrynets ${ }^{117}$, G. Cibinetto ${ }^{123,262}$, A.K. Ciftci $^{141}$,

R. Ciftci $^{59}$, R. Cimino ${ }^{116}$, M. Ciuchini ${ }^{136,274}$, P.J. Clark ${ }^{303}$, Y. Coadou ${ }^{4,26,32}$,

M. Cobal ${ }^{138,276}$, A. Coccaro ${ }^{125}$, J. Cogan ${ }^{32,33}$, E. Cogneras $^{30}$, F. Collamati ${ }^{134,272}$,

C. Colldelram 7 , P. Collier ${ }^{65}$, J. Collot ${ }^{33,283}$, R. Contino ${ }^{216}$, F. Conventi ${ }^{129}$,

C.T.A. Cook $^{65}$, L. Cooley ${ }^{11,178}$, G. Corcella ${ }^{116,117}$, A.S. Cornell ${ }^{330}$, G.H. Corral ${ }^{36}$,

H. Correia-Rodrigues ${ }^{65}$, F. Costanza ${ }^{33}$, P. Costa Pinto ${ }^{65}$, F. Couderc ${ }^{40}$,

J. Coupard ${ }^{65}$, N. Craig ${ }^{297}$, I. Crespo Garrido ${ }^{336}$, A. Crivellin ${ }^{192}$, J.F. Croteau ${ }^{84}$,

M. Crouch ${ }^{65}$, E. Cruz Alaniz ${ }^{143}$, B. Curé ${ }^{65}$, J. Curti ${ }^{168}$, D. Curtin ${ }^{331}$, M. Czech ${ }^{65}$,

C. Dachauer ${ }^{162}$, R.T. D'Agnolo ${ }^{226}$, M. Daibo ${ }^{74}$, A. Dainese ${ }^{130,268}$, B. Dalena ${ }^{40}$,

A. Daljevec ${ }^{65}$, W. Dallapiazza ${ }^{86}$, L. D'Aloia Schwartzentruber ${ }^{27}$, M. Dam ${ }^{185}$,

G. D'Ambrosio ${ }^{129}$, S.P. $\operatorname{Das}^{250}$, S. DasBakshi ${ }^{88}$, W. da Silva ${ }^{33}$, G.G. da Silveira ${ }^{252}$,

V. D'Auria ${ }^{57}$, S. D'Auria ${ }^{267}$, A. David ${ }^{65}$, T. Davidek ${ }^{69}$, A. Deandrea ${ }^{33,337}$,

J. de Blas ${ }^{130,268}$, C.J. Debono ${ }^{312}$, S. De Curtis ${ }^{124,263}$, N. De Filippis ${ }^{118,258}$,

D. de Florian ${ }^{110}$, S. Deghaye ${ }^{65}$, S.J. de Jong ${ }^{95,176}$, C. Del Bo ${ }^{267}$, V. Del Duca ${ }^{137,275}$,

D. Delikaris ${ }^{65}$, F. Deliot ${ }^{40}$, A. Dell'Acqua ${ }^{65}$, L. Delle Rose ${ }^{124,263}$, M. Delmastro ${ }^{153}$,

E. De Lucia ${ }^{116}$, M. Demarteau ${ }^{12}$, D. Denegri ${ }^{40}$, L. Deniau ${ }^{65}$, D. Denisov ${ }^{71}$,

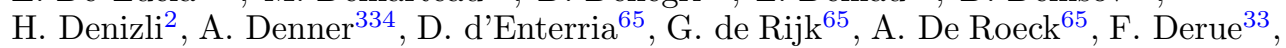

O. Deschamps ${ }^{33}$, S. Descotes-Genon ${ }^{33}$, P.S.B. Dev ${ }^{343}$, J.B. de Vivie de Régie ${ }^{33}$,

R.K. Dewanjee ${ }^{179}$, A. Di Ciaccio ${ }^{135,273}$, A. Di Cicco ${ }^{116}$, B.M. Dillon ${ }^{102}$,

B. Di Micco ${ }^{136,274}$, P. Di Nezza ${ }^{116}$, S. Di Vita ${ }^{128,267}$, A. Doblhammer ${ }^{233}$,

A. Dominjon ${ }^{153}$, M. D'Onofrio ${ }^{310}$, F. Dordei ${ }^{65}$, A. Drago ${ }^{116}$, P. Draper ${ }^{306}$,

Z. Drasal ${ }^{69}$, M. Drewes ${ }^{148}$, L. Duarte ${ }^{249}$, I. Dubovyk ${ }^{85}$, P. Duda ${ }^{348}$, A. Dudarev ${ }^{65}$,

L. Dudko ${ }^{222}$, D. Duellmann ${ }^{65}$, M. Dünser ${ }^{65}$, T. du Pree ${ }^{176}$, M. Durante ${ }^{40}$,

H. Duran Yildiz ${ }^{10}$, S. Dutta ${ }^{225}$, F. Duval ${ }^{65}$, J.M. Duval ${ }^{41,283}$, Y. Dydyshka ${ }^{56}$,

B. Dziewit ${ }^{326}$, S. Eisenhardt ${ }^{303}$, M. Eisterer ${ }^{233}$, T. Ekelof $^{338}$, D. El Khechen ${ }^{65}$,

S.A. Ellis ${ }^{226}$, J. Ellis ${ }^{150}$, J.A. Ellison ${ }^{318}$, K. Elsener ${ }^{65}$, M. Elsing ${ }^{65}$, Y. Enari ${ }^{237}$,

C. Englert ${ }^{211}$, H. Eriksson ${ }^{165}$, K.J. Eskola ${ }^{308}$, L.S. Esposito ${ }^{65}$, O. Etisken ${ }^{10}$,

E. Etzion ${ }^{234}$, P. Fabbricatore ${ }^{125,264}$, A. Falkowski ${ }^{33}$, A. Falou ${ }^{154}$, J. Faltova ${ }^{69}$,

J. Fan ${ }^{22}$, L. Fanò ${ }^{132,271}$, A. Farilla ${ }^{136,274}$, R. Farinelli ${ }^{123,262}$, S. Farinon ${ }^{125,264}$,

D.A. Faroughy ${ }^{102}$, S.D. Fartoukh ${ }^{65}$, A. Faus-Golfe ${ }^{33}$, W.J. Fawcett ${ }^{299}$,

G. Felici ${ }^{116}$, L. Felsberger ${ }^{164}$, C. Ferdeghini ${ }^{43}$, A.M. Fernandez Navarro ${ }^{35}$,

A. Fernández-Téllez ${ }^{16}$, J. Ferradas Troitino ${ }^{65,305}$, G. Ferrara ${ }^{128,267}$, R. Ferrari $^{131,270}$,

L. Ferreira ${ }^{65}$, P. Ferreira da Silva ${ }^{65}$, G. Ferrera ${ }^{127,267}$, F. Ferro ${ }^{125,264}$,

M. Fiascaris ${ }^{65}$, S. Fiorendi ${ }^{127}$, C. Fiorio ${ }^{267}$, O. Fischer ${ }^{147,287}$, E. Fischer ${ }^{78}$,

W. Flieger ${ }^{326}$, M. Florio ${ }^{267}$, D. Fonnesu ${ }^{65}$, E. Fontanesi ${ }^{119,259}$, N. Foppiani ${ }^{80}$, 
K. Foraz ${ }^{65}$, D. Forkel-Wirth ${ }^{65}$, S. Forte ${ }^{267}$, M. Fouaidy ${ }^{90}$, D. Fournier ${ }^{33}$,

T. Fowler ${ }^{65}$, J. Fox ${ }^{227}$, P. Francavilla ${ }^{133,216}$, R. Franceschini ${ }^{136,274}$, S. Franchino ${ }^{278}$,

E. Franco ${ }^{134,272}$, A. Freitas ${ }^{197}$, B. Fuks ${ }^{157}$, K. Furukawa ${ }^{82}$, S.V. Furuseth ${ }^{57}$,

E. Gabrielli ${ }^{138,276}$, A. Gaddi ${ }^{65}$, M. Galanti ${ }^{321}$, E. Gallo ${ }^{50}$, S. Ganjour ${ }^{40}$,

J. Gao ${ }^{192}$, J. Gao ${ }^{98}$, V. Garcia Diaz ${ }^{117}$, M. García Pérez ${ }^{65}$, L. García Tabarés ${ }^{35}$,

C. Garion ${ }^{65}$, M.V. Garzelli ${ }^{277,281}$, I. Garzia ${ }^{123,262}$, S.M. Gascon-Shotkin ${ }^{33,337}$,

G. Gaudio ${ }^{131,270}$, P. Gay ${ }^{31,33}$, S.-F. Ge ${ }^{237,293}$, T. Gehrmann ${ }^{335}$, M.H. Genest ${ }^{33,283}$,

R. Gerard ${ }^{65}$, F. Gerigk ${ }^{65}$, H. Gerwig ${ }^{65}$, P. Giacomelli19 ${ }^{119,259}$, S. Giagu ${ }^{134,272}$,

E. Gianfelice-Wendt ${ }^{71}$, F. Gianotti ${ }^{65}$, F. Giffoni ${ }^{29,267}$, S.S. Gilardoni ${ }^{65}$,

M. Gil Costa ${ }^{35}$, M. Giovannetti ${ }^{116}$, M. Giovannozzi ${ }^{65}$, P. Giubellino ${ }^{78,137}$,

G.F. Giudice ${ }^{65}$, A. Giunta ${ }^{255}$, L.K. Gladilin ${ }^{222}$, S. Glukhov ${ }^{24}$, J. Gluza ${ }^{326}$,

G. Gobbi ${ }^{65}$, B. Goddard ${ }^{65}$, F. Goertz ${ }^{169}$, T. Golling ${ }^{305}$, V.P. Goncalves ${ }^{253}$,

R. Gonçalo ${ }^{159}$, L.A. Gonzalez Gomez ${ }^{116}$, S. Gorgi Zadeh ${ }^{322}$, G. Gorine ${ }^{57}$,

E. Gorini ${ }^{126,257}$, S.A. Gourlay ${ }^{161}$, L. Gouskos ${ }^{297}$, F. Grancagnolo ${ }^{126,266}$,

A. Grassellino ${ }^{71}$, A. Grau ${ }^{147}$, E. Graverini ${ }^{335}$, H.M. Gray ${ }^{161}$, Ma. Greco ${ }^{136,274}$,

Mi. Greco ${ }^{136,274}$, J.-L. Grenard ${ }^{65}$, O. Grimm ${ }^{60}$, C. Grojean ${ }^{50}$, V.A. Gromov ${ }^{144}$,

J.F. Grosse-Oetringhaus ${ }^{65}$, A. Grudiev ${ }^{65}$, K. Grzanka ${ }^{326}$, J. Gu ${ }^{142}$,

D. Guadagnoli ${ }^{153}$, V. Guidi ${ }^{123,262}$, S. Guiducci ${ }^{116}$, G. Guillermo Canton ${ }^{36}$,

Y.O. Günaydin ${ }^{146}$, R. Gupta ${ }^{21}$, R.S. Gupta ${ }^{55}$, J. Gutierrez ${ }^{89}$, J. Gutleber ${ }^{65}$,

C. Guyot ${ }^{40}$, V. Guzey ${ }^{195}$, C. Gwenlan ${ }^{191}$, C. Haberstroh ${ }^{231}$, B. Hacışahinoğlu ${ }^{113}$,

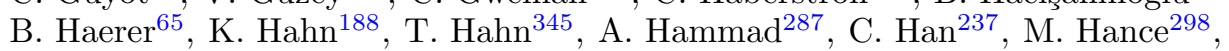

A. Hannah ${ }^{212}$, P.C. Harris ${ }^{168}$, C. Hati ${ }^{31,282}$, S. Haug ${ }^{290}$, J. Hauptman ${ }^{111}$,

V. Haurylavets ${ }^{96}$, H.-J. He ${ }^{220}$, A. Hegglin ${ }^{218,221}$, B. Hegner ${ }^{21}$, K. Heinemann ${ }^{318}$,

S. Heinemeyer ${ }^{106}$, C. Helsens ${ }^{65}$, A. Henriques ${ }^{65}$, A. Henriques ${ }^{65}$, P. Hernandez $^{105}$,

R.J. Hernández-Pinto ${ }^{246}$, J. Hernandez-Sanchez ${ }^{16}$, T. Herzig ${ }^{99}$, I. Hiekkanen ${ }^{165}$,

W. Hillert ${ }^{277}$, T. Hoehn ${ }^{232}$, M. Hofer ${ }^{233}$, W. Höfle ${ }^{65}$, F. Holdener ${ }^{221}$, S. Holleis ${ }^{233}$,

B. Holzer ${ }^{65}$, D.K. Hong ${ }^{200}$, C.G. Honorato ${ }^{16}$, S.C. Hopkins ${ }^{65}$, J. Hrdinka ${ }^{65}$,

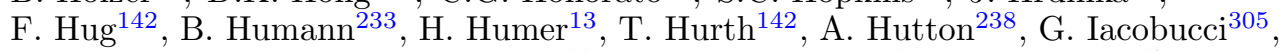

N. Ibarrola ${ }^{65}$, L. Iconomidou-Fayard ${ }^{33}$, K. Ilyina-Brunner ${ }^{65}$, J. Incandela ${ }^{297}$,

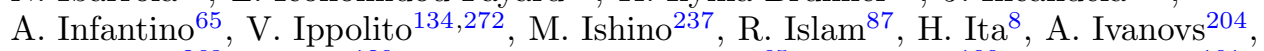

S. Iwamoto ${ }^{268}$, A. Iyer ${ }^{129}$, S. Izquierdo Bermudez ${ }^{65}$, S. Jadach ${ }^{100}$, D.O. Jamin ${ }^{101}$,

P. Janot ${ }^{65}$, P. Jarry ${ }^{40}$, A. Jeff ${ }^{37,65}$, P. Jenny ${ }^{166}$, E. Jensen ${ }^{65}$, M. Jensen ${ }^{67}$,

X. Jiang ${ }^{280}$, J.M. Jiménez ${ }^{65}$, M.A. Jones ${ }^{65}$, O.R. Jones ${ }^{65}$, J.M. Jowett ${ }^{65}$, S. Jung ${ }^{217}$,

W. Kaabi ${ }^{33}$, M. Kado ${ }^{65,134,272}$, K. Kahle ${ }^{65}$, L. Kalinovskaya ${ }^{56}$, J. Kalinowski ${ }^{332}$,

J.F. Kamenik ${ }^{102}$, K. Kannike ${ }^{179}$, S.O. Kara ${ }^{10,186}$, H. Karadeniz ${ }^{76}$, V. Karaventzas ${ }^{65}$,

I. Karpov ${ }^{65}$, S. Kartal ${ }^{113}$, A. Karyukhin ${ }^{94}$, V. Kashikhin ${ }^{71}$, J. Katharina Behr ${ }^{50}$,

U. Kaya ${ }^{10,239}$, J. Keintzel ${ }^{233}$, P.A. Keinz ${ }^{340}$, K. Keppel ${ }^{117}$, R. Kersevan ${ }^{65}$,

K. Kershaw ${ }^{65}$, H. Khanpour ${ }^{210,325}$, S. Khatibi ${ }^{49,210}$, M. Khatiri Yanehsari ${ }^{210}$,

V.V. Khoze ${ }^{55}$, J. Kieseler ${ }^{65}$, A. Kilic ${ }^{245}$, A. Kilpinen ${ }^{165}$, Y.-K. Kim ${ }^{300}$,

D.W. Kim ${ }^{75}$, U. Klein ${ }^{310}$, M. Klein ${ }^{310}$, F. Kling ${ }^{295}$, N. Klinkenberg ${ }^{65,68}$,

S. Klöppel ${ }^{231}$, M. Klute ${ }^{168}$, V.I. Klyukhin ${ }^{222}$, M. Knecht ${ }^{32,33}$, B. Kniehl ${ }^{85}$,

F. Kocak ${ }^{245}$, C. Koeberl ${ }^{184}$, A.M. Kolano ${ }^{65}$, A. Kollegger ${ }^{285}$, K. Kołodziej ${ }^{326}$,

A.A. Kolomiets ${ }^{144}$, J. Komppula ${ }^{65}$, I. Koop ${ }^{24}$, P. Koppenburg ${ }^{176}$, M. Koratzinos ${ }^{168}$,

M. Kordiaczyńska ${ }^{326}$, M. Korjik ${ }^{96}$, O. Kortner ${ }^{345}$, P. Kostka ${ }^{310}$, W. Kotlarski ${ }^{231}$,

C. Kotnig ${ }^{65}$, T. Köttig ${ }^{65}$, A.V. Kotwal ${ }^{54}$, A.D. Kovalenko ${ }^{144}$, S. Kowalski ${ }^{326}$,

J. Kozaczuk ${ }^{306}$, G.A. Kozlov ${ }^{144}$, S.S. Kozub ${ }^{144}$, A.M. Krainer ${ }^{65}$, T. Kramer ${ }^{65}$,

M. Krämer ${ }^{203}$, M. Krammer ${ }^{65}$, A.A. Krasnov ${ }^{24}$, F. Krauss ${ }^{55}$, K. Kravalis ${ }^{204}$,

L. Kretzschmar ${ }^{340}$, R.M. Kriske ${ }^{168}$, H. Kritscher ${ }^{184}$, P. Krkotic ${ }^{7}$, H. Kroha ${ }^{170}$,

M. Kucharczyk ${ }^{100}$, S. Kuday ${ }^{112}$, A. Kuendig' ${ }^{162}$, G. Kuhlmann ${ }^{72}$, A. Kulesza ${ }^{346}$,

M. Kumar ${ }^{57}$, M. Kumar ${ }^{330}$, A. Kusina ${ }^{100}$, S. Kuttimalai ${ }^{226}$, M. Kuze ${ }^{240}$,

T. Kwon ${ }^{217}$, F. Lackner ${ }^{65}$, M. Lackner ${ }^{285}$, E. La Francesca ${ }^{116,272}$, M. Laine ${ }^{290}$,

G. Lamanna ${ }^{153}$, S. La Mendola ${ }^{65}$, E. Lançon ${ }^{21}$, G. Landsberg ${ }^{22}$, P. Langacker ${ }^{91}$,

C. Lange ${ }^{65}$, A. Langner ${ }^{65}$, A.J. Lankford ${ }^{295}$, J.P. Lansberg ${ }^{33}$, T. Lari ${ }^{127}$, 
P.J. Laycock ${ }^{310}$, P. Lebrun ${ }^{66}$, A. Lechner ${ }^{65}$, K. Lee ${ }^{217}$, S. Lee ${ }^{25,152}$, R. Lee ${ }^{24}$,

T. Lefevre ${ }^{65}$, P. Le Guen ${ }^{65}$, T. Lehtinen ${ }^{202}$, S.B. Leith ${ }^{280}$, P. Lenzi ${ }^{124,263}$,

E. Leogrande ${ }^{65}$, C. Leonidopoulos ${ }^{299}$, I. Leon-Monzon ${ }^{246}$, G. Lerner ${ }^{65}$,

O. Leroy ${ }^{32,33}$, T. Lesiak ${ }^{100}$, P. Lévai ${ }^{173}$, A. Leveratto ${ }^{44}$, E. Levichev ${ }^{24}$, G. Li ${ }^{98}$,

S. $\mathrm{Li}^{220}$, R. $\mathrm{Li}^{351}$, D. Liberati ${ }^{42}$, M. Liepe ${ }^{45}$, D.A. Lissauer ${ }^{21}$, Z. Liu ${ }^{314}$,

A. Lobko ${ }^{96}$, E. Locci ${ }^{40}$, E. Logothetis Agaliotis ${ }^{65,183}$, M.P. Lombardo ${ }^{124,263}$,

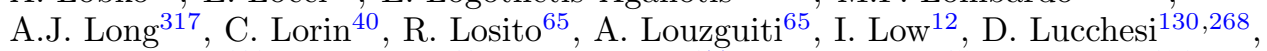

M.T. Lucchini ${ }^{198}$, A. Luciani ${ }^{62}$, M. Lueckhof ${ }^{277}$, A.J.G. Lunt ${ }^{65}$, M. Luzum ${ }^{251}$,

D.A. Lyubimtsev ${ }^{144}$, M. Maggiora ${ }^{137,275}$, N. Magnin ${ }^{65}$, M.A. Mahmoud ${ }^{70}$,

F. Mahmoudi ${ }^{33,337}$, J. Maitre ${ }^{28}$, V. Makarenko ${ }^{96}$, A. Malagoli ${ }^{44}$, J. Malclés ${ }^{40}$,

L. Malgeri ${ }^{65}$, P.J. Mallon ${ }^{40}$, F. Maltoni ${ }^{148}$, S. Malvezzi ${ }^{127}$, O.B. Malyshev ${ }^{212}$,

G. Mancinelli ${ }^{32,33}$, P. Mandrik ${ }^{94}$, P. Manfrinetti ${ }^{44,264}$, M. Mangano ${ }^{65}$, P. Manil ${ }^{40}$,

M. Mannelli ${ }^{65}$, G. Marchiori ${ }^{33,155}$, F. Marhauser ${ }^{238}$, V. Mariani ${ }^{132,271}$,

V. Marinozzi ${ }^{128,267}$, S. Mariotto ${ }^{128,267}$, P. Marquard ${ }^{51}$, C. Marquet ${ }^{33}$,

T. Marriott-Dodington ${ }^{65}$, R. Martin ${ }^{65}$, O. Martin ${ }^{171}$, J. Martin Camalich ${ }^{103,248}$,

T. Martinez ${ }^{35}$, H. Martinez Bruzual ${ }^{131,270}$, M.I. Martínez-Hernández ${ }^{16}$,

D.E. Martins ${ }^{254}$, S. Marzani ${ }^{125,264}$, D. Marzocca ${ }^{138}$, L. Marzola ${ }^{179}$,

S. Masciocchi ${ }^{78,278}$, I. Masina ${ }^{123,262}$, A. Massimiliano ${ }^{128}$, A. Massironi ${ }^{65}$,

T. Masubuchi ${ }^{237}$, V.A. Matveev ${ }^{144}$, M.A. Mazzoni ${ }^{134}$, M. McCullough ${ }^{65}$,

P.A. McIntosh ${ }^{212}$, P. Meade ${ }^{228}$, L. Medina ${ }^{247}$, A. Meier ${ }^{162}$, J. Meignan ${ }^{65}$,

B. Mele ${ }^{134,272}$, J.G. Mendes Saraiva ${ }^{159}$, F. Menez ${ }^{28}$, M. Mentink ${ }^{65}$, E. Meoni ${ }^{122,256}$,

P. Meridiani ${ }^{128,267}$, M. Merk ${ }^{176}$, P. Mermod ${ }^{305}$, V. Mertens ${ }^{65}$, L. Mether ${ }^{57}$,

E. Métral ${ }^{65}$, M. Migliorati ${ }^{134,272}$, A. Milanese ${ }^{65}$, C. Milardi ${ }^{116}$, G. Milhano $^{159}$,

B.L. Militsyn ${ }^{212}$, F. Millet ${ }^{41,283}$, I. Minashvili ${ }^{140,144}$, J.V. Minervini ${ }^{168}$,

L.S. Miralles ${ }^{65}$, D. Mirarchi ${ }^{65}$, S. Mishima ${ }^{82}$, D.P. Missiaen ${ }^{65}$, G. Mitselmakher ${ }^{304}$,

T. Mitshuhashi ${ }^{82}$, J. Mnich ${ }^{50}$, M. Mohammadi Najafabadi ${ }^{210}$, R.N. Mohapatra ${ }^{314}$,

N. Mokhov ${ }^{71}$, J.G. Molson ${ }^{65}$, R. Monge ${ }^{7}$, C. Montag $^{21}$, G. Montagna ${ }^{131,270}$,

S. Monteil ${ }^{31,33}$, G. Montenero ${ }^{192}$, E. Montesinos ${ }^{65}$, F. Moortgat ${ }^{65}$, N. Morange ${ }^{154}$,

G. Morello ${ }^{116}$, M. Moreno Llácer ${ }^{65}$, M. Moretti ${ }^{123,262}$, S. Moretti $^{213}$, A.K. Morley ${ }^{65}$,

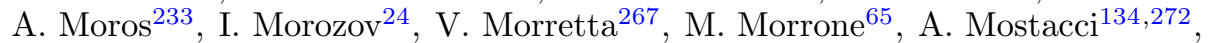

S. Muanza ${ }^{32,33}$, N. Muchnoi ${ }^{24}$, M. Mühlegger ${ }^{162}$, M. Mulder ${ }^{176}$, M. Mulders ${ }^{65}$,

B. Müller ${ }^{21,54}$, F. Müller ${ }^{99}$, A.-S. Müller ${ }^{147}$, J. Munillä ${ }^{35}$, M.J. Murray ${ }^{309}$,

Y. Muttoni ${ }^{65}$, S. Myers ${ }^{65}$, M. Mylona ${ }^{65}$, J. Nachtman ${ }^{307}$, T. Nakamoto ${ }^{82}$,

M. Nardecchia ${ }^{65}$, G. Nardini ${ }^{327}$, P. Nason ${ }^{127}$, Z. Nergiz ${ }^{239}$, A.V. Nesterenko ${ }^{144}$,

J.A. Netto ${ }^{318}$ A. Nettsträter ${ }^{72}$, C. Neubüser ${ }^{65}$, J. Neundorf ${ }^{50}$, F. Niccoli ${ }^{65}$,

O. Nicrosini ${ }^{131,270}$, Y. Nie ${ }^{65}$, U. Niedermayer ${ }^{229}$, J. Niedziela ${ }^{65}$, A. Niemi ${ }^{65}$,

S.A. Nikitin ${ }^{24}$, A. Nisati ${ }^{134,272}$, J.M. No ${ }^{106}$, M. Nonis ${ }^{65}$, Y. Nosochkov ${ }^{226}$,

M. Novák ${ }^{173}$, A. Novokhatski ${ }^{226}$, J.M. O'Callaghan ${ }^{279}$, C. Ochando ${ }^{158}$,

S. Ogur ${ }^{20}$, K. Ohmi ${ }^{82}$, K. Oide ${ }^{65}$, V.A. Okorokov ${ }^{181}$, Y. Okumura ${ }^{237}$,

C. Oleari ${ }^{127}$, F.I. Olness ${ }^{224}$, Y. Onel ${ }^{307}$, M. Ortino ${ }^{233}$, J. Osborne ${ }^{65}$,

P. Osland ${ }^{289}$, T. Otto ${ }^{65}$, K.Y. Oyulmaz ${ }^{2}$, A. Ozansoy ${ }^{10}$, V. Özcan ${ }^{20}$,

K. Özdemir ${ }^{196}$, C.E. Pagliarone ${ }^{53,113,115}$, H.F. Pais da Silva ${ }^{65}$, E. Palmieri ${ }^{117}$,

L. Palumbo ${ }^{134,272}$, A. Pampaloni ${ }^{125,264}$, R.-Q. Pan ${ }^{350}$, M. Panareo ${ }^{126,266}$,

O. Panella ${ }^{132,271}$, G. Panico ${ }^{263}$, G. Panizzo ${ }^{138,276}$, A.A. Pankov ${ }^{77}$, V. Pantsyrny ${ }^{17}$,

C.G. Papadopoulos ${ }^{177}$, A. Papaefstathiou ${ }^{176}$, Y. Papaphilippou ${ }^{65}$, M.A. Parker ${ }^{299}$,

V. Parma ${ }^{65}$, M. Pasquali ${ }^{65}$, S.K. Patra ${ }^{88}$, R. Patterson ${ }^{45}$, H. Paukkunen ${ }^{308}$,

F. Pauss ${ }^{60}$, S. Peggs ${ }^{21}$, J.-P. Penttinen ${ }^{202}$, G. Peón ${ }^{65}$, E.E. Perepelkin ${ }^{144}$,

E. Perez ${ }^{65}$, J.C. Perez ${ }^{65}$, G. Perez ${ }^{344}$, F. Pérez ${ }^{7}$, E. Perez Codina ${ }^{65}$,

J. Perez Morales ${ }^{35}$, M. Perfilov ${ }^{222}$, H. Pernegger ${ }^{65}$, M. Peruzzi ${ }^{65}$, C. $^{2}$ Pes $^{40}$,

K. Peters ${ }^{50}$, S. Petracca ${ }^{114}$, F. Petriello ${ }^{188}$, L. Pezzotti ${ }^{131,270}$, S. Pfeiffer ${ }^{233}$,

F. Piccinini ${ }^{131,270}$, T. Pieloni ${ }^{57}$, M. Pierini ${ }^{65}$, H. Pikhartova ${ }^{205}$, G. Pikurs ${ }^{204}$,

E. Pilicer ${ }^{245}$, P. Piminov ${ }^{24}$, C. Pira ${ }^{117}$, R. Pittau ${ }^{47}$, W. Płaczek ${ }^{167}$,

M. Plagge ${ }^{65,190}$, T. Plehn ${ }^{278}$, M.-A. Pleier ${ }^{21}$, M. Płoskoń ${ }^{161}$, M. Podeur ${ }^{328}$, 
H. Podlech ${ }^{92}$, T. Podzorny ${ }^{65}$, L. Poggioli ${ }^{33}$, A. Poiron $^{58}$, G. Polesello ${ }^{131,270}$, M. Poli Lener ${ }^{116}$, A. Polini ${ }^{119,259}$, J. Polinski ${ }^{348}$, S.M. Polozov ${ }^{181}$, L. Ponce ${ }^{65}$, M. Pont ${ }^{7}$, L. Pontecorvo ${ }^{134,272}$, T. Portaluri ${ }^{60}$, K. Potamianos ${ }^{50}$, C. Prasse ${ }^{72}$, M. Prausa ${ }^{8}$, A. Preinerstorfer ${ }^{13}$, E. Premat ${ }^{27}$, T. Price $^{291}$, M. Primavera ${ }^{126}$, F. Prino ${ }^{137,275}$, M. Prioli ${ }^{128}$, J. Proudfoot ${ }^{12}$, A. Provino ${ }^{44}$, T. Pugnat ${ }^{40}$, N. Pukhaeva ${ }^{144}$, S. Puławski ${ }^{326}$, D. Pulikowski ${ }^{65,347}$, G. Punzi ${ }^{133,216}$, M. Putti ${ }^{264}$, A. Pyarelal ${ }^{286}$, H. Quack ${ }^{231}$, M. Quispe ${ }^{7}$, A. Racioppi ${ }^{179}$, H. Rafique ${ }^{313}$, V. Raginel ${ }^{78}$, M. Raidal ${ }^{179}$, N.S. Ramírez-Uribe ${ }^{104}$, M.J. Ramsey-Musolf ${ }^{315}$, R. Rata ${ }^{65}$, P. Ratoff ${ }^{38}$, F. Ravotti ${ }^{65}$, P. Rebello Teles ${ }^{34}$, M. Reboud ${ }^{153}$, S. Redaelli ${ }^{65}$, E. Renner ${ }^{233}$, A.E. Rentería-Olivo ${ }^{105}$, M. Rescigno ${ }^{134,272}$, J. Reuter ${ }^{50}$, A. Ribon ${ }^{65}$, A.M. Ricci ${ }^{125,264}$, W. Riegler ${ }^{65}$, S. Riemann ${ }^{51}$, B. Riemann ${ }^{230}$, T. Riemann ${ }^{326}$, J.M. Rifflet ${ }^{40}$, R.A. Rimmer ${ }^{238}$, R. Rinaldesi ${ }^{65}$, L. Rinolf ${ }^{65}$, O. Rios Rubiras ${ }^{65}$, T. Risselada ${ }^{65}$, A. Rivetti ${ }^{137,275}$, L. Rivkin ${ }^{192}$,

T. Rizzo ${ }^{226}$, T. Robens ${ }^{206}$, F. Robert ${ }^{27}$, A.J. Robson ${ }^{305}$, E. Rochepault ${ }^{40}$, C. Roda ${ }^{133,216}$, G. Rodrigo ${ }^{107}$, M. Rodríguez-Cahuantzi ${ }^{16}$, C. $\operatorname{Rogan}^{309}$, M. Roig $^{3}$, S. Rojas-Torres ${ }^{246}$, J. Rojo ${ }^{176}$, G. Rolandi ${ }^{133,216}$, G. Rolando ${ }^{65,192}$, P. Roloff ${ }^{65}$, A. Romanenko ${ }^{71}$, A. Romanov ${ }^{89}$, F. Roncarolo ${ }^{65}$, A. Rosado Sanchez ${ }^{16}$, G. Rosaz ${ }^{65}$, L. Rossi ${ }^{65,267}$, A. Rossi ${ }^{132,271}$, R. Rossmanith ${ }^{50,147}$, B. Rousset ${ }^{41,283}$, C. Royon ${ }^{309}$, X. Ruan ${ }^{330}$, I. Ruehl ${ }^{65}$, V. Ruhlmann-Kleider ${ }^{40}$, R. Ruiz ${ }^{55}$, L. Rumyantsev ${ }^{56,223}$, R. Ruprecht ${ }^{147}$, A.I. Ryazanov ${ }^{180}$, A. Saba ${ }^{44}$, R. Sadykov ${ }^{56}$, D. Saez de Jauregui ${ }^{147}$, M. Sahin ${ }^{339}$, B. Sailer ${ }^{23}$, M. Saito ${ }^{237}$, F. Sala ${ }^{50}$, G.P. Salam ${ }^{191}$, J. Salfeld-Nebgen ${ }^{198}$, C.A. Salgado ${ }^{109}$, S. Salini ${ }^{267}$, J.M. Sallese ${ }^{57}$, T. Salmi ${ }^{202}$, A. Salzburger ${ }^{65}$, O.A. Sampayo ${ }^{108}$, S. Sanfilippo ${ }^{192}$, J. Santiago ${ }^{47}$, E. Santopinto ${ }^{125}$, R. Santoro ${ }^{128,265}$, A. Sanz Ull ${ }^{61}$, X. Sarasola ${ }^{192}$, I.H. Sarpün ${ }^{6}$, M. Sauvain ${ }^{160}$, S. Savelyeva ${ }^{231}$, R. Sawada ${ }^{237}$, G.F.R. Sborlini ${ }^{46,110}$, A. Schaffer ${ }^{33}$, M. Schaumann ${ }^{65}$, M. Schenk ${ }^{65}$, C. Scheuerlein ${ }^{65}$, I. Schienbein ${ }^{156}$, K. Schlenga ${ }^{23}$, H. Schmickler ${ }^{65}$, R. Schmidt ${ }^{65,229}$, D. Schoerling ${ }^{65}$, A. Schoning ${ }^{207}$, T. SchörnerSadenius $^{50}$, M. Schott ${ }^{199}$, D. Schulte ${ }^{65}$, P. Schwaller ${ }^{142}$, C. Schwanenberger ${ }^{50}$, P. Schwemling ${ }^{40}$, N. Schwerg ${ }^{65}$, L. Scibile ${ }^{65}$, A. Sciuto ${ }^{121,261}$, E. Scomparin ${ }^{137,275}$, C. Sebastiani ${ }^{134,272}$, B. Seeber ${ }^{214,305}$, M. Segreti ${ }^{40}$, P. Selva ${ }^{162}$, M. Selvaggi ${ }^{65}$, C. Senatore ${ }^{305}$, A. Senol ${ }^{2}$, L. Serin ${ }^{33}$, M. Serluca ${ }^{153}$, N. Serra ${ }^{335}$, A. Seryi ${ }^{143}$, L. Sestini ${ }^{130,268}$, A. Sfyrla ${ }^{305}$, M. Shaposhnikov ${ }^{57}$, E. Shaposhnikova ${ }^{65}$, B.Y. Sharkov ${ }^{144}$, D. Shatilov ${ }^{24}$, J. Shelton ${ }^{306}$, V. Shiltsev ${ }^{71}$, I.P. Shipsey ${ }^{191}$, G.D. Shirkov ${ }^{144}$, A. Shivaji 131,270 , D. Shwartz ${ }^{24}$, T. Sian ${ }^{212,303,313}$, S. Sidorov ${ }^{192}$, A. Siemko ${ }^{65}$, L. Silvestrini ${ }^{134,272}$, N. Simand ${ }^{28}$, F. Simon ${ }^{170}$, B.K. Singh ${ }^{14}$, A. Siódmok ${ }^{100}$, Y. Sirois ${ }^{33}$, E. Sirtori ${ }^{29}$, R. Sirvinskaite ${ }^{163,212}$, B. Sitar ${ }^{39}$, T. Sjöstrand ${ }^{311}$, P. Skands ${ }^{172}$, E. Skordis ${ }^{65,310}$, K. Skovpen ${ }^{342}$, M. Skrzypek ${ }^{100}$, E. Slade ${ }^{191}$, P. Slavich ${ }^{157}$, R. Slovak ${ }^{69}$, V. Smaluk ${ }^{21}$, V. Smirnov ${ }^{222}$, W. Snoeys ${ }^{65}$, L. Soffi ${ }^{45}$, P. Sollander ${ }^{65}$, O. Solovyanov ${ }^{94}$, H.K. Soltveit ${ }^{278}$, H. Song ${ }^{286}$, P. Sopicki ${ }^{100}$, M. Sorbi ${ }^{128,267}$, L. Spallino ${ }^{116}$, M. Spannowsky ${ }^{55}$, B. Spataro ${ }^{134,272}$, P. Sphicas ${ }^{65}$, H. Spiesberger ${ }^{199}$, P. Spiller ${ }^{78}$, M. Spira ${ }^{192}$, T. Srivastava ${ }^{88}$, J. Stachel ${ }^{278}$, A. Stakia ${ }^{65}$, J.L. Stanyard ${ }^{65}$, E. Starchenko ${ }^{180}$, A.Y. Starikov ${ }^{144}$, A.M. Staśto ${ }^{236}$, M. Statera ${ }^{128,267}$, R. Steerenberg ${ }^{65}$, J. Steggemann ${ }^{65}$, A. Stenvall ${ }^{202}$, F. Stivanello ${ }^{117}$, D. Stöckinger ${ }^{231}$, L.S. Stoel ${ }^{65}$, M. Stöger-Pollach ${ }^{233}$, B. Strauss ${ }^{48,97}$, M. Stuart ${ }^{65}$, G. Stupakov ${ }^{26}$, S. Su ${ }^{286}$,

A. Sublet ${ }^{65}$, K. Sugita ${ }^{78}$, L. Sulak ${ }^{19}$, M.K. Sullivan ${ }^{226}$, S. Sultansoy ${ }^{239}$,

T. Sumida ${ }^{151}$, K. Suzuki ${ }^{82}$, G. Sylva ${ }^{44}$, M.J. Syphers ${ }^{187}$, A. Sznajder ${ }^{252}$, M. Taborelli6 ${ }^{65}$, N.A. Tahir ${ }^{78}$, M. Takeuchi ${ }^{237}$, E. Tal Hod ${ }^{234}$, C. Tambasco ${ }^{57}$, J. Tanaka ${ }^{237}$, K. Tang ${ }^{168}$, I. Tapan ${ }^{245}$, S. Taroni ${ }^{319}$, G.F. Tartarelli ${ }^{128,267}$, G. Tassielli ${ }^{126,266}$, L. Tavian ${ }^{65}$, T.M. Taylor ${ }^{65}$, G.N. Taylor ${ }^{316}$, A.M. Teixeira ${ }^{31,33}$, G. Tejeda-Muñoz ${ }^{16}$, V.I. Telnov ${ }^{24,189}$, R. Tenchini ${ }^{133,216}$, H.H.J. ten Kate ${ }^{65}$,

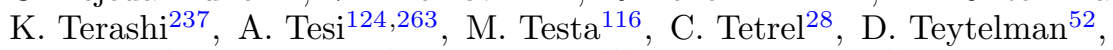
J. Thaler ${ }^{168}$, A. Thamm ${ }^{65}$, S. Thomas ${ }^{208}$, M.T. Tiirakari ${ }^{65}$, V. Tikhomirov ${ }^{96}$, 
D. Tikhonov ${ }^{81}$, H. Timko ${ }^{65}$, V. Tisserand ${ }^{31,33}$, L.M. Tkachenko ${ }^{144}$,

J. Tkaczuk ${ }^{41,283}$, J.P. Tock ${ }^{65}$, B. Todd ${ }^{65}$, E. Todesco ${ }^{65}$, R. Tomás Garcia ${ }^{65}$,

D. Tommasini6 ${ }^{65}$, G. Tonelli ${ }^{133,216}$, F. Toral ${ }^{35}$, T. Torims ${ }^{204}$, R. Torre ${ }^{65}$,

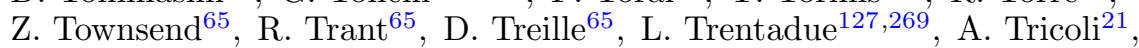

A. Tricomi ${ }^{121,261}$, W. Trischuk ${ }^{331}$, I.S. Tropin ${ }^{71}$, B. Tuchming ${ }^{40}$, A.A. Tudora ${ }^{65}$,

B. Turbiarz ${ }^{100}$, I. Turk Cakir ${ }^{76}$, M. Turri ${ }^{267}$, T. Tydecks ${ }^{65}$, J. Usovitsch ${ }^{241}$,

J. Uythoven ${ }^{65}$, R. Vaglio ${ }^{44}$, A. Valassi ${ }^{65}$, F. Valchkova ${ }^{65}$, M.A. Valdivia Garcia ${ }^{247}$,

P. Valente ${ }^{128,267}$, R.U. Valente ${ }^{272}$, A.-M. Valente-Feliciano ${ }^{238}$, G. Valentino ${ }^{312}$,

L. Vale Silva ${ }^{329}$, J.M. Valet ${ }^{28}$, R. Valizadeh ${ }^{212}$, J.W.F. Valle ${ }^{107}$, S. Vallecorsa ${ }^{75}$,

G. Vallone ${ }^{161}$, M. van Leeuwen ${ }^{176}$, U.H. van Rienen ${ }^{322}$, L. van Riesen-Haupt ${ }^{143}$,

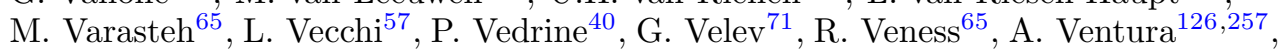

W. Venturini Delsolaro ${ }^{65}$, M. Verducci ${ }^{136,274}$, C.B. Verhaaren ${ }^{294}$, C. Vernieri ${ }^{71}$,

A.P. Verweij ${ }^{65}$, O. Verwilligen ${ }^{118,258}$, O. Viazlo ${ }^{65}$, A. Vicini ${ }^{128,267}$, G. Viehhauser $^{191}$,

N. Vignaroli ${ }^{130,268}$, M. Vignolo ${ }^{44}$, A. Vitrano ${ }^{40}$, I. Vivarelli ${ }^{329}$, S. Vlachos ${ }^{183}$,

M. Vogel ${ }^{280}$, D.M. Vogt ${ }^{328}$, V. Völkl ${ }^{93}$, P. Volkov ${ }^{222}$, G. Volpini ${ }^{128,267}$,

J. von Ahnen ${ }^{50}$, G. Vorotnikov ${ }^{222}$, G.G. Voutsinas ${ }^{65}$, V. Vysotsky ${ }^{9}$, U. Wagner ${ }^{65}$,

R. Wallny ${ }^{60}$, L.-T. Wang ${ }^{300}$, R. Wang ${ }^{12}$, K. Wang ${ }^{349}$, B.F.L. Ward ${ }^{15,345}$,

T.P. Watson ${ }^{139}$, N.K. Watson ${ }^{291}$, Z. Wąs ${ }^{100}$, C. Weiland ${ }^{197}$, S. Weinzierl ${ }^{199}$,

C.P. Welsch ${ }^{310}$, J. Wenninger ${ }^{65}$, M. Widorski ${ }^{65}$, U.A. Wiedemann ${ }^{65}$,

H.-U. Wienands ${ }^{12}$, G. Wilkinson ${ }^{191}$, P.H. Williams ${ }^{212}$, A. Winter ${ }^{291}$,

A. Wohlfahrt ${ }^{72}$, T. Wojtoń ${ }^{100}$, D. Wollmann ${ }^{65}$, J. Womersley ${ }^{67}$, D. Woog ${ }^{65}$,

X. Wu ${ }^{305}$, A. Wulzer ${ }^{130,268}$, M.K. Yanehsari ${ }^{210}$, G. Yang ${ }^{149}$, H.J. Yang ${ }^{220,244}$, W.-M. Yao ${ }^{161}$, E. Yazgan ${ }^{98}$, V. Yermolchik ${ }^{96}$, A. Yilmaz ${ }^{113}$, A. Yilmaz ${ }^{76}$,

H.-D. Yoo ${ }^{217}$, S.A. Yost ${ }^{235}$, T. You ${ }^{299}$, C. Young ${ }^{226}$, T.-T. Yu ${ }^{320}$, F. Yu ${ }^{142}$,

A. Zaborowska ${ }^{65}$, S.G. Zadeh ${ }^{322}$, M. Zahnd ${ }^{58}$, M. Zanetti ${ }^{130,268}$, L. Zanotto ${ }^{117}$,

L. Zawiejski ${ }^{100}$, P. Zeiler ${ }^{64}$, M. Zerlauth ${ }^{65}$, S.M. Zernov ${ }^{73}$, G. Zevi Dell Porta ${ }^{296}$,

Z. Zhang ${ }^{33}$, Y. Zhang ${ }^{343}$, C. Zhang ${ }^{193}$, H. Zhang ${ }^{98}$, Z. Zhao ${ }^{324}$, Y.-M. Zhong ${ }^{19}$,

J. Zhou ${ }^{131,270}$, D. Zhou ${ }^{82}$, P. Zhuang ${ }^{243}$, G. Zick ${ }^{3}$, F. Zimmermann ${ }^{65}$, J. Zinn-

Justin $^{40}$, L. Zivkovic ${ }^{288}$, A.V. Zlobin ${ }^{71}$, M. Zobov ${ }^{116}$, J. Zupan ${ }^{301}$, J. Zurita ${ }^{147}$, and the FCC Collaboration ${ }^{352}$

${ }^{1}$ A.I. Alikhanyan National Science Laboratory (YerPhi), Yerevan, Armenia

${ }^{2}$ Abant Izzet Baysal University (AIBU), Bolu, Turkey

${ }^{3}$ Air Liquide Advanced Technologies (ALAT), Sassenage, France

${ }_{5}^{4}$ Aix-Marseille Université (AMU), Marseille, France

${ }^{5}$ Aix-Marseille Univ., Université de Toulon, CNRS, CPT (AMU/UTLN/CNRS/CPT), Marseille, France

${ }^{6}$ Akdeniz University (UAKDENIZ), Antalya, Turkey

7 ALBA Synchrotron - Consorcio para la Construcción, Equipamiento y Explotación del Laboratorio de Luz Sincrotrón, Cerdanyola del Vallès (CELLS-ALBA), Cerdanyola del Vallès, Spain

${ }^{8}$ Albert-Ludwigs-Universität Freiburg (UFreiburg), Freiburg, Germany

${ }^{9}$ All-Russian Scientific Research and Development Cable Institute (VNIIKP), Moscow, Russia

${ }^{10}$ Ankara University (Ankara U), Tandogan, Ankara, Turkey

${ }^{11}$ Applied Superconductivity Center (ASC), Tallahassee, FL, USA

12 Argonne National Laboratory (ANL), Argonne, IL, USA

${ }^{13}$ Austrian Institute of Technology (AIT), Vienna, Austria

${ }^{14}$ Banaras Hindu University (BHU), Varanasi, India

${ }^{15}$ Baylor University (Baylor), Waco, TX, USA

${ }^{16}$ Benemérita Universidad Autónoma de Puebla (BUAP), Puebla, Mexico

${ }^{17}$ Bochvar Institute of Inorganic Materials (VNIINM), Mosocw, Russia

${ }_{18}$ Bogoliubov Laboratory of Theoretical Physics (BLTP JINR), Dubna, Russia

19 Boston University (BU), Boston, MA, USA 
${ }^{20}$ Boğaziçi University (BOUN), Istanbul, Turkey

${ }^{21}$ Brookhaven National Laboratory (BNL), Upton, NY, USA

${ }^{22}$ Brown University (Brown), Providence, RI, USA

${ }^{23}$ BRUKER EST (Bruker), Hanau, Germany

${ }^{24}$ Budker Institute of Nuclear Physics (BINP), Novosibirsk, Russia

${ }^{25}$ Center for High Energy Physics (CHEP), Daegu, Republic of Korea

${ }^{26}$ Centre de Physique des Particules de Marseille (CPPM), Marseille, France

${ }^{27}$ Centre d'Etudes des Tunnels (CETU), Bron, France

${ }^{28}$ Centre d'études et d'expertise sur les risques, l'environnement, la mobilité et l'aménagement (CEREMA), Lyon, France

${ }^{29}$ Centre for Industrial Studies (CSIL), Milan, Italy

${ }^{30}$ Centre National de la Recherche Scientifique (CNRS), Aubière, France

${ }^{31}$ Centre National de la Recherche Scientifique (CNRS/IN2P3), Clermont-Ferrand, France

${ }^{32}$ Centre National de la Recherche Scientifique (CNRS), Marseille, France

${ }^{33}$ Centre National de la Recherche Scientifique (CNRS), Paris, France

${ }^{34}$ Centro Brasileiro de Pesquisas Físicas (CBPF), Rio de Janeiro, Brazil

${ }^{35}$ Centro de Investigaciones Energéticas, Medioambientales y Tecnológicas (CIEMAT), Madrid, Spain

${ }^{36}$ Centro de Investigacion y de Estudios Avanzados (CINVESTAV), Merdia, Mexico

${ }^{37}$ Cockcroft Institute (CI Daresbury), Daresbury, UK

${ }^{38}$ Cockcroft Institute (CI Lancaster), Lancaster, UK

${ }^{39}$ Comenius University (CU), Bratislava, Slovakia

${ }^{40}$ Commissariat à l'énergie atomique et aux énergies alternatives - Institut de Recherche sur les lois Fondamentales de l'Univers Saclay (CEA/DSM/Irfu Saclay), Gif-sur-Yvette, France

${ }^{41}$ Commissariat à l'énergie atomique et aux énergies alternatives - Institut Nanosciences et Cryogénie (CEA), Grenoble, France

${ }^{42}$ Consiglio Nazionale delle Ricerche (CNR), Milan, Italy

${ }^{43}$ Consiglio Nazionale delle Ricerche - Superconducting and other Innovative materials and devices institute (CNR-SPIN), Genoa, Italy

${ }^{44}$ Consiglio Nazionale delle Ricerche - Superconducting and other Innovative materials and devices institute (CNR-SPIN), Naples, Italy

${ }^{45}$ Cornell University (Cornell), Ithaca, NY, USA

${ }^{46}$ Departamento de Física Teórica, Universidad de València (UV), València, Spain

${ }^{47}$ Departamento de Física Teórica y del Cosmos and CAFPE, Universidad de Granada (UGR), Granada, Spain

${ }^{48}$ Department of Energy (DoE), Washington, DC, USA

${ }^{49}$ Department of Physics, University of Tehran (UT), Tehran, Iran

${ }^{50}$ Deutsches Elektronen Synchrotron (DESY), Hamburg, Germany

${ }^{51}$ Deutsches Elektronen Synchrotron (DESY ZEU), Zeuthen, Germany

${ }^{52}$ Dimtel, Inc. (Dimtel), San Jose, CA, USA

${ }^{53}$ Dipartimento di Ingegneria Civile e Meccanica, Università degli Studi di Cassino e del Lazio Meridionale (DICEM), Cassino, Italy

${ }^{54}$ Duke University (DU), Durham, NC, USA

${ }^{55}$ Durham University, Institute for Particle Physics Phenomenology (IPPP), Durham, UK

${ }^{56}$ Dzhelepov Laboratory of Nuclear Problems (DLNP JINR), Dubna, Russia

${ }^{57}$ Ecole polytechnique fédérale de Lausanne (EPFL), Lausanne, Switzerland

${ }^{58}$ Ecotec Environnement SA (Ecotec), Geneva, Switzerland

${ }^{59}$ Ege University (EgeU), Izmir, Turkey

${ }^{60}$ Eidgenössische Technische Hochschule Zürich (ETHZ), Zürich, Switzerland

${ }^{61}$ Eindhoven University of Technology (TU/e), Eindhoven, Netherlands

${ }^{62}$ Elle Marmi SARL (EM), Carrara, Italy

${ }^{63}$ Eskişehir Technical University (ESTU), Istanbul, Turkey

${ }^{64}$ Esslingen University of Applied Sciences (HS Esslingen), Göppingen, Germany

${ }^{65}$ European Organization for Nuclear Research (CERN), Geneva, Switzerland 
${ }^{66}$ European Scientific Institute (ESI), Archamps, France

${ }^{67}$ European Spallation Source (ESS), Lund, Sweden

${ }^{68}$ Fachhochschule Südwestfalen (FH-SWF), Gelsenkirchen, Germany

${ }^{69}$ Faculty of Mathematics and Physics, Charles University Prague (CU), Prague, Czech Republic

${ }^{70}$ Fayoum University (FU), El-Fayoum, Egypt

${ }^{71}$ Fermi National Accelerator Laboratory (FNAL), Batavia, IL, USA

${ }^{72}$ Fraunhofer-Institut für Materialfluss und Logistik (FIML), Dortmund, Germany

${ }^{73}$ Fuel Company of Rosatom TVEL (TVEL), Moscow, Russia

${ }^{74}$ Fujikura Ltd. (Fujikura), Sakura City, Japan

${ }^{75}$ Gangneung-Wonju National University (GWNU), Gangneung-Wonju, Republic of Korea

${ }^{76}$ Giresun University (Giresun), Giresun, Turkey

${ }^{77}$ Gomel State Technical University (GSTU), Gomel, Belarus

${ }^{78}$ GSI Helmholtz Zentrum für Schwerionenforschung (GSI), Darmstadt, Germany

${ }^{79}$ Gümüşhane University (Gumushane), Gümüşhane, Turkey

${ }^{80}$ Harvard University (Harvard), Cambridge, MA, USA

${ }^{81}$ Helmholtz-Zentrum Berlin (HZB), Berlin, Germany

${ }^{82}$ High Energy Accelerator Research Organization (KEK), Tsukuba, Japan

${ }^{83}$ Işı University (Isikun), Istanbul, Turkey

${ }^{84}$ I-Cube Research (I-Cube), Toulouse, France

${ }^{85}$ II. Institut für Theoretische Physik, Universität Hamburg (UNITH), Hamburg, Germany

${ }^{86}$ ILF Consulting Engineers (ILF), Zürich, Switzerland

${ }^{87}$ Indian Institute of Technology Guwahati (IITG), Guwahati, India

${ }^{88}$ Indian Institute of Technology Kanpur (IITK), Uttar Pradesh, India

${ }^{89}$ Institut de Ciència de Materials de Barcelona (ICMAB-CSIC), Barcelona, Spain

${ }^{90}$ Institut de Physique Nucléaire d'Orsay (CNRS/IN2P3/IPNO), Orsay, France

${ }^{91}$ Institute for Advanced Study (IAS), Princeton, NJ, USA

${ }^{92}$ Institute for Applied Physics, Goethe University (IAP), Frankfurt, Germany

${ }^{93}$ Institute for Astro and Particle Physics, University of Innsbruck (UIBK), Innsbruck, Austria

${ }^{94}$ Institute for High Energy Physics of NRC "Kurchatov Institute" (IHEP), Protvino, Russia

${ }^{95}$ Institute for Mathematics, Astrophysics and Particle Physics, Radboud University (IMAPP), Nijmegen, Netherlands

${ }^{96}$ Institute for Nuclear Problems of Belarusian State University (INP BSU), Minsk, Belarus

${ }^{97}$ Institute of Electrical and Electronic Engineers (IEEE), Piscataway, NJ, USA

${ }^{98}$ Institute of High Energy Physics, Chinese Academy of Science, Beijing (IHEP CAS), Beijing, P.R. China

${ }^{99}$ Institute of Machine Components, University of Stuttgart (IMA), Stuttgart, Germany

${ }^{100}$ Institute of Nuclear Physics Polish Academy of Sciences (IFJ PAN), Krakow, Poland

${ }^{101}$ Institute of Physics, Academia Sinica (AS), Taipei, Taiwan

${ }^{102}$ Institut Jožef Stefan (IJS), Ljubljana, Slovenia

${ }^{103}$ Instituto de Astrofísica de Canarias (IAC), La Laguna, Spain

${ }^{104}$ Instituto de Física Corpuscular (CSIC-UV), Paterna, Spain

${ }^{105}$ Instituto de Física Corpuscular (CSIC-UV), València, Spain

${ }^{106}$ Instituto de Física Teórica, Universidad Autonoma de Madrid (IFT-UAM), Madrid, Spain

${ }^{107}$ Instituto de Física, Universitat de València (CSIC), València, Spain

${ }^{108}$ Instituto de Investigaciones Físicas de Mar del Plata (IFIMAR), Mar del Plata, Argentina

${ }^{109}$ Instituto Galego de Física de Altas Enxerxías, Universidade de Santiago de Compostela (IGFAE), Santiago de Compostela, Spain

${ }^{110}$ International Center for Advanced Studies, Universidad Nacional de San Martin (ICAS-UNSAM), San Martin, Argentina

111 Iowa State University (ISU), Ames, IA, USA

${ }^{112}$ Istanbul Aydin University (IAU), Istanbul, Turkey

${ }^{113}$ Istanbul University (IÜ), Istanbul, Turkey 
${ }^{114}$ Istituto Nazionale di Fisica Nucleare, Gruppo Collegato di Salerno - Sezione di Napoli (INFN SA), Salerno, Italy

115 Istituto Nazionale di Fisica Nucleare, Laboratori Nazionali del Gran Sasso (INFN LNGS), Assergi (L'Aquila), Italy

${ }^{116}$ Istituto Nazionale di Fisica Nucleare, Laboratori Nazionali di Frascati (INFN LNF), Frascati, Italy

${ }^{117}$ Istituto Nazionale di Fisica Nucleare, Laboratori Nazionali di Legnaro (INFN LNLN), Legnaro, Italy

118 Istituto Nazionale di Fisica Nucleare Sezione di Bari (INFN BA), Bari, Italy

${ }^{119}$ Istituto Nazionale di Fisica Nucleare, Sezione di Bologna (INFN BO), Bologna, Italy

${ }^{120}$ Istituto Nazionale di Fisica Nucleare, Sezione di Cagliari (INFN CA), Cagliari, Italy

${ }^{121}$ Istituto Nazionale di Fisica Nucleare, Sezione di Catania (INFN CT), Catania, Italy

${ }^{122}$ Istituto Nazionale di Fisica Nucleare, Sezione di Cosenza (INFN CS), Cosenza, Italy

${ }^{123}$ Istituto Nazionale di Fisica Nucleare, Sezione di Ferrara (INFN FE), Ferrara, Italy

${ }^{124}$ Istituto Nazionale di Fisica Nucleare, Sezione di Firenze (INFN FI), Florence, Italy

${ }^{125}$ Istituto Nazionale di Fisica Nucleare, Sezione di Genova (INFN GE), Genoa, Italy

${ }^{126}$ Istituto Nazionale di Fisica Nucleare, Sezione di Lecce (INFN LE), Lecce, Italy

${ }^{127}$ Istituto Nazionale di Fisica Nucleare, Sezione di Milano Bicocca (INFN MIB), Milan, Italy

${ }^{128}$ Istituto Nazionale di Fisica Nucleare, Sezione di Milano (INFN MI), Milan, Italy

${ }^{129}$ Istituto Nazionale di Fisica Nucleare, Sezione di Napoli (INFN NA), Naples, Italy

${ }^{130}$ Istituto Nazionale di Fisica Nucleare, Sezione di Padova (INFN PD), Padua, Italy

${ }^{131}$ Istituto Nazionale di Fisica Nucleare, Sezione di Pavia (INFN PV), Pavia, Italy

${ }^{132}$ Istituto Nazionale di Fisica Nucleare, Sezione di Perugia (INFN PG), Perugia, Italy

133 Istituto Nazionale di Fisica Nucleare, Sezione di Pisa, Università di Pisa (INFN PI), Pisa, Italy

${ }^{134}$ Istituto Nazionale di Fisica Nucleare, Sezione di Roma 1 (INFN Roma 1), Rome, Italy

135 Istituto Nazionale di Fisica Nucleare, Sezione di Roma Tor Vergata (INFN Roma 2), Rome, Italy

${ }^{136}$ Istituto Nazionale di Fisica Nucleare, Sezione di Roma Tre (INFN Roma 3), Rome, Italy

137 Istituto Nazionale di Fisica Nucleare, Sezione di Torino (INFN TO), Turin, Italy

${ }^{138}$ Istituto Nazionale di Fisica Nucleare, Sezione di Trieste (INFN TS), Trieste, Italy

${ }^{139}$ ITER (ITER), Cadarache, France

${ }^{140}$ Ivane Javakhishvili T'bilisi State University (TSU), T'bilisi, Georgie

${ }^{141}$ Izmir University of Economics (IUE), Izmir, Turkey

142 Johannes-Gutenberg-Universität (JGU), Mainz, Germany

143 John Adams Institute for Accelerator Science, The Chancellor, Masters and Scholars of the University of Oxford (JAI), Oxford, UK

144 Joint Institute for Nuclear Research (JINR), Dubna, Russia

145 Julius-Maximilians-Universität Würzburg (UWUERZBURG), Würzburg, Germany

${ }^{146}$ Kahramanmaras Sutcu Imam University (KSU), Kahramanmaras, Turkey

147 Karlsruher Institut für Technologie (KIT), Karlsruhe, Germany

${ }^{148}$ Katholieke Universiteit Leuven Research \& Development (LRD), Louvain, Belgium

149 Key Laboratory of Theoretical Physics, Chinese Academy of Science (SKLTP ITP CAS), Beijing, P.R. China

${ }^{150}$ King's College London (KCL), London, UK

${ }^{151}$ Kyoto University (Kyodai), Kyoto, Japan

${ }^{152}$ Kyungpook National University (KNU), Sankyuk-dong, Republic of Korea

${ }^{153}$ Laboratoire d'Annecy-Le-Vieux de Physique des Particules (CNRS/IN2P3/LAPP), Annecy, France

${ }^{154}$ Laboratoire de l'Accélérateur Linéaire, Université de Paris Sud (CNRS/IN2P3/UPSUD/ LAL), Orsay, France

${ }^{155}$ Laboratoire de Physique Nucléaire et de Hautes Energies (LPNHE), Paris, France

${ }^{156}$ Laboratoire de Physique Subatomique et de Cosmologie Grenoble (LPSC), Grenoble, France 
${ }^{157}$ Laboratoire de Physique Théorique et Hautes Energies (CNRS/Sorbonne/LPTHE), Paris, France

${ }^{158}$ Laboratoire Leprince-Ringuet, Ecole Polytechnique (LLR), Palaiseau, France

${ }^{159}$ Laboratório de Instrumentação e Física Experimental de Partículas (LIP), Lisbon, Portugal

${ }^{160}$ Latitude Durable (LD), Geneva, Switzerland

${ }^{161}$ Lawrence Berkeley National Laboratory (LBNL), Berkeley, CA, USA

${ }^{162}$ Linde Kryotechnik AG (Linde), Pfungen, Switzerland

${ }^{163}$ Loughborough University (LBoro), Loughborough, UK

${ }^{164}$ Ludwig Maximilians University of Munich (LMU), Munich, Germany

${ }^{165}$ Luvata Pori Oy (Luvata), Pori, Finland

${ }^{166}$ MAN Energy Solutions Schweiz AG (MAN ES), Zürich, Switzerland

${ }^{167}$ Marian Smoluchowski Institute of Physics, Jagiellonian University (UJ), Kraków, Poland

${ }^{168}$ Massachusetts Institute of Technology (MIT), Cambridge, MA, USA

169 Max-Planck-Institut für Kernphysik (MPIK), Heidelberg, Germany

170 Max-Planck-Institut für Physik (MPP), Munich, Germany

${ }^{171}$ Ministère de l'Europe et des Affaires étrangères (MEAE), Paris, France

172 Monash University (Monash), Melbourne, Australia

${ }^{173}$ MTA Wigner Research Centre for Physics (Wigner), Budapest, Hungary

${ }^{174}$ Mustafa Kemal Üniversitesi (MKU), Hatay, Turkey

${ }^{175}$ Nankai University (NKU), Tianjin, P.R. China

${ }^{176}$ Nationaal instituut voor subatomaire fysica (NIKHEF), Amsterdam, Netherlands

177 National Centre for Scientific Research Demokritos (NCSRD), Athens, Greece

178 National High Magnetic Field Laboratory, Florida State University (MagLab), Tallahassee, FL, USA

${ }^{179}$ National Institute of Chemical Physics and Biophysics (NICPB), Tallin, Estonia

${ }^{180}$ National Research Center Kurchatov Institute (NRCKI), Moscow, Russia

${ }^{181}$ National Research Nuclear University MEPhI (MEPhI), Moscow, Russia

182 National Science Centre Kharkov Institute of Physics and Technology (KIPT), Kharkov, Ukraine

183 National Technical University of Athens (NTUA), Athens, Greece

${ }^{184}$ Naturhistorisches Museum Wien (NHM), Vienna, Austria

185 Niels Bohr Institute, Copenhagen University (NBI), Copenhagen, Denmark

${ }^{186}$ Nigde Ömer Halisdemir University (OHU), Nigde, Turkey

187 Northern Illinois University (NIU), DeKalb, IL, USA

${ }^{188}$ Northwestern University (NU), Evanston, IL, USA

189 Novosibirsk State University (NSU), Novosibirsk, Russia

190 Otto-von-Guericke-Universityät Magdeburg (OVGU), Magdeburg, Germany

191 Oxford University (UOXF), Oxford, UK

192 Paul Scherrer Institute (PSI), Villigen, Switzerland

193 Peking University (PU), Beijing, P.R. China

194 Perimeter Institute for Theoretical Physics (PI), Watterloo, Canada

195 Petersburg Nuclear Physics Institute, NRC "Kurchatov Institute" (PNPI), Gatchina, Russia

${ }^{196}$ Piri Reis University (PRU), Istanbul, Turkey

${ }^{197}$ Pittsburgh Particle physics, Astrophysics \& Cosmology Center and Department of Physics \& Astronomy, University of Pittsburgh (PITT PACC), Pittsburgh, PA, USA

198 Princeton University (PU), Princeton, NJ, USA

199 PRISMA Cluster of Excellence, Inst. für Physik, Johannes-Gutenberg-Universität (PRISMA), Mainz, Germany

${ }^{200}$ Pusan National University (PNU), Busan, Republic of Korea

201 Queen's University (Queens U), Kingston, Canada

202 Tampere University (TAU), Tampere, Finland

${ }^{203}$ Rheinisch-Westfälische Technische Hochschule Aachen (RWTH), Aachen, Germany

${ }^{204}$ Riga Technical University (RTU), Riga, Latvia 
${ }^{205}$ Royal Holloway University (RHUL), London, UK

${ }^{206}$ Ruder Boskovic Institute (RBI), Zagreb, Croatia

${ }^{207}$ Ruprecht Karls Universität Heidelberg (RKU), Heidelberg, Germany

${ }^{208}$ Rutgers, The State University of New Jersey (RU), Piscataway, NJ, USA

${ }^{209}$ Sapienza Università di Roma (UNIROMA1), Rome, Italy

${ }^{210}$ School of Particles and Accelerators, Institute for Research in Fundamental Sciences (IPM), Tehran, Iran

${ }^{211}$ School of Physics and Astronomy, University of Glasgow (SUPA), Glasgow, UK

${ }^{212}$ Science and Technology Facilities Council, Daresbury Laboratory (STFC DL), Warrington, UK

${ }^{213}$ Science and Technology Facilities Council, Rutherford Appleton Laboratory (STFC RAL), Didcot, UK

214 scMetrology SARL (scMetrology), Geneva, Switzerland

215 Scuola Int. Superiore di Studi Avanzati di Trieste (SISSA), Trieste, Italy

${ }^{216}$ Scuola Normale Superiore (SNS), Pisa, Italy

217 Seoul National University (SNU), Seoul, Republic of Korea

218 Sevaplan und Wurm Schweiz AG (WURM), Winterthur, Switzerland

${ }^{219}$ Shahid Beheshti University (SBUT), Tehran, Iran

${ }^{220}$ Shanghai Jiao Tong University (SJTU), Shanghai, P.R. China

${ }^{221}$ Shirokuma GmbH (Shirokuma), Wetzikon, Switzerland

${ }^{222}$ Skobeltsyn Institute of Nuclear Physics, Lomonosov Moscow State University (SINP MSU), Moscow, Russia

${ }^{223}$ Southern Federal University (SFU), Rostov, Russia

${ }^{224}$ Southern Methodist University (SMU), Dallas, TX, USA

${ }^{225}$ Sri Guru Tegh Bahadur Khalsa College, University of Delhi (SGTB Khalsa College), New Delhi, India

${ }^{226}$ Stanford National Accelerator Center (SLAC), Menlo Park, CA, USA

${ }^{227}$ Stanford University (SU), Stanford, CA, USA

${ }^{228}$ Stony Brook University (SBU), Stony Brook, NY, USA

${ }^{229}$ Technische Universität Darmstadt (TU Darmstadt), Darmstadt, Germany

${ }^{230}$ Technische Universität Dortmund (TU Dortmund), Dortmund, Germany

${ }^{231}$ Technische Universität Dresden (TU Dresden), Dresden, Germany

${ }^{232}$ Technische Universität Graz (TU Graz), Graz, Austria

${ }^{233}$ Technische Universität Wien (TU Wien), Vienna, Austria

${ }^{234}$ Tel Aviv University (TAU), Tel Aviv, Israel

${ }^{235}$ The Citadel, The Military College of South Carolina (Citadel), Charleston, SC, USA

${ }^{236}$ The Pennsylvania State University (PSU), University Park, PA, USA

237 The University of Tokyo (Todai), Tokyo, Japan

238 Thomas Jefferson National Accelerator Facility (JLab), Newport News, VA, USA

${ }^{239}$ TOBB University of Economics and Technology (TOBB ETU), Ankara, Turkey

${ }^{240}$ Tokyo Institute of Technology (Tokyo Tech), Tokyo, Japan

${ }^{241}$ Trinity College Dublin (TCD), Dublin, Ireland

${ }^{242}$ Tri-University Meson Facility (TRIUMF), Vancouver, Canada

${ }^{243}$ Tsinghua University (THU), Beijing, P.R. China

${ }^{244}$ Tsung-Dao Lee Institute (TDLI), Shanghai, P.R. China

${ }^{245}$ Uludag University (ULUÜ), Bursa, Turkey

${ }^{246}$ Universidad Autónoma de Sinaloa (UAS), Culiacán, Mexico

${ }^{247}$ Universidad de Guanajuato (UGTO), Guanajuato, Mexico

${ }^{248}$ Universidad de La Laguna (ULL), La Laguna, Spain

${ }^{249}$ Universidad de la República (Udelar), Montevideo, Uruguay

${ }^{250}$ Universidad de los Andes (Uniandes), Bogotá, Colombia

${ }^{251}$ Universidade de São Paulo (USP), São Paulo, Brazil

${ }^{252}$ Universidade do Estado do Rio de Janeiro (UERJ), Rio de Janeiro, Brazil

${ }^{253}$ Universidade Federal de Pelotas (UFPel), Pelotas, Brazil

${ }^{254}$ Universidade Federal de Rio de Janeiro (UFRJ), Rio de Janeiro, Brazil 
${ }^{255}$ Università degli Studi Roma Tre - Centro Ricerche Economiche e Sociali Manlio Rossi-Doria (EDIRC), Rome, Italy

${ }^{256}$ Università della Calabria (UNICAL), Arcavacata, Italy

${ }^{257}$ Università del Salento (UNISALENTO), Lecce, Italy

${ }^{258}$ Università di Bari (UNIBA), Bari, Italy

${ }^{259}$ Università di Bologna (UNIBO), Bologna, Italy

${ }^{260}$ Università di Cagliari (UNICA), Cagliari, Italy

${ }^{261}$ Università di Catania (UNICT), Catania, Italy

${ }^{262}$ Università di Ferrara (UNIFE), Ferrara, Italy

${ }^{263}$ Università di Firenze (UNIFI), Florence, Italy

${ }^{264}$ Università di Genova (UNIGE), Genoa, Italy

${ }^{265}$ Università di Insubria (UNINSUBRIA), Milan, Italy

${ }^{266}$ Università di Lecce (UNILE), Lecce, Italy

${ }^{267}$ Università di Milano (UNIMI), Milan, Italy

${ }^{268}$ Università di Padova (UNIPD), Padua, Italy

${ }^{269}$ Università di Parma (UNIPR), Parma, Italy

${ }^{270}$ Università di Pavia (UNIPV), Pavia, Italy

${ }^{271}$ Università di Perugia (UNIPG), Perugia, Italy

${ }^{272}$ Università di Roma Sapienza (UNIROMA1), Rome, Italy

${ }^{273}$ Università di Roma Tor Vergata (UNIROMA2), Rome, Italy

${ }^{274}$ Università di Roma Tre (UNIROMA3), Rome, Italy

${ }^{275}$ Università di Torino (UNITO), Turin, Italy

${ }^{276}$ Università di Udine (UNIUD), Udine, Italy

${ }^{277}$ Universität Hamburg (UHH), Hamburg, Germany

${ }^{278}$ Universität Heidelberg (HEI), Heidelberg, Germany

${ }^{279}$ Universitat Politecnica de Catalunya (UPC), Barcelona, Spain

${ }^{280}$ Universität Siegen (U Siegen), Siegen, Germany

${ }^{281}$ Universität Tübingen (TU), Tübingen, Germany

${ }^{282}$ Université Clermont Auvergne (UCA), Aubière, France

${ }^{283}$ Université Grenoble Alpes (UGA), Grenoble, France

${ }^{284}$ University College London (UCL), London, UK

${ }^{285}$ University of Applied Sciences Technikum Wien (UAS TW), Vienna, Austria

${ }^{286}$ University of Arizona (UA), Tucson, AZ, USA

${ }^{287}$ University of Basel (UNIBAS), Basel, Switzerland

${ }^{288}$ University of Belgrade (UB), Belgrade, Serbia

${ }^{289}$ University of Bergen (UiB), Bergen, Norway

${ }^{290}$ University of Bern (UNIBE), Bern, Switzerland

${ }^{291}$ University of Birmingham (UBIRM), Birmingham, UK

${ }^{292}$ University of Bristol (UOB), Bristol, UK

${ }^{293}$ University of California Berkeley (UCB), Berkeley, CA, USA

${ }^{294}$ University of California, Davis (UCD), Davis, CA, USA

${ }^{295}$ University of California, Irvine (UCI), Irvine, CA, USA

${ }^{296}$ University of California, San Diego (UCSD), San Diego, CA, USA

${ }^{297}$ University of California Santa Barbara (UCSB), Santa Barbara, CA, USA

${ }^{298}$ University of California Santa Cruz (UCSC), Santa Cruz, CA, USA

${ }^{299}$ University of Cambridge (CAM), Cambridge, UK

${ }^{300}$ University of Chicago (UCHI), Chicago, IL, USA

${ }^{301}$ University of Cincinnati (UC), Cincinnati, OH, USA

${ }^{302}$ University of Colorado Boulder (UCB), Boulder, Co, USA

${ }^{303}$ University of Edinburgh (ED), Edinburgh, UK

${ }^{304}$ University of Florida (UF), Gainesville, FL, USA

${ }^{305}$ University of Geneva (UniGE), Geneva, Switzerland

${ }^{306}$ University of Illinois at Urbana Champaign (UIUC), Urbana Champaign, IL, USA

${ }^{307}$ University of Iowa (UIowa), Iowa City, IA, USA

${ }^{308}$ University of Jyväskylä (JYU), Jyväskylä, Finland 
${ }^{309}$ University of Kansas (KU), Lawrence, KS, USA

${ }^{310}$ University of Liverpool (ULIV), Liverpool, UK

${ }^{311}$ University of Lund (ULU), Lund, Sweden

312 University of Malta (UM), Msida, Malta

${ }^{313}$ University of Manchester (UMAN), Manchester, UK

${ }^{314}$ University of Maryland (UMD), College Park, MD, USA

${ }^{315}$ University of Massachusetts-Amherst (UMass), Amherst, MA, USA

${ }^{316}$ University of Melbourne (UniMelb), Melbourne, Australia

${ }^{317}$ University of Michigan (UMich), Ann Arbor, MI, USA

${ }^{318}$ University of New Mexico (NMU), Albuquerque, NM, USA

${ }^{319}$ University of Notre Dame du Lac (ND), South Bend, IA, USA

${ }^{320}$ University of Oregon (UO), OR Eugene, USA

${ }^{321}$ University of Rochester (Rochester), Rochester, NY, USA

${ }^{322}$ University of Rostock (U Rostock), Rostock, Germany

${ }^{323}$ University of Saskatchewan (USASK), Saskatoon, Canada

${ }^{324}$ University of Science and Technology of P.R. China (USTC), Hefei, P.R. China

${ }^{325}$ University of Science and Technology of Mazandaran (USTM), Behshahr, Iran

${ }^{326}$ University of Silesia (USKAT), Katowice, Poland

${ }^{327}$ University of Stavanger (UiS), Stavanger, Norway

${ }^{328}$ University of Stuttgart (USTUTT), Stuttgart, Germany

${ }^{329}$ University of Sussex (US), Brighton, UK

${ }^{330}$ University of the Witwatersrand (WITS), Johannesburg, South Africa

${ }^{331}$ University of Toronto (UToronto), Toronto, Canada

${ }^{332}$ University of Warsaw (UW), Warszawa, Poland

${ }^{333}$ University of Wisconsin-Madison (WISC), Madison, WI, USA

${ }^{334}$ University of Würzburg (U Würzburg), Würzburg, Germany

${ }^{335}$ University of Zürich (UZH), Zürich, Switzerland

${ }^{336}$ University Rey Juan Carlos (URJC), Madrid, Spain

${ }^{337}$ Univ. Lyon 1, CNRS/IN2P3, Institut de Physique Nucléaire de Lyon (CNRS/IN2P3/ IPNL), Lyon, France

${ }^{338}$ Uppsala University (UU), Uppsala, Sweden

${ }^{339}$ Usak University (Usak), Usak, Turkey

${ }^{340}$ Vienna University of Economics and Business (WU), Vienna, Austria

${ }^{341}$ Vinca Institute of Nuclear Sciences (Vinca), Belgrade, Serbia

${ }^{342}$ Vrije Universiteit Brussel (VUB), Brussels, Belgium

${ }^{343}$ Washington University (WUSTL), St. Louis, MO, USA

${ }^{344}$ Weizmann Institute (Weizmann), Rehovot, Israel

${ }^{345}$ Werner-Heisenberg-Institut, Max-Planck-Institut für Physik (MPP), Munich, Germany

${ }^{346}$ Westfälische Wilhelms-Universität Münster (WWU), Münster, Germany

${ }^{347}$ West Pomeranian University of Technology (ZUT), Szczecin, Poland

${ }^{348}$ Wroclaw University of Science and Technology (PWR), Wroclaw, Poland

${ }^{349}$ Wuhan University of Technology (WHUT), Wuhan, P.R. China

${ }^{350}$ Zhejiang Institute of Modern Physics, Department of Physic (ZIMP), Hangzhou, P.R. China

${ }^{351}$ Zhejiang University (ZJU), Hangzhou, P.R. China

352 fcc.secretariat@cern.ch

Received 20 December 2018

Published online 5 July 2019 


\begin{abstract}
In response to the 2013 Update of the European Strategy for Particle Physics (EPPSU), the Future Circular Collider (FCC) study was launched as a world-wide international collaboration hosted by CERN. The FCC study covered an energy-frontier hadron collider (FCC-hh), a highest-luminosity high-energy lepton collider (FCC-ee), the corresponding $100 \mathrm{~km}$ tunnel infrastructure, as well as the physics opportunities of these two colliders, and a high-energy LHC, based on FCC-hh technology. This document constitutes the third volume of the FCC Conceptual Design Report, devoted to the hadron collider FCC-hh. It summarizes the FCC-hh physics discovery opportunities, presents the FCC-hh accelerator design, performance reach, and staged operation plan, discusses the underlying technologies, the civil engineering and technical infrastructure, and also sketches a possible implementation. Combining ingredients from the Large Hadron Collider (LHC), the high-luminosity LHC upgrade and adding novel technologies and approaches, the FCC-hh design aims at significantly extending the energy frontier to $100 \mathrm{TeV}$. Its unprecedented centreof-mass collision energy will make the FCC-hh a unique instrument to explore physics beyond the Standard Model, offering great direct sensitivity to new physics and discoveries.
\end{abstract}

${ }^{a}$ e-mail: Michael.Benedikt@cern.ch 


\section{Preface}

The 2013 Update of the European Strategy for Particle Physics (ESPPU) [1] stated, inter alia, that "... Europe needs to be in a position to propose an ambitious post$L H C$ accelerator project at CERN by the time of the next Strategy update" and that "CERN should undertake design studies for accelerator projects in a global context, with emphasis on proton-proton and electron-positron high-energy frontier machines. These design studies should be coupled to a vigorous accelerator R\&D programme, including high-field magnets and high-gradient accelerating structures, in collaboration with national institutes, laboratories and universities world-wide".

In response to this recommendation, the Future Circular Collider (FCC) study was launched [2] as a world-wide international collaboration under the auspices of the European Committee for Future Accelerators (ECFA). The FCC study was mandated to deliver a Conceptual Design Report (CDR) in time for the following update of the European Strategy for Particle Physics.

European studies of post-LHC circular energy-frontier accelerators at CERN had actually started a few years earlier, in 2010-2013, for both hadron [3-5] and lepton colliders [6-8], at the time called HE-LHC/VHE-LHC and LEP3/DLEP/TLEP, respectively. In response to the $2013 \mathrm{ESPPU}$, in early 2014 these efforts were combined and expanded into the FCC effort.

Since its inception, the international FCC collaboration has been working on the design of a hadron collider (FCC-hh) for proton-proton collisions at the centre-ofmass energy of $100 \mathrm{TeV}$ that would offer the broadest discovery potential at the energy frontier. The FCC-hh physics programme also has the potential to be expanded to include heavy-ion, electron-proton and electron-ion collisions.

Five years of intense work and a steadily growing international collaboration have resulted in the present Conceptual Design Report, consisting of four volumes covering the physics opportunities, technical challenges, cost and schedule of several different circular colliders, some of which could be part of an integrated programme extending until the end of the 21st century.

Geneva, December 2018

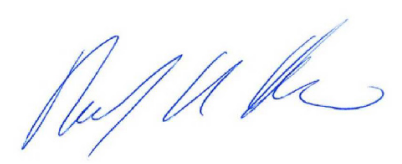

Rolf Heuer

CERN Director-General 2009-2015

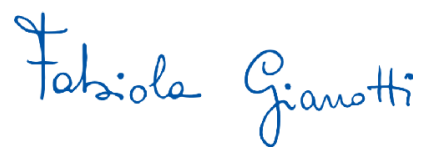

Fabiola Gianotti

CERN Director-General since 2016 


\section{Contents}

1 Physics opportunities and reach . . . . . . . . . . . . . . . 786

1.1 Introduction . . . . . . . . . . . . . . . . . . . . 786

1.2 Why $100 \mathrm{TeV} ? \ldots \ldots \ldots$. . . . . . . . . . . . . . . . . . . . . . . .

1.2.1 The nature of the Higgs potential . . . . . . . . . . . . 787

1.2 .2 Dark matter . . . . . . . . . . . . . . . . 787

1.2.3 Finding the origin of new physics exposed by indirect evidence 788

1.3 Setting the luminosity goals . . . . . . . . . . . . . . . 788

1.4 Discovery reach at the highest masses . . . . . . . . . . . . . . . 789

1.5 Precision Higgs studies and the exploration of EWSB . . . . . . . . 790

1.5.1 Higgs couplings . . . . . . . . . . . . . . . . . . . . . 790

1.5.2 The nature of the EW phase transition . . . . . . . . . . . 791

1.6 Dark matter searches . . . . . . . . . . . . . . . . . . . . . . . . 792

1.7 Lepton-Hadron physics . . . . . . . . . . . . . . . . . . . . . . . . . . . . . . . . . . . . . . . .

1.7.1 QCD and Hadron structure . . . . . . . . . . . . . . . . . 794

1.7.2 Higgs measurements . . . . . . . . . . . . . . . . . . . 795

1.7.3 Precision EW and top physics . . . . . . . . . . . . . . 796

1.7.4 Production of new particles . . . . . . . . . . . 796

1.8 The physics goals and prospects of the heavy ion programme at FCC-hh796

1.8.1 Novel access to QCD thermodynamics and QCD equilibration

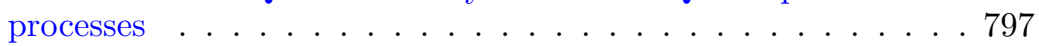

1.8.2 Characterisation of dense QCD matter through hard-scattering processes .......................... 797

1.8.3 Parton saturation in an ultra-dense kinematic domain . . . . 798

2 Collider design and performance . . . . . . . . . . . . . . . . . 799

2.1 Requirements and design considerations . . . . . . . . . . . . . . 799

2.2 Key parameters and layout . . . . . . . . . . . . . . . . . . 799

2.2.1 Layout and beam energy . . . . . . . . . . . . . . . . . 799

2.2.2 Luminosity and beam parameters . . . . . . . . . . . . . . . . 802

2.2 .3 Integrated luminosity . . . . . . . . . . . . . . . . . . . . . . . . 803

2.2.4 Additional experiments . . . . . . . . . . . . . . . 805

2.2.5 Alternative bunch spacings . . . . . . . . . . . . . . . . 805

2.2 .6 Injection considerations . . . . . . . . . . . . . . . . . . . . . . 805

2.3 Design challenges and approaches . . . . . . . . . . . . 806

2.3.1 Lattice considerations . . . . . . . . . . . . . . . . 806

2.3.2 Arc vacuum . . . . . . . . . . . . . . . . . 807

2.3.3 Experimental areas . . . . . . . . . . . . . . . . . 808

2.3.4 Machine protection . . . . . . . . . . . . . . . 809

2.3 .5 Injection . . . . . . . . . . . . . . . . . . . . . . . . . . . . . . . . .

2.3.6 Extraction and dump . . . . . . . . . . . . . . . . . 810

2.3.7 Collimation . . . . . . . . . . . . . . . . . . 811

2.3 .8 RF system . . . . . . . . . . . . . . . . . . . . . 814

2.4 Optics design and beam dynamics . . . . . . . . . . . . . . . 814

2.4.1 Lattices . . . . . . . . . . . . . . . . . . . . . . 814

2.4.2 Main experiment insertions . . . . . . . . . . . . . . . . . . . . . . . . . . . . . .

2.4.3 Collimation insertions . . . . . . . . . . . . . . . . . . . . . . . . . . . . .

2.4.4 RF insertions . . . . . . . . . . . . . . . . . . . . . . . . . . . . . . . . . .

2.4.5 Injection and extraction . . . . . . . . . . . . . . 815

2.4.6 Dynamic aperture and correction schemes . . . . . . . . . . 816

2.4 .7 Impedances and mitigation . . . . . . . . . . . . . . . . . 817

2.4.8 Electron cloud considerations . . . . . . . . . . . . . . . . . . . . . . . . . . . . . . . . . .

2.4 .9 Beam-beam effects . . . . . . . . . . . . . . . . 821 
2.5 Operation and performance . . . . . . . . . . . . . . . . 822

2.6 Energy upgrade . . . . . . . . . . . . . . . . . . . . . . . . . . . . . . . . . . . . . . . . . . . .

2.7 Ion operation . . . . . . . . . . . . . . . . . . . . 826

2.8 Lepton-Hadron operation . . . . . . . . . . . . . . . . . . . . . . . . . . . . . . . . . . . . . . .

3 Collider technical systems . . . . . . . . . . . . . . . . . 834

3.1 Overview . . . . . . . . . . . . . . . . . . . . 834

3.2 Main magnet system . . . . . . . . . . . . . . . . . . . . 834

3.2 .1 Introduction . . . . . . . . . . . . . . . . . . . 8 834

3.2.2 Superconducting main dipole . . . . . . . . . . . . . . . . . 834

3.2 .3 Field quality . . . . . . . . . . . . . . . . . . . . 838

3.2.4 Magnet protection . . . . . . . . . . . . . . . . 839

3.2 .5 Other design options . . . . . . . . . . . . . . . . . . . . . 840

3.2.6 Low temperature superconductors . . . . . . . . . . . . . . . 841

3.2.7 Superconducting main quadrupole . . . . . . . . . . . . . . . . 841

3.2 .8 Other magnets in the arcs . . . . . . . . . . . . . . . . . . . . 842

3.2 .9 Low-beta triplets . . . . . . . . . . . . . . . . . . . . . . 844

3.2 .10 Other magnets . . . . . . . . . . . . . . . . . . . . 844

3.3 Cryogenic beam vacuum system . . . . . . . . . . . . . . . . . . . . . . . . . . . . . . . . . . . . . . . .

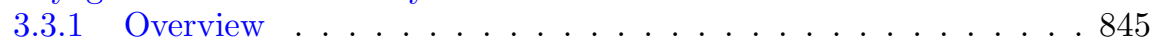

3.3 .2 Beamscreen . . . . . . . . . . . . . . . . . . . 845

3.3.3 Vacuum . . . . . . . . . . . . . . . . . . . . . . . 849

3.4 Radiofrequency system . . . . . . . . . . . . . . . . . . 851

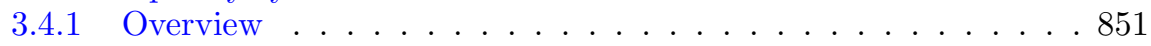

3.4 .2 Superconducting cavities . . . . . . . . . . . . . . . . . . . . . . . . . . . . . . . . . . . . .

3.4 .3 RF Powering . . . . . . . . . . . . . . . . . . . 854

3.4 .4 Feedback . . . . . . . . . . . . . . . . . 856

3.4.5 R\&D challenges . . . . . . . . . . . . . . . . . . . . . . . . . . . . . . . . . . . . . . . . . . .

3.5 Beam transfer systems . . . . . . . . . . . . . . . . . 859

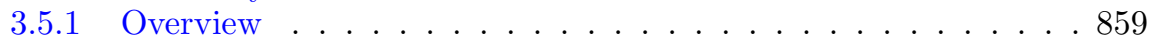

3.5 .2 Injection . . . . . . . . . . . . . . . . . . 859

3.5 .3 Extraction . . . . . . . . . . . . . . . . . . . . . . . . . . . . . . . . . . . . . . . . . . . . .

3.5.4 Dumps and absorbers . . . . . . . . . . . . . 8 866

3.6 Beam diagnostics requirements and concepts . . . . . . . . . . . . 868

3.6.1 Beam position monitoring . . . . . . . . . . . . . . 868

3.6.2 Beam loss monitoring . . . . . . . . . . . . . . . . 869

3.6.3 Beam current and intensity measurements . . . . . . . . . . . . 869

3.6.4 Tune, chromaticity and coupling . . . . . . . . . . . . . . 870

3.6.5 Transverse profile measurements . . . . . . . . . . . . . 870

3.7 Hollow electron lens . . . . . . . . . . . . . . . . . . 871

3.8 Magnet powering requirements and concepts . . . . . . . . . . . . . . 872

3.8.1 Circuit powering . . . . . . . . . . . . . . 872

3.8.2 Quench detection technologies . . . . . . . . . . . . 874

3.8.3 Energy extraction system technologies . . . . . . . . . . . . 874

3.8.4 Power converters for magnet powering . . . . . . . . . . . . 875

3.8.5 Energy storage in power converters . . . . . . . . . . . . . 877

3.9 Machine protection management concepts . . . . . . . . . . . . . 878

3.10 Controls requirements and concepts . . . . . . . . . . . . . . . . 880

3.11 Radiation environment . . . . . . . . . . . . . . . . . . . . . . . . . . . . . . . . . . . . . . . . .

3.11 .1 Introduction . . . . . . . . . . . . . . . . . . 882

3.11 .2 Reference radiation levels . . . . . . . . . . . . . . . . 883

3.11 .3 Radiation hardness . . . . . . . . . . . . . . . . . . . . 883

3.11.4 Radiation-hard technology trends . . . . . . . . . . . . . . 885

4 Civil engineering . . . . . . . . . . . . . . . . . . 888 
4.1 Requirements and design considerations . . . . . . . . . . . . . . . 888

4.2 Layout and placement . . . . . . . . . . . . . . . . . . 889

4.2.1 Layout . . . . . . . . . . . . . . . . . . . . . . . 889

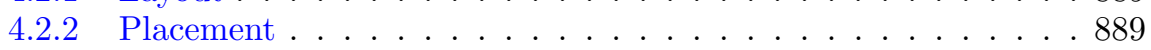

4.2 .3 Necessary site investigations . . . . . . . . . . . . . . . 8891

4.3 Underground structures . . . . . . . . . . . . . . . . . . . . . . . . . . . . . . . . . . . . . . . . . . . . .

4.3 .1 Tunnels . . . . . . . . . . . . . . . . . . . . . . . . . 892

4.3 .2 Shafts . . . . . . . . . . . . . . . . . . 894

4.3 .3 Alcoves . . . . . . . . . . . . . . . . . . 895

4.3.4 Experiment caverns . . . . . . . . . . . . . . . . 895

4.3.5 Service caverns . . . . . . . . . . . . . . . . . . . 895

4.3 .6 Junction caverns . . . . . . . . . . . . . . . . . . . . . . . . . . . . . . . . . . . . . . . . . .

4.3.7 FCC-eh requirements . . . . . . . . . . . . . . 896

4.4 Surface sites . . . . . . . . . . . . . . . . . . . . 897

4.4.1 Experiment surface sites . . . . . . . . . . . . . . . 8897

4.4 .2 Technical surface sites . . . . . . . . . . . . . . . 897

4.4 .3 Access roads . . . . . . . . . . . . . . . . . . . 898

5 Technical infrastructure . . . . . . . . . . . . . . . . . 898

5.1 Requirements and design considerations . . . . . . . . . . . . . 898

5.2 Piped utilities . . . . . . . . . . . . . . . . . . . . . . . 898

5.2 .1 Introduction . . . . . . . . . . . . . . . . . 898

5.2 .2 Water cooling plants . . . . . . . . . . . . . . . . . . . . . 899

5.2 .3 Operational parameters . . . . . . . . . . . . . . . . . . . . . . . . . . . . . . . .

5.2 .4 Chilled water . . . . . . . . . . . . . . . 902

5.2 .5 Drinking water . . . . . . . . . . . . . . . . . . . . . . . . . . 902

5.2 .6 Fire fighting network . . . . . . . . . . . . . . . . . . . . . . . . . . . . . . . . . . . . .

5.2 .7 Reject water . . . . . . . . . . . . . . . . . . . . 903

5.2.8 Compressed air . . . . . . . . . . . . . . . . . 903

5.3 Heating, ventilation, air conditioning . . . . . . . . . . . . . . 903

5.3 .1 Overall design concept . . . . . . . . . . . . . . . . . . . . . . . . . . . . . . . . . .

5.3 .2 Interior conditions . . . . . . . . . . . . . . . . . . . . . . . . . . . . . . . . . . . . . . . . .

5.3.3 Ventilation of underground areas . . . . . . . . . . . . . . . . . . . . . . . . . . . . . . . . .

5.3 .4 Machine tunnel . . . . . . . . . . . . . . . . . . . . 904

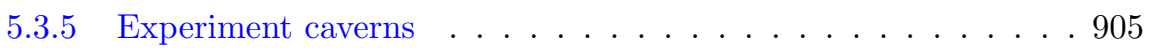

5.3 .6 Other areas . . . . . . . . . . . . . . . . . 906

5.3 .7 Operating modes . . . . . . . . . . . . . . . . 906

5.3 .8 Working parameters . . . . . . . . . . . . . . . 906

5.3 .9 Ventilation of surface buildings . . . . . . . . . . . . . . . . . . . . . . . . . . . . . . . . . . . .

5.3 .10 Safety . . . . . . . . . . . . . . . . . . . 908

5.4 Electricity distribution . . . . . . . . . . . . . . . . . . . . . . . . . . . . . . . . . . . . . . . .

5.4 .1 Conceptual layout . . . . . . . . . . . . . . . . . . 998

5.4 .2 Source of electrical energy . . . . . . . . . . . . . . . . . . 908

5.4 .3 Transmission network topology . . . . . . . . . . . . . . . . 908

5.4.4 Distribution network topology . . . . . . . . . . . . . . 9910

5.4.5 Power quality and transient voltage dip mitigation . . . . . . . 911

5.5 Emergency power . . . . . . . . . . . . . . . . . . 913

5.6 Cryogenic system . . . . . . . . . . . . . . . . . . . . 915

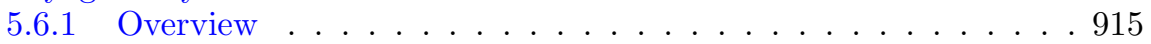

5.6 .2 Proximity cryogenics and heat loads . . . . . . . . . . . . . 917

5.6 .3 Cryogenic plants . . . . . . . . . . . . . . . . 921

5.6.4 Cryogen inventory and storage . . . . . . . . . . . . . . . 926

5.7 Equipment transport and handling . . . . . . . . . . . . . . . . . . 927

5.7.1 Underground vehicles . . . . . . . . . . . . . . 927 
5.7 .2 Overhead cranes . . . . . . . . . . . . . . . . . . . . . . . . . . . . . . . . . . .

5.7 .3 Lifts . . . . . . . . . . . . . . . . . . . . . 930

5.8 Personnel transport . . . . . . . . . . . . . . . . . 931

5.8.1 Transport for emergency services . . . . . . . . . . . . . . 931

5.9 Geodesy, survey and alignment . . . . . . . . . . . . . . . . 932

5.9 .1 Alignment tolerances . . . . . . . . . . . . . . . . . . . 9 932

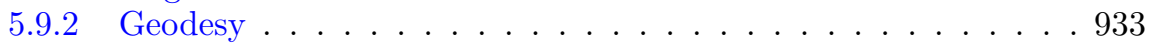

5.9 .3 Metrological aspects . . . . . . . . . . . . . . . . 933

5.9.4 Alignment of accelerator components . . . . . . . . . . . . . . . 934

5.9.5 Interaction regions and collimator areas . . . . . . . . . . . . 934

5.9.6 Experiments . . . . . . . . . . . . . . . . 934

5.10 Communications, computing and data services . . . . . . . . . 935

5.11 Safety and access management systems . . . . . . . . . . . . . 938

6 Injector scenarios . . . . . . . . . . . . . . . . . . . . . . . 940

6.1 Introduction . . . . . . . . . . . . . . . . . . . . . 940

6.1.1 Requirements . . . . . . . . . . . . . . . . . . 9940

6.1 .2 HEB options . . . . . . . . . . . . . . . . . . . . . . . . . 9940

6.1.3 Pre-injector chain . . . . . . . . . . . . . . . . . 941

$6.1 .45 \mathrm{~ns}$ bunch spacing . . . . . . . . . . . . . . . . . . . . . . . . 942

6.2 Transfer lines . . . . . . . . . . . . . . . . . . . . . . . 942

6.3 Reuse of LHC as HEB . . . . . . . . . . . . . . . . . . . . . . . 942

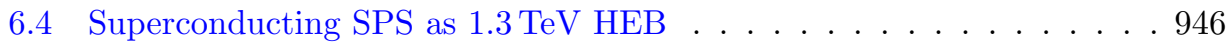

6.5 Other HEB options . . . . . . . . . . . . . . . . . . . . . . . . . . . . 949

6.5.1 3.3 TeV superconducting (4 T) HEB in the $27 \mathrm{~km}$ LHC tunnel . 950

6.5.2 $3.3 \mathrm{TeV}$ normal conducting (1 T) $100 \mathrm{~km}$ HEB in the FCC tunnel950

6.6 Summary and comparison of HEB options . . . . . . . . . . . . . . . . 950

7 Experiments and detectors . . . . . . . . . . . . . . . 951

7.1 Physics and detector performance considerations . . . . . . . . . . . . 951

7.2 Detector reference design . . . . . . . . . . . . . . . . . . . . . . . . . . . . . . . . . . . . . . .

7.3 Radiation environment . . . . . . . . . . . . . . . . . . . . . . . . . . . . . . . . . . . . . . . . .

7.4 Magnet system . . . . . . . . . . . . . . . . . . . . . . 959

7.5 Detector sub-system concepts . . . . . . . . . . . . . . . 962

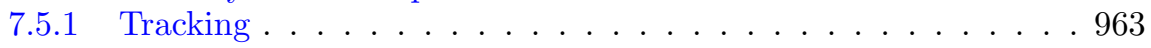

7.5.2 Calorimetry . . . . . . . . . . . . . . . . 967

7.5 .3 Muon system . . . . . . . . . . . . . . . . . . 971

7.5.4 Trigger and data acquisition . . . . . . . . . . . . . . . . 973

7.6 Detector performance and physics benchmark studies . . . . . . . . . 975

7.6.1 The Higgs self-coupling . . . . . . . . . . . . . . . . 9975

7.6.2 High mass resonances decaying to leptons . . . . . . . . . . 977

7.6 .3 Top squarks . . . . . . . . . . . . . . . . . . . . . . . . . . . . . . . . . . .

7.6 .4 Disappearing tracks . . . . . . . . . . . . . . . . . . 978

7.7 Special purpose experiments: ions . . . . . . . . . . . . . . . . . . 980

7.8 Special purpose experiments: Lepton-Hadron . . . . . . . . . . . . . . 981

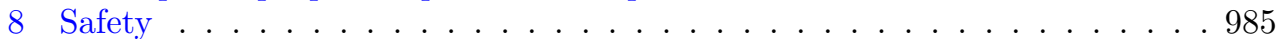

8.1 Safety policy and regulatory framework . . . . . . . . . . . 985

8.1.1 Legal context of CERN . . . . . . . . . . . . . . . 9855

8.1.2 Hazard register and safety performance based design . . . . . . 986

8.2 Occupational health and safety . . . . . . . . . . . . . . . . 986

8.2 .1 Fire hazard . . . . . . . . . . . . . . . . . . . . 9887

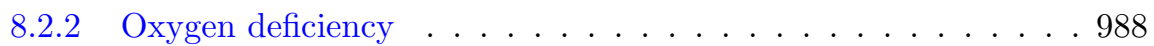

8.3 Radiation protection . . . . . . . . . . . . . . . . . . . . 989

8.3.1 Particle beam operation . . . . . . . . . . . . . . . . . . . . . . . . . . . . . . . . . . . . . .

8.3 .2 Activation of solids . . . . . . . . . . . . . . . . 990 
8.3.3 Activated or contaminated liquids . . . . . . . . . . . . . 990

8.3.4 Activated or radioactive gases and radioactive aerosols . . . . . 991

9 Energy efficiency . . . . . . . . . . . . . . . . . . . 991

9.1 Requirements and design considerations . . . . . . . . . . . . . . 991

9.2 Power requirements . . . . . . . . . . . . . . . . . . . . . . . . . . . . . . . . . . . . . . . . . . . . . .

9.3 Energy management and saving . . . . . . . . . . . . . . . . . . 994

9.4 Waste heat recovery . . . . . . . . . . . . . . . . . . 995

10 Environment . . . . . . . . . . . . . . . . . . . 997

10.1 Requirements and approach considerations . . . . . . . . . . . 997

10.1.1 Legal context . . . . . . . . . . . . . . . . . . 9997

10.1.2 Environmental compatibility management concept . . . . . . . 998

10.2 Environmental impact . . . . . . . . . . . . . . . . . . . . . 999

10.2.1 Radiological impact . . . . . . . . . . . . . . . . . 999

10.2.2 Conventional impact . . . . . . . . . . . . . . . . . . . . 1001

10.3 Waste management . . . . . . . . . . . . . . . . . . . . . . . . . 1002

10.3.1 Radioactive waste management . . . . . . . . . . . . . . 1002

10.3.2 Conventional waste management . . . . . . . . . . . . 1003

11 Education, economy and society . . . . . . . . . . . . . 1005

11.1 Implementation with the host states . . . . . . . . . . . . . . . . 1005

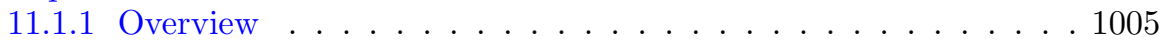

11.1 .2 France . . . . . . . . . . . . . . . . . . . 1006

11.1.3 Switzerland . . . . . . . . . . . . . . . . . . . 1008

11.2 Socio-Economic opportunities . . . . . . . . . . . . . . . . . . . 1010

11.2.1 Introduction and motivation . . . . . . . . . . . . . . 1010

11.2.2 The value of training . . . . . . . . . . . . . . . . . 1011

11.2.3 Opportunities for industries and technological spillover . . . . . 1011

11.2 .4 Cultural effects . . . . . . . . . . . . . . . . . . . . . 1014

11.2 .5 Impact potential . . . . . . . . . . . . . . . . . . . 1016

12 Strategic research and development . . . . . . . . . . . . . . 1017

12.1 Introduction . . . . . . . . . . . . . . . . . . . . . . . . . . . . . . . . . . . . . . . . . . .

12.216 tesla superconducting magnet . . . . . . . . . . . . . . . . . 1018

$12.3 \mathrm{Nb}_{3} \mathrm{Sn}$ wire . . . . . . . . . . . . . . . . . . . . 1021

12.4 High temperature superconductors . . . . . . . . . . . . . . . . 1025

12.5 Efficient and cost-effective cryogenic refrigeration . . . . . . . . . . . 1028

12.6 Cryogenic distribution line . . . . . . . . . . . . . . . . . . 1030

12.7 Superconducting septum magnets . . . . . . . . . . . . . . . . . 1031

12.8 Solid state generators . . . . . . . . . . . . . . . . . . . . . . . . . . . . . . . . . . . . . . . . .

12.9 Particle detector technologies . . . . . . . . . . . . . . . . 1035

12.10Energy storage and release R\&D . . . . . . . . . . . . 1038

12.11Efficient power distribution infrastructure . . . . . . . . . . . . 1039

12.12Efficient use of excavation materials . . . . . . . . . . . . . . . . . . . 1042

Appendix A: Collider parameters . . . . . . . . . . . . . . . . . . . . . 1046

Appendix B: Uncertainties . . . . . . . . . . . . . . . . . . . 1046

B.1 Accelerator and technologies . . . . . . . . . . . . . . . . 1047

B.2 Implementation . . . . . . . . . . . . . . . . . . . . . . . . . . . . 1049

Appendix C: Communities . . . . . . . . . . . . . . . . . 1053

Appensix D: Timeline . . . . . . . . . . . . . . . . . . . . . 1055

D.1 Timeline for FCC-hh without prior implementation of FCC-ee . . . . . 1055

D.2 Timeline for FCC-hh with prior implementation of FCC-ee . . . . . . . 1056

Appendix E: Costs . . . . . . . . . . . . . . . . . . . . 1057

E.1 Construction costs . . . . . . . . . . . . . . . . . . . . 1057

E.1.1 Construction cost for FCC-hh without prior implementation of FCC-ee . . . . . . . . . . . . . . . . 1057 
E.1.2 Construction cost for FCC-hh with prior implementation of FCC-ee . . . . . . . . . . . . . . . 1057

E.2 Operation costs . . . . . . . . . . . . . . . . . 1059

\section{Executive summary}

\section{Overview}

Particle physics has arrived at an important moment in its history. The discovery of the Higgs boson, with a mass of $125 \mathrm{GeV}$, completes the matrix of particles and interactions that has constituted the "Standard Model" for several decades. This model is a consistent and predictive theory, which has so far proven successful at describing all phenomena accessible to collider experiments. However, several experimental facts require the extension of the Standard Model and explanations are needed for observations such as the abundance of matter over antimatter, the striking evidence for dark matter and the non-zero neutrino masses. Theoretical issues such as the hierarchy problem, and, more in general, the dynamic origin of the Higgs mechanism, likewise point to the existence of physics beyond the Standard Model.

This report contains the description of a novel research infrastructure based on a highest-energy hadron collider with a centre-of-mass collision energy of $100 \mathrm{TeV}$ and an integrated luminosity of at least a factor of 5 larger than the HL-LHC. It will extend the current energy frontier by almost an order of magnitude. The mass reach for direct discovery will reach several tens of $\mathrm{TeV}$, and allow, for example, the production of new particles whose existence could be indirectly exposed by precision measurements during the earlier preceding $\mathrm{e}^{+} \mathrm{e}^{-}$collider phase. This collider will also precisely measure the Higgs self-coupling and thoroughly explore the dynamics of electroweak symmetry breaking at the TeV scale, to elucidate the nature of the electroweak phase transition. WIMPs as thermal dark matter candidates will be discovered, or ruled out.

As a single project, this particle collider infrastructure will serve the world-wide physics community for about 25 years and, in combination with a lepton collider (see FCC Conceptual Design Report volume 2), will provide a research tool until the end of the 21st century. The European Strategy for Particle Physics (ESPP) update 2013 stated "To stay at the forefront of particle physics, Europe needs to be in a position to propose an ambitious post-LHC accelerator project at CERN by the time of the next Strategy update". The FCC study has implemented the ESPP recommendation by developing a long-term vision for an "accelerator project in a global context". This document describes the detailed design and preparation of a construction project for a post-LHC circular energy frontier collider "in collaboration with national institutes, laboratories and universities world-wide", and enhanced by a strong participation of industrial partners. A coordinated preparatory effort can now be based on a core of an ever-growing consortium of already more than 135 institutes world-wide.

\section{Accelerator}

The FCC-hh is designed to provide proton-proton collisions with a centre-of-mass energy of $100 \mathrm{TeV}$ and an integrated luminosity of $\approx 20 \mathrm{ab}^{-1}$ in each of the two main experiments for 25 years of operation. The combined data sets will thus significantly exceed the total of $30 \mathrm{ab}^{-1}$ required by the foreseen physics studies. In addition, the FCC-hh offers the potential of colliding ions with protons and ions with ions. The design also allows one interaction point to be upgraded to electron-proton and electron-ion collisions. In this case, an additional recirculating, energy-recovery linac will provide the electron beam that collides with one circulating proton or ion beams. The other experiments can operate concurrently with hadron collisions. 


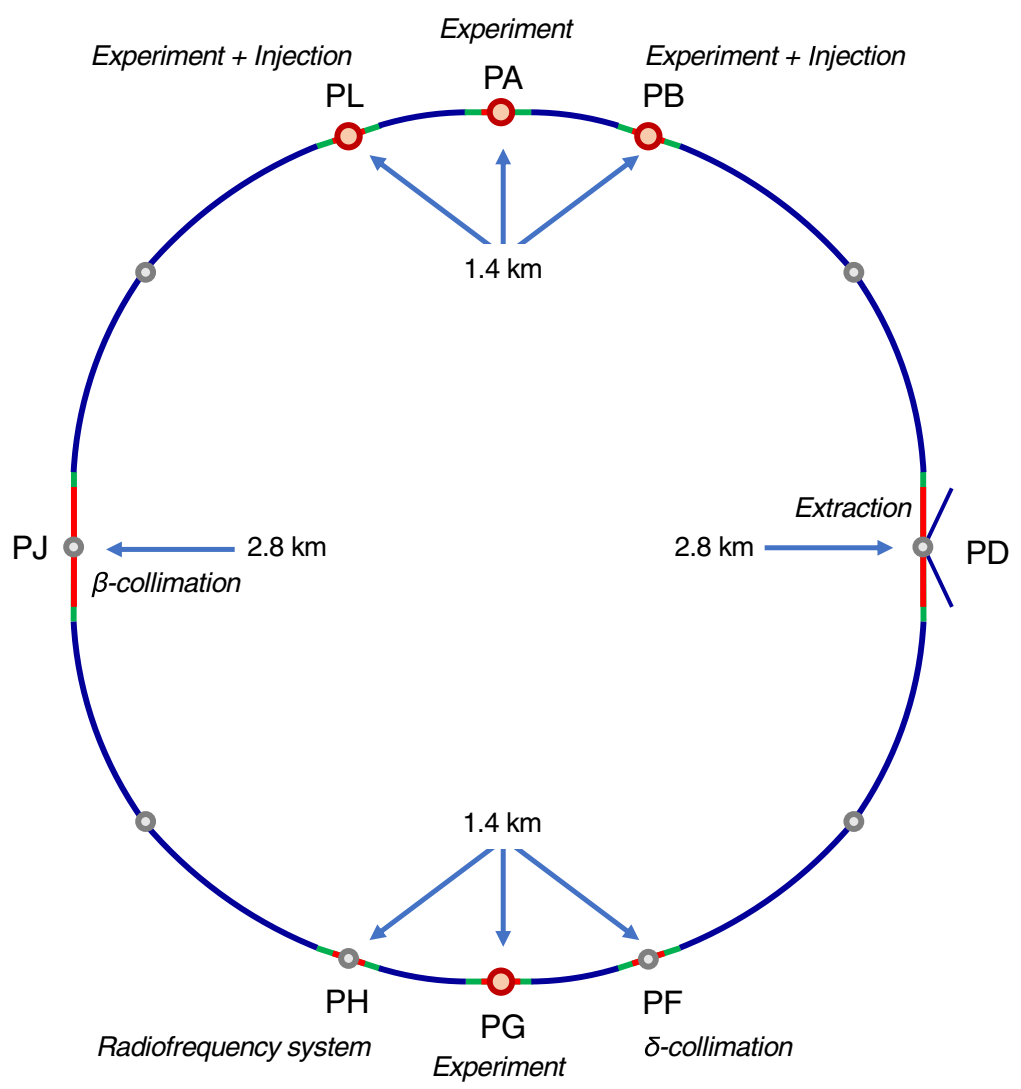

Fig. 1. Conceptual layout of the collider ring, indicating the main insertions. The experiment interaction points are at PA, PB, PG and PL. Injection takes place at PL and PB. $\mathrm{PD}$ hosts the beam extraction. Collimation takes place at $\mathrm{PF}$ and $\mathrm{PJ}$. The radiofrequency and feedback systems are installed at PH.

\section{Layout and design}

The layout of the collider is shown in Figure 1 and key parameters are given in Table 1. The circumference of the collider is $97.75 \mathrm{~km}$ and the insertions are $1.4 \mathrm{~km} \mathrm{long}$, with the exception of the $2.8 \mathrm{~km}$-long transverse collimation and beam extraction areas. This additional length facilitates the mitigation of the technological challenges generated by the high beam energies. An integrated lattice, which includes all the required functions has been developed.

Two high luminosity experiments are located in opposite insertions (PA and PG). This ensures the highest luminosity, reduces unwanted beam-beam effects and is independent of the beam-filling pattern. The main experiments are located in $66 \mathrm{~m}$ long halls, sufficient for the detector that has been studied and ensuring that the final focus system can be integrated into the available length of the insertion. Two additional, lower luminosity experiments are located together with the injection systems in insertions PB and PL. In contrast to the LHC, these experiments are not located in the centre of their insertion but are further away from PA, placing the injection points close to PA. This allows better protection from the injected beam for the 
Table 1. Key FCC-hh baseline parameters compared to LHC and HL-LHC parameters.

\begin{tabular}{|c|c|c|c|c|}
\hline & \multirow[t]{2}{*}{ LHC } & \multirow[t]{2}{*}{ HL-LHC } & \multicolumn{2}{|c|}{ FCC-hh } \\
\hline & & & Initial & Nominal \\
\hline \multicolumn{5}{|c|}{ Physics performance and beam parameters } \\
\hline Peak luminosity ${ }^{1}\left(10^{34} \mathrm{~cm}^{-2} \mathrm{~s}^{-1}\right)$ & 1.0 & 5.0 & 5.0 & $<30.0$ \\
\hline $\begin{array}{l}\text { Optimum average integrated luminos- } \\
\text { ity/day }\left(\mathrm{fb}^{-1}\right)\end{array}$ & 0.47 & 2.8 & 2.2 & 8 \\
\hline Assumed turnaround time $(\mathrm{h})$ & & & 5 & 4 \\
\hline Target turnaround time $(\mathrm{h})$ & & & 2 & 2 \\
\hline Peak number of inelastic events/crossing & 27 & 135 levelled & 171 & 1026 \\
\hline $\begin{array}{l}\text { Total/inelastic cross section } \sigma \text { proton } \\
\text { (mbarn) }\end{array}$ & \multicolumn{2}{|c|}{$111 / 85$} & \multicolumn{2}{|c|}{$153 / 108$} \\
\hline Luminous region RMS length $(\mathrm{cm})$ & \multirow{2}{*}{\multicolumn{2}{|c|}{23}} & 5.7 & 5.7 \\
\hline Distance IP to first quadrupole, $\mathrm{L}^{*}(\mathrm{~m})$ & & & 40 & 40 \\
\hline \multicolumn{5}{|l|}{ Beam parameters } \\
\hline Number of bunches $n$ & \multicolumn{2}{|c|}{2808} & \multicolumn{2}{|c|}{10400} \\
\hline Bunch spacing (ns) & 25 & 25 & \multicolumn{2}{|c|}{25} \\
\hline Bunch population $N\left(10^{11}\right)$ & 1.15 & 2.2 & \multicolumn{2}{|c|}{1.0} \\
\hline $\begin{array}{l}\text { Nominal transverse normalised emit- } \\
\text { tance }(\mu \mathrm{m})\end{array}$ & 3.75 & 2.5 & 2.2 & 2.2 \\
\hline Number of IPs contributing to $\Delta \mathrm{Q}$ & 3 & 2 & $2+2$ & 2 \\
\hline Maximum total b-b tune shift $\Delta \mathrm{Q}$ & 0.01 & 0.015 & 0.011 & 0.03 \\
\hline Beam current $(\mathrm{A})$ & 0.584 & 1.12 & \multicolumn{2}{|c|}{0.5} \\
\hline RMS bunch length ${ }^{2}(\mathrm{~cm})$ & \multicolumn{2}{|c|}{7.55} & \multicolumn{2}{|c|}{8} \\
\hline IP beta function $(\mathrm{m})$ & 0.55 & $0.15(\min )$ & 1.1 & 0.3 \\
\hline RMS IP spot size $(\mu \mathrm{m})$ & 16.7 & $7.1(\min )$ & 6.8 & 3.5 \\
\hline Full crossing angle $(\mu \mathrm{rad})$ & 285 & 590 & 104 & $200^{3}$ \\
\hline
\end{tabular}

experiments. The collider will use the existing CERN accelerator complex as injector facility and the baseline is to inject beam at $3.3 \mathrm{TeV}$ from the LHC. Injection from a new, superconducting SPS can be considered as an alternative. The transverse beam cleaning is located in insertion PJ and the beam extraction in insertion PD. The longitudinal beam cleaning is placed in insertion PF and finally, the RF systems and the fast feedback are placed in insertion $\mathrm{PH}$. The long arcs also contain technical points (PC, PE, PI and PK, not shown in Figure 1).

This layout increases the collider robustness because the high radiation experiment and collimation sections are separated from the radiation sensitive equipment, i.e. the RF, instrumentation and the extraction kickers. Also the short arcs between the main experiment in PA and the additional experiments in $\mathrm{PB}$ and $\mathrm{PL}$ are long enough to avoid background from the collision debris in the main experiment, which would perturb the other experiments. Similarly, the RF insertion is protected from the main experiment in $\mathrm{PG}$.

\section{Energy reach}

The total length of the arcs is $83.75 \mathrm{~km}$. The lattice in the arc consists of $90^{\circ}$ FODO cells with a length of about $213 \mathrm{~m}$ and six $14 \mathrm{~m}$-long dipoles between quadrupoles. The the dipole filling factor is about 0.8 , hence a dipole field just below $16 \mathrm{~T}$ is required to keep the nominal beams on the circular orbit. 
The dipoles use $\mathrm{Nb}_{3} \mathrm{Sn}$ conductors at a temperature of $2 \mathrm{~K}$ to reach this field and are a key cost item. A focused R\&D programme to increase the maximum current density in the conductors to at least $1500 \mathrm{~A} / \mathrm{mm}^{2}$ at $4.2 \mathrm{~K}$ temperature started in 2014 (currently $1200 \mathrm{~A} / \mathrm{mm}^{2}$ has been achieved). Based on this performance, several optimised dipole designs have been developed in the EuroCirCol H2020 EC funded project - each implementing a different design concept. This allowed the amount of conductor material to be minimised and led to the choice of the cosine-theta design as the baseline. Collaboration agreements are in place with the French CEA, the Italian INFN, the Spanish CIEMAT, the Swiss PSI and the Russian BINP organisations, to build short model magnets based on the designs. In addition, a US DOE Magnet Development Programme is working to demonstrate a $15 \mathrm{~T}$ superconducting accelerator magnet.

If the FCC-hh is implemented following a lepton collider (FCC-ee) in the same underground infrastructure, the time scale for design and R\&D for FCC-hh is lengthened by 15-20 years. This additional time will be used to develop alternative technologies, e.g. magnets based on high temperature superconductors with potentially, a significant impact on the collider parameters (e.g. increase of beam energy), relaxed infrastructure requirements (cryogenics system) and increased energy efficiency (temperature of magnets and beamscreen).

\section{Luminosity performance}

It is planned to reach the initial parameters with a maximum luminosity of $5 \times$ $10^{34} \mathrm{~cm}^{-2} \mathrm{~s}^{-1}$ in the first years. In the following years the luminosity will be increased to reach the nominal parameters with a luminosity of up to $3 \times 10^{35} \mathrm{~cm}^{-2} \mathrm{~s}^{-1}$. The estimated integrated luminosity is $2 \mathrm{fb}^{-1}$ per day of operation for the initial and $8 \mathrm{fb}^{-1}$ for the nominal parameters. A generic design for the additional experiments shows that a luminosity of $2 \times 10^{34} \mathrm{~cm}^{-2} \mathrm{~s}^{-1}$ can be reached, but further studies are required.

The high luminosity is achieved with high brightness beams, a high beam current comparable to LHC parameters, and a small $\beta^{*}$ at the collision points. Crossing the beams at an angle limits the impact of parasitic beam-beam interactions and the associated luminosity reduction is compensated by using crab cavities. Electron lenses and current carrying wire compensators may further improve the performance of the machine.

In the nominal phase, the beams can only be used for about $3.5 \mathrm{~h}$ for collision due to the fast beam burn-off. Consequently, the turn-around time from one luminosity run to the next is critical to achieve the target integrated luminosity. Theoretically a time of about $2 \mathrm{~h}$ can be achieved. However, to include sufficient margin, turn-around times of $5 \mathrm{~h}$ and $4 \mathrm{~h}$ are assumed for initial and nominal parameters, respectively. An availability of $70 \%$ at flat top for physics operation is assumed for the estimate of the overall integrated luminosity.

The various beam dynamics effects that can compromise the beam stability and quality have been investigated and modelled for the conceptual design and mitigation methods were devised where required. A combination of fast transverse feedback and octupoles is used to stabilise the beam against parasitic electromagnetic interaction with the beamline components. Electron cloud build-up, which could render the beam unstable, is suppressed by appropriate hardware design. The impact of main magnet field imperfections on the beam is mitigated by high quality magnet design and use of corrector magnets. 


\section{Technical systems}

Many technical systems and operational concepts for FCC-hh can be scaled up from HL-LHC or can be based on technology demonstrations carried out in the frame of ongoing R\&D projects. Particular technological challenges arise from the higher total energy in the beam (20 times that of LHC), the much increased collision debris in the experiments (40 times that of HL-LHC) and far higher levels of synchrotron radiation in the arcs (200 times that of LHC).

The high luminosity and beam energy will produce collision debris with a power of up to $0.5 \mathrm{MW}$ in the main experiments and a significant fraction of this will be lost in the machine near the experiment. A sophisticated shielding system, similar to HL-LHC but thicker, protects the final focusing triplet, avoids quenches and reduces the radiation dose. The current radiation limit of $30 \mathrm{MGy}$ for the magnets, due to the resin used, will be reached for an integrated luminosity of $13 \mathrm{ab}^{-1}$. However, improvement of both the shielding and the radiation hardness of the magnets appears possible. It is therefore likely that the magnets will not have to be replaced during the entire lifetime of the project.

The robust collimation and beam extraction system protects the machine from the energy stored in the beam. The collimation system design is based on the LHC system, with a number of improvements to make it viable. Additional protection has been added to mitigate losses in the arcs that would otherwise quench magnets. Also improved conceptual designs of collimators and dogleg dipoles have been developed to reduce the beam induced stress to acceptable levels. Further R\&D should allow the margin in the design to be increased to comfortable levels.

The extraction system uses a segmented, dual-plane dilution kicker system to distribute all bunches of the beam onto a multi-branch spiral on the absorber block. Novel superconducting septa capable of deflecting the rigid beams are currently being developed. The system design is fault tolerant, in particular, the most critical failure mode - erratic firing of a single extraction kicker element - has limited impact since the system has a high granularity. Investigations of suitable absorber materials including $3 \mathrm{D}$ carbon composites and carbon foams are ongoing in the HL-LHC project.

The cryogenics system must compensate the continuous heat loads in the arcs of $1.4 \mathrm{~W} / \mathrm{m}$ at a temperature below $2 \mathrm{~K}$ and the $30 \mathrm{~W} / \mathrm{m}$ /aperture due to synchrotron radiation at a temperature of $50 \mathrm{~K}$, as well as absorbing the transient loads from ramping of the magnets. The system must also be able to fill and cool down the cold mass of the machine $\left(230 \times 10^{6} \mathrm{~kg}\right)$ in less than 20 days, while avoiding thermal gradients higher than $50 \mathrm{~K}$ in the cryomagnet structure. It must also cope with resistive transitions of the superconducting magnets and recover sufficiently fast from such situations that the operational availability of the collider remains at an adequate level.

The number of active cryogenic components distributed around the ring is minimised for reasons of simplicity, reliability and maintenance. The cooling power required at each temperature will be produced by 10 refrigeration plants at 6 technical sites, and distributed to the adjacent sectors over distances of up to $10 \mathrm{~km}$. At each site, an interconnection box couples the various pieces of refrigeration equipment to the cryogenic distribution line and where possible, they also facilitate redundancy. To limit the pressure build-up during helium discharge in case of a sector quench and to mitigate the risks of equipment failures, helium storage is provided at all 12 surface sites. The cooling of the two high-luminosity insertions requires additional cryoplants.

Today, helium cryogenic refrigeration only reaches efficiencies of about $30 \%$ with respect to an ideal Carnot cycle which leads to high electrical power consumption. 
Part of the FCC study is to perform R\&D on novel refrigeration down to $40 \mathrm{~K}$ based on a neon-helium (nelium) gas mixture, which has the potential to reach efficiencies higher than $40 \%$. This technology could reduce the electrical energy consumption of the cryogenics system by $20 \%$.

The cryogenic beam vacuum system ensures excellent vacuum to limit beam-gas scattering. It also protects the magnets from the synchrotron radiation of the high energy beam and efficiently removes the heat. In addition, it avoids beam instabilities due to parasitic beam-surface interactions and electron cloud effects. The LHC vacuum system design is not suitable for FCC-hh, hence a novel design has been developed in the scope of the EuroCirCol H2020 funded project. It is as compact as possible to minimise the magnet aperture and consequently magnet cost. The beamscreen features an ante-chamber and is copper coated to limit the parasitic interaction with the beam. The shape also reduces seeding of the electron cloud by backscattered photons and additional carbon coating or laser treatment prevents the build-up. This novel system is operated at $50 \mathrm{~K}$ and a prototype has been validated experimentally in the KARA synchrotron radiation facility at KIT (Germany).

The RF system is similar to the one of LHC with an RF frequency of $400 \mathrm{MHz}$, but it provides a higher maximum total voltage of $48 \mathrm{MV}$. To adjust the bunch length in the presence of longitudinal damping by synchrotron radiation, controlled longitudinal emittance blow-up by band-limited $\mathrm{RF}$ phase noise is implemented. The current design uses 24 single-cell cavities.

\section{Injection}

The baseline injection concept reuses CERN's Linac4, PS, PSB, SPS and the LHC at $3.3 \mathrm{TeV}$ as pre-accelerators and connects the LHC to FCC-hh with transfer lines using $7 \mathrm{~T}$ superconducting magnets. This choice also permits the continuation of CERN's rich and diverse fixed-target physics programme in parallel with FCC-hh operation. The necessary modifications for the LHC are considered limited and feasible and in particular, the ramp speed can be increased as needed.

Reliability and availability studies have confirmed that operation can be optimised such that the FCC-hh collider will have an adequate availability for luminosity production. However, the power consumption of the ageing LHC cryogenic system is a concern. The required $80 \%-90 \%$ availability of the injector chain could best be achieved with a new high energy booster. As an alternative, direct injection from a new superconducting synchrotron at $1.3 \mathrm{TeV}$ that would replace the current $6.7 \mathrm{~km}$ long SPS could be considered. In this case, simpler normal conducting transfer lines with magnets operating at $1.8 \mathrm{~T}$ are sufficient. For this scenario more studies on beam stability in the collider at injection are required.

\section{Ion operation}

A first parameter set for ion operation has been developed based on current injector performance. If two experiments operate simultaneously for 30 days, one can expect an integrated luminosity in each of them of $6 \mathrm{pb}^{-1}$ and $18 \mathrm{pb}^{-1}$ for proton-lead ion operation with initial and nominal parameters, respectively. For lead-ion lead-ion operation $23 \mathrm{nb}^{-1}$ and $65 \mathrm{nb}^{-1}$ could be expected. More detailed studies are being carried out to address the key issues in ion production and collimation and to review the luminosity predictions. 

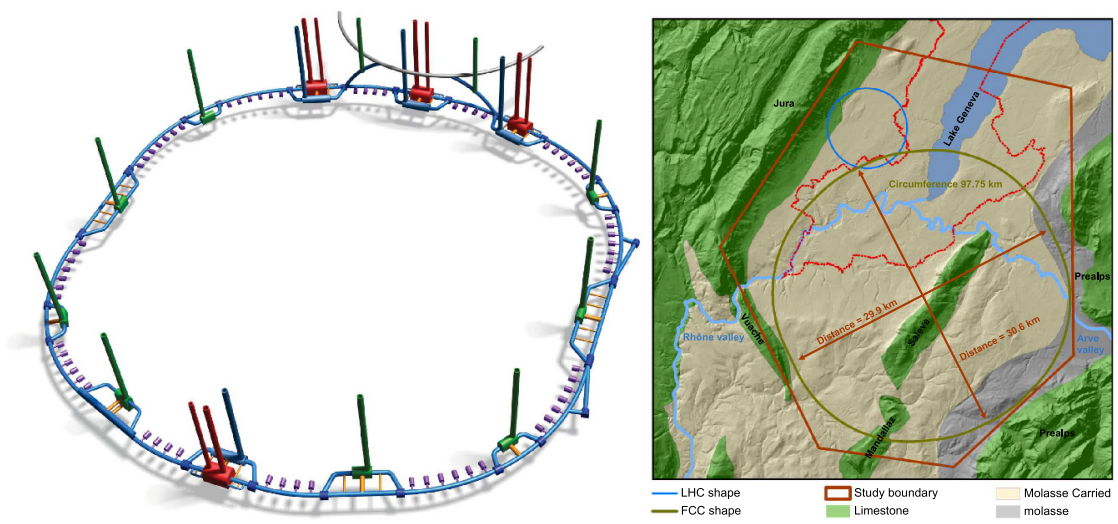

Fig. 2. Left: 3D, not-to-scale schematic of the underground structures. Right: study boundary (red polygon), showing the main topographical and geological structures, LHC (blue line) and FCC tunnel trace (olive green line).

\section{Civil engineering}

The principal structure of the FCC-hh collider is a quasi-circular tunnel composed of arc segments interleaved with straight sections with a diameter of $5.5 \mathrm{~m}$ and a circumference of $97.75 \mathrm{~km}$. Approximately $8 \mathrm{~km}$ of bypass tunnels, 22 shafts, 14 large caverns and 12 new surface sites are also planned. On the left Figure 2 shows a 3D, not-to-scale schematic of the underground structures. The chosen layout satisfies the requirements of the FCC-hh and the FCC-ee machines.

Many different considerations played a role in choosing a suitable position for the machine. The underground structures should be located as much as possible in the sedimentary rock of the Geneva basin, known as Molasse (which provides good conditions for tunnelling) and avoid the limestone of the nearby Jura. Another aim was to limit the depth of the tunnel and shafts to control the overburden pressure on the underground structures and to limit the lengths of service infrastructure (cables, ducts, pipes). These requirements, along with the need to connect to the existing accelerator chain through new beam transfer lines, led to the definition of the study boundary, within the Jura range to the north-west, the Vuache mountain to the south-west and the Pre-Alps to the south-east and east. An additional boundary is placed to the north due to the increasing depth of Lake Geneva (see Fig. 2 right).

In order to evaluate different layouts and positions within the boundary area, a software tool incorporating a 3D geological model was developed and used. The tunnel will be constructed with a slope of $0.2 \%$ in a single plane, partly to optimise the geology intersected by the tunnel and the shaft depths, and partly to implement a gravity drainage system. It is anticipated that the majority of the machine tunnel will be constructed using tunnel boring machines. One sector passing through limestone will be mined. Different lining designs have been developed corresponding to the excavations' rock conditions.

The study was based on geological data from previous projects and data available from national services. Based on this information, the civil engineering project is considered feasible, both in terms of technology and project risk control. Dedicated ground and site investigations are required during the early stage of the preparatory phase to confirm the findings, to provide a comprehensive technical basis for an optimised placement and as preparation for project planning and implementation processes. 


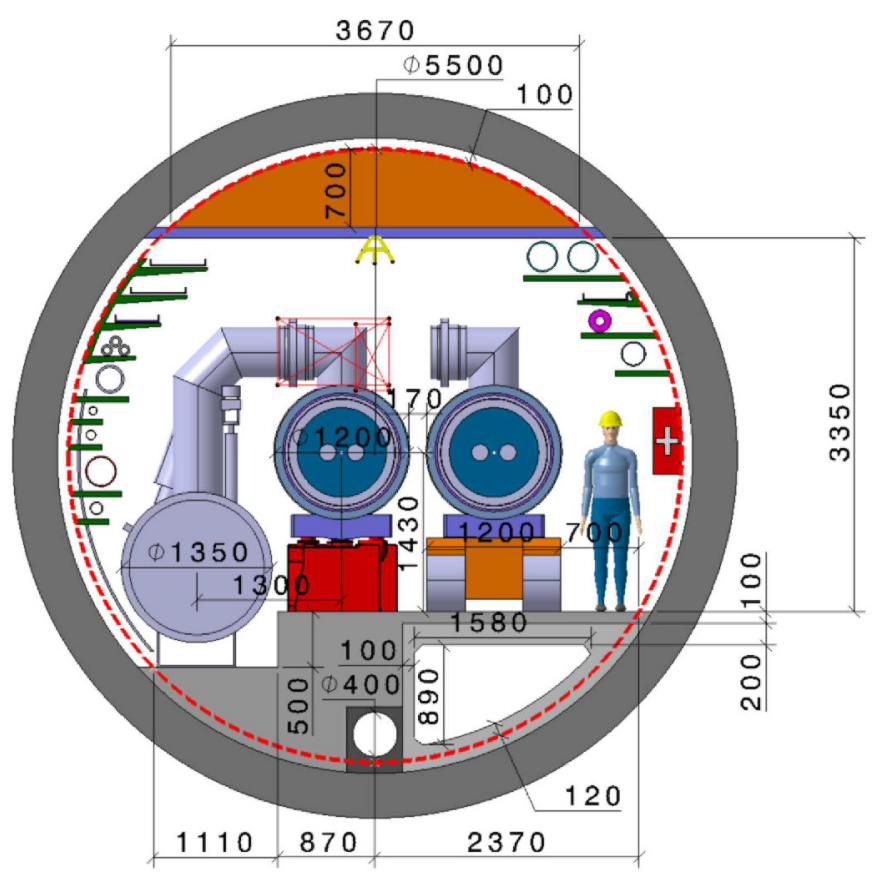

Fig. 3. Cross section of an FCC-hh arc. The grey equipment on the left side of the tunnel represents the cryogenic distribution line. A $16 \mathrm{~T}$ superconducting magnet can be seen in the middle, mounted on a red support element. Another superconducting magnet on a transport vehicle is shown next to it, in the transport passage.

For the access points and their associated surface structures, the focus has been on identifying possible locations which are feasible from socio-urbanistic and environmental perspectives. The construction methods, and hence the technical feasibility of construction have been studied and are deemed achievable.

A $5.5 \mathrm{~m}$ internal diameter tunnel is required to house all necessary equipment for the machine, while providing sufficient space for transport (this diameter is also compatible with the FCC-ee requirements). Figure 3 shows the cross section of the tunnel in a typical arc segment, including the integrated air supply and smoke extraction ducts, the cryogenic distribution line as well as the other services required.

\section{Detector considerations}

A $100 \mathrm{TeV}$ proton collider is a discovery machine as well as a precision measurement machine. The factor 7 higher energy over the present LHC increases the mass reach by a similar factor. The much larger cross sections for SM processes together with the higher luminosity lead to a significant increase in measurement precision. The detector must therefore be able to measure multi-TeV jets, leptons and photons from heavy resonances with masses up to $50 \mathrm{TeV}$, while at the same time be able to measure the known SM processes with high precision and be sensitive to a broad range of BSM signatures at moderate $p_{T}$. Since the SM particles are at low mass compared to the $100 \mathrm{TeV}$ collision energy, many SM processes show significant forward boost while still having transverse momentum distributions that are comparable to LHC energies. A detector for $100 \mathrm{TeV}$ must therefore increase the acceptance for precision tracking and calorimetry to $|\eta| \approx 4$, and keep at the same time the $p_{T}$ thresholds 


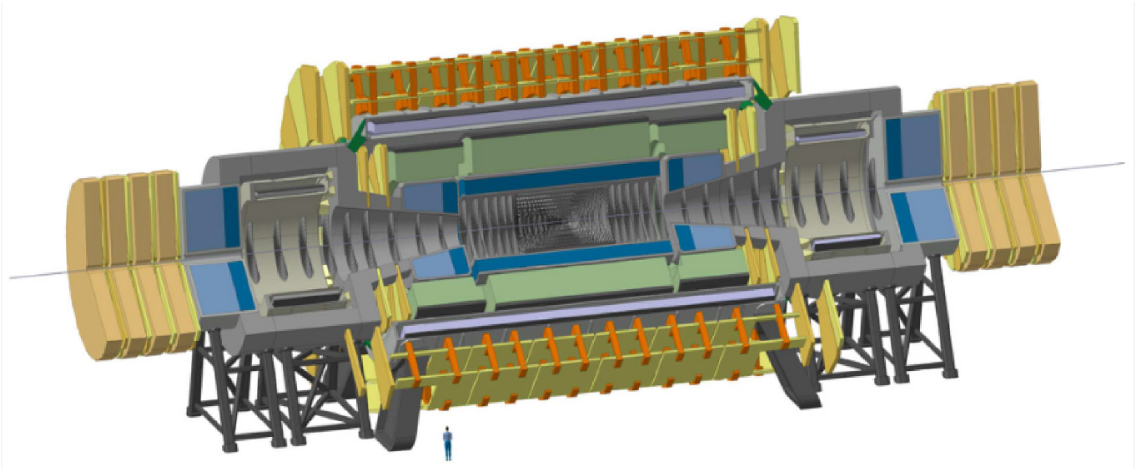

Fig. 4. The FCC-hh reference detector with an overall length of $50 \mathrm{~m}$ and a diameter of $20 \mathrm{~m}$. A central solenoid with $10 \mathrm{~m}$ diameter bore and two forward solenoids with $5 \mathrm{~m}$ diameter bore provide a $4 \mathrm{~T}$ field for momentum spectroscopy in the entire tracking volume.

for triggering and reconstruction at levels close to the present LHC detectors. The large number of p-p collisions per bunch-crossing, leading to the so called "pile-up", imposes stringent criteria on the detector design. While the present LHC detectors have to cope with pile-up numbers up to 60 , the HL-LHC will produce values of up to 200. The projected value of 1000 for the FCC-hh creates a technological challenge that needs to be addressed with novel approaches. Continuing technology advancements, specifically in the context of high precision timing detectors, will likely allow such numbers to be handled.

Figure 4 shows the conceptual FCC-hh reference detector. It does not represent a specific technical design for an implementation, but it serves as a concrete example used for subsystem and physics studies to identify those topics where dedicated R\&D efforts are needed. The detector has a diameter of $20 \mathrm{~m}$ and a length of $50 \mathrm{~m}$, comparable to the dimensions of the ATLAS detector at the LHC. The central detector with coverage of $|\eta|<2.5$ houses the tracking, electromagnetic calorimetry and hadron calorimetry inside a $4 \mathrm{~T}$ solenoid with a free bore diameter of $10 \mathrm{~m}$. In order to reach the required performance for $|\eta|>2.5$, the forward parts of the detector are displaced from the interaction point along the beam axis and two forward magnet coils with an inner bore of $5 \mathrm{~m}$ provide the required bending power. These forward magnets are also solenoids with a $4 \mathrm{~T}$ field, providing a total solenoid volume of $32 \mathrm{~m}$ length for high precision momentum spectroscopy up to rapidity values of $|\eta| \approx 4$ and tracking up to $|\eta| \approx 6$. An alternative consisting of 2 dipole magnets placed in the forward regions with a field integral along the $z$-axis of about $4 \mathrm{Tm}$, similar to the value for the ALICE and LHCb dipoles, is also studied. Although such a magnet system shows better tracking performance in the very forward region, the fact of losing the rotational symmetry and the requirement of a compensation system for the hadron beam are seen as drawbacks. The tracker is specified to provide better than $20 \%$ momentum resolution for $p_{T}=10 \mathrm{TeV} / \mathrm{c}$ for heavy $Z^{\prime}$ type particles and better than $0.5 \%$ momentum resolution at the multiple scattering limit at least up to $|\eta|=3$.

The tracker cavity has a radius of $1.7 \mathrm{~m}$ with the outermost layer at around $1.6 \mathrm{~m}$ from the beam in the central and the forward regions, providing the full spectrometer arm up to $|\eta|=3$. The electromagnetic calorimeter (EMCAL) uses a thickness of around 30 radiation lengths $\left(X_{0}\right)$ and together with the hadron calorimeter (HCAL) provides an overall calorimeter thickness of more than 10.5 nuclear interaction lengths $(\lambda)$ to ensure $98 \%$ containment of high energy showers and to limit punch-through to the muon system. 
The EMCAL is based on liquid argon (LAr) due to its intrinsic radiation hardness. The barrel HCAL is a scintillating tile calorimeter with steel and $\mathrm{Pb}$ absorbers, divided into a central barrel and two extended barrels. The HCALs for the endcap and forward regions are also based on LAr. The requirement of calorimetry acceptance up to $|\eta| \approx 6$ translates into an inner active radius of only $8 \mathrm{~cm}$ at a $z$-distance of $16.6 \mathrm{~m}$ from the IP. The EMCAL is specified to have an energy resolution around $10 \% / \sqrt{E}$ and the hadron calorimetry around $50 \% / \sqrt{E}$ for single particles.

The considerations for the muon system have a significant impact on the overall detector design. When the LHC detectors were designed, it was still unclear whether precision tracking close to the interaction point would be possible. Therefore significant emphasis was put on the standalone performance of muon systems. Today there is little doubt that large scale silicon trackers will be core parts of future detectors. Hence, the emphasis on standalone muon performance is less pronounced and the focus should be on the aspects of muon trigger and muon identification.

The reference detector does not assume any shielding of the magnetic field. A massive iron yoke, in this case much heavier than the one used for CMS at the LHC, has significant cost and weight for such a large magnet system and alternative solutions with active shielding coils increase the system size to dimensions that require access shafts in excess of $25 \mathrm{~m}$ diameter for lowering these coils to the detector cavern. The unshielded coil can be lowered through a shaft of $15 \mathrm{~m}$ diameter and the detector can be installed in a cavern of $37 \mathrm{~m}$ height and $35 \mathrm{~m}$ width, similar to the present ATLAS cavern. The magnetic stray field reaches $5 \mathrm{mT}$ at a radial distance of $50 \mathrm{~m}$ from the beamline. Therefore there is no relevant stray field in the service cavern, which is placed at a distance of $50 \mathrm{~m}$ from the experiment cavern and separated by rock. The shower and absorption processes inside the forward calorimeter produce a large quantity of low energy neutrons of which a significant fraction enters the tracker volume. In order to keep these neutrons from entering the muon system and the detector cavern, a heavy radiation shield is placed around the forward solenoid magnets to close the gap between the endcap and forward calorimeters.

The significant radiation levels create strong constraints on the technologies for the various subsystems. On the first silicon layer at $r=2.5 \mathrm{~cm}$ the charged particle rate is around $10 \mathrm{GHz} / \mathrm{cm}^{2}$. The number drops to about $3 \mathrm{MHz} / \mathrm{cm}^{2}$ at the outer radii of the tracker. Inside the forward EMCAL the number rises to levels of $100 \mathrm{GHz} / \mathrm{cm}^{2}$.

The $1 \mathrm{MeV}$ neutron equivalent fluence ( $1 \mathrm{MeV}$-neq) which is a key number for long term damage of silicon sensors and electronics in general evaluates to a value of $6 \times 10^{17} / \mathrm{cm}^{2}$ for the first silicon layer. Beyond a radius of $40 \mathrm{~cm}$ the number drops below $10^{16} / \mathrm{cm}^{2}$ and in the outer parts of the tracker this number is around $5 \times 10^{15} / \mathrm{cm}^{2}$. Technologies used for the HL-LHC detectors are therefore applicable to radii $>40 \mathrm{~cm}$. Novel sensors and readout electronics have to be developed for the innermost parts of the tracker.

The charged particle rate in the muon system is dominated by electrons, created from high energy photons in the $\mathrm{MeV}$ range. These photons are created by processes related to thermalisation and capture of neutrons that are produced in hadron showers mainly in the forward region. These processes immerse the muon system and the cavern in a "gas" of high energy photons that convert to electrons in the muon system material. In the barrel muon system and the outer endcap muon system the charged particle rate does not exceed $500 \mathrm{~Hz} / \mathrm{cm}^{2}$, the rate in the inner endcap muon system goes up to $10 \mathrm{kHz} / \mathrm{cm}^{2}$ and in the forward muon system the rate increases to $500 \mathrm{kHz} / \mathrm{cm}^{2}$ at a distance of $1 \mathrm{~m}$ from the beam. These rates are comparable to those in the ATLAS, CMS and LHCb muon systems [9-11] and gaseous detectors used in these experiments can therefore be adopted in these regions. 


\section{Cost and schedule}

The construction cost for FCC-hh as a single project (i.e. without prior construction of an FCC-ee lepton collider) amounts to 24000 million CHF for the entire project. All particle collider and injector related investments amount to 13600 million CHF or $57 \%$ of the total cost. The major part of the accelerator cost corresponds to the $4700 \mathrm{Nb}_{3} \mathrm{Sn} 16 \mathrm{~T}$ main dipole magnets, totalling $9400 \mathrm{MCHF}$, at a cost target of 2 $\mathrm{MCHF} /$ magnet. Civil engineering accounts for 25\% (6000 million CHF). The capital cost for the technical infrastructure is 4400 million CHF.

If the FCC-hh project is preceded by an intensity-frontier lepton collider (FCCee), both, civil engineering and general technical infrastructures can be fully reused for this project, thus substantially lowering the necessary investments to $17000 \mathrm{MCHF}$. The operation costs are expected to remain within limits, since the evolution from LEP to LHC operation today shows a steady decrease in the effort needed to operate, maintain and repair the equipment. The cost-benefit analysis of the LHC/HL-LHC programme reveals that a research infrastructure project of such a scale and hitech level has the potential to generate significant socio-economic value throughout its lifetime, in particular if the tunnel, surface and technical infrastructures from a preceding project have been amortised. For such a scenario, a detailed assessment remains to be performed.

The FCC-hh programme will commence with a preparatory phase of 8 years, followed by the construction phase (all civil and technical infrastructure, machines and detectors including commissioning) lasting 15 years. A duration of 25 years is projected for the subsequent operation of the FCC-hh facility, to complete the currently envisaged physics programme. This makes a total of nearly 50 years for construction and operation of FCC-hh as a standalone project.

As a combined project (FCC-ee followed by FCC-hh), the FCC covers a total of 70 years, i.e. until the end of the 21 st century.

\section{Outlook}

The technology for constructing a high-energy circular hadron collider can be brought to the technology readiness level required for constructing within the next ten years through a focused R\&D programme. The FCC-hh baseline concept comprises a power-saving, low-temperature superconducting magnet system based on an evolution of the $\mathrm{Nb}_{3} \mathrm{Sn}$ technology pioneered at the HL-LHC, an energy-efficient cryogenic refrigeration infrastructure based on a neon-helium (nelium) light gas mixture, a high-reliability and low loss cryogen distribution infrastructure based on Invar, highly segmented kicker, superconducting septa and transfer lines and local magnet energy recovery, and use of technologies that are already being gradually introduced at other CERN accelerators. On a longer time scale, high-temperature superconductor R\&D together with industrial partners has the potential to achieve an even more energy efficient particle collider or to reach even higher collision energies. The reuse of the entire CERN accelerator chain, which also serve a concurrent physics programme, is an essential lever to come to an overall sustainable research infrastructure at the energy frontier.

Strategic R\&D for FCC-hh aims at minimising construction cost and energy consumption, while maximising the socio-economic impact. It will mitigate technologyrelated risks and ensure that industry can benefit from an acceptable utility. For the implementation, a preparatory phase of about eight years is both necessary and adequate to establish the project governing bodies and organisational structures, to build the international machine and experiment consortia, to develop a territorial 
implantation plan in agreement with the host states' requirements, to optimise the disposal of land and underground volumes, and to prepare the civil engineering project.

Such a large-scale, international fundamental research infrastructure, tightly involving industrial partners and providing training at all education levels, will be a strong motor of economic and societal development in all participating nations. The FCC study has implemented a set of actions towards a coherent vision for the worldwide high-energy and particle physics community, providing a collaborative framework for topically complementary and geographically well-balanced contributions. This conceptual design report lays the foundation for a subsequent infrastructure preparatory and technical design phase.

\section{Physics opportunities and reach}

\subsection{Introduction}

After answering the question "where is the Higgs?" with the LHC, particle physics is now faced with an even more challenging question: "what is next and where is it?". Additional particles and interactions must extend the Standard Model (SM), to explain for example, the existence of dark matter (DM), neutrino masses and the observed matter/antimatter asymmetry. The SM itself calls for a broader theoretical framework, to provide a rationale for the dynamical origin of electroweak (EW) symmetry breaking (EWSB) and to justify the otherwise unnatural fine tuning needed to prevent quantum corrections pushing the Fermi scale up to the Planck scale. Most of the theories proposed in the last few decades require these extensions of the SM to appear at the $\mathrm{TeV}$ scale, predicting that the LHC would discover new particles beyond the Higgs.

That such predictions have not been fulfilled so far, can be due to two reasons: either the scale of new physics is higher than expected, possibly well beyond what even the HL-LHC will be able to explore; or this scale is indeed within the LHC's reach, but the related phenomena are very elusive, either because of their small rates, large backgrounds, or of the lack of outstanding and triggerable features. The FCC project, in its globality, aims to address both of these scenarios, by pushing the potential for discovery through enhanced sensitivity to elusive phenomena and by extending the mass reach through higher precision and larger energy. The FCC-hh, defined by the target of $100 \mathrm{TeV}$ proton-proton collisions with a total integrated luminosity of 20-30 $\mathrm{ab}^{-1}$, has a unique and all encompassing role: it extends the LHC mass reach for direct observation of new phenomena by nearly an order of magnitude, and enriches the indirect sensitivity of lepton collider FCC-ee through its own complementary potential for precision measurements.

While data in the next few years (from the LHC, DM experiments, flavour and neutrino facilities, etc.) will hopefully provide evidence of new phenomena at energy scales well below $100 \mathrm{TeV}$ and possibly within the range of HE-LHC, the high energy physics community must meet the challenge of planning for a future collider at the highest energies compatible with technological and financial realities. The FCC-hh $100 \mathrm{TeV}$ pp collider fulfils this ambition and, as discussed in the following, is properly dimensioned in terms of energy and luminosity, to achieve key scientific goals [12].

Aside from its fundamental role in the exploration of new physics beyond the SM (BSM), the FCC-hh will provide the infrastructure required for a broader and diverse research programme, including the continued study of the proton structure and of strong interactions at the highest densites and temperature. 


\subsection{Why $100 \mathrm{TeV}$ ?}

While the LHC and other experiments do not concretely point to any specific BSM scenario and mass scale today, rather general arguments support $100 \mathrm{TeV}$ as a sensible target for FCC-hh. There is clearly no upper limit to how high the community would like the energy to be. If $120 \mathrm{TeV}$ could be achievable, FCC-hh should go for it. But there is evidence that $100 \mathrm{TeV}$ is necessary and sufficient to achieve crucial measurements and give clear yes/no answers to some of the important questions that might still be left open after the HL-LHC and to explore scenarios that could emerge from the FCC-ee phase. The basis for this evidence is summarised here. Additional details are provided in the following sections, and a more complete discussion is contained in Volume 1 of the Conceptual Design Report (CDR).

\subsubsection{The nature of the Higgs potential}

Until new physics is found, the complete exploration of Higgs properties will remain the top priority of high energy physics. Progress is rapidly being made by the LHC in improving the knowledge of Higgs couplings, but a precise answer to two key issues will remain open even after the HL-LHC and the FCC-ee: how does the Higgs couple to itself? What was the nature of the phase transition that, approximately $10^{-10} \mathrm{~s}$ after the Big Bang, accompanied EWSB and the creation of the Higgs vacuum expectation value? This last question has implications for the possibility that this EW phase transition (EWPT), if strongly of 1st order, played a role in establishing the observed excess of matter over antimatter, and on the possible release of detectable gravitational waves generated by the collision of bubbles of the new vacuum during the Big Bang. The questions of the Higgs self-coupling and of the nature of the EWPT are tightly related, since their answer depends on the parameters of the Higgs potential. These are fixed in the SM, but could be altered in BSM scenarios. These modifications to the SM are necessary to achieve a strong 1st order phase transition. FCC-hh at $100 \mathrm{TeV}$ has a $5 \%$ precision target for the measurement of the Higgs cubic self-coupling in the SM (the precision could be better in BSM models with an enhanced self-coupling), whereas the HL-LHC will only be sensitive to $\mathcal{O}(50 \%)$ deviations from the SM. On the basis of the BSM models considered so far, this $5 \%$ precision, together with the FCC-hh discovery reach for additional particles that could affect the EWSB transition, have been shown to be necessary and sufficient to decide whether the EW phase transition was of strong 1st order or not.

\subsubsection{Dark matter}

Very different scenarios exist to explain the origin of DM, ranging from sub-eV axion particles to the class of heavy black holes whose merging was recently detected by gravitational antennas. An important large class of models is that of thermal weakly interacting massive particles (WIMPs): the DM abundance observed is in fact consistent with the densities predicted by the cosmological evolution of a class of particles coupled to the SM via weak interactions and with masses of order of the weak scale. Thermal WIMPs are the targets of both direct and indirect detection searches in underground and space facilities. Possible signals detected by these facilities would call for a direct confirmation by accelerators. Furthermore, accelerators are needed to cover possible blind spots in the direct/indirect searches. The conclusive confirmation or rejection of the thermal WIMP hypothesis by accelerator experiments is therefore a fundamental step in the understanding of the origin of DM. 
Thermal WIMPs cannot be too heavy, or their cosmological abundance would exceed the DM density. The upper limit of their mass is in the range of $1-3 \mathrm{TeV}$, depending on whether they couple via weak or Higgs bosons. Due to their weak coupling and small production rates, only a fraction of this mass range is within the reach of the LHC. As discussed below, the $100 \mathrm{TeV}$ of FCC-hh are necessary and just sufficient, to fully cover the rest.

\subsubsection{Finding the origin of new physics exposed by indirect evidence}

Most of what is known about the EW gauge bosons, the Higgs and the top quark was learned by producing them directly, even though their existence had been clearly substantiated indirectly. Future evidence for the existence of heavier particles, even if coming indirectly from flavour observables or precision EW and Higgs observables, will likewise require direct production to precisely determine their properties. This will be much more the case in the future than it has been for the top quark or the Higgs, when the SM provided a unique and robust interpretation framework to extract their properties from indirect measurements.

As discussed in more detail in Volume 1, precision measurements at FCC-ee will be sensitive to possible new phenomena at scales of tens of $\mathrm{TeV}$. New physics at these scales can be revealed in the near future by flavour phenomena, as suggested by the studies of the current flavour anomalies in B decays, and might therefore call for direct searches of massive particles possibly well beyond the LHC reach. Direct particle production at the scale of a few tens $\mathrm{TeV}$ can only be achieved, within the current technological landscape, by a hadron collider in the energy range of $100 \mathrm{TeV}$.

\subsection{Setting the luminosity goals}

Cross sections for the production of a state of mass $M$ typically scale like $1 / M^{2}$. The FCC-hh luminosity should therefore increase w.r.t. the LHC by a factor of $(100 / 14)^{2} \sim 50$, for its discovery reach to be sensitive to masses 100/14 7 times larger than at the LHC. The baseline FCC-hh integrated luminosity of 20-30 ab-1 exceeds what is obtained with this factor, if rescaled from $300 \mathrm{fb}^{-1}$, the target of the nominal LHC luminosity [13] programme. As argued in [14], this is sufficient to practically saturate the discovery reach at the highest masses, since a further increase of the luminosity by a factor of 10 beyond these values would only extend the discovery reach by less than $20 \%$.

Statistics are crucial to improve the measurements' precision and to increase the sensitivity to rare or elusive processes. The $20-30 \mathrm{ab}^{-1}$ goal is sufficient to push the target precision below the percent level for several Higgs couplings that cannot

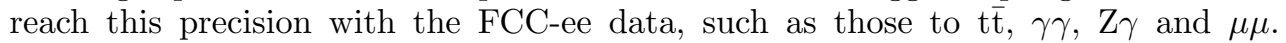
This luminosity allows also to measure the Higgs cubic self-coupling with a precision around 5\%. More in general, the goals highlighted in the previous section can all be achieved with this luminosity. Specific measurements may set more aggressive luminosity goals, but generic arguments to justify them have not been found. The needs of precision physics arising from new physics scenarios to be discovered at the HL-LHC, to be suggested by anomalies observed by FCC-ee, or to be discovered at $100 \mathrm{TeV}$, may well drive the need for even higher statistics. While extra statistics are always welcome, there is, however, no strong argument today that calls for an integrated luminosity higher than indicated here.

Furthermore, for a large class of new-physics scenarios that may arise from the LHC, less ambitious luminosity goals are acceptable as a compromise between physics 

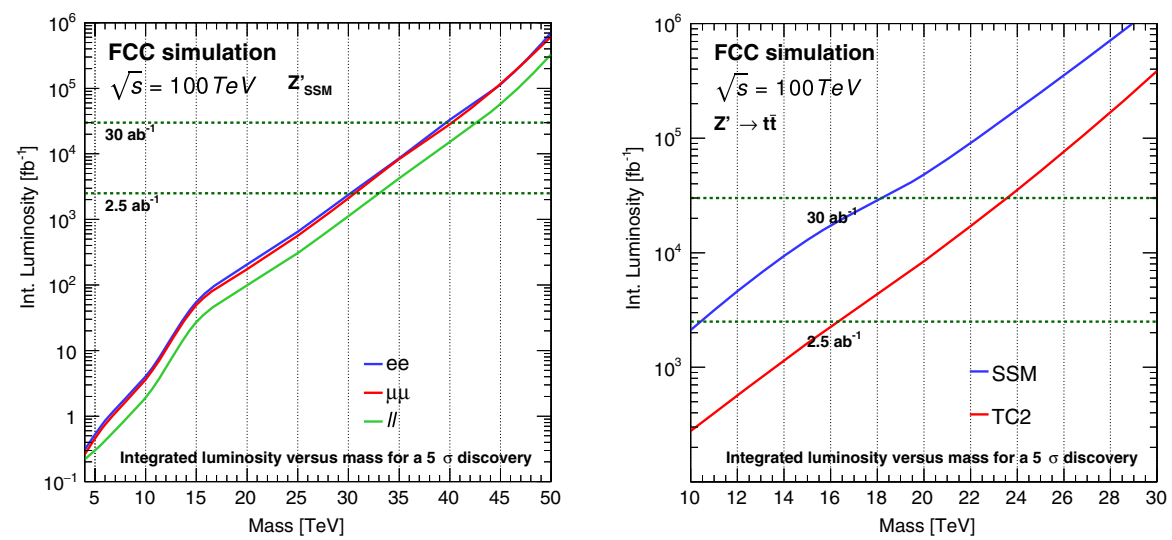

Fig. 1.1. Integrated luminosity required for a $5 \sigma$ discovery, as a function of the mass, for a $\mathrm{Z}^{\prime}$ gauge boson coupled with SM couplings and decaying to leptons (left) and to t $\overline{\mathrm{t}}$ (right).

return and technical or experimental challenges. In particular, even luminosities of few $\mathrm{fb}^{-1}$ are enough to greatly extend the discovery reach of the $100 \mathrm{TeV}$ collider over that of the HL-LHC, or to enhance the precision in the measurement of discoveries made at the HL-LHC. Note that, while the luminosity goal of the accelerator is $20 \mathrm{ab}^{-1}$, most of the analyses done in the context of the CDR studies use $30 \mathrm{ab}^{-1}$, assuming that the results of two experiments will be at least partly combined, for the most important analyses limited by statistics.

Finally, the luminosity goals of FCC-hh make it also possible to achieve the $2 \mathrm{ab}^{-1}$ integrated luminosity that defines the target of the FCC-eh programme, as discussed below.

\subsection{Discovery reach at the highest masses}

The increase in centre of mass energy with respect to the LHC obviously leads to a comparable extension of the discovery reach. Some concrete examples are given below. Figure 1.1 shows the luminosity required to discover a SM-coupled $\mathrm{Z}^{\prime}$ gauge boson, in its leptonic decay, as a function of its mass. The projection, described in detail in [15], is based on the simulation of the baseline FCC detector presented in Chapter 7. The reach extends to $43 \mathrm{TeV}$ at the ultimate integrated luminosity of $30 \mathrm{ab}^{-1}$. A few tens of $\mathrm{fb}^{-1}$, within the reach of the first year of data taking, are sufficient to exceed the discovery reach of the HL-LHC by a factor of 2 . The figure also shows the impact of increased luminosity: a factor of $\sim 10$, from 2.5 to $30 \mathrm{ab}^{-1}$, leads to an increase in reach by over $30 \%$, while going from 30 to $300 \mathrm{ab}^{-1}$ reduces the further gain to less than $20 \%$. Requiring a comparable sensitivity in the electron and muon channels (as shown in Fig. 1.1), sets the resolution requirement for the muon $p_{T}$ to the $\sim 20 \%$ level at $p_{T}=10 \mathrm{TeV}$, which is implemented in the detector design presented in Chapter 7. The right plot in Figure 1.1 shows the results for

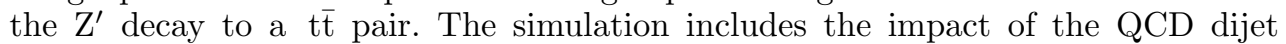
and $t \bar{t}$ continuum backgrounds, and exploits the detector calorimetric granularity (for the reconstruction of the top-jet substructure and the suppression of the dijet backgrounds) and of the tracking performance (for the b-tagging). The reach extends to a $\mathrm{Z}^{\prime}$ mass value of $17(23) \mathrm{TeV}$ for $2.5(30) \mathrm{ab}^{-1}$. All studies performed so far (e.g. for supersymmetric particles such as squarks, gluinos or stops [16]) show a similar increase by factors of 5 or larger in the discovery reach, with respect to the reach of the HL-LHC. 
Table 1.1. Higgs production event rates for selected processes at $100 \mathrm{TeV}\left(N_{100}\right)$ and statistical increase with respect to the statistics of the HL-LHC $\left(N_{100}=\sigma_{100 \mathrm{TeV}} \times 30 \mathrm{ab}^{-1}\right.$, $\left.N_{14}=\sigma_{14 \mathrm{TeV}} \times 3 \mathrm{ab}^{-1}\right)$.

\begin{tabular}{|l|r|c|c|c|c|c|}
\hline & $\mathrm{gg} \rightarrow \mathrm{H}$ & $\mathrm{VBF}$ & $\mathrm{WH}$ & $\mathrm{ZH}$ & $\mathrm{t} \overline{\mathrm{t}} \mathrm{H}$ & $\mathrm{HH}$ \\
\hline$N_{100}$ & $24 \times 10^{9}$ & $2.1 \times 10^{9}$ & $4.6 \times 10^{8}$ & $3.3 \times 10^{8}$ & $9.6 \times 10^{8}$ & $3.6 \times 10^{7}$ \\
$N_{100} / N_{14}$ & 180 & 170 & 100 & 110 & 530 & 390 \\
\hline
\end{tabular}

Table 1.2. Target precision for the parameters relative to the measurement of various Higgs decays, ratios thereof, and of the Higgs self-coupling $\lambda$.

\begin{tabular}{|l|c|c|c|}
\hline Observable & Parameter & $\begin{array}{c}\text { Precision } \\
\text { (stat) }\end{array}$ & $\begin{array}{c}\text { Precision } \\
\text { (stat+syst+lumi) }\end{array}$ \\
\hline$\mu=\sigma(\mathrm{H}) \times \mathrm{B}(\mathrm{H} \rightarrow \gamma \gamma)$ & $\delta \mu / \mu$ & $0.1 \%$ & $1.45 \%$ \\
$\mu=\sigma(\mathrm{H}) \times \mathrm{B}(\mathrm{H} \rightarrow \mu \mu)$ & $\delta \mu / \mu$ & $0.28 \%$ & $1.22 \%$ \\
$\mu=\sigma(\mathrm{H}) \times \mathrm{B}(\mathrm{H} \rightarrow 4 \mu)$ & $\delta \mu / \mu$ & $0.18 \%$ & $1.85 \%$ \\
$\mu=\sigma(\mathrm{H}) \times \mathrm{B}(\mathrm{H} \rightarrow \gamma \mu \mu)$ & $\delta \mu / \mu$ & $0.55 \%$ & $1.61 \%$ \\
$\mu=\sigma(\mathrm{HH}) \times \mathrm{B}(\mathrm{H} \rightarrow \gamma \gamma) \mathrm{B}(\mathrm{H} \rightarrow \mathrm{b} \overline{\mathrm{b}})$ & $\delta \lambda / \lambda$ & $5 \%$ & $7.0 \%$ \\
$R=\mathrm{B}(\mathrm{H} \rightarrow \mu \mu) / \mathrm{B}(\mathrm{H} \rightarrow 4 \mu)$ & $\delta R / R$ & $0.33 \%$ & $1.3 \%$ \\
$R=\mathrm{B}(\mathrm{H} \rightarrow \gamma \gamma) / \mathrm{B}(\mathrm{H} \rightarrow 2 \mathrm{e} 2 \mu)$ & $\delta R / R$ & $0.17 \%$ & $0.8 \%$ \\
$R=\mathrm{B}(\mathrm{H} \rightarrow \gamma \gamma) / \mathrm{B}(\mathrm{H} \rightarrow 2 \mu)$ & $\delta R / R$ & $0.29 \%$ & $1.38 \%$ \\
$R=\mathrm{B}(\mathrm{H} \rightarrow \mu \mu \gamma) / \mathrm{B}(\mathrm{H} \rightarrow \mu \mu)$ & $\delta R / R$ & $0.58 \%$ & $1.82 \%$ \\
$R=\sigma(\mathrm{t} \overline{\mathrm{t}} \mathrm{H}) \times \mathrm{B}(\mathrm{H} \rightarrow \mathrm{b} \overline{\mathrm{b}}) / \sigma(\mathrm{t} \overline{\mathrm{t}} \mathrm{Z}) \times \mathrm{B}(\mathrm{Z} \rightarrow \mathrm{b} \overline{\mathrm{b}})$ & $\delta R / R$ & $1.05 \%$ & $1.9 \%$ \\
$B(\mathrm{H} \rightarrow$ invisible $)$ & $B @ 95 \% \mathrm{CL}$ & $1 \times 10^{-4}$ & $2.5 \times 10^{-4}$ \\
\hline
\end{tabular}

Notes. Notice that Lagrangian couplings have a precision that is typically half that of what is shown here, since all rates and branching ratios depend quadratically on the couplings.

\subsection{Precision Higgs studies and the exploration of EWSB}

\subsubsection{Higgs couplings}

Two elements characterise the Higgs physics programme of FCC-hh: the large statistics (see Tab. 1.1), and the large kinematic range, which probes Higgs production at very large $p_{T}$. As shown in Table 1.2, these factors allow the measurement of Higgs couplings with (sub)percent-level precision that FCC-ee can probe with limited statistics and the precision of the Higgs self-coupling to below $10 \%$.

The results in Table 1.2 represent the target uncertainties due to statistics (taking into account analysis cuts, expected efficiencies, and the possible irreducible backgrounds) and to systematics (limited here to the identification efficiencies for the relevant final states, plus an overall $1 \%$ to account for luminosity and modelling uncertainties). The full details of the analyses are presented in [17]. While these estimates do not reflect the full complexity of the experimental analyses in the huge pileup environment of FCC-hh, the systematics assumptions that were used are rather conservative. The projections given here are considered to be reasonable targets for the ultimate precision and useful benchmarks to define the goals of the detector performance.

The $\mu$ parameters shown in Table 1.2 are typically affected by systematics related to the theoretical uncertainty in the production cross sections and the luminosity measurement. It is reasonable to expect that these will be reduced to the percent level by the time of operation. These systematics, however, cancel entirely in the ratio of branching ratios, which are derived from events with identical Higgs kinematics. The measurement of the $t \bar{t} \mathrm{H}$ process allows the extraction of the top Yukawa coupling, 

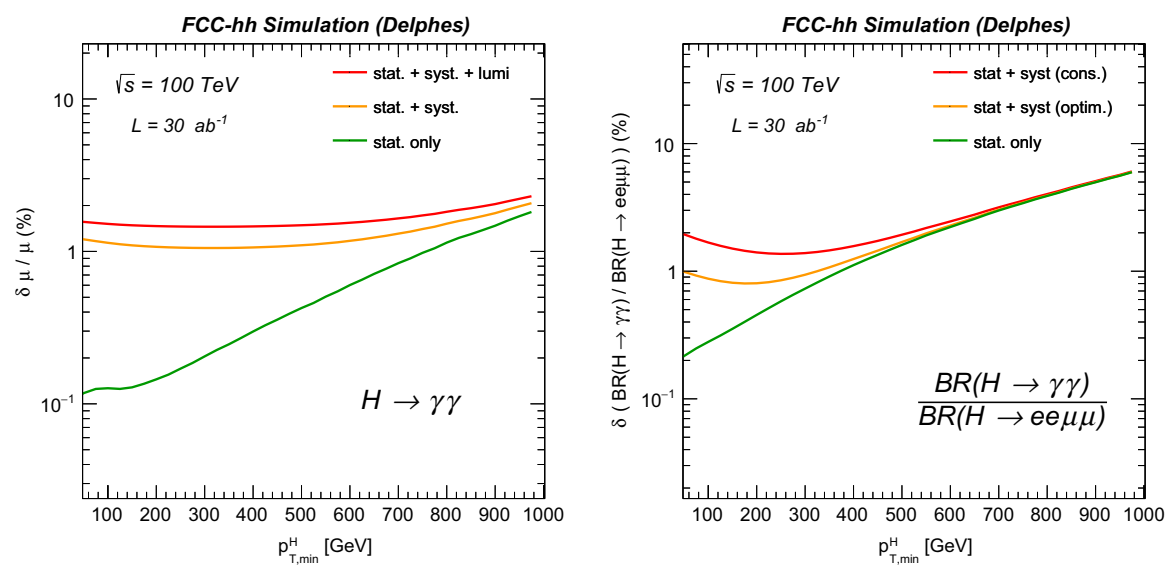

Fig. 1.2. Examples of projected precision for Higgs measurements with $30 \mathrm{ab}^{-1}$. Left: $\mathrm{H} \rightarrow$ $\gamma \gamma$ production rate at large $p_{T}$. Right: ratio of $\mathrm{H} \rightarrow \gamma \gamma$ and $\mathrm{H} \rightarrow 2 \mathrm{e} 2 \mu$ rates. The results labelled as "cons" are obtained by doubling the default systematic uncertainties for $\gamma$, e and $\mu$ efficiencies.

once $B(\mathrm{H} \rightarrow \mathrm{b} \bar{b})$ is known from FCC-ee. The ratio to the $\mathrm{t} \overline{\mathrm{t} Z}$ process allows the removal of the production and luminosity uncertainties. For the studies shown here, samples of Higgs bosons produced at large $p_{T}$ were used to reduce backgrounds and systematics. An example of the power of using such high- $p_{T}$ Higgses is given in Figure 1.2(left), which shows the expected precision of the measurements relative to Higgs produced above a given minimum $p_{T}$ and decaying to $\gamma \gamma$. The measurement of the ratio of $\gamma \gamma$ and 4 lepton final states, where production, luminosity and part of the detection systematics cancel, is shown as a function of $p_{T}(\min )$ in the right side of Figure 1.2. These measurements can be performed with a precision better than $10 \%$ up to $p_{T}$ values in excess of $1 \mathrm{TeV}$, allowing to probe the possible existence of higher-dimension operators affecting Higgs dynamics up to scales of several $\mathrm{TeV}$.

Note that the precision of the ratios of branching ratios allows the extraction of absolute determinations of the Higgs couplings, if use is made of the HZZ coupling measured by FCC-ee.

\subsubsection{The nature of the EW phase transition}

As noted above, mapping out the thermal history of EWSB addresses two important questions: was the matter-antimatter asymmetry generated in connection with EWSB? and should one anticipate the generation of gravitational waves (GW) that could be observed with future GW probes? If the EWPT was first order with sufficient strength, the answer to both questions may be "yes". Lattice studies imply that, for a $125 \mathrm{GeV}$ Higgs boson, the EWSB transition in the SM is of a cross-over type, thereby failing to provide the out-of-equilibrium conditions needed to create of the matter-antimatter asymmetry. On the other hand, BSM scenarios that contain new bosonic degrees of freedom that interact with the Higgs boson, could change the picture dramatically. A strongly first order phase transition (SFOPT) may arise through BSM-induced changes in the zero-temperature vacuum structure of the scalar potential and/or finite-temperature quantum corrections. In addition, the presence of "BSM Higgses" may allow a richer thermal history than in the SM universe, including the presence of new symmetry-breaking phases that preceded the present "Higgs phase". 
Searches for BSM scalars at the LHC have begun to explore this possibility, largely precluding a SFOPT if the new scalars - such as top squarks - have strong interactions. On the other hand, the presence of scalars that carry only electroweak quantum numbers (EW multiplets) or no SM quantum numbers at all (singlets), is more challenging to observe at the LHC, even by the end of the HL-LHC. Direct production cross sections can be as small as a few $\mathrm{fb}$ in regions of parameter space consistent with a SFOPT. Studies completed to date, however, indicate that the FCC-hh with $30 \mathrm{ab}^{-1}$ should provide a definitive probe of these new states in these regions of parameter space. Indirect effects entering through modifications of the Higgs self-coupling or the rate for $\mathrm{H} \rightarrow \gamma \gamma$ may be observable at the HL-LHC or even the HE-LHC, but can be as small as a few percent, pointing to the precision achievable only with the FCC-hh.

The simplest illustration of these possibilities is the extension of the SM scalar sector with a single real singlet scalar, the "xSM" [18]. Singlet scalars occur copiously in ultraviolet-complete embeddings of the SM, but it is not essential to examine the additional degrees of freedom and interactions for the identification of the EWPT dynamics. The xSM contains two Higgs-like scalars, $\mathrm{h}_{1}$ and $\mathrm{h}_{2}$ that are admixtures of the neutral component of the SM Higgs doublet and the singlet. Unlike the pure $\mathrm{SM}$ case, the associated interactions in the scalar potential can readily lead to a SFOPT when the SM-like state $\mathrm{h}_{1}$ has a mass of $125 \mathrm{GeV}$. The associated collider signatures include modifications of the Higgs self-coupling, which may be as small as a few percent; a shift in the associated production $\left(\mathrm{Zh}_{1}\right)$ cross section; and direct production of two SM-like scalars $h_{1}$ through an on-shell $h_{2}$. Each $h_{1}$ then decays to the conventional Higgs boson decay products, yielding various combinations. The possibilities for discovery through the "resonant di-Higgs production" process are illustrated in Figure 1.3, where the results are obtained by combining the $4 \tau$ and $\mathrm{b} \overline{\mathrm{b}} \gamma \gamma$ final states [19]. Each coloured band gives the projected significance $N_{\sigma}$ of observation as a function of the $\mathrm{h}_{2}$ mass, with the $N_{\sigma}$ range obtained by varying over all other model parameters consistent with a SFOPT, constraints from electroweak precision observables and present LHC Higgs signal strength determinations. The maximum $\mathrm{h}_{2}$ mass consistent with a SFOPT is just below $900 \mathrm{GeV}$. As one can see, the HL-LHC discovery potential is limited to a relatively modest portion of the light $\mathrm{h}_{2}$ parameter space, whereas the FCC-hh with $30 \mathrm{ab}^{-1}$ would enable discovery over the entire first order EWPT-viable parameter space. Complementary parameter scenarios, where the dominantly-singlet $\mathrm{h}_{2}$ boson is stable and invisible, can give rise to missing $E_{T}$ signatures in (possibly off-shell) Higgs decays and to departures in the Higgs self-coupling at one-loop. The FCC-hh target energy and luminosity have been shown to be adequate to fully cover such scenarios as well [20].

\subsection{Dark matter searches}

The FCC-hh would play a crucial role in the ongoing search for dark matter. As a direct consequence of its increased center-of-mass-energy, it will be the first collider capable of producing weakly-interacting particles with masses up to a few TeV. For example, SU(2) triplet DM, also known as "wino" DM, has a thermal relic mass of $\sim 2-3 \mathrm{TeV}$ and will not be found except by very futuristic direct detection experiments, which probe DM-nucleon cross sections down to the neutrino floor. While traditional monojet DM searches at FCC-hh would only uncover wino DM up to $\sim 0.5 \mathrm{TeV}$ mass, using disappearing charged track searches, the FCC-hh would discover winos up to $3 \mathrm{TeV}$ in mass, with $3 \mathrm{ab}^{-1}$ luminosity [21,22]. These conclusions are corroborated by the detailed study of the disappearing track signatures in reference [23], which simulated the performance of possible tracking layouts in the presence of the large FCC-hh pile-up. 


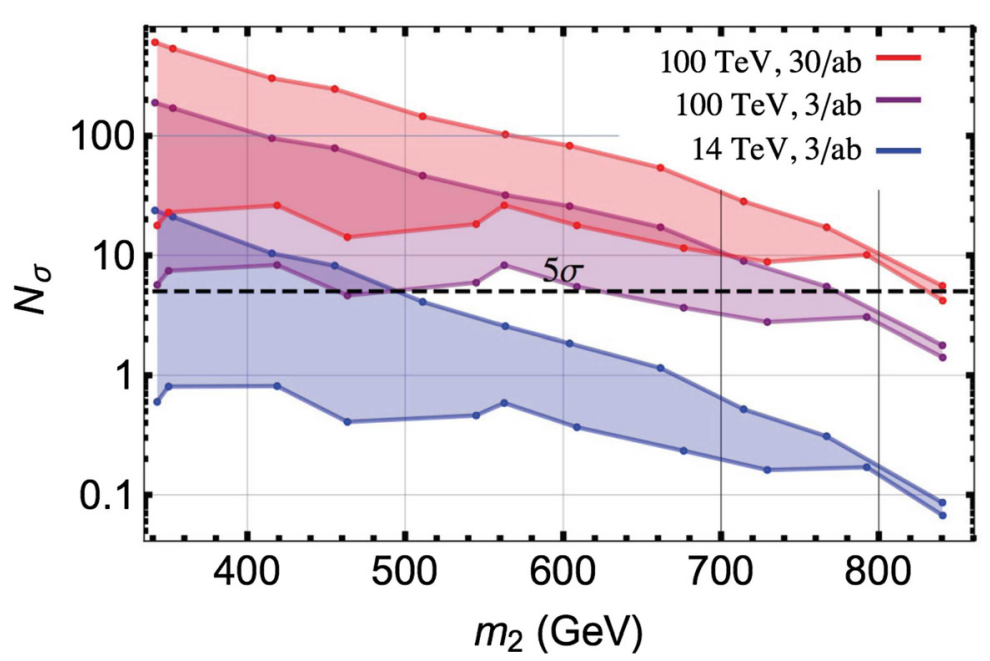

Fig. 1.3. Discovery potential for the singlet-induced SFOPT using resonant di-Higgs production combining $4 \tau$ and $b b \gamma \gamma$ final states [19]. The vertical axis gives the significance as a function of the singlet-like scalar mass $m_{2}$ for the HL-LHC (blue band) and the FCC-hh with $3 \mathrm{ab}^{-1}$ and $30 \mathrm{ab}^{-1}$ (purple and red bands, respectively).

FCC-hh's role in uncovering weakly-interacting DM is reinforced by its coverage of "simplified" DM models, which couple to SM particles via new scalar and vector currents. For example, Dirac fermion DM, universally coupled to SM fermions through an axial-vector mediator, can be found in multijet searches, for mediator masses up to $23 \mathrm{TeV}$ and Dirac DM masses up to $3 \mathrm{TeV}[24,25]$, after one $\mathrm{ab}^{-1}$ of luminosity. These models cannot be found by direct detection experiments for mediator masses heavier than $\sim 5 \mathrm{TeV}$.

Perhaps most importantly, it has become clear that many canonical weaklyinteracting DM models would only be discovered at FCC-hh. Some canonical DM particles that may not be found without the FCC-hh, include "Higgsinos", "bino-winos", and "coannihilated singlets". These DM models all include a light, extremely-weakly interacting neutral state separated in mass by $\sim 10^{-3}-100 \mathrm{GeV}$ from a heavier, more strongly interacting state. The reason that indirect astrophysical searches and underground detectors cannot find such DM, even after direct detection experiments reach the neutrino floor, is that the lightest DM state has tiny elastic interactions. Despite these tiny interactions with SM particles, the DM state nevertheless annihilates to the correct relic abundance in the early universe through co-annihilation with the heavier, more strongly interacting DM state. The FCC-hh is uniquely capable of producing this heavier, more strongly-interacting dark sector state.

Specifically, Higgsinos, which fill out the relic abundance for masses between 1 and 1.2 TeV, have a nucleon scattering cross section that lies well below the neutrino floor. On the other hand, charged track searches at the FCC-hh could find these particles up to the thermal relic mass of $1.2 \mathrm{TeV}[23,26]$. Another example of canonical DM that only the FCC-hh can find is "bino-wino" DM, which spans the mass range $0.2-3 \mathrm{TeV}$. While bino-winos with masses between $\sim 1.6$ and $3 \mathrm{TeV}$ will be explored by indirect and direct detection, bino-winos in the mass range $\sim 0.2-1.6 \mathrm{TeV}$ cannot be found by any currently planned experiment. The FCC-hh would explore masses up to $1.6 \mathrm{TeV}$ [27] after $10 \mathrm{ab}^{-1}$, in events where the wino produces a moderate amount of missing energy along with soft photons and leptons. Finally, another case of co-annihilated singlet DM, beyond the reach of planned experiments, is a bino 
co-annihilated by a gluino. Monojet searches at the FCC-hh allow for discovery of bino-gluino DM up to masses of $6 \mathrm{TeV}$ after $3 \mathrm{ab}^{-1}$ luminosity, compared with a thermal relic mass range which extends to $8 \mathrm{TeV}$ [21]. It remains to be assessed whether the full luminosity of $30 \mathrm{ab}^{-1}$ could completely explore such co-annihilated thermal DM.

In summary, a $100 \mathrm{TeV}$ proton-proton collider would be the first facility broadly capable of searching for weakly-interacting dark matter via direct production and, in certain scenarios, is the only facility capable of uncovering DM like Higgsinos, binowinos and thermal relic DM which freezes out by co-annihilating with a heavier, strongly interacting dark sector state.

\subsection{Lepton-Hadron physics}

The FCC-eh is designed to collide $60 \mathrm{GeV}$ electrons from an energy recovery linac (as planned for the LHeC [28]) with the $50 \mathrm{TeV}$ FCC-hh protons, for an ep centre of mass energy of $3.5 \mathrm{TeV}$. The upper reach in the $t$-channel momentum transfer is $Q_{\max }^{2}=12 \mathrm{TeV}^{2}$, and Bjorken $x$ extends from $10^{-7}$ (at $Q^{2}=1 \mathrm{GeV}^{2}$ ) to $x \sim 1$. The ep and pp colliders can operate concurrently and $2 \mathrm{ab}^{-1}$ of ep luminosity can therefore be integrated in two decades of operation, exceeding HERA's luminosity [29] by more than a factor of $10^{3}$. A few $\mathrm{fb}^{-1}$ may also be achieved in electron-ion collisions. Deep inelastic scattering is the cleanest probe to resolve the substructure and dynamics of hadronic matter. FCC-eh's high energy and luminosity enhance this sensitivity substantially, with a rich potential for discovery and for precision measurements of the Higgs and EW sectors and of the top quark. Operating together with FCC-hh, FCC-eh opens new horizons for hadron collider physics that are complementary to the prospects of FCC-ee. These areas of investigation are briefly reviewed below.

\subsubsection{QCD and Hadron structure}

QCD exhibits the richest range of manifestations among all fundamental interactions, from the short-distance perturbative dynamics of high energy processes, to the non-perturbative dynamics responsible for hadronic matter, at distance scales going from the proton and its substructure to a neutron star. In between these extremes, a large variety of phenomena await a quantitative, first-principle description, and QCD related discoveries may be made. Precision QCD measurements in the extended kinematic range of FCC-eh, in addition to shedding further light on a whole set of issues from gluon saturation at small $x$ to the nature of the pomeron, will be possible and necessary to maximise the physics return of FCC-hh by providing, among other, the most detailed knowledge of the proton parton distribution functions (PDFs) and a value of $\alpha_{s}$ with $1-2 \times 10^{-3}$ relative precision.

The FCC-eh can extract the PDFs of all individual quark flavours and of the gluon without ad hoc assumptions or constraints, thanks to the high energy, which provides comparable event rates from all three channels of $\gamma, \mathrm{Z}$ and $\mathrm{W}^{ \pm}$exchange. At the largest $x$ values the PDFs will be determined with a few percent precision for the $\mathrm{u}$ and $\mathrm{d}$ valence quarks. This will then allow, for example, accurate prediction of the FCC-hh jet production cross section at the highest energies, improving the sensitivity to possible new interactions, or substructures, of quarks. At the lowest values of $x$, the FCC-eh data will reduce the PDF systematics to the $\mathrm{pp} \rightarrow \mathrm{H}$ inclusive cross section to the $0.5 \%$ level, challenging the precision of theoretical calculations. A summary of the precision projections for the partonic luminosity extracted from FCC-eh is shown in Figure 1.4. 


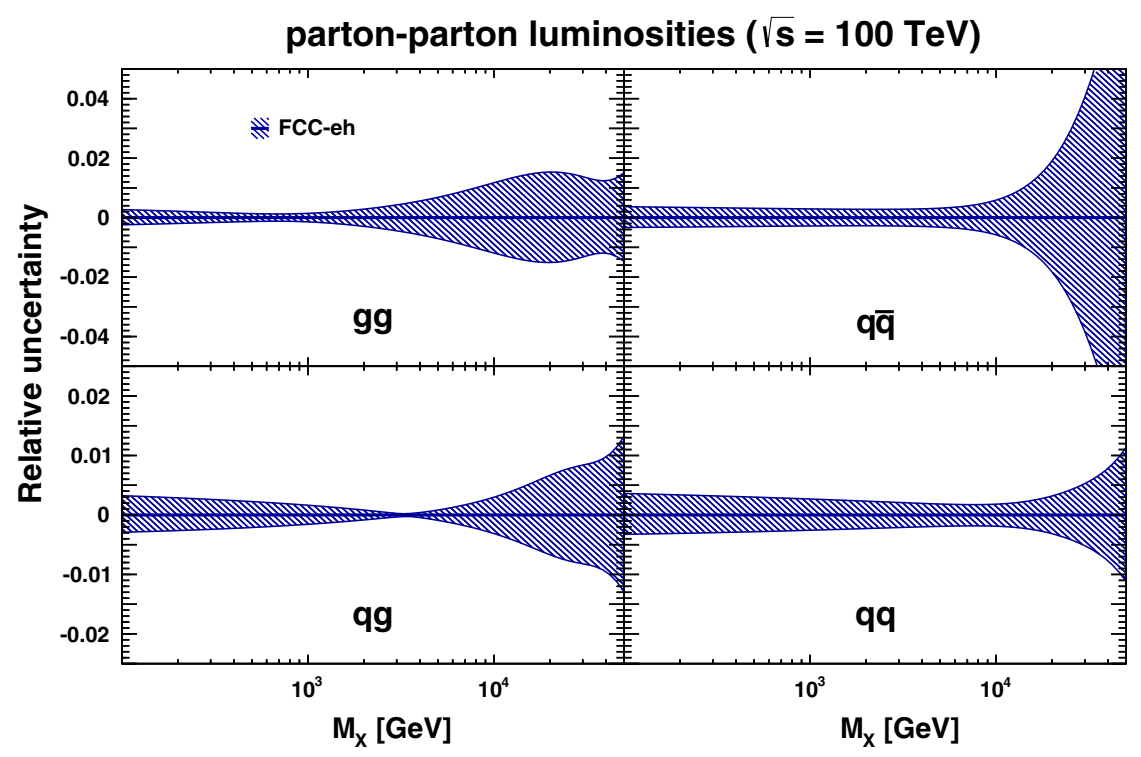

Fig. 1.4. Relative PDF uncertainties on parton-parton luminosities from the FCC-eh PDF set, as a function of the mass of the produced heavy object, $M_{X}$, at $\sqrt{s}=100 \mathrm{TeV}$. Shown are the gluon-gluon (top left), quark-antiquark (top right), quark-gluon (bottom left) and quark-quark (bottom right) luminosities.

The kinematic range for electron-nucleus (eA) scattering accessible to FCC-eh extends what has been studied so far by a factor of the order of $10^{4}$, opening new windows on the study of nuclear structure. For example, a future observation of nonlinear effects at small $x$ in ep collisions, through measurements of $F_{2}$ and $F_{L}$, can then be confronted with the superposition of non-linear and nuclear effects in eA, to test the expected amplification $\propto A^{1 / 3}$. The high FCC-eh energy is crucial, in order to be in a perturbative regime where saturation and nuclear effects can be properly disentangled. Furthermore, FCC-eh will measure nuclear and proton PDFs independently of each other at high precision, to deliver flavour and kinematic dependent nuclear-to-proton ratio functions. That will lead to new theoretical developments on, e.g. shadowing, and shed further light on the mechanism of hadronisation and on the collective phenomena recently observed at the LHC in high-density final states from AA, pA and pp collisions.

\subsubsection{Higgs measurements}

The main Higgs production mechanism at FCC-eh is ep $\rightarrow \mathrm{H} \nu(\mathrm{e}) \mathrm{X}$, via WW(ZZ) fusion, with $\sigma \simeq 1 \mathrm{pb}$, and a total statistics of a few million events. The ep environment is very clean, with a low pile-up and robust theoretical calculations, which allow for absolute and precise predictions of the production rates. The scattered $\nu$ or e distinguish $\mathrm{H}$ production via $\mathrm{WW}$ and $\mathrm{ZZ}$ fusion, providing two independent measurements of the accessible decay modes at once. This redundancy enhances the precision prospects to (sub)percent level, as summarised in Figure 1.5 for the seven strongest $\mathrm{H}$ decay couplings. Furthermore, a first cut-based analysis, using only the $\mathrm{HH} \rightarrow 4 \mathrm{~b}$ final state, shows high sensitivity to anomalous HWW and HHWW couplings and a $3-4 \sigma$ sensitivity to the Higgs self-coupling. This conservative estimate will improve with refined analysis techniques and the inclusion of all other major 


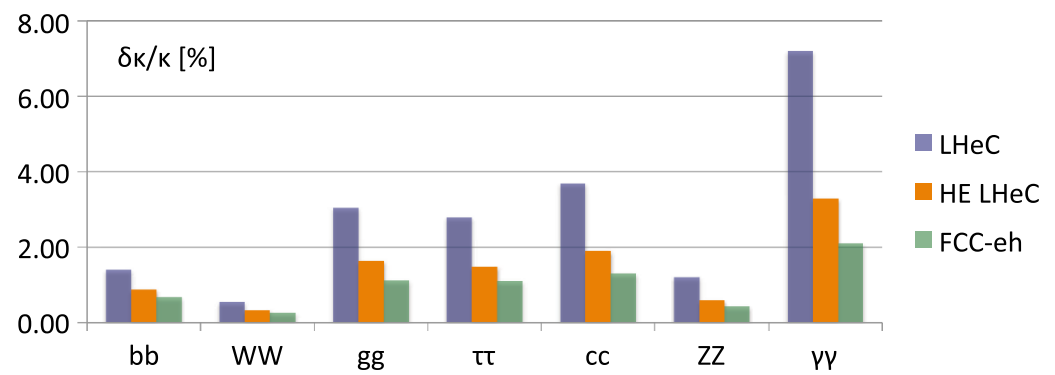

Fig. 1.5. Determination of SM Higgs couplings in the seven most abundant decay channels, from a fit to simulated $\mathrm{WW}$ and $\mathrm{ZZ}$ fusion production channels, including acceptance, background and efficiency effects. The statistics is assumed to be collected in simultaneous ep operation with pp at the LHC (HL, HE) and FCC.

decay channels and of the neutral current production will provide genuine discovery potential for $\mathrm{H} \rightarrow \mathrm{HH}[30]$.

\subsubsection{Precision EW and top physics}

At $Q^{2} \gg M_{Z, W}^{2}$ weak interactions dominate the deep inelastic scattering interaction, and top quarks are copiously produced in the $\mathrm{Wb} \rightarrow \mathrm{t}$ and $\gamma \mathrm{g} \rightarrow \mathrm{t} \overline{\mathrm{t}}$ processes. Unique measurements of the EW sector become possible, like the scale dependence of the weak mixing angle up to a few $\mathrm{TeV}$ and the precise measurement of the $\mathrm{tWb}$ and tt $\gamma$ vertices, which could expose anomalous couplings. Flavour physics studies are also possible: direct, sub-percent precision measurements of $V_{t b}$ and $V_{t d}$ from single top production; $V_{c s}$ from charm production; probes of FCNC interactions beyond the SM through processes such as $\mathrm{Z} / \gamma \mathrm{q} \rightarrow \mathrm{t}$ production or $\mathrm{t} \rightarrow \mathrm{Hq}$ decay $(q=u, c)$.

\subsubsection{Production of new particles}

The high-energy and clean environment of FCC-eh collisions offers additional windows for discovery. Examples include the detection of exotic Higgs decays to DM, of neutralinos in $R$-parity violating supersymmetry, or of exotic scalars. Vector-boson scattering yields singly and doubly charged Higgs particles, should they exist. New physics may appear through long-lived Higgsinos, which for small (large) wino-bino mixing may be favourably discovered in ep (pp) interactions at the FCC. Finite neutrino masses may lead to heavier neutrino mass eigenstates, which can be discovered in ep collisions through novel charged-current interactions. The so-called compressed scenarios of supersymmetry, where soft decay products may escape searches in FCChh, could be seen by FCC-eh. The scattering of electrons and quarks at the highest $Q^{2}$ values can probe new contact interactions, or a lepton/quark substructure, up to scales in the $100 \mathrm{TeV}$ range. Likewise lepto-quark resonances can be discovered, and their spectroscopy explored, should their mass be smaller than $\sim 3 \mathrm{TeV}$.

\subsection{The physics goals and prospects of the heavy ion programme at FCC-hh}

The operation of the FCC-hh with heavy-ion beams is part of the accelerator design studies, as discussed in Section 2.7. For a centre-of-mass energy $\sqrt{s}=100 \mathrm{TeV}$ for 
pp collisions, the relation $\sqrt{s_{\mathrm{NN}}}=\sqrt{s} \sqrt{Z_{1} Z_{2} / A_{1} A_{2}}$ gives the energy per nucleonnucleon collision of $\sqrt{s_{\mathrm{NN}}}=39 \mathrm{TeV}$ for $\mathrm{PbPb}(Z=82, A=208)$ and $63 \mathrm{TeV}$ for $\mathrm{pPb}$ collisions. The present estimate of the integrated luminosity per month of running for $\mathrm{PbPb}$ collisions results in about $35 \mathrm{nb}^{-1}$ in the baseline scenario and $110 \mathrm{nb}^{-1}$ in the ultimate scenario. The estimate for the $\mathrm{pPb}$ integrated luminosity is 8 and $29 \mathrm{pb}^{-1}$ per month, in the baseline and ultimate scenarios, respectively. For both colliding systems, the luminosity is larger than the current projection for the future LHC runs [31] by factors of 10-30. The possibility of using nuclei smaller than $\mathrm{Pb}$, like ${ }^{40} \mathrm{Ar},{ }^{84} \mathrm{Kr}$ or ${ }^{129} \mathrm{Xe}$, to achieve larger instantaneous luminosity is also being considered.

Collisions of heavy ions at ultra-relativistic energies allow the investigation of the collective properties of the elementary quark and gluon fields of QCD, providing a unique test bed for the study of strongly-interacting matter and, in general, of the many-body properties of non-Abelian quantum field theory (QFT). The increase in the centre-of-mass energy and integrated luminosity with respect to the LHC opens up novel opportunities for physics studies of the Quark-Gluon Plasma (QGP), described in detail in [32]. While unexpected discoveries, by their very nature, cannot be anticipated, the main focus is on those fundamental questions about the nature of QCD matter at high temperature and density for which qualitative advances are expected. The main scientific motivations to carry out measurements with heavy ions are summarised in the next section.

\subsubsection{Novel access to QCD thermodynamics and QCD equilibration processes}

Substantially increasing the centre-of-mass energy leads to the creation of initially denser and hotter systems, which expand for a longer duration and over a larger volume, developing stronger collective phenomena. In particular, extrapolations of LHC measurements indicate that, going from $\sqrt{s_{\mathrm{NN}}}=5.5 \mathrm{TeV}$ to $39 \mathrm{TeV}$, increases both the initial energy density and the volume of the system by a factor of about two, up to values of about $40 \mathrm{GeV} / \mathrm{fm}^{3}$ (at $\tau=1 \mathrm{fm} / c$ ) and $11000 \mathrm{fm}^{3}$, respectively. Beyond expected quantitative gains, this may bring novel qualitative phenomena into experimental reach. For instance, FCC ion collisions reach temperatures $(T \sim 1 \mathrm{GeV})$ where charm quarks start to contribute as active thermal degrees of freedom in the QGP equation of state, thus playing a novel role in QCD equilibration processes. In addition, the large increase in the particle multiplicity density and in integrated luminosity will allow the systematic study of flow-like features in smaller collision systems (including pp and pA collisions), facilitating the characterisation of important signatures of collectivity at the level of single events, rather than of event samples only. This opens novel opportunities to understand the equilibration processes that lead to hydrodynamisation and thermalisation in QCD and, in general, in non-Abelian QFTs.

\subsubsection{Characterisation of dense QCD matter through hard-scattering processes}

High-energy partons produced in heavy-ion collisions undergo strong mediuminduced modifications, referred to as jet quenching, up to the highest transverse momenta of the order of $1 \mathrm{TeV}$ explored at the LHC so far. Jet quenching measurements provide quantitative information on the transport properties of hot and dense QCD matter and on the mechanism of thermalisation of a strongly-interacting probe that is initially very far from equilibrium. The FCC-hh will greatly expand the set of hard-scattering processes available for studies at the LHC, including novel 
(both experimentally and theoretically) probes such as the top quark and the Higgs boson [33-35], which require the large FCC-hh statistics.

The $\mathrm{t} \overline{\mathrm{t}}$ cross section increases by a factor 80 from $\sqrt{s_{\mathrm{NN}}}=5.5 \mathrm{TeV}$ to $39 \mathrm{TeV}$, leading e.g. to $3-10 \times 10^{5} \mathrm{t} \overline{\mathrm{t}} \rightarrow \mathrm{b} \overline{\mathrm{b}} \ell \ell \nu \nu$ reconstructed events in a one-month $\mathrm{PbPb}$ run. The products of the $\mathrm{t} \rightarrow \mathrm{W} \rightarrow \mathrm{q} \overline{\mathrm{q}}$ decay will be injected in the QGP at times up to $10 \mathrm{fm} / c$ after the collision, depending on the top quark transverse momentum. The time evolution of the QGP density and of the role of colour coherence [33] can then be monitored through the jet-quenching-induced decrease of the measured $\mathrm{W} \rightarrow \mathrm{q} \overline{\mathrm{q}}$ mass. Multiple parton interactions in the initial state will produce a large multiplicity of charm and bottom quark pairs [36]. In addition, the secondary production of charm quarks in scattering between partonic constituents of the QGP is expected to be as large as $80 \%$ of the initial hard scattering production and is a novel observable sensitive to the medium temperature evolution $[37,38]$.

The yields and kinematic distributions of heavy quarkonium carry information about the produced QCD matter, since quarkonia states are expected to dissociate above critical energy densities (that depend on the binding energy of the state) and to form in recombination processes (depending on the density of heavy quarks in the system). The aforementioned secondary production of $c \bar{c}$ pairs could lead to a global enhancement of the $\mathrm{J} / \psi$ yield in $\mathrm{PbPb}$ compared to pp collisions, rather than the suppression that is observed at LHC energies. In addition, a first observation of $\Upsilon$ formation from $\mathrm{b} \overline{\mathrm{b}}$ recombination is expected. Finally, the increase in energy and luminosity for $\mathrm{pA}$ collisions could prove decisive for the search of parton energy loss (e.g. with correlations of electroweak bosons and jets) and thermal radiation in high-multiplicity small-system collisions, where several QGP-like effects are observed in the particle yield and flow measurements.

\subsubsection{Parton saturation in an ultra-dense kinematic domain}

Parton densities increase strongly with decreasing momentum fraction $x$. At any given $\sqrt{s}$, the nuclear parton densities are larger than those in the proton due to geometric enhancement. On general grounds, this growth at small $x$ is expected to saturate once parton densities reach non-perturbative values of parametric order $\sim 1 / \alpha_{s}$. In the context of heavy-ion collisions, the study of saturated QCD is of fundamental interest, mainly because it fixes the initial conditions for the collective dynamics. For instance, the accuracy reached in the quantitative properties of hot and dense QCD matter depends ultimately on the accuracy with which one can characterise the incoming nuclear wave functions at small $x$. More generally, saturated QCD is of fundamental interest as it is a qualitatively-novel kinematic regime where QCD scale dependence is governed by non-linear evolution equations and where bulk properties of QCD become amenable to perturbative calculations. The higher centre-of-mass energy of FCC-hh allows the exploration of a wide and previously uncharted kinematic range in the $\left(x, Q^{2}\right)$ plane, where gluon saturation is expected to manifest itself. Complementary pA and eA collision programmes at the FCC-hh/eh are both needed to fully explore this opportunity. pA collisions have the advantage of a larger coverage at small $x$, because of the larger centre-of-mass energy, e.g. down to $x \sim 10^{-6}$ at a rapidity of about 5 in $\mathrm{pPb}$ collisions at $63 \mathrm{TeV}$. On the other hand, electron-nucleus collisions have the advantage of a fully constrained kinematics (precise determination of $x$ and $Q^{2}$ ). Among others, promising observables in pA collisions include: (i) photon production and photon-hadron correlations at forward rapidity, which are sensitive to the small $x$ and small $Q^{2}$ region where saturation is expected to set in; (ii) heavy quarkonium production in $\gamma \mathrm{A}$ collisions (ultra-peripheral nucleus-nucleus collisions); (iii) the production of very heavy objects like $\mathrm{W}, \mathrm{Z}$ and $\mathrm{t}$, which can provide strong 
constraints on the modification of the parton density functions in nuclei at small $x$ and large $Q^{2}$. For example, the nuclear modification factor of muons from $\mathrm{t} \overline{\mathrm{t}} \rightarrow \mathrm{b} \overline{\mathrm{b}} \ell \ell \nu \nu$ in $\mathrm{pPb}$ collisions $\left(8 \mathrm{pb}^{-1}\right)$ would reduce the nuclear parton distribution function uncertainties by a factor of about two in $0.05<x<0.7$ at $Q=m_{\text {top }}$ [34].

In addition to the three key motivations for a heavy-ion beam programme at the FCC-hh listed above, further opportunities are discussed in [32], including contributions to other sectors of high energy physics, such as searches for new particles in photon-photon scattering processes induced at very high rates by the strong electromagnetic fields of the colliding $\mathrm{Pb}$ nuclei [39], the contribution to the physics of ultra-high-energy cosmic rays and the physics with fixed-target collisions using FCC proton or heavy-ion beams.

\section{Collider design and performance}

\subsection{Requirements and design considerations}

The FCC-hh will provide proton-proton collisions with a centre-of-mass energy of $100 \mathrm{TeV}$, about seven times higher than in LHC. Within 25 years of operation it can accumulate an integrated luminosity of $\approx 20 \mathrm{ab}^{-1}$ in each of the two main experiments. The combined data sets will thus easily surpass the total of $30 \mathrm{ab}^{-1}$ required by the physics studies. With the initial parameters it is planned to reach a maximum luminosity of $5 \times 10^{34} \mathrm{~cm}^{-2} \mathrm{~s}^{-1}$ in the first years. In the following years the luminosity will be increased reaching the final parameters with a luminosity of up to $3 \times 10^{35} \mathrm{~cm}^{-2} \mathrm{~s}^{-1}$.

The layout of the collider has been developed to integrate with the existing CERN accelerator complex as injector facility. The beam quality from the existing injector complex is sufficient. Care has been taken to ensure easy beam transfer from either the LHC or the SPS to the future collider tunnel (see Chap. 6).

The FCC-hh also offers the potential of colliding ions with protons and ions with ions. The existing accelerator complex can provide the required ion beams. Additional protection in the collider is needed to mitigate the very localised beam loss that ions can induce.

The design also allows for an upgrade to electron-proton and electron-ion collisions. In these cases, one of the circulating proton or ion beams collides with an electron beam provided by a new energy recovery linac.

The collider design provides a robust solution that achieves the performance goals and with sufficient margins. With the availability of the cost model, the design can be further optimised to balance risk and cost.

\subsection{Key parameters and layout}

The machine layout is shown in Figure 2.1 and the key parameters are listed in Table 2.1. The performance will increase from the initial to the nominal parameters.

\subsubsection{Layout and beam energy}

The collider has been placed adjacent to the CERN LHC and SPS accelerators and the two injection insertions (at $\mathbf{P B}$ and $\mathbf{P L}$ ) are positioned such that one can inject beam from either the LHC or the SPS. 


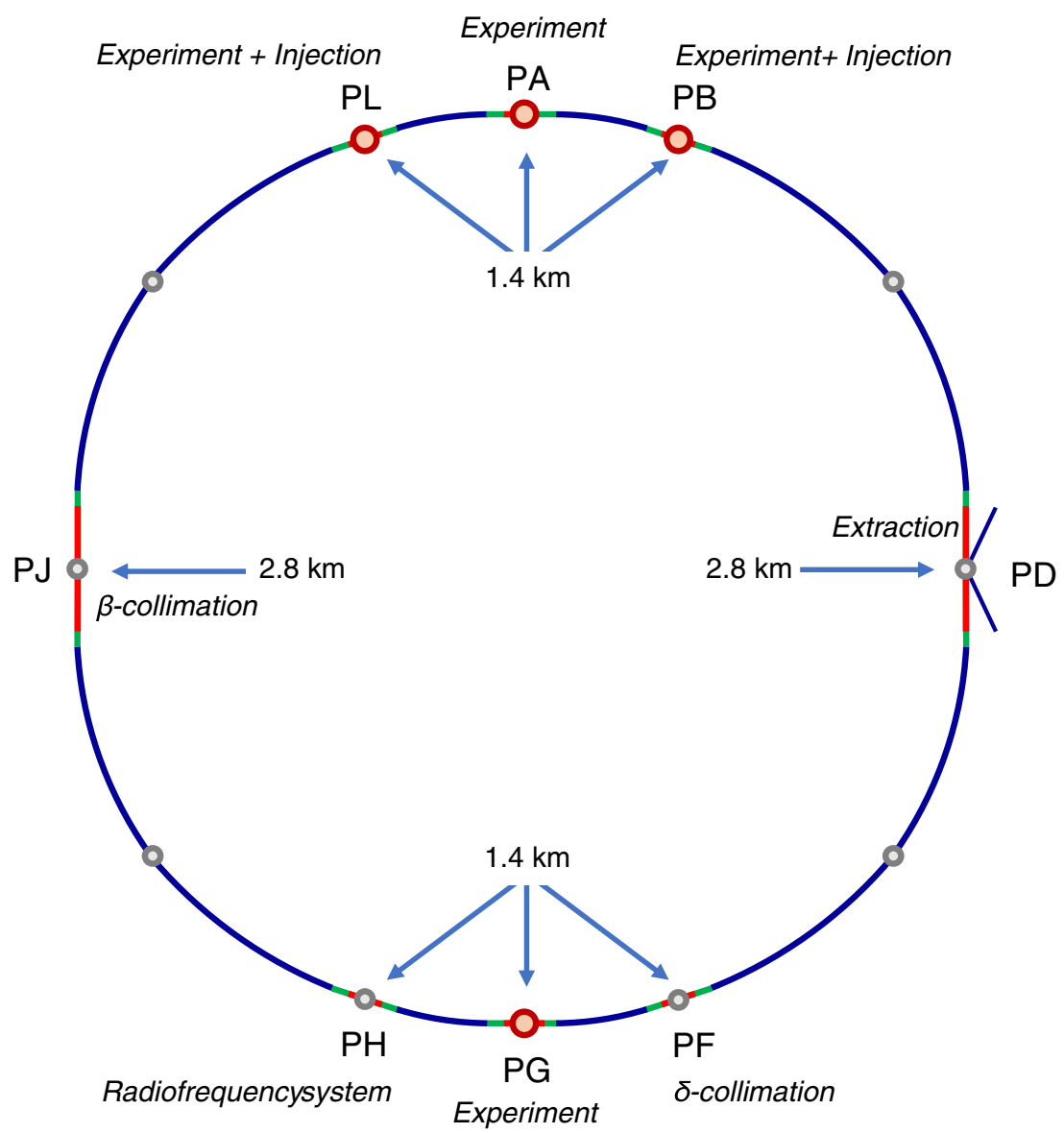

Fig. 2.1. The FCC-hh conceptual layout. The two main experiments are located in PA and $\mathbf{P G}$ and the additional experiments in $\mathbf{P B}$ and $\mathbf{P L}$, the latter combined with the injection. The momentum and betatron collimation systems are in insertions $\mathbf{P F}$ and $\mathbf{P J}$, respectively. The beam extraction is located in PD. The radiofrequency system and the main feedback are integrated in $\mathbf{P H}$.

Two high luminosity experiments are located in opposite insertions (PA and PG). This ensures the highest luminosity, reduces unwanted beam-beam effects and is independent of the beam-filling pattern. Two additional, lower luminosity experiments are located together with the injection systems in insertions PB and PL. The transverse beam cleaning is located in insertion PJ and the beam extraction in insertion $\mathrm{PD}$. The longitudinal beam cleaning is placed in insertion PF and finally, the RF systems and the fast feedback are placed in insertion PH. The long arcs also contain technical points (PC, PE, PI and PK).

The total circumference of the collider is $97.75 \mathrm{~km}$ and most of the insertions are $1.4 \mathrm{~km}$ long. Only the transverse collimation and the beam extraction areas are $2.8 \mathrm{~km}$ long. This additional length facilitates the mitigation of the challenges posed in these systems by the high stored energy. The arcs of the collider have a total length of $83.75 \mathrm{~km}$ and a dipole filling factor of about 0.8 . A dipole field of slightly less than $16 \mathrm{~T}$, which is the target for the superconducting, $\mathrm{Nb}_{3} \mathrm{Sn}$-based magnets, is required to keep the beams on the circular orbit. Figure 2.2 depicts the cross section of a typical arc segment. 
Table 2.1. FCC-hh baseline parameters compared to the LHC and HL-LHC parameters.

\begin{tabular}{|c|c|c|c|c|}
\hline & \multirow[t]{2}{*}{ LHC } & \multirow[t]{2}{*}{ HL-LHC } & \multicolumn{2}{|c|}{ FCC-hh } \\
\hline & & & Initia & Nominal \\
\hline \multicolumn{5}{|c|}{ Main parameters and geometrical aspects } \\
\hline c.m. Energy $(\mathrm{TeV})$ & \multicolumn{2}{|c|}{14} & \multicolumn{2}{|c|}{100} \\
\hline Circumference C (km) & \multicolumn{2}{|c|}{26.7} & \multicolumn{2}{|c|}{97.75} \\
\hline Dipole field $(\mathrm{T})$ & \multicolumn{2}{|c|}{8.33} & \multicolumn{2}{|c|}{$<16$} \\
\hline Arc filling factor & \multicolumn{2}{|c|}{0.79} & \multicolumn{2}{|c|}{0.8} \\
\hline Straight sections & \multicolumn{2}{|c|}{$8 \times 528 \mathrm{~m}$} & \multicolumn{2}{|c|}{$6 \times 1400 \mathrm{~m}+2 \times 2800 \mathrm{~m}$} \\
\hline Number of IPs & \multicolumn{2}{|c|}{$2+2$} & \multicolumn{2}{|c|}{$2+2$} \\
\hline Injection energy $(\mathrm{TeV})$ & \multicolumn{2}{|c|}{0.45} & \multicolumn{2}{|c|}{3.3} \\
\hline \multicolumn{5}{|c|}{ Physics performance and beam parameters } \\
\hline Peak luminosity ${ }^{1}\left(10^{34} \mathrm{~cm}^{-2} \mathrm{~s}^{-1}\right)$ & 1.0 & 5.0 & 5.0 & $<30.0$ \\
\hline Optimum average integrated & 0.47 & 2.8 & 2.2 & 8 \\
\hline $\begin{array}{l}\text { lumi/day }\left(\mathrm{fb}^{-1}\right) \\
\text { Assumed turnaround time }(\mathrm{h})\end{array}$ & & & 5 & \\
\hline Target turnaround time $(\mathrm{h})$ & & & 2 & 2 \\
\hline Peak no. of inelastic events/crossing & 27 & $135(\mathrm{lev})$ & 171 & 1026 \\
\hline $\begin{array}{l}\text { Total/inelastic cross section } \sigma \text { proton } \\
\text { (mbarn) }\end{array}$ & \multirow{2}{*}{\multicolumn{2}{|c|}{$111 / 85$}} & \multicolumn{2}{|c|}{$153 / 108$} \\
\hline Luminous region RMS length $(\mathrm{cm})$ & & & 5.7 & 5.7 \\
\hline $\begin{array}{l}\text { Distance IP to first quadrupole, } L^{*} \\
(\mathrm{~m})\end{array}$ & \multicolumn{2}{|c|}{23} & 40 & 40 \\
\hline \multicolumn{5}{|l|}{ Beam parameters } \\
\hline Number of bunches $n$ & \multicolumn{2}{|c|}{2808} & \multicolumn{2}{|c|}{10400} \\
\hline Bunch spacing (ns) & 25 & 25 & & \\
\hline Bunch population $N\left(10^{11}\right)$ & 1.15 & 2.2 & & \\
\hline $\begin{array}{l}\text { Nominal transverse normalised emit- } \\
\text { tance }(\mu \mathrm{m})\end{array}$ & 3.75 & 2.5 & 2.2 & 2.2 \\
\hline Number of IPs contributing to $\Delta \mathrm{Q}$ & 3 & 2 & $2+2$ & 2 \\
\hline Maximum total b-b tune shift $\Delta \mathrm{Q}$ & 0.01 & 0.015 & 0.011 & 0.03 \\
\hline Beam current $(\mathrm{A})$ & 0.584 & 1.12 & & \\
\hline RMS bunch length $^{2}(\mathrm{~cm})$ & & & & \\
\hline IP beta function (m) & 0.55 & $0.15(\min )$ & 1.1 & 0.3 \\
\hline RMS IP spot size $(\mu \mathrm{m})$ & 16.7 & $7.1(\min )$ & 6.8 & 3.5 \\
\hline Full crossing angle $(\mu \mathrm{rad})$ & 285 & 590 & 104 & $200^{3}$ \\
\hline Other beam and machine parame & ers & & & \\
\hline Stored energy per beam (GJ) & 0.392 & 0.694 & & \\
\hline SR power per ring $(\mathrm{MW})$ & 0.0036 & 0.0073 & & \\
\hline Arc SR heat load (W/m/aperture) & 0.17 & 0.33 & & \\
\hline Energy loss per turn (MeV) & & & & \\
\hline Critical photon energy (keV) & & & & \\
\hline $\begin{array}{l}\text { Longitudinal emittance damping } \\
\text { time }(\mathrm{h})\end{array}$ & & & & \\
\hline $\begin{array}{l}\text { Transverse emittance damping time } \\
\text { (h) }\end{array}$ & & & & \\
\hline Dipole coil aperture (mm) & & & & \\
\hline $\begin{array}{l}\text { Minimum arc beam half aperture } \\
(\mathrm{mm})\end{array}$ & & & & \\
\hline $\begin{array}{l}\text { Installed RF voltage }(400.79 \mathrm{MHz}) \\
(\mathrm{MV})\end{array}$ & & & & \\
\hline Harmonic number & & & & 680 \\
\hline
\end{tabular}

Notes. ${ }^{1}$ For the nominal parameters, the peak luminosity is reached during the run. ${ }^{2}$ The HL-LHC assumes a different longitudinal distribution; the equivalent Gaussian is $9 \mathrm{~cm} .{ }^{3}$ The crossing angle will be compensated using the crab crossing scheme. 


\subsubsection{Luminosity and beam parameters}

The luminosity is a function of the beam current $I$, the beam-beam tune shift $\xi$, the beam gamma factor $\gamma$ and the beta-functions at the collision point $\beta^{\star}$. For a round beam it is given by:

$$
\mathcal{L}=\xi \frac{I}{q} \frac{\gamma}{\beta^{\star}} \frac{1}{r_{p}} F
$$

Here, $r_{p}$ is the classical proton radius and $q$ the elementary charge. The form factor $F$ allows for geometric luminosity reduction effects like the hour glass effect; it is neglected in the following discussion. Hence, to reach high luminosity, one has to use a high brightness beam (i.e. large $\xi$ ), achieve small beta functions and have a high beam current.

Larger beam-beam tuneshifts can reach more luminosity but also have undesired effects. In particular, large beam-beam tuneshifts can turn small amplitude beambeam jitter into emittance growth which results in an increase of beam size and hence a decrease of the luminosity. They can also lead to an increase of particle losses due to non-linear effects and consequently a decrease in the beam lifetime. In FCC-hh a maximum beam-beam tuneshift of $\xi \leq 0.03$ is expected. In the initial phase each of the four experiments contributes about 0.0055 to the total remains well below the maximum. In the nominal phase each of the two main experiments reaches up to 0.013 and the tuneshift of the additional experiments has to be reduced, e.g. by separating the beams. Simulations [40] and LHC experience with the current working point confirm that this value is acceptable. The fraction of beam lost in the transverse tails remains below $10^{-3}$ per hour and also the emittance growth induced by beambeam jitter remains limited. In contrast, a slightly larger tuneshift would rapidly increase the loss rate and the beam emittance would increase significantly faster for the same jitter amplitudes. Studies [40] indicate that other working points might allow even larger tuneshifts but detailed work to evaluate the impact on other parts of the machine remains to be done. The crossing angle of about $\theta_{c} \approx 200 \mu \mathrm{rad}$ limits the impact of parasitic beam-beam crossings and the associated luminosity reduction is mitigated by the use of crab cavities. Other means that could be added to mitigate the long-range and head-on beam-beam effects include electron lenses and current carrying wire compensators.

A small beta function, $\beta^{*}$, at the collision point makes the optics design for the experiment insertion demanding and requires a large aperture in the focus triplets. This poses challenges for the magnet and protection design and for the collimation system which has to scrape off tails that could hit the triplets.

There are three main potential limitations for the beam current: synchrotron radiation, single beam collective effects and machine protection considerations. At the high energy of the FCC-hh, even proton beams emit significant synchrotron radiation power: together both beams emit about $5 \mathrm{MW}$. Beamscreens protect the superconducting magnets from this power, in the same way as in the LHC. The cryogenic system cools these screens to a temperature of $50 \mathrm{~K}$. Due to the Carnot inefficiency and the limited technical efficiency of the cryogenic system, about $100 \mathrm{MW}$ of electrical power is required to remove the synchrotron radiation power. A lower operating temperature would increase the power required to cool the beamscreen even further whilst a higher temperature would increase the heat transferred from the beamscreen (see Sect. 3.3.2) into the magnet and hence increase the power required to cool the magnet (see Sect. 5.6.2).

The second limitation, single beam collective effects, requires advanced methods for its mitigation. These techniques include a novel beamscreen design that ensures very good vacuum. This screen also reduces the potential for single bunch instabilities by making the pumping holes invisible to the beam, a marked improvement compared 


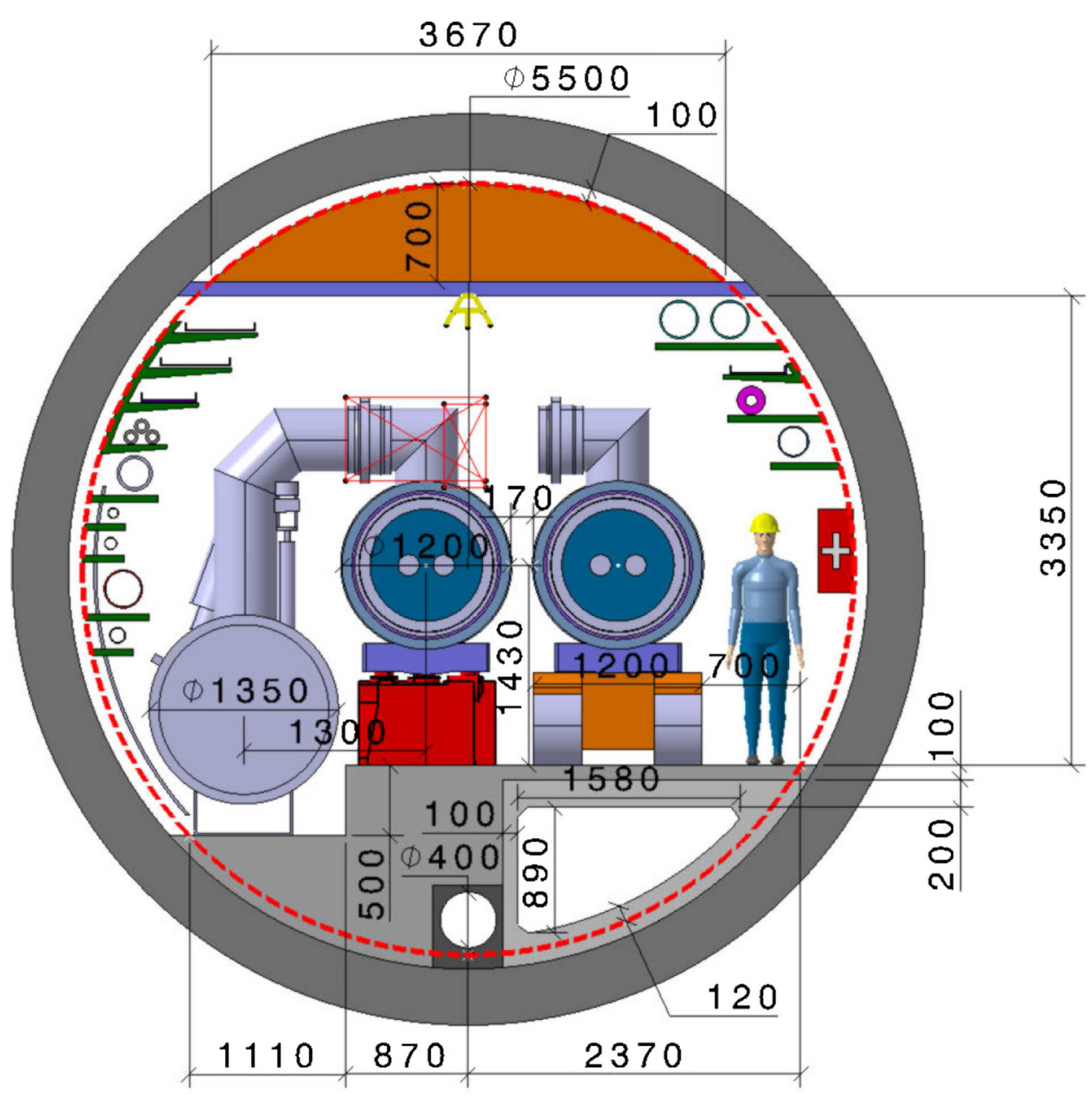

Fig. 2.2. Cross section of an FCC-hh arc. The grey equipment on the left side of the tunnel represents the cryogenic distribution line. A $16 \mathrm{~T}$ superconducting magnet can be seen in the middle, mounted on a red support element. Another superconducting magnet on a transport vehicle is shown next to it, in the transport passage.

to LHC [41]. A carbon layer on the surface and the specific geometry suppress the build-up of electron clouds, one of the important issues in the LHC. The same goal could be achieved by using a laser treatment to roughen the surface. Additional means to mitigate the collective effects include feedback, octupoles, and potentially $\mathrm{RF}$ quadrupoles and electron lenses. They are instrumental in permitting the use of a small free aperture. Combined with a compact beamscreen design a magnet aperture of only $50 \mathrm{~mm}$ becomes possible, which helps to limit cost.

A robust collimation and beam extraction system protect the machine from the energy stored in the beam.

\subsubsection{Integrated luminosity}

The beam parameters vary significantly during luminosity production [42]. Figure 2.3 illustrates the evolution of the main beam parameters taking into account the effect of intra-beam scattering, synchrotron radiation damping and quantum excitations, 

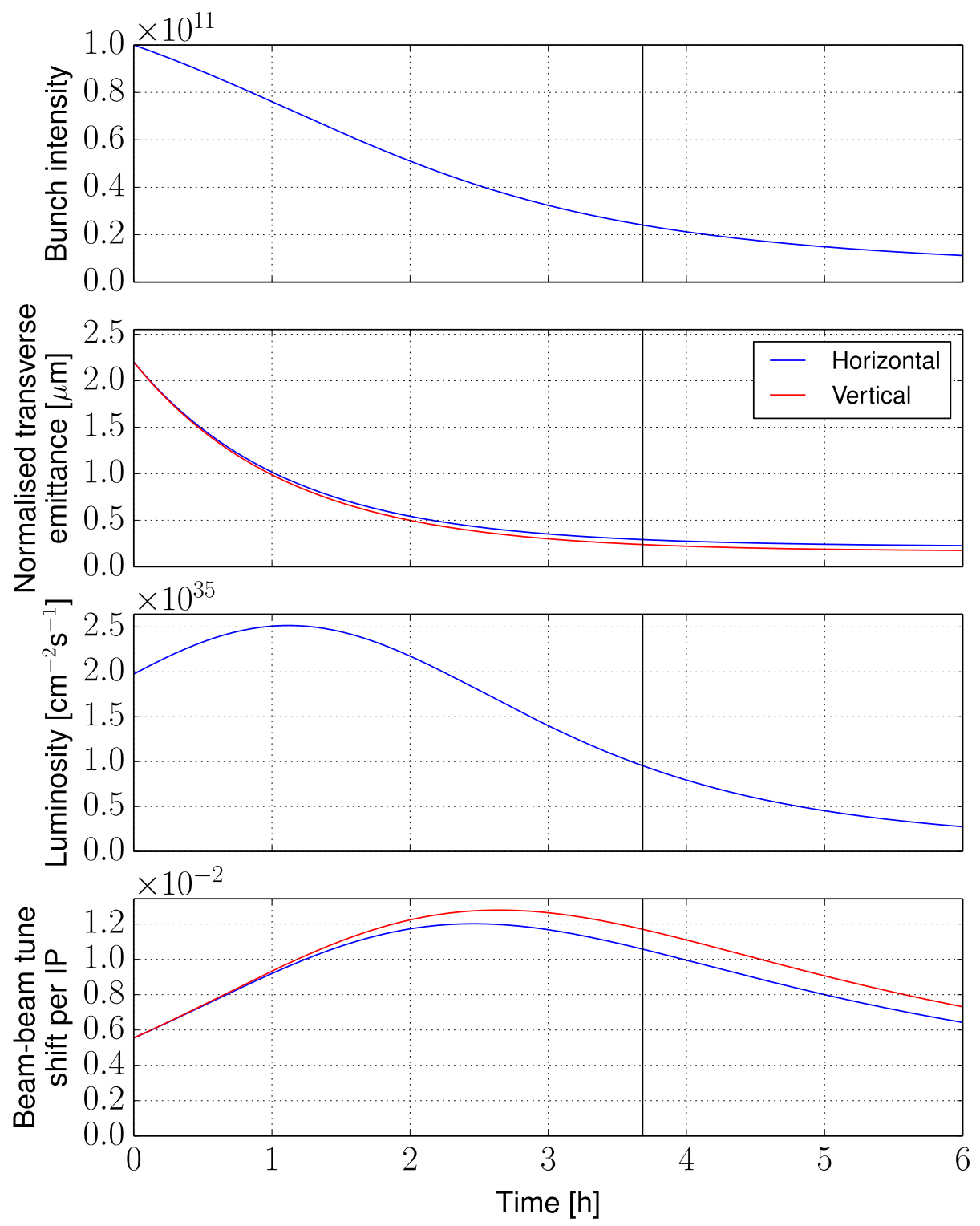

Fig. 2.3. Evolution of the beam parameters and luminosity during luminosity production, for the nominal $25 \mathrm{~ns}$ parameter set (see Tab. 2.1). A vertical black line marks the optimum fill length for a turn around time of $4 \mathrm{~h}$. The corresponding average luminosity production rate (without down time) is $8.2 \mathrm{fb}^{-1}$ per day.

luminosity burn-off and an absolute emittance growth rate of $0.15 \mu \mathrm{m} / \mathrm{h}$. The mathematical description of the model can be found in [43]. It is assumed that the longitudinal emittance is kept constant to maintain the size of the luminous region inside the detector. The budget for an additional source of emittance growth due to external sources of noise is conservatively based on LHC experience, where about $0.05 \mu \mathrm{m} / \mathrm{h}$ is routinely observed [44]. The short period of the collide-and-squeeze scheme, which brings the beams already into collision before the squeeze, is not included here as it has almost no impact on the integrated luminosity. 
During the run, the emittance reduction dominates first and hence the luminosity increases up to $2.5 \times 10^{35} \mathrm{~cm}^{-2} \mathrm{~s}^{-1}$. Then the beam burn-off starts to dominate and the luminosity decreases. A maximum beam-beam tune shift of 0.013 per IP is reached after $2 \mathrm{~h}$ of luminosity production. During the run the intensity decreases by a factor of 4 and the transverse emittances by a factor of 8 .

The experience in LHC shows that the turnaround time from the end of luminosity production to the start of the next could be reduced to $2 \mathrm{~h}$ with some modifications of the injector complex [45]. The turnaround times assumed for the initial and nominal parameters are $5 \mathrm{~h}$ and $4 \mathrm{~h}$, respectively, see Section 2.5. These times include a margin for limited availability. An optimum average production rate of $8.2 \mathrm{fb}^{-1}$ per day is obtained in these conditions - meeting the target of $8 \mathrm{fb}^{-1}$. The beams should be renewed after $3.7 \mathrm{~h}$ in collision.

\subsubsection{Additional experiments}

The injection insertions allow the installation of two additional experiments in the collider. The first half of the insertion in the direction of the injected beam is used for injection and the detector is placed in the middle of the second half. This configuration avoids the need to bypass the detector with the transfer line and affords ample space to protect the detector from potential beam losses at injection. Currently, no specific performance requirements have been established for these experiments. A generic insertion design gives an indication of the expected performance [46]. It provides a free distance from the collision point to the focusing triplets of $25 \mathrm{~m}$ and can reach luminosities in the $2 \times 10^{34} \mathrm{~cm}^{-2} \mathrm{~s}^{-1}$ range. The shielding to protect the triplet can be less massive than for the main experiments thanks to the lower luminosity.

\subsubsection{Alternative bunch spacings}

With the nominal parameters, a maximum of about 1000 events per bunch crossing can be reached. Novel detector design and data analysis methods need to be developed to allow the interesting physics to be extracted from this high background. Other operation modes based on reducing the bunch spacing from $25 \mathrm{~ns}$ to $12.5 \mathrm{~ns}$ or $5 \mathrm{~ns}$ together with proportionate changes to the charge and emittance can facilitate this task by reducing the number of events per bunch crossing. Table 2.2 shows three tentative scenarios. These options have not been considered in detail and more work is required to judge their feasibility and performance. They are more demanding for the hardware design and lead to a reduction of the integrated luminosity. The first option requires an adaptation of the injector chain to produce $12.5 \mathrm{~ns}$ beams. The second uses a $5 \mathrm{~ns}$ beam that could potentially be provided by the current injector complex, whilst the third option needs major modifications in the injector to produce the very small emittances.

If the experiments require these smaller bunch spacings an appropriate R\&D programme can be launched.

The alternative bunch spacings will increase the electron cloud effect, with the $12.5 \mathrm{~ns}$ option being the most critical. This requires additional mitigation and improvements of the existing mitigation measures. Furthermore, the instrumentation and feedback systems must have higher bandwidth.

\subsubsection{Injection considerations}

The injection energy is $3.3 \mathrm{TeV}$ and beams at this energy can be provided by the LHC. The choice of this energy ensures that the beam size is small enough to provide a 
Table 2.2. Key beam parameters.

\begin{tabular}{|l|c|c|c|c|c|}
\hline & Initial & Nominal & Opt 1 & Opt 2 & Opt 3 \\
\hline Bunch spacing $(\mathrm{ns})$ & 25 & 25 & 12.5 & 5 & 5 \\
Protons per bunch $\left(10^{11}\right)$ & 1 & 1 & 0.5 & 0.2 & 0.2 \\
Init. hor. transv. emittance $(\mu \mathrm{m})$ & 2.2 & 2.2 & 1.1 & 1.1 & 0.44 \\
Init. vert. transv. emittance $(\mu \mathrm{m})$ & 2.2 & 2.2 & 1.1 & 1.1 & 0.44 \\
Final hor. transv. emittance $(\mu \mathrm{m})$ & 1.28 & 0.29 & 0.25 & 0.22 & 0.22 \\
Final vert. transv. emittance $(\mu \mathrm{m})$ & 1.28 & 0.24 & 0.2 & 0.17 & 0.17 \\
Max. total beam-beam tuneshift & 0.01 & 0.03 & 0.03 & 0.03 & 0.03 \\
IP beta-function $(\mathrm{m})$ & 1.1 & 0.3 & 0.3 & 0.3 & 0.3 \\
Peak luminosity $\left(10^{34} \mathrm{~cm}^{-2} \mathrm{~s}^{-1}\right)$ & 5.01 & 25.2 & 23.2 & 14.5 & 20.1 \\
Max. number events per crossing & 170 & 857 & 394 & 99 & 137 \\
Optimum integrated luminosity/day $\left(\mathrm{fb}^{-1}\right)$ & 2.27 & 8.2 & 7.5 & 5.5 & 6.2 \\
\hline
\end{tabular}

Notes. The initial and ultimate baseline parameters are shown together with the phase two parameters for some alternative bunch spacing scenarios.

sufficient number of sigmas of beam stay clear for collimation and machine protection. The dynamic aperture and beam stability are also guaranteed. Some modifications of the powering scheme will allow the LHC to be ramped quickly providing the short turnaround times required. Lower injection energies have also been studied, in particular a choice of $1.3 \mathrm{TeV}$. This would allow injection from a new accelerator, either a superconducting $6 \mathrm{~T}$ machine in the SPS tunnel or a superferric $1.5 \mathrm{~T}$ one in the LHC tunnel. However, the lower energy will reduce the dynamic aperture at injection, not only due to the larger beam but also because of the increased degradation of the magnetic field quality. In addition, the collimation system and the impedance effects will become more challenging. Further design effort would therefore be required to judge whether one can adopt this injection energy.

\subsection{Design challenges and approaches}

\subsubsection{Lattice considerations}

The lattice layout facilitates the separation of the various functions which ensures that sensitive equipment is not affected by systems which generate significant quantities of radiation. During physics periods with stable beams in collision, the main experiments are the principle source of radiation, since most of the beam is consumed in collisions. The short arcs between the main experiment in PA and the additional experiments in PB and PL ensure that this collision debris does not cause significant background in the smaller experiments.

The collimation systems can also generate significant quantities of radiation under certain operating conditions. Therefore they are separated from all other systems. The momentum collimation system takes advantage of the dispersion from the arcs to remove energy tails. In the betatron collimation system the dispersion from the arcs is suppressed to more easily remove transverse tails.

The relatively sensitive RF systems, electron lenses and the feedback systems are located in a dedicated insertion $(\mathrm{PH})$ where the radiation levels are low.

Similarly, the position of the beam extraction systems in a separate insertion (PD) ensures that the extraction kicker systems are only exposed to low levels of radiation, to limit the likelihood of erroneous kicker firing. 
The injection systems are placed close to the additional experiments but the experiments will only produce significant amounts of radiation at collision energy, when injection is switched off.

With the exception of PD and PJ, the length of each insertion is $1400 \mathrm{~m}$ which corresponds to a scaling of the LHC insertion lengths with the square root of the beam energy. This choice allows the LHC and HL-LHC optics solutions to be implemented in the FCC-hh with the same magnet technology. Improved technologies are used to push the performance beyond that which can be achieved by scaling. In particular the final triplet magnets in the experiment insertions use the same technology as the HL-LHC but with higher fields. This allows a relatively smaller $\beta$-function at the collision point - which naturally scales with the square root of the energy - and also increases the protection of the magnets against the higher levels of collision debris in the FCC-hh. Optics design studies have confirmed that the full $1400 \mathrm{~m}$ is required to achieve the performance goals.

The betatron collimation and the extraction systems are the most sensitive to beam losses under these higher performance scenarios since the increased total particle energy, the higher beam brightness and the larger number of stored protons increase the risk. Consequently, longer insertions of $2800 \mathrm{~m}$ has been allocated for these systems. Further studies will optimise the insertion lengths taking into account the civil engineering and implementation aspects.

\subsubsection{Arc vacuum}

The arc vacuum system defines the aperture of the magnet, which is a key cost driver and has a strong impact on the beam performance. The proposed design allows the magnet aperture to be reduced to $50 \mathrm{~mm}$ and still provides good conditions for the beam, i.e. good vacuum, low impedance and suppression of electron cloud effects.

The beamscreen protects the cold bore of the magnets from the $30 \mathrm{~W} / \mathrm{m}$ of synchrotron radiation emitted by each beam at collision energy (see Sect. 3.3.2). The cryogenic system cools this screen to $50 \mathrm{~K}$; at this temperature the heat can be removed more efficiently than at the $2 \mathrm{~K}$ system of the magnets.

A vacuum pressure of $10^{15} \mathrm{~m}^{-3}$ hydrogen equivalent, similar to the LHC, ensures a beam lifetime of about $100 \mathrm{~h}$, which is compatible with the integrated luminosity target. The power deposited by beam scattering on the rest gas is low enough to avoid quenches of the superconducting magnets due to induced heating. However, with this lifetime up to about $40 \mathrm{~kW}(0.45 \mathrm{~W} / \mathrm{m})$ are still being deposited in the arc dipoles. To remove it requires about $30 \mathrm{MW}$ of cooling power. Therefore the vacuum design aims at a significantly lower vacuum pressure of $0.2 \times 10^{15} \mathrm{~m}^{-3}$ hydrogen, which is feasible.

The copper coating of the inner surface of the beamscreen in combination with the chosen aperture ensures that the impedance is low enough for beam stability, although a high-temperature superconductor (HTS) coating option is being considered to provide a higher stability margin. Pumping holes in the screen facilitate a good vacuum and in contrast to the LHC, they do not generate significant impedance because they are located in two small antechambers. The antechambers also help to reduce the amount of synchrotron radiation that is reflected back into the beam aperture. This arrangement reduces the production of seed electrons that could form an electron cloud. A thin coating of amorphous carbon on the inner part of the chamber hinders any existing electrons from producing electron showers thereby suppressing any electron cloud build-up. 


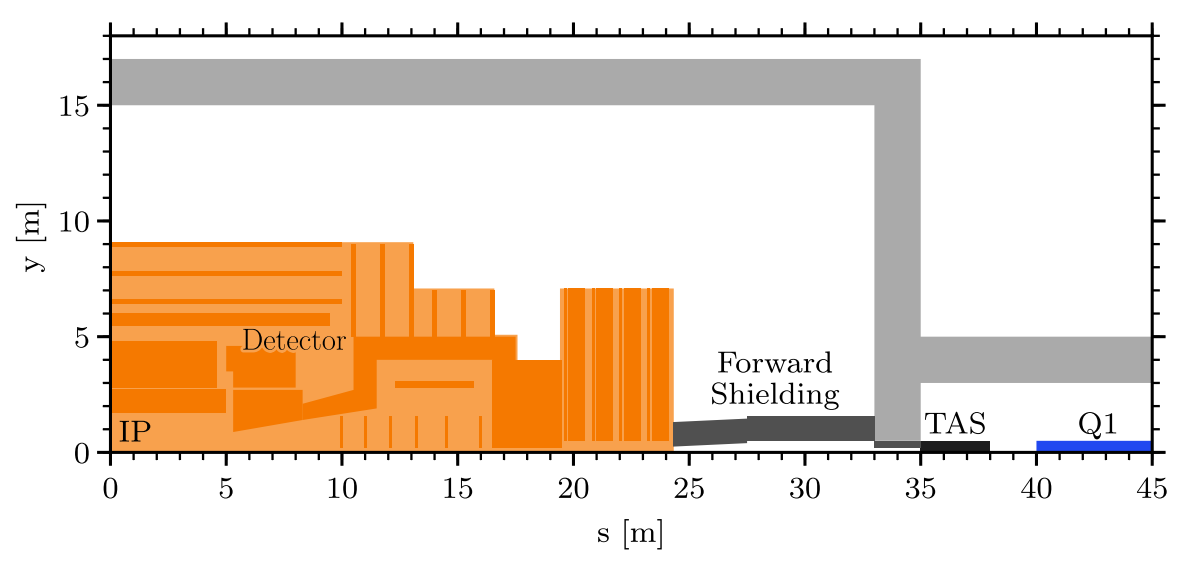

Fig. 2.4. Detector region layout.

\subsubsection{Experimental areas}

The design of the interaction region follows the structure of the LHC interaction region: the final focus system consists of a quadrupole triplet on each side of the interaction point (IP) with a single aperture shared by the counter-rotating beams. Dipoles in the following section separate the two beams into the individual apertures used in the rest of the ring. Four quadrupoles in the straight section, the dispersion suppressor quadrupoles and trim quadrupoles in the first arc cell are used to match the optical functions between the final focus system and the regular arcs. The design of the final focus system is driven by energy deposition from collision debris from the IP. It requires a short drift space between the IP and the final focus system, as well as long, large aperture final focus quadrupoles. The opening of the detector design requires a $33 \mathrm{~m}$ space on either side of the IP, resulting in a total cavern length of $66 \mathrm{~m}$ (Fig. 2.4). The cavern is closed by a $2 \mathrm{~m}$ thick shielding wall followed by an absorber to protect the front of the final focus system from collision debris. This leads to a minimum drift space between the IP and the final focus system of $40 \mathrm{~m}$. Systems with longer drift spaces were also considered but they featured larger chromaticity and matching of the optical functions to the arcs proved difficult within the designated insertion length. The total magnetic length of the triplet quadrupoles is $107.2 \mathrm{~m}$ and allows the use of relatively low gradient, large aperture quadrupoles. This also allows the inside of the magnets to be protected with up to $35 \mathrm{~mm}$ of tungsten shielding, while still leaving enough free aperture to accommodate a beam with a beta function at the IP of almost $\beta^{*}=0.2 \mathrm{~m}$ without violating the collimation hierarchy. This beta function offers a comfortable margin to the nominal goal of $\beta^{*}=0.3 \mathrm{~m}$.

The high luminosity leads to collision debris reaching a total power of about $500 \mathrm{~kW}$ in each of the high luminosity detectors and a significant amount is lost in the machine section next to the detectors. A sophisticated shielding system protects the final triplet magnets from these losses. It consists of a copper shield in front of the triplet and a tungsten tube inside the magnet bore. This tube protects the magnets from charged particles (mainly pions) that enter the magnet aperture and are defocused by the field. Figure 2.5 shows the conceptual design of this shielding [47].

The shielding inside the quadrupoles increases the aperture, which in turn reduces the achievable field. Therefore the absorber design is optimised to provide a good balance between aperture and protection. Detailed simulation studies have confirmed the effectiveness of the shielding of the triplet magnets from the collision debris. For the peak luminosity of $3 \times 10^{35} \mathrm{~cm}^{-2} \mathrm{~s}^{-1}$, the maximum power in the cold mass of 


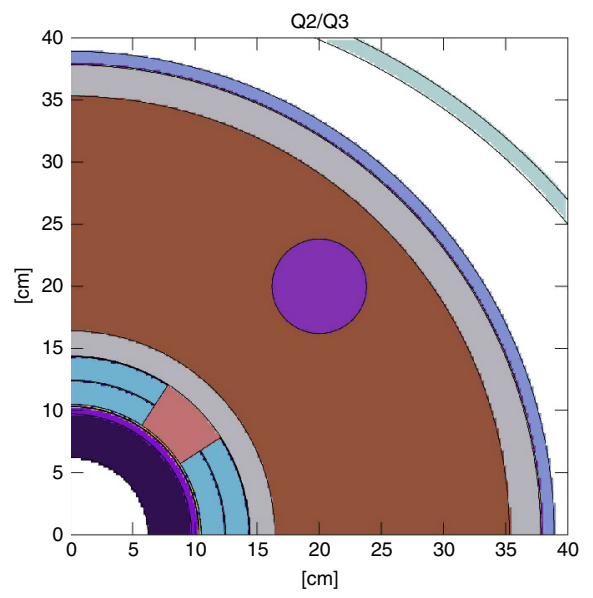

(a)

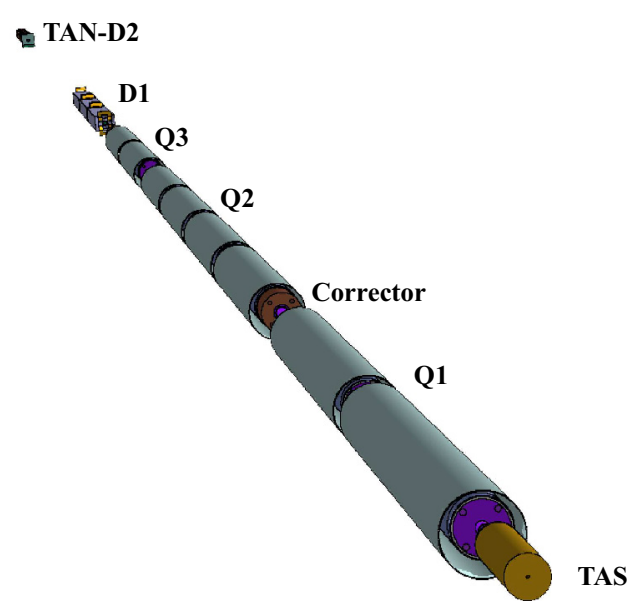

(b)

Fig. 2.5. Conceptual design of the protection system for the focusing triplets next to the collision points. (a) Q2 and Q3 cross section, (b) 3D diagram of the insertion region.

the triplet magnets remains at the level of a few $\mathrm{kW}$ and this can be handled by the cryogenic system. The corresponding maximum local power density in the cold mass reaches $4.5 \mathrm{~mW} / \mathrm{cm}^{-3}$ and thus remains below the safe quench limit of $5 \mathrm{~mW} / \mathrm{cm}^{-3}$. Over the project lifetime, the collision debris deposits a peak integrated radiation dose in the triplet quadrupoles of $70 \mathrm{MGy}$, assumes an integrated luminosity of $30 \mathrm{ab}^{-1}$. The current safe limit is $30 \mathrm{MGy}$ in order to avoid damage to the epoxy resin used in current magnet. New developments, which profit from experiences in the ITER project, show a potential to withstand about 200 MGy by using, for example, by using blends of cynate ester and epoxy resin [48]. Additional means exist to reduce the maximum dose and to increase the radiation hardness of the magnets. In particular, the shielding could be further optimised to distribute the radiation more, which is occurs only in a small part of the magnet. Operating part of the time with vertical crossing angle and the other part with horizontal crossing would further reduce the local dose.

The dipoles next to the triplet are normal conducting. Their peak dose from the collision debris is $90 \mathrm{MGy}$. As for the superconducting magnets this exceeds the current safe limit of 30 MGy but will become acceptable with improved, radiation hard technology. Also in this case further improvement of the shielding design is possible to minimise the dose.

\subsubsection{Machine protection}

After injection at $3.3 \mathrm{TeV}$, each beam has a total energy of $560 \mathrm{MJ}$. At collision energy of $50 \mathrm{TeV}$ this increases to $8.3 \mathrm{GJ}$. These values exceed those of LHC by more than a factor 20. An adequately designed machine protection system must be conceived to mitigate this risk. Dedicated systems will predict and detect equipment failures rapidly enough to engage protection systems that can extract the beam cleanly before it can generate harmful losses. Passive protection mitigates the impact from failure of active components, such as injection and extraction kickers. The collimation system removes the beam halo and tails and protects the machine from losses which might otherwise cause magnet quenches. The collimation system also allows 
continued operation of the machine with large losses in order to be able to diagnose the origin of the problem.

\subsubsection{Injection}

An injection system is located upstream of each of the two low-luminosity experiments within a straight section of $1.4 \mathrm{~km}$. This layout allows three of the four experiments to be close to the CERN site. The injection $[49,50]$ utilises a fast bunch-to-bucket transfer with a normal conducting Lambertson septum to deflect the beam vertically onto the collider orbit. The horizontal angle is reduced by off-centre passage through a defocussing quadrupole and is then fully compensated by a fast kicker system. A specific injection protection system is required to protect the machine and the experiments from badly injected beam. The main constraints for the injection system at $3.3 \mathrm{TeV}$ come from the total beam energy of $560 \mathrm{MJ}$ of the high energy booster (HEB) to FCC transfer, coupled with the beam rigidity of $11 \mathrm{~T} \mathrm{~km}$.

Investigations of LHC injection protection absorber blocks with the HL-LHC beam parameters [51] show that the threshold for absorbers to survive beam impact is around $5 \mathrm{MJ}$ with the expected beam sizes. A burst-mode transfer of 130 batches, each consisting of 80 bunches, is therefore envisaged. The target filling factor of the collider is $80 \%$ (10 400 bunches) and hence the gaps between batches needed for the injection kicker rise time have to be shorter than $425 \mathrm{~ns}$. A fast recharging kicker system allows the full LHC beam to be transferred within several seconds and thus, the time for the injection process is negligible in comparison to the overall filling time. Many kickers will be used to achieve a fast rise time and this requires high reliability in order to achieve a low probability of any kicker misfiring. Novel solid-state kicker generator concepts are being developed to reach these short rise times. These very low failure rates will be achieved by having a very modular and easily maintainable system (see Sect. 3.5.2).

\subsubsection{Extraction and dump}

The dump systems $[52,53]$ have to abort the beam in a safe and reliable way at any energy level between injection and collision (see Sect. 3.5.3). There is one system per beam which extracts the full beam in one turn onto an external absorber block. Both active and passive beam dilution is required in the dump line to avoid damaging this block. The extraction systems for both beams are located in a dedicated $2.8 \mathrm{~km}$ long straight section. At collision energy, the extreme beam rigidity and stored beam energy pose serious challenges for the performance of the extraction kickers, septa and dilution kickers. In order for the dump block to survive the impact of a nominal beam dump, a dual plane dilution kicker system is required. The kicker is excited with a damped voltage pulse oscillating at $50 \mathrm{kHz}$ which distributes the full beam of 10400 bunches onto a multi-branch spiral on the front face of the absorber block which is $20 \mathrm{~m}$ long and $55 \mathrm{~cm}$ in radius. Novel superconducting septa capable of deflecting these rigid beams with a reasonable power consumption are being developed.

Failures within the system which could lead to an asynchronous beam dump are of special concern due to the potential for damage and the difficulty of designing passive absorbers. These failure cases dominate the system design and dictate many of the detailed layout and parameter choices. The extraction kicker system is highly segmented. Hence, the erratic firing of a single kicker leads only to a limited level of orbit oscillation of the beam which can be accepted in the machine for a substantial fraction of a turn. This allows waiting for the next abort gap to fully extract the beam 
Table 2.3. Collimator families, lengths, materials and gaps for both the betatron $(\beta)$ and momentum $(\delta)$ collimation systems in the collision configuration.

\begin{tabular}{|c|c|c|c|}
\hline Type & Length $(\mathrm{m})$ & Material & Half gap $\sigma$ \\
\hline TCP $\beta$ & 0.3 & CFC & 7.6 \\
TCSG $\beta$ & 1.0 & CFC $/$ MoGR & 8.8 \\
TCLA $\beta$ & 1.0 & Iner180 & 12.6 \\
\hline TCP $\delta$ & 0.3 & CFC & 10.8 \\
TCSG $\delta$ & 1.0 & MoGr & 13.0 \\
TCLA $\delta$ & 1.0 & Iner180 & 14.4 \\
\hline TCT & 1.0 & Iner180 & 10.5 \\
TCLD & 1.0 & Iner180 & 35.1 \\
\hline
\end{tabular}

without losses. It is therefore planned to have several abort gaps in the filling pattern. This scheme prevents an asynchronous dump caused by a single kicker misfiring which could create beam losses around the ring during the extraction. The system is designed to survive other failure modes that still might lead to a full asynchronous firing of the extraction kickers, by using passive protection elements. Since a single bunch at $50 \mathrm{TeV}$ is already close to the damage limit of state-of-the-art composites, the extraction kicker rise time has to be as low as $1 \mu$ s to avoid absorber damage caused by particles being swept across the surface during a rising kicker pulse. This constraint could be significantly relaxed if sacrificial absorbers could be used for asynchronous dump protection.

\subsubsection{Collimation}

As in any high-energy hadron collider, a collimation system is needed to intercept and safely dispose of unavoidable beam losses. It should clean the beam halo, to keep losses in the superconducting magnets below the quench limit and also provide passive protection during fast beam failures. The design of the collimation system also has implications for controlling the background in the experiments and/or the machine impedance. The collimation is particularly challenging due to the unprecedented stored beam energy of 8.3 GJ, which is more than a factor 20 higher than the LHC [54].

The backbone of the collimation system is housed in two dedicated insertions: PJ for betatron collimation which is $2.8 \mathrm{~km}$ long and $\mathrm{PF}$ for momentum cleaning which is $1.4 \mathrm{~km}$ long. The design and optics of these insertions $[55,56]$ are scaledup versions of the LHC collimation system [57-60], which has been optimised and demonstrated a very good performance during operation $[61,62]$. The length of the betatron system, as well as its $\beta$-functions, have been scaled up by a factor 5 from LHC to achieve collimator gaps that are similar to the LHC both in units of beam $\sigma$ and in $\mathrm{mm}$. This avoids excessive impedance and guarantees mechanical stability while keeping the $\sigma$-setting small enough to protect the aperture. The optics in PJ is shown in Figure 2.6 and the collimator parameters are in Table 2.3. The magnets in the insertion are normal conducting - to limit their sensitivity to losses - with a design similar to the one for LHC. Future studies will attempt to improve the system design.

As in the LHC, the multi-stage system consists of robust primary collimators (TCP) closest to the beam, followed by secondary collimators (TCSG), also robust, which intercept the secondary halo from the TCPs. In the LHC, the TCPs and TCSGs are made of carbon-fibre-composite (CFC), however, this would induce 

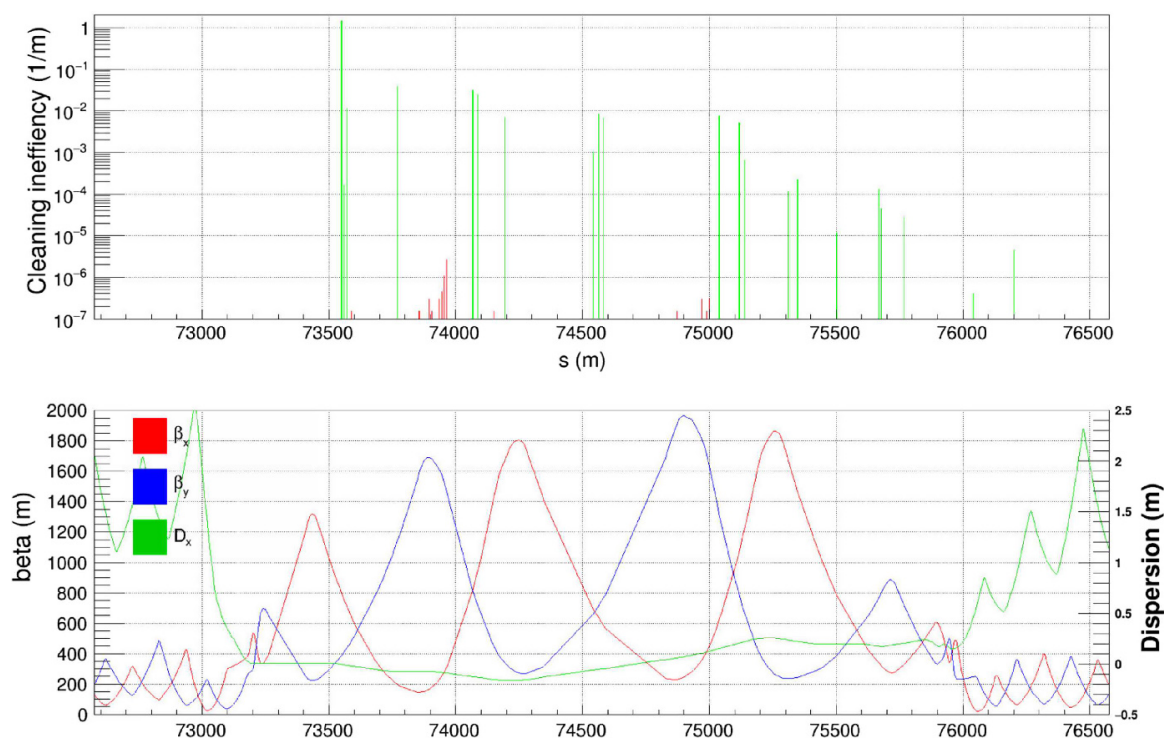

Fig. 2.6. Top: The simulated betatron losses in $\mathrm{PJ}$ at $50 \mathrm{TeV}$ with collision optics for a horizontal beam loss, normalised to cleaning inefficiency as in [60]. Green bars represent losses on collimators, and red bars are losses on warm magnets. No losses in the downstream cold elements (after $s=76000$ ) are observed. Bottom: the optics in PJ.

Table 2.4. The parameters used in the MAD-X model for FCC-hh studies at top energy and injection.

\begin{tabular}{|lrr|}
\hline Parameter set & FCC-hh injection $(3.3 \mathrm{TeV})$ & FCC-hh top energy $(50 \mathrm{TeV})$ \\
\hline Normalised emittance $\epsilon_{n}$ & $2.2 \mu \mathrm{m}$ & $2.2 \mu \mathrm{m}$ \\
\hline $\begin{array}{l}\text { Radial closed orbit } \\
\text { excursion } x_{\mathrm{co}}\end{array}$ & $2 \mathrm{~mm}$ & $2 \mathrm{~mm}$ \\
\hline Momentum offset $\delta_{p}$ & $6 \times 10^{-4}$ & $2 \times 10^{-4}$ \\
\hline $\begin{array}{l}\beta \text {-beating fractional } \\
\text { beam size change } k_{\beta}\end{array}$ & 1.05 & 1.1 \\
\hline $\begin{array}{l}\text { Relative parasitic } \\
\text { dispersion } f_{\text {arc }}\end{array}$ & 0.14 & 0.1 \\
\hline Protected aperture $(\sigma)$ & 13.4 & 15.5 \\
\hline
\end{tabular}

too high an impedance for FCC-hh. Therefore, the majority of the TCSGs are made of molybdenum-graphite instead, but with a $5 \mu \mathrm{m}$ molybdenum coating as in HL-LHC [63]. Active tungsten absorbers (TCLA) intercept the tertiary halo and showers from the TCSGs and are a third stage of collimation. In the same way as in the HL-LHC $[64,65]$, further collimators (TCLD) are introduced in the dispersion suppressor (DIS), to intercept single-diffractive protons leaking from the TCP. Furthermore, tertiary collimators (TCTs) upstream of the experiments provide local protection for the aperture bottlenecks, in particular the final focus triplets. The proposed collimation layout provides sufficient protection of the full machine at injection and also in the most demanding situation at the smallest $\beta^{*}$ at collision.

The betatron cleaning performance of the baseline system has been assessed through tracking simulations [66] and energy deposition studies $[67,68]$. The system is conservatively designed to withstand a temporary beam lifetime drop to $12 \mathrm{~min}$ 
over a period of $10 \mathrm{~s}$ with a beam loss power of $11.8 \mathrm{MW}$. The cleaning simulated by the tracking in the most critical part of the ring, the betatron cleaning insertion at PJ, is shown in Figure 2.6. With the TCLDs and fixed masks, no direct proton impacts are observed in the DIS, which is the most critical area due to the high losses. Nevertheless, showers reach the superconducting magnet coils, however, the simulated power in the most loaded magnets stays well below the estimated quench limit of about $5 \mathrm{~mW} / \mathrm{cm}^{3}$ at $50 \mathrm{TeV}$ (see Sect. 3.2).

With the $11.8 \mathrm{MW}$ power impacting on the system, the loads on the collimators and all other elements in PJ are very challenging. FLUKA $[69,70]$ simulations show that, as for LHC, only up to $10 \%$ of the total energy is deposited in the collimator jaws and absorbers $[67,71-74]$. Almost half of the power is lost in the tunnel wall while the rest is mostly deposited in the warm magnets of the insertion and in the beam pipe. With the horizontal and vertical TCPs shortened to $30 \mathrm{~cm}$ from the initial $60 \mathrm{~cm}$ (LHC design), without skew TCP, and increased thickness of the TCP and first TCSG jaws, the power deposited on any single collimator stays below $100 \mathrm{~kW}$. Thermo-mechanical studies of the first TCSGs [75], under the estimated load of $92 \mathrm{~kW}$, show a temperature of $330^{\circ} \mathrm{C}$ and a temporary deflection of $375 \mu \mathrm{m}$, which is deemed acceptable in the sense that no permanent jaw deformation or damage is induced. Nevertheless, the cleaning performance under this load must be studied in more detail, as well as the cooling pipes which could be plastically deformed if the LHC design were used. Furthermore, at such high temperatures significant outgassing is expected from both the TCP and TCSG. Therefore, the vacuum should be studied in detail and the pumping capabilities reviewed.

Other elements in the warm collimation section also receive a high power deposition. Warm dipoles absorb up to around $1 \mathrm{MW}$, which can be reduced with shielding and handled by a suitably powerful cooling system. The tunnel wall and other elements receive about $50 \%$ of the power intercepted by the collimation system. The distribution of this must be studied in detail to quantify any possible bottlenecks.

The absence of a skew TCP, which is necessary to keep the power load on single collimators at a tractable level, has the downside of potentially introducing losses in the dispersion suppressor just upstream of the TCLD. Tracking simulations indicate that beam tails in the skew plane cause about a factor 5-10 higher losses than tails in the horizontal plane. These losses, if not alleviated, could introduce a stronger limitation on the minimum beam lifetime if tails are present in the skew plane. Further studies will refine the estimates of the energy deposition studies and the potential mitigation and should also be used to study the power load on the TCSGs. However, it should be noted, that for the LHC, a similar stringent requirement on beam lifetime for skew losses would not introduce an operational bottleneck. It is nevertheless under study whether these DS losses can be reduced by either introducing an additional TCLD and masks in front of the affected magnets, or by placing the skew TCP at a different location further upstream.

In summary, a simulation campaign $[56,67,71-76]$ has shown that the proposed collimation system design can protect the magnets against quenches and survive without damage during the pessimistic scenario of a beam lifetime drop to $12 \mathrm{~min}$. Further studies are required to quantify the change in cleaning efficiency during the collimator deformation, as well as studies to quantify the risks associated with removing the skew TCP, the outgassing and vacuum and the loads on the warm magnets.

The design of the momentum cleaning, which is less critical than the betatron cleaning, and the protection of the aperture at injection are under study. The quench limit is less challenging at injection than at top energy but the size of the beamscreen, which is significantly smaller than in LHC, results in normalised apertures of about $11.4 \sigma$. This is lower than the design criterion for HL-LHC, however, it is believed [77] 
that the aperture can be brought within tolerance through a careful study of error tolerances and local optics. First studies of an asynchronous beam dump indicate that the collimation system can protect the machine without being damaged if a single kicker module fails. Other failure scenarios, radiation damage, shielding design and advanced collimation concepts remain to be studied.

\subsubsection{RF system}

The baseline RF system design is similar to that of the LHC and has a frequency of $400.8 \mathrm{MHz}$. The installed voltage will be $48 \mathrm{MV}$, three times more than in the LHC. In the longitudinal plane, an inductive longitudinal impedance budget of $\operatorname{Im}(Z) / n=$ $0.2 \Omega$ is expected, similar to the one of LHC $(0.1 \Omega)$. At injection $12 \mathrm{MV}$ is used and ensures beam stability, at collision energy $42 \mathrm{MV}$. During the ramping a maximum of $43.5 \mathrm{MV}$ is used. At injection the longitudinal beam emittance is $2.3 \mathrm{eVs}$, i.e. a foursigma bunch length of $1.42 \mathrm{~ns}$ and a two-sigma energy spread of $\Delta E / E=3.25 \times 10^{-4}$ and the synchrotron tune is $2.77 \times 10^{-3}$. At collision the corresponding values are $10.2 \mathrm{eVs}, 1.07 \mathrm{~ns}, \Delta E / E=1.24 \times 10^{-4}$ and $1.33 \times 10^{-3}$, respectively. Controlled longitudinal emittance blow-up (by band-limited RF phase noise) will be required not only during the ramp but also in the coast at $50 \mathrm{TeV}$ to balance synchrotron radiation damping.

\subsection{Optics design and beam dynamics}

\subsubsection{Lattices}

The arcs are made of $213.030 \mathrm{~m}$ long FODO cells with a phase advance of $90^{\circ}$ in both planes [78]. The phase advances in the cells of the long arcs are adjusted to obtain global tunes of $110.28 / 108.31$ at injection and 110.31/108.32 at collision. Each arc cell hosts 2 short straight sections (SSS) and 12 cryo-dipoles (see Fig. 2.7). Each SSS is $12 \mathrm{~m}$ long and contains from left to right one beam position monitor (BPM), one $1.2 \mathrm{~m}$ long sextupole, one $7.2 \mathrm{~m}$ long quadrupole, one $0.5 \mathrm{~m}$ long multipole corrector (trim quadrupole, skew quadrupole, or octupole), and one $1.2 \mathrm{~m}$ long dipole corrector. Each cryo-dipole contains one $14.069 \mathrm{~m}$ long dipole and one $0.11 \mathrm{~m}$ long sextupole corrector. The interconnection distance between dipoles is $1.5 \mathrm{~m}$. The interconnection distance between one dipole and the SSS is $1.3 \mathrm{~m}$. The interconnection distance between two elements of the SSS is $0.35 \mathrm{~m}$. The cell length is optimised to maximise the dipole filling factor resulting in the dipole magnetic field of $15.96 \mathrm{~T}$ at $50 \mathrm{TeV}$ beam energy [79].

Each arc begins and ends with a dispersion suppressor (DIS). The DIS cancels the dispersion coming from the arcs and matches the optical functions to the insertions. The LHC-like configuration is the best compromise between compactness and flexibility [79]. The DIS is made of 6 sections. Each of the three first sections, starting from the insertion, is $75.376 \mathrm{~m}$ long and contains one SSS and four cryo-dipoles. Here, the SSS contains one BPM, one $9.7 \mathrm{~m}$ long quadrupole and one $1.2 \mathrm{~m}$ long dipole corrector. The fourth section has the same length as the arc half-cell but contains only four cryo-dipoles. The two last sections are similar to arc half-cells. The SSS is slightly modified and contains one BPM, one $7.2 \mathrm{~m}$ quadrupole, one $2 \mathrm{~m}$ trim quadrupole and one $1.2 \mathrm{~m}$ long dipole corrector. Collimators are inserted in the first and third sections to protect the arcs from the debris coming from the insertions [80] (see Sect. 2.3.7).

The total lengths of the LSS and ESS insertion are 1.4 and $2.8 \mathrm{~km}$, respectively. 

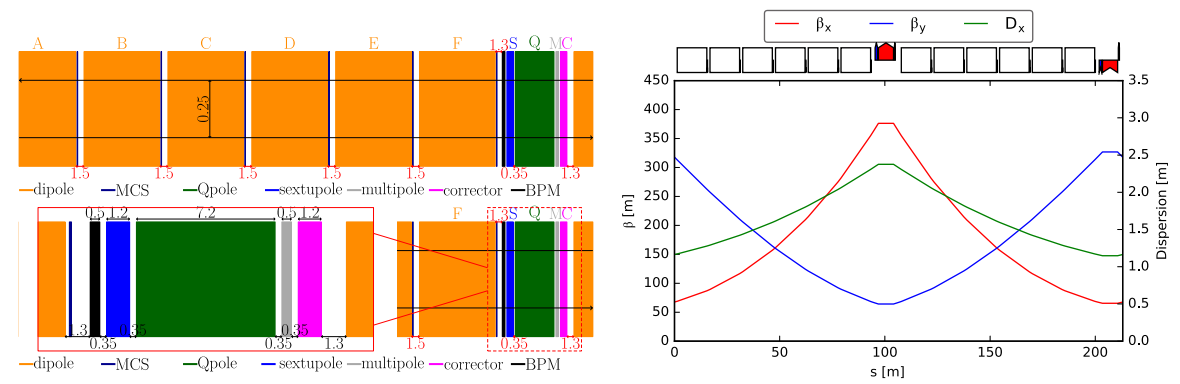

Fig. 2.7. Layout of an arc half-cell (top left) and of the SSS (bottom left). Optical functions in the inner arc cell are shown on the right.

\subsubsection{Main experiment insertions}

The optics of the two high-luminosity experiments insertion regions (EIR), named LSS-PA-EXP and LSS-PG-EXP, is shown at the top left of Fig. 2.8 for $\beta^{*}=0.3 \mathrm{~m}$ [81]. The distance between the IP and the inner triplet of the EIR is $40 \mathrm{~m}$. The separation dipoles are assumed to be normal conducting. A space of $20 \mathrm{~m}$ is reserved for crab cavities. The superconducting quadrupoles of the inner triplet are optimised to handle the radiation coming from the interaction point [47].

\subsubsection{Collimation insertions}

Collimation sections for momentum and betatron cleaning are scaled from LHC and located in LSS-PF-COL and ESS-PJ-COL, respectively. The optics is shown in Figures $2.8 \mathrm{~d}$ and $2.8 \mathrm{e}$. In order to handle the debris losses downstream of the collimators, the magnets of these two sections are warm. The collimation inefficiency is presented in Section 2.3.7 [80].

\subsubsection{RF insertions}

The RF cavities are located in a dedicated section in LSS-PH-RFS. This insertion is made of $230 \mathrm{~m}$ long FODO cells with a phase advance of $90^{\circ}$. This section may also host electron lenses in some upgrade schemes of the machine.

\subsubsection{Injection and extraction}

The low-luminosity EIRs, named LSS-PB-EXP and LSS-PL-EXP, each contain an injection system and an IP. The protection of cold elements from miss-kicked injected beams requires specific phase advances between magnets and reduces optics flexibility. The distance between the IP and the inner triplet is $25 \mathrm{~m}$. The optics of the lowluminosity EIR is shown in Figures $2.8 \mathrm{~b}$ and $2.8 \mathrm{f}$ for $\beta^{*}=3 \mathrm{~m}$. A beam stay-clear region of more than 15 root mean square sizes $(\sigma)$ is achieved in all EIRs. The extraction is located in ESS-PD-EXT. The optics of the extraction section are derived from LHC and are given in Figure 2.8c. Surviving absorbers are inserted near the middle quadrupoles to protect them. 


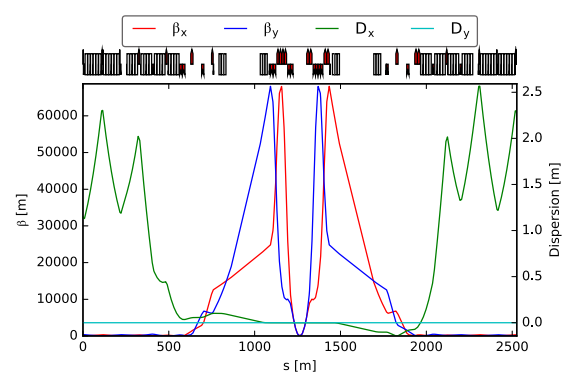

(a)

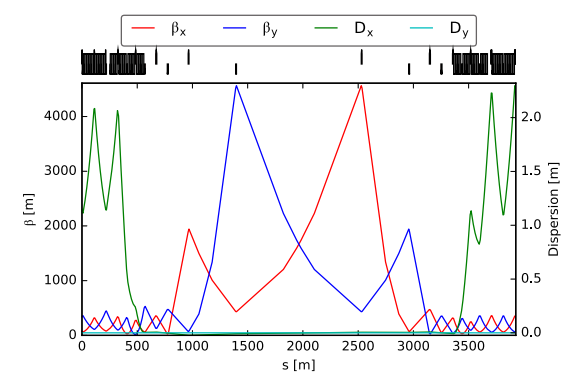

(c)

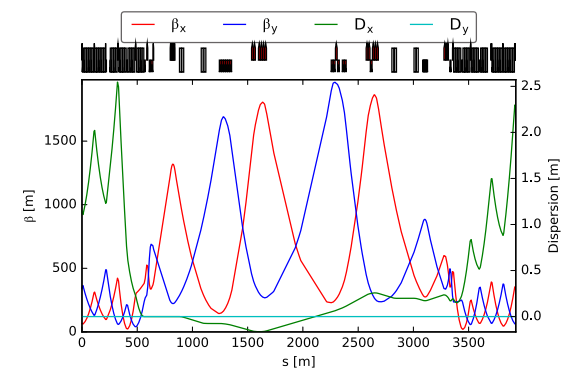

(e)

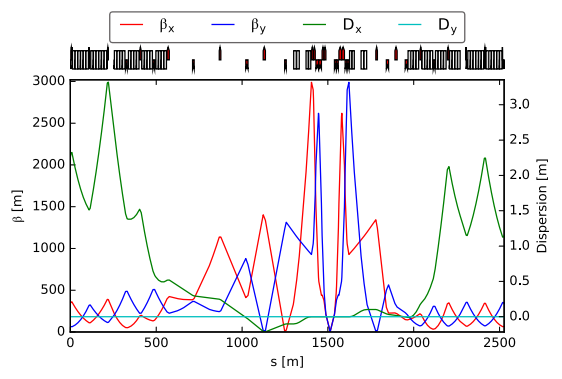

(b)

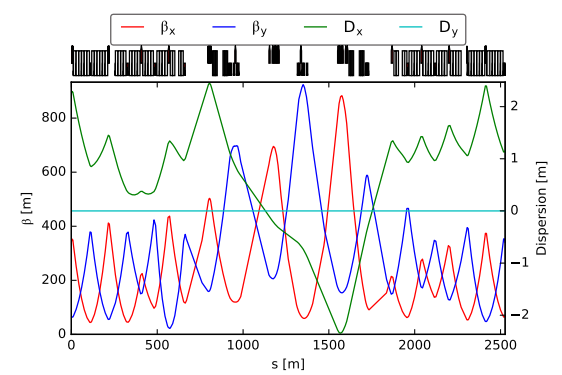

(d)

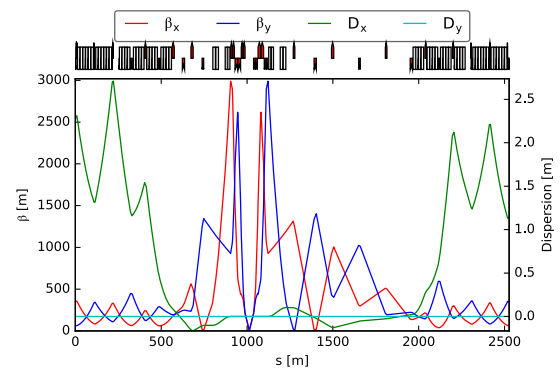

(f)

Fig. 2.8. Optical functions in the insertions LSS-PA-EXP, LSS-PB-EXP, ESS-PD-EXT, LSS-PF-COL, ESS-PJ-COL, and LSS-PL-EXP. (a) Interaction region: LSS-PA-EXP \& LSS-PG-EXP. (b) Injection+interaction region: LSS-PB-EXP. (c) Extraction section: ESSPD-EXT. (d) Collimation section: LSS-PF-COL. (e) Collimation section: ESS-PJ-COL. (f) Injection+interaction region: LSS-PL-EXP.

\subsubsection{Dynamic aperture and correction schemes}

Dynamic aperture (DA) is the maximum phase-space amplitude within which no particle gets lost as a consequence of single-particle-dynamic effects. The DA is given at injection and at collision energies without beam-beam effects in Table 2.5. Beambeam effects are given in Section 2.4.9. The target value for the DA of due to field errors at injection is fixed at $12 \sigma$, similar to LHC, to provide a safety factor of about 2 taking into account the uncertainties of SixTrack calculations [82]. At injection, the multipole components of the main dipoles drive the DA. The DA is above the target value of $12 \sigma$ provided the main dipoles are equipped with local correctors to compensate the $b_{3}$ and $b_{5}$ components. In addition to the field errors, the octupoles that stabilise the beam can reduce the DA. Powering them to a level that provides a safety factor three for beam stability, an DA of $8.1 \sigma$ is achieved. At collision energy, 
Table 2.5. Average and minimum $10^{5}$ turn DA for 5 directions $\phi=\arctan \left(\sqrt{\epsilon_{y} / \epsilon_{y}}\right)$ of the real space $(x, y)$ and 60 seeds at collision (nominal tunes 110.31/108.32) and injection (nominal tunes 110.28/108.31) for the dipole errors given in Table 3.1.

\begin{tabular}{|l|l|l|l|l|l|l|}
\hline Case & DA $(\boldsymbol{\sigma})$ & $\mathbf{1 5}^{\circ}$ & $\mathbf{3 0}^{\circ}$ & $\mathbf{4 5}^{\circ}$ & $\mathbf{6 0}^{\circ}$ & $\mathbf{7 5}^{\circ}$ \\
\hline Injection & minimum & 14.4 & 14.3 & 14.0 & 15.2 & 16.9 \\
$(3.3 \mathrm{TeV})$ & average & 17.6 & 17.6 & 17.8 & 18.3 & 18.4 \\
\hline Injection & minimum & 8.1 & 8.4 & 8.8 & 8.9 & 9.5 \\
(arc, IRs errors and MO -15 A) & average & 9.9 & 10.0 & 11.0 & 10.8 & 10.9 \\
\hline Collision (no beam-beam) & minimum & 27.2 & 25.9 & 24.7 & 24.9 & 25.1 \\
$\left(\beta^{*}=0.3 \mathrm{~m}\right)$ & average & 31.9 & 31.3 & 30.3 & 29.0 & 29.9 \\
\hline
\end{tabular}

Notes. At injection, DA due to errors in dipoles only is reported. At collision, the high luminosity IR errors and non-linear correctors are also included.

non-linear errors in the EIR drive the DA. Non-linear correctors are mandatory in the EIRs to achieve a DA above 20 $\sigma$ 's [83] for the non-colliding beam.

The cross-talk through the yoke between the two dipole chambers gives a systematic $b_{2}$ component of 6 units in the dipoles for a beam separation of $250 \mathrm{~mm}$. The sign of $b_{2}$ is negative/positive in the inner/outer side for cos-theta or block configuration. This $b_{2}$ component is corrected by the main arc quadrupoles to keep the phase advances in the FODO cells.

In the current magnet design, the systematic component of $b_{3}$ in the dipoles reaches up to 25 units at injection. To first order, this is mitigated by the spool pieces. Orbit uncertainties in the dipoles and spool pieces create feed-down effects with a random $b_{2}$ component, and are a source of beta-beating [84]. To keep this beta-beating contribution at the same level as the one of random $b_{2}$ in the dipoles and quadrupoles, the relative RMS misalignment between the spool pieces and the dipoles should be below $0.3 \mathrm{~mm}[85]$.

The orbit correction scheme uses dipole correctors and BPMs located in each SSS. Horizontal/vertical dipole correctors are located near the focusing/defocusing quadrupoles, respectively. A maximum integrated field value of $4.8 \mathrm{Tm}$ in the correctors requires the alignment tolerances shown in Table 2.6 [86]. The tune is corrected by matching the gradients of the main quadrupoles in the long arcs or alternatively by trims at the entrance and exit of the long arcs. Sets of four skew quadrupoles separated in phase by $90^{\circ}$ are located in the long arcs and correct the coupling introduced by the $a_{2}$ components of the dipoles. A set of trim and skew quadrupoles at the short arc entrances, at phase advances of $180^{\circ}$, correct the spurious dispersion coming from the EIRs [87]. The maximum gradient in the trim and skew quadrupoles is $220 \mathrm{~T} / \mathrm{m}$. The 90 -percentile beta-beating is $22 \%$ and $25 \%$ in the horizontal and vertical planes respectively and has to be corrected. The 90 -percentile parasitic dispersion $\Delta D / \sqrt{\beta}$ is $2.9 / 2.8 \times 10^{-2} \mathrm{~m}^{1 / 2}$ and may be corrected.

\subsubsection{Impedances and mitigation}

Coupling impedances can drive beam instabilities and cause heating of machine components, ultimately limiting the beam intensity. As the heat load will be dominated by synchrotron radiation, transverse instabilities and the induced coupled-bunch and single-bunch instabilities are the most critical. To ensure operation at the intensity of $10^{11}$ protons per bunch with some safety margin, the transverse impedances are limited to the allowed "impedance budget" determined by the balance of the instability growth rate and the active and passive damping mechanisms. The effective dipolar 
Table 2.6. Misalignment errors in the arcs.

\begin{tabular}{|l|l|l|l|l|l|}
\hline Element & Error & Error desc. & Units & FCC & LHC \\
\hline Dipole & $\sigma_{x}, \sigma_{y}$ & Position & $\mathrm{mm}$ & 0.5 & 0.5 \\
& $\sigma_{\psi}$ & Roll angle & $\mathrm{mrad}$ & 0.5 & $\mathrm{n} / \mathrm{a}$ \\
& $\sigma_{b_{1}}$ & Random $b_{1}$ & $\%$ & 0.1 & 0.08 \\
& $\sigma_{b_{2}}$ & Random $b_{2}$ & $10^{-4}$ & 0.92 & 0.8 \\
& $\sigma_{a_{2}}$ & Random $a_{2}$ & $10^{-4}$ & 1.04 & 1.6 \\
& $\sigma_{a_{2}}$ & Uncert. $a_{2}$ & $10^{-4}$ & 1.04 & 0.5 \\
\hline Quadrupole & $\sigma_{x}, \sigma_{y}$ & Position & $\mathrm{mm}$ & 0.5 & 0.36 \\
& $\sigma_{\psi}$ & Roll angle & $\mathrm{mrad}$ & 1.0 & 0.5 \\
& $\sigma_{b_{2}}$ & Random $b_{2}$ & $\%$ & 0.1 & 0.1 \\
\hline $\mathrm{BPM}$ & $\sigma_{x}, \sigma_{y}$ & Position & $\mathrm{mm}$ & 0.3 & 0.24 \\
& $\sigma_{r}$ & Read error & $\mathrm{mm}$ & 0.2 & 0.5 \\
\hline
\end{tabular}

Table 2.7. Effective transverse coupled-bunch (CB) and single-bunch (SB) impedances for the rigid mode $(k=0)$ at zero chromaticity.

\begin{tabular}{|l|c|c|}
\hline Source of impedance & $\begin{array}{c}\text { Re }\left(Z_{\mathrm{dip}}^{\mathrm{CB}}\right)(\mathrm{M} \Omega / \mathrm{m}) \\
\text { injection/top energy }\end{array}$ & $\begin{array}{c}\text { Im }\left(Z_{\mathrm{dip}}^{\mathrm{SB}}\right)(\mathrm{M} \Omega / \mathrm{m}) \\
\text { injection/top energy }\end{array}$ \\
\hline $\begin{array}{l}\text { Resistive impedance of beamscreen + coat- } \\
\text { ing }\end{array}$ & $-940 /-1610$ & $4.5 / 5.7$ \\
Pumping holes & Negligible & $\leq 0.1$ \\
Resistive impedance of warm beam pipe & $-100 /-400$ & $0.4 / 1.5$ \\
Resistive impedance of collimators & $-30 /-150$ & $1.0 / 34.5$ \\
Geometrical impedance of collimators & Negligible & $0.9 / 11.9$ \\
Dipole-dipole interconnects & Negligible & 2.4 \\
RF cavities & Negligible & Negligible \\
Crab cavities & Negligible & Negligible $/ 0.5$ \\
\hline Total & $-1070 /-2160$ & $9.2 / 56.5$ \\
\hline Instability threshold & $-4070 /-7260$ & $35 / 220$ \\
\hline
\end{tabular}

Notes. Impedances in the most critical (vertical) plane are shown. The chamber is fully copper coated and has an additional carbon layer to suppress electron cloud.

transverse impedances are shown in Table 2.7. The present transverse impedance model includes the most critical components: the beamscreen together with the contributions of the surface coating and the pumping holes, the warm beam pipe in the straight sections, the collimators, the interconnects between the dipole magnets, the RF system and the crab cavities.

The transverse resistive wall impedance of the beamscreen sampled at a fraction of the revolution frequency is the primary driver for the coupled-bunch instability. Its value per unit length at the lowest betatron sideband is about an order of magnitude larger than that of the LHC beamscreen due to the smaller aperture, the higher wall temperature, and the lower revolution frequency. The beamscreen impedance can also contribute to the transverse mode coupling instability (TMCI). This contribution is especially important at injection when the beamscreen together with the interconnects dominate the impedance budget. A novel design, shielding the pumping holes from the beam, significantly reduces the impedance of the beamscreen. The beamscreen surface coating is expected to give a $30 \%$ increase in impedance at the relevant frequencies if an amorphous carbon or a titanium-nitride coating is used for electron cloud mitigation. Alternatively, a laser treatment of the beamscreen surface $[88,89]$ can be used. However, the impedance considerations may limit the treated area to only a small fraction of the surface. The collimators are another critical 


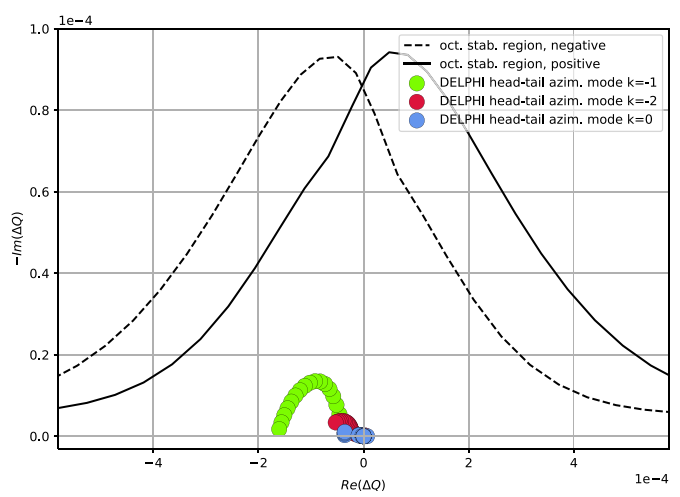

Fig. 2.9. Stability diagram (top energy, $y$-plane). Real and imaginary tune shifts caused by the impedance are shown in round dots for different chromaticities in the range $0<Q^{\prime}<20$. The transverse feedback is operating at a damping rate of 460 turns. The lines represent the stability region provided by the 480 Landau octupoles, assuming no long range beam-beam effects.

contributor to the impedance budget and they are the primary driver of TMCI at collision energy. Making the jaws of some collimators out of a low-resistivity material (such as molybdenum-graphite) brings their impedance down to an acceptable level. The intensity threshold for single-bunch TMCI instabilities has been estimated for zero chromaticity. The threshold is found to be several times greater than the nominal bunch intensity, provided that the screen is coated with carbon (Tab. 2.7). If necessary, the TMCI threshold can be increased further by using the feedback system in the reactive mode [90].

For the rigid $(k=0)$ coupled-bunch instability, the tune spread required for Landau damping is estimated to be about an order of magnitude larger than in the LHC (at top energy). Therefore, in order to limit the number and strength of Landau octupoles required to produce the tune spread in particular at collision energy, stability of the rigid mode is ensured by the feedback system. The planned feedback gain corresponds to a damping rate of 20 turns at injection and 150 turns at collision energy and is sufficient to stabilise the rigid bunch modes at zero chromaticity with a safety factor of three. The stability of the rigid mode at zero chromaticity also guarantees stability at all positive chromaticities. The higher order $|k| \geq 1$ head-tail modes are stabilised by the combination of the transverse feedback and the 480 installed Landau octupole magnets. The octupoles alone would be sufficient to stabilise the $|k| \geq 1$ modes only in a narrow window of chromaticities. However, at collision energy, with the transverse feedback strong enough to completely damp the rigid mode (damping rate of 460 turns), the $|k| \geq 1$ modes lie well within the octupole stability region in the entire chromaticity range $0<Q^{\prime}<20$, as shown in Figure 2.9 [91]. A stronger damping rate can bring the growth rates even lower as long as feedback-amplified noise does not cause an excessive emittance growth. A negative octupole polarity is chosen because it provides higher safety margins for the single-beam stability and is favourable for the dynamic aperture. The beam-beam forces can significantly impact the stability limits during the betatron squeeze [92-94], as has been shown by LHC experience [95]. Therefore, the "collide and squeeze" scheme is implemented [96,97] in order to prevent the long-range beam-beam effect from reducing the stability region. In this scheme, the head-on collision provides improved stability during the squeeze when the increasing effect of the parasitic long-range interactions reduces the stabilising effect of the octupoles. 
Table 2.8. SEY thresholds for multipacting leading to electron cloud build-up in the arcs at injection and collision energy.

\begin{tabular}{|c|c|c|c|}
\hline Arc element & \begin{tabular}{|c|}
$25 \mathrm{~ns}$ \\
injection/top energy
\end{tabular} & $\begin{array}{c}12.5 \mathrm{~ns} \\
\text { injection/top energy }\end{array}$ & $\begin{array}{c}5 \mathrm{~ns} \\
\text { injection/top energy }\end{array}$ \\
\hline Dipole & $1.5 / 1.5$ & $1.1 / 1.1$ & $1.5 / 1.5$ \\
\hline Quadrupole & $1.1 / 1.2$ & $1.0 / 1.0$ & $1.1 / 1.0$ \\
\hline Drift & $2.0 / 2.0$ & $1.3 / 1.3$ & $1.6 / 1.6$ \\
\hline
\end{tabular}

At injection the octupoles can provide beam stability with lower field. A level that provides a safety factor three for beam stability is acceptable for the dynamic aperture, which remains above $8 \sigma$.

A number of solutions exist to even further increase the safety margins. The octupoles can be made more effective by increasing the field gradient and/or by increasing the $\beta$-function in the arcs. The octupoles can also be replaced with an electron lens as the source of a tune spread [98,99]. Studies have shown that an electron lens of $2 \mathrm{~m}$ powered with $600 \mathrm{~mA}$ will easily damp the higher order modes for $Q^{\prime}=20$. Other passive damping mechanisms such as RF quadrupoles [100] have been studied. Finally, optimisation of the lattice (i.e. working point and phase advance), coating of the beamscreen with a high-temperature superconductor (HTS), or the use of an intra-bunch feedback [101], can also be considered.

\subsubsection{Electron cloud considerations}

Electron clouds may cause several unwanted effects, in particular beam instabilities, emittance growth, tune shifts, additional heat load on the cryogenic system and vacuum degradation [102]. The build-up of electron clouds through multipacting can be suppressed by having a sufficiently low secondary electron yield (SEY) of the beamscreen surface. Simulations of electron cloud build-up and its effect on beam stability have been performed to identify the conditions required for electron cloud suppression.

The SEY threshold values, above which electron cloud build-up occurs, have been estimated in arc dipoles, quadrupoles and drift sections at injection and collision energies and are summarised in Table 2.8. For $25 \mathrm{~ns}$ bunch spacing, the build-up can be suppressed with a low-SEY surface treatment, such as an amorphous carbon coating [103]. In the dipoles the coating is applied over the top and bottom surface of the beamscreen, while in the quadrupoles the coating is required on the sides, at $45^{\circ}$ to the horizontal plane. In the field-free regions, where an SEY of up 2.0 is allowed, no dedicated coating is necessary.

Electron cloud build-up has also been studied for the alternative options of $12.5 \mathrm{~ns}$ and $5 \mathrm{~ns}$ bunch spacing. The multipacting thresholds for these shorter spacings, listed in Table 2.8, are considerably lower than for the $25 \mathrm{~ns}$ bunch spacing. To suppress electron cloud build-up for these bunch spacings, a surface treatment with SEY no larger than 1.0 may be required.

Threshold electron densities for inducing single-bunch instabilities have been estimated with analytical calculations and beam dynamics simulations to be around $1 \times 10^{11} \mathrm{~m}^{-3}$ at injection and $1 \times 10^{12} \mathrm{~m}^{-3}$ at collision energy. In general, electron densities are well below the instability thresholds when the SEY is below the multipacting threshold. However, if a significant quantity of photoelectrons are produced by the synchrotron radiation, the electron density can exceed the instability threshold without multipacting. The constraints on the quantity of photoelectrons determined 
by e-cloud studies have been taken into account in the beamscreen design and were met by having a saw-tooth surface at the point of impact of the radiation.

\subsubsection{Beam-beam effects}

The beam-beam interaction is known to be an important factor limiting the performance reach of particle colliders. Three of the most significant effects of beam-beam interactions are: (i) the induced particle losses that decrease the beam lifetime, create a high background load for physics experiments and elevate the heat and radiation load on the collimation system; (ii) the degradation of beam quality manifesting itself through the beam size blow-up which decreases the luminosity delivered to the experiments; and (iii) the impact on the coherent beam stability.

The collider design is driven by the LHC experience where beam-beam effects have been studied both theoretically and experimentally [104-106]. However, the FCC-hh represents a quantitative as well as a qualitative leap into unknown territory with respect to beam-beam effects. The baseline configuration makes use of some novel concepts that have not yet been used to their full extent in hadron colliders and thus require careful evaluation. The concepts related to beam-beam effects are: (i) tilting bunches in the main interaction points with crab cavities; (ii) radiation damping in the presence of large beam-beam parameters; (iii) noise sources and interplay with beam-beam head-on; and (iv) the impact on Landau damping and impedance mode coupling.

The expected impact of the beam-beam interactions on the machine performance has been studied with weak-strong as well as strong-strong models employing the SixTrack [107] and COMBI [108] codes, which have been successfully used for the design and optimisation of the LHC and HL-LHC [109-112]. SixTrack is capable of calculating the area of stable motion in real space, the DA, and has been benchmarked for LHC experimental data and a detailed lattice description, with and without beam-beam effects $[112,113]$. The COMBI code evaluates the evolution of macroscopic beam parameters including intensity, emittance and luminosity, together with a detailed description of the Landau damping properties of two beams $[108,114]$, in a self consistent treatment, but with a simplified lattice description. The study uses criteria similar to those in the LHC and HL-LHC design studies $[115,116]$. Benchmarking of the simulations with LHC observations indicate that losses and reduction of beam lifetime start to appear at values of the simulated DA equal to or lower than $4 \sigma$ [104]. More detailed studies show a strong correlation between the long term beam intensity lifetimes and the computed DA for different beam configurations with and without beam-beam interactions [111-113]. The FCC-hh design ensures a DA of $7.2 \sigma$ for the nominal emittance of $2.2 \mu \mathrm{m}$ - well above $4 \sigma$, in order to provide sufficient margin. This also ensures that no extra particles are lost on the collimation system due to beam-beam diffusive mechanisms. Studies are valid for particles with relative momentum deviation of $1.0 \times 10^{-4}$.

In the nominal scenario ( $25 \mathrm{~ns}$ spacing), bunches with $10^{11}$ protons per bunch and transverse normalised emittance of $2.2 \mu \mathrm{m}$ collide at four Interaction Points (IPs). The bunches will be tilted by crab cavities at points PA and PG to ensure headon collisions despite the trajectories crossing with a finite angle. Assuming constant transverse emittances, the separation of beams at the first parasitic encounter (104116 long-range interactions per IP are expected) in PA and PG will be $17 \sigma$ while for $\mathrm{PB}$ and PL (60 long-range interactions per IP) the separation will be $30 \sigma$ to keep the impact of such low-luminosity experiments in the shadow of the two main ones. This configuration corresponds to a crossing angle of $200 \mu \mathrm{rad}$ at PA and PG and of $160 \mu \mathrm{rad}$ at PB and PL. 
Studies performed at the constant crossing angle of $200 \mu \mathrm{rad}[117,118]$ show that the target DA of $7.2 \sigma$ can be maintained during the entire operation cycle. In addition, margins are also left for (i) operating at a larger value of chromaticity (up to 20 units) if so demanded by the coherent beam stability, (ii) operating in the presence of the multipolar lattice errors [119], and (iii) account for the presence of collision points in PB and PL. PACMAN effects have been evaluated and shown not to have a significant impact assuming the passive compensation of tune and chromaticity shifts introduced when the PA and PG have alternating crossing planes [120]. Alternative crossing schemes to dilute the energy deposited in the IR are also feasible [117] and open the possibility for variable crossing planes. Bunch-by-bunch orbit offsets of the order of $0.2-0.3 \sigma$ are expected at the IPs due to the long-range effects with a consequent loss of luminosity of less than $1 \%$.

The total head-on beam-beam tune shift $Q_{b b h o}$ is limited to less than 0.03 based on experience gained from LHC Runs 1 and 2 [121,122] and in agreement with simulation results [123]. This will require $\mathrm{PB}$ and $\mathrm{PL}$ to operate with a transverse offset resulting in a maximum tune shift of $1-2 \times 10^{-3}$. The beam-beam head-on tune spread together with transverse noise sources could lead to emittance blow-up. Transverse feedback, necessary for coherent stability, should be used to reduce the impact of transverse noise on colliding beams [124]. To obtain such a condition it is essential to improve the amplitude of the transverse feedback pick-up noise floor to maintain a tolerable growth rate $[125,126]$. The impact of active devices such as crab cavities and electron lenses must be evaluated together with the impact of radiation damping on the emittance evolution.

The Landau octupoles are preferentially powered with negative polarity for two reasons: they provide the maximum threshold for single beam stability at flat-top energy and allow global compensation of the long-range beam-beam interactions $[127,128]$. In this configuration the DA improves and reaches a maximum value of $8.5 \sigma$, leaving margins to reduce the crossing angles or increase the intensity. Further improvement of the lattice appears possible to optimise the global compensation of the long-range effects with multipoles. To maximise the beam stability, the beams will be brought into collision using the "collide and squeeze" scheme that is planned for HL-LHC [96,97].

\subsection{Operation and performance}

In the 25-year life-cycle of the collider, the machine performance will evolve from the initial parameters to the nominal design values [129]. For the estimate of the total produced integrated luminosity, the initial parameters are assumed to be used during the first ten years and the nominal parameters during the last 15 years of operations. Considering the overall production goal of $17.5 \mathrm{ab}^{-1}$ over the lifetime, the integrated luminosity goals for the individual phases have been set to: (i) baseline parameters: $5 \mathrm{ab}^{-1}$ and (ii) nominal parameters: $15 \mathrm{ab}^{-1}$. The only hardware modification required between the two phases is the installation of crab cavities. Therefore, a gradual evolution of the beam parameters that follows the progress of the detector performance to handle pile-up and high amounts of data is possible.

Figure 2.10 shows a reference schedule for collider operation [130]. The first run starts with an extensive commissioning period, which could overlap with the construction phase, as some of the systems can be brought into operation while the collider installation is being completed. Before each further physics operation period, a technical re-qualification of the individual systems and the entire machine takes place. During the first long shutdown, work on the collider take place to achieve operation with nominal parameters. The second long shutdown is placed in the middle of phase two. Alternating proton and ion physics operation periods, a total of 


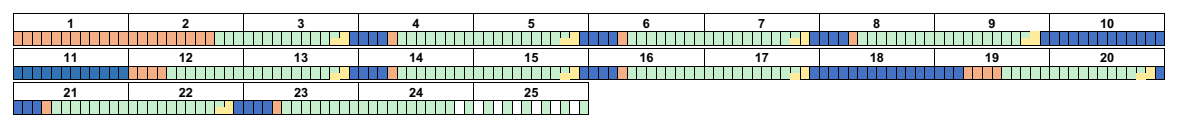

Fig. 2.10. Operation schedule. The numbers in the boxes indicate the operation year. The coloured sub-divisions indicate months. Blue indicates machine stops, orange denotes commissioning and machine development, green shows time for proton physics and yellow indicates operation for ion physics.

165 months of beam production for physics is achieved during a time span of 25 years. This includes 15 months of operation with ions and time for machine commissioning with beam, beam optimisation studies and scheduled short technical stops.

The operation schedule illustrated imposes several challenges on machine design, requiring (i) an efficient commissioning strategy; (ii) system availability according to target reliability requirements that lead to extended maintenance-free operation; (iii) optimised management of maintenance and repair activities during technical stops, including injectors (e.g. addressing present limitations related to the technical infrastructure); (iv) efficient machine cycles with turnaround times that help meeting the overall collider goals.

Operational cycles consist of a collision phase, or "physics production phase", and a turnaround phase, required to reach collisions. The minimum turnaround is $1.8 \mathrm{~h}$ (Tab. 2.9). Nevertheless, luminosity estimates are based on $4 \mathrm{~h}$ (nominal) and $5 \mathrm{~h}$ (initial) turnaround time to account for inefficiencies and faults [129]. Table 2.9 shows the breakdown of the cycle phases, including the LHC experience. The LHC ramp-down time is artificially longer, as it is dominated by failure recovery times, which are mostly associated with this phase. Given the fact that the optimum collision time will be only $3.5 \mathrm{~h}$ for nominal parameters, the production efficiency will strongly depend on the average turnaround time. This implies an even higher importance of the injection phase and of the injectors' performance. New injection schemes are under study [131]. Based on LHC experience, the current injection process could be improved by (i) adding beam diagnostics in the injectors to help identify beam quality issues as close as possible to the source, (ii) having fast diagnostics to understanding the cause of rejected injections, and (iii) improved synchronisation and coordination with the injectors [132].

Reaching the physics goals set for FCC-hh requires about $70 \%$ availability [129] for generating particle collisions with beams that have the necessary quality for physics operation. Availability is therefore defined as the ability for the machine to perform physics operation cycles (collision and turnaround beam cycle phases), i.e. the probability of not being in another state. Comparable availability figures have been reached with the LHC in the 2016-2017 runs [136,137]. Considering the higher number of subsystems, the potential complexity increase that arises from the higher machine performance requirements and the introduction of an additional injector in the baseline scenario, calls for a careful sensitivity analysis during the technical design phase that permits specifying the subsystem reliability and availability requirements needed to meet the overall operation goals. A preliminary first scaling from LHC operation performance observations that accounts for the increased system complexity indicates, that for new subsystems it is mandatory to consider novel designs, built for reliability and availability. Figure 2.11(left) shows the evolution of the integrated luminosity as a function of the global machine Mean Time To Failure (MTTF) and Mean Time To Repair (MTTR). This allows an specification of the acceptable availability parameter space for accelerator subsystems. Figure 2.11(right) shows the sensitivity of the integrated luminosity to the availability of the injector chain, setting an overall injector availability goal of $80-90 \%$, depending on the MTTF. These considerations make a 
Table 2.9. Technical performance targets for FCC-hh turnaround cycle [133], theoretical limit for LHC turnaround [134] and observed minimum and mean turnaround times in 2017 [135] in minutes.

\begin{tabular}{|l|l|l|l|l|}
\hline Phase & FCC target & LHC theoretical & LHC min 2017 & LHC mean 2017 \\
\hline Setup & 10 & 10 & - & - \\
\hline Injection & 40 & $16^{a}$ & 28.0 & 77.1 \\
\hline $\begin{array}{l}\text { Prepare } \\
\text { ramp }\end{array}$ & 5 & - & 2.3 & 5.0 \\
\hline $\begin{array}{l}\text { Ramp- } \\
\text { squeeze- } \\
\text { flat top }\end{array}$ & $20+5+3$ & 20 & $20.2+13.4+2.8$ & $20.5+18.1+4.5$ \\
\hline Adjust & 5 & - & 3.3 & 7.9 \\
\hline $\begin{array}{l}\text { Ramp } \\
\text { down }\end{array}$ & 20 & 20 & 36 & $153.2^{b}$ \\
\hline Total & $108(1.8 \mathrm{~h})$ & $\approx 70(1.2 \mathrm{~h})$ & $106.0(1.8 \mathrm{~h})$ & $286.3(4.8 \mathrm{~h})$ \\
\hline
\end{tabular}

Notes. ${ }^{a}$ This assumes 20 s-long SPS cycles. ${ }^{b}$ The ramp-down phase includes the recovery time from failures.
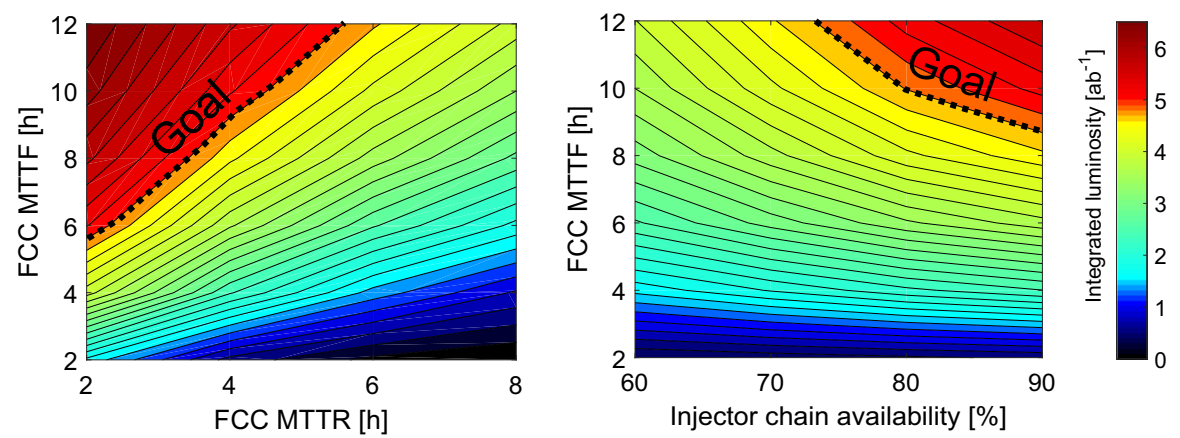

Fig. 2.11. Effect of reliability, recoverability and injector availability on integrated luminosity with nominal parameters.

new superconducting SPS an interesting alternative to the baseline scenario [138], which assumed the LHC as injector. Further studies would be required to identify the best injector option taking into account availability, beam quality, available magnet technologies, capital investment to build a new superconducting machine or to consolidate the existing accelerator complex, and operational expenditures.

In addition, some general guidelines have been established as an outcome of these preliminary studies: (i) design intrinsically reliable systems with built in redundancy and remote diagnostic capabilities, to reduce the number of spurious beam aborts; (ii) minimise the number of systems exposed to radiation in the tunnel to limit the number of single event effects (SEE); (iii) invest in advanced diagnostic and remote and autonomous maintenance to reduce intervention and logistics time, (iv) define a strategy for spare part management, with high priority for critical systems.

The potential impact that design changes driven by such considerations would have on operation and production can be evaluated with accelerator availability modelling based on Monte Carlo simulations [139]. These analyses allow the integrated luminosity for different operating scenarios to be predicted and the availability budgets for individual systems to be derived and these can then be used as input to their design. Such models should be maintained and updated as the machine design evolves. 
The aim should globally optimise the machine design while taking into account constraints like costs and technical feasibility of the different options. An approach for collider availability modelling has been established for these analyses [140,141].

\subsection{Energy upgrade}

Magnets based on high-temperature superconductor (HTS) technology can potentially reach higher fields than those based on $\mathrm{Nb}_{3} \mathrm{Sn}$ technology. This would allow the construction of an even higher energy collider in the FCC-hh tunnel. Such a scenario relies however on the development of high-field HTS dipole and quadrupole magnets at an affordable cost, which is today estimated to take around one to two decades longer than the development of $16 \mathrm{~T} \mathrm{Nb}_{3} \mathrm{Sn}$ magnets (see also Sect. 12.4). This section provides some considerations about the technical feasibility of such a higher-energy collider in a new tunnel, with $24 \mathrm{~T}$ strong magnets that would aim at $150 \mathrm{TeV}$ centre of mass energy.

At $75 \mathrm{TeV}$ beam energy, the synchrotron radiation emitted by each particle increases by about a factor 5 compared to $50 \mathrm{TeV}$ beam energy. To compensate this loss, an RF voltage of $25 \mathrm{MV}$ is required, compared to $5 \mathrm{MV}$ at $50 \mathrm{TeV}$ beam energy. This goes together with a limited increase of the installed voltage. In the $50 \mathrm{TeV}$ design the cryogenics system requires about $100 \mathrm{MW}$ of power to absorb the effects of the synchrotron radiation. If the same beam current, vacuum system and magnet operating temperature were used at the higher energy, the power consumption would increase by a factor 5 . The resulting impact could be mitigated by increasing the operating temperature of the magnets and the beamscreen and by limiting the beam current. An optimisation will have to be done based on a re-design of the vacuum system, a review of the magnet performance for different temperatures and on re-evaluation of the planned integrated luminosity.

The minimum affordable magnet aperture in the arcs is governed by two main factors: first, the aperture of the beamscreen has to be large enough to limit its impedance effects on the beam, avoiding instabilities. Second, sufficient space is needed between the beamscreen aperture and the magnet aperture for vacuum and cooling. A higher beam energy and in particular a lower beam current will allow the beamscreen aperture to be reduced slightly. If the total heat load increases, more space will be needed for the cooling system. Therefore, the aperture size change is expected to remain within reasonable limits. Since the quadrupole gradient is expected to scale in proportion to the dipole field, the arc optics will not diverge significantly from the current design. With the exception of the transverse collimation and beam extraction, the lengths of the insertions should first scale with the square root of the beam energy, assuming the same aperture size.

HTS technology will also allow the quadrupole strength to be increased. Because the quadrupole gradient and the dipole strength scale together, in proportion to the beam energy, the length of the final triplet could remain unchanged with the same magnet aperture and the same beta function could be achieved at the collision point. The collision debris produced in the interaction point is approximately proportional to the product of centre-of-mass energy and luminosity. Assuming the same luminosity as for $50 \mathrm{TeV}$ beams, the debris would only increase by a small amount. The design of a workable experiment insertion therefore seems feasible. Studies are of course required to determine if a larger fraction of the debris deposits energy in the triplets. Since the beam needs slightly less space in the triplets, it might be possible to increase the thickness of the shielding and to close the hole in the TAS that protects the triplets from the direct losses from the collision point. Similarly, the RF insertion could maintain its optics using the stronger magnets. 
The higher beam energy at the same beam current will render machine protection more critical. However, the two most challenging insertions are the transverse collimation system and the beam extraction system. These systems have the longest insertions, therefore providing some flexibility for the development of a means that can address the higher risks. In particular, losses in the collimation systems can be high at certain times during machine operation. LHC experience shows that this is mostly the case for the transverse collimation system, therefore normal conducting magnets are used in this insertion to avoid magnet quenches. However, this technology does not allow the fields to be increased. With an increase of beam energy, it could therefore be difficult to maintain the current optics design. This problem is observed in the design of the HE-LHC scenario. The beam energy doubles compared to LHC, but the length of the collimation insertion increases. For the FCC-hh baseline design this problem is avoided, because the collimation insertion is twice as long as the scaling from LHC suggests. Hence it has sufficient margin in the magnet strength to accommodate the higher beam energy. Some R\&D will also have to be done on the momentum cleaning insertion to ensure that an efficient beam optics is developed.

\subsection{Ion operation}

The machine could also operate as an ion-ion or proton-nucleus collider, similar to the LHC. Considering the current baseline assumptions for the proton-proton programme, the main focus of this chapter lies on collisions of lead nuclei with each other $(\mathrm{Pb}-\mathrm{Pb})$ and with protons $(\mathrm{p}-\mathrm{Pb})$. Beam and luminosity evolution, as well as estimates for the integrated luminosity are given. The performance projections assume the LHC to be the final injector. In order to provide a broad overview of the potential as an ion collider, several operational scenarios are compared throughout the chapter:

- Two beam parameter cases (see Tab. 2.10): initial and nominal, which differ in the $\beta$-function at the interaction point (IP) and the bunch spacing, which defines the maximum number of circulating bunches.

- Collisions at one or two experiments, which share the total luminosity available.

In the initial scenario (see Tab. 2.10), the parameters of the injected beam are based on those obtained in the $2016 \mathrm{p}-\mathrm{Pb}$ run of the LHC [142]. This could be easily achieved by maintaining the present source and injector chain of the LHC at the performance levels of LHC Run 2. The nominal scenario assumes a shorter bunch spacing of $50 \mathrm{~ns}$ - the principal improvement of the LHC injector chain planned for high-luminosity (HL-LHC) operation in LHC Run 3. The choices of $\beta^{*}$ ( $\beta$-function at the IP) follow the $\mathrm{p}-\mathrm{p}$ proposal presented in Section 2.2 .

Previous studies [143-148] have shown that the FCC-hh enters a new, highlyefficient operating regime, in which a large fraction of the injected intensity can be converted to useful integrated luminosity. Thanks to strong synchrotron radiation damping, the beam emittances shrink rapidly and compensate the rapid decay of initial luminosity seen at lower-energy colliders. As shown in the left plots of Figure 2.12, the luminosity may even increase during a fill until the beams are exhausted. Not only is this natural beam cooling twice as fast for lead ions as for protons, it can also be better exploited since the lower overall bunch charges do not lead to high beam-beam tune-shifts.

How much luminosity can be integrated over a run strongly depends on the turnaround time. In particular the time needed to inject all bunches into the accelerator is critical, since it is directly proportional to the cycle time and availability of 
Table 2.10. Beam and machine parameters for operation with ions.

\begin{tabular}{|c|c|c|c|c|c|}
\hline & Unit & \multicolumn{2}{|c|}{ Initial } & \multicolumn{2}{|c|}{ Nominal } \\
\hline Operation mode & - & $\mathrm{Pb}-\mathrm{Pb}$ & $\mathrm{p}-\mathrm{Pb}^{a}$ & $\mathrm{~Pb}-\mathrm{Pb}$ & $\mathrm{p}-\mathrm{Pb}^{a}$ \\
\hline Beam energy & $(\mathrm{TeV})$ & 4100 & 50 & 4100 & 50 \\
\hline Centre-of-mass energy per nucleon pair & $(\mathrm{TeV})$ & 39.4 & 62.8 & 39.4 & 62.8 \\
\hline No. of bunches & - & \multicolumn{2}{|c|}{2760} & \multicolumn{2}{|c|}{5400} \\
\hline Bunch spacing & (ns) & \multicolumn{2}{|c|}{100} & \multicolumn{2}{|c|}{50} \\
\hline No. of particles per bunch & $\left(10^{8}\right)$ & 2 & 164 & 2 & 164 \\
\hline Transverse normalised emittance & $(\mu \mathrm{m} . \mathrm{rad})$ & $1.5^{b}$ & $3.75^{b}$ & $1.5^{b}$ & $3.75^{b}$ \\
\hline Stored energy per beam & $(\mathrm{MJ})$ & \multicolumn{2}{|c|}{362} & \multicolumn{2}{|c|}{709} \\
\hline Stored energy per beam at injection & $(\mathrm{MJ})$ & \multicolumn{2}{|c|}{24} & \multicolumn{2}{|c|}{47} \\
\hline$\beta$-function at the IP & $(\mathrm{m})$ & \multicolumn{2}{|c|}{1.1} & \multicolumn{2}{|c|}{0.3} \\
\hline Number of IPs in collision & - & \multicolumn{2}{|c|}{1 or 2} & \multicolumn{2}{|c|}{1 or 2} \\
\hline Initial luminosity & $\left(10^{27} \mathrm{~cm}^{-2} \mathrm{~s}^{-1}\right)$ & 34 & 2800 & 248 & 20400 \\
\hline Peak luminosity $^{c}$ & $\left(10^{27} \mathrm{~cm}^{-2} \mathrm{~s}^{-1}\right)$ & 80 & 13300 & 320 & 55500 \\
\hline Integrated luminosity $^{d}(1$ experiment) & $\left(\mathrm{nb}^{-1} / \mathrm{run}\right)$ & 35 & 8000 & 110 & 29000 \\
\hline Integrated luminosity $^{d}$ (2 experiments) & $\left(\mathrm{nb}^{-1} / \mathrm{run}\right)$ & 23 & 6000 & 65 & 18000 \\
\hline Total cross-section & (b) & 597 & 2 & 597 & 2 \\
\hline Peak BFPP beam power & $(\mathrm{kW})$ & 19 & 0 & 75 & 0 \\
\hline
\end{tabular}

Notes. ${ }^{a} \mathrm{p}-\mathrm{Pb}$ operation uses the same $\mathrm{Pb}$ beam as in $\mathrm{Pb}-\mathrm{Pb}$ operation. The parameters listed in this column correspond to the proton beam. ${ }^{b} \mathrm{~Pb}$ emittances are based on LHC experience. Proton emittances are chosen to feature the same geometric beam size, which is larger than in p-p operation, where $\epsilon_{n}=2.2 \mu \mathrm{m}$. ${ }^{c}$ One experiment in collisions. ${ }^{d}$ Per experiment. Including a performance efficiency factor of $50 \%$ to take account of down-time due to failures.

its injectors. In 2016 the LHC achieved a minimum turnaround time of $2.5 \mathrm{~h}$ [149]. Therefore any measures envisaged to shorten the LHC cycle (see Sect. 2.5) boost the peak and integrated luminosities. The effect of this is shown Figure 2.12 (right), which illustrates the luminosity that would be integrated in an ideal 30-day run at full performance with perfect efficiency (no down-time or other interruptions). The solid lines show the projections for a single experiment taking data; the dashed lines assume two experiments with the same configuration. The integrated luminosity per experiment goes down, because the intensity burn-off becomes twice as strong and the total available luminosity has to be shared between the experiments.

Table 2.10 summarises key parameters for $\mathrm{Pb}-\mathrm{Pb}$ and $\mathrm{p}-\mathrm{Pb}$ operation at $E_{b}=50 Z \mathrm{TeV}$. In the case of $\mathrm{p}-\mathrm{Pb}$ operation the $\mathrm{Pb}$ beam is assumed to be the same as for $\mathrm{Pb}-\mathrm{Pb}$, so the corresponding column only quotes the proton beam parameters. The calculated luminosity values assume an optimised theoretical turnaround time of around $26 \mathrm{~min}$ per LHC ion cycle and an additional preparation time in the collider of $1.2 \mathrm{~h}$ per filling, as quoted in [150]. It was assumed that the first LHC beam is prepared during the collider preparation time, such that the total turnaround time sums up to $105 \mathrm{~min}$. This represents a theoretical minimum. In reality, early beam aborts and other faults will increase this time and somewhat reduce the integrated luminosity.

The final values for the integrated luminosity in a typical one-month run quoted in Table 2.10 assume a "performance efficiency factor" of $50 \%$ to allow for set-up time, down-time and other deviations from the idealised running described in Figure 2.12 (a similar factor was observed during LHC operation [137] and applied in HL-LHC performance projections). For the moment, no studies of upgrades to the heavy-ion injectors (source, linac, accumulation ring, PS and SPS synchrotrons) have been 


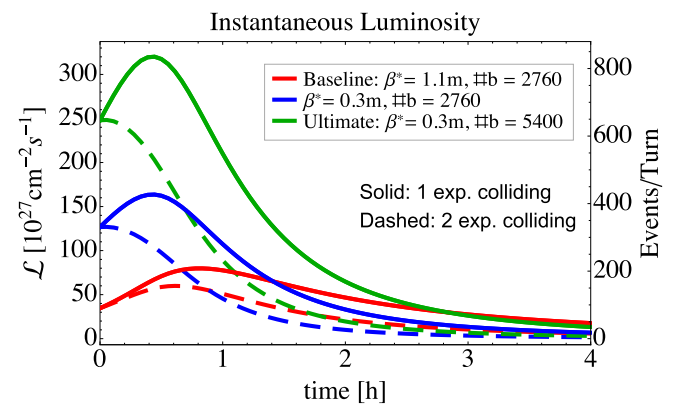

(a)

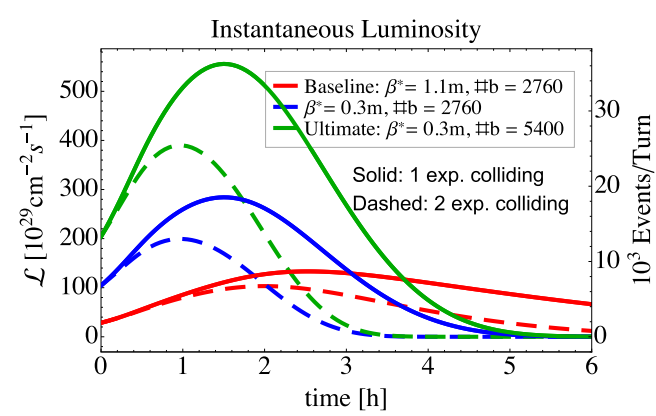

(c)

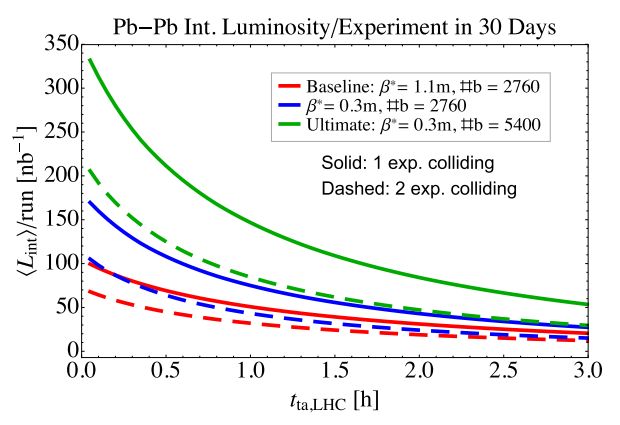

(b)

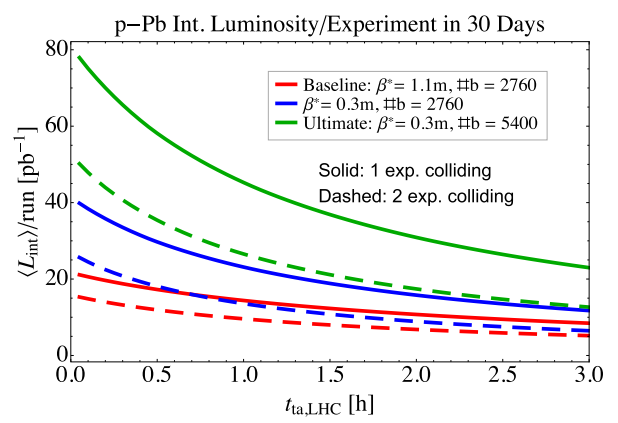

(d)

Fig. 2.12. Left: instantaneous luminosity evolution. Right: optimum average integrated luminosity as a function of LHC turnaround time, assuming $100 \%$ efficiency and machine availability. In 2016 the LHC achieved a minimum turnaround time of $2.5 \mathrm{~h}$ [149]. With a faster cycling LHC (see Sect. 2.5) the theoretical optimum of the LHC turnaround for an ion cycle could be around 28 min, depending on the filling scheme. (a) $\mathrm{Pb}-\mathrm{Pb}$ instantaneous luminosity. (b) $\mathrm{Pb}-\mathrm{Pb}$ integrated luminosity. (c) $\mathrm{p}-\mathrm{Pb}$ instantaneous luminosity. (d) $\mathrm{p}-\mathrm{Pb}$ integrated luminosity.

performed. If upgrades to these machines can be made in time for collider operation, then still higher luminosities are likely to be available.

Heavy-ion operation imposes challenging specifications for the collimation system, including certain adaptations of the collider main ring, e.g., special absorbers in key locations for the high flux of modified ions from the bound-free pair-production process at the interaction point. The cross-sections of these processes are so high that power exceeding a few tens of $\mathrm{kW}$ is continuously deposited in localised positions. These special absorbers need to be installed in the cold area of the dispersion suppressor on both sides of each interaction region where ions collide. They must not only ensure that the power deposited by the ions is discharged safely, but also that fragmentation products do not escape and reach the subsequent superconducting magnet area. An absorber design still remains to be studied. Collimation of the heavy-ion beams will also be difficult and require further absorbers or, possibly, the application of new collimation technologies such as bent crystals or electron lenses. The potential of these technologies is being studied at the LHC.

If the demands on the collimation system are too extreme for lead ion operation, an ion species with a lower charge number $(Z)$ could be used. The cross-sections of the ultra-peripheral electromagnetic processes are proportional to high powers of $Z$. Therefore the power of the secondary beams emerging from the collision point would be reduced, while providing a longer luminosity lifetime, since more particles 
are available for hadronic interactions. The physics case and choice of lower $Z$ ion species is under study by the experiments.

\subsection{Lepton-Hadron operation}

The high energy hadron beam of FCC-hh facilitates the next step for Deep Inelastic Scattering physics with the construction of a new, higher energy electron facility. The FCC-eh configuration closely follows the design work for the LHeC, an eh collider based on the LHC and it's high luminosity upgrade. The LHeC physics, a detector design and two options for machines and their infrastructure have been studied in a series of workshops supported by CERN, ECFA and NuPECC; they are described in detail in a conceptual design report (CDR) which was published in 2012 [28]. The FCC-eh has been designed for synchronous operation with the hadron collider and uses a $60 \mathrm{GeV}$ three-turn intense energy-recovery linac (ERL) in racetrack configuration as electron accelerator. To enable precision Higgs physics [151] and support a novel deep inelastic scattering programme, recently described in [152], the FCC-eh aims for a luminosity in excess of $10^{34} \mathrm{~cm}^{-2} \mathrm{~s}^{-1}$ in parallel operation with the FCChh programme. This is more than ten times higher than that considered in the LHeC CDR. FCC-eh includes options for high energy electron-ion $(e A)$ scattering. The feasibility of the FCC-eh electron beam accelerator complex, a high current, multi-turn ERL, can be investigated through the proposed lower energy test and development facility at LAL Orsay, called PERLE [153].

The ERL configuration features two superconducting linacs to accelerate an electron beam to $60 \mathrm{GeV}$ in a 3-pass racetrack configuration, as illustrated in Figure 2.13. This arrangement is outside the FCC tunnel and thus minimises any interference with the main hadron beam infrastructure. The electron accelerator may be built largely independently of the operating status of the proton machine. The chosen energy of $60 \mathrm{GeV}$ leads to a circumference of the electron racetrack of approximately $9 \mathrm{~km}$. This length is $1 / 11$ of the FCC circumference and is chosen to limit the energy loss in the last return arc.

The luminosity $L$, in a simplified model, is given by the following formula

$$
L=\frac{N_{p} N_{e} f \gamma_{p}}{4 \pi \epsilon_{p} \beta_{p}} \cdot H_{\text {geom }} H_{b-b} H_{\text {coll }}
$$

Here, $N_{p}$ is the number of protons per bunch and $\epsilon_{p}$ and $\beta_{p}$ are the proton emittance and beta-functions, respectively. It is assumed that the proton beam parameters $N_{p}$ and $\epsilon_{p}$ are defined by the main experiments that collide protons on protons because the default assumption is for concurrent ep and pp operation. For the proton $\beta$-function in the electron-proton collision point a challenging target value of $\beta_{p}=15 \mathrm{~cm}$ is assumed. This may be achievable because only one proton beam needs to be focused, which is a simplification compared to the proton-proton case. $f=1 / \Delta$ denotes the bunch frequency, which for the default bunch spacing of $\Delta=25 \mathrm{~ns}$ is $40 \mathrm{MHz} . N_{e}$ is the number of electrons per bunch which determines the electron current $I_{e}=e N_{e} f$. The electron current for HE-LHC and FCC-eh is assumed ${ }^{1}$ to be

\footnotetext{
1 The numbers quoted hold for unpolarised electron beams. Given further developments such as those ongoing for weak interaction measurements at MESA, one may expect a polarised electron source to provide half of that current. In order to achieve luminosities of order $10^{33}$ with positrons, significant developments are required. For positrons, dedicated operation at very high luminosity may be a particularly attractive option as the loss in lepton intensity is compensated by a gain in proton intensity and operational performance as indicated below.
} 


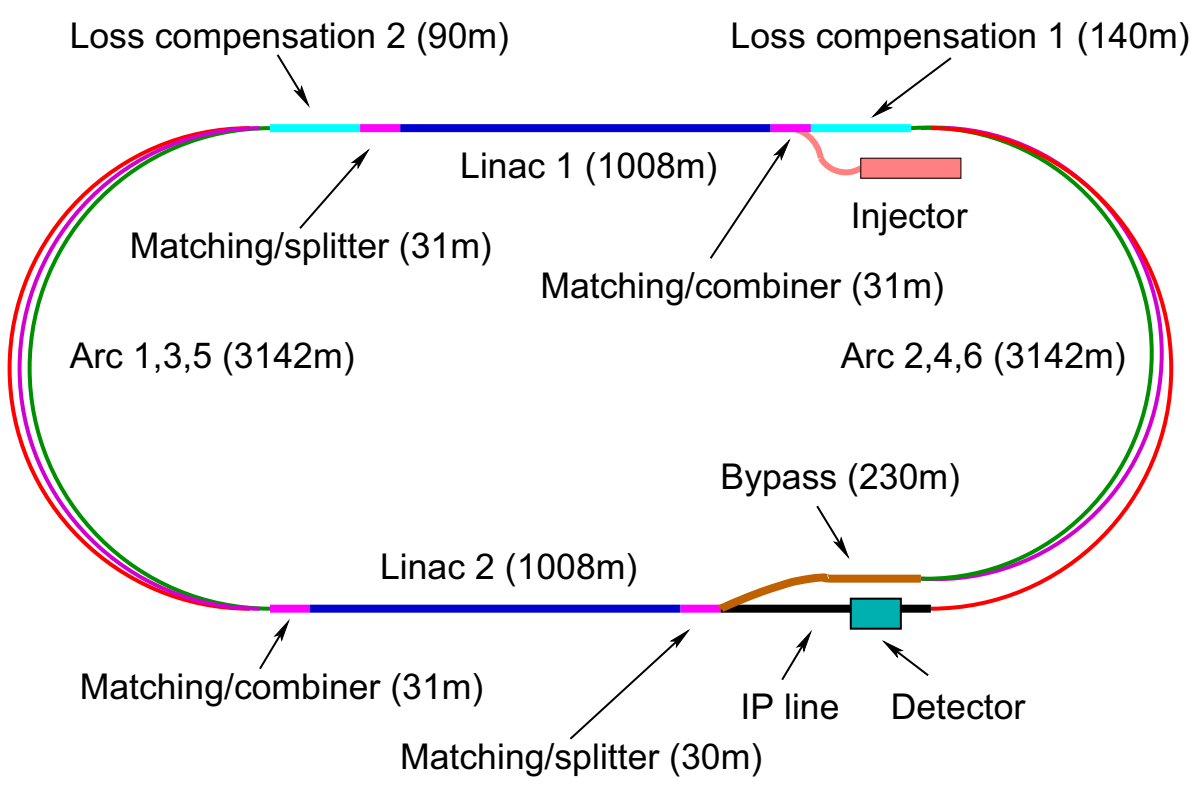

Fig. 2.13. Schematic view of the electron accelerator configuration. Each linac accelerates the beam to $10 \mathrm{GeV}$, which leads to a $60 \mathrm{GeV}$ electron energy at the interaction point after three passes through the opposite lying linac structures each made of 60 -cavity cryomodules. The arc radius is about $1 \mathrm{~km}$ and the circumference chosen to be $1 / 3$ of that of the LHC. The beam is decelerated to recover the beam power after having passed through the IP.

$I_{e}=20 \mathrm{~mA}$, a slight increase compared to the $15 \mathrm{~mA}$ assumed for the LHeC in the HL-LHC scenario and three times the value of $6.4 \mathrm{~mA}$ used in the LHeC CDR. This will yield a total synchrotron radiation of about $40 \mathrm{MW}$ in the return arcs. To compensate for this power loss through the beam, a grid power of the order of $65 \mathrm{MW}$ may be required. A value of $20 \mathrm{~mA}$ is already feasible or has even been surpassed with intense DC photocathodes. Since, however, a cavity has to withstand six times the value of $I_{e}$ due to the (de)acceleration in three turns, $I_{e}$ should not to be too large.

The factors $H_{\text {geom }}, H_{b-b}$ and $H_{\text {coll }}$ are geometric correction factors with values typically close to unity. $H_{\text {geom }}$ is the reduction of the luminosity due to the hourglass effect, $H_{b-b}$ is the increase of the luminosity by the strong attractive beam-beam forces and $H_{\text {coll }}$ is a factor that takes the filling patterns of the electron and the proton beam into account. Estimates for these parameters are shown in Table 2.11. Unless discussed above, further parameters used for the four ep collider configurations considered can be found (i) for the LHeC as evaluated in its conceptional design in reference [28], (ii) for the high luminosity version of the LHeC in references [154-156], (iii) for the HE-LHC in references $[157,158]$ and for the FCC-eh in reference $[157,158]$. One observes that compared to the 2012 LHeC CDR, it seems possible to achieve peak luminosities near to or larger than $10^{34} \mathrm{~cm}^{-2} \mathrm{~s}^{-1}$, which makes these future ep colliders the most exciting and efficient machines for the study of new physics at the accelerator energy frontier.

It should be noted that the collider proton beam parameters vary during a run. The protons emit synchrotron radiation, which reduces their emittance, $\epsilon_{p}$. Their number, $N_{p}$, decreases as they are destroyed by colliding in the main experiments. Hence the proton beam size and intensity change during the run, which leads to a weak variation of the luminosity. The electron beam current is distributed in bunches 
Table 2.11. Baseline parameters and estimated peak luminosities of future electron-proton collider configurations for the electron ERL when used in concurrent ep and pp operating mode.

\begin{tabular}{|l|c|c|c|c|}
\hline Parameter (unit) & LHeC CDR & ep at HL-LHC & ep at HE-LHC & FCC-eh \\
\hline$E_{p}(\mathrm{TeV})$ & 7 & 7 & 12.5 & 50 \\
$E_{e}(\mathrm{GeV})$ & 60 & 60 & 60 & 60 \\
$\sqrt{s}(\mathrm{TeV})$ & 1.3 & 1.3 & 1.7 & 3.5 \\
Bunch spacing $(\mathrm{ns})$ & 25 & 25 & 25 & 25 \\
Protons per bunch $\left(10^{11}\right)$ & 1.7 & 2.2 & 2.5 & 1 \\
$\epsilon_{p}(\mu \mathrm{m})$ & 3.7 & 2 & 2.5 & 2.2 \\
Electrons per bunch $\left(10^{9}\right)$ & 1 & 2.3 & 3.0 & 3.0 \\
Electron current $(\mathrm{mA})$ & 6.4 & 15 & 20 & 20 \\
IP beta function $\beta_{p}^{*}(\mathrm{~cm})$ & 10 & 7 & 10 & 15 \\
Hourglass factor $H_{\text {geom }}$ & 0.9 & 0.9 & 0.9 & 0.9 \\
Pinch factor $H_{b-b}$ & 1.3 & 1.3 & 1.3 & 1.3 \\
Proton filling $H_{\text {coll }}$ & 0.8 & 0.8 & 0.8 & 0.8 \\
Luminosity $\left(10^{33} \mathrm{~cm}^{-2} \mathrm{~s}^{-1}\right)$ & 1 & 8 & 12 & 15 \\
\hline
\end{tabular}

with a default spacing of $25 \mathrm{~ns}$, leading to $N=3 \times 10^{9}$ particles per bunch. Studies of the beam stability showed that a charge of $N=4 \times 10^{9}$ is still stable. The electron beta-function and the position of the electron beam waist are the result of the overall optimisation of the collision which affects the product $H_{\text {geom }} H_{b-b}$. This optimisation is dominated by the strong beam-beam forces. In general, smaller electron emittances lead to larger luminosity. The electron beam emittance from the source can be of the order of $\epsilon_{e} \approx 1 \mu \mathrm{m}$. In the arcs of the recirculating electron linac, the horizontal emittance will increase by about $7.5 \mu \mathrm{m}$ and the vertical only by $0.8 \mu \mathrm{m}$. A target of $\epsilon_{e}=10 \mu \mathrm{m}$ is set at the collision point for both planes. The possibility of colliding flat electron beams remains to be studied.

The collision of the two beams has little impact on the proton beam. The electron bunch charge is quite small and the proton energy is high. However, the electron beam is strongly affected by the proton beam. During the collision the electron bunch is focused by the protons, which leads to a significant reduction of the transverse electron beam size. As a consequence, the luminosity is larger than for rigid beams. Also, the conventional matching of the sizes of the two beams does not work because the electron bunch size is changing by a factor of two or so during the collision. The beambeam effect has been simulated with GUINEA-PIG [159]. The longitudinal position of the waist and the beta-functions were varied in order to optimise luminosity.

Finally, the factor $H_{\text {coll }}$ is given by the fraction of electron bunches that collide with a proton bunch. Only $80 \%$ of the circumference is filled with proton bunches and therefore $20 \%$ of the electron bunches will not collide with a proton bunch. This leads to a collision factor $H_{\text {coll }}=0.8$. Depending on the filling pattern of the proton ring, it could be possible to use an electron bunch pattern which has no bunches in non-colliding positions. This would reduce the rate of electron bunches by $20 \%$ and allow their charge to be increased by $25 \%$. The luminosity would increase by $25 \%$. However, this option is not in the baseline. Accelerating the non-colliding bunches may be useful to limit the fluctuations of the RF power stored in the linacs. A small fraction of non-colliding bunches is also known to be of interest for the understanding of backgrounds and detector response. The bunch distribution of the electron beam could be affected by another process: the electron beam ionises the rest gas in the linacs and arcs. Positive ions may then be trapped in the electron beam which can lead to an instability which can be suppressed by introducing a gap in the electron 
Table 2.12. Parameters and estimated peak and integrated luminosities of the FCC-eh, when the $50 \mathrm{TeV}$ proton and the $60 \mathrm{GeV}$ electron beams collide, in an operational mode where pp data may be taken simultaneously.

\begin{tabular}{|l|c|c|c|}
\hline Parameter & Unit & Protons & Electrons \\
\hline Beam energy & $\mathrm{GeV}$ & 50000 & 60 \\
Normalised emittance & $\mu \mathrm{m}$ & $2.2 \rightarrow 1.1$ & 10 \\
IP beta function & $\mathrm{mm}$ & 150 & $42 \rightarrow 52$ \\
Nominal RMS beam size & $\mu \mathrm{m}$ & $2.5 \rightarrow 1.8$ & $1.9 \rightarrow 2.1$ \\
Waist shift & $\mathrm{mm}$ & 0 & $65 \rightarrow 70$ \\
Bunch population & $10^{10}$ & $10 \rightarrow 5$ & 0.31 \\
Bunch spacing & $\mathrm{ns}$ & 25 & 25 \\
\hline Luminosity & $10^{33} \mathrm{~cm}^{-2} \mathrm{~s}^{-1}$ & \multicolumn{2}{|c|}{$18.3 \rightarrow 14.3$} \\
Int. luminosity per 10 years & $\left(\mathrm{ab}^{-1}\right)$ & \multicolumn{2}{|c|}{1.2} \\
\hline
\end{tabular}

beam which causes the ions to be lost [28]. The results of the simulation study are summarised in Table 2.12. They are in good agreement with the rough estimate presented above (Tab. 2.11).

There could be an interest in dedicated ep operation because significant gains in the instantaneous and integrated luminosity performance are potentially possible. A first estimate indicates a potential for a 10 fold higher proton beam brightness and a beta function reduced by a factor of two, with only one beam present and squeezed and fewer aperture constraints. A factor of two may also be obtained from the much enhanced operation efficiency in dedicated mode, mainly because the proton beam lifetime would be hugely increased without the pp collisions, which lead to $\tau_{p}<5 \mathrm{~h}$. Therefore, dedicated ep runs could be typically a day long; overall, in dedicated mode, luminosities in excess of the order of $10^{35} \mathrm{~cm}^{-2} \mathrm{~s}^{-1}$ appear to be not unrealistic. An integrated luminosity of $1 \mathrm{ab}^{-1}$ annually would be possible to achieve. Such a scenario could be specially relevant for taking a large amount of positron-proton data in a period of operation which is not too long, since the $\mathrm{e}^{+}$currents will be much lower than the $\mathrm{e}^{-}$currents by one or even two orders of magnitude.

The heavy ion beams provide a unique opportunity for high energy, high luminosity deep inelastic electron-ion scattering physics explorations. Since HERA was restricted to protons only, the FCC-eh extends the kinematic range in $Q^{2}$ and $1 / x$ by 4 (5) orders of magnitude. This is a significant increase in coverage and would be set to radically change the understanding of parton dynamics in nuclei and of the formation of the quark-gluon plasma. An initial set of parameters in the maximum energy configurations was given in [158]. This did not yet take account of the intense beams of ${ }^{208} \mathrm{~Pb}^{82+}$ nuclei that have already been largely demonstrated [160] and are planned for HL-LHC. Combining these with the default $60 \mathrm{GeV}$ electron ERL, an updated parameter set is presented in Table 2.13. Radiation damping of $\mathrm{Pb}$ beams in the hadron rings is about twice as fast as for protons and can be fully exploited. For the FCC-hh case, the emittance values in Table 2.13 are estimates of effective average values during a fill in which $\mathrm{Pb}-\mathrm{Pb}$ collisions are being provided at one other interaction point [32].

The design of the FCC-eh interaction region follows that of LHeC [28]: strong quadrupole septa focus the colliding proton beam at the interaction point. Since the electron beam has a significantly lower beam rigidity it must pass these magnets in the field-free region. This leads to an angle of $8 \mathrm{mrad}$ between the two incoming colliding beams. Dipoles around the IP bend the electron beam into head-on collisions with the proton beam and separate the two beams after the IP. 
Table 2.13. Baseline parameters of future electron-ion collider configurations based on the electron ERL, in concurrent $\mathrm{e} A$ and $A A$ operation mode.

\begin{tabular}{|l|c|c|c|}
\hline Parameter (unit) & LHeC (HL-LHC) & eA at HE-LHC & FCC-eh \\
\hline$E_{\mathrm{Pb}}(\mathrm{PeV})$ & 0.574 & 1.03 & 4.1 \\
$E_{e}(\mathrm{GeV})$ & 60 & 60 & 60 \\
$\sqrt{s_{e N}}$ electron-nucleon $(\mathrm{TeV})$ & 0.8 & 1.1 & 2.2 \\
Bunch spacing $(\mathrm{ns})$ & 50 & 50 & 100 \\
No. of bunches & 1200 & 1200 & 2072 \\
Ions per bunch $\left(10^{8}\right)$ & 1.8 & 1.8 & 1.8 \\
$\gamma \epsilon_{A}(\mu \mathrm{m})$ & 1.5 & 1.0 & 0.9 \\
Electrons per bunch $\left(10^{9}\right)$ & 4.67 & 6.2 & 12.5 \\
Electron current $(\mathrm{mA})$ & 15 & 20 & 20 \\
IP beta function $\beta_{A}^{*}(\mathrm{~cm})$ & 7 & 0.9 & 15 \\
Hourglass factor $H_{\text {geom }}$ & 0.9 & 1.3 & 0.9 \\
Pinch factor $H_{b-b}-b$ & 0.8 & 1.3 \\
Bunch filling $H_{\text {coll }}$ & 1.3 & 18 & 0.8 \\
Luminosity $\left(10^{32} \mathrm{~cm}^{-2} \mathrm{~s}^{-1}\right)$ & 0.8 & & 54 \\
\hline
\end{tabular}

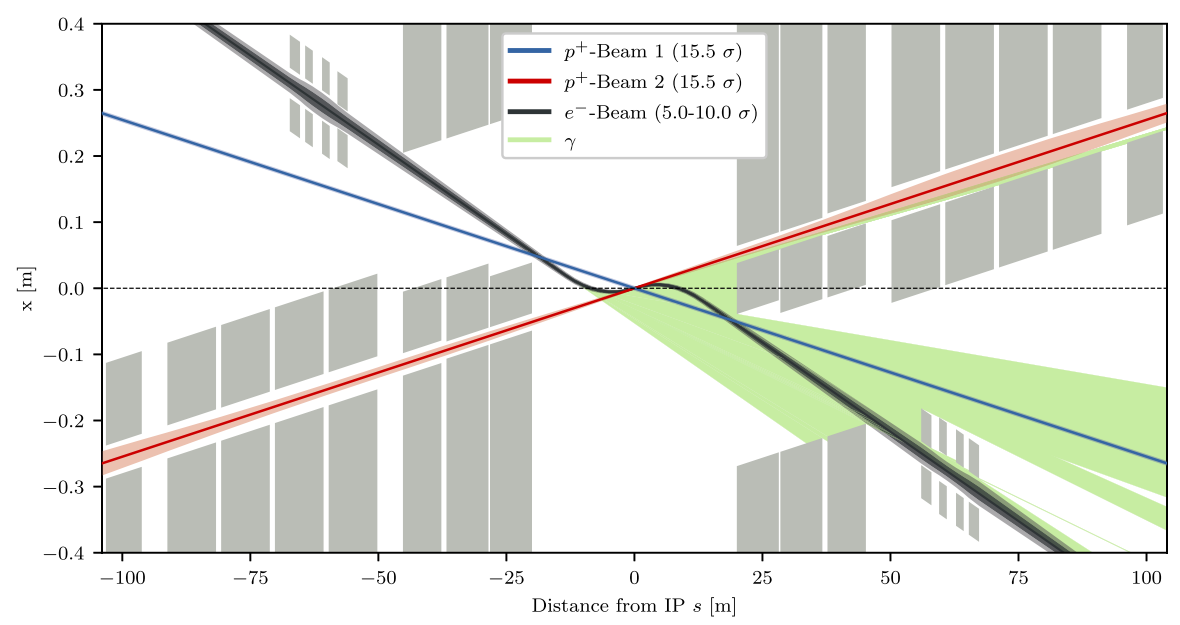

Fig. 2.14. Layout of the FCC-eh interaction region. The red and blue lines indicate the crossing FCC-hh proton beams. The black slope indicates the electron beam that is provided by an energy recovery linac and which is collided with the red-marked proton beam. The green area indicates the fan of the synchrotron radiation. The quadrupole parameters are estimates based on magnets designed for LHeC.

The FCC-eh is considered an option for the "low luminosity" experimental regions in FCC-hh. Geological constraints of the Geneva area for the ERL tunnel probably leave PL as the only viable option. In the current layout of FCC-hh, PL not only houses an experiment but also the injection section for the colliding proton beam. The interface between the injection section and FCC-eh IR requires further study as it will dictate the minimum reachable $\beta^{*}$. The current design of the interaction region is shown in Figure 2.13. With magnet parameters estimated from updated LHeC quadrupole septa designs, it achieves a minimum $\beta^{*}$ of $30 \mathrm{~cm}$. A lower $\beta^{*}$ might be achievable but will require significant changes to the injection scheme in FCC-hh to make space for longer, larger aperture triplet quadrupoles. The FCC-eh IR design has to account for large electron currents of about $20 \mathrm{~mA}$ and a beam separation 
constraint in the quadrupole septa. Choosing $L^{*}$ of $20 \mathrm{~m}$, together with an optimised dipole length [161], one obtains a synchrotron radiation load of $35 \mathrm{~kW}$ at a critical energy of $286 \mathrm{keV}$. The shown electron beam optics were chosen to simply match the beam size of the proton beam at the IP, i.e. $\beta_{e^{-}}^{*} \cdot \epsilon_{e^{-}}=\beta_{p^{+}}^{*} \cdot \epsilon_{p^{+}}$.

An earlier design of the FCC-eh interaction region was integrated into the FCChh ring and showed that chromaticity correction of the $\beta^{*}=30 \mathrm{~cm}$ optics requires arc sextupoles only about $14 \%$ stronger than the current FCC-hh specification.

\section{Collider technical systems}

\subsection{Overview}

This chapter presents details of those technical infrastructure systems for which substantial research and development is required. It is known that it will be possible to scale up many systems from LHC for use in FCC-hh and these systems are not presented below. Various major systems such as magnets, cryogenics, RF, beam transfer and vacuum are described here as well as many smaller essential devices. Finally there is a description of the radiation environment in which they will have to perform. Details for all technical systems will be presented at a later stage in the design process but systems which require particular attention at this conceptual design stage have been identified and are presented here.

\subsection{Main magnet system}

\subsubsection{Introduction}

The magnetic system will profit greatly from the experience gained with the LHC, which has demonstrated the feasibility and effectiveness of operating a large number of superconducting magnets cooled by using superfluid helium at $1.9 \mathrm{~K}$. There will be about four times more magnets in FCC-hh than in LHC and the field amplitude produced by the arc dipoles will be increased by almost a factor of two, whilst maintaining a similar beam aperture and twin configuration. The field increase will be enabled by using $\mathrm{Nb}_{3} \mathrm{Sn}$ superconductor instead of the $\mathrm{Nb}-\mathrm{Ti}$ used in the LHC arc dipoles. With respect to the conductor properties, the superconducting magnets will operate in a similar condition than the LHC magnets, with $14 \%$ margin on the load line and at about $60 \%$ of the maximum upper critical field $\mathrm{B}_{\mathrm{c} 20}$. With an appropriate R\&D programme, and if all magnets are cold tested before installation, this margin can be considered sufficient to achieve the nominal energy with limited magnet training. This technology, though not yet used in particle colliders, is being implemented for dipoles and quadrupoles of the HL-LHC project, where they will be operating at peak fields of between 11 and $12 \mathrm{~T}$. One can expect that this technology will be ready to start mass production of $16 \mathrm{~T}$ magnets within a decade from the manufacture of a first long model.

\subsubsection{Superconducting main dipole}

The main dipoles (MD) are twin-aperture magnets of cosine-theta coil layout assembled in a helium-tight cold mass (CM) structure, integrated in a cryostat. A cross section of the system is presented in Figure 3.1 and a $3 \mathrm{D}$ of the assembly is shown in Figure 3.2. 


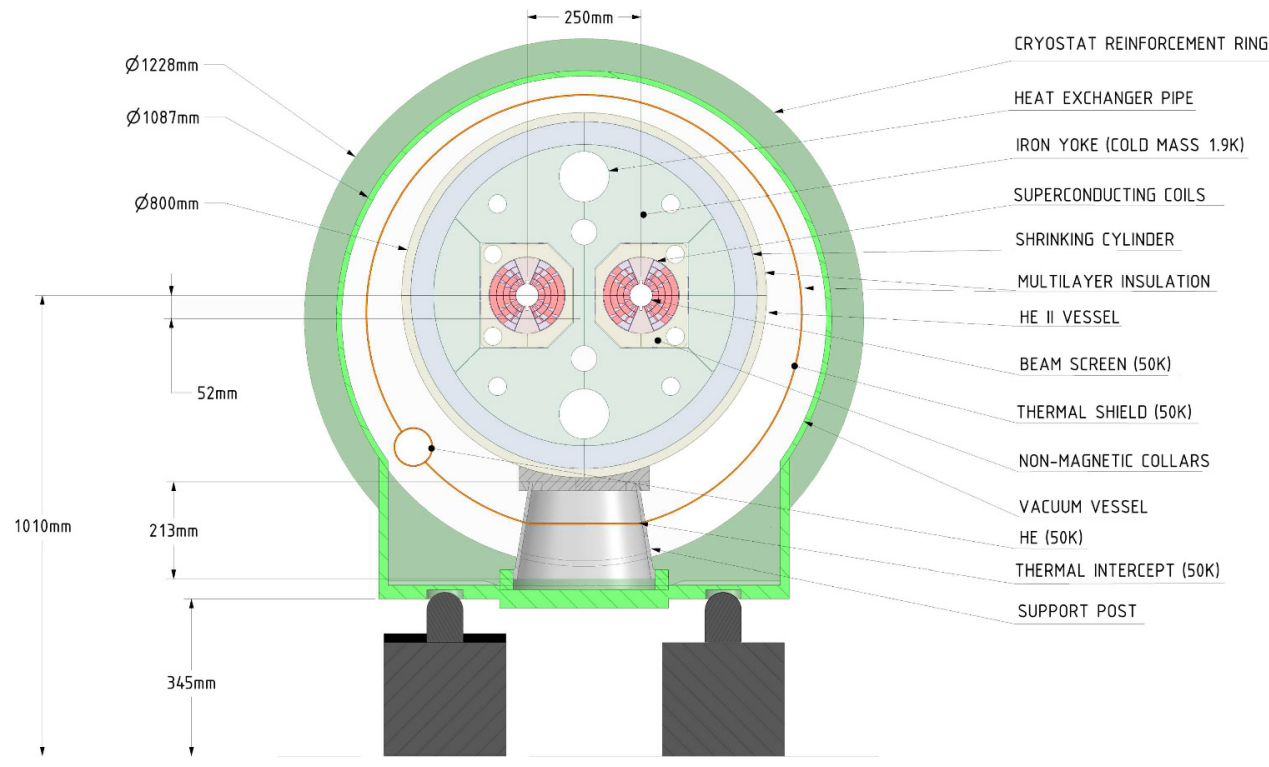

Fig. 3.1. Main dipole cross-section.

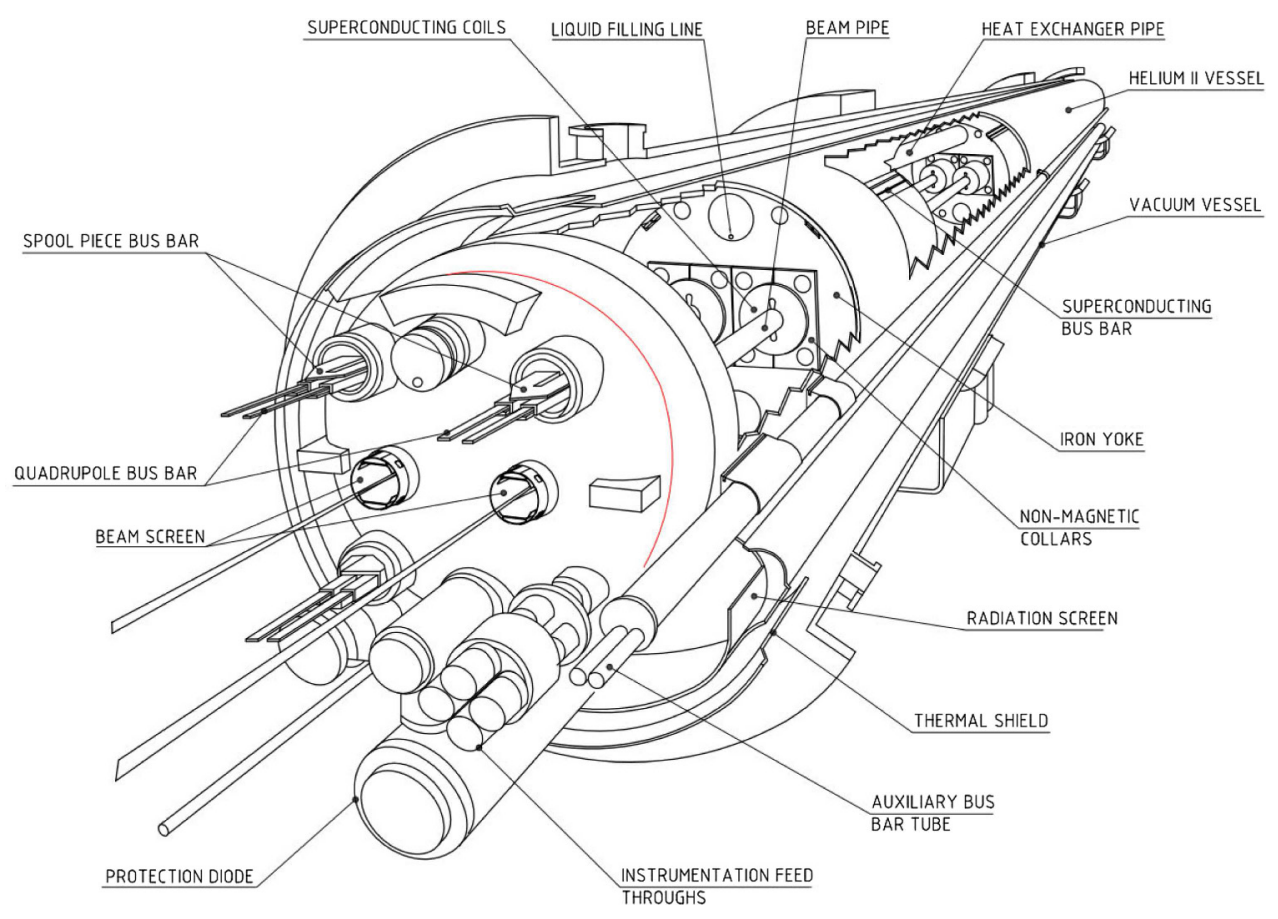

Fig. 3.2. 3D-view of main dipole cold mass assembly. 
Table 3.1. Main dipole parameters.

\begin{tabular}{|l|l|l|}
\hline Item & Unit & Value \\
\hline Number of units & & 4668 \\
\hline Operating field & $\mathrm{T}$ & 16.0 \\
\hline Coil physical aperture & $\mathrm{mm}$ & 50.0 \\
\hline Operating current & $\mathrm{A}$ & 11441 \\
\hline Operating temperature & $\mathrm{K}$ & 1.9 \\
\hline Magnetic length @ 1.9 K & $\mathrm{mm}$ & 14069 \\
\hline Stored energy at 16 T (entire magnet) & MJ & 37 \\
\hline Self-inductance at 16 T (entire magnet) & $\mathrm{mH}$ & 570 \\
\hline Field margin on the load line at 16 T & $\%$ & 14 \\
\hline $\begin{array}{l}\text { Magnetisation losses (two apertures) over a full exci- } \\
\text { tation cycle }\end{array}$ & $\mathrm{kJ} / \mathrm{m}$ & 5 \\
\hline Distance between aperture axes at $1.9 \mathrm{~K}$ & $\mathrm{~mm}$ & 250 \\
\hline Number of coil turns per aperture & & 200 \\
\hline Surface of conductor $(2$ apertures $)$ & $\mathrm{cm}{ }^{2}$ & 131 \\
\hline Cold mass length beam pipe flange-to-flange at $1.9 \mathrm{~K}$ & $\mathrm{~m}$ & 15.8 \\
\hline Mass of the cold mass & $\mathrm{t}$ & 55 \\
\hline Mass of the cryostat & $\mathrm{t}$ & 6 \\
\hline Geometric field harmonics $\mathrm{b}_{2}, \mathrm{~b}_{3}, \mathrm{~b}_{4}, \mathrm{~b}_{5}$ & $\mathrm{units}$ & $3.7,-2.4,0.95,0.30$ \\
\hline Contribution of persistent $c u r r e n t s ~ \mathrm{~b}_{2}, \mathrm{~b}_{3}, \mathrm{~b}_{4}, \mathrm{~b}_{5}$ & $\mathrm{units}$ & $2.0,-23,-0.16,4.9$ \\
\hline Contribution of saturation $\mathrm{b}_{2}, \mathrm{~b}_{3}, \mathrm{~b}_{4}, \mathrm{~b}_{5}$ & $\mathrm{units}$ & $-3.7,2.5,-0.64,-0.11$ \\
\hline $\begin{array}{l}\text { Total field harmonics } \mathrm{b}_{2}, \mathrm{~b}_{3}, \mathrm{~b}_{4}, \quad \mathrm{~b}_{5} \text { at injection } \\
(1.06 \mathrm{~T})\end{array}$ & units & $5.6,-25,0.79,5.2$ \\
\hline $\begin{array}{l}\text { Total field harmonics } \mathrm{b}_{2}, \mathrm{~b}_{3}, \mathrm{~b}_{4}, \mathrm{~b}_{5} \text { at nominal field } \\
(16 \mathrm{~T})\end{array}$ & units & $0.025,0.11,0.31,0.18$ \\
\hline Random harmonics $\mathrm{b}_{2}, \mathrm{~b}_{3}, \mathrm{~b}_{4}, \mathrm{~b}_{5}$ & $\mathrm{units}$ & $0.93,0.67,0.47,0.28$ \\
\hline Random harmonics $(\mathrm{skew}) \mathrm{a}_{2}, \mathrm{a}_{3}, \mathrm{a}_{4}, \mathrm{a}_{5}$ & $\mathrm{units}$ & $1.1,0.75,0.48,0.33$ \\
\hline
\end{tabular}

The CM for the main dipole is straight, and has a total length between the two extremities of the beam pipe flanges of $15.8 \mathrm{~m}$ and a magnetic length of $14.069 \mathrm{~m}$. Its external diameter is $800 \mathrm{~mm}$. It is installed in a cryostat structure composed of a radiation shield, a thermal screen and a vacuum vessel. It is supported on three feet made from a composite material and a flange is bolted to the vacuum vessel.

All parts between the beam pipe and the shrinking cylinder, which defines the outer envelope of the cold mass, are immersed in superfluid helium at atmospheric pressure and cooled by a heat-exchanger tube, in which two-phase low-pressure helium circulates. The next temperature stage is that of the beamscreen, cooled at a reference temperature of $50 \mathrm{~K}$, which is also the temperature level for cooling the thermal screen and the support posts. The fact that the additional intermediate temperature level used in the LHC, in the range between 4 and $20 \mathrm{~K}$, is missing, results in larger static losses from the cold mass and the support posts than in the LHC. The total heat loads of a cryodipole operating in steady state mode are estimated to be about $0.5 \mathrm{~W} / \mathrm{m}$ at $1.9 \mathrm{~K}$ and about $10 \mathrm{~W} / \mathrm{m}$ at $50 \mathrm{~K}$. The target losses during a full cycle from nominal field, down to injection and up to nominal field again, which mainly come from the magnetisation of the superconductor, are set to $5 \mathrm{~kJ} / \mathrm{m}$ at $1.9 \mathrm{~K}$ for the two apertures. The operating field of $16 \mathrm{~T}$ is generated by a current of $11441 \mathrm{~A}$ in a coil with a physical aperture of $50 \mathrm{~mm}$. The distance between the axis of the two apertures is $250 \mathrm{~mm}$. The magnet design is described in [162]. The main parameters of the MD, including the expected field quality, are listed in Table 3.1. 


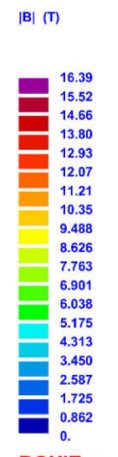

$\operatorname{ROXIE}_{10.2}$
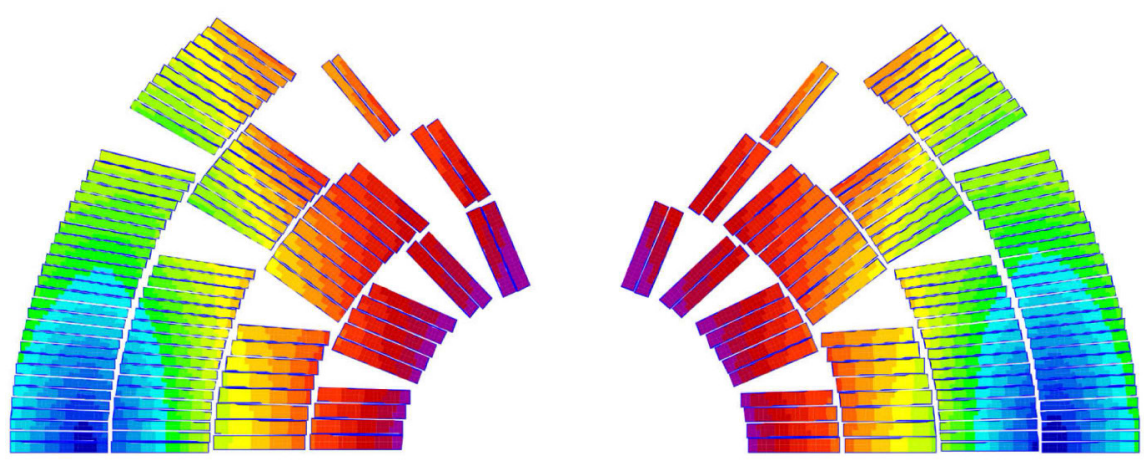

Fig. 3.3. Conductor distribution and field amplitude in the coil (half aperture).

The so called "margin on the load line" has been set to $14 \%$, which is the same as the one adopted for the LHC at the nominal field of 8.3 T. Each MD aperture has 200 cable turns distributed in one upper and one lower pole, and each pole comprises two double layer (inner and outer) coils. Since the magnetic flux density varies considerably in the coil (it is much higher in the inner than in the outer pole), the design exploits the principle of grading (see below). The inner pole comprises 32 turns of a $0.5^{\circ}$ keystoned Rutherford cable, made from 22 strands of $1.1 \mathrm{~mm}$ diameter, the outer pole has 68 turns of a $0.5^{\circ}$ keystoned Rutherford cable, made from 38 strands of $0.7 \mathrm{~mm}$ diameter (see Tab. 3.3). The conductor distribution and the field amplitude in the coil is shown in Figure 3.3, where one quarter of an aperture is shown. The coil cross-section is asymmetric, to compensate the quadrupole component of the magnetic field coming from the interaction between the two magnet apertures.

The current density in the outer coil is larger than that in the inner coil because the two coils are connected in series and the cable in the inner layers has a larger conductor area than that in the outer layers. Thanks to grading, the current density, where the magnet field is lower, is increased, resulting in a considerable saving of conductor for a given margin on the load line. The structure is based on the socalled "key and bladders" concept together with the use of an aluminium cylinder surrounded by a stainless steel welded shell. The aluminium shell provides the increase of coil loading required from assembly to the operational temperature and during magnet powering. The stainless shell, as well as adding stiffness to the structure, provides helium tightness, alignment fiducials and support for the magnet end covers. The CM assembly and its main components are shown in Figure 3.4.

The field distribution in the magnet cross section for a central field in the magnet aperture of $16 \mathrm{~T}$, is shown on the left side of Figure 3.5. The von Mises stress distribution in the structure at the same field of $16 \mathrm{~T}$ is shown on the right side of the same picture. The detail of the stress distribution in the coil cross section is shown in Figure 3.6. Concerning the electromagnetic section, the ferromagnetic yoke is saturated, thus producing a stray field which is about $0.1 \mathrm{~T}$ at the boundary of a non-magnetic cryostat. Concerning the structural section, the coil remains entirely under azimuthal compression (with a minimum pressure of $6 \mathrm{MPa}$ ) up to the $16 \mathrm{~T}$ field amplitude. In these conditions the peak stress on the coil does not exceed $180 \mathrm{MPa}$. The stress in the other part of the structure remains well below the limits of the magnet components.

Each magnet will be cold tested prior to installation in the tunnel. Depending on its training performance, the magnet may also be submitted to a thermal cycle to confirm that, once installed, the magnet can be powered up to nominal field without experiencing training quenches. As was successfully done for the LHC, a warm-cold 


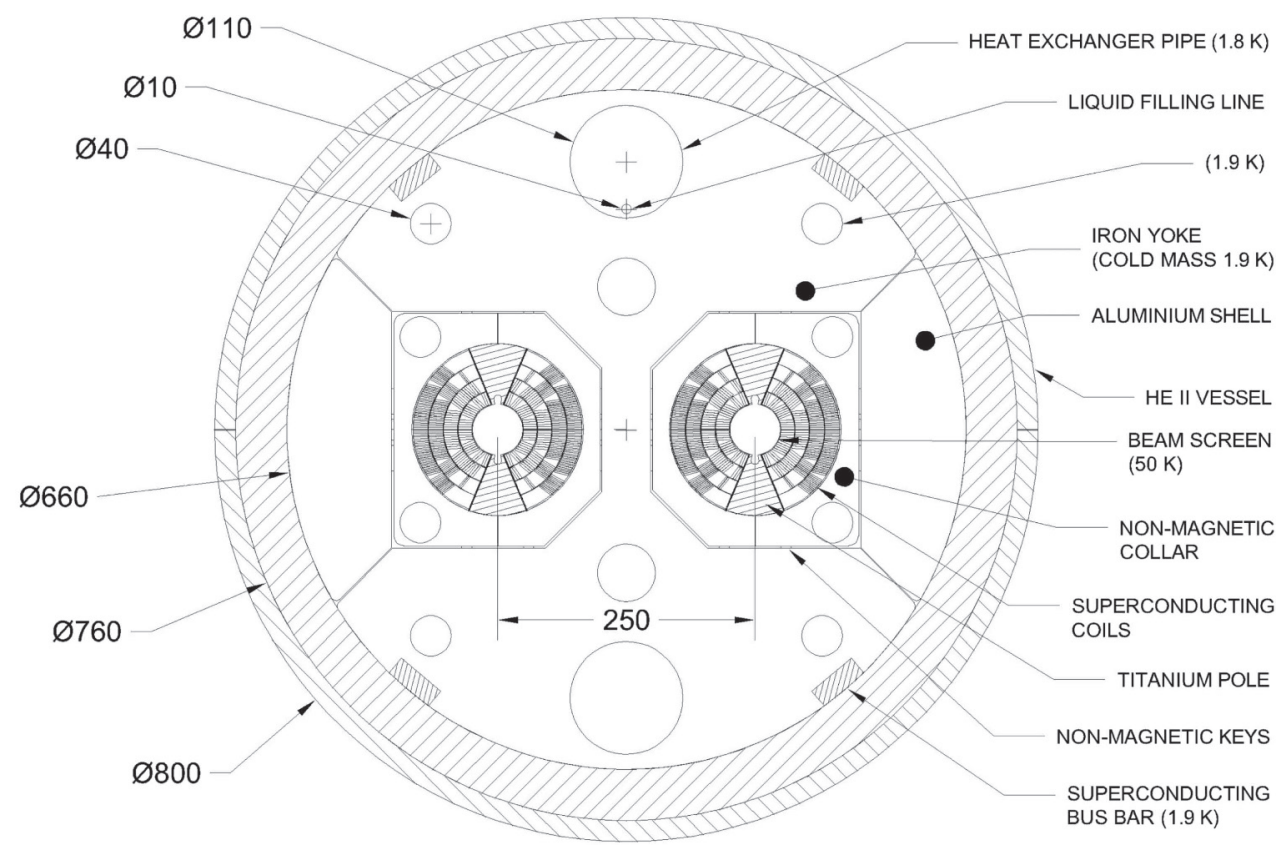

Fig. 3.4. Main dipole cold mass.
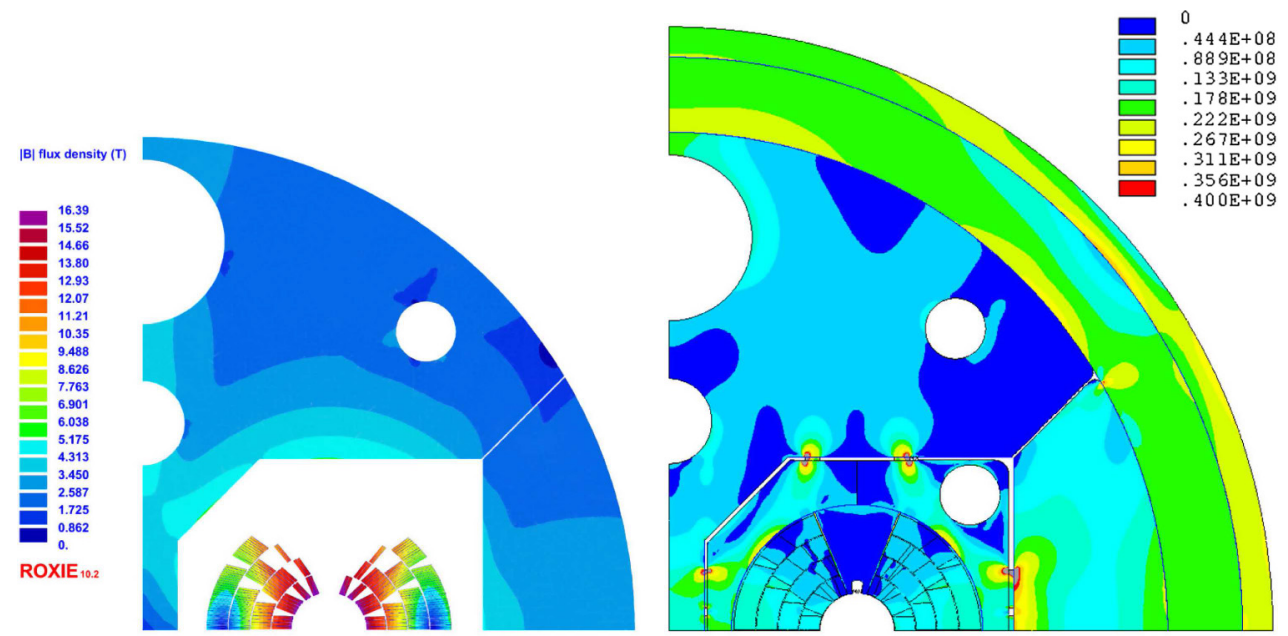

Fig. 3.5. Electromagnetic (left) and structural (right) cross-section for a central field of $16 \mathrm{~T}$.

magnet measurement correlation will be established, based on the statistics from preseries magnets. All series magnets will be then magnetically measured warm. Only a small percentage of them will also measured at operating (cryogenic) temperatures.

\subsubsection{Field quality}

The field error naming convention follows the one adopted for the LHC [163]. The systematic field error values are deterministic and computed with ROXIE: 

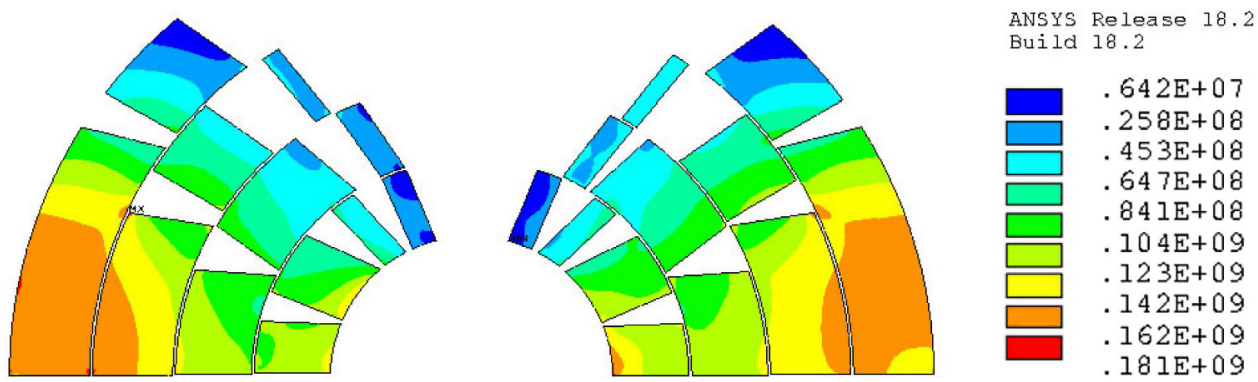

Fig. 3.6. Simulated "Von Mises" stress distribution in the coil for a central field amplitude of $16 \mathrm{~T}$.

Table 3.2. Salient features of design options for $16 \mathrm{~T}$ magnets.

\begin{tabular}{|l|l|l|l|l|}
\hline Parameter & Cos-theta & Block-coil & CCT & Common-coil \\
\hline Peak field on conductor $(\mathrm{T})$ & 16.40 & 16.73 & 16.35 & 16.57 \\
\hline Operating current $(\mathrm{A})$ & 11441 & 10176 & 18135 & 15880 \\
\hline Inductance @ 16 T $(\mathrm{mH} / \mathrm{m})$ & 38 & 48 & 18 & 26 \\
\hline Outer yoke diameter $(\mathrm{mm})$ & 660 & 616 & 750 & 650 \\
\hline Mass of conductor $(\mathrm{kg} / \mathrm{m})$ & 115 & 120 & 148 & 145 \\
\hline
\end{tabular}

they comprise a geometric contribution, a contribution coming from persistent currents and the effect of saturation of the ferromagnetic yoke. The contribution from the persistent currents [164] has been computed using the conductor parameters of Table 3.2 and assuming that artificial pinning has been implemented which allows the critical current (thus the magnetisation) to be reduced at low fields. Considering that it is very unlikely that a perfect point pinning can be achieved, the contribution reported in Table 3.1 has been obtained considering $50 \%$ of perfect point pinning. The random values are due to the spread of the geometric and persistent current contributions. The geometric random errors have been determined by means of Monte-Carlo simulations which include a random displacement of the coil blocks with a root-mean-square (RMS) amplitude $\mathrm{d}=50 \mu \mathrm{m}$. The uncertainty errors are linked to the production line. At this stage, it is assumed, as for HL-LHC, that the production is uniform; therefore the uncertainties are equal to the random values. Further optimisation is on-going to passively correct the $b_{3}$ error from persistent currents by using iron shims. The yoke shape will also be further optimised to minimise the saturation effects.

\subsubsection{Magnet protection}

The magnet and its protection system are conceived to limit the hot spot temperature to below $350 \mathrm{~K}$ in the case of a quench and the peak voltage to ground in the coil below $2.5 \mathrm{kV}$. This voltage limit includes up to $1.2 \mathrm{kV}$ due to the quench evolution in the magnet itself and up to $1.3 \mathrm{kV}$ from the circuit. The protection system can be based on the coupling-loss-induced quench method (CLIQ), on quench-heaters alone or on a combination of both. On paper, all options effectively protect the magnets within the above limits [165]. Experiments on FCC models and prototypes will show if CLIQ can be implemented in real conditions with the required reliability and redundancy for every quench situation. For the reasons above, though it is believed that CLIQ has the potential to quench the entire magnet in $30 \mathrm{~ms}$ after the initiation 

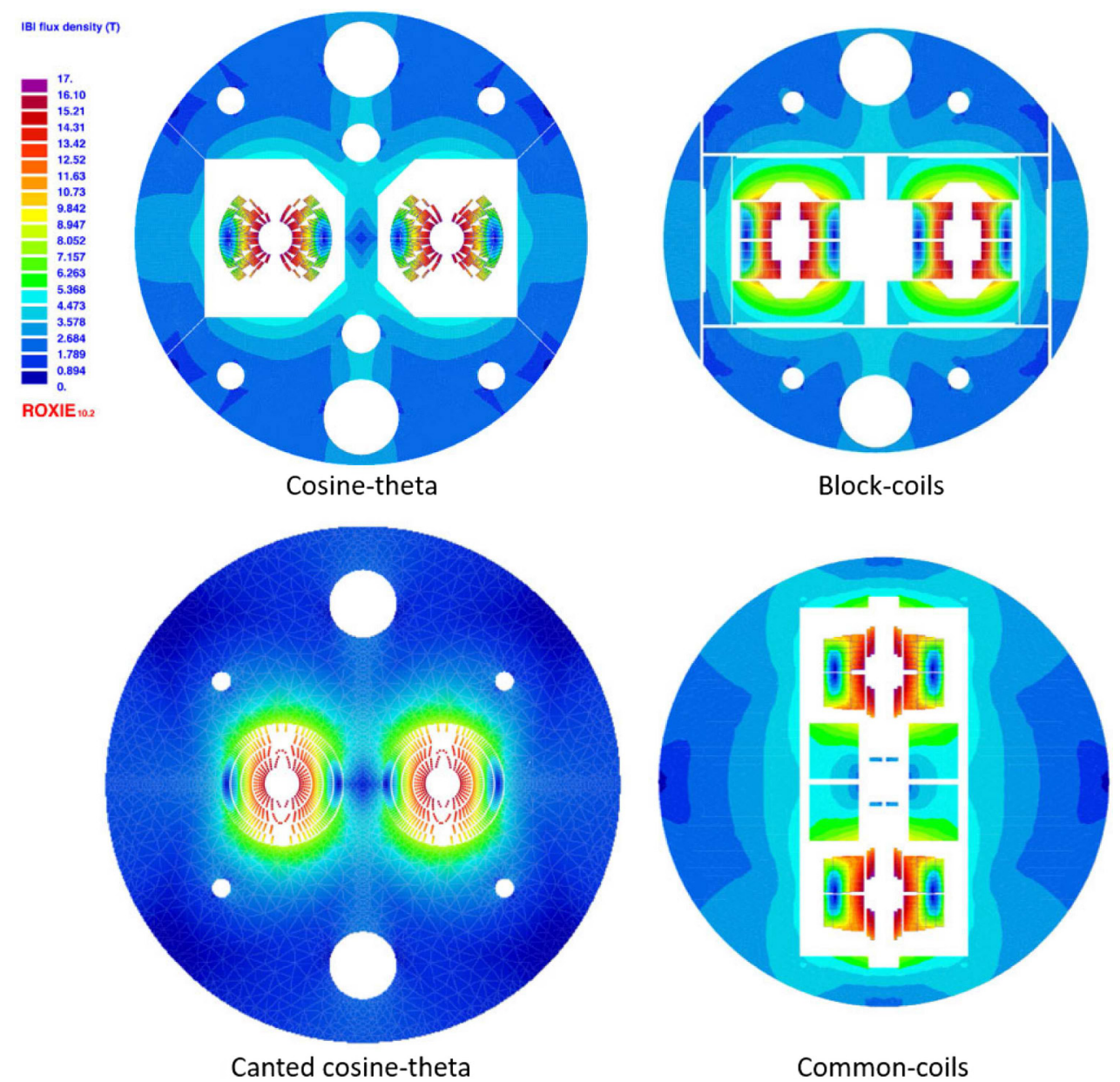

Fig. 3.7. Electromagnetic cross sections of the $16 \mathrm{~T}$ dipole design variants.

of a quench (time delay), the $16 \mathrm{~T}$ magnets have been designed assuming a time delay of $40 \mathrm{~ms}$, which is compatible with the use of quench-heaters.

\subsubsection{Other design options}

In addition to the baseline design of the cosine-theta coil type, other design options have been studied in detail; they will be tested experimentally in the coming years. These other designs are the block-type [166], the common-coil [167] and the cantedcosine-theta (CCT) [168] configurations. All options have been explored considering the same assumptions, in particular concerning the magnet aperture $(50 \mathrm{~mm})$, the field amplitude $(16 \mathrm{~T})$, the conductor performance (assuming the availability of a conductor with a target critical current density of $1500 \mathrm{~A} / \mathrm{mm}^{2} @ 4.2 \mathrm{~K} @ 16 \mathrm{~T}$, corresponding to $\left.2300 \mathrm{~A} / \mathrm{mm}^{2} @ 1.9 \mathrm{~K} @ 16 \mathrm{~T}\right)$, the margin on the load line $(>14 \%)$ and the allowed mechanical constraints on the superconducting coil $(<150 \mathrm{MPa}$ at warm and $<200 \mathrm{MPa}$ at cold). The electromagnetic cross section of each of these options is shown in Figure 3.7. Their salient features, with respect to the baseline cosine-theta, are shown in Table 3.2. 
Each of these alternatives features some interesting characteristics which may have a potential to become competitive to the baseline cosine-theta design in terms of performance, in particular if they would allow operation at a lower margin on the load-line, thus reducing the required amount of conductor.

\subsubsection{Low temperature superconductors}

The $16 \mathrm{~T}$ dipole magnets for the FCC rely on $\mathrm{Nb}_{3} \mathrm{Sn}$. Experience has been gained in the use of this technology in both the U.S and Europe, not only on R\&D magnets but, more recently also on accelerator magnets, thanks to the HL-LHC project. Both the electrical performance and filament size are currently beyond state-of-the-art for $\mathrm{Nb}_{3} \mathrm{Sn}$ wire. A dedicated $\mathrm{R} \& \mathrm{D}$ programme has been launched worldwide, with some promising results already [169]. This programme has three phases. In the first phase, the focus is on increasing the critical current by $50 \%$ with respect to HLLHC $\left(1500 \mathrm{~A} / \mathrm{mm}^{2}\right.$ at $4.2 \mathrm{~K}$ and $\left.16 \mathrm{~T}\right)$, maintaining high RRR (150). This requires a major breakthrough and work on novel methods, such as artificial pinning centres (APC), grain refinement and architectures. In the second phase, the conductor will be optimised to reduce the magnetisation, in particular at low fields, by modifying the effective filament diameter and possibly using APC. The third phase can be considered the preparation to industrialisation, focusing on achieving long unit length $(5 \mathrm{~km})$ and competitive cost (5 kEuro/kAm at $4.2 \mathrm{~K}$ and $16 \mathrm{~T})$. As reported in [169], despite the short time since the start of the programme, high-performing $\mathrm{Nb}_{3} \mathrm{Sn}$ conductors have been already produced by new collaborating partner institutes and companies, achieving a $\mathrm{J}_{\mathrm{c}}$ performance of the order of the specification for HL-LHC. Work performed on grain refinement and APC has shown promising results, nearly doubling the $\mathrm{J}_{\mathrm{c}}$ at $12 \mathrm{~T}, 4.2 \mathrm{~K}$ on small samples. Finally, to improve the training of magnets, the introduction of materials with high heat capacity $\left(\mathrm{Gd}_{2} \mathrm{O}_{3}\right)$ directly within the $\mathrm{Nb}_{3} \mathrm{Sn}$ wire is being considered.

Two distinct conductors are used for the $16 \mathrm{~T}$ dipoles: a high-field (HF) conductor used for the inner pole and a low-field (LF) conductor used for the outer pole. The parameters of the HF and LF conductors are summarised in Table 3.3. It is assumed that the insulated conductor can be subjected to pressures of up to $150 \mathrm{MPa}$ at ambient temperature and $200 \mathrm{MPa}$ when cold, without experiencing an irreversible degradation. Based on the information coming from tailored experiments and from magnet tests, these values are considered to be challenging but realistic. Finally, due to the high $\mathrm{J}_{\mathrm{c}}$, the large filament diameter and the large amplitude of a magnet cycle, the magnetisation losses of these magnets have a considerable impact on the design of the cryogenic system, which assumes $5 \mathrm{~kJ} / \mathrm{m}$ at $1.9 \mathrm{~K}$ for the two apertures. This limit can be respected with filament diameters of around $20 \mu \mathrm{m}$ and if new manufacturing techniques have been developed, e.g. the aforementioned APC, and if the reset current during the machine powering cycle will be optimised.

\subsubsection{Superconducting main quadrupole}

The main quadrupoles (MQ) of FCC are twin-aperture magnets based on a cosine2theta coil configuration assembled in a $20 \mathrm{~mm}$ thick helium II vessel. Their design is detailed in [170]. The cooling system and the cryogenic features in the iron yoke are linked to the MD magnet characteristics. Like the MD magnet, the inter-beam distance is $250 \mathrm{~mm}$ and the physical aperture is $50 \mathrm{~mm}$ in diameter. Each aperture is mechanically independent from the other due to the use of a collar-and-key mechanical assembly. The main parameters of the MQ are listed in Table 3.4. Each double pancake is made of 18 turns of $\mathrm{Nb}_{3} \mathrm{Sn}$ Rutherford cable with a $0.4^{\circ}$ keystone angle. 
Table 3.3. Target parameters for the main dipole conductor.

\begin{tabular}{|l|l|l|}
\hline Property & Unit & Value \\
\hline Wire & & \\
\hline Critical current density at 16 T and 1.9 K & $\mathrm{A} / \mathrm{mm}^{2}$ & 1500 \\
\hline Strand diameter HF conductor & $\mathrm{mm}$ & 1.1 \\
\hline Strand diameter LF conductor & $\mathrm{mm}$ & 0.7 \\
\hline Filament size HF conductor & $\mu \mathrm{m}$ & 20 \\
\hline Filament size LF conductor & $\mu \mathrm{m}$ & 20 \\
\hline $\mathrm{Cu} /$ nonCu HF conductor & & $0.8: 1$ \\
\hline $\mathrm{Cu} /$ nonCu LF conductor & & $2.1: 1$ \\
\hline Cable & & \\
\hline Number of strands HF cable & & 22 \\
\hline Number of strands LF cable & & 38 \\
\hline Width of HF cable & $\mathrm{mm}$ & 13.2 \\
\hline Width of LF cable & $\mathrm{mm}$ & 14.0 \\
\hline Keystone angle of HF/LF cable & $\mathrm{degrees}$ & 0.5 \\
\hline Average thickness of HF cable & $\mathrm{mm}$ & 1.950 \\
\hline Average thickness of LF cable & $\mathrm{mm}$ & 1.265 \\
\hline
\end{tabular}

Table 3.4. Main quadrupole parameters.

\begin{tabular}{|l|l|l|}
\hline Item & Unit & Value \\
\hline Number of units & & 744 \\
\hline Operating gradient & $\mathrm{T} / \mathrm{m}$ & 367 \\
\hline Coil physical aperture & $\mathrm{mm}$ & 50.0 \\
\hline Peak field & $\mathrm{T}$ & 10.51 \\
\hline Operating current & $\mathrm{A}$ & 22500 \\
\hline Operating temperature & $\mathrm{K}$ & 1.9 \\
\hline Magnetic length @ 1.9 K & $\mathrm{mm}$ & 7063 \\
\hline Stored energy at 16 T (entire magnet) & MJ & 3.7 \\
\hline Self-inductance at 16 T (entire magnet) & $\mathrm{mH}$ & 14.4 \\
\hline Field margin on the load line at nominal & $\%$ & 20 \\
\hline Temperature margin at nominal & $\mathrm{K}$ & 4.6 \\
\hline Distance between aperture axes at 1.9 K & $\mathrm{mm}$ & 250 \\
\hline Number of coil turns per aperture & & 72 \\
\hline Mass of the cold mass & $\mathrm{t}$ & 17 \\
\hline Total field harmonics at nominal $\mathrm{b}_{6}, \mathrm{~b}_{10}$ & units & $-0.47,0.41$ \\
\hline Total field harmonics at injection $\mathrm{b}_{6}, \mathrm{~b}_{10}$ & units & $-22.3,2.40$ \\
\hline
\end{tabular}

The cable consists of 35 strands, $0.85 \mathrm{~mm}$ in diameter; the filament size is $20 \mu \mathrm{m}$. The CLIQ system protects the magnet with a hot spot limited to $350 \mathrm{~K}$ and a peak voltage to ground below $900 \mathrm{~V}$.

\subsubsection{Other magnets in the arcs}

For the FCC-hh, the FODO cell length in the arc was chosen to be $213 \mathrm{~m}$, roughly double the length of the LHC FODO cell. The FCC-hh has 8 long arcs, each with 36.5 FODO cells, and 4 short arcs, each with 15 FODO cells. Each FODO cell has 12 dipoles and 2 Short Straight Sections (SSS). As in the LHC, each SSS contains 


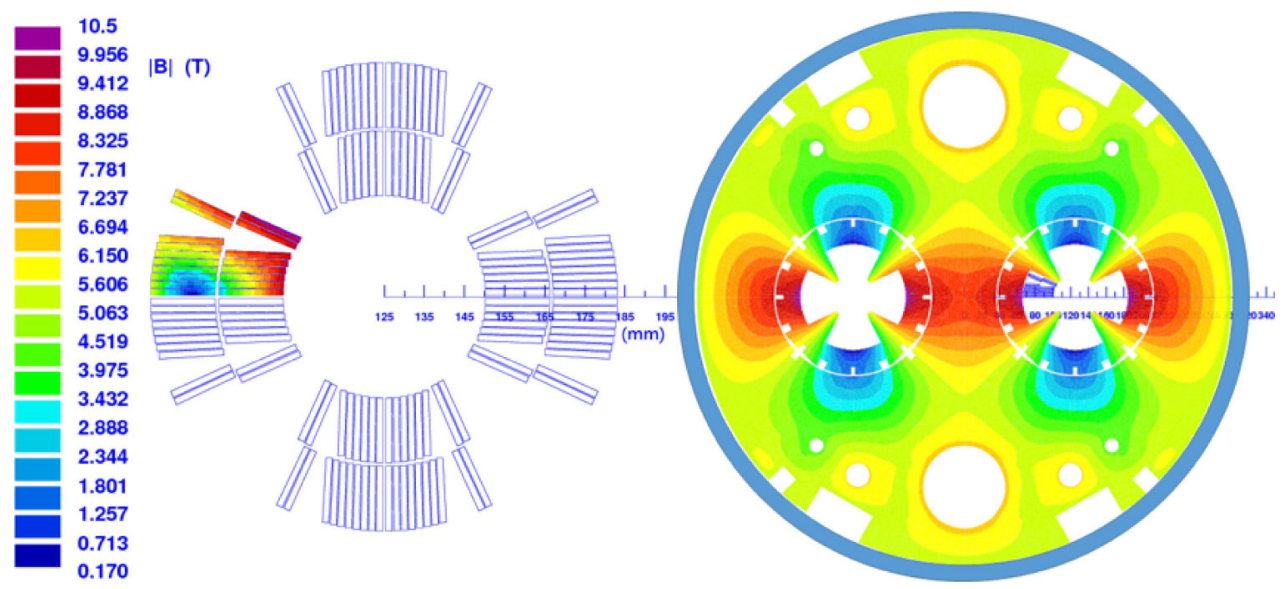

Fig. 3.8. Left: conductor distribution and magnetic field $[\mathrm{T}]$ in the coil. Right: iron yoke, steel collar layout and HeII vessel.

Table 3.5. Other magnets in the Arcs.

\begin{tabular}{|l|l|l|l|l|}
\hline Magnet type & Technology & Number & Strength & Length \\
\hline Lattice sextupoles (MS) & $\mathrm{NbTi}$ & 696 & $7000 \mathrm{~T} / \mathrm{m}^{2}$ & $1.2 \mathrm{~m}$ \\
\hline Lattice octupoles (MO) & $\mathrm{NbTi}$ & 480 & $200000 \mathrm{~T} / \mathrm{m}^{3}$ & $0.5 \mathrm{~m}$ \\
\hline Dipole correctors (MCB) & $\mathrm{NbTi}$ & 792 & $4 \mathrm{~T}$ & $1.2 \mathrm{~m}$ \\
\hline Trim quadrupoles (MQT) & $\mathrm{NbTi}$ & 120 & $220 \mathrm{~T} / \mathrm{m}$ & $0.5 \mathrm{~m}$ \\
\hline Skew quadrupoles (MQS) & $\mathrm{NbTi}$ & 96 & $220 \mathrm{~T} / \mathrm{m}$ & $0.5 \mathrm{~m}$ \\
\hline MCS & $\mathrm{NbTi}^{\mathrm{N}}$ & $2 \times 4668$ & $3000 \mathrm{~T} / \mathrm{m}^{2}$ & $0.11 \mathrm{~m}$ \\
\hline DIS quadrupole (MQDA) & $\mathrm{Nb}_{3} \mathrm{Sn}$ & 48 & $360 \mathrm{~T} / \mathrm{m}$ & $9.7 \mathrm{~m}$ \\
\hline DIS trim quadrupole (MQTL) & $\mathrm{NbTi}$ & 48 & $220 \mathrm{~T} / \mathrm{m}$ & $2.0 \mathrm{~m}$ \\
\hline
\end{tabular}

Table 3.6. Distances between magnets (magnetic lengths).

\begin{tabular}{|l|l|l|}
\hline Magnet types & Distance $(\mathbf{m})$ & Remarks \\
\hline MB-MB & 1.5 & For 0.11 m long MCS \\
\hline MB-BPM & 1.3 & $\begin{array}{l}\text { BPM stands for beam position } \\
\text { monitor }\end{array}$ \\
\hline MQ-other & 0.35 & Other magnetic elements in SSS \\
\hline Other-other & 0.35 & \\
\hline
\end{tabular}

one MQ, and sextupole (MS) and dipole corrector magnets (MC). Depending on the SSS location in the arc, there may be in addition octupole corrector magnets (MO), tuning quadrupoles (MQT) or skew quadrupoles (MQS). It is planned to have 3510 MB-MB, 704 MB-SSS and 704 SSS-MB interconnections in the arcs. The magnet types and their main parameters are listed in Table 3.5. All these magnets, except the DIS quadrupole (MQDA), use Nb-Ti technology. The MQDA relies on $\mathrm{Nb}_{3} \mathrm{Sn}$. The space required for the interconnections and the magnet extremities is summarised in Table 3.6, which shows the target distance between the magnetic ends of the different magnets. 
Table 3.7. Low-beta triplets.

\begin{tabular}{|l|l|l|l|l|l|}
\hline $\begin{array}{l}\text { Magnet } \\
\text { type }\end{array}$ & Technology & Number/IP & Strength & Length & Aperture \\
\hline Q1 high lumi & $\mathrm{Nb}_{3} \mathrm{Sn}$ & 4 & $130 \mathrm{~T} / \mathrm{m}$ & $14.3 \mathrm{~m}$ & $164 \mathrm{~mm}$ \\
\hline Q2 high lumi & $\mathrm{Nb}_{3} \mathrm{Sn}$ & 8 & $105 \mathrm{~T} / \mathrm{m}$ & $12.5 \mathrm{~m}$ & $210 \mathrm{~mm}$ \\
\hline Q3 high lumi & $\mathrm{Nb}_{3} \mathrm{Sn}$ & 4 & $105 \mathrm{~T} / \mathrm{m}$ & $14.3 \mathrm{~m}$ & $210 \mathrm{~mm}$ \\
\hline Q1 low lumi & $\mathrm{Nb}-\mathrm{Ti}$ & 4 & $270 \mathrm{~T} / \mathrm{m}$ & $10.0 \mathrm{~m}$ & $64 \mathrm{~mm}$ \\
\hline Q2 low lumi & $\mathrm{Nb}-\mathrm{Ti}$ & 4 & $270 \mathrm{~T} / \mathrm{m}$ & $15.0 \mathrm{~m}$ & $64 \mathrm{~mm}$ \\
\hline Q3 low lumi & $\mathrm{Nb}-\mathrm{Ti}$ & 4 & $270 \mathrm{~T} / \mathrm{m}$ & $10.0 \mathrm{~m}$ & $64 \mathrm{~mm}$ \\
\hline
\end{tabular}

\subsubsection{Low-beta triplets}

The low-beta triplets are composed of quadrupole magnets and corrector magnets. There are two types of low-beta triplets for installation in the high- and lowluminosity interaction regions, respectively. The magnet types and their main parameters are listed in Table 3.7. The target distance between the magnetic length of each of these magnets is two metres, ignoring the corrector magnets.

The magnets around the collision points will be exposed to high radiation levels which may adversely affect their performance. It is assumed that the conductor performance can be maintained until a displacement-per-atom (DPA) value of $2 \times 10^{-3}$ and that the magnet insulation can withstand an accumulated radiation dose of 30 MGy. These values may be exceeded over the machine lifetime, going up to 40-50 MGy assuming the use of the baseline $35 \mathrm{~mm}$ thick tungsten shield. Some optimisation to reduce this value in the collision optics is ongoing. This radiation load may be still affordable if either a more radiation resistant impregnation system is developed in the magnets or if the triplet magnets are replaced once in the machine lifetime. Furthermore, the estimated static heat load using the baseline shield and at the nominal operation conditions, is $4.5 \mathrm{~mW} / \mathrm{cm}^{3}$, which corresponds to a temperature increase of the coil of about $0.5 \mathrm{~K}$.

\subsubsection{Other magnets}

Depending on their location, the matching and dispersion suppressor quadrupoles have a cross section similar to the MQ but with apertures increased to up to $70 \mathrm{~mm}$. Around 156 matching quadrupoles are distributed as follows: 96 in the dispersion suppressors, 16 in the high-luminosity experiment insertions, 20 in the low-luminosity experiment insertions and injections, 6 in the injection and extraction sections, 8 in the RF section and 10 in the collimation section. Furthermore, 48 trim quadrupoles are installed in the 16 dispersion suppressors. The same type of magnets as those in the LHC are required in the collimation insertion. However, the radiation load in the betatron collimation region is large. Normal-conducting dipole magnets with bedstead coils are proposed in order to reduce the radiation dose by one order of magnitude compared to magnets with racetrack coils. Finally, there are 12 normalconducting and 8 superconducting separation and recombination dipoles. These magnets are listed in in Table 3.8 . 
Table 3.8. Other magnets.

\begin{tabular}{|l|l|l|l|l|l|}
\hline Magnet type and location & Tech & Number & Strength & Length & Aperture \\
\hline Separation dipoles D1 high lumi & Copper & $6 / \mathrm{IP}$ & $1.91 \mathrm{~T}$ & $3 \times 12.5 \mathrm{~m}$ & $168 \mathrm{~mm}$ \\
\hline Recombination dipoles D2 high lumi & Copper & $6 / \mathrm{IP}$ & $1.91 \mathrm{~T}$ & $3 \times 12.5 \mathrm{~m}$ & $58 \mathrm{~mm}$ \\
\hline Separation dipoles D1 low lumi & $\mathrm{Nb}_{3} \mathrm{Sn}$ & $4 / \mathrm{IP}$ & $12 \mathrm{~T}$ & $12.5 \mathrm{~m}$ & $100 \mathrm{~mm}$ \\
\hline Recombination dipoles D2 low lumi & $\mathrm{Nb}_{3} \mathrm{Sn}$ & $4 / \mathrm{IP}$ & $10 \mathrm{~T}$ & $15.0 \mathrm{~m}$ & $60 \mathrm{~mm}$ \\
\hline Q4 high lumi & $\mathrm{Nb}-\mathrm{Ti}$ & $2 / \mathrm{IP}$ & $175 \mathrm{~T} / \mathrm{m}$ & $9.1 \mathrm{~m}$ & $70 \mathrm{~mm}$ \\
\hline Q5 high lumi & $\mathrm{Nb}-\mathrm{Ti}$ & $2 / \mathrm{IP}$ & $260 \mathrm{~T} / \mathrm{m}$ & $12.8 \mathrm{~m}$ & $60 \mathrm{~mm}$ \\
\hline Q6 high lumi & $\mathrm{Nb}-\mathrm{Ti}$ & $2 / \mathrm{IP}$ & $225 \mathrm{~T} / \mathrm{m}$ & $12.8 \mathrm{~m}$ & $60 \mathrm{~mm}$ \\
\hline Q7 high lumi & $\mathrm{Nb}$ 3 $\mathrm{Sn}$ & $4 / \mathrm{IP}$ & $400 \mathrm{~T} / \mathrm{m}$ & $14.3 \mathrm{~m}$ & $50 \mathrm{~mm}$ \\
\hline Q4 low lumi & $\mathrm{Nb}-\mathrm{Ti}$ & $2 / \mathrm{IP}$ & $200 \mathrm{~T} / \mathrm{m}$ & $9.1 \mathrm{~m}$ & $70 \mathrm{~mm}$ \\
\hline Q5 low lumi & $\mathrm{Nb}-\mathrm{Ti}$ & $2 / \mathrm{IP}$ & $275 \mathrm{~T} / \mathrm{m}$ & $12.8 \mathrm{~m}$ & $50 \mathrm{~mm}$ \\
\hline Q6 low lumi, extraction side & $\mathrm{Nb}-\mathrm{Ti}$ & $1 / \mathrm{IP}$ & $170 \mathrm{~T} / \mathrm{m}$ & $12.8 \mathrm{~m}$ & $50 \mathrm{~mm}$ \\
\hline Q6 low lumi, injection side & $\mathrm{Nb}-\mathrm{Ti}$ & $2 / \mathrm{IP}$ & $170 \mathrm{~T} / \mathrm{m}$ & $9.1 \mathrm{~m}$ & $70 \mathrm{~mm}$ \\
\hline Q7 low lumi & $\mathrm{Nb}-\mathrm{Ti}$ & $2 / \mathrm{IP}$ & $84 \mathrm{~T} / \mathrm{m}$ & $12.8 \mathrm{~m}$ & $50 \mathrm{~mm}$ \\
\hline Q8 low lumi & $\mathrm{Nb}-\mathrm{Ti}$ & $2 / \mathrm{IP}$ & $120 \mathrm{~T} / \mathrm{m}$ & $9.1 \mathrm{~m}$ & $70 \mathrm{~mm}$ \\
\hline MCB2 (single ap) high lumi & $\mathrm{Nb}-\mathrm{Ti}$ & 8 & $3 \mathrm{~T} \mathrm{H} / \mathrm{V}$ & $1.3 \mathrm{~m}$ & $240 \mathrm{~mm}$ \\
\hline MCB3 (single ap) high lumi & $\mathrm{Nb}-\mathrm{Ti}$ & 4 & $3 \mathrm{~T} \mathrm{H} / \mathrm{V}$ & $1.3 \mathrm{~m}$ & $240 \mathrm{~mm}$ \\
\hline MCB4h high lumi & $\mathrm{Nb}-\mathrm{Ti}$ & 4 & $3 \mathrm{~T}$ & $3 \mathrm{~m}$ & $70 \mathrm{~mm}$ \\
\hline MCB4v high lumi & $\mathrm{Nb}-\mathrm{Ti}$ & 4 & $3 \mathrm{~T}$ & $3 \mathrm{~m}$ & $70 \mathrm{~mm}$ \\
\hline Orbit Correctors low lumi & $\mathrm{Nb}-\mathrm{Ti}$ & 12 & $3 \mathrm{~T}$ & $1 \mathrm{~m}$ & $64 \mathrm{~mm}$ \\
\hline
\end{tabular}

\subsection{Cryogenic beam vacuum system}

\subsubsection{Overview}

Vacuum stability at cryogenic temperature is a key element for the design of the hadron collider. Significant levels of synchrotron radiation are produced in this machine that result in heat power depositions of the order of $30 \mathrm{~W} / \mathrm{m}$. Early analysis has revealed that it is unlikely to be able to design a beamscreen akin to the one in the LHC that can cope with the operation conditions. A novel design is needed that can effectively shield the cold bore of the superconducting magnets operating at $1.9 \mathrm{~K}$ from the heat load. The concept must also help mitigating electron cloud, resistive and impedance effects from the beginning. The proposed design is currently being experimentally validated. Simulations have shown that it can keep the heat load below $300 \mathrm{~mW} / \mathrm{m}$.

\subsubsection{Beamscreen}

Synchrotron radiation, impedance and cryogenic considerations

The synchrotron radiation (SR) power and flux are higher than those of the LHC. Figure 3.9 shows a comparison between the LHC, FCC-ee, and FCC-hh flux spectra from $4 \mathrm{eV}$ to $1 \mathrm{MeV}$. A $4 \mathrm{eV}$ cut-off has been chosen because photons below $4 \mathrm{eV}$, the typical value of work-function for metals, are incapable of extracting photo-electrons and producing molecular desorption from the walls of the beamscreen. While the linear photon flux for FCC-hh is only a factor of 3.5 times higher than that of LHC, the linear SR power density at $50 \mathrm{TeV}$ is almost 200 times higher, ruling out a scaled version of the LHC beamscreen. Calculations [171] have ruled out the possibility of using LHC-sized capillaries $(<4 \mathrm{~mm})$ because the supercritical helium flow rate 


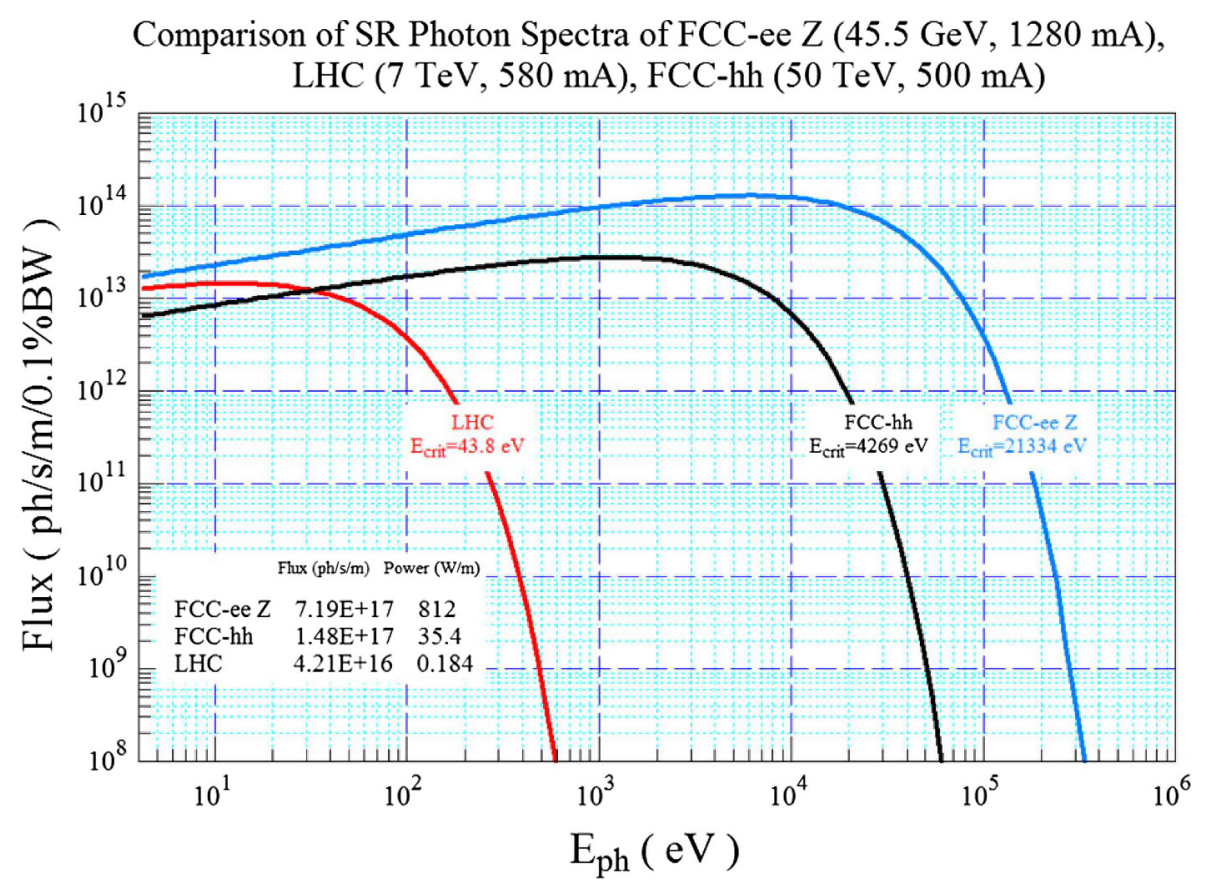

Fig. 3.9. Comparison of the SR flux spectra for LHC, FCC-ee (Z-pole) and FCC-hh.

would not be sufficient. The required number of pumping slots would also affect the impedance budget too much [172]. For these reasons the design concept has longitudinal slots along the external part of the beamscreen in the plane of the orbit, to where the highly collimated SR photon fan is directed.

\section{Beamscreen design}

Figure 3.10 shows the beamscreen design concept. With respect to initial designs, the height of the two longitudinal slots has been increased to limit effects on the impedance budget. This improvement is also beneficial for the vacuum quality, since it captures a larger fraction of the primary SR photon fan during the acceleration phase. At lower beam energies the rms vertical aperture of the SR photon fan is bigger, as it depends on the inverse of the relativistic factor [173]. The number and position of the pumping slots has been optimised taking into account the leakage of scattered SR power reaching the magnet cold-bore. The internal surface of the beamscreen would be treated using Laser-Ablated Surface Engineering (LASE), creating $\mu \mathrm{m}$ sized patterns on the surface. This treatment has proven [174] to greatly reduce the secondary-electron yield (SEY) of the bare surface. Experimental validation of LASE treated surfaces on the resistive wall impedance to reduce SEY and photodesorption yield are ongoing in the EC-funded EuroCirCol project [174]. Details of the measurements on 3 variations of the beamscreen were reported at the 2018 FCC week [175]. If HTS coatings are chosen for improved impedance reduction, e-cloud mitigation would be provided by an a-C coating.

\section{Beamscreen temperature}

The total SR load is $\sim 2.3 \mathrm{MW} /$ beam. If the SR power were to be dumped on a beamscreen at a temperature between $5 \mathrm{~K}$ (inlet) and $20 \mathrm{~K}$ (outlet) as in the LHC 

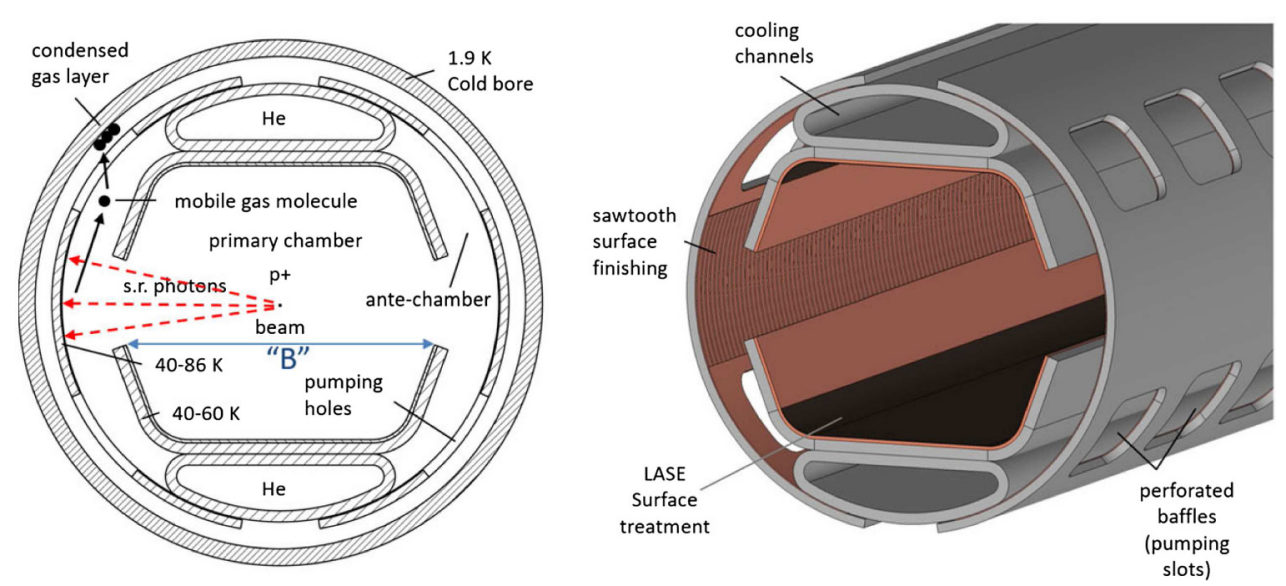

Fig. 3.10. Latest design of the FCC-hh beamscreen. The main functions and parts of the BS are indicated with details of the temperature distribution. The internal part has a height of $26.9 \mathrm{~mm}$ and a width "B" of $27.55 \mathrm{~mm}$. The large pumping slots provide an effective pumping speed for $\mathrm{H}_{2}$ inside the beamscreen of $\sim 860 \mathrm{l} / \mathrm{m} / \mathrm{s}$ at $50 \mathrm{~K}$ (LHC: $\sim 480$ $\mathrm{l} / \mathrm{s} / \mathrm{m}$ at $15 \mathrm{~K}$, as derived from [13]). The thickness of the copper layer on the inside of the ante-chamber areas, including the sawtooth, has been reduced to $80 \mu \mathrm{m}$.

Table 3.9. Cold bore heat load sources.

\begin{tabular}{|l|c|}
\hline Source & $\begin{array}{c}\text { FCC-hh Cold bore heat load } \\
\mathbf{5 0} \mathbf{~ T e V ~ 5 0 0 ~} \mathbf{~ m A}\end{array}$ \\
\hline Beam-gas scattering for baseline MD & $191 \mathrm{~mW} / \mathrm{m}$ \\
\hline Thermal conduction BC 50K-CB 1.9K & $100 \mathrm{~mW} / \mathrm{m}$ \\
\hline Grey body thermal radiation from 50K BS & $3 \mathrm{~mW} / \mathrm{m}$ \\
\hline Leaked radiation power through p. holes & $0.5 \mathrm{~mW} / \mathrm{m}$ \\
\hline Total heat load & $\mathbf{2 9 4 . 5} \mathbf{~ m W} / \mathbf{m}$ \\
\hline
\end{tabular}

arc cells, then given the Carnot efficiency, the cryo-compressor electric power input at room temperature would exceed several GW, making it impossible to operate [171]. A detailed analysis has determined the most appropriate operating temperature for the beamscreen for a workable system. The temperature range for cryogenic cooling at the inlet and outlet of each cell should be between $40 \mathrm{~K}$ and $60 \mathrm{~K}$. A reference temperature of $50 \mathrm{~K}$ is close to the optimum working point for a cryogenic refrigeration system, considering the temperature-dependent resistivity of $\mathrm{Cu}[176]$, and also ideal in the option of deploying HTS coatings [177]. This temperature range is also advantageous for the vacuum system. It leads to higher conductance, which is proportional to the square root of the absolute temperature. It also guarantees that no particular vapour pressure instability is excited [178].

\section{Heat load to cold bore}

Table 3.9 gives an overview of all beamscreen heat load sources, assuming that the beamscreen is mounted using elastic springs in the cold-bore [179] and excluding electron cloud contributions. The results show that the total heat load is below the allowed threshold of $300 \mathrm{~mW} / \mathrm{m}$ [180]. 


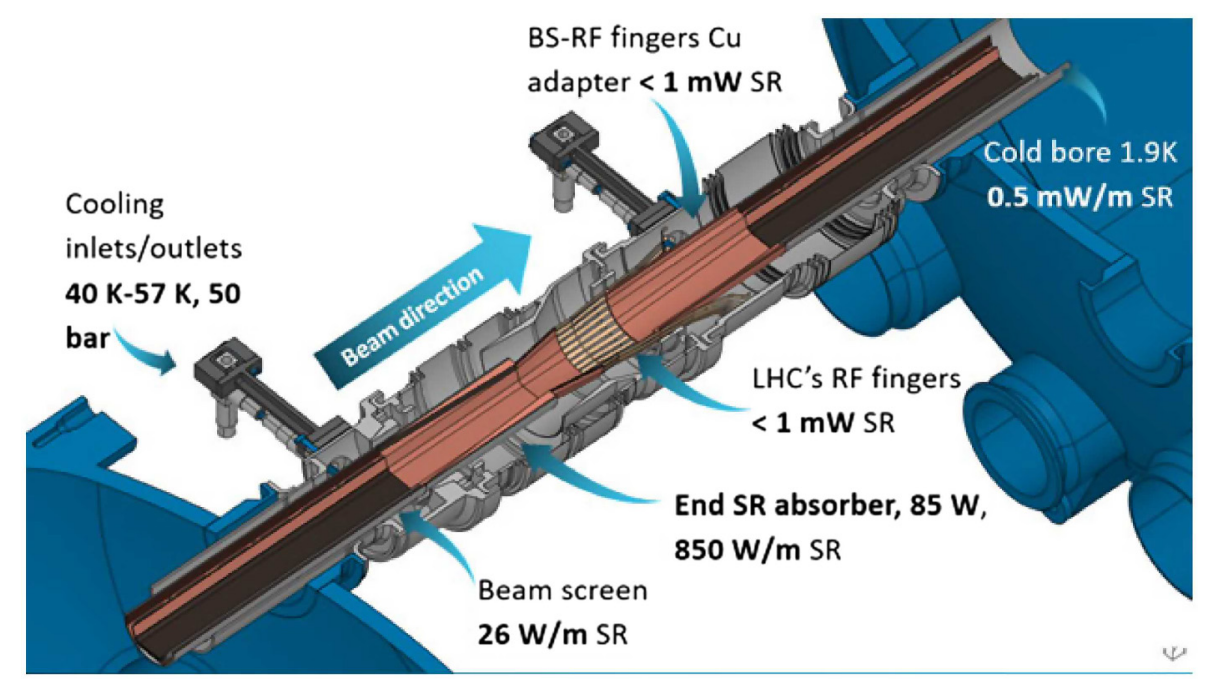

Fig. 3.11. Cut-away, in the plane of the orbit, of the dipole-to-dipole short interconnect area model. This model does not show the BPM block.

\section{Residual gas density}

The requirements in terms of residual gas density are similar to those of the LHC. For the LHC the acceptable level was specified for an $\mathrm{H}_{2}$-equivalent gas density of $1.2 \times 10^{15} \mathrm{~m}^{-3}$ for a $100 \mathrm{~h}$ beam-gas nuclear scattering lifetime, i.e. a gas density of a mixture of gases usually released by $\mathrm{SR}$ irradiation, consisting of $\mathrm{H}_{2}, \mathrm{CO}$, $\mathrm{CO}_{2}, \mathrm{CH}_{4}$, each weighted with its radiation length and percentage molecular content and referred to that of $100 \% \mathrm{H}_{2}$ [181]. At $50 \mathrm{TeV}$ the rate is only slightly larger and an equivalent gas density of $1.2 \times 10^{15} \mathrm{~m}^{-3}$ yields $100 \mathrm{~h}$ lifetime, sufficent not not compromise luminosity. A lifetime of $500 \mathrm{~h}$ is preferred to minimise the heat load from losses due to beam-gas scattering. The target is to remain below $2 \times 10^{14}$ $\mathrm{H}_{2}$-equivalent $\mathrm{m}^{-3}$.

A 3D CAD model of the arc dipole and interconnect area has been created [181] based on LHC. It takes into account the new requirements, SR photon flux and linear power densities. It has been found that the RF fingers installed after each dipole and quadrupole, compensating displacements and thermal contraction during cooldown, need to be shielded from the primary SR photon fan. Otherwise they could be damaged as a result of the $35 \mathrm{~W} / \mathrm{m}$ power density. A symmetric SR absorber upstream of the RF contact fingers, as used in modern light sources, mitigates that risk. The absorber intercepts and concentrates up to $85 \mathrm{~W}$ of SR power, casting a shadow downstream for about 2-3 m, up to the subsequent beamscreen. Along the shadowed area, the racetrack-shaped beamscreen evolves gradually into the circular shape which is needed for the LHC-style RF contact fingers and, eventually, for the BPM button blocks.

Figure 3.11 shows a cut-away view of the interconnect area. The left part of Figure 3.12 shows that even at rather low beam current (roughly $5 \%$ of the nominal) the FCC-hh vacuum system should condition rather quickly to a level below the $100 \mathrm{~h}$ nuclear beam-gas lifetime limit, within about 3 months. The right picture of Figure 3.12 shows a residual gas density profile generated by the SR fan after a certain conditioning time. At each point along the beamscreen, the molecular desorption rate depends on the locally accumulated photon dose. This means that one always has to specify for which photon dose a pressure or density profile is being 


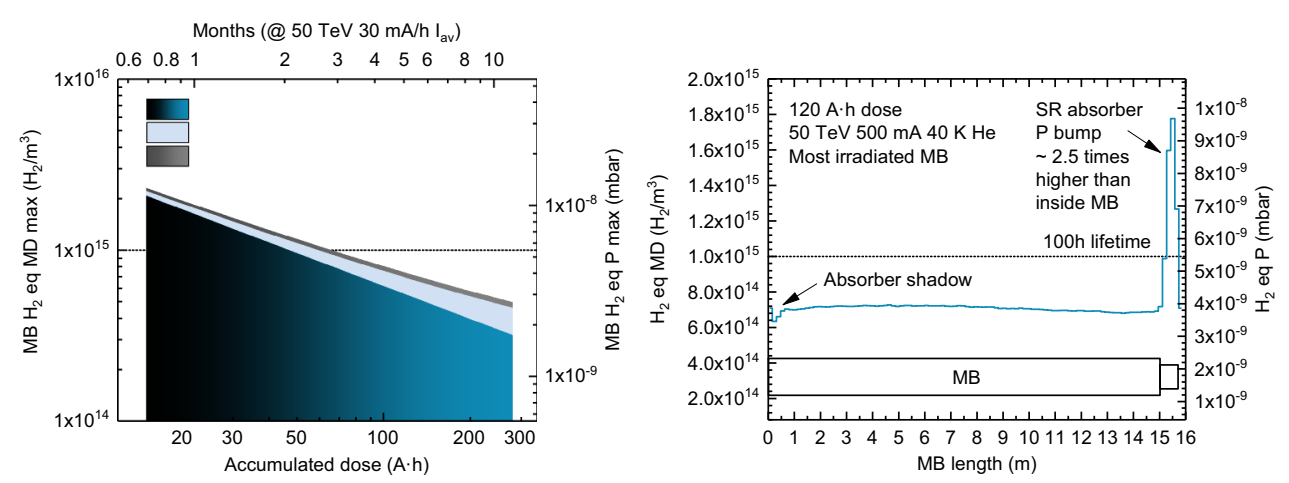

Fig. 3.12. Left: vacuum conditioning versus the accumulated beam dose in units of Ampèrehour, for the $\mathrm{H}_{2}$-equivalent pressure, i.e. including contributions from $\mathrm{CO}, \mathrm{CO}_{2}$ and $\mathrm{CH}_{4}$ [182]. The second $\mathrm{H}$ scale, at the top of the picture, indicates the conditioning time in months, assuming a very low constant beam current of only $30 \mathrm{~mA}$ [182]. Right: density profile in $\mathrm{H}_{2}$-equivalent, i.e. taking into account the gas composition, along one arc dipole [182]. All of the $\mathrm{H}_{2}$ pressure is due to SR, assuming that the static outgassing and the e-cloud contributions are negligible. The density spike visible in the plot is located at the photon absorber position; it is followed (on the left-hand-side of the picture) by a shallow density minimum corresponding to the shadowing of the absorbers and reduced photo-desorption. PSD designates the photon-stimulated desorption; $\mathrm{PSD}^{\prime}$ is the same for the "recycled" molecules, i.e. those molecules which are re-desorbed from their absorption sites on the cold bore due to the tiny fraction of low-energy photons which, after having been scattered many times, are able to reach the cold bore; ESD+ISD is the sum of the contribution from electrons and ions, computed using published data.

calculated. The photon dose at each point along the beamscreen is calculated using 3D ray-tracing Monte Carlo simulation that has been validated with experimental data [181].

\section{Electron cloud mitigation}

The beamscreen design addresses the electron cloud by reducing the rate of synchrotron radiation photons that are scattered into the main part of the screen around the beam. In addition is it necessary to reduce the secondary emission yield to a level of about 1.0 to avoid a build-up of the cloud; this requires coating or treatment of the copper. One path currently being considered is using amorphous carbon (a-C) coating, which has been successfully tested in the SPS at CERN. Another option is LASE treatment (see Fig. 3.10), which is also being assessed experimentally. Figure 3.13 shows the areas which would need to be treated, the corresponding percentage of copper-coated beamscreen area and the electron cloud distribution for each type of magnetic element: dipole, quadrupole and field-free drift [183].

\subsubsection{Vacuum}

\section{Insulation vacuum design}

The insulation vacuum system creates a vacuum barrier between the magnet cryostat and the cryogenic distribution lines. The design will be based on the LHC system [171], which has by now been operating successfully for more than ten years. 

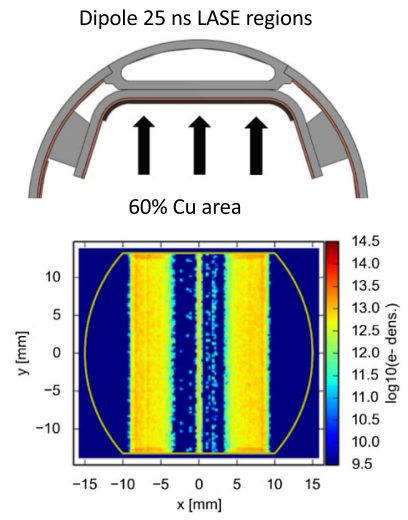
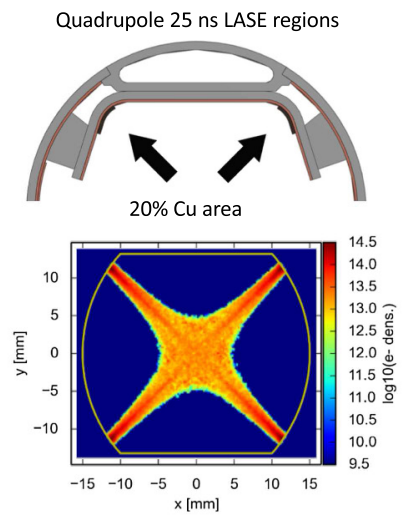

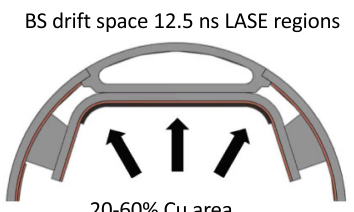

$20-60 \%$ Cu area

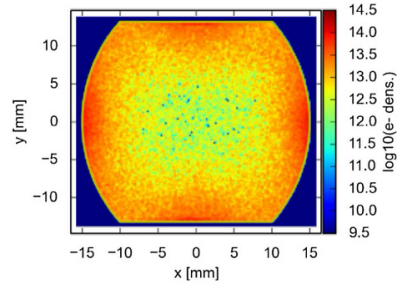

Fig. 3.13. The transverse density of the electron cloud in different components of the collider arc. The location of the surface coating in each component is also shown.

By-passes will be possible. Mobile, rough pumping groups will evacuate the large volumes, starting from atmospheric pressure level. Permanent turbo-molecular pumping groups installed at the vacuum barrier by-pass will be used to reach the required target pressure levels and will evacuate helium gas if there are leaks in the cryogenic pipes.

Equipping the arcs with turbo pumps at a spacing comparable to the LHC would be prohibitive due to the cost of pumps, cables, controllers, and power supplies. The number of fixed turbo-molecular pumping groups would be four times higher than at the LHC. Longer sectors on the contrary, have a reduced effective pumping speed in the central region. Therefore, the reduced pumping capacity will be compensated by He gas absorbers installed at suitable locations and cooled to the lowest possible temperature. Other gas species are absorbed efficiently by the cold walls. The permanent turbo-molecular groups will also guarantee pumping during thermal transients of the cryostat which lead to excessive gas release from the absorbers.

\section{Helium absorbers}

The absorber material, its shape and its assembly in the cryostat are under investigation. The current conceptual design is based on compact nano-porous materials fixed in thermal contact with the cold mass support cooling pipes at $4.5 \mathrm{~K}$ (the $\mathrm{C}^{\prime}$ line in LHC terminology).

The development of the He absorbers can benefit from the large spectrum of studies performed over the last ten years, in particular for the ITER project $[172,176,181]$. He pumping has been measured for activated charcoal fixed on metallic surfaces. Surface areas available for absorption regularly exceed $3000 \mathrm{~m}^{2}$ per gram of absorber; this means that $30 \mathrm{~g}$ of activated charcoal would have the same surface area available as the total multi-layer insulation (MLI) in a $400 \mathrm{~m}$ long vacuum sector. In the coming years, the focus will be to develop a material with a well defined porous structure for He pumping at affordable cost for quantities around $\sim 100 \mathrm{~kg}$. The activation of the absorbers in the cryostats is currently also under investigation. 


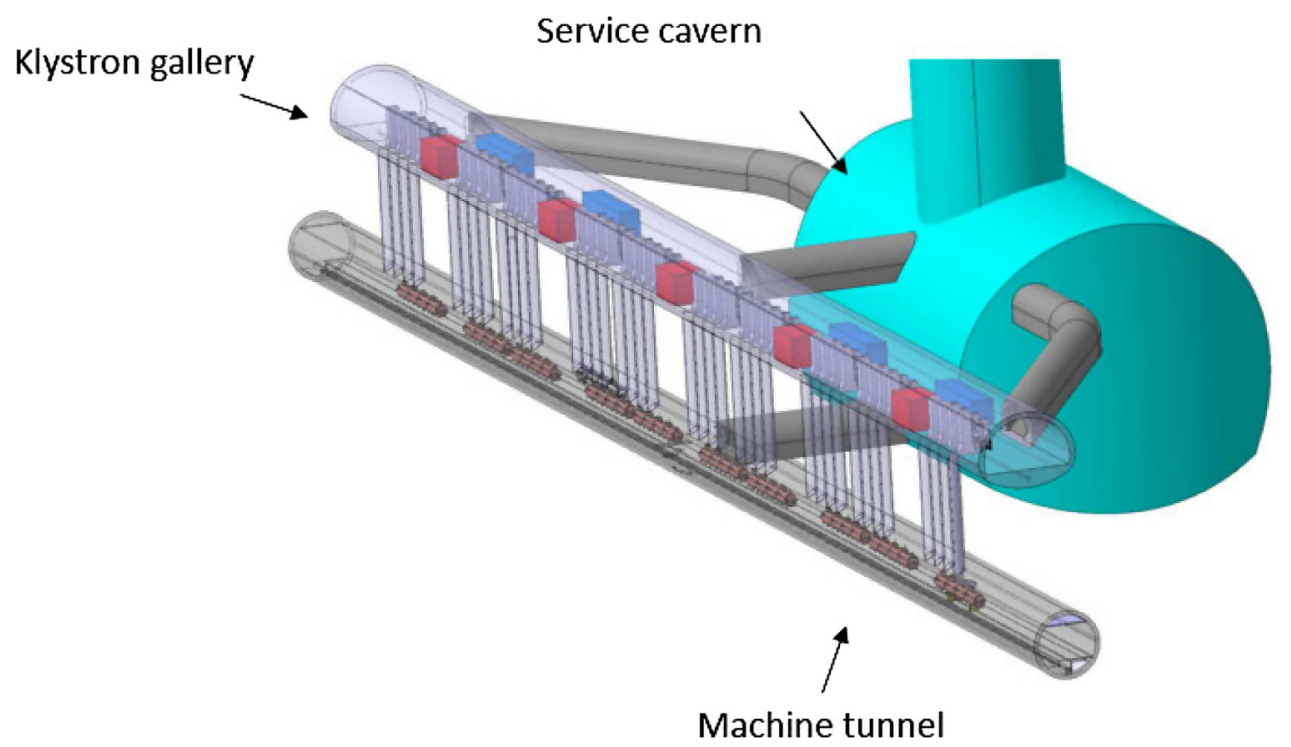

Fig. 3.14. A schematic view of the RF system at point PH.

\subsection{Radiofrequency system}

\subsubsection{Overview}

\section{Introduction}

The injected beam will be captured, accelerated and stored using a $400 \mathrm{MHz}$ superconducting cavity system, which is also used to damp the longitudinal injection errors. A system of electrostatic deflectors will be used to damp the transverse injection instabilities/errors and thus ensure transverse stability. The radiofrequency (RF) and beam feedback systems are located in point PH. The klystron power plant and equipment racks are located in a parallel tunnel several meters above the machine tunnel. Figure 3.14 illustrates the concept.

Two independent RF systems must each provide at least $48 \mathrm{MV}$. A continuous wave (CW) $400 \mathrm{MHz}$ system, the same as the one used in the LHC, leads to a design based on proven technology. It comprises 24 single-cell cavities per beam, operating at $2 \mathrm{MV}$. This requires low RF power through the RF windows and corresponds to a moderate accelerating gradient of $5.3 \mathrm{MV} / \mathrm{m}$. Each cavity is driven by an individual powering system with its klystron, circulator and load. Several feedback loops around each cavity permit precise control of the field in each cavity, an important feature needed to manage the high-intensity proton beam.

\section{System parameters}

The beam and machine parameters that are directly relevant to the design of the RF and beam feedback systems are given in Table 3.10. The minimum bunch spacing of $5 \mathrm{~ns}$ defines the maximum bucket length and gives the lower limit of the RF frequency, which is $200 \mathrm{MHz}$. The bunch length during physics limits the maximum RF frequency to $400 \mathrm{MHz}$, which corresponds to $2.5 \mathrm{~ns}$ bucket length. For the same bunch length at $50 \mathrm{TeV}$, the $200 \mathrm{MHz}$ RF system requires a significantly larger RF voltage than the $400 \mathrm{MHz} R \mathrm{RF}$ system to provide sufficient Landau damping. Thus an 
Table 3.10. Main ring and beam parameters.

\begin{tabular}{|l|l|}
\hline Parameter & Value \\
\hline Circumference $(\mathrm{km})$ & 97.75 \\
\hline Energy (injection/collision) $(\mathrm{TeV})$ & $3.3 / 50$ \\
\hline Transition gamma & $99 \& 71$ \\
\hline Energy loss per turn $(\mathrm{MeV})$ & 4.67 \\
\hline Bunch spacing (ns) & $25(5)$ \\
\hline RMS bunch length during physics $(\mathrm{cm})$ & 8 \\
\hline $4 \sigma$ bunch length during physics $(\mathrm{ns})$ & 1.07 \\
\hline Bunch intensity $(\mathrm{ppb})$ & $10^{11}$ \\
\hline
\end{tabular}

RF frequency of $400 \mathrm{MHz}$ was chosen and the synchronisation with the injectors sets the harmonic number $h=130,680$.

\section{Stability limit}

The minimum RF voltage and the longitudinal emittance at $50 \mathrm{TeV}$ and during the ramp are defined by the following criteria:

- Based on LHC experience, the maximum momentum filling factor will be 0.9 during the ramp and 0.8 in physics.

- The longitudinal emittance should be sufficiently large to prevent loss of Landau damping.

The longitudinal beam stability was evaluated at $50 \mathrm{TeV}$ for the effective impedance $\operatorname{Im} Z / n=0.2 \Omega$ (in the LHC calculated and measured $\operatorname{Im} Z / n=0.1 \Omega$ ) and for both values of transition gamma. For the optics with $\gamma_{t}=99$ the $\mathrm{RF}$ voltage of $42 \mathrm{MV}$ provides the stability with $\pm 5 \%$ bunch length spread and the longitudinal emittance required is about $10.2 \mathrm{eVs}$ [184]. For smaller transition gamma $\left(\gamma_{t}=71\right)$, the same RF voltage is required while the longitudinal emittance can be reduced to $7.3 \mathrm{eVs}$. The threshold impedance, $\operatorname{Im} Z / n_{\mathrm{th}}$, which corresponds to loss of Landau damping, strongly depends on the bunch length (to the power 5). During physics the bunch length would be quickly reduced by synchrotron radiation damping. For the given parameters this means that controlled emittance blow-up is needed to maintain longitudinal beam stability and an additional $800 \mathrm{MHz}$ RF system might be beneficial for this purpose.

The proposed $20 \mathrm{~min}$ ramp from $3.3 \mathrm{TeV}$ injection energy to $50 \mathrm{TeV}$ in collision consists of parabolic, linear, and again parabolic parts with $10 \%, 80 \%$, and $10 \%$ of total energy difference, correspondingly. The controlled emittance blow-up with the square root dependence of longitudinal emittance on energy is required for longitudinal beam stability. For both values of transition gamma the maximum RF voltage is about $43.5 \mathrm{MV}$. Further optimisation of beam stability and particle losses from the $\mathrm{RF}$ bucket during the ramp requires increase of this value up to $48 \mathrm{MV}$.

\subsubsection{Superconducting cavities}

\section{Cavities}

The frequency of $400 \mathrm{MHz}$ naturally calls for the $\mathrm{Nb} / \mathrm{Cu}$ technology operated at $4.5 \mathrm{~K}$. It is anticipated that a vigorous $\mathrm{R} \& \mathrm{D}$ programme on $\mathrm{Nb} / \mathrm{Cu}$ films will eventually decrease the surface resistance by a factor two to three, and hence further 
increase the advantage of this technology [185]. Efforts are ongoing to push the cavity construction technology beyond existing limits to produce seamless cavities within the very tight tolerances required [186]. Such cavities will no longer suffer from performance limitations related to various defects induced by the presence of welds and have less scatter in their electro-magnetic performance [187].

The A15 compounds have the potential to outperform Nb as their BCS surface resistance is much lower due to the higher critical temperatures. $\mathrm{Nb}_{3} \mathrm{Sn}$ cavities obtained by thermal diffusion of $\mathrm{Sn}$ in bulk $\mathrm{Nb}$ have similar performance at $4.5 \mathrm{~K}$ to state of the art bulk $\mathrm{Nb}$ cavities at $2 \mathrm{~K}$. A programme aimed at the synthesis of $\mathrm{Nb}_{3} \mathrm{Sn}$ films on copper substrates is ongoing at CERN and has already produced high quality films on small samples $[188,189]$.

\section{RF power couplers}

The FCC-hh RF system can profit from the FCC-ee power coupler development of the proposed configuration for the FCC-ee Z-pole and W-threshold machines. The RF coupler technology requires R\&D efforts to increase the $\mathrm{CW}$ power transfer capability. The higher order mode couplers will have to deal with high beam loading and must extract $\mathrm{kW}$ of RF power. Progress on fundamental power couplers is essential to limit the cost and size of the RF system. The target value for fixed couplers is $1 \mathrm{MW}$ CW per power coupler at $400 \mathrm{MHz}$ [190]. A suitable fixed power coupler (FPC) design must ensure that the coupling coefficient can be easily adapted to the different machines. The machine parameters and the proposed timeline impose the use of "adaptable" power couplers. The external Q of the coupler must be easily adapted "in situ", without venting the cavities.

Fundamental power couplers for superconducting cavities are among the most important and most complex auxiliary systems. They must simultaneously deliver RF power to the beam and separate the cavity ultra-high vacuum, ultra-low temperature environment from air-filled, room temperature transmission lines, as illustrated in Figure 3.15. A wide variety of techniques is involved in achieving these goals: RF design, cryogenic and mechanical engineering, materials science, vacuum technology, and electromagnetic field modelling.

\section{Crab cavities}

Crab cavities are used to increase the luminosity by tilting the bunches to maximise their geometrical overlap at the collision point. In FCC-hh, the limited impedance budget calls for new concepts and innovative developments. Unlike the existing HLLHC crab cavity prototypes [191], the design for FCC crab cavities is based on $\mathrm{Nb} / \mathrm{Cu}$ superconducting technology. As illustrated in Figure 3.16a, it consists of a ridged waveguide resonator with wide open apertures to make the $\mathrm{Nb}$ coating process possible and also optimise the RF performance.

Figures $3.16 \mathrm{~b}$ and $3.16 \mathrm{c}$ show one quarter of the transverse cross-section at the cavity centre together with the electric and magnetic field distributions, respectively. The particle deflection is caused by the transverse electric field of the TE111-like fundamental mode between the two mushroom-shaped ridges (see Fig. 3.16a). Details of the optimised cavity design and the relevant RF parameters can be found in [192].

This design has good performance; the simulated beam coupling impedances are shown in Figure 3.17. It has only half the effective impedance of the double quarter wave and one third of that of the RF dipole crab cavity [193]. 


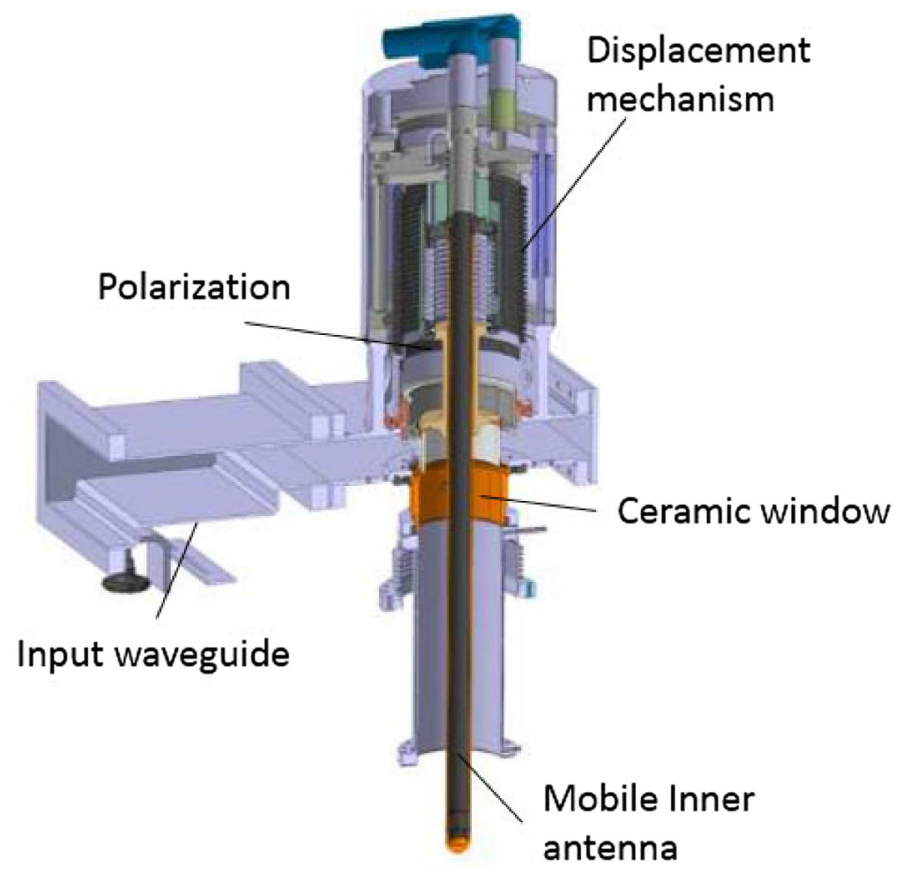

Fig. 3.15. Schematic of the LHC power coupler.

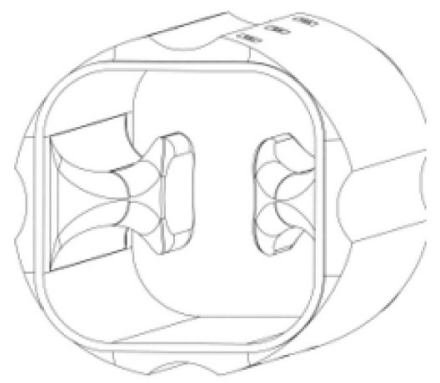

(a)

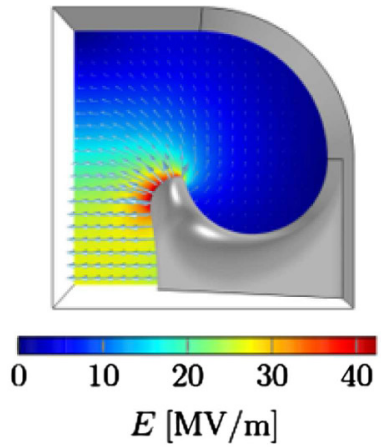

(b)

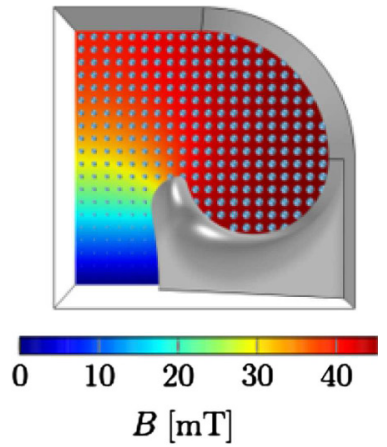

(c)

Fig. 3.16. (a) Design of the wide-open-waveguide crab cavity. The electric (b) and magnetic (c) fields of the fundamental mode are scaled to the nominal deflecting voltage of $V_{\perp}=3 \mathrm{MV}$.

\subsubsection{RF Powering}

\section{Power requirements}

The RF system needs to compensate the significant energy loss of $4.67 \mathrm{MeV}$ per turn at $50 \mathrm{TeV}$. It means that the maximum RF power required at the end of the ramp strongly depends on the voltage program required for beam stability and acceleration. The magnetic ramp should be optimised to minimise the total RF power.

The RF power also depends on the number of RF cavities, which is given by accelerating voltage per cavity (24 cavities/beam with $2 \mathrm{MV} /$ cavity or 48 with 1 $\mathrm{MV} /$ cavity). It is assumed to be about $500 \mathrm{~kW} /$ cavity with $12 \mathrm{MW}$ per beam during the ramp with full transient beam loading compensation. 


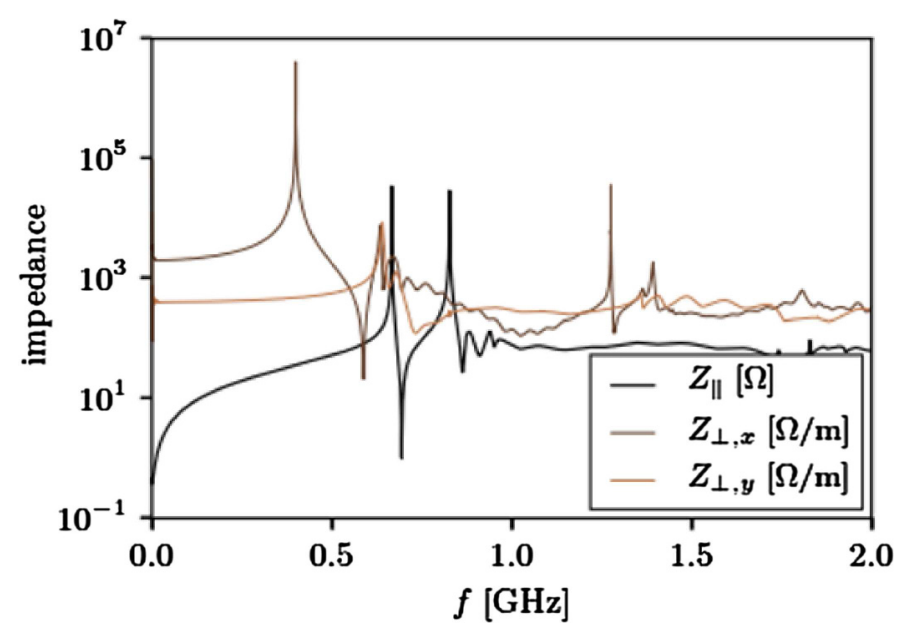

Fig. 3.17. Longitudinal and transverse beam coupling impedances.

Table 3.11. Design parameters of klystrons operating at 400 and $800 \mathrm{MHz}$.

\begin{tabular}{|l|l|l|l|l|l|l|}
\hline Frequency & $\begin{array}{l}\text { Beam } \\
\text { voltage }\end{array}$ & $\begin{array}{l}\text { Beam } \\
\text { current }\end{array}$ & $\begin{array}{l}\text { Peak RF } \\
\text { power }\end{array}$ & Efficiency & $\begin{array}{l}\text { Power } \\
\text { gain }\end{array}$ & $\begin{array}{l}\text { Tube } \\
\text { length }\end{array}$ \\
\hline $400 \mathrm{MHz}$ & $54 \mathrm{kV}$ & $9 \mathrm{~A}$ & $357 \mathrm{~kW}$ & $73.5 \%$ & $38.5 \mathrm{~dB}$ & $1.26 \mathrm{~m}$ \\
\hline $800 \mathrm{MHz}$ & $134 \mathrm{kV}$ & $12.6 \mathrm{~A}$ & $1.35 \mathrm{MW}$ & $80.0 \%$ & $38 \mathrm{~dB}$ & $1.74 \mathrm{~m}$ \\
\hline
\end{tabular}

\section{RF power generation}

Improving energy efficiency and reducing energy demand is absolutely crucial for any future big accelerator and the development of high-efficiency RF power sources must be at the core of the R\&D program [194], see also Section 12. The High Efficiency International Klystron Activity (HEIKA) was initiated at CERN in 2014 [195] to evaluate and develop new klystron bunching technologies for high-efficiency klystrons [196-198]. In the light of these developments, efficiencies are expected to rise from $65 \%$ to potentially above $80 \%$. Gaining efficiency also means reducing costs [198].

This success can be attributed to the very high performance computer code called KlyC [199] which has recently been developed at CERN. This code is both accurate and capable of performing massive optimisations within a reasonable amount of time.

Table 3.11 displays the main parameters obtained for an $800 \mathrm{MHz}$ high-efficiency klystron, optimised for FCC-ee and a scaled version at $400 \mathrm{MHz}$ adapted for HL-LHC. The performance of this $400 \mathrm{MHz}$ klystron is constrained by the fact that the parameters of the existing LHC klystron modulator were preserved. FCC-hh will be equipped with better adapted modulators and the same level of performance as for the $800 \mathrm{MHz}$ tube should be possible. The bunching technology of these klystrons is based on the core stabilisation method described in [199]. The gain and power transfer curves of the $400 \mathrm{MHz}$ tube, simulated by KlyC for different voltages, are shown in Figure 3.18. The tube has a good dynamic range, preserving efficiency above $70 \%$ for the range of output power from 200 to $430 \mathrm{~kW}$ and a comfortable 1-dB bandwidth of $2 \mathrm{MHz}$. 

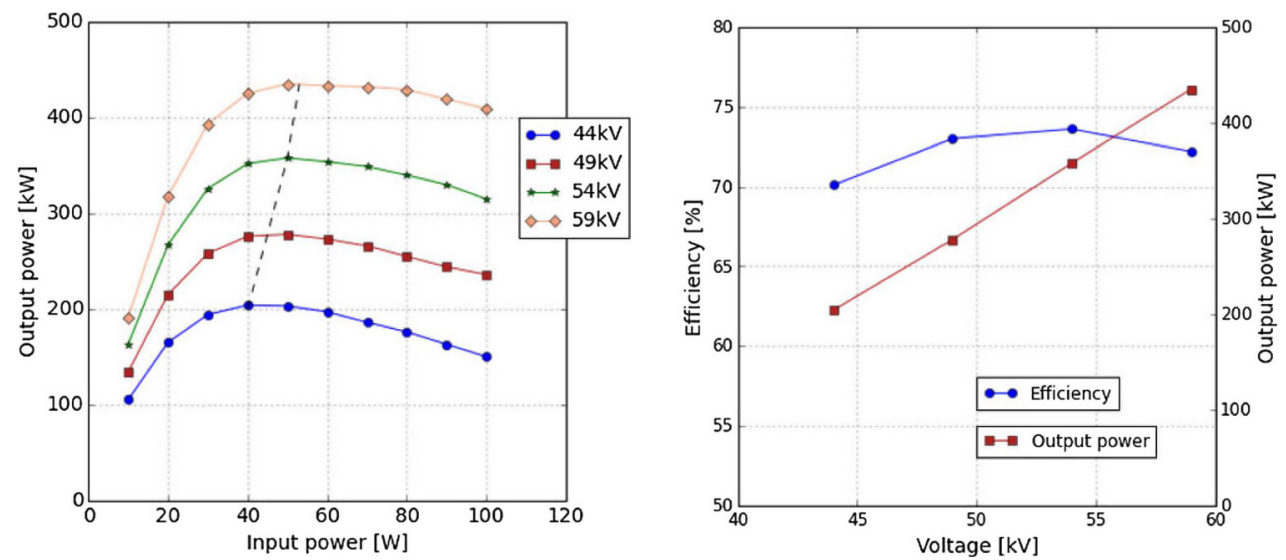

Fig. 3.18. The power gain curves simulated by KlyC for different voltages (left) and the transfer curves for saturated power (right). The dashed line in the left plot traces the saturated power.

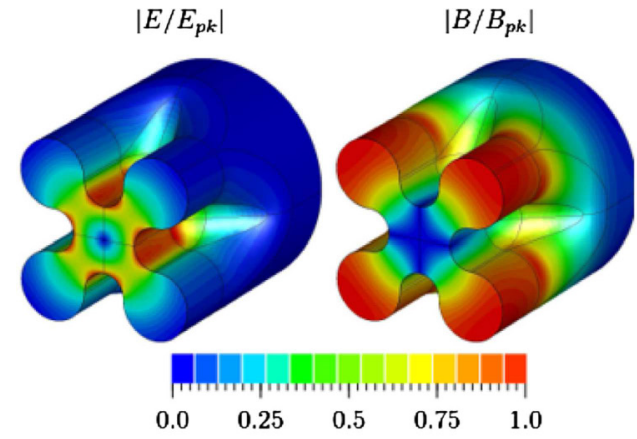

(a)

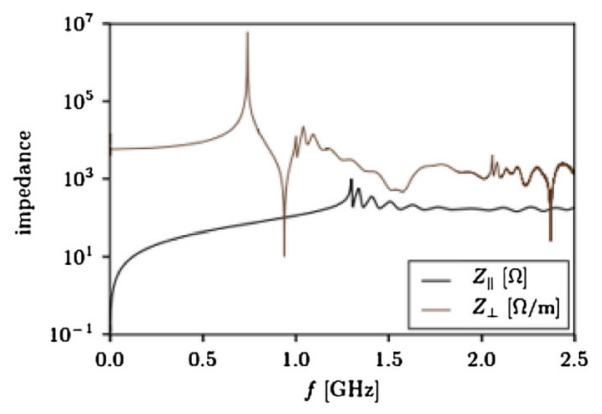

(b)

Fig. 3.19. (a) Normalised field of the TE like quadrupole mode on the surface and in the transverse plane at the centre of a four-vane cavity. (b) Longitudinal and transverse beam coupling impedances.

\section{Design of the RF quadrupole resonator for Landau damping}

The four-vane quadrupole resonator shown in Figure 3.19a is based on superconducting technology in order to provide sufficient tune spread with a feasible number of cavities [200].

It provides a stronger transverse kick than the conventional superconducting elliptical cavities operating in the $\mathrm{TM}_{210}$ mode [201] and the open-left beampipe ports yield lower effective longitudinal and transverse impedances, as shown in Figure 3.19b. The design is sufficiently compact to consider their manufacture in bulk Nb. Details of the cavity design and the relevant RF parameters can be found in [200].

\subsubsection{Feedback}

\section{Low-Level RF}

Longitudinal coupled-bunch instabilities due to the fundamental cavity impedance will be an issue at nominal beam current where the cavity detuning approaches the 
revolution frequency $(3 \mathrm{kHz})$. Strong feedback around the cavities will therefore be required for high intensities. A direct RF feedback will be supplemented by a one-turn delay feedback giving extra impedance reduction around the revolution frequency sidebands. Transient power requirements can be kept to a minimum by the use of a cavity phase modulation scheme as in LHC.

Controlled longitudinal emittance blow-up to maintain stability by Landau damping, both during the ramp and in physics, is achieved by the injection of band-limited $\mathrm{RF}$ phase noise.

Most of the low-level RF issues for FCC-hh have been addressed in LHC [202], or studied for HL-LHC [203]. Modern designs implement most of the signal processing in the digital domain and even better performance will be achieved in the future with the continuous growth of processing power.

\section{Transverse damping and feedback system}

With bunch intensities of $10^{11}$ protons per $25 \mathrm{~ns}$, a strong transverse feedback system similar to the feedback already operating in LHC will be needed to damp injection errors and to cure transverse instabilities caused by the resistive wall impedance. The impedance is dominated by the beamscreen impedance. Details are given together with the projected transverse feedback performance in Section 2.4.7.

From the technology point of view, the transverse feedback system needed to cure the coupled-bunch instabilities at $25 \mathrm{~ns}$ bunch spacing will resemble the ADT system deployed in LHC. The bandwidth of this system has to be at least $20 \mathrm{MHz}$ to cover all possible coupled-bunch modes and - similar to LHC - with bunch lengths in the order of ns, the choice is for a system to operate in the base-band, i.e. from the lowest betatron frequency up to $20 \mathrm{MHz}$, half the bunch repetition frequency.

Optionally the system can be complemented with separate kickers to cover higher frequencies well beyond $20 \mathrm{MHz}$. This is necessary in two scenarios, firstly for the alternative case with $5 \mathrm{~ns}$ bunch spacing and secondly for the case where mitigation of intra-bunch motion is required. The latter can be caused by electron-cloud effects or impedance-driven intra-bunch instabilities (Transverse Mode Coupling Instability, TMCI). In the first case, i.e. for $5 \mathrm{~ns}$ bunch spacing, it is appropriate to choose stripline kickers, while the mitigation of intra-bunch motion requires either dedicated wideband kickers which reach into the $\mathrm{GHz}$ range or sets of highly damped cavitylike kickers with a bandwidth inversely proportional to the bunch spacing and a spacing in frequency inversely proportional to the bunch or RF bucket length. Such kickers were studied to mitigate intra-bunch motion in the SPS as part of the LHC Injector Upgrade Programme (LIU).

The baseline system for $25 \mathrm{~ns}$ bunch spacing can be easily scaled from the LHC parameters. From a technological point of view, it can be implemented using tetrode power amplifiers directly installed under the kicker tanks, which contain electrodes to supply kicks in the horizontal and vertical planes. The advantage of this system over a matched $50 \Omega$ strip-line system is that the tetrode amplifier can deliver strong kicks at low frequencies in the multi-kV range essentially because it is a high impedance device $(\approx 1 \mathrm{k} \Omega$ load in the case of LHC). It is not straight forward to replace this tetrode based system by a system using solid state devices. Solid state devices can usually provide high currents at low impedance determined by their internal capacitances and are therefore not suitable for this application. A strip-line kicker system with long strip-lines can be considered as an alternative to a tetrode-based system.

Different options are proposed for the injection energy ranging from $450 \mathrm{GeV}$ up to the baseline $3.3 \mathrm{TeV}$. Two variants of the bunch spacing are under study, $5 \mathrm{~ns}$ and as baseline $25 \mathrm{~ns}$. The total current is the same for both options, leading 
to similar rise times for the coupled-bunch instabilities at low frequency. However, emittances in the $5 \mathrm{~ns}$ option are approximately 5 times smaller to maintain the same total luminosity when the bunch intensity is reduced by a factor 5 with respect to the $25 \mathrm{~ns}$ baseline. Overall the $5 \mathrm{~ns}$ option puts more stringent requirements on the transverse feedback system, both with respect to strength (faster damping needed to preserve the emittance) and bandwidth (100 MHz minimum) when compared to a $25 \mathrm{~ns}$ bunch spacing $(20 \mathrm{MHz})$. This has also implications for the kicker design. The most challenging is the 5 ns option in combination with an injection energy of $450 \mathrm{GeV}$, leading to very high growth rates.

For the baseline option of $3.3 \mathrm{TeV}$ injection energy, an instability growth time of 45 turns and a damping time of 20 turns is suggested. This allows damping of a maximum injection error of $1 \mathrm{~mm}$ (reference $\beta=200 \mathrm{~m}$ ), fast enough to keep the transverse emittance blow-up from this injection error below 5\%, assuming a decoherence time of $100 \mathrm{~ms}\left(\Delta Q=3.3 \times 10^{-3}\right)$ and a normalised transverse emittance of $2.2 \mu \mathrm{m}$. A damping time of 20 turns is in line with what has been achieved in the LHC and consequently the system layout can be derived from a scaling of the LHC transverse damper system (ADT).

Due to the closer spacing of batches by a factor of three compared to the LHC (300 ns versus $925 \mathrm{~ns}$ in LHC), scaling the tetrode system requires a faster rise-time at the expense of reducing the maximum kick strength per kicker from $7.5 \mathrm{kV}$ to $2.5 \mathrm{kV}$. Keeping the factor three for this scaling and the same kicker length of $1.5 \mathrm{~m}$, 22 kicker units would be required per beam and plane. It is possible to assume a staggered installation despite the close beam spacing, about $\sim 100 \mathrm{~m}$ is required for the complete transverse kicker system. Each of the 88 amplifiers would integrate two tetrodes in push-pull configuration as in the SPS and LHC with supply voltages similar to the SPS damper $(6 \mathrm{kV}, 15$ A peak per tube). About $6 \mathrm{MW}$ of installed electrical power would be required for the tetrode amplifiers $(30 \mathrm{~kW}$ maximum anode dissipation) and a matched cooling system (demineralised water) is required. Tetrode amplifiers would be installed directly under the kicker vacuum tanks.

A pick-up system similar to the LHC standard coupler type (four per plane and beam), ideally spaced at $90^{\circ}$ phase advance, is needed. Kickers should be placed at different betatron phases to achieve smooth damping at tunes close to the half-integer or the integer.

As previously mentioned, the $5 \mathrm{~ns}$ bunch spacing option requires a second system with longer strip-lines and matched solid state amplifiers. The amplifiers would be located in a gallery next to the accelerator with an extra $50 \mathrm{~m}$ required. Additional $\mathrm{R} \& \mathrm{D}$ is needed to design this system which also has the potential to replace the tetrode system. For the $5 \mathrm{~ns}$ option and the wideband $(\mathrm{GHz})$ option, different sets of pick-ups are needed, either exponential couplers or slotted structures for the very high frequencies.

\subsubsection{R\&D challenges}

Two independent RF systems must each provide at least $48 \mathrm{MV}$. The frequency of $400 \mathrm{MHz}$, which is the same as the LHC frequency, allows the RF system to be based on the proven technology developed for LHC. In its present form, a $400 \mathrm{MHz}$ CW RF system made up of 24 single-cell cavities per beam, operating at $2 \mathrm{MV}$, is proposed. This ensures minimum power through the RF windows and corresponds to a moderate accelerating gradient of $5.3 \mathrm{MV} / \mathrm{m}$. Each cavity is driven by an individual RF system with its klystron, circulator and load. Complex feedback loops around each cavity allow precise control of the field in each cavity, which is important for the high-intensity proton beam. 


\subsection{Beam transfer systems}

\subsubsection{Overview}

The baseline scenario for the injection system assumes a beam energy of $3.3 \mathrm{TeV}$, with lower injection energy only considered as an option. Injection will be a conventional bunch-to-bucket transfer with boxcar stacking, with the main challenges being the limitations from machine protection, the fast rise times of the kicker systems and the precision in delivery which is needed to preserve the transverse emittance. The three main subsystems are the injection septum used to provide most of the deflection into the FCC ring, the injection kickers to accurately deposit the beam on the closed orbit and the beam-intercepting protection devices to prevent damage in the event of anomalous kicker operation. The following paragraphs present the concepts for each of these subsystems.

\subsubsection{Injection}

\section{Kicker system}

Most kicker systems today use thyratron switches and pulse-forming networks/lines (PFNs/PFLs). For thyratrons, long-term availability is a real concern. PFNs are built of many discrete components making it difficult to optimise the pulse shapes. PFLs rely on difficult-to-obtain low-loss cables for the ultra high $80 \mathrm{kV}$ voltages [204]. Solid-state technology combined with topologies such as the inductive adder or Marx generator permit the series and parallel connection of power semiconductor switches to achieve high pulse power designs. Both store energy in capacitors, instead of PFNs/PFLs. Solid-state switch technology results in good reliability, modularity and maintainability for kicker generators. Since the capacitors are not significantly discharged between pulses, considerable amounts of energy remain stored in the generator once the collider ring has been filled. The potential effects of this condition remain to be studied further.

\section{Solid-state kicker generators}

The modularity of the inductive adder and Marx generator permit different functional and performance requirements to be met with the same design [205]. The major challenge for solid-state technology is to achieve sufficiently fast pulse riseand fall-times [204]. The overall length of a solid-state generated pulse, and hence its propagation delay, depends on the output voltage. The allowable field rise-time of the injection kicker magnet is $425 \mathrm{~ns}$ (see Sects. 2.3.5 and 2.3.6): thus, allowing $75 \mathrm{~ns}$ for the rise-time of the output pulse of the modulator, the propagation delay of an individual magnet module can be $350 \mathrm{~ns}$. To achieve the required pulse current magnitude and rise-time, a reduced output voltage is required: hence a relatively low characteristic impedance is needed, but consistent with the rise-time requirements. A detailed study of the expected capabilities of the pulse generator $(2.5 \mathrm{kA}$ pulse current), achievable current rise-time ( $75 \mathrm{~ns}$ ) and obtaining a reasonably short-length for the injection kicker magnets (to limit their total beam coupling impedance), has resulted in an optimised value for the characteristic impedance of $6.25 \Omega$ [206].

MOSFETs are presently the most promising technology for the solid-state pulse generators, as fast switching elements $(\sim 10$ 's ns) are available commercially today. Silicon carbide ( $\mathrm{SiC}$ ) MOSFETs are becoming more widely available: this technology 

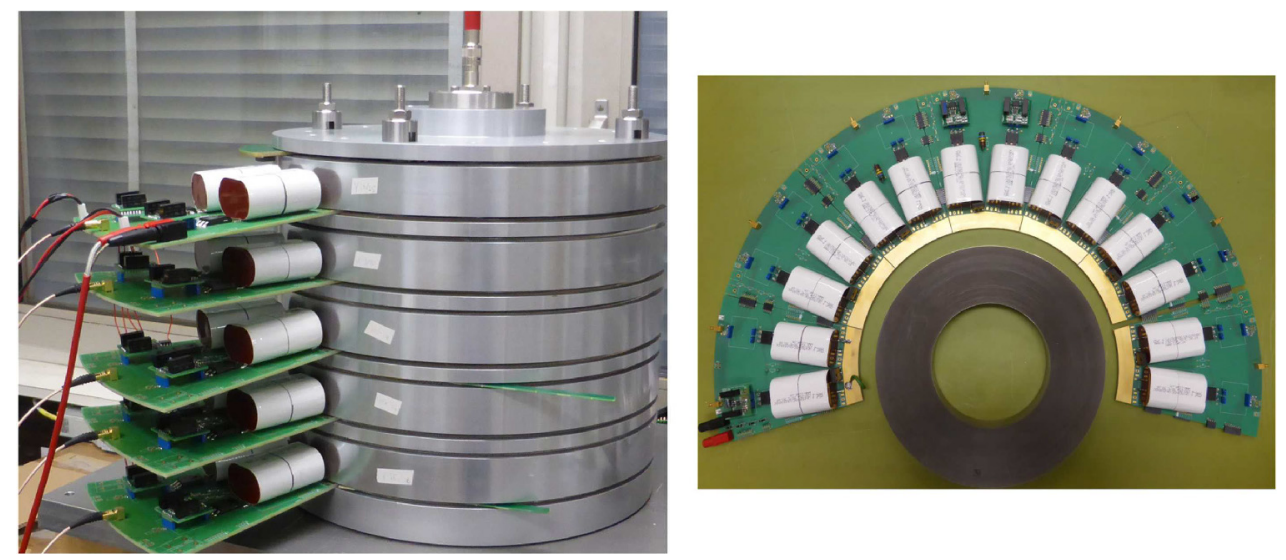

Fig. 3.20. Left: prototype inductive adder being developed for FCC. Right: PCBs and magnetic core of the prototype inductive adder.

is preferred over Silicon as the $\mathrm{SiC}$ has significantly lower on-state resistance: this is desirable for a pulse generator with low characteristic impedance (e.g. 6.25 $\Omega$ ). Commercial-of-the-shelf MOSFETs still have limited voltage rating, generally up to $1.2 \mathrm{kV}$ [206]. MOSFETs rated at $3.3 \mathrm{kV}$, with pulse current ratings of $250 \mathrm{~A}$ or more, would lead to more compact generators, with potentially faster rise- and fall-times of the output pulse. Hence the evolution of the commercially available devices needs to be followed attentively. However, a real breakthrough in MOSFET technology is needed.

To make use of higher voltage ratings of MOSFETs in a cost-effective way and therefore reduce the number of layers needed for the inductive adder pulse generator, new magnetic core materials would help to achieve the required pulse length with reasonable core cross-sectional area. Again, in this case, a breakthrough in the technology is required and developments with nano-crystalline magnetic materials are being followed.

Figure 3.20 shows a prototype inductive adder that is being developed in the scope of the study: the prototype is a significant evolution of a design conceived for CLIC [207]. Key components including SiC MOSFETs, MOSFET gate drivers, pulse capacitors and magnetic materials, have been tested and prototype PCBs designed and successfully tested. Initial results show that the required inductive adder performance can be achieved. In addition, a novel concept for an inductive adder has been designed to limit current following an electrical breakdown in a kicker magnet. Detailed tests and measurements will be carried out on the prototype, before upgrading it to a full-voltage version.

Figure 3.21 shows a prototype, four-stage, Marx generator developed by ISEL University and the EPS company, both of Lisbon [208]. This Marx generator is assembled in a coaxial type structure in order to limit the loop inductance: the current flows to the load through the MOSFETs and capacitors, placed around the sides of this structure and returns back through external, insulated, wires. Each stage operates at a voltage of up to $800 \mathrm{~V}$, and consists of 24 SiC MOSFETs in parallel. The four stage Marx generator was successfully tested with a load current pulse of $2.7 \mathrm{kA}$ (which meets the specifications) into a $1.18 \Omega$ resistive load: the measured load voltage pulse is shown in Figure 3.22. The measured rise- and fall-times, 10\%-90\%, were both approximately $50 \mathrm{~ns}$. This prototype allows the proposed design to be tested and evaluated, in order to assess the technological advantages of such a system and limitations for the scale-up to full voltage. 


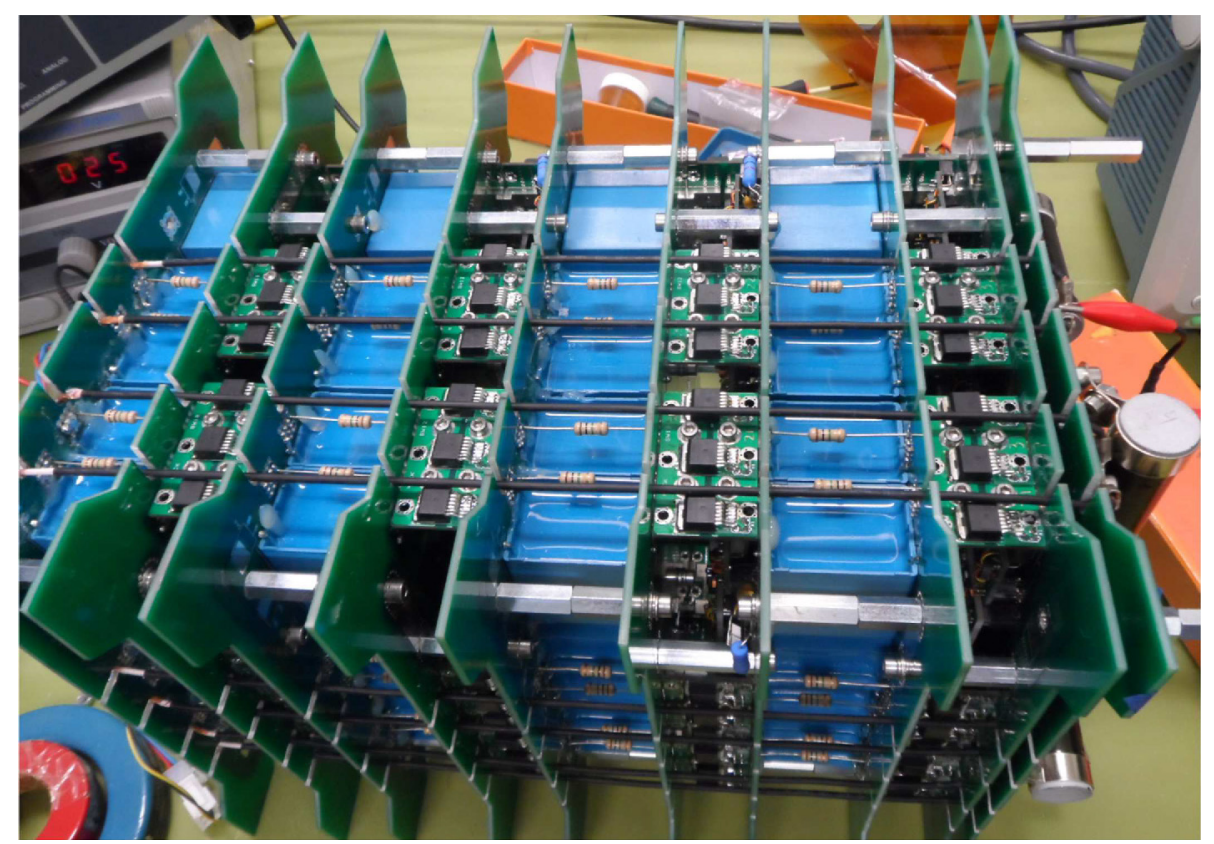

Fig. 3.21. Four stage Marx generator assembled in a coaxial structure (four return conductors per side).

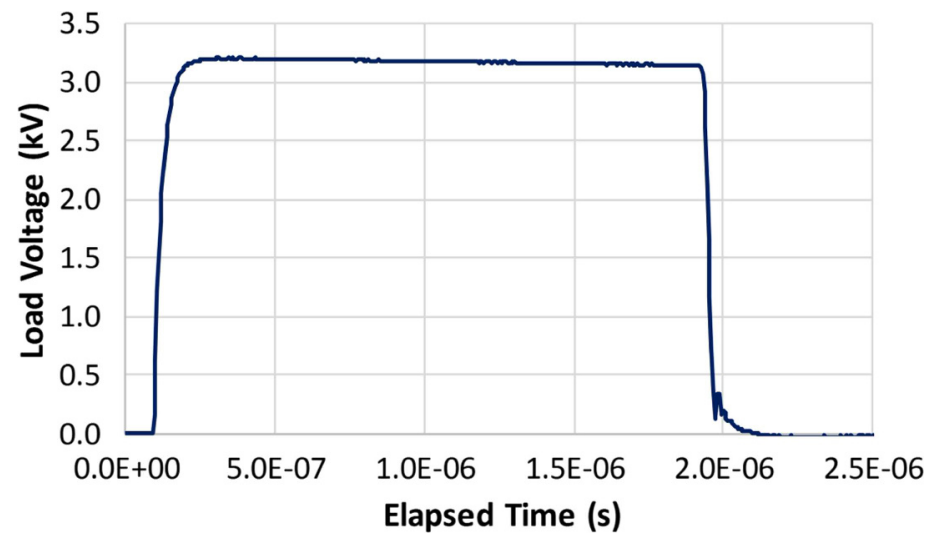

Fig. 3.22. Measured load voltage pulse for four Marx stages (pre-charge $=800 \mathrm{~V} /$ stage).

Scaling-up this concept requires further significant effort such as separating the main circuit and wires carrying the return current [208]. Modifications of the Marx generator are under consideration so that it can safely limit the current in the event of a short-circuit load (e.g. electrical breakdown in a kicker magnet). Future tests will include long-term stability and reliability tests and measurements, as well as testing under fault conditions.

\section{Kicker magnet}

High-intensity accelerators are very sensitive to longitudinal and transverse beamcoupling impedance. Kicker magnets, with their ferrite yoke, can be one of the 
dominant sources of impedance [209], which limits the beam intensity. In addition, the real part of the longitudinal beam impedance, together with the beam spectrum, determines beam induced power deposition and beam induced kicker heating. It will be necessary to screen the yoke of the injection kicker magnet from the beam, which has the consequence that the aperture of the magnet has to increase. In addition, the overall length of the injection kicker magnets has been reduced from $130 \mathrm{~m}$ to approximately $40 \mathrm{~m}$ magnetic length, to decrease the beam coupling impedance for the installation [210].

High purity alumina will be required to mechanically support the screen conductors: the alumina will also provide high voltage insulation. This has the undesirable side-effect of creating a vacuum-metal-insulator triple junction, which requires special attention to avoid electrical breakdown. The alumina, if untreated, will provoke electron cloud generation. The $\mathrm{Cr}_{2} \mathrm{O}_{3}$ coating developed for the LHC injection kickers can help mitigate this effect and should also improve the high voltage performance [211].

\section{Septum}

The baseline for the injection septa is to use three families of normal-conducting Lambertson septa at $0.7,1$ and $1.2 \mathrm{~T}$ with septum thicknesses of 8,12 and $18 \mathrm{~mm}$, respectively, due to their robustness and simplicity. Alternatively, superconducting septa with the same septum thicknesses would reduce both power consumption and the installed length. However, today it is difficult to achieve the smallest septum thickness. At $3.3 \mathrm{TeV}$ injection energy, it may be advantageous to supplement the normal conducting septum with a 4 or $6 \mathrm{~T}$ superconducting septum system. The studies ongoing for the extraction septa are relevant in this context. For a $\cos \theta$ device [212] the superconducting cables could be made of readily available NbTi. Bulk $\mathrm{MgB}_{2}$ [213] is being considered as an alternative material. The power considerations are less relevant for the injection septum than the dump septum, since it can be ramped down to zero current between injections (i.e. the total energy consumption is much less because the injection septum is only switched on for $1 \%$ of the time, whereas the dump septum is on all of the time).

Protection of the septa has to be included in the design of the injection system at the very end of the transfer line - in the case of a superconducting septum, additional studies are needed for quench protection and beam loss.

After the septa, the beam's horizontal angle is reduced by off-centre passage through a ring quadrupole and finally compensated by the fast kicker system. Massless septa are under consideration to complement the injection dump in case of a mis-injected beam.

\subsubsection{Extraction}

The extraction kickers and dilution kickers have to direct proton beams with stored energies in the range of 8 GJ reliably to the beam dump for the safe operation of the collider. A dumping system similar to the LHC concept [54] has been developed, but it requires a combination of very different technologies for the kickers and septa. This approach also creates challenges for the control system in terms of triggering, erratic rate and fault isolation.

The extraction kicker system faces stringent requirements for field rise-time, reliability and availability. The flattop duration of the extraction system is $326 \mu$ s. The extreme beam rigidity requires large $\int \mathrm{B} \cdot \mathrm{dl}$, which imposes a segmented system architecture comprising about 100 units per beam. The overall extraction kicker system 
a)

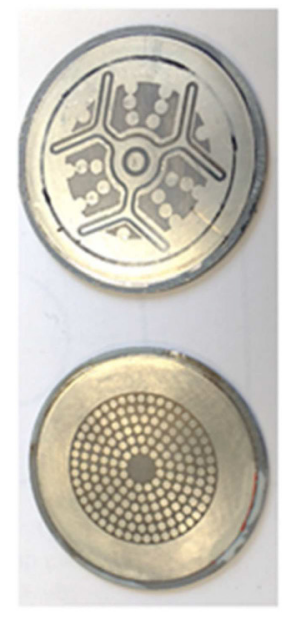

b)

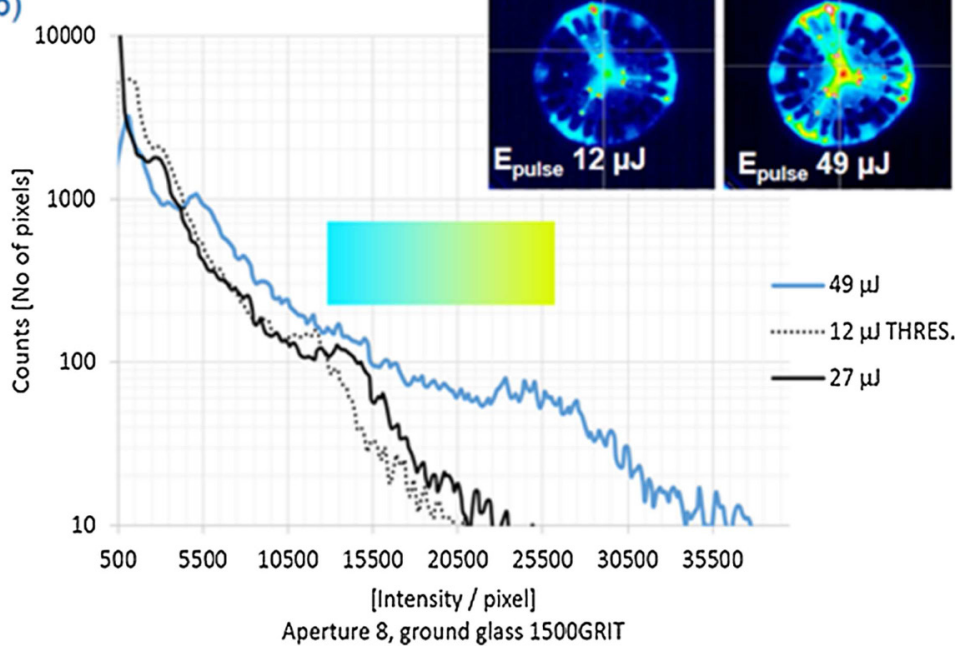

Fig. 3.23. (a) Anode and cathode sides of a thyristor wafer that has been etched; (b) CCD camera images and histograms of the optical interface output light projected on the thyristor wafer.

length available is $120 \mathrm{~m}$. This approach leads to an acceptable loss level of individual kicker elements. Nevertheless, a system reliability analysis needs to be carried out in order to identify the detailed requirements and to be able to develop an appropriate technical design. The short magnet module also provides a low inductance and hence allows for a rise-time of $1 \mu \mathrm{s}(0-100 \%)$, imposing significantly less beam load on the protection devices during an asynchronous extraction event. In addition, the relatively low current $(3 \mathrm{kA})$ and voltage per unit result in reasonable generator design values, beneficially influencing the switch selection and system reliability. Lumped inductance magnets, similar to those in the LHC beam extraction kicker magnets, are planned.

\section{Triggering and re-triggering}

In the case of an erratic triggering of a single extraction kicker, the baseline plan is for a dump synchronous with the next abort gap. However, for simultaneous multiple kicker magnet erratics, immediate re-triggering and therefore an asynchronous beam abort is necessary to protect the machine from damage. The probability of multiple erratics due to common-cause failures (e.g. electromagnetic coupling) needs to be kept as low as possible. Several concepts for the extraction kicker generators are under study. One of them is the use of optically triggered thyristors [214]: this technology potentially avoids false-triggering of thyristors by electromagnetic coupling in cables used for electrically triggered devices. Figure 3.23 shows the anode and cathode sides of a thyristor wafer that has been etched for improved optical triggering of the device.

\section{Extraction kicker generators}

Generator topologies based on stacked arrays of solid state switches have been studied. Alternative switch topologies, namely series-connected switches and crowbar architecture, are being studied to reduce the impact of a pre-firing kicker on the 


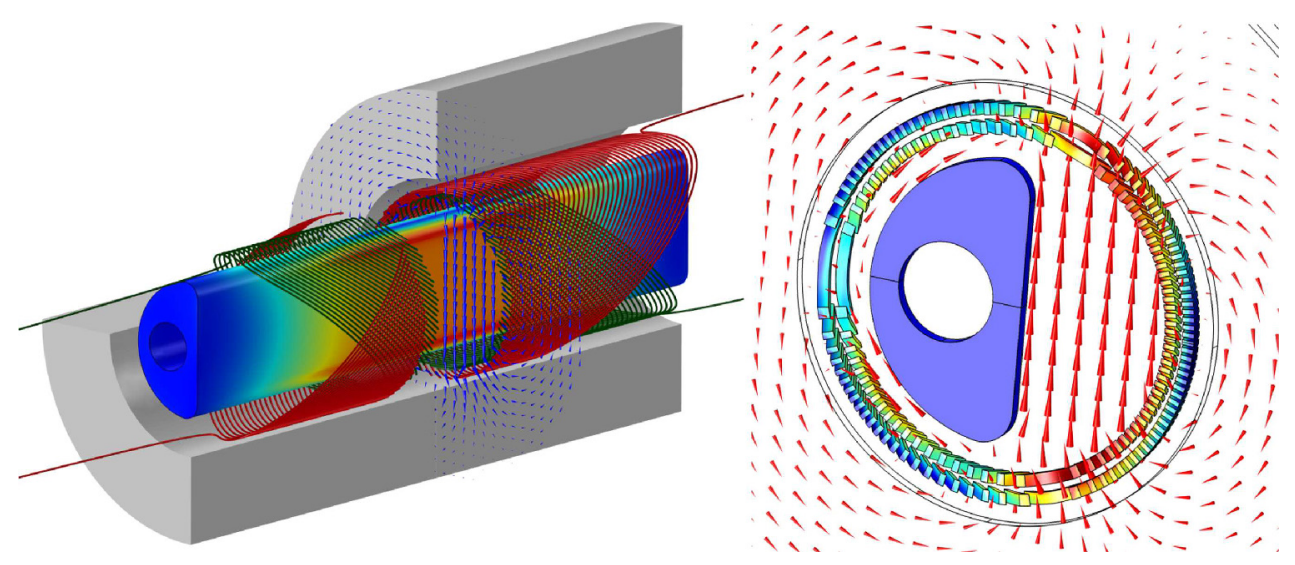

Fig. 3.24. Illustration of the 3D concept of the CCT-SuShi septum magnet.

beam. Proof of one of these concepts would allow alternative reaction strategies to erratics and reduced segmentation.

\section{Extraction kicker magnet}

To reduce beam coupling impedance it is planned to use Ti-coated alumina tubes in the aperture of each magnet. The thickness and resistivity of the coating is a compromise between adequate beam shielding and the required field rise-time: a relatively short field rise-time, of less than $1 \mu \mathrm{s}$, could potentially limit the thickness of the coating to several hundred $\mathrm{nm}$. A novel idea that remains to be studied in detail allows a thicker coating: overshooting the pulse generator current to compensate for the reaction field due to the eddy-currents in the coating.

\section{Extraction septum}

The extraction septa need to produce a very large deflection - the baseline for the dump septa is a two-stage single-plane extraction consisting of $10 \mathrm{~m}$ of superconducting shield septum (SuShi) and $40 \mathrm{~m}$ of truncated cosine-theta septa. The SuShi can shield up to $3 \mathrm{~T}$ with an effective septum thickness of $20 \mathrm{~mm}$, and the truncated cosine-theta can provide up to $4 \mathrm{~T}$ with a septum thickness of $30 \mathrm{~mm} \quad[215,216]$. The main challenges of the SuShi technology are to produce a geometrically complex shield shape and to increase the maximum shield length that can be manufactured. A model and simulation of a SuShi system has been developed, see Figure 3.24, and proof-of-principle devices have been built using $\mathrm{MgO}_{2}$ and HTS tape topologies.

The devices were tested at CERN in the frame of the EUCARD2 H2020 project [217]. A $70 \mathrm{~mm}$ bore LHC MCBY corrector magnet reaching $2.5 \mathrm{~T}$ (corresponding to $5 \mathrm{~T}$ on the shield surface) was used. The prototype flux shield tubes were instrumented with Hall probes inside and outside the shield. The shield was supported inside the magnet with one end only outside the field. The $\mathrm{MgB}_{2}$ shielded up to $2.6 \mathrm{~T}$, measured by the external Hall sensors (around $2.8 \mathrm{~T}$ on the shield's surface, according to simulations), with a small relaxation of shielding currents on the plateaus. The field smoothly penetrated above this level, without a flux jump, but showing a significant creep: a typical scan is shown in Figure 3.25.

The technological difficulties for the truncated cosine-theta septum are the complex coil geometry and mechanical structure, in particular the coil ends. Simulations 


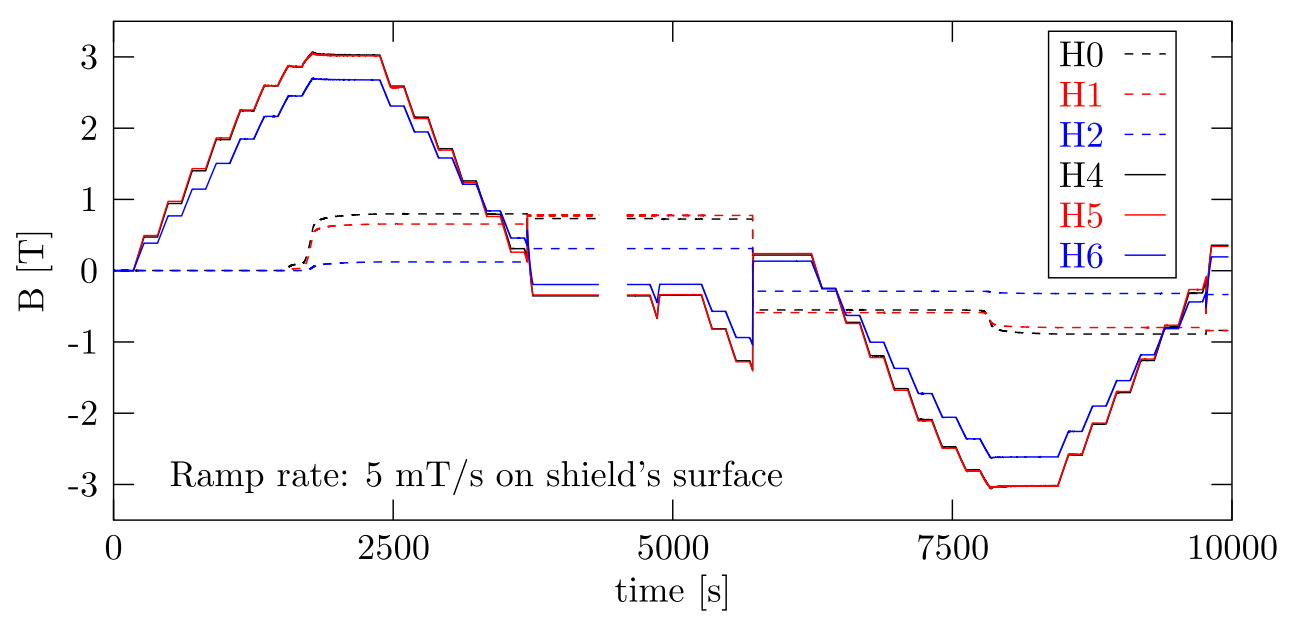

Fig. 3.25. Field measurements inside and outside the $\mathrm{MgB}_{2}$ shield prototype.

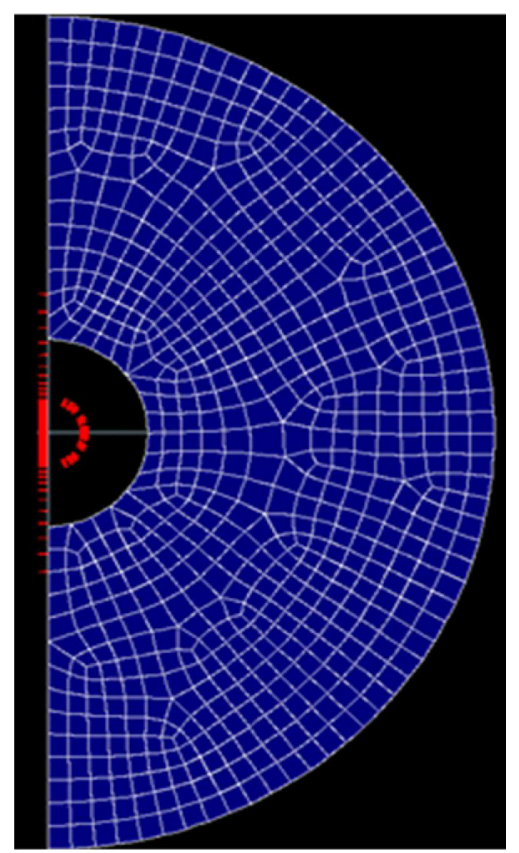

Fig. 3.26. Cross-section of the truncated cosine-theta implemented in the computer software Roxie.

are ongoing for the 3D geometry, see Figure 3.26, and a preliminary conceptual design exists.

The extraction septum system needs an upstream passive protection device. It is difficult to design the object so that it can survive a beam abort. As an alternative, a design for a sacrificial absorber should also be investigated. This may result in significant improvements of the beam optics and reduced layout and performance requirements for the extraction kicker. As is the case for the superconducting injection septa option, the implications for quench protection and machine availability remain to be studied. 


\section{Dilution kickers}

The dilution kicker system is the most technologically challenging part of the beam dump system. The overall magnetic length is $130 \mathrm{~m}$ : approximately $45 \mathrm{~m}$ for the horizontal and $85 \mathrm{~m}$ for the vertical dilution kickers. To give a reasonable inductance $(\sim 2 \mu \mathrm{H})$, individual magnets would be $1.5 \mathrm{~m}$ long. The maximum currents required are $12 \mathrm{kA}$ and $16 \mathrm{kA}$ per horizontal and vertical magnet, respectively. An oscillation frequency of $50 \mathrm{kHz}$ is needed to sweep the beam over the dump block. LHC-like lumped-inductance magnets are suitable. The design of the generators and kicker magnets needs to be studied in the detailed design phase.

\subsubsection{Dumps and absorbers}

The energy deposited by $50 \mathrm{TeV}$ proton bunches in absorber materials poses a challenge for the robustness of protection devices and dumps. Compared to the LHC, the energy density in absorber materials increases due to the higher proton energy and due to the smaller angular opening of particle showers. Depending on the transverse bunch size at the absorber location, a single $50 \mathrm{TeV}$ bunch with $10^{11}$ protons can damage today's most resistant materials such as graphite and carbon-fibre reinforced carbon (CfC) [52]. This imposes a limit on the number of bunches per train which can be transferred from the injectors, creates constraints on the injection and extraction region optics and determines design criteria for extraction and dilution kickers. Absorber materials must combine good mechanical strength with low material density in order to reduce the shower-induced peak load. Beam impact tests [218] are essential to probe material limits and to quantify the dynamic response to extreme thermal shocks. Promising materials for future absorbers are 3D carbon composites and carbon foams. Some of these materials are already being tested in the framework of the LHC injector upgrade and HL-LHC projects [219,220]. An alternative approach could be the use of sacrificial absorbers which need to be exchanged in case of an accidental beam loss.

\section{Energy deposition challenges for protection absorbers}

In order to safely absorb bunches swept across the aperture in the case of an asynchronous beam dump, the bunch size needs to be sufficiently large and bunches need to be sufficiently diluted across the front faces of the absorbers. FLUKA [69,221] shower simulations show that the peak temperature induced by a single bunch in an LHC-like CfC absorber $\left(1.4 \mathrm{~g} / \mathrm{cm}^{3}\right)$ exceeds $1500^{\circ} \mathrm{C}$ if $\sqrt{\beta_{x} \beta_{y}}$ is less than $\sim 300 \mathrm{~m}$ [52]. Detailed thermo-structural calculations are necessary to assess the stresses generated by the thermal shock but experience from LHC shows that tensile stresses can exceed the tensile strength of such absorbers if peak temperatures are as high as $1500^{\circ} \mathrm{C}$. This implies that if LHC-like absorbers are used, minimum $\beta$-functions of several hundreds of meters would be needed at the absorber locations together with fastrising extraction kickers in order to separate swept bunches sufficiently in the case of an asynchronous beam dump. The validation of new or improved materials with a better resistance to high-energy particle beams will to some extent relax these requirements.

\section{Dilution requirements for beam dumping}

The absorber blocks needs to be surrounded by heavy shielding to limit the activation of the machine environment. Sweeping the beam transversely across the absorber 

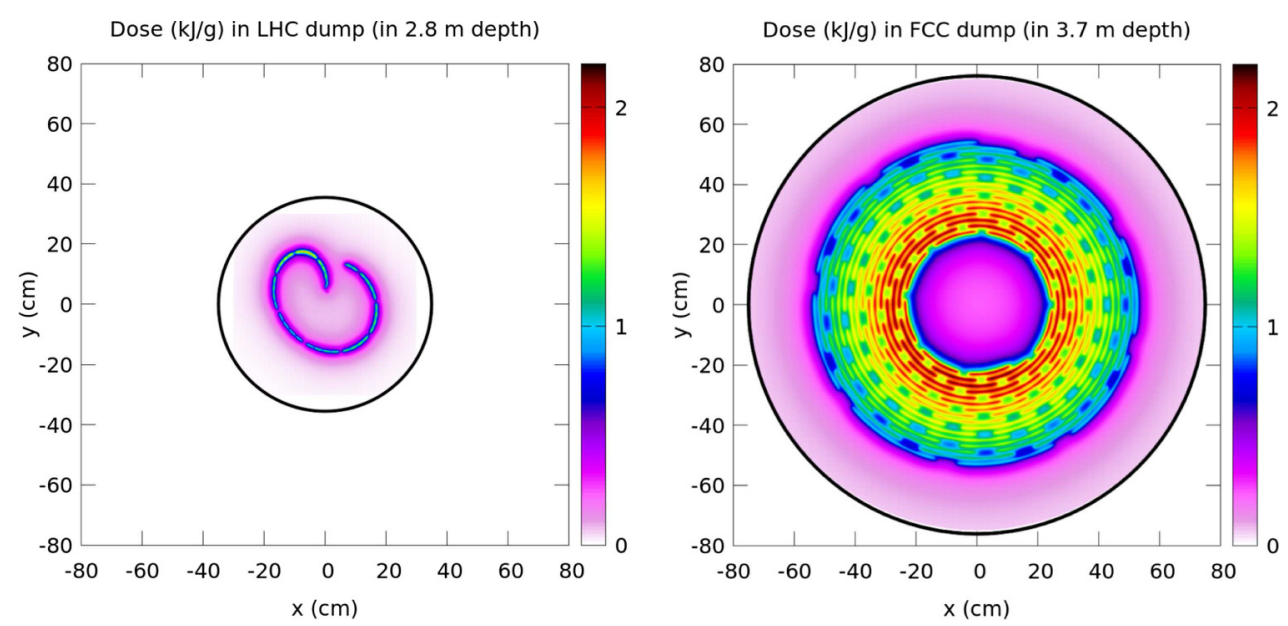

Fig. 3.27. Transverse dose distribution in the LHC dump (2748 bunches, $1.2 \times 10^{11}$ protons per bunch, $7 \mathrm{TeV}$ ) and in a LHC-like FCC dump (10 400 bunches, $1 \times 10^{11}$ protons ber bunch, $50 \mathrm{TeV}$ ). The longitudinal position of the maps corresponds to the depths where the dose is maximum. The dump diameter is $70 \mathrm{~cm}$ in the LHC case and must be at least $150 \mathrm{~cm}$ for the FCC. Results were obtained with the FLUKA code.

front face by dilution kickers avoids excessive temperatures and stresses in the dump core. Graphite has so far been the preferred choice for high-energy hadron dumps at CERN [222,223] and other facilities [224-226]) because of its low atomic number, its high thermal shock resistance and its high melting point. Low-density variants like graphite foams $\left(<0.5 \mathrm{~g} / \mathrm{cm}^{3}\right)$ or innovative solutions like carbon powder-based dumps are promising possibilities, but a first estimate of the dilution requirements and the minimum dump dimensions can be derived by considering a conventional LHC-like graphite core.

An Archimedean spiral is a suitable sweep pattern, which can be generated by orthogonally deflecting kickers from the outside to the inside [227]. It has an optimised footprint on the dump without trace crossing. Large $\beta$-functions of the order of hundreds of $\mathrm{km}$ only moderately relax the dilution requirements since the maximum temperature is determined by the transverse shower overlap of many bunches. For an LHC-like dump made of low-density graphite sheets $\left(\sim 1 \mathrm{~g} / \mathrm{cm}^{3}\right)$ the path length of the sweep must be at least 20 times longer than for the LHC $(1.2 \mathrm{~m})$ in order to keep the maximum temperature around $1500^{\circ} \mathrm{C}$. The sweep path length required can be even longer if the kicker frequency is not modulated.

Figure 3.27 illustrates the energy deposition profile for a fixed kicker frequency of $50 \mathrm{kHz}$ and an outer radius of $55 \mathrm{~cm}$. The diameter of the dump needs to be at least twice as large as that of the LHC dump. With the assumed kicker parameters, the energy density in the dump core remains acceptable in the case of an asynchronous beam dump when the beam is extracted while the dilution kickers are still rising. The same applies for a partial dilution failure with $10 \%$ less dilution in one plane. Since the shower length scales logarithmically with beam energy, the length of the dump increases only moderately compared to the LHC, i.e. the length of a LHC-like graphite dump would be of the order of 10-20 m. Some benefits can be expected from foamor powder-based dumps, but the dilution requirements are still substantial. Dilutionfree dumping systems based on water or gas dumps have been studied [228,229], but these systems lead to other challenges such as dump vessels which are hundreds of metres long. 
Table 3.12. Basic parameters of relevance for operation of FCC-hh beam diagnostic devices.

\begin{tabular}{|c|c|c|c|c|c|}
\hline $\begin{array}{c}\text { Particle } \\
\text { type }\end{array}$ & $\begin{array}{c}\text { Bunch } \\
\text { spacing }(\mathrm{ns})\end{array}$ & Bunch charge (e) & $\begin{array}{c}\text { Number of } \\
\text { bunches }\end{array}$ & $\begin{array}{c}\text { Transverse size }(\mu \mathrm{m}) \\
\beta=200 \mathrm{~m} E=3.3 \mathrm{TeV}\end{array}$ & $\begin{array}{c}\text { Transverse size }(\mu \mathrm{m}) \\
\beta=200 \mathrm{~m} E=50 \mathrm{TeV}\end{array}$ \\
\hline $\mathrm{p}^{+}$ & 25 & $5 \times 10^{8} \rightarrow 1 \times 10^{11}$ & $1 \rightarrow 10600$ & $350[\varepsilon=2.2]$ & $90 \rightarrow 30[\varepsilon=2.2 \rightarrow 0.2]$ \\
& $(5)$ & $\left(5 \times 10^{8} \rightarrow 2 \times 10^{10}\right)$ & $(53000)$ & $(150[\varepsilon=0.44])$ & $(40 \rightarrow 10[\varepsilon=0.44 \rightarrow 0.04])$ \\
\hline
\end{tabular}

\subsection{Beam diagnostics requirements and concepts}

The beam diagnostic requirements are to a large extent based on the experience gained from LHC operation [230]. These requirements (with respect to the relevant parameters in Tab. 3.12) are compared to what is achievable with present state-ofthe-art technologies used for modern beam instrumentation systems. Based on the outcome of this comparison a baseline suite of beam diagnostics is proposed. Any areas, where further research and development is required to fulfil specifications that cannot be met using existing technologies are highlighted.

The operational scenarios considered for beam diagnostics are:

- Initial beam commissioning, including the need for beam threading to establish circulating beams, the first ramp and squeeze cycle and optics measurement and correction; all typically using a low intensity, single pilot bunch.

- Machine protection validation for injection and beam dump systems, collimator set-up and loss-map validation, all using a single, nominal intensity setup bunch.

- Operation with nominal beams.

The LHC notion of a "setup beam" is defined as a beam with an intensity that can never harm machine components as a result of total beam loss. For LHC, the setup beam is defined as $5 \times 10^{11}$ protons at $450 \mathrm{GeV}$ and $\times 10^{10}$ protons at $7 \mathrm{TeV}$. Assuming an injection energy of $3.3 \mathrm{TeV}$ and top energy of $50 \mathrm{TeV}$, with a nominal transverse beam size of $0.35 \mathrm{~mm}(1 \sigma$ at $3.3 \mathrm{TeV})$ and $0.09 \mathrm{~mm}(1 \sigma$ at $50 \mathrm{TeV})$ for a typical $\beta=200 \mathrm{~m}$ in the arc, the FCC setup beam is estimated as $1 \times 10^{10}$ and $5 \times 10^{8}$ protons at injection and top energy, respectively. This sets the lower intensity limit for the operation of all beam diagnostic devices.

\subsubsection{Beam position monitoring}

The requirements for beam position monitoring are similar to those of LHC [231]. It is proposed to equip all quadrupoles with dual plane beam position monitors (BPMs) as this significantly improves the global measurement capabilities of the system and makes it less susceptible to single BPM failures. Although the ratio of FCC-hh to LHC circumference is 3.75, the longer FODO cell in FCC-hh implies that the number of BPMs is doubled rather than quadrupled. This results in a total of $\sim 2000$ BPMs ( 1000 per beam). Due to the significant synchrotron radiation emitted at high energy, the BPM sensors will need to be placed at $45^{\circ}$ (similar to synchrotron radiation facilities), which has a slight impact on availability compared to an orthogonal arrangement as the loss of a single sensor implies the inoperability of both BPM planes. It also reduces the active sensor area to avoid the synchrotron radiation fan, making the BPM less sensitive. This could be an issue for accurate measurement of the pilot bunch, as this requires an order of magnitude improvement over what is currently achieved in the LHC.

An absolute alignment accuracy with respect to the quadrupoles of better than $200 \mu \mathrm{m}$ is needed in the arcs, with tighter tolerances in the insertion regions. The 
system must be capable of providing measurements of the closed orbit with submicron resolution and a fill-to-fill reproducibility of better than $20 \mu \mathrm{m}$. The data will be provided in real-time to allow orbit and radial position feedback. Discussions are ongoing about the tolerances required to ensure alignment of the beam orbit with the synchrotron radiation slits in the beamscreen and the impact that this has on the BPM alignment.

The system will also provide turn-by-turn data for injection oscillations, optics measurements and post mortem analysis. A few special BPMs will provide bunchby-bunch and turn-by-turn, sub-micron resolution data for special purposes such as instability observations or interlock purposes.

A modern, state-of-the-art BPM acquisition system should be able to meet all of these requirements. The sensor and front-end electronics, comprising analogue signal shaping and digitisation circuitry, will be located in the tunnel. A radiation-hard, bidirectional, fibre-optic link [232] connects each station to the processing electronics located out of the radiation environment, on the surface. The challenge will be to design a cost-effective solution, where the front-end electronics can function in a tunnel radiation environment with a high energy hadron fluence 500 times higher than in the LHC and a total dose a 200 times higher. Such BPM systems have already been proven to work in environments with similar total dose requirements, but the impact of the significantly higher fluence will need further study.

One other limitation of the current LHC BPM system is the crosstalk between the BPMs used to measure the two beams in the common beam pipe regions near the interaction points. These are also the BPMs where the precision and reproducibility requirements are the most stringent. One option is to use optical BPMs relying on highly directional Čerenkov diffraction radiation [233], something that becomes feasible at large distances at such high energies. It is estimated that a factor 20 improvement could be achieved using such a technique, but this still needs to be validated through further study.

\subsubsection{Beam loss monitoring}

A lack of data on the quench behaviour of the $16 \mathrm{~T}$ dipoles prevents an in-depth analysis of the beam loss monitor (BLM) requirements. It is therefore assumed that a copy of the LHC BLM system [234] would be adequate for machine protection purposes, with some 6000 ionisation chambers (6 per arc half-cell as for the LHC) distributed throughout the tunnel and read-out via optical fibres using radiationqualified electronics.

The latest magnet design has compact $16 \mathrm{~T}$ dipoles with a reduced iron yoke, which could lead to the stray field reaching $0.2 \mathrm{~T}$ on the outside of the cryostat. The detectors and electronics used for loss monitoring would therefore need to work under such conditions. This should be the case for ionisation chambers, where there can be further magnetic shielding around the detector, if necessary.

\subsubsection{Beam current and intensity measurements}

The requirements for beam current and intensity measurement are to provide the total circulating current and the relative bunch-by-bunch intensities with an accuracy better than $1 \%$. These systems should also provide the overall beam lifetime and bunch-by-bunch lifetimes.

For the total circulating current the main challenge lies in the large dynamic range to be covered, from $0.25 \mu \mathrm{A}$ (single bunch of $5 \times 10^{8}$ charges) to $0.5 \mathrm{~A}$ (10600 
bunches of $1 \times 10^{11}$ charges). From LHC experience an accuracy of better than $1 \%$ is required for medium to high current operation (to allow accurate luminosity calibration), while this is significantly relaxed for lower current operation. Nevertheless, for machine protection purposes an interlock on the setup beam intensity is required, implying a system that has an accuracy and resolution of at least $\sim 20 \%$ of this lower limit $(\sim 0.05 \mu \mathrm{A})$. While direct current current transformers (DCCTs) are suitable for the accurate measurement of medium to high currents, they have an intrinsic noise floor at around the $1 \mu \mathrm{A}$ level, believed to be the result of properties of the magnetic core material such as Barkhausen noise. This makes them unsuitable as machine protection devices. A cryogenic current comparator [235] could therefore be considered for this functionality. At present this is the only device capable of directly measuring beam currents with a resolution below the $50 \mathrm{nA}$ required.

For the bunch-by-bunch measurement, a wall-current transformer coupled to fast digitisation electronics is proposed. A recent upgrade of the LHC system using this technology, combined with numerical integration, has already reached the required performance for $25 \mathrm{~ns}$ beams [236]. The challenge will be to achieve this $1 \%$ bunch-bybunch resolution with the $5 \mathrm{~ns}$ bunch spacing, where a considerable improvement to both the bandwidth of the sensor and digitisation readout electronics will be required.

\subsubsection{Tune, chromaticity and coupling}

The requests for tune, chromaticity and coupling measurements are the same as for LHC. The principal problem to be overcome, already present in the LHC, is the incompatibility of tune measurements with a high-gain active transverse damping system. Study of the possible implementation of a non-linear transfer function for the transverse damper is therefore proposed. This may lead to some selfexcitation of betatron oscillations at low amplitudes that will result in slow emittance growth, but this may be fully compensated by the radiation damping in the FCC-hh.

\subsubsection{Transverse profile measurements}

The target accuracy for normalised emittance $(\varepsilon)$ derivation is $1 \%$. This folds in the errors from the measurement of the beam size $(\sigma)$ and the knowledge of the beta function $(\beta)$ at the measurement location. If it is assumed that both have a similar contribution $\left(\varepsilon=\beta \sigma^{2}\right)$ then this translates into an admissible error of $0.7 \%$ on the beta function and $0.5 \%$ on the beam size. For the beam size, this is certainly a challenge towards the end of the fill, where the average size in the arcs is expected to be in the $10-30 \mu \mathrm{m}$ range depending on the bunch spacing. In addition to this absolute emittance requirement, a bunch-to-bunch emittance with a relative accuracy of $1 \%(0.5 \%$ on the transverse bunch size) is requested. Both the absolute emittance measurement and the bunch-by-bunch measurement must be continuous, i.e. capable of providing on-line results from injection, throughout the ramp and during physics.

Due to the high energy density contained in most operational beams, nonintercepting devices will be required to measure the beam size. A synchrotron light monitor is the instrument of choice for such a high-energy hadron accelerator [237], with plenty of visible light generated from a standard bending dipole at all energies and hard X-rays generated above $40 \mathrm{TeV}$. The variety of sources required to generate synchrotron radiation in the LHC (undulator at injection, edge radiation during the ramp and dipole radiation at top energy) can therefore be avoided, along with the 
difficulties in reliable calibration that this poses. Nevertheless, measuring the small beam size at top energy will be a challenge and requires the synchrotron radiation instrument to be placed in a location with high beta-function and the use of an X-ray pin-hole camera. The heat deposition on the extraction mirror is significantly larger than for the LHC due to the hard X-ray components, but not larger than at existing synchrotron light facilities. Direct cooling of the mirror will be necessary.

Since the visible radiation observed is close to the diffraction limit for a large part of the energy range, cross calibration with other instruments is mandatory to ensure reliable measurements. This is currently provided by intercepting devices (wire scanners) working with a low number of nominal bunches, but this may no longer be feasible at top energy. Alternative, non-intercepting calibration devices should therefore be studied, such as gas-jet scanners [238] or a beam-gas vertex detector like those being developed for HL-LHC [239].

\subsection{Hollow electron lens}

High-energy high-luminosity colliders like the HL-LHC and the FCC-hh aim at peak luminosities in excess of $5 \times 10^{34} \mathrm{~cm}^{-2} \mathrm{~s}^{-1}$, requiring a total proton beam current between $0.4 \mathrm{~A}$ (FCC-hh) and $0.85 \mathrm{~A}$ (HL-LHC) and implying a total beam power of up to $700 \mathrm{MJ}$ (HL-LHC) and $8400 \mathrm{MJ}$ (FCC-hh) per beam in the storage ring. Scaling from LHC Run2 experience to that expected for the HL-LHC and the FCC-hh, one estimates a stored energy of up to $35 \mathrm{MJ}$ (HL-LHC) and $420 \mathrm{MJ}$ (FCC-hh) above $3.5 \sigma[240]$ in the beam halo of these future machines! This represents a significant damage potential for both cases $(\sim 1 \mathrm{MJ}$ can melt $\sim 2 \mathrm{~kg}$ of copper) and calls for mitigation measures in order to protect the machine against fast changes in the central beam orbit or beam size. In such a case, the halo particles could be deposited at a single aperture bottleneck and the changes can occur too fast to be detected by the machine protection system (MPS) to initiate a beam abort before damage can occur. One attractive mitigation measure is to actively deplete the beam and in this way "buy" more time for the MPS to react before significant losses can occur in a single aperture bottleneck.

Several options for halo depletion have been discussed during the design process of the HL-LHC, ranging from active tune modulation as demonstrated in HERA [241] and tested in the LHC [242], selective frequency excitation of the halo particles by the transverse damper system and finally, the depletion of the beam halo through the electromagnetic interaction of the protons with a hollow electron beam. A review of the requirements for active beam halo control held at CERN [240] in 2016 concluded that active halo control is highly desirable for the HL-LHC and recommended the use of a hollow electron lens (HEL) as the best tool. The HEL is the only device under study that acts directly on the beam distribution in the physical space of the transverse plane. The other two options considered only act indirectly on the beam halo particles via convolution with the tune spectrum.

Electron lenses were first developed for applications in the Tevatron storage ring where they have been successfully operated to deplete the beam abort gaps and at the RHIC hadron collider where they are successfully used for compensation of the head-on beam-beam tune spread. Both of these applications relied on the use of "solid" electron beams and were able to benefit from the expertise developed for the construction of electron cooler devices for hadron beams. Beam tests carried out at the Tevatron to demonstrate the feasibility of beam collimation with hollow e-lenses were very positive and provided a convincing demonstration that this technique can be used at the LHC [243]. The application for the HL-LHC and FCC-hh machines requires further development of these devices and the inclusion of operation with 


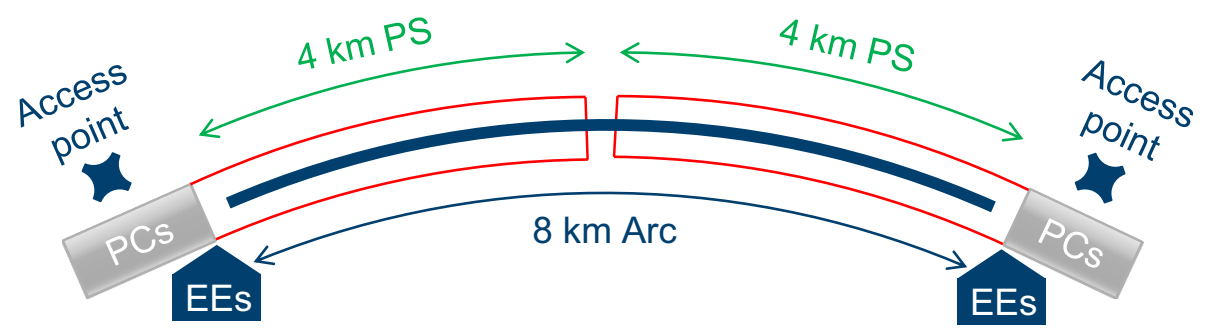

Fig. 3.28. Definition of the powering sector. The locations of power converters (PCOs) and energy extractors (EESs) are also shown.

pulsed electron beams. The operation with DC currents and high non-linearities (chromaticity and Landau octupoles) is also being studied. Operation with hollow electron beams implies new challenges in the form of new electron beam instabilities that do not occur in electron-cooler devices. Also, the operation with pulsed electron beams imposes more challenging requirements for the "field-free" region in the centre of the hollow electron beam in order to minimise detrimental effects to the hadron beam core.

Efficient beam halo cleaning requires significantly large electron beam intensities and sufficiently long interaction regions. For the HL-LHC HEL, the design aims at a $\sim 3 \mathrm{~m}$ long region for the electron beam to interact with the proton beams and an electron beam current of the order of $5 \mathrm{~A}$. These parameter choices are challenging for the cathode which has to generate the electron beam and they bring the risk of creating beam instabilities in the electron beam. Stabilising the electron beam at such high beam currents requires the use of strong solenoid fields. The HL-LHC HEL design aims to have a peak solenoid field of $5 \mathrm{~T}$ in the central interaction region, requiring the use of superconducting magnets. Non-invasive beam diagnostics capable of measuring both electron and proton beam position and profile within such a solenoid field need to be developed in order to guarantee precise centring and detect possible deformation of the electron beam.

\subsection{Magnet powering requirements and concepts}

\subsubsection{Circuit powering}

The stored energy at nominal current in a cos-theta dipole magnet (37 MJ) is 5 times higher than in an LHC main dipole and the arc between two access points is almost 3 times longer. In order to avoid excessive voltages to ground developing during fast power aborts, there must be multiple circuits per arc and/or energy extractors distributed along the tunnel. Each circuit would be equipped with a power converter (PCO) and an energy extraction system (EES) located close to the access points. This approach helps optimising space in the tunnel and makes the maintenance of PCOs and EESs easier. Superconducting links in the tunnel, already under study for the HL-LHC project [244], would greatly simplify the powering of multiple circuits.

As shown in Figure 3.28, a powering sector (PSE) is defined as half of the arc. Including the $3.2 \mathrm{~km}$ long short arcs, the number of PS for the entire accelerator is $N_{\mathrm{PSE}}=20$. As shown in Figure 3.29 , each PS is subdivided into $N_{\text {cir } / \text { PS }}=5$ independent circuits so that the total number of dipole circuits is $N_{\text {cir }}=N_{\mathrm{PS}}$. $N_{\text {cir/PSE }}=100$. Since one PCO and one EES is present for each circuit, $N_{\text {cir }}$ also identifies the total number of these devices. The main parameters of the proposed dipole circuit layout are given in Table 3.13 . 


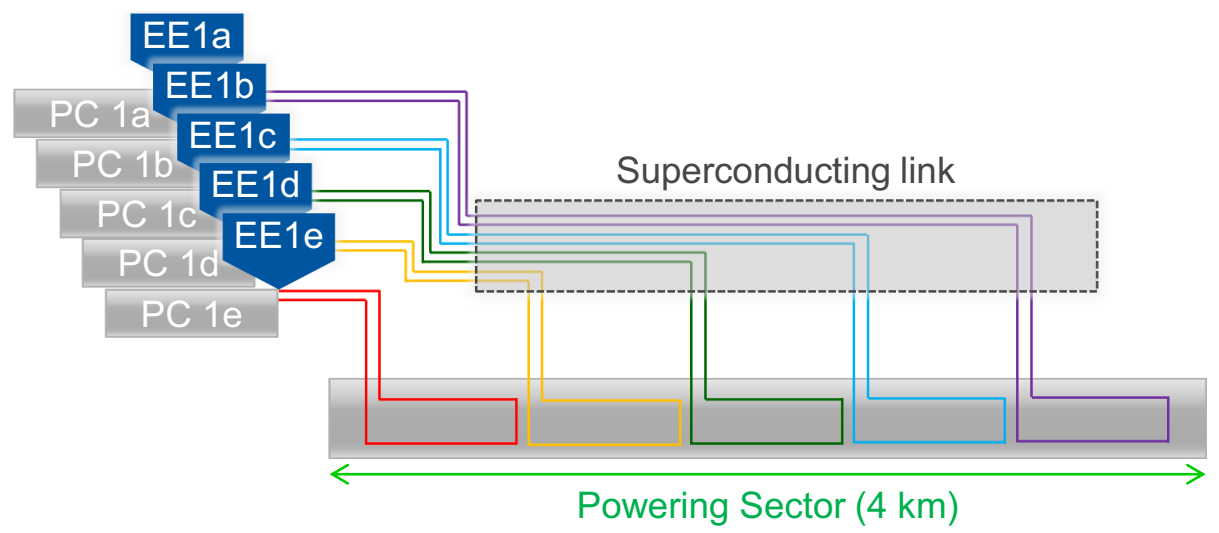

Fig. 3.29. Schematic of the dipole circuit architecture.

Table 3.13. FCC-hh and LHC dipole circuit parameters.

\begin{tabular}{|cccccc|}
\hline & $\begin{array}{c}\text { Number of } \\
\text { circuits } N_{\text {cir }}\end{array}$ & $\begin{array}{c}\text { Nominal } \\
\text { current }\end{array}$ & $\begin{array}{c}\text { Magnets in } \\
\text { series }\end{array}$ & Energy $^{\dagger}$ & $\begin{array}{c}\text { Apparent } \\
\text { inductance }\end{array}$ \\
\hline FCC & 100 & $11.2 \mathrm{kA}$ & 44 & $1.6 \mathrm{GJ}$ & $26 \mathrm{H}$ \\
LHC & 8 & $11.9 \mathrm{kA}$ & 154 & $1.1 \mathrm{GJ}$ & $15 \mathrm{H}$ \\
\hline
\end{tabular}

Notes. ${ }^{\dagger}$ Energy per circuit at nominal current.

Table 3.14. FCC and LHC dipole circuit parameters.

\begin{tabular}{|cccc|}
\hline & Ramp-up time & Max PCO voltage & Max PCO net power \\
\hline FCC-hh & $20 \mathrm{~min}$ & $240 \mathrm{~V}$ & $2.7 \mathrm{MW}$ \\
LHC & $20 \mathrm{~min}$ & $150 \mathrm{~V}$ & $1.8 \mathrm{MW}$ \\
\hline
\end{tabular}

Table 3.15. Fast power abort of a dipole circuit compared to the LHC.

\begin{tabular}{|lcccc|}
\hline & $\begin{array}{c}\text { Half of } \\
\text { EES voltage }\end{array}$ & $\begin{array}{c}\text { Discharge } \\
\text { time constant }\end{array}$ & MIITs & $\begin{array}{c}\text { Busbar copper } \\
\text { cross-section }\end{array}$ \\
\hline FCC-hh & $1.3 \mathrm{kV}$ & $110 \mathrm{~s}$ & $7 \times 10^{3} \mathrm{MA}^{2} \mathrm{~s}$ & $270 \mathrm{~mm}^{2}$ \\
LHC & $0.45 \mathrm{kV}$ & $100 \mathrm{~s}$ & $7 \times 10^{3} \mathrm{MA}^{2} \mathrm{~s}$ & $270 \mathrm{~mm}^{2}$ \\
\hline
\end{tabular}

In order to keep the turnaround time of the accelerator low, the goal is to keep the same ramp-up time as the LHC (see Tab. 3.14). Another relevant circuit parameter is the maximum voltage to ground, which is the sum of half of the EES voltage (provided that the EES is grounded in the middle point) and the internal magnet voltage developed during quench. Table 3.15 shows that, if half of the EE voltage is $1.3 \mathrm{kV}$ (EuroCirCol specification as in Section 3.2.4), the time constant for the fast power abort is $110 \mathrm{~s}$, slightly higher than the LHC one. A fast discharge limits the number of neighbouring quenching magnets, thereby reducing the recovery time determined by the cryogenic system. The higher time constant and lower nominal current imply that the integral of $\mathrm{I}^{2}$ during discharge (commonly referred to as MIITs) and that the copper cross section of the busbars can be maintained at levels similar to those in the LHC.

The main drawback of the proposed circuit layout is the high number of PCO and EES devices and the many supply circuits (superconducting cables). This could 
lead to a reduction of the overall circuit availability. This aspect has been studied and the results are presented in Section 2.5

The circuit architecture presented above is based on the parameters of the baseline magnet design, i.e. the cos- $\vartheta$ option. The block-coil design has a similar nominal current and energy and, therefore, leads to the same circuit layout. Common coil and canted $\cos -\vartheta$ options have higher stored energies, which is a disadvantage for the circuit protection. On the other hand, they have the advantage of lower inductances. Applying the same circuit layout to these magnets would lead to a larger MIITs (almost twice) and to a faster discharge (about 20\% lower time constant).

The powering of quadrupole magnets and correctors is considered less critical and will follow the same strategy as adopted for the dipole circuits.

\subsubsection{Quench detection technologies}

The core strategy for the quench detection system is based on the concept of centralised data processing and quench detection as presented in Figure 3.30. This approach simplifies the instrumentation units located in the tunnel (QS), thus reducing their susceptibility to ionising radiation. Due to the low latency, high sensitivity and reconfigurability requirements, a distributed system of intelligent sensors is needed. A high level of standardisation of the equipment located in the tunnel will facilitate repair and maintenance. Ideally the system is highly fault tolerant and can be maintained with robots. Therefore, mechanical form factors, interfaces and a suitable integration of the mechanical and electronic systems must be considered from a very early stage onwards. The central quench detection data processing units (QPU) will be located outside of the tunnel and must therefore be connected to the individual sensor units with low-latency real-time links. The system must be open to accommodate an ever growing number and new types of quench instrumentation technologies to improve reliability and accuracy of the decision process. Correlation across multiple channels of the superconducting circuits will be one factor towards this goal via a software-defined approach.

Machine learning approaches could complement the scheme, in order to provide an increasingly accurate assessment of the operational state of the system. Eventually, the entire decision process could be centralised, including the information from the distributed instrumentation units. Such a global protection approach relies on a high performance real-time communication network in the tunnel. Another benefit of this approach is the comprehensive acquisition and logging of all information that led to the quench protection action. This will lead to an ever improving, autonomous analysis of faults and their causes in the machine. This in turn helps implementing a continuous machine availability improving process.

\subsubsection{Energy extraction system technologies}

Two methods are proposed for the extraction of energy from the main dipole circuits: (i) discharging the energy into passive resistors as done at LHC, and (ii) active energy extraction and recuperation. In the first scenario, a classical electromechanical circuit breaker or a switch based on a quenching superconductor will be connected in series to the magnet chain, dissipating the stored energy in a dump resistor. For the second method a converter module, maintaining a constant voltage across the magnet chain during the extraction process will transfer the energy to a temporary buffer unit. The stored energy can later be used e.g. for the subsequent energy ramp.

The first scenario has a lengthy energy extraction, limited by the maximum extraction voltage allowed and an important cost to be able to cope with the 


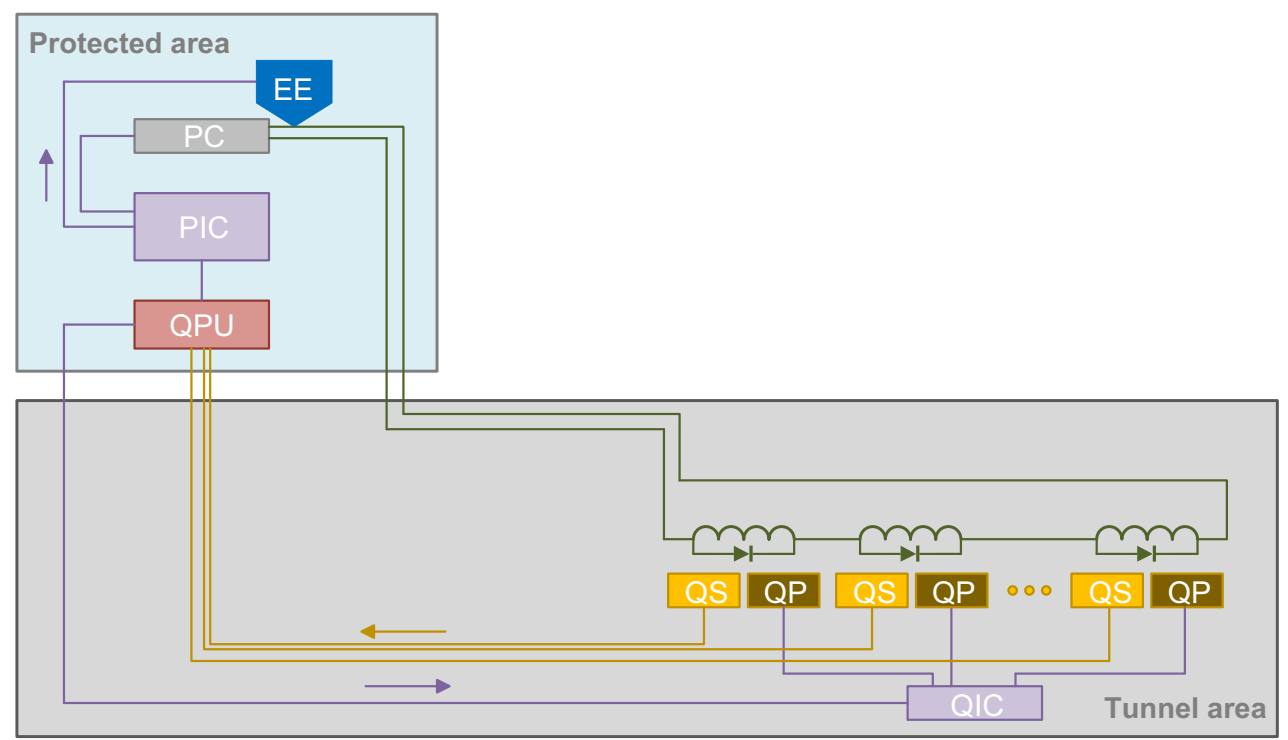

Fig. 3.30. Overview of a potential quench detection architecture for an individual dipole circuit in an arc. The tunnel area houses a distributed system of quench sensors (QS) and magnet quench protection devices (QP). The QS signals are concentrated in a quench processing unit (QPU) that is located in a protected area. It communicates in turn to a powering interlock controller (PIC) that serves as control input to the magnet power converter (PC). It also controls an energy extraction system (EE). The QPU drives also quench protection devices (QP), which are located in the tunnel through a quench interlock controller (QIC). This controller could also be located in a protected area, depending on the available technology.

heat induced by the lost energy. This second scenario addresses the two disadvantages effectively. By performing the extraction at constant maximum voltage, the extraction time can be considerably reduced compared to a resistor-based system. The energy recovery leads to reduced operational losses and therefore higher operational efficiency and lower infrastructure requirements. In addition, it will save energy. Although the powering architecture mentioned earlier will not be altered, the reduced extraction time could permit fewer independent circuits: their number can be reduced by $33 \%$ while keeping the same circuit MIITs. The latter solution will require an additional power converter as well as an intermediate energy storage unit, coupled with the proposed DC-grid powering (see Sect. 3.8.5) capable of handling the large energies. To ensure safe extraction of the energy at all times, the system reliability must remain high in spite of the added complexity.

\subsubsection{Power converters for magnet powering}

The principal challenge for the powering of the magnets is the high peak power during ramp-up and the energy return during ramp-down. The system can be optimised by eliminating or reducing these large power swings and, at the same time, minimising the energy consumption. 


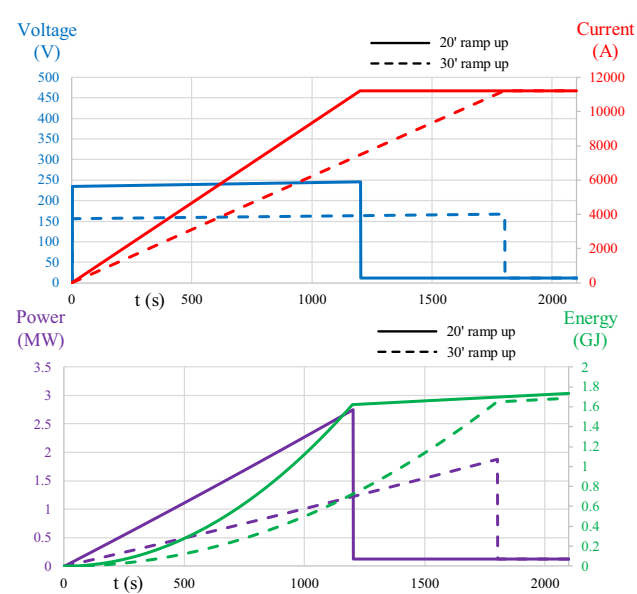

(a) constant-voltage ramp up

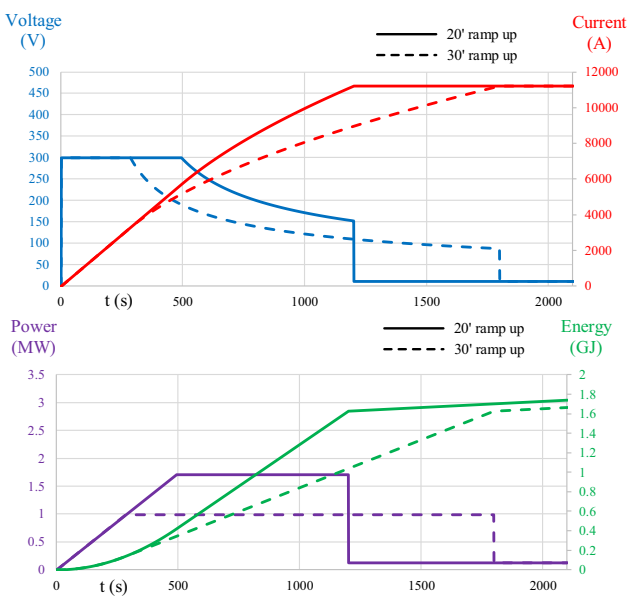

(b) constant-power ramp up

Fig. 3.31. Voltage applied to the dipole circuit, current in the dipole circuit, power requirement and energy supplied to the magnets for: (a) constant-voltage ramp; (b) constant-power ramp.

\section{Power requirements for the main dipole converters}

With five circuits per powering sector, there will be 44 magnets per circuit, resulting in a stored energy per circuit of about 1.6 GJ. The rated current for the dipole magnets is $11.39 \mathrm{kA}$ and the estimated equivalent resistance for each magnet circuit is $1 \mathrm{~m} \Omega$. There are two options for the ramp-up strategy.

- Constant voltage: a fixed output voltage is applied to the magnets and the current ramps up with a constant slope (di/dt). The main drawback of this option is that the power requirement during the end of the ramp-up is very high. This can be seen from the solid curves in Figure 3.31a.

- Constant power: the output voltage applied to the magnets is controlled in order to limit the maximum power during ramp-up. A higher voltage is applied in the first part of the ramp which is reduced as the current increases (solid curves in Figure 3.31b).

The duration of the ramp has an important influence on the power converter requirements: the shorter the ramp, the higher the power requirements. The difference in power ratings of the converters for a $20^{\prime}$ ramp-up and a $30^{\prime}$ ramp-up is shown in Figure 3.31 where the solid lines are for a $20^{\prime}$ ramp and the dashed lines for a $30^{\prime}$ ramp. The main parameters corresponding to these ramps are given in Table 3.16.

The total power requirements for all dipole circuits will be 100 times those given in Table 3.16; therefore, reducing the power requirements of each power converter is extremely important. In conclusion, to limit the power consumption from the AC network as much as possible, the ramp-up should be as long as possible and the most appropriate strategy should be selected for the converter topology.

\section{Topology and ratings of main dipole converters}

The LHC uses twelve-pulse thyristor rectifier technology (13 kA @ 190 V) in the dipole magnet power converters. For the FCC, it is proposed to use switch-mode converters which will provide several advantages over thyristor converters: 
Table 3.16. Maximum voltage, di/dt and active power requirement for the two different ramp-up strategies and the two different ramp-up durations.

\begin{tabular}{|l|l|l|l|l|}
\hline Ramp strategy & $\begin{array}{l}\text { Ramp- } \\
\text { up time }\end{array}$ & Max. Voltage & Max. di/dt & Max. Power req. \\
\hline Constant voltage & $20^{\prime}$ & $245 \mathrm{~V}$ & $9.50 \mathrm{~A} / \mathrm{s}$ & $2.75 \mathrm{MW}$ \\
\hline Constant voltage & $30^{\prime}$ & $167 \mathrm{~V}$ & $6.30 \mathrm{~A} / \mathrm{s}$ & $1.87 \mathrm{MW}$ \\
\hline Constant power & $20^{\prime}$ & $300 \mathrm{~V}$ & $11.55 \mathrm{~A} / \mathrm{s}$ & $1.71 \mathrm{MW}$ \\
\hline Constant power & $30^{\prime}$ & $300 \mathrm{~V}$ & $11.55 \mathrm{~A} / \mathrm{s}$ & $0.98 \mathrm{MW}$ \\
\hline
\end{tabular}

Notes. The power is given per circuit.

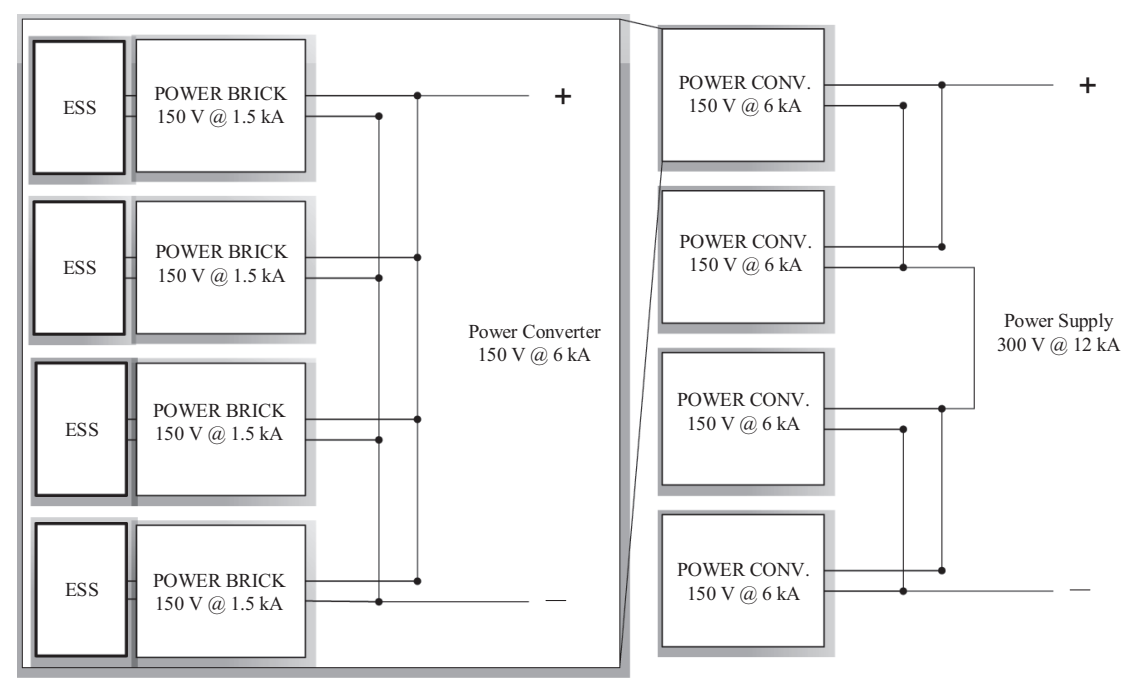

Fig. 3.32. Simplified diagram of a dipole power converter circuit.

- Fewer harmonics will be injected into the AC network;

- There is no need for reactive power compensation;

- DC storage can be incorporated, which offers the possibility of storing part or all of the peak energy for the dipole magnets and to reuse it during the ramp-up;

- It is possible to downsize the AC connection if energy storage is integrated since the AC network will only have to compensate the system losses.

The power supply for the dipole circuits will be rated for $12 \mathrm{kA} @ 300 \mathrm{~V}$. Each power supply will be composed of four $6 \mathrm{kA} @ 150 \mathrm{~V}$ converters, connected two in parallel in series with another two in parallel (2P2S) as shown in Figure 3.32. Each converter will be composed of 4 bricks of $1.5 \mathrm{kA} @ 150 \mathrm{~V}$ in parallel. The modularity of this converter topology provides excellent availability $(N+1)$ and easy repair and maintenance capabilities.

\subsubsection{Energy storage in power converters}

In order to minimise the power consumption from the $\mathrm{AC}$ network, it is essential to integrate energy storage systems (ESS) in the power converters. At CERN, the use of energy storage in power converters has been implemented in devices and is becoming the general practice for all new projects CERN-wide e.g. POPS, POPS-B 
or the new SIRIUS class converter series. Today, these converters use capacitors for energy storage.

For HL-LHC and FCC-hh, the cycles are much slower than in the injectors however they require much more energy due to superconducting magnets. In this case, capacitors cannot be used as energy storage medium due to their volume. Alternative energy storage technologies are under consideration, of which only two technologies were identified as most suitable:

- Super-capacitors: this type of capacitor is characterised by a much greater energy density than electrolytic capacitors. Within this family, electrical double-layer capacitors (EDLCs) are the most interesting. However, for large-scale energy storage this technology is still very expensive: they are at least ten times more expensive than equivalent lithium-ion batteries.

- Batteries: due to their superior energy density, battery storage seems to be the best technology. Significant developments in this area, driven by the automotive sector, are expected in the coming years.

Battery systems have been analysed in detail for magnet powering applications. The most suitable battery technology is based on lithium batteries and in particular the lithium titanium oxide (LTO) type. The main issue with lithium batteries is their limited lifetime, which is typically a maximum of 3000 charge-discharge cycles. For LTO batteries, the lifetime ranges from 5000 up to 20000 cycles, which would be suitable for HL-LHC, as well as for the FCC. For the new inner triplet magnets of HL-LHC, the ESS needs to be able to recover $55 \mathrm{MJ}$ from the magnets, which requires only one $19^{\prime}$ rack of LTO batteries. This solution seems very promising, since the energy storage system is very compact and cheap. For FCC, the energy to manage is two orders of magnitude higher. The dipole magnets require an energy of $1.6 \mathrm{GJ}$, which means an effective energy of $445 \mathrm{kWh}$ which corresponds to about $550 \mathrm{kWh}$ in the energy storage system. Two options are proposed for the ESS:

- One common storage system for each power sector: this system of $2750 \mathrm{kWh}$ $(5 \times 550 \mathrm{kWh} /$ circuit $)$ would be placed on the surface and linked to the converters in the tunnel by a common DC cable passing down the shaft. This configuration implies high transmission losses and the need for a DC breaker with high switching capability;

- Distributed amongst all power bricks: the energy storage systems would be installed in the tunnel, in separate racks or even integrated in the converter racks. This option requires more space in the tunnel (approximately 25-30\% more than the space requirements for the converters alone), however, it provides the best performance and reliability.

Using current LTO batteries, the energy for each circuit ( $550 \mathrm{kWh}$ ) could be easily installed in the equivalent volume of ten $19^{\prime \prime}$ racks, weighing a total of $6.7 \mathrm{t}$. Nevertheless, research on energy storage is currently intense and significant improvements in energy density, size and weight as probably cost are expected in the near future.

\subsection{Machine protection management concepts}

The energy stored in the magnets and beams are 20 times higher than in the LHC, while the quench limit of the superconducting magnets is 15 times lower. In order to conceive an approach for the management of the protection of the entire collider, it is crucial to assess the consequences of the beam on the different machine components for controlled and uncontrolled beam losses. The energy deposition of protons in solid copper and graphite targets - two materials widely used in magnets, beamscreens, 
Table 3.17. Number of protons needed to melt copper at the maximum energy deposition point for different energies and beam sizes.

\begin{tabular}{|lcc|}
\hline Energy $(\mathrm{GeV})$ & RMS beam size $(\mathrm{mm})$ & $N p$ \\
\hline 450 & 0.1 & $6.4 \times 10^{11}$ \\
& 0.8 & $3.1 \times 10^{12}$ \\
3300 & 0.1 & $4.6 \times 10^{10}$ \\
& 0.4 & $1.5 \times 10^{11}$ \\
7000 & 0.1 & $1.8 \times 10^{10}$ \\
& 0.4 & $6.2 \times 10^{10}$ \\
50000 & 0.1 & $1.9 \times 10^{9}$ \\
& 0.4 & $7.5 \times 10^{9}$ \\
\hline
\end{tabular}

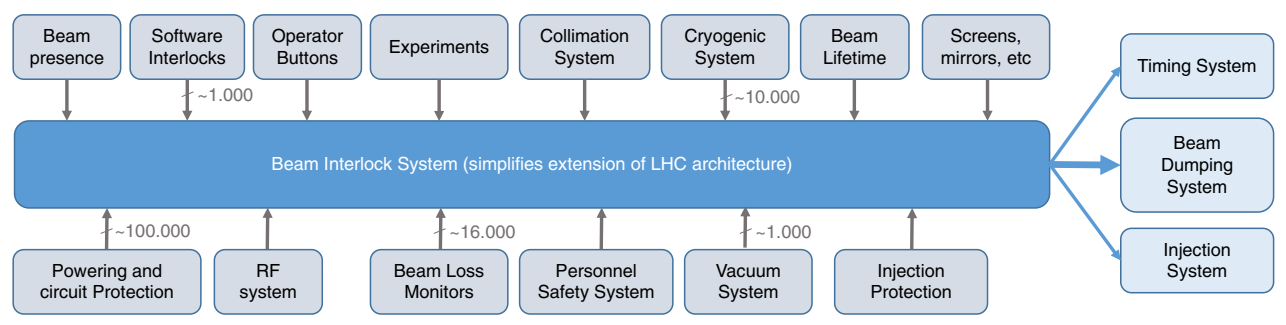

Fig. 3.33. Machine protection system architecture and client systems.

collimators and beam absorbers - has been studied [245]. The simulation at nominal injection and extraction energies estimate the damage limits for different beam sizes. Results are presented in Table 3.17. Based on these results, a safe beam intensity, a vital concept for the initial commissioning and setup of the machine at $50 \mathrm{TeV}$, has been defined as $5.0 \times 10^{8}$ protons, maintaining a reasonable safety margin with respect to the estimated damage limit. This number is also important for the definition of the dynamic range of beam instrumentation which will interact with the machine protection systems.

Beam permit loops will transmit the beam dump requests from a variety of critical equipment to the beam extraction system. The number of input channels potentially triggering a beam dump is estimated to exceed 100000 . A symbolic presentation of this beam interlock system with its connections is shown in Figure 3.33. Three important requirements for the beam interlock system are reliability, availability and latency. The system must be reliable due to the large energy stored in the beam and the damage it can cause. Redundancy, starting with the design of the user system requesting the beam dump up to the design of the beam extraction system, needs to be included in all parts of the system. Continuous self-testing will be an important feature required to meet the protection requirements. At the same time, the system needs to be highly available to make sure that the entire accelerator complex can be used efficiently. A balance between availability and reliability can be achieved by a voting logic on redundant interlock channels. A voting logic of at least three redundant connections will also help to implement a continuous testing of the system.

The latency requirement mainly concerns the permitted period of time between the occurrence of an event requiring a beam dump and the time by when the beam has been completely extracted. This duration is composed of the following elements:

- The time needed to detect the situation calling for a beam abort. Beam loss monitors are the most important triggers of a beam dump. Today their reaction 
time is more than $80 \mu \mathrm{s}$, but these times could be shortened significantly to around $1 \mu \mathrm{s}$ by using faster detectors like the so called "diamond BLMs".

- The time to transmit the signal to the decision logic and to take the decision to extract the beam. It is estimated to be around $300 \mu \mathrm{s}$, determined by the signal propagation in optical fibres. Some time can be gained by making one or several "direct" connections across the ring or by maintaining a short distance between the collimation system and the beam extraction system since losses will most frequently be seen first at this aperture limitation.

- The synchronisation between the extraction kickers and the particle-free abort gap. This delay can amount to $330 \mu$ s if there is one abort gap as in the LHC, but this can be shortened by having multiple abort gaps (an option also studied for absorber survival in the case of erratic kicker triggering, see Sect. 2.3.6).

- The time to extract the beam. This time is also equal to the time it takes to travel once around the circumference, around $330 \mu \mathrm{s}$, and can only be shortened by having multiple beam dumping systems, a hypothesis that is not part of the baseline concept.

The acceptable delay between the beam dump request and the extraction of the last particles is determined by the speed at which the core of the beam can be moved by the fault event and the beam density of the beam halo. Reducing the beam halo population, e.g. by using an electron lens, can increase the acceptable delay between the occurrence of the failure and the moment of beam dump. On the other hand, the presence of a beam halo allows early detection of beam movements or instabilities by beam loss monitors at the collimators. This could be guaranteed by relying on a few witness bunches, which have a larger halo population than the bunches which have been cleaned. Quenches induced by an unidentified falling object (UFO) can be avoided more effectively if beam losses are detected in the area between the beam and the superconducting coils (compared to LHC's beam loss monitors which are outside the cryostat). Options to be studied are fast diamond detectors behind the beamscreens distributed over the superconducting magnets, a continuous optical fibre close to the beam aperture or a superconducting cable with a very low quench threshold located in the magnet cryostat, close to the beam aperture. In the last case, the beam would be dumped if there was a quench of this superconducting wire which would happen before the quench threshold of the magnets would be reached.

\subsection{Controls requirements and concepts}

With the ever larger particle accelerator facilities that have been constructed over the last two decades [246-249] and the entry of versatile particle accelerators in industry and healthcare, the development of control systems for particle accelerators has become a well understood task. The work of companies worldwide towards an Industry 4.0 architecture [250] extends the traditional architecture with enterprise resource planning and intelligent device tiers in the hierarchy and includes life cycle and functional layers (see Fig. 3.34).

With a steady increase of computing and communication technology capacities, a move to wireless communication including safety-related data exchange and ever more flexible embedded computing systems and re-programmable hardware, functionality is shifting from the upper tiers of the system architecture to the lower ones. Front-end systems that are cooperating more autonomously can overcome the constraints that result from centralised control over long distances. Scalable synchronisation and the reliable coordination of actions on a timescale of nanoseconds to support acquired 


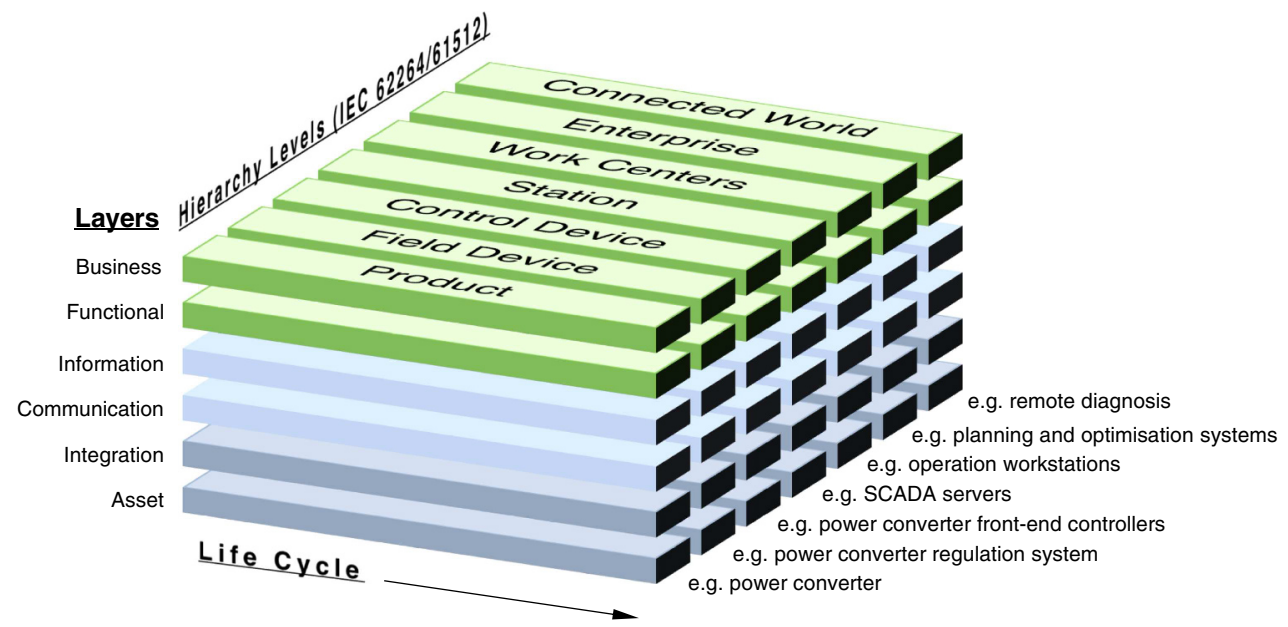

Fig. 3.34. The "Industry 4.0 reference architecture" can serve as a model for an integrated particle accelerator and experiment control ecosystem. For each system tier (connected world, enterprise, work centers, station, control device) that is needed to integrate the accelerator and detector equipment (field device and product tiers), services, interfaces and integration guidelines are currently being conceived by industry players at the different functional levels (business, functional, information, communication, integration and asset). Together with a lifecycle management framework that spans from concept over requirements, design, implementation/procurement, transition to operation, operation, maintenance/repair and retirement, this model represents an ideal reference for establishing a control and data acquisition architecture for the entire project, limiting project-internal developments to those parts, which cannot be covered by existing products, services, standards and guidelines.

Table 3.18. Number of front-end control units in different systems.

\begin{tabular}{|lcc|}
\hline Category & System & $\begin{array}{c}\text { Number of front-end } \\
\text { control units }\end{array}$ \\
\hline Particle accelerator & CERN PS Booster & 81 \\
Particle accelerator & CERN PS & 115 \\
Particle accelerator & XFEL & ca. 200 \\
Particle accelerator & CERN SPS & 257 \\
Particle accelerator & CERN LHC & 525 \\
Aerospace & International Space Station & 660 \\
Particle accelerator & Future Circular Collider & ca. 1500 \\
Fusion research & ITER & ca. 5000 expected \\
\hline
\end{tabular}

data correlation will soon be widely available [251-255]. Femtosecond timing distributions over kilometre distances, as are currently used in free electron lasers [256,257], demonstrate that the performance of timing systems are up to FCC collider (hh, ee, and $\mathrm{HE}$ ) demands today. An ever better understanding of how to adapt off-the-shelf designs to radiation environments is leading to more affordable intelligent controls in equipment that has to be close to the accelerator [258]. Industry is asking for greater use of COTS in mission-critical applications [259-263] and the factory of the future [264] has comparable requirements and these facts will drive the development of commercially available technologies within a few years [265].

Although the system sizes are gradually increasing (see Tab. 3.18), the main challenges for a control system infrastructure are therefore gradually shifting from tech- 
nical to organisational domains. On the time scale of a future collider project, the key persons who conceived CERN's accelerator complex operation and control concepts may no longer be available. This potential loss of expertise needs to be mitigated by establishing a systems engineering process [266,267] with adequate documentation support to capture operation requirements, architecture and design components as well as end-to-end test scenarios, well before a new facility is constructed.

Developers of industrial embedded systems have understood that traditional programming is a time consuming, costly and error prone activity [268]. Particle accelerators are no exception and therefore new development paradigms are needed to come to a sustainable long-term operation concept, reducing loss of knowledge, easing the management of software and its evolution and facilitating working with external partners in a cost effective way. Medical particle accelerator facilities have been successfully applying this approach for several years [269-274]. The need for continuous operation of CERN's particle accelerator complex calls for a gradual modernisation that has to be mastered from a managerial perspective. Such a scheme indicates a gradual evolution towards a new generation of accelerator control systems. It allows suitable concepts to be assessed early using the existing accelerators as test beds for new concepts.

Although fault-tolerant designs can help to meet the reliability requirements of these geographically extended machines, automated and remote maintenance/repair will play a role in ensuring the overall availability of a particle accelerator. As embedded systems become more powerful and flexible they also become more vulnerable to intentional and unintentional misuse. Cybersecurity became an important activity during the development of the control systems for the LHC [275]. For a future facility, this topic needs to be included from the beginning in the systems engineering process in close cooperation with the planning for the IT infrastructure. A well coordinated, complex-wide information and communication technology management environment covering requirements, planning, procurement, maintenance and upgrades will help accelerator groups to focus on the provision of the core control functionality. This approach will ease the transition towards the concept of "Controls as a Service" which can evolve and scale with the underlying technology platforms, focusing on technology independence as much as is reasonably possible.

As is the case in other domains [276], support for closed-loop settings optimisation and including an accelerator physics point of view towards controls will help to improve the efficiency of the accelerator complex [277]. This will become possible with the higher computing power and data exchange capacities, more flexible analysis using big-data approaches, the introduction of machine learning, model driven approaches and an end-to-end cost/benefit sensitivity analysis.

\subsection{Radiation environment}

\subsubsection{Introduction}

Radiation levels in the collider scale with energy and, as LHC has shown, degradation of components exposed to radiation can become a show stopper. Two complementary approaches are needed: the reduction of the dose to equipment by shielding and developing fault tolerant or radiation resistant electronics and equipment. A structured approach for radiation hardness assurance (RHA) will ensure that the electronics and materials developed perform to their design specifications after exposure to the radiation in the collider environment. 


\subsubsection{Reference radiation levels}

Radiation to electronics (R2E) is an issue in the design of any high energy and high intensity machine [278]. Radiation effects in electronic devices can be divided into two main categories: cumulative effects and stochastic effects (Single Event Effects SEE). Cumulative effects are proportional to the total ionising dose (TID) - the damage induced by ionising radiation, and the $1 \mathrm{MeV}$ neutron-equivalent fluence which concerns displacement damage. On the other hand, SEE, which are proportional to the high energy hadron fluence ( $\mathrm{HEH}$, i.e. hadrons with energies $>20 \mathrm{MeV}$ ), are due to the direct or indirect ionisation by a single particle which is able to deposit sufficient energy to disturb the operation of the device. SEE can only be defined by their probability to occur and the effect strongly depends on the device, the intensity and the kind of radiation field.

An accurate specification of the radiation levels in areas critical for electronics is essential for the design of the accelerator. Monte Carlo (MC) simulation is an indispensable tool to evaluate the impact of radiation on the machine equipment, but it relies on both a refined implementation of physics models of the particle interaction with matter and an accurate 3D-description of the region of interest. In this context, FLUKA [69,70] which is widely employed at CERN, is a well benchmarked, multi-purpose and fully integrated particle physics $\mathrm{MC}$ code for calculations of particle transport and interactions with matter. For a high intensity and high energy machine like the FCC, typical sources of radiation are luminosity debris, direct losses on collimators and dumps and beam interactions with the residual gas in the vacuum [279].

A FLUKA model of the collider arc, comprising 12 main dipoles and 2 quadrupoles, has been created. The source term considered in this study was the beam-gas interactions, for a residual gas-density averaging $1.0 \times 10^{15} \mathrm{H}_{2} / \mathrm{m}^{3}$ (corresponding to beam lifetime of $100 \mathrm{~h}$ ). The $\mathrm{HEH}$ and dose distribution in the arc and in a lateral alcove were evaluated through a Cartesian mesh. Variance reduction techniques were applied to reach a statistically meaningful result. Finally, all the results were normalised for $10^{7} \mathrm{~s}$, corresponding to a standard year's operation (see Fig. 3.35).

The reference radiation levels for the tunnel, which are reported in Table 3.19, can be compared with measurements made in the LHC. The comparison results in an increase by factors of $\sim 500$ and $\sim 200$ with respect to the LHC for the HEH and the dose, respectively. These factors are affected by the quality of the vacuum, for example, for an average gas-density profile of $2.0 \times 10^{14} \mathrm{H}_{2} / \mathrm{m}^{3}$ the data of Table 3.19 decrease proportionately. Nevertheless, the shielded alcoves for the electronics will further reduce the radiation levels, increasing the lifetime of the devices and reducing the probability of stochastic effects. The shielded alcoves concept leads to a feasible design for the collider which is compatible with today's knowledge the behaviour of commercial of the shelf electronics in radiation environments and with our current knowledge of fault tolerant electronics. The radiation levels for the detector are discussed in Section 7.3.

\subsubsection{Radiation hardness}

To avoid degradation of components, the dose to equipment has to be reduced by shielding and at the same time, fault tolerant or radiation resistant electronics and equipment has to be developed. The structured approach for radiation hardness assurance (RHA) for the electronics and materials in the collider environment is described below. 


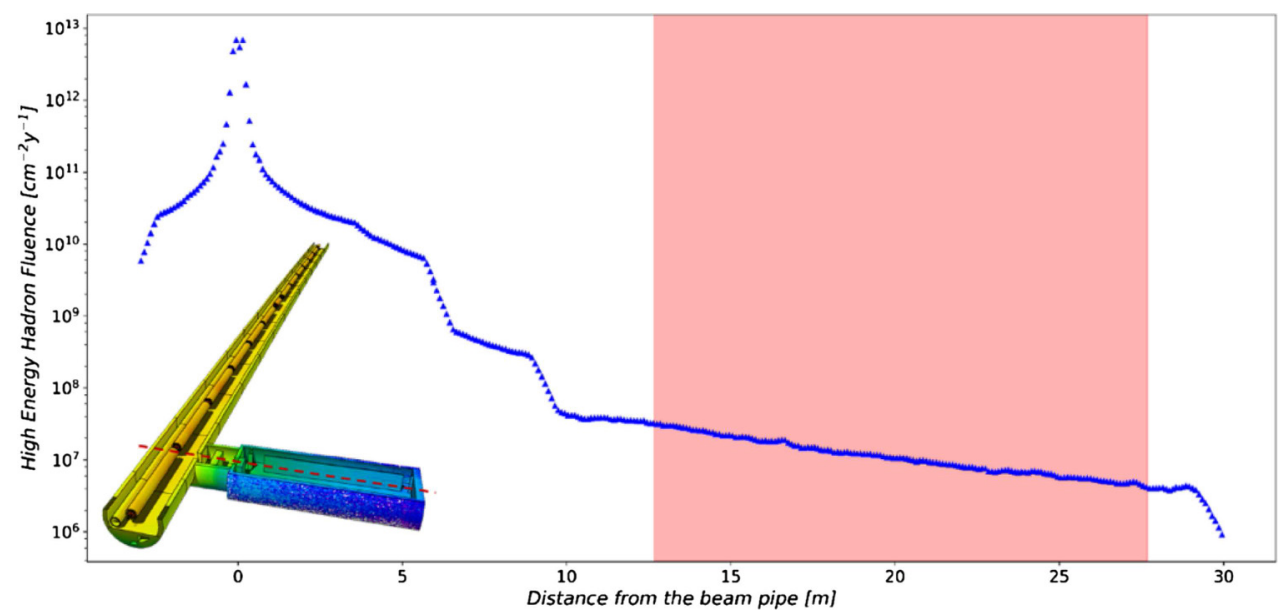

Fig. 3.35. HEH distribution, along the dashed red line shown at the bottom-left of the drawing, across a generic arc cell and into a lateral alcove. The pink shaded area represents the locations of the electronics racks within the alcove. A similar distribution can be retrieved for the dose.

Table 3.19. Reference radiation levels for the tunnel and the alcove.

\begin{tabular}{|l|l|l|}
\hline Location & HEH $\left(\mathrm{cm}^{-2} \mathrm{y}^{-1}\right)$ & Dose $\left(\mathrm{Gy} \mathrm{y}^{-1}\right)$ \\
\hline Tunnel (below magnet) & $\sim 1.0 \times 10^{11}$ & $\sim 200$ \\
\hline Alcove (Racks) & $\sim 4-30 \times 10^{6}$ & $<1$ \\
\hline
\end{tabular}

As is the case for the present LHC machine, the power converters, beam position and beam loss monitors (BPM and BLM) and quench protection system (QPS) have to be close to the accelerator itself. Such equipment is mainly based on commercialoff-the-shelf (COTS) components and therefore the equipment needs to be qualified for use in the radiation environment [280]. Given the expected radiation levels in the arc regions (see Tab. 3.19), a distributed system comprising $\sim 4000$ electronic units and subject to the agreed maximum annual limit for radiation induced dumps of one per system, an upper limit to the SEE cross section of $10^{-14} \mathrm{~cm}^{2}$ is imposed. This is roughly two orders of magnitude beyond what can reasonably be achieved in today's CHARM mixed-field facility [281] used for the qualification of LHC and HL-LHC systems e.g. the regulation loop for the power converters and the power converters [282].

The FCC-hh RHA strategy is founded on a full-availability approach based on: (i) remote control, moving the processing tasks away from the equipment under control and (ii) failure self-diagnosis, online hot swapping and remote handling. Therefore system designs are based on a modular approach that will allow switching to a redundant sub-system without any impact on operation. This will be particularly beneficial for transient errors, which can typically be corrected with a reset. The approach will also relax the constraints on the error qualification limits, which will be obtained through accelerated radiation testing.

In the case of events which cause permanent effects such as hard SEEs (occurring stochastically) or cumulative damage, online hot-swapping will need to be complemented by the substitution of the faulty board. This procedure will need to be carefully optimised, especially for cumulative damage, where similar sub-systems exposed to similar radiation levels are expected to fail at around the same time. The annual 
radiation levels expected in the tunnel of roughly $200 \mathrm{~Gy} / \mathrm{y}$ are above the TID limits for COTS in many cases such as linear and mixed-signal ICs, flash memories, DRAMs, microprocessors or FPGAs. Therefore, remote handling and the possibility of replacement of faulty units with spares which have been stored in radiation-safe areas, is one way to mitigate the risk.

The proposed scheme will bring benefits from the use of a selected set of semiconductor components that can be used in different sub-systems. The related procurement and qualification processes can be optimised and the impact of variability in sensitivity across batches and deliveries can be reduced. In specific cases, the use of radiation-hardened solutions at component level (e.g. FPGA) can be considered in combination with the use of COTS devices.

The radiation levels in optimised alcoves are comparable to the shielded areas in the present LHC machine, therefore the existing LHC qualification approach can be applied. In addition, the TID levels expected in the alcoves are low enough for them to store spares for the tunnel sub-systems.

CERN's current irradiation facilities are an essential means to independently assess the radiation hardness of electronic components, assemblies and systems in the collider environment. An analysis of the main shortcomings of CERN's facilities and possible solutions has been carried out and the main conclusions are outlined below [283].

For cumulative TID effects, facilities such as the CC60 [284] and GIF ++ [285] will have to be upgraded with more powerful sources. This will allow higher doses to be reached in shorter times and it has the potential of running more users in parallel. A source of $100 \mathrm{TBq}$ for the CC60 source will allow dose rates of $2 \mathrm{kGy} / \mathrm{h}$ to be reached so that the assessment of total ionising dose effects for tunnel electronics can be reached in a few hours instead of several days. In addition, higher doses, in the MGy range, which cannot currently be achieved, can be reached in around a month.

For the assessment of the single event effects, the CHARM facility can be upgraded by increasing the beam intensity and the space available for the users. This will make parallel and multiple testing possible, which is important because the number of systems to be tested for SEEs is proportional to the number of systems deployed, which will be much larger than for LHC.

\subsubsection{Radiation-hard technology trends}

In parallel with the rapid advance of electronics development and market trends, intensive work on radiation hardening is ongoing for electronics, components, materials and detectors with the main focus on HL-LHC. Continuous technology scouting and early technology analysis throughout the FCC design phase will be an important activity.

\section{Communication links}

A reliable, high performance communication link is a fundamental component of a new collider. It helps to move processing and control logic away from the radiation areas. Possibilities include fibre optic links and wireless technologies. A first study has been carried out on an Ethernet based solution. The basic building blocks of such a system can be seen in Figure 3.36. In this case, three components need to be radiation tolerant: the Ethernet physical layer component (PHY), a transceiver that bridges the digital world (including processors); field-programmable gate arrays (FPGAs); and application-specific integrated circuits (ASICs), which bridge to the 


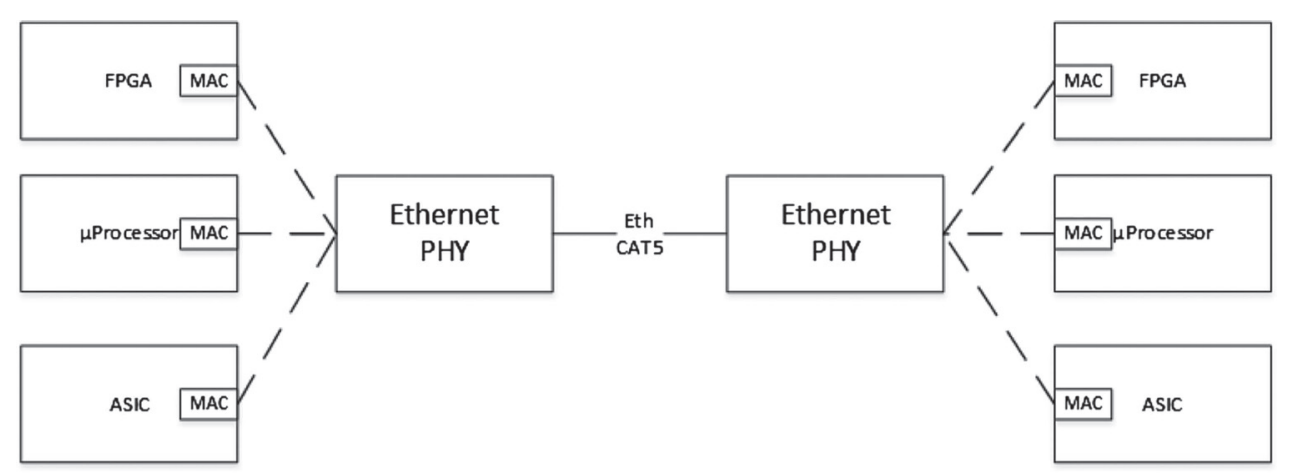

Fig. 3.36. Ethernet Subsystem.

analogue world. The MAC is usually integrated in a processor, FPGA or ASIC and controls the data-link-layer portion of the OSI model. Finally, an FPGA, a processor or an ASIC is needed to implement the application protocol. This solution will allow rates of several tens of Mbps with a low packet loss/failure factor to be reached

Preliminary studies to evaluate the feasibility of using such a system to reliably transmit data over long distances in a radiation environment have been conducted [286]. This solution would use either hard or soft processors in microcontrollers or FPGAs so that the system is able to conduct additional operations. The processor-based solution is not only chosen for the simplification of the implementation of transmission protocols, but also for the processing of the input/output data. In order to achieve higher radiation tolerance in terms of single event upset, the best choice would be the use of a radiation tolerant flash-based FPGA with a radiation mitigated soft-core processor implementing the application protocol.

\section{CMOS technologies}

Most of the on-detector application-specific integrated circuits (ASICs) being developed for HL-LHC make use of CMOS technologies in the 130 and $65 \mathrm{~nm}$ nodes. The study of radiation tolerance of these technologies has revealed that parasitic oxides used in the manufacturing processes are responsible for a significant degradation which limits their application. This is the case even in the pixel detector of HL-LHC, where the current plan is to replace the inner detector layers after 5 years of operation. As an example, Figure 3.37 shows the dramatic degradation in the current capability of small size $65 \mathrm{~nm}$ transistors. This study is now extended to 40 and $28 \mathrm{~nm}$ technologies, where preliminary results show different phenomenology and demonstrate slightly more promising radiation tolerance.

From the available data, it is unlikely that circuits designed with these technologies could survive the total ionising dose (TID) levels (100-5000 MGy) expected in the inner tracker layers and the forward calorimeters of FCC detectors. TID has traditionally limited the radiation tolerance of CMOS ASICs, however these technologies have not been tested for displacement damage at the levels expected in these detectors $\left(>5 \times 10^{17} 1 \mathrm{MeV}\right.$ neutrons $\left./ \mathrm{cm}^{2}\right)$ and this might lead to additional failure mechanisms. Testing to these levels is very challenging: with the current X-ray generator, the irradiation of a single sample to $100 \mathrm{MGy}$ would require more than 40 days.

CMOS technologies have been shifted from planar to bulk FINFETs starting from a nominal gate length of about $22 \mathrm{~nm}$ and have now reached the $7 \mathrm{~nm}$ pattern size. 


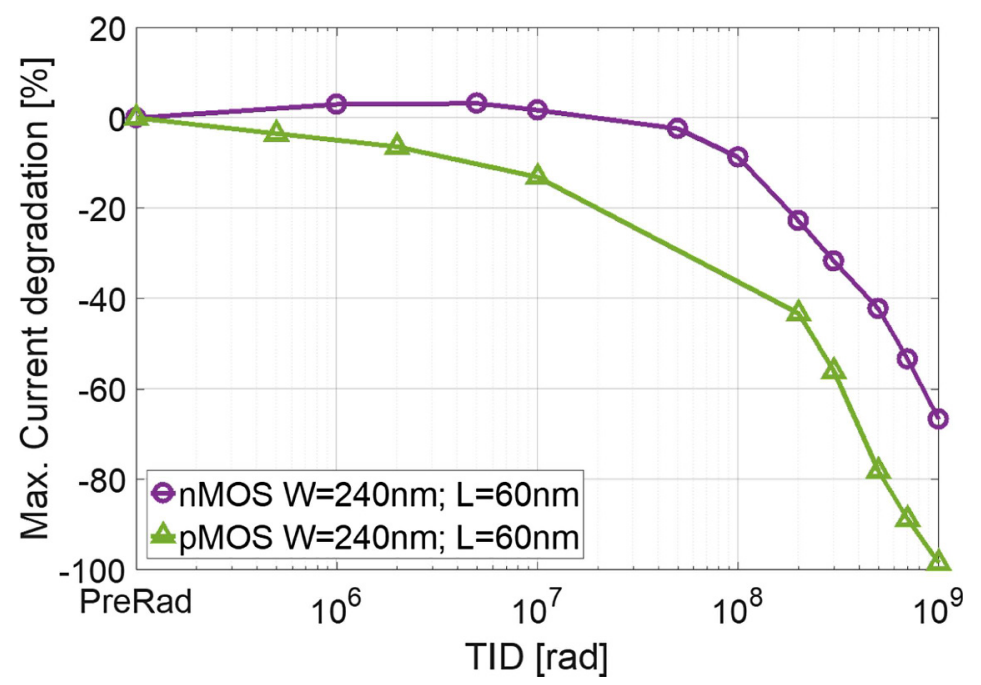

Fig. 3.37. Percent degradation of the current capability for small-size nMOS and pMOS transistors in the $65 \mathrm{~nm}$ technology node up to $10 \mathrm{MGy}$.

The literature consistently [287] shows that TID tolerance has decreased with this miniaturisation due to radiation-induced leakage currents in the neck region of these devices, a characteristic that cannot be addressed by any design technique. This evidence shows that the construction of reliable electronics systems for FCC detectors cannot simply rely on the improved radiation performance which accompanies miniaturisation, a concept exploited largely for LHC and HL-LHC. The situation calls for an R\&D programme on technologies and front-end systems, possibly nurturing new concepts such as disposable detectors.

\section{Ultra high radiation dosimetry}

Existing solid-state technologies for radiation measurements do not work for the radiation levels expected in certain sections of the FCC tunnel and within the volume of the experiments. In order to overcome these limitations, innovative solutions have to be developed. Structures based on thin metal films (see Fig. 3.38), in which the electrical resistivity varies according to the integrated particle fluence, are being studied. The probability of interaction between highly energetic particles and matter is known to scale with the volume of the medium and at the same time, metals are less sensitive to isolated lattice defects than semiconductors. The optical and electrical properties of the first prototypes of radiation dependent resistors (RDR), manufactured at the EPFL Centre of Micronanotechnology, have been studied before and after irradiation with high fluence of protons, photons, mixed-field radiation and neutrons. These tests confirm the possibility of using thin metal films for ultrahigh particle fluence monitoring. In particular, copper films have shown a greater response in the FCC radiation range [288]. Additional research will lead to a greater understanding of the impact of RDR geometry (width, length, thickness) on the sensitivity to non-ionising energy loss, and of the underlying phenomena that result in the resistivity increase.

The promising results with RDR suggest that a similar approach using a miniaturised sensor, should be considered for the implementation of a dosimeter for the total ionising dose. In this case, thin layers of oxides sandwiched as nano-capacitors 


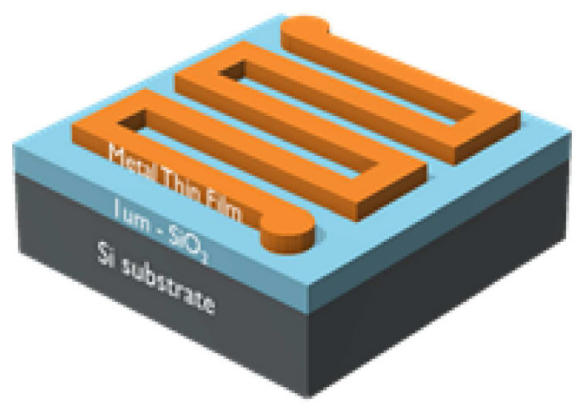

(a)

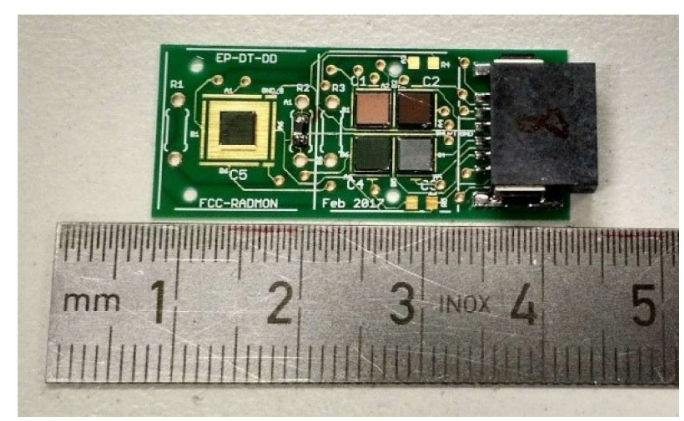

(b)

Fig. 3.38. An example of an FCC class radiation monitor. (a) Model of a radiation dependent resistor (RDR). (b) Example of an FCC-hh radiation monitor with a temperature sensor and RDRs made of different metals ready for irradiation tests.

to form radiation dependent capacitors (RDC), would facilitate the measurement of very high ionising doses based on a variation in their capacitance.

Finally, in order to implement an integrated radiation monitoring sensor (a proof of concept is shown in Fig. 3.38), an on-board radiation-hard readout circuit should be implemented and this will allow on-chip signal treatment and eventually wireless transmission of the readout data. For this reason further R\&D has been carried out on a vacuum transistor (VFET). Currently this device is being studied for high speed electronics and space applications and is intrinsically radiation hard since no oxides are used in its channel.

Implementing an RDR, an RDC and a circuit made of VFET, will help coming to a suitable radiation monitoring sensor and it could also be used as building block for radiation-hard electronic circuits with on-chip dosimetry.

\section{Civil engineering}

\subsection{Requirements and design considerations}

The civil engineering design and planning is a key factor in establishing the feasibility of the FCC-hh. The machine tunnel will be one of the longest tunnels in the world; only water supply tunnels with smaller diameters are longer. It will be similar in scope to the recently completed Gotthard Base Tunnel (total of $151.84 \mathrm{~km}$ including two $57 \mathrm{~km}$ long tunnel tubes) in Switzerland.

Following analysis of various options, the tunnel layout is now quasi-circular. The current baseline layout has a circumference of $97.75 \mathrm{~km}$. In addition to that, there are approximately $8 \mathrm{~km}$ of bypass tunnels, 22 shafts, 14 large caverns (two in addition to the 12 of the FCC-ee lepton collider) and 12 new surface sites.

The emphasis for the study of the underground structures has been on locating the machine within the natural boundaries defined by the geological formations of the Geneva basin, with as short as possible connections to the existing CERN accelerator complex. For the access points and their associated surface structures, the focus has been on identifying possible locations which are feasible from socio-urbanistic and environmental perspectives. The construction methods, and hence the technical feasibility of construction has been studied and deemed achievable.

The underground structures for LEP were designed for a lifespan of 50 years. They were completed in 1989 and will thus reach the end of their specified lifetime around 
2040. Refurbishment, continuous monitoring and maintenance is therefore necessary to extend their lifetime for use with a future particle collider. Such activities have already started in order to meet the requirements of the HL-LHC project.

\subsection{Layout and placement}

\subsubsection{Layout}

The principal structure for the FCC-hh collider is a quasi-circular $97.75 \mathrm{~km}$ long tunnel with $5.5 \mathrm{~m}$ internal diameter, comprising straight sections and arcs. In addition, large caverns are required at points PA, PB, PG and PL to house the experiments. The caverns at PA and PG have a maximum clear span of $35 \mathrm{~m}$, which is at the limit of what is possible today, given the ground conditions. The caverns at PB and PL have a maximum clear span of $30 \mathrm{~m}$. At each of the 12 access points around the ring, a service cavern with a span of $25 \mathrm{~m}$ is required. These caverns are connected to the surface via shafts with diameters ranging from $10 \mathrm{~m}$ to $18 \mathrm{~m}$. Auxiliary structures are required in the form of connection and bypass tunnels and of alcoves, to house electrical equipment. Figure 4.1 shows a 3D, not-to-scale schematic of the underground structures. The chosen layout equally satisfies the requirements of the FCC-hh and the FCC-ee machine.

The excavation of the underground structures will produce approximately 9 million cubic metres of spoil. With the current placement, this will primarily be made up of sedimentary deposits, a mixture of marls and sandstone. A small fraction of the tunnel (approximately 5\%) will be excavated in limestone.

\subsubsection{Placement}

Experience from the LEP and LHC construction has shown that the sedimentary rock in the Geneva Basin, known as molasse, provides good conditions for tunnelling. In contrast, during the excavation of the LEP tunnel, water ingress from the limestone formations in the Jura mountains caused significant problems. For this reason, one of the primary aims when positioning the FCC tunnel was to maximise the fraction of the tunnel in the molasse and minimise that in the limestone. Another prominent concern was to orientate the tunnel in a way that limited the depth around its perimeter, thereby minimising the depth of the shafts, reducing the overburden pressure on the underground structures, and reducing the length of service infrastructure (cables, ducts, pipes). These concerns, along with the need to connect to the existing accelerator chain, led to the definition of the study boundary, within the Jura range to the north-west, the Vuache mountain to the south-west and the Pre-Alps to the south-east and east. An additional boundary is placed to the north due to the increasing depth of Lake Geneva in that direction. Figure 4.2 shows the boundary of the study in red.

In order to evaluate different layouts and positions within the boundary area, a software tool incorporating a 3D geological model was developed. This tunnel optimisation tool (TOT) is based on an open source driven Geographical Information system (GIS), which enables multiple sets of data to be arranged spatially, together with a topographical map. The ensemble can then be manipulated, managed and analysed as one. This means that the TOT user is able to input any size, shape and position of the tunnel and quickly see how this interacts with the geology, the terrain, the environment and the surface structures in the study area.

The geological data for the tool were collected from various sources [289], but not limited to: previous underground projects at CERN, the French Bureau de Recherches 


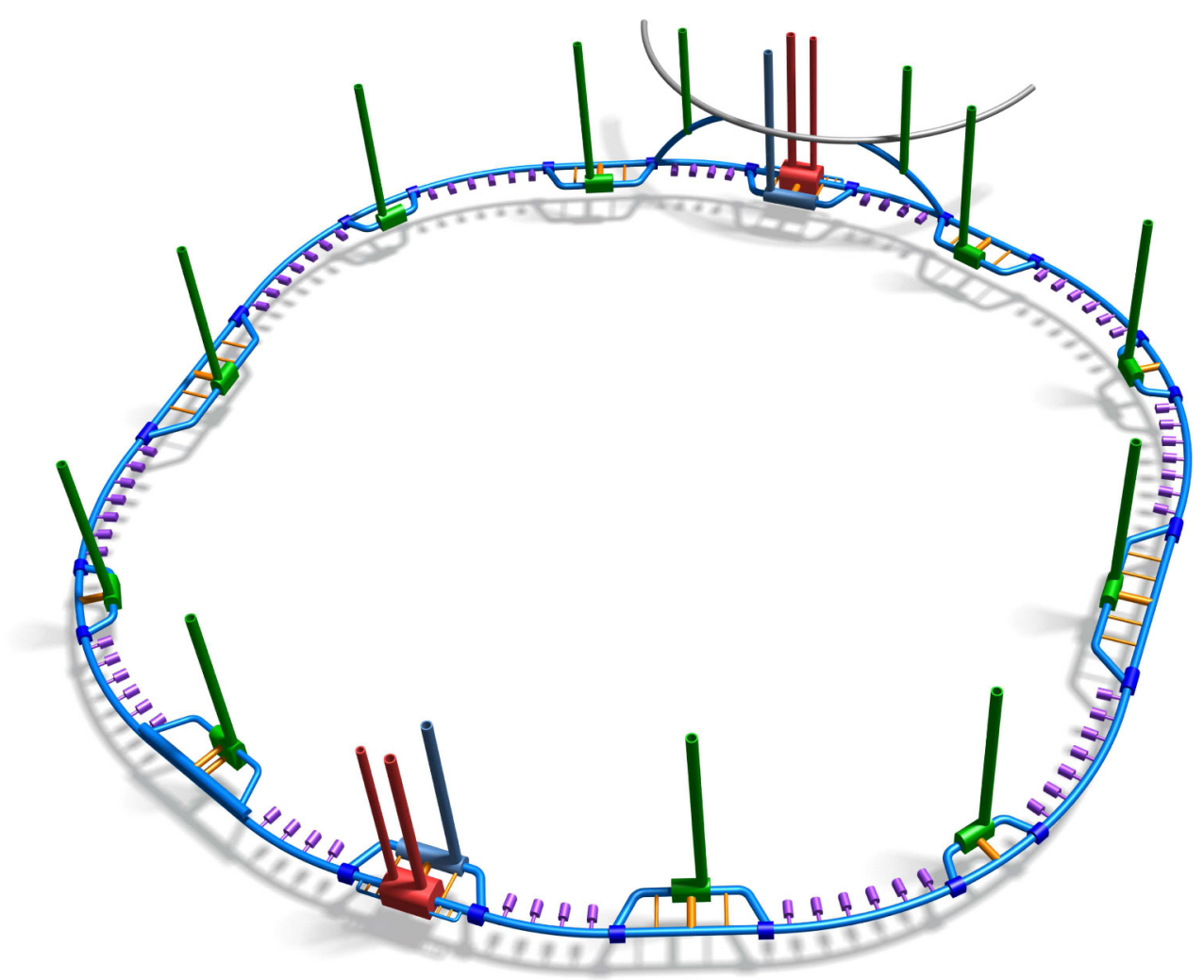

Fig. 4.1. 3D, not-to-scale schematic of the underground structures. The main collider tunnel and the service tunnels are indicated in blue colour. Connection caverns between main and service tunnels are indicated in dark blue colour. The two red caverns indicate the experiment caverns. They are reached by two, red marked, shafts: a large one for the detector elements and a smaller one for the service. Secondary caverns and shafts for detector-related equipment and services are shown in blue color next to the experiments. Technical equipment caverns and the shafts to access them are indicated in green colour. Yellow tubes indicate the connections between the machine tunnel and certain equipment galleries, for instance to connect the power sources to the radiofrequency cryomodules. Equipment alcoves along the entire machine tunnel are indicated in violet colour. The upper part of the image shows an exemplary connection to the injector accelerator.

Géologiques et Minières (BRGM), and existing geological maps and boreholes for geothermal and petroleum exploration. The data was processed to produce rockhead maps that form the basis of TOT. All of the geological data for the study has come from previous projects and existing data. No specific ground investigations have yet been conducted for FCC-hh.

The machine studies demonstrated that it was necessary to have a circumference of $\sim 100 \mathrm{~km}$ in order to meet the physics goals. The alignment of the tunnel has been optimised based on criteria such as geology along the tunnel, overburden, shaft depth and surface locations. A good solution has been found in which the tunnel is located primarily in the molasse (90\%). It avoids the limestone of the Jura mountains and the Prealps but passes through the Mandallaz limestone formation, which is unavoidable. The tunnel passes through the moraines under Lake Geneva at a depth where they are believed to be well consolidated. Whilst there will be some additional challenges during excavation, the long-term stability of the tunnel is not a major concern. The 


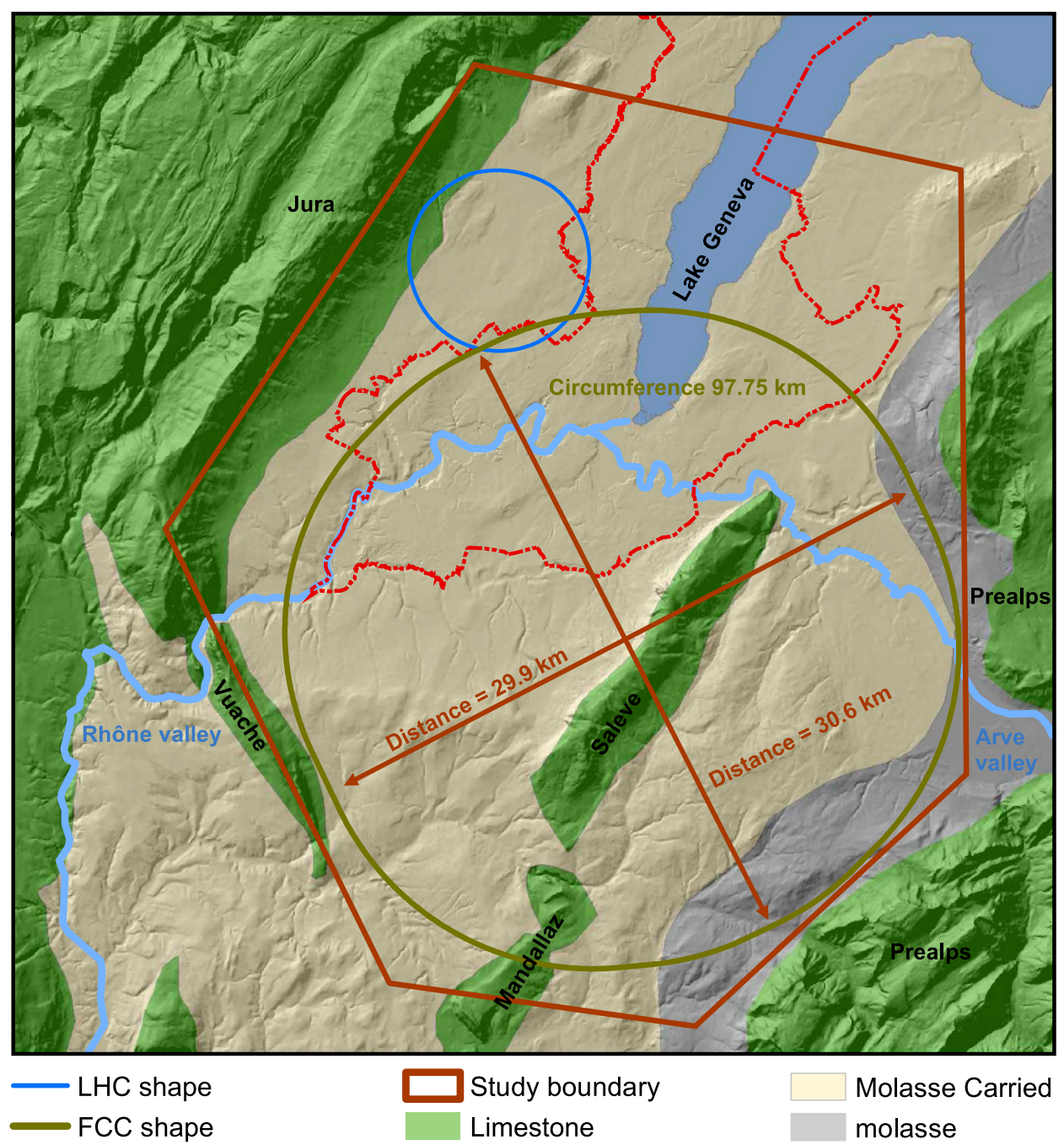

Fig. 4.2. Study boundary (red polygon), showing the main topographical and geological structures and the layout of the current FCC tunnel baseline with a perimeter of $97.75 \mathrm{~km}$. This version with an approximate inner diameter of $30 \mathrm{~km}$ serves as the baseline for the planned layout and placement optimisation.

topographical and geological profile of the tunnel in the chosen position is shown in Figure 4.3.

The tunnel position places the shafts in suitable positions with acceptable depths of less than $300 \mathrm{~m}$, apart from the shaft at $\mathrm{PF}$ which requires special attention as it is $558 \mathrm{~m}$ deep. It is being considered to replace this shaft with an inclined access tunnel.

\subsubsection{Necessary site investigations}

Based on the available geological data for the region, the civil engineering project is deemed feasible. However, in order to confirm this and to provide a comprehensive technical basis for further detailed design iterations, to establish a comprehensive project risk management and to improve the accuracy of the construction cost esti- 


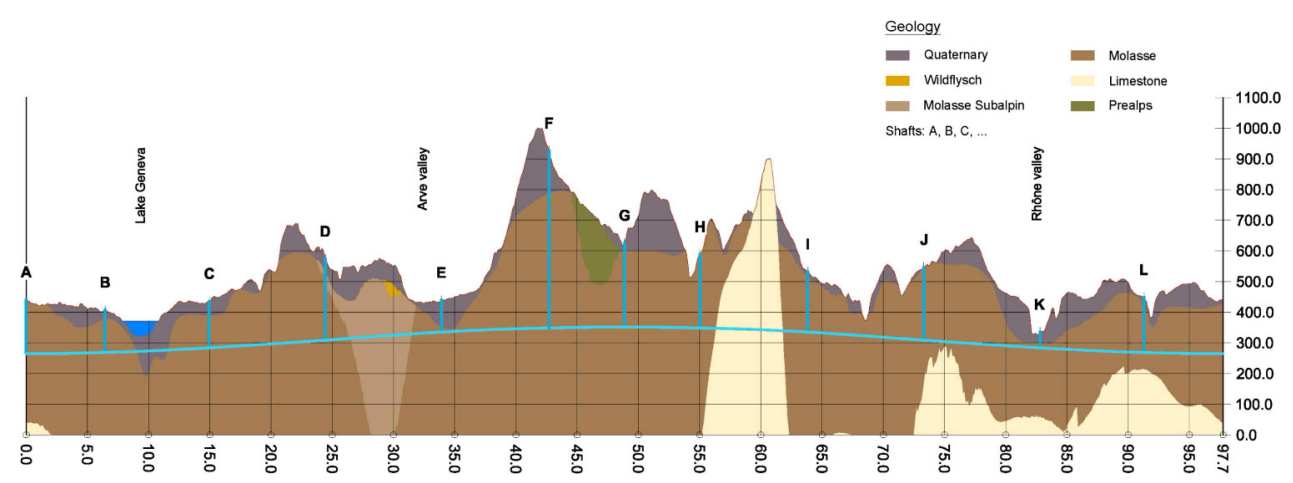

Fig. 4.3. Geological profile along the collider circumference. The horizontal axis labels the kilometers along the entire collider circumference starting with $\mathrm{km} 0$ at point $\mathrm{A}$. The so called "alignment elevation" is shown in meters above seal level (mASL) on the vertical axis. Different brown shades indicate different soil types such as molasse (dark brown) and limestone (light beige). The collider ring and the access shafts are indicated in light blue. Access points are labelled with letters in alphabetical order (A-L). Point A is close to the CERN Meyrin site. The collider tunnel is visualised along a sinuidal shape, since the tunnel in a single plane is tilted. The lake Geneva zone is shown in blue at about kilometer 10 .

mates, dedicated ground and site investigations are required during the early stage of a preparatory phase. These investigations will rely on non-invasive techniques such as walkover surveys and geophysical analysis, and will also use invasive techniques, such as the analysis of samples from boreholes that need to be constructed at regular distances. A combination of in-situ tests, such as the standard penetration test (SPT) and the permeability test, in combination with laboratory testing of samples, will give a comprehensive understanding of the geological situation.

The initial site investigations must focus on the highest risk areas: the crossing of Lake Geneva, the Rhône and the Arve valleys. In addition, the access point location candidates should be investigated in order to optimise the placements. This can be conducted via geophysical investigations and could lead to a recommendation to adjust the alignment in order to optimise the construction cost and reduce residual project-related risks.

\subsection{Underground structures}

\subsubsection{Tunnels}

A $5.5 \mathrm{~m}$ internal diameter tunnel can house all the necessary equipment for the machine, while providing sufficient space for transport. Figure 4.4 shows the crosssection of the empty tunnel but with the air supply and smoke extraction ducts, which have been integrated into the civil engineering design (these are discussed in Sect. 5.3). The air-supply duct in the floor is a pre-cast structure. The rest of the floor will be cast in concrete around it.

The tunnel will be constructed with a slope of $0.2 \%$ in a single plane, in part to optimise for the geology intersected by the tunnel and the shaft depths and in part to use a gravity drainage system.

It is anticipated that the majority of the machine tunnel will be constructed using tunnel boring machines (TBM). The sector passing through the Mandallaz limestone will be mined. For the TBM excavations, different lining designs have been developed corresponding to "good" or "poor" conditions of the rock. For TBM 


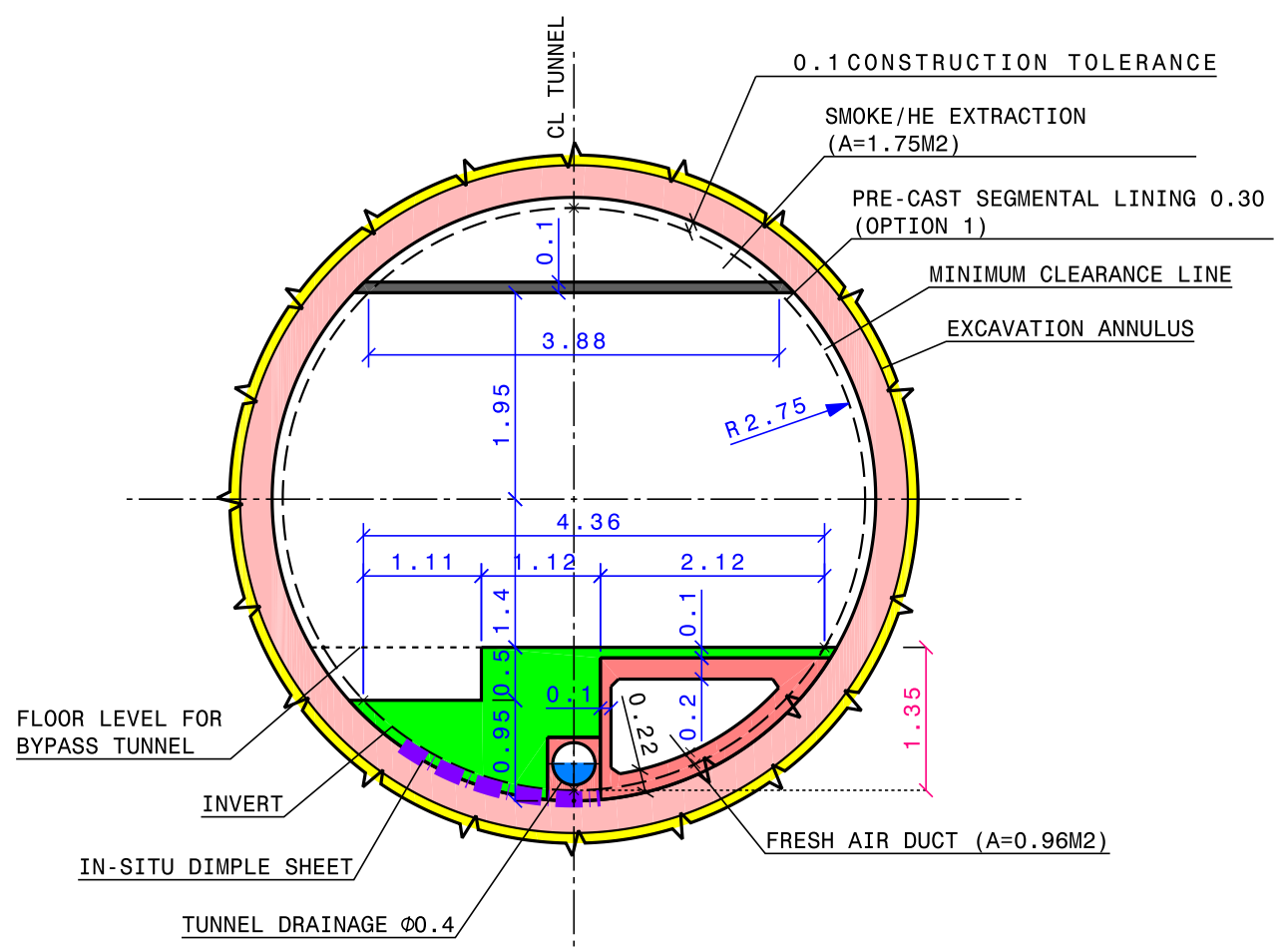

Fig. 4.4. Machine tunnel cross-section in "good" molasse.

excavation in a sector with "good" conditions, a single-pass pre-cast lining is adopted. This is the fastest and cheapest construction method but is reserved for sectors that are completely located in molasse with a good rock coverage and hence a low risk of water inflow. For sectors in "poor" conditions to be excavated with a TBM, an optional second in-situ cast lining can be incorporated. This reduces the risk of water inflow in sectors which are located in the molasse, but where the depth of rock to the water bearing layers is minimal. Construction under the lake presents another situation which is similar to "poor" conditions but with a thicker initial pre-cast lining. Table 4.1 shows the excavation and lining parameters for each of the TBM lining cases.

The tunnel under Lake Geneva will be excavated in water bearing moraines in sector PB-PC (see Fig. 4.3). In order to achieve this, it is necessary to employ a slurry shield TBM. During excavation with this type of machine, the excavated material behind the cutter face is pressurised to support the tunnel face. Consequently, the excavation can be achieved safely and efficiently in wet and unstable conditions. It is anticipated that the layer of moraines to be excavated is impermeable enough that the tunnel would not be affected by the fluctuating depth of the lake and hence would not impact on accelerator operation, however, this risk must be evaluated once additional ground investigations have been conducted.

In addition to the machine tunnel, auxiliary tunnels are required for bypasses, connections, beam dumps and transfer lines. These have requirements similar to the machine tunnel and, depending on their diameter and position in relation to the TBM launch points, will be constructed using a TBM or roadheaders. 
Table 4.1. Proposed TBM excavation and lining parameters.

\begin{tabular}{|c|c|c|c|}
\hline Parameter & $\begin{array}{c}\text { TBM tunnel in } \\
\text { "good" rock }\end{array}$ & $\begin{array}{c}\text { TBM tunnel in } \\
\text { "poor" rock }\end{array}$ & $\begin{array}{c}\text { TBM tunnel in } \\
\text { moraines }\end{array}$ \\
\hline $\begin{array}{c}\text { Minimum internal diameter } \\
(\mathrm{m})\end{array}$ & 5.5 & 5.5 & 5.5 \\
\hline $\begin{array}{c}\text { Characteristic compressive } \\
\text { concrete strength for pre-cast } \\
\text { concrete, fck (MPa) }\end{array}$ & 50 & 50 & 50 \\
\hline Pre-cast concrete thickness (m) & 0.30 & 0.30 & 0.45 \\
\hline $\begin{array}{c}\text { Reinforcement density for } \\
\text { pre-cast concrete }\end{array}$ & $\begin{array}{c}\text { Steel fibre }(50 \%) \\
\text { and bars at } \\
80 \mathrm{~kg} / \mathrm{m}^{3}\end{array}$ & $\begin{array}{c}\text { Steel fibre }(50 \%) \\
\text { and bars at } \\
80 \mathrm{~kg} / \mathrm{m}^{3}\end{array}$ & $150 \mathrm{~kg} / \mathrm{m}^{3}$ \\
\hline Gasketed segments & Yes & Yes & Yes \\
\hline $\begin{array}{c}\text { Cast in-situ concrete thickness } \\
\text { (m) }\end{array}$ & None & 0.25 & 0.25 \\
\hline $\begin{array}{c}\text { Characteristic compressive } \\
\text { concrete strength for in-situ } \\
\text { concrete, fck (MPa) }\end{array}$ & - & 40 & 40 \\
\hline $\begin{array}{c}\text { Reinforcement for in-situ } \\
\text { concrete }\end{array}$ & - & $\begin{array}{c}\text { Local } \\
\text { reinforcement } \\
\text { cages }\end{array}$ & $\begin{array}{c}\text { reinforcement } \\
\text { cages }\end{array}$ \\
\hline $\begin{array}{c}\text { Total radial construction } \\
\text { tolerance (m) }\end{array}$ & 0.10 & 0.10 & 0.10 \\
\hline Excavation diameter (m) & 6.3 & 6.8 & 7.1 \\
\hline
\end{tabular}

\subsubsection{Shafts}

Twenty-two large-diameter shafts are included in the design:

- one $12 \mathrm{~m}$ diameter shaft at each of the 12 surface sites for access and service requirements;

- one $15 \mathrm{~m}$ diameter and one $10 \mathrm{~m}$ diameter shaft at each of four experiment points PA, PB, PG and PL;

- two shafts located near the existing CERN accelerators to facilitate the removal of spoil during construction of the beam transfer tunnels from the LHC or SPS.

At least one of the access and service shafts will have a wider diameter to lower large accelerator or infrastructure elements.

The shafts depths range from $52 \mathrm{~m}$ to $274 \mathrm{~m}$ (without point PF). As mentioned, the shaft at $\mathrm{PF}$ is considered to be replaced with an inclined access tunnel, with a length of $2750 \mathrm{~m}$ and a gradient of $15 \%$. This is deemed a better solution than a $578 \mathrm{~m}$ vertical shaft for which a lift system would not be easy. A better location for the access portal can be found and an inclined access tunnel would be slightly faster and cheaper to build. The feasibility to route all services through it needs to be verified.

Internal structures in the form of staircases and lift shafts are required within the service shafts. Figure 4.5 shows the layout of these items; the lift shafts and staircases are pre-fabricated concrete structures. The initial excavation for each shaft will be through the moraines. This will be achieved either by using a diaphragm wall or a vertical shaft sinking machine (VSM). The remainder of each shaft will be constructed using traditional excavation techniques with shotcrete followed by an in-situ cast lining. 


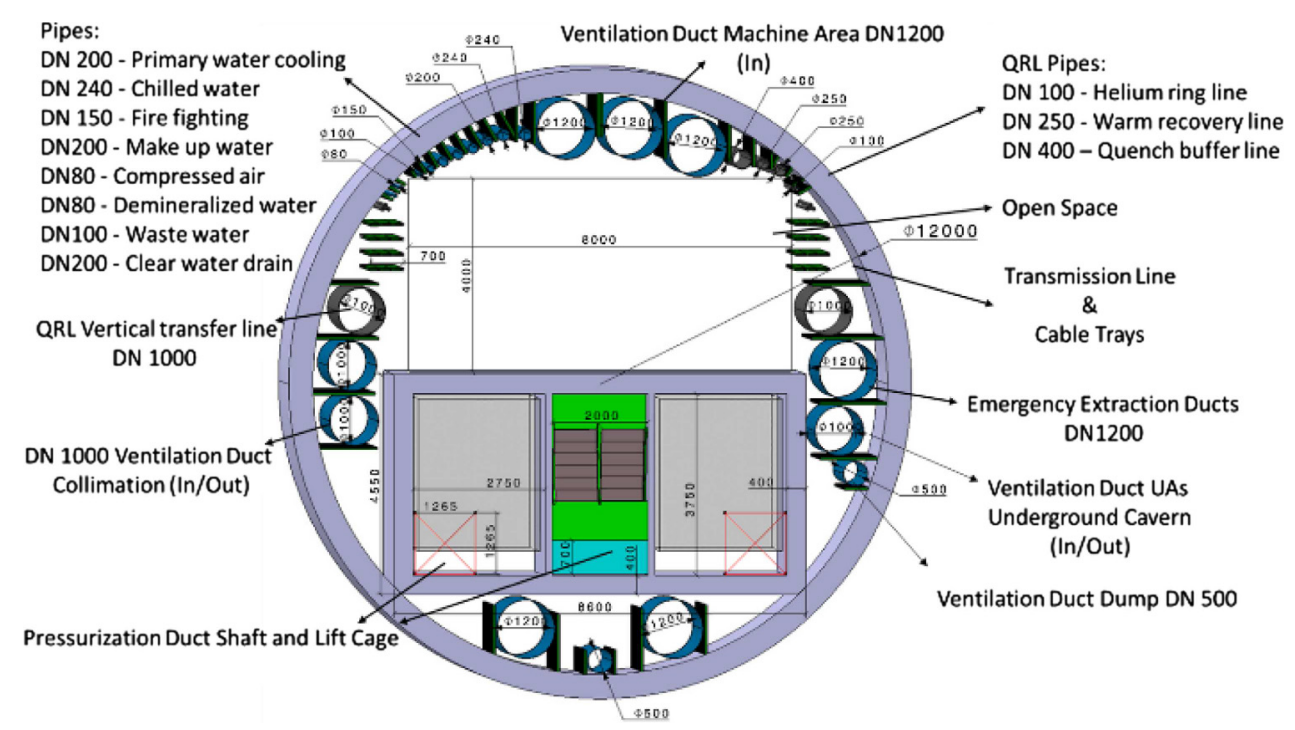

Fig. 4.5. Service shaft cross-section.

\subsubsection{Alcoves}

Alcoves for electrical equipment are required every $1.5 \mathrm{~km}$ around the machine circumference. These are $25 \mathrm{~m}$ long, $6 \mathrm{~m}$ wide and $6 \mathrm{~m}$ high, located on the inside of the ring at $90^{\circ}$ to the machine. The excavation for these will be carried out after the machine tunnel drives. This will be followed by the inner lining work for the alcoves, before the secondary lining of the machine tunnel.

\subsubsection{Experiment caverns}

Large span caverns are required at the experiment points $\mathrm{PA}, \mathrm{PB}, \mathrm{PG}$ and $\mathrm{PL}$ to accommodate the detectors. The dimensions for these are $66 \mathrm{~m} \times 35 \mathrm{~m} \times 35 \mathrm{~m}$ $(\mathrm{L} \times \mathrm{W} \times \mathrm{H})$ at $\mathrm{PA}$ and $\mathrm{PG}$ and $66 \mathrm{~m} \times 30 \mathrm{~m} \times 35 \mathrm{~m}$ at PB and PL. They will be constructed at a depth of up to $274 \mathrm{~m}$ in the molasse layer. The exact construction sequence is yet to be confirmed, however, it will include benched excavations using a rockbreaker and roadheader with the primary support being provided by rock bolts, cable bolts and layers of steel fibre concrete. During the widening of the crown area of the experiment cavern, additional girder lattices and layers of steel fibre reinforced shotcrete will be installed. The lattice girders for the various excavation steps can be bolted together to ensure continuous rock support along the excavated area. The final lining will be in-situ cast concrete.

\subsubsection{Service caverns}

A service cavern at machine level with dimensions of $100 \mathrm{~m} \times 25 \mathrm{~m} \times 15 \mathrm{~m}$ $(\mathrm{L} \times \mathrm{W} \times \mathrm{H})$ is required near the experiment caverns. Shorter services caverns are planned for the remaining 8 access points, depending on the requirements for housing infrastructure equipment underground. These caverns will be constructed in a similar manner to those for the experiments. At the experiment points, the spacing between the two caverns is $50 \mathrm{~m}$ as this allows the structures to be independent and 


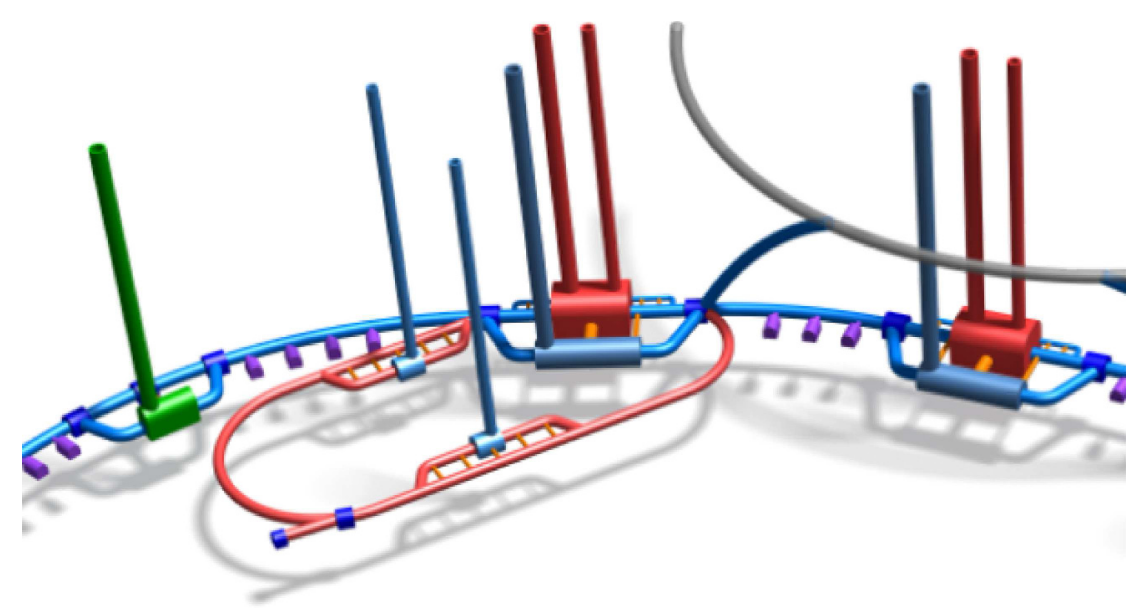

Fig. 4.6. Schematic diagram of an FCC-eh facility providing collisions at point PL (not to scale).

hence minimises the structural support needs and reduces the risk and complexity during construction.

\subsubsection{Junction caverns}

There are three types of junction caverns which are required for structural purposes when tunnels of similar size connect, for example a bypass tunnel connecting to the machine tunnel. There are 26 locations which require a junction cavern, ranging in length from $30 \mathrm{~m}$ to $400 \mathrm{~m}$ and with a cross-section of approximately $16 \mathrm{~m} \times 8 \mathrm{~m}$ $(\mathrm{W} \times \mathrm{H})$. The longest junction caverns also serve as reception points or crossing points for the TBMs. A $400 \mathrm{~m}$ long junction cavern is required for each of the beam dumps to accommodate the dump beamline up to the point where it is possible to have a separate tunnel for it.

\subsubsection{FCC-eh requirements}

A facility allowing collisions between high energy hadrons and leptons is also being studied. This will require a separate lepton accelerator, for example an energy recovery linac (ERL), as described in Section 2.8.

For the present tunnel alignment, point PL seems to be the most appropriate location for this machine because it provides good geological conditions, being fully based in the molasse layer with an overburden of approximately $180 \mathrm{~m}$. Point PG, in comparison, has much larger overburdens. Point PA is challenging due to the proximity of the LHC and PB is located in a congested urban environment, so finding a location for the additional shafts would be difficult. Figure 4.6 shows a schematic drawing of the eh machine.

The collision point is inside the cavern. Two additional caverns are necessary for access, with dimensions $20 \mathrm{~m} \times 20 \mathrm{~m} \times 30 \mathrm{~m}(\mathrm{~W} \times \mathrm{H} \times \mathrm{L})$ as well as two shafts with $9 \mathrm{~m}$ diameter. Bypass tunnels, at an approximate spacing of $40 \mathrm{~m}$ and parallel to the linacs, are required along with connections back to the machine tunnel for waveguides. Additional underground infrastructure is needed to house injection facilities and a beam dump. 
Table 4.2. Example of the main surface structures at a typical experiment site.

\begin{tabular}{|c|c|c|}
\hline Structure name & Structure type & $\begin{array}{c}\text { Dimensions } \\
(\mathbf{W} \times \mathbf{H} \times \mathbf{L})\end{array}$ \\
\hline Shaft head/detector building & Steel-frame & $25 \times 25 \times 100 \mathrm{~m}$ \\
\hline Reception/office building & & $10 \times 11 \times 30 \mathrm{~m}$ \\
\hline Gas building & Concrete & $15 \times 4 \times 40 \mathrm{~m}$ \\
\hline Data centre & & $20 \times 10 \times 40 \mathrm{~m}$ \\
\hline Workshop & & $30 \times 12 \times 15 \mathrm{~m}$ \\
\hline Cryogenic plant building & $\begin{array}{c}\text { Noise insulated } \\
\text { steel-frame }\end{array}$ & $15 \times 12 \times 40 \mathrm{~m}$ \\
\hline Ventilation building & Steel-frame & $25 \times 14 \times 40 \mathrm{~m}$ \\
\hline Electrical building & Steel-frame & $20 \times 6 \times 80 \mathrm{~m}$ \\
\hline Power converter building & Steel-frame & $25 \times 14 \times 40 \mathrm{~m}$ \\
\hline Access control building & & $10 \times 4 \times 10 \mathrm{~m}$ \\
\hline
\end{tabular}

The connection to the FCC is challenging for the integration of the machines because, on one side, five tunnels will need to be combined at one junction cavern. A cavern with dimensions of $25 \mathrm{~m} \times 10.5 \mathrm{~m} \times 30 \mathrm{~m}(\mathrm{~W} \times \mathrm{H} \times \mathrm{L})$ is proposed for this purpose.

\subsection{Surface sites}

\subsubsection{Experiment surface sites}

The conceptual designs for surface sites range from classical sites similar to the LHC to semi-underground installations. Specific designs will reflect the particular machine and environmental requirements. It is anticipated that each site will be approximately 6-9 hectares in size, housing buildings and spaces for infrastructure needs (e.g. electrical distribution, cooling and ventilation, cryogenics). There will be a large shaft head building at the experiment sites, which will act as detector assembly hall during installation. The surface sites will also serve as temporary spoil storage during construction and storage and assembly space during installation. Every effort will be made during the design process to minimise the visual, environmental and acoustic impact of these sites, which could mean building parts of the site below ground.

Using TOT, it has been possible to quickly identify candidate surface sites, considering the proximity to existing infrastructures, protected areas and transport links. With the current machine placement, site PA is located near the CERN Meyrin campus. Points PL, PA, PB and PC are located in Switzerland, the others are located in France. Table 4.2 gives an impression of the typical structures for surface sites and their dimensions. Most of the buildings will use standard industrial construction techniques, unless additional considerations dictate other designs.

\subsubsection{Technical surface sites}

The 8 access points without experiments will require surface sites for the technical facilities. The requirements are similar to those for the experimental surface sites, while experiment-related buildings will be omitted and the shaft head building will be smaller. 


\subsubsection{Access roads}

It is preferable for the surface sites to be largely accessible via existing roads. It is however anticipated that additional roads and even tunnels or bridges may be necessary for the more remote sites. Large accelerator and detector components will need to be transported along these roads, as well as the vehicles and machinery for construction, hence the roads must be able to withstand heavy loads. For the purpose of the siting study it has been assumed a new $5 \mathrm{~km}$ long road is required for each surface site.

\section{Technical infrastructure}

\subsection{Requirements and design considerations}

The technical infrastructure for FCC-hh comprises a large and diverse set of services to enable and support the operation of the collider, the beam transfer lines and the experiments. These include the supply of electrical energy and cryogens, the air and water cooling systems, facilities to transport people and material, the geodetic network, survey and alignment, control of accelerator equipment, data acquisition, computing and networking, as well as access control and other safety related functions.

As is customary for other facilities at CERN, the FCC-hh will make as much use of the existing chain of pre-accelerators as possible. As a new large-scale accelerator facility, the FCC will require new infrastructure systems. Some of them, like computer networks, will integrate with the existing infrastructure; others, like the supply of electrical energy, will extend existing facilities.

Building a large research infrastructure which crosses borders in a densely populated area like the Geneva basin requires that a wide range of conditions and regulations are respected to ensure its environmental and socio-cultural compatibility. The whole FCC-hh, including its technical infrastructure, must be designed and built for safe, high-performance operation, with high reliability and availability in mind. The equipment must generally be energy- and cost-effective. Future-oriented, yet technically solid approaches must be chosen to ensure enduring high performance and affordable operation.

\subsection{Piped utilities}

\subsubsection{Introduction}

The piping systems concern the various water systems for the machine and its infrastructure. Accelerator and detector equipment such as electronic racks, cryogenics plants, conventional magnets will require industrial and demineralised water. Chilled water will be needed for the air handling units of the ventilation systems. Raw water will be used in primary cooling circuits and for fire fighting purposes. Drinking water will be needed for sanitary purposes as well as for the make-up of raw water cooling circuits. There will also be a drainage network for the waste water which comprises the rejected and drained water from the surface facilities and the underground areas. The other main piped utility is for compressed air which will be used both underground and on the surface. 


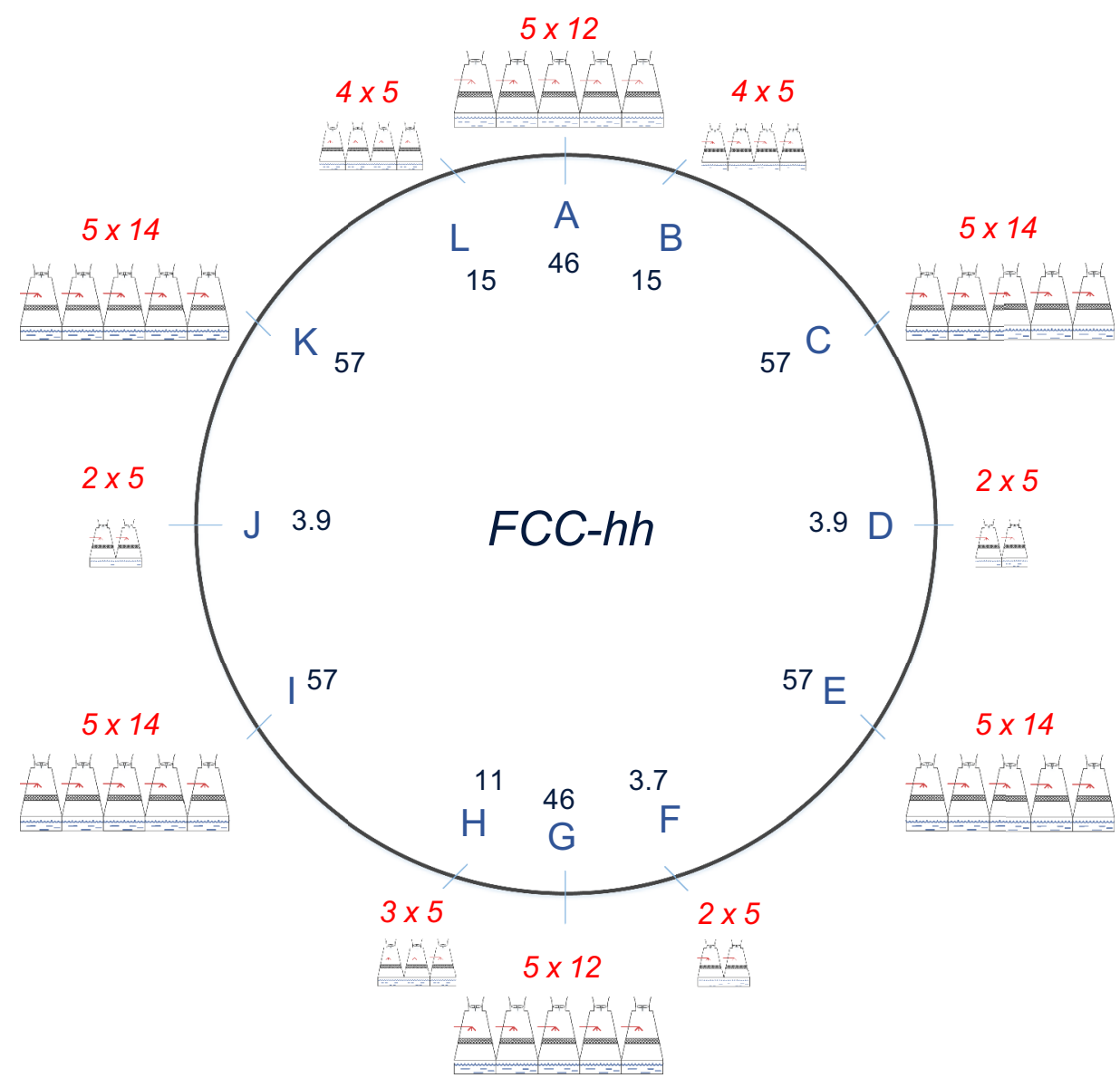

Fig. 5.1. Schematic view of the primary water cooling system (black numbers: primary cooling power needed per point in MW; red numbers: cooling cells per point (including backup) $\times$ cooling capacity per cell in MW).

\subsubsection{Water cooling plants}

Using raw water, the cooling plants will remove most of the heat generated by the accelerator equipment, the detectors and in the technical areas. It is planned to install one plant in each of the 12 surface sites. The current baseline scenario has the primary circuit of the water cooling plant using raw industrial water which will be cooled by means of open wet cooling towers. Some equipment, in particular cryogenics systems, will be cooled directly by the primary circuit. The secondary circuit will be connected to the primary system through a heat exchanger. In most cases the secondary circuit will use demineralised water in a closed loop. Figure 5.1 gives a schematic view of the primary water cooling system and its requirements.

Distribution circuits will be grouped according to the typology of the equipment to be cooled and to the equipment pressure ratings. Since the underground areas will be up to $400 \mathrm{~m}$ below ground level (PF), it will be necessary to install an underground cooling station in the service cavern at each point. Here a heat exchanger will separate the circuit coming from the surface (with a static pressure of up to 40 bar) from the underground distribution circuit. Wherever possible this separation will correspond to the separation between the primary and secondary circuit, but it will also be 
applied for other circuits such as those of the underground cryogenic equipment. The decoupling of the surface from the underground circuit will allow safer operation of the underground circuit as well as a cost reduction resulting from the ability to use pipes with a lower nominal pressure rating. For operability and maintenance purposes, both surface and underground cooling stations will be accessible during accelerator running.

The cooling area of all service caverns hosts secondary circuit stations to cool adjacent sectors or special equipment such as power converters. Points PA and PG also host the secondary circuit station to cool the equipment in the experiment areas. In point $\mathrm{PH}$ a similar area will be dedicated to the cooling of RF equipment. The secondary circuit in each sector will also cool the air handling units in the alcoves.

Primary circuits will use raw industrial water with a make-up of drinking water, to compensate for evaporation, losses and blow-down. Continuous water treatment against legionellae, scaling and proliferation of algae will also be included. The makeup is assumed to be provided by the local water supplier from the network serving each point.

Secondary circuits will use demineralised water with a maximum conductivity of $0.5 \mu \mathrm{S} / \mathrm{cm}$ in a closed loop. A set of demineralisation cartridges will be installed in each circuit to control the conductivity. The demineralised water will be produced in a central station. However, given the long distances, it will not be possible to have automatic refill pipework from this station to all the circuits in the various points via the tunnel without degrading the quality of the water. If there is a leak, the refill will be made by transporting the required volume of water in tanks from the production station to the point concerned.

To ensure continuous operation if one plant stops, the level of redundancy of the primary and secondary circuits is set at $N+1$ for pumps, chillers and cooling towers. No redundancy is needed for plate heat exchangers, power or control cubicles. The safe power supply is at present only planned for systems related to safety. No equipment needs continuous cooling to prevent damage. Some cooling towers with a lower capacity will be installed to backup the main towers in order to allow essential cryogenic equipment to be kept at low temperature during mandatory stops for maintenance and cleaning.

Each cooling tower will contain a plant to concentrate the chemicals in the rejected water and to recycle most of it. This will allow the amount of make-up water, compared to today, to be reduced by about $50 \%$ and the rejected volume by more than $70 \%$.

\subsubsection{Operational parameters}

The design parameters for the cooling plants are:

- primary circuit: $40^{\circ} \mathrm{C}$ at the inlet of the cooling towers and $25^{\circ} \mathrm{C}$ at their outlet; - secondary circuit: $42^{\circ} \mathrm{C}$ at the inlet of the heat exchanger and $27^{\circ} \mathrm{C}$ at its outlet.

The temperature difference between inlet and outlet is $15 \mathrm{~K}$ with a tolerance of about $0.5 \mathrm{~K}$. Tables 5.1 and 5.2 list the total power and the nominal diameter for the circuits in the underground area and at the surface of each point, respectively.

The diameter of pipework in the tunnel is optimised with respect to the pressure loss of the circuit due to its length. This permits the reduction of the pressure rating for the pipeline or to avoid installing booster pumps at regular intervals in the sectors. 
Table 5.1. Cooling power and pipe diameter for circuits in the FCC-hh underground areas.

\begin{tabular}{|c|c|c|c|c|c|c|c|c|}
\hline Point & & Cryogenics & Experiment & $\begin{array}{c}\text { Power } \\
\text { converters }\end{array}$ & $\mathbf{R F}$ & $\begin{array}{c}\text { Tunnel } \\
\text { left }\end{array}$ & $\begin{array}{c}\text { Tunnel } \\
\text { right }\end{array}$ & $\begin{array}{c}\text { Total } \\
\text { underground }\end{array}$ \\
\hline PA & P (MW) & 1.5 & 2.7 & 0.15 & & 0.7 & 0.7 & $\mathbf{5 . 8}$ \\
\hline & ND (mm) & 125 & 150 & 50 & & 150 & 150 & $\mathbf{2 5 0}$ \\
\hline PB, PL & P (MW) & & 2.0 & 0.3 & & & & $\mathbf{2 . 3}$ \\
\hline & ND (mm) & & 200 & 80 & & & & $\mathbf{2 0 0}$ \\
\hline PC, PK & P (MW) & 2.9 & & 0.3 & & 1.4 & 1.7 & $\mathbf{6 . 3}$ \\
\hline & ND (mm) & 200 & & 80 & & 200 & 200 & $\mathbf{2 5 0}$ \\
\hline PD, PJ & P (MW) & & & 0.3 & & & & $\mathbf{0 . 3}$ \\
\hline & ND (mm) & & & 80 & & & & $\mathbf{8 0}$ \\
\hline PE, PI & P (MW) & 2.9 & & 0.3 & & 1.6 & 1.3 & $\mathbf{6 . 1}$ \\
\hline & ND (mm) & 200 & & 80 & & 200 & 200 & $\mathbf{2 5 0}$ \\
\hline PF & P (MW) & & & 0.14 & & & & $\mathbf{0 . 1 4}$ \\
\hline & ND (mm) & & & 50 & & & & $\mathbf{5 0}$ \\
\hline PG & P (MW) & 1.5 & 2.7 & 0.14 & & 0.65 & 0.65 & $\mathbf{5 . 6}$ \\
\hline & ND (mm) & 125 & 150 & 50 & & 100 & 100 & $\mathbf{2 5 0}$ \\
\hline PH & P (MW) & 1.5 & & 0.14 & 4.5 & & & $\mathbf{6 . 1}$ \\
\hline & ND (mm) & 125 & & 50 & 250 & & & $\mathbf{2 5 0}$ \\
\hline
\end{tabular}

Notes. "Tunnel left" designates the counter-clock-wise adjacent machine tunnel sector; "Tunnel right" the clock-wise adjacent machine tunnel sector.

Table 5.2. Cooling power and pipe diameter for circuits at FCC-hh surface sites ("Total underground" is identical to the last column of Tab. 5.1).

\begin{tabular}{|c|c|c|c|c|c|c|c|}
\hline Point & & Cryogenics & Experiment & $\begin{array}{c}\text { Gen. } \\
\text { services }\end{array}$ & $\begin{array}{c}\text { Chilled } \\
\text { water }\end{array}$ & $\begin{array}{c}\text { Total } \\
\text { underground }\end{array}$ & $\begin{array}{c}\text { Total } \\
\text { point }\end{array}$ \\
\hline PA & P (MW) & 22.0 & 10.5 & 2.0 & 5.8 & 5.8 & $\mathbf{4 6 . 2}$ \\
\hline & ND $(\mathrm{mm})$ & 400 & 300 & 200 & 350 & 250 & $\mathbf{6 0 0}$ \\
\hline PB, PL & P (MW) & & 7.3 & 2.0 & 3.1 & 2.3 & $\mathbf{1 4 . 7}$ \\
\hline & ND (mm) & & 300 & 200 & 250 & 200 & $\mathbf{3 5 0}$ \\
\hline PC, PK & P (MW) & 43.0 & & 2.0 & 5.5 & 6.3 & $\mathbf{5 6 . 8}$ \\
\hline & ND (mm) & 650 & & 200 & 350 & 250 & $\mathbf{6 5 0}$ \\
\hline PD, PJ & P (MW) & & & 2.0 & 1.6 & 0.3 & $\mathbf{3 . 9}$ \\
\hline & ND (mm) & & & 200 & 200 & 80 & $\mathbf{2 0 0}$ \\
\hline PE, PI & P (MW) & 43 & & 2.0 & 5.5 & 6.1 & $\mathbf{5 6 . 6}$ \\
\hline & ND (mm) & 600 & & 200 & 300 & 250 & $\mathbf{6 5 0}$ \\
\hline PF & P (MW) & & & 2.0 & 1.5 & 0.14 & $\mathbf{3 . 6}$ \\
\hline & ND (mm) & & & 200 & 200 & 50 & $\mathbf{2 5 0}$ \\
\hline PG & P (MW) & 22 & 10.5 & 2.0 & 5.8 & 5.6 & $\mathbf{4 5 . 9}$ \\
\hline & ND $(\mathrm{mm})$ & 400 & 300 & 200 & 400 & 250 & $\mathbf{6 0 0}$ \\
\hline PH & P (MW) & 0.6 & & 2.0 & 1.9 & 6.1 & $\mathbf{1 0 . 7}$ \\
\hline & ND $(\mathrm{mm})$ & 100 & & 200 & 200 & 250 & $\mathbf{3 5 0}$ \\
\hline
\end{tabular}


Table 5.3. Main characteristics of chilled water circuits.

\begin{tabular}{|c|c|c|c|c|}
\hline Point & $\begin{array}{c}\text { Cooling } \\
\text { power }(\mathbf{k W})\end{array}$ & $\begin{array}{c}\text { Flow } \\
\text { rate }\left(\mathbf{m}^{\mathbf{3}} \mathbf{/ h}\right)\end{array}$ & $\begin{array}{c}\text { Number of } \\
\text { chillers }\end{array}$ & $\begin{array}{c}\text { Cooling power/ } \\
\text { chiller }(\mathbf{k W})\end{array}$ \\
\hline PA & 4410 & 633 & 5 & 1100 \\
\hline PB, PL & 2340 & 336 & 3 & 1200 \\
\hline PC, PK & 4150 & 595 & 5 & 1100 \\
\hline PD, PJ & 1220 & 175 & 2 & 1200 \\
\hline PE, PI & 4130 & 593 & 5 & 1100 \\
\hline PF & 1140 & 163 & 2 & 1200 \\
\hline PG & 4360 & 626 & 5 & 1100 \\
\hline PH & 1480 & 212 & 3 & 750 \\
\hline
\end{tabular}

\subsubsection{Chilled water}

The cooling for ventilation plants (dehumidification or air cooling) will require the installation of chilled water production stations at each surface point and some distribution circuits for the air handling units on the surface and in the underground areas. At present, no chilled water is needed in the accelerator arcs.

The chilled water will be produced at a temperature of $6^{\circ} \mathrm{C}$ and return at $12^{\circ} \mathrm{C}$. The chillers will be water cooled and connected to the cooling towers at each point.

The redundancy level for the cooling circuits ensures continuous operation in the case of a breakdown of a single element (chiller or distribution pump). In case of a general power failure, a buffer tank in each production circuit will ensure sufficient autonomy of part of the plant for a limited period of time. The distribution pumps will therefore be connected to the secure electrical network.

Table 5.3 presents the total power and the main characteristics of the chilled water circuits at each point.

\subsubsection{Drinking water}

Drinking water will be used by personnel and as make-up water for cooling towers. The current baseline scenario includes provisioning by the local water network at each point. If the water network does not have enough capacity to provide the required flow for the cooling tower make-up, this water will be provided from the closest point with sufficient capacity via a pipeline in the tunnel. In such cases, the same pipe will also be used for fire fighting purposes in the sector concerned.

\subsubsection{Fire fighting network}

It is planned to install a water network for fire fighting purposes in the underground areas and tunnel. This will comprise a pipe connected to fire hoses with Storz connections at regular intervals. In the case of major damage to this pipe, valves installed along the sector will allow isolation of the damaged part whilst maintaining the rest of the circuit operational for the fire brigade. It is planned to keep this pipe dry to avoid stagnation and corrosion during normal operation, In case of a fire, water will be supplied from the surface by opening isolation valves. Each sector can be supplied by the adjacent points, thereby guaranteeing a redundant supply.

Surface premises will be protected by a hydrant network and by dedicated water hoses inside the buildings where necessary. 
It is planned that the fire fighting water will be delivered by the local public water network at each point. If this network is not able to deliver the required water flow, volume or redundancy level, the water can be supplied from the adjacent point via a pipe in the tunnel.

\subsubsection{Reject water}

Two separate pump systems to lift clear water and sewage will be installed underground at each point. They will be connected to the local drainage network of the point. All underground equipment (tunnel and caverns) must be redundant in order to avoid affecting operation in case of breakdown. Alarms for "high level" and "level too high" will be implemented in all basins.

The main parameters (e.g. temperature, $\mathrm{pH}$ ) of the rejected water will be monitored before release. If the rejected water does not comply with the quality level required, or it presents a risk of environmental pollution, compensatory measures, such as retention basins at each point, will be implemented.

\subsubsection{Compressed air}

The compressed air for all equipment and actuators will be provided by compressed air stations located on the surface at each point. These will supply both surface and underground premises. It is planned to have a level of redundancy of $N+1$ to ensure the reliability and maintainability of the plant.

\subsection{Heating, ventilation, air conditioning}

\subsubsection{Overall design concept}

The installations have to provide fresh air for personnel working within the facilities as well as heating for the working environment. At the same time, the ambient temperature has to be suitable for the installed accelerator equipment. It is important that the air supplied has been dehumidified in order to prevent condensation on equipment and structures. In addition to being able to purge the air in the tunnel before access is allowed, the extraction systems have to be capable of removing smoke and gases. The extracted volumes have to be filtered before release.

\subsubsection{Interior conditions}

The interior conditions to be provided by the ventilation system are the following:

- FCC-hh tunnels (with maximum heat load): $\max .32^{\circ} \mathrm{C}$;

- Experiment caverns: $18 / 32^{\circ} \mathrm{C}$ - from floor to ceiling;

- Surface buildings with controlled temperature: $18^{\circ} \mathrm{C}$ during winter, $25^{\circ} \mathrm{C}$ during summer.

The values for surface buildings are mean values at heights where people and equipment are expected.

The relative humidity does not need to be regulated except for some specific areas which might require it (Faraday cage, clean rooms or other laboratories). If needed, 


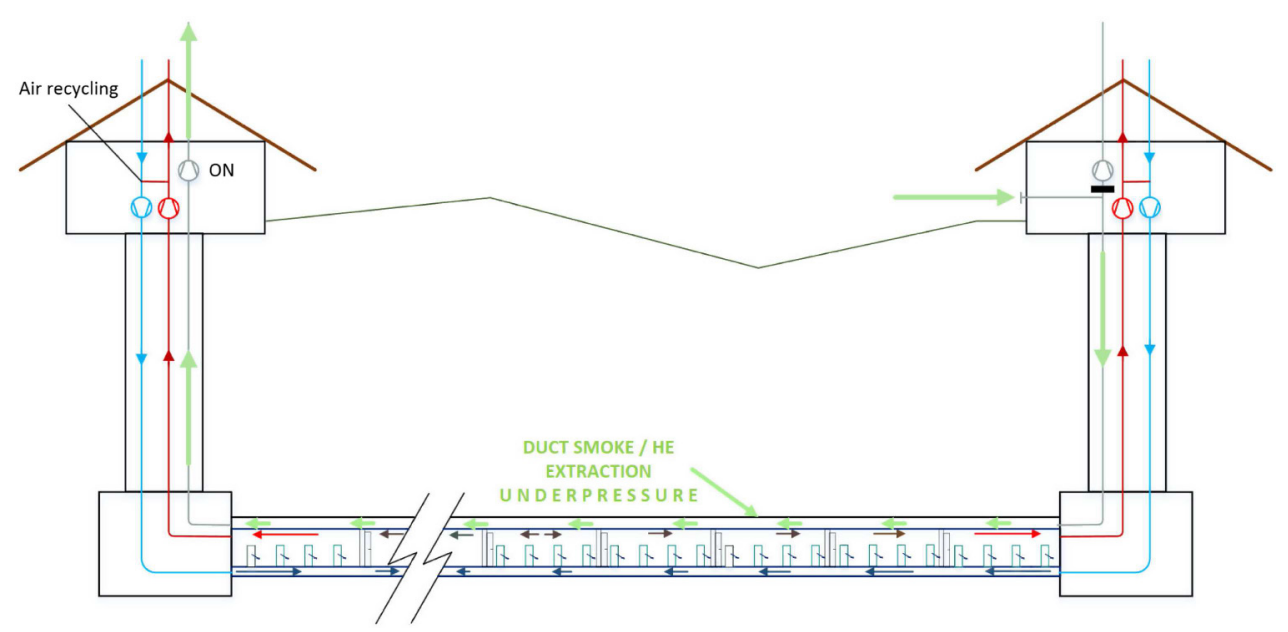

Fig. 5.2. Schematic view of the main ventilation elements of a machine sector.

such systems will be designed at a later stage. The dew point will be kept below $12^{\circ} \mathrm{C}$ to avoid condensation.

The outdoor conditions for the Geneva region which were used to specify the air handling equipment are $32^{\circ} \mathrm{C}$ for dry bulb temperature and $40 \%$ for relative humidity during summer and $-12^{\circ} \mathrm{C}$ and $90 \%$ during winter.

As a general principle, a free cooling and air recycling approach will be adopted in order to reduce the electrical consumption (the term "free cooling" refers to the use of outside air at ambient temperature to cool equipment or areas, instead of using artificial air cooling).

\subsubsection{Ventilation of underground areas}

The underground areas are generally ventilated by air handling units located on the surface, which are therefore accessible at all times. It is planned to have redundant units (level $N+1$ ) everywhere in order to avoid affecting accelerator operation if there is a breakdown. One of the two air extraction units will not be equipped with filters since it will be used to extract smoke, which could clog them. All systems related to safety will be powered by the secure electrical network.

\subsubsection{Machine tunnel}

The tunnel will be compartmentalised every $440 \mathrm{~m}$ by fixed fireproof panels and mobile doors, to control the propagation of smoke in case of fire or of helium gas in case of an accidental leak on a magnet cryostat, and RF module or the cryogenic distribution line. In addition, a semi-transverse ventilation scheme has been adopted. The air is supplied via a specific duct throughout the sector and extracted either through the tunnel itself, or by an emergency extraction duct. Air is supplied to each sector from both end points to ensure an air supply even if there is a duct failure; the same configuration has been adopted for the extraction. In the case of the failure of one unit, the other will accelerate to ventilate both adjacent sectors. A schematic view of the main ventilation elements of a sector of the machine is given in Figure 5.2.

The air supply duct runs in the concrete floor slab and supplies air to the tunnel about every $100 \mathrm{~m}$ via diffusers at floor level. For emergency extraction, a closed 


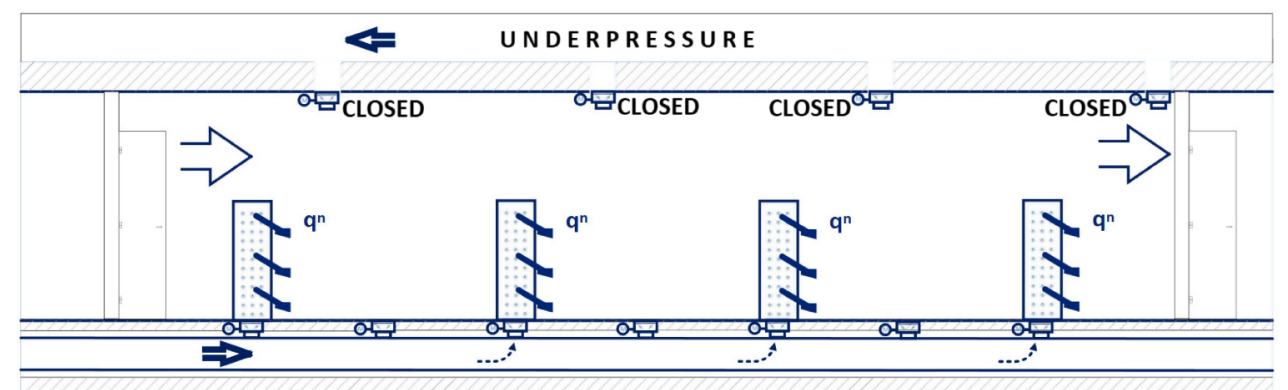

Fig. 5.3. Operation of the ventilation elements in one compartment of the machine tunnel during normal operation.

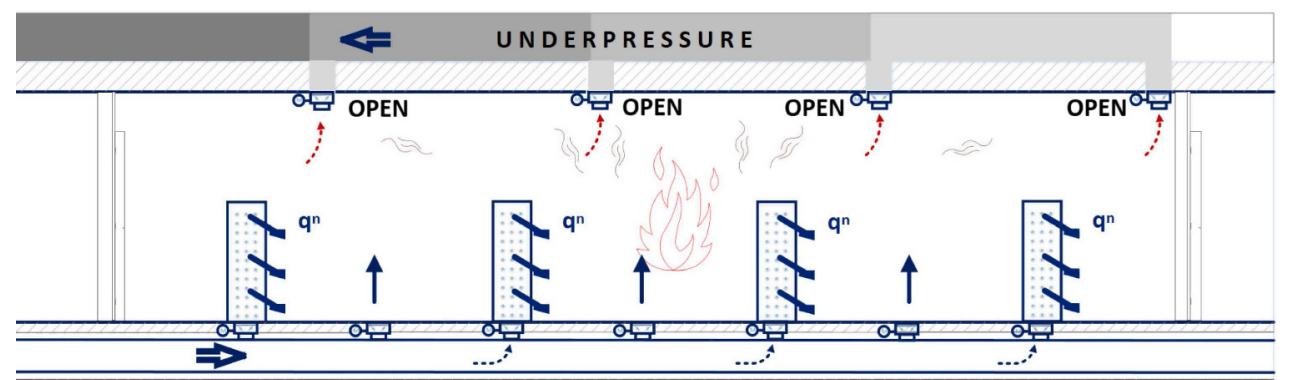

Fig. 5.4. Operation of the ventilation elements in one compartment of the machine tunnel in an emergency situation (in this case a fire).

circular segment in the upper part of the tunnel is used. It is formed by a $70 \mathrm{~mm}$ thick steel structure with passive fire protection on both sides, connected to the lining using post-drilled anchors (Fig. 4.4). Inlet diffusers and extraction grills are offset with respect to each other in order to ensure better distribution of the air in the tunnel and to avoid shortcuts between supply and extraction. Fire resistant dampers will be installed at every connection with diffusers and grills for the extraction. In the case of a fire or helium release, they will allow better management of the ventilation in the concerned tunnel compartment.

During normal operation, all emergency extraction dampers are closed and the doors are open in the whole sector (Fig. 5.3). The emergency duct will be underpressured by extracting air from one extremity of the sector. When smoke or helium is detected, only the dampers of the extraction duct in the compartment affected and the adjacent ones will open; the doors of these compartments will be closed (Fig. 5.4). Additional air supply to the affected and adjacent compartments will be achieved by opening other dampers in the supply duct. For the remaining compartments, the air supply will still be maintained. The extraction will be done via different air handling units on the surface.

\subsubsection{Experiment caverns}

For the ventilation of the experiment caverns, the air is blown in through diffusers at floor level (or the different floor levels) and extracted through one or more ducts located on the ceiling. Dedicated gas extraction systems will be installed where needed. 
Table 5.4. Operating modes and conditions for ventilation systems.

\begin{tabular}{|c|c|}
\hline Mode & Conditions \\
\hline Run & No access, accelerators running and equipment powered, full air recycling \\
\hline Shutdown & $\begin{array}{c}\text { Open access, accelerator stopped, maintenance interventions, fresh } \\
\text { air/partial recycling }\end{array}$ \\
\hline Purge & $\begin{array}{l}\text { Where needed, before allowing access to personnel, accelerator stopped, } \\
\text { fresh air }\end{array}$ \\
\hline
\end{tabular}

Table 5.5. Working parameters for the ventilation of a long machine tunnel sector.

\begin{tabular}{|c|c|}
\hline Air flow from each side in run and shutdown modes $\left(\mathrm{m}^{3} / \mathrm{h}\right)$ & 25000 \\
\hline Air flow from each side in purge mode $\left(\mathrm{m}^{3} / \mathrm{h}\right)$ & 50000 \\
\hline Number of diffusers and extraction grills per compartment & 4 \\
\hline Air flow per diffuser $\left(\mathrm{m}^{3} / \mathrm{h}\right)$ & 520 \\
\hline Supply duct nominal diameter $(\mathrm{mm})$ & 1200 \\
\hline
\end{tabular}

\subsubsection{Other areas}

Local air handling units will be added in areas housing equipment with particularly high heat dissipation. These units will be fitted with coils cooled by chilled water produced on the surface.

\subsubsection{Operating modes}

It is planned to have different modes for the ventilation systems depending on the machine operating conditions; these are presented in Table 5.4. All motors for ventilators will be equipped with variable speed drives in order to adjust the flow rates, to adapt the working conditions to the operational needs and to achieve the requested dynamic confinement between adjacent areas, where requested.

\subsubsection{Working parameters}

Table 5.5 shows the main ventilation parameters of a long FCC-hh tunnel sector, e.g. sector PH-PI. The parameters of the ventilation plants for the underground points are presented in Table 5.6.

The dissipated heat that needs to be removed by the ventilation systems for various types of equipment on the surface is shown in Table 5.7. Table 5.8 specifies the underground thermal loads.

\subsubsection{Ventilation of surface buildings}

Each surface building will be ventilated by a dedicated air handling unit. Where the building size requires it, it is planned to have several units in the same building, each of them taking care of a part of the building.

At present, it is not considered necessary to have redundant units in these buildings. Should this be needed, redundancy can easily be implemented. All surface buildings will be equipped with a mechanical system on the roof to extract smoke, designed and certified for operation at $400^{\circ} \mathrm{C}$ for a minimum period of $2 \mathrm{~h}$. 
Table 5.6. Working parameters of air handling units for underground areas.

\begin{tabular}{|c|c|c|c|}
\hline $\begin{array}{c}\text { Underground } \\
\text { structure }\end{array}$ & $\begin{array}{l}\text { Nominal flow } \\
\text { rate }\left(\mathrm{m}^{3} / \mathrm{h}\right)\end{array}$ & $\begin{array}{l}\text { Nominal duct } \\
\text { diameter }(\mathrm{mm})\end{array}$ & $\begin{array}{c}\text { Air } \\
\text { recycling }\end{array}$ \\
\hline Shaft and safe area pressurisation & 45000 & 1200 & No \\
\hline $\begin{array}{l}\text { Ventilation of service caverns in } \\
\text { experiment points }(\mathrm{PA}, \mathrm{PB}, \mathrm{PG} \text {, } \\
\mathrm{PL})\end{array}$ & 45000 & 1200 & Possible \\
\hline $\begin{array}{l}\text { Ventilation of service caverns in } \\
\text { technical points (PC, PE, PI, PK) }\end{array}$ & 15000 & 1000 & No \\
\hline $\begin{array}{c}\text { Ventilation of collimation areas } \\
\text { (PF, PJ) }\end{array}$ & 6000 & 700 & No \\
\hline Ventilation of $\mathrm{RF}$ area $(\mathrm{PH})$ & 6000 & 700 & Possible \\
\hline Ventilation of dump areas (PD) & 6000 & 700 & No \\
\hline
\end{tabular}

Table 5.7. Main heat dissipation on surface (in $(\mathrm{kW}))$.

\begin{tabular}{|c|c|c|c|}
\hline Point & Cryogenics & Experiment & General services \\
\hline PA, PG & 1100 & 1050 & 500 \\
\hline PB, PL & & 730 & 500 \\
\hline PC, PE, PI, PK & 2150 & & 500 \\
\hline PD, PF, PJ & & & 500 \\
\hline PH & 30 & & 500 \\
\hline Total & $\mathbf{1 0 8 3 0}$ & $\mathbf{3 5 6 0}$ & $\mathbf{6 0 0 0}$ \\
\hline
\end{tabular}

Table 5.8. Main heat dissipation underground in (kW) ("Adjacent Sector" designates the adjacent clock-wise sector).

\begin{tabular}{|c|c|c|c|c|c|c|c|c|}
\hline Point & Dump & Cryogenics & Experiment & $\begin{array}{c}\text { Power } \\
\text { converters }\end{array}$ & $\mathbf{R F}$ & Collimator & $\begin{array}{c}\text { Adjacent } \\
\text { Sector }\end{array}$ & $\begin{array}{c}\text { Underground } \\
\text { cooling } \\
\text { station area }\end{array}$ \\
\hline $\mathrm{PA}$ & & 75 & 370 & 7 & & & 110 & 200 \\
\hline $\mathrm{PB}$ & & & 300 & 14 & & & 200 & \\
\hline $\mathrm{PC}$ & & 140 & & 14 & & & 240 & 200 \\
\hline $\mathrm{PD}$ & 50 & & & 14 & & & 220 & \\
\hline $\mathrm{PE}$ & & 140 & & 14 & & & 190 & 200 \\
\hline $\mathrm{PF}$ & & & & 7 & & 50 & 100 & \\
\hline $\mathrm{PG}$ & & 70 & 370 & 7 & & & 100 & 200 \\
\hline $\mathrm{PH}$ & & 75 & & 7 & 225 & & 190 & \\
\hline $\mathrm{PI}$ & & 145 & & 14 & & & 220 & 200 \\
\hline PJ & & & & 14 & & 50 & 200 & 200 \\
\hline $\mathrm{PK}$ & & 145 & & 14 & & & 240 & \\
\hline PL & & & 300 & 14 & & & 110 & \\
\hline Total & 50 & 790 & 1340 & 140 & 225 & 100 & 2120 & 1200 \\
\hline
\end{tabular}




\subsubsection{Safety}

In general, smoke extraction is planned for all facilities with a risk due to fire loads or where it is necessary to ensure the safety of personnel. If there is a fire, in addition to the automatic actions, the fire brigade will be able to switch off or manually reconfigure the ventilation system.

All air supply handling units are equipped with smoke detection sensors downstream of the ventilator in order to avoid injection of smoke into underground areas.

The concrete module for the lift and staircase in the shafts is kept over-pressure with respect to the surrounding underground areas and will therefore be used as a safe area in emergencies.

In compliance with safety norms, to prevent the passage of activated air from areas with higher levels of activation to areas with lower levels, a pressure cascade will be established. Therefore it is planned that the machine tunnel will be at a lower pressure with respect to the experiment caverns and adjacent areas. Volumes with higher activation risk are separated from less activated areas by airlocks kept pressurised by dedicated fans installed in the less activated areas.

Exhaust air ducts will have branches to connect air monitoring equipment for radiation protection monitoring of the air before its release into the atmosphere.

\subsection{Electricity distribution}

\subsubsection{Conceptual layout}

The concept for the design of the FCC-hh electrical network is driven by three factors:

- The estimated electrical power requirements (Tab. 9.1 in Sect. 9.2);

- The location and type of equipment to be supplied;

- The expected level of electrical network availability and operability.

The electrical network is composed of a transmission and a distribution level. The transmission level transmits the power from three sources to three FCC-hh points and between the 12 FCC-ee points. This level operates at voltages of $400 \mathrm{kV}$ and $135 \mathrm{kV}$. The distribution level distributes the power from the transmission level to the end users at medium and low voltage levels comprised between $36 \mathrm{kV}$ and $400 \mathrm{~V}$. The present baseline uses conventional AC schemes. Emerging new technologies based on DC schemes, which could improve the power quality and power consumption efficiency, are presented in Section 12.11.

\subsubsection{Source of electrical energy}

The estimated $580 \mathrm{MW}$ electrical power requirement of FCC-hh is supplied from the European grid. The current configuration of the European grid provides three $400 \mathrm{kV}$ sources in France in the area of the collider facilities (Fig. 5.5). The three sources are self-redundant and, according to French network provider RTE (Reseau Transport Electricité), each of them is capable of providing $200 \mathrm{MW}$ on top of their current load by the year 2035 (the present maximum daily load by CERN on source I is $191 \mathrm{MW}$ ).

\subsubsection{Transmission network topology}

The transmission network includes:

- The $400 \mathrm{kV}$ transmission lines connecting the three $400 \mathrm{kV}$ sources on the European grid to three incoming substations; 


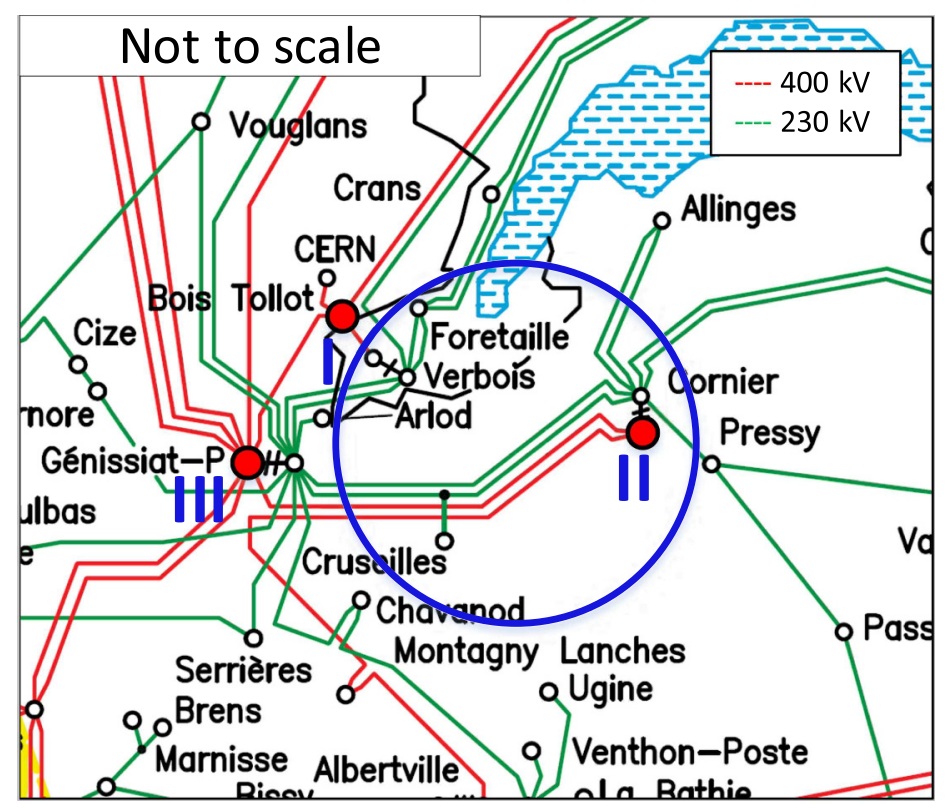

Fig. 5.5. Schematic representation of the $400 \mathrm{kV}$ and $230 \mathrm{kV}$ lines in the region. The blue circle indicates the FCC collider ring. Red dots denote the three possible $400 \mathrm{kV}$ sources.

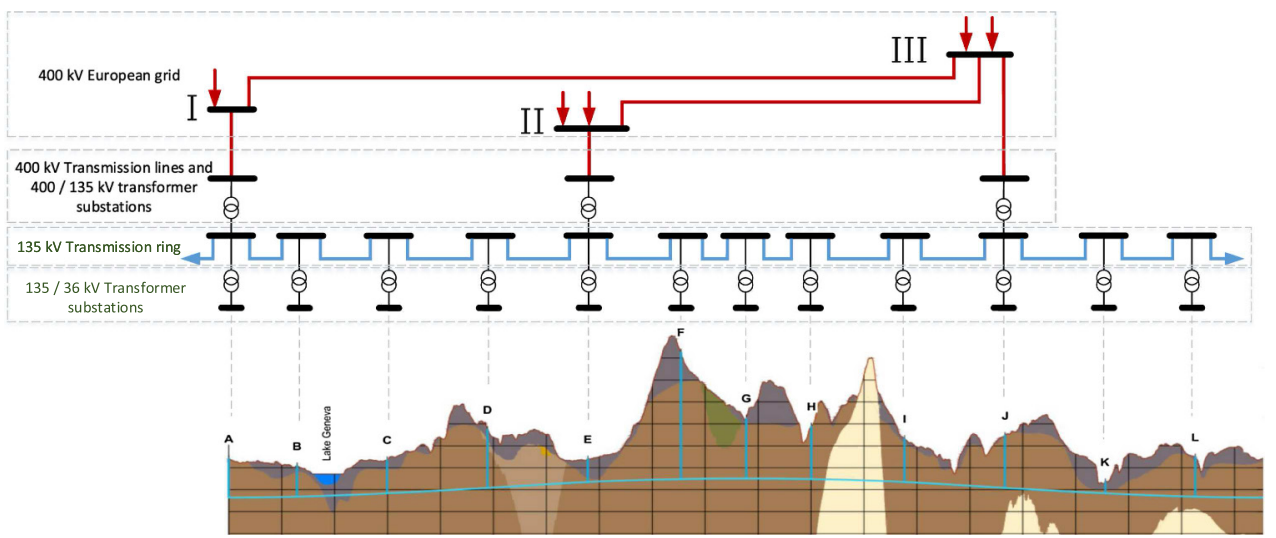

Fig. 5.6. Schematic representation of the transmission network.

- Three 400/135 kV transformer substations;

- The $135 \mathrm{kV}$ transmission line ring composed of 12 segments connecting each of the 12 surface points to its two neighbouring points;

- A $135 / 36 \mathrm{kV}$ transformer substation at each point.

Figure 5.6 shows a schematic view of the transmission network. Analysing the power requirements for each point with nominal beam operation, the highest power is demanded at points PA, PC, PE, PG, PI and PK, each requiring between 53 and $64 \mathrm{MW}$, predominately for the cryogenics plants. The remaining 6 points require less power - between 16 and $38 \mathrm{MW}$ each. The three $400 \mathrm{kV}$ sources supply PA, PD and PJ directly. 


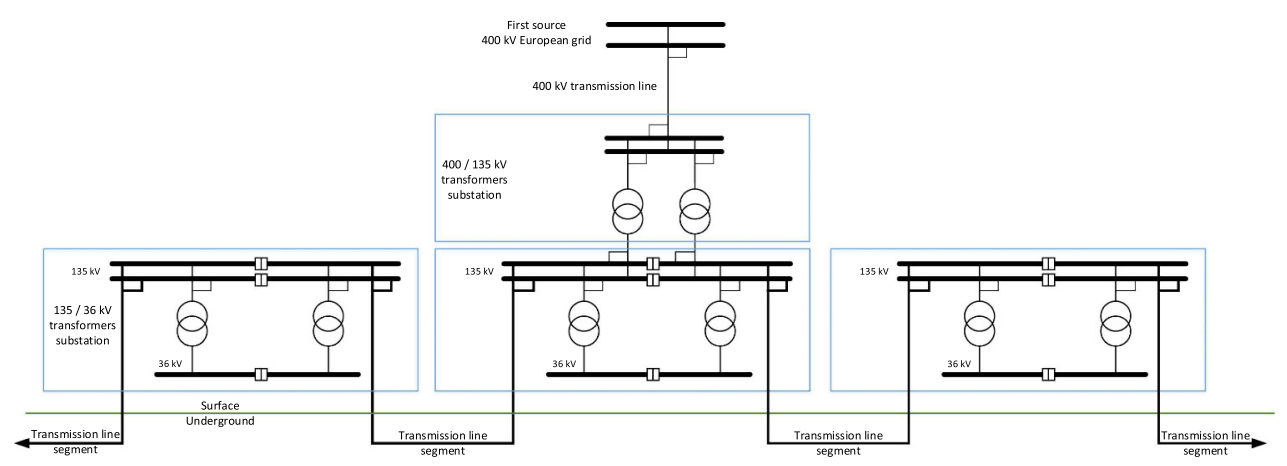

Fig. 5.7. Simplified scheme of a $400 \mathrm{kV}$ incoming substation and the connection to the two $135 \mathrm{kV}$ substations at the adjacent points.

Through the transmission line ring, each of the three incoming substations supplies four neighbouring points. This transmission network layout provides full redundancy, enhanced availability and operability in case of a fault on one of the transmission line segments. A redundant scheme of $400 / 135 \mathrm{kV}$ voltage step-down transformers supplies the transmission line segments connecting two adjacent points. In $\mathrm{PB}, \mathrm{PC}, \mathrm{PD}, \mathrm{PF}, \mathrm{PG}, \mathrm{PH}, \mathrm{PI}, \mathrm{PK}$ and $\mathrm{PL}$ a substation will receive the incoming $135 \mathrm{kV}$ transmission line segments. In all points $135 / 36 \mathrm{kV}$ step-down transformers supply the distribution networks level. Figure 5.7 shows a simplified scheme of a $400 \mathrm{kV}$ incoming substation and the connection to the two adjacent points with the corresponding step-down transformers.

\subsubsection{Distribution network topology}

The distribution networks connect the transmission network to the surface and underground equipment and systems. During nominal operation, the transmission network supplies the distribution network. Alternative sources of supply are needed to meet the required level of network availability and to cope with a degraded scenario such as a disruption of the general or local power supply. Therefore, the distribution network includes a second supply, rated between 2 and 10 MVA, fed from a regional grid node (due to the limited power request one can connect directly to the medium voltage level $-20 \mathrm{kV}$ in France and $18 \mathrm{kV}$ in Switzerland), a third source of supply rated 1-MVA from local diesel power stations and a fourth source which provides uninterruptable power. Figure 5.8 shows the single line diagram of the baseline distribution network of one point including the alternative power sources.

The distribution network is composed of a primary indoor substation comprising five bus bars located on the surface. The incoming feeders are the two redundant $135 / 36 \mathrm{kV}$ transformers supplied from the transmission network, the second supply from a regional source and the third supply from the local diesel power station. The outgoing feeders supply secondary substations. These are located either on the surface or underground, near the load. The operating voltage of the distribution network is typically $36 \mathrm{kV}$ for the power distribution over distances greater than $750 \mathrm{~m}$. Voltage step-down transformers feed end users from the secondary substations over a maximum cable length of $750 \mathrm{~m}$. End users are supplied from the secondary substations at voltage levels between $400 \mathrm{~V}$ for wall plug equipment and $3.3 \mathrm{kV}$ for high power motors for cooling, ventilation and cryogenic systems. 


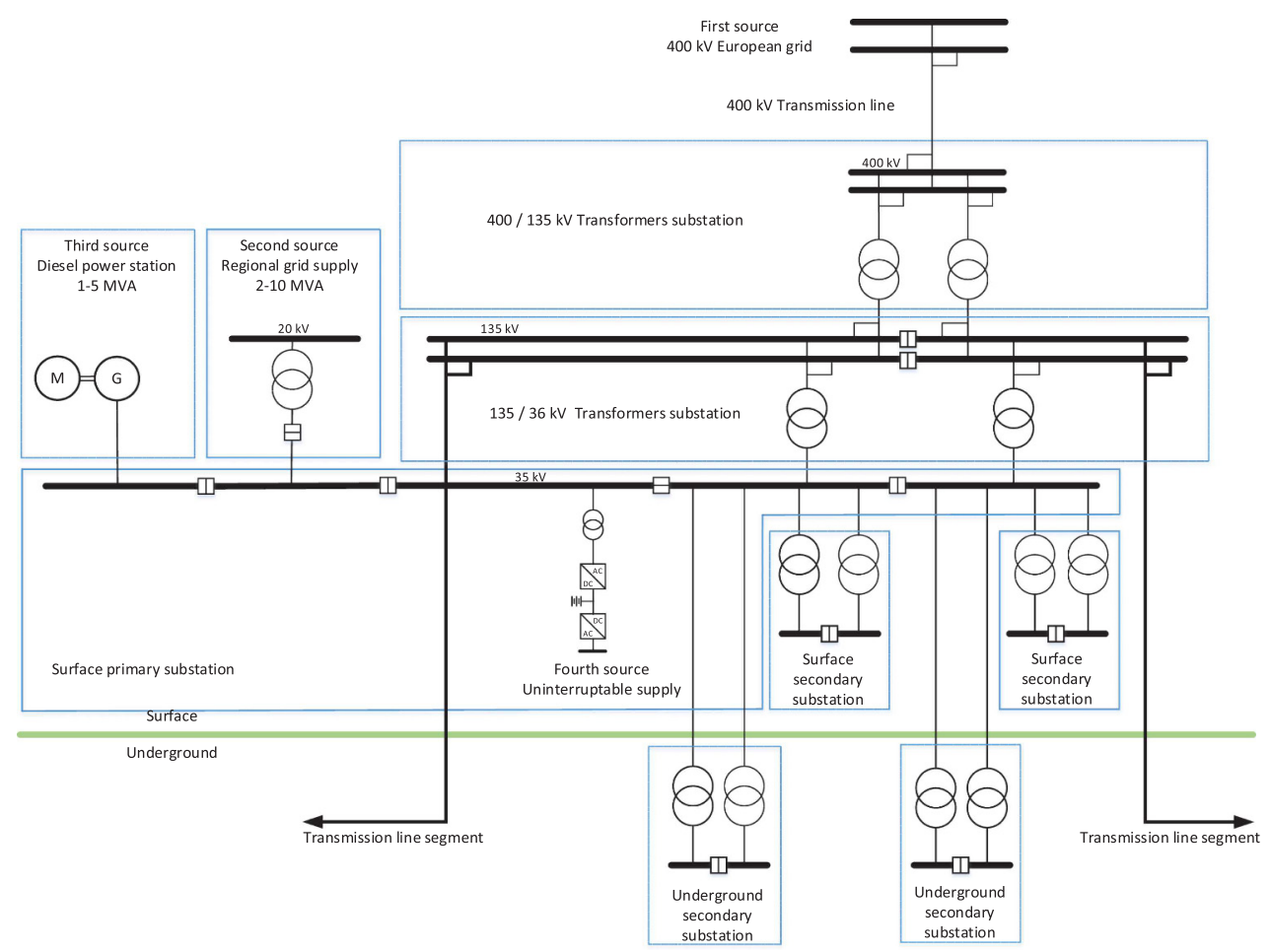

Fig. 5.8. Diagram of the baseline distribution network of one FCC point including the alternative power sources.

\subsubsection{Power quality and transient voltage dip mitigation}

The main issues concerning power quality are voltage stabilisation, harmonic filtering and reactive power compensation as well as the mitigation of transient voltage dips. Transient voltage dips as shown in Figure 5.9, which are typically caused by lightning strikes on the $400 \mathrm{kV}$ network overhead lines, often cause undesired stops of CERN's accelerators. Due to its geographic extent, the FCC-hh infrastructure will be exposed to a higher number of transient network disturbances than the current CERN accelerators. The powering system design must include mitigation measures against these. Extrapolation from LHC operation forecasts a total of 100-200 transient voltage dips per year.

The following mitigation measures are being studied:

- Dynamic Voltage Restorer (DVR) technology: the voltage will be restored by dynamic series injection of the phase voltage between the distribution network and the loads. An integrated energy storage system provides the required energy to restore the load voltage during transient voltage dips (Fig. 5.10a).

- High-Voltage DC (HVDC) back-to-back link: HVDC is a well-established technology for long distance transmission of large powers and for decoupling different high voltage networks. Combined with energy storage, an HVDC system provides performance similar to a very large uninterruptable power supply (UPS). Such a system would prevent transient voltage dips in the $400 \mathrm{kV}$ transmission network from entering the collider network. In addition it would facilitate control of the reactive power (see Fig. 5.10b). 


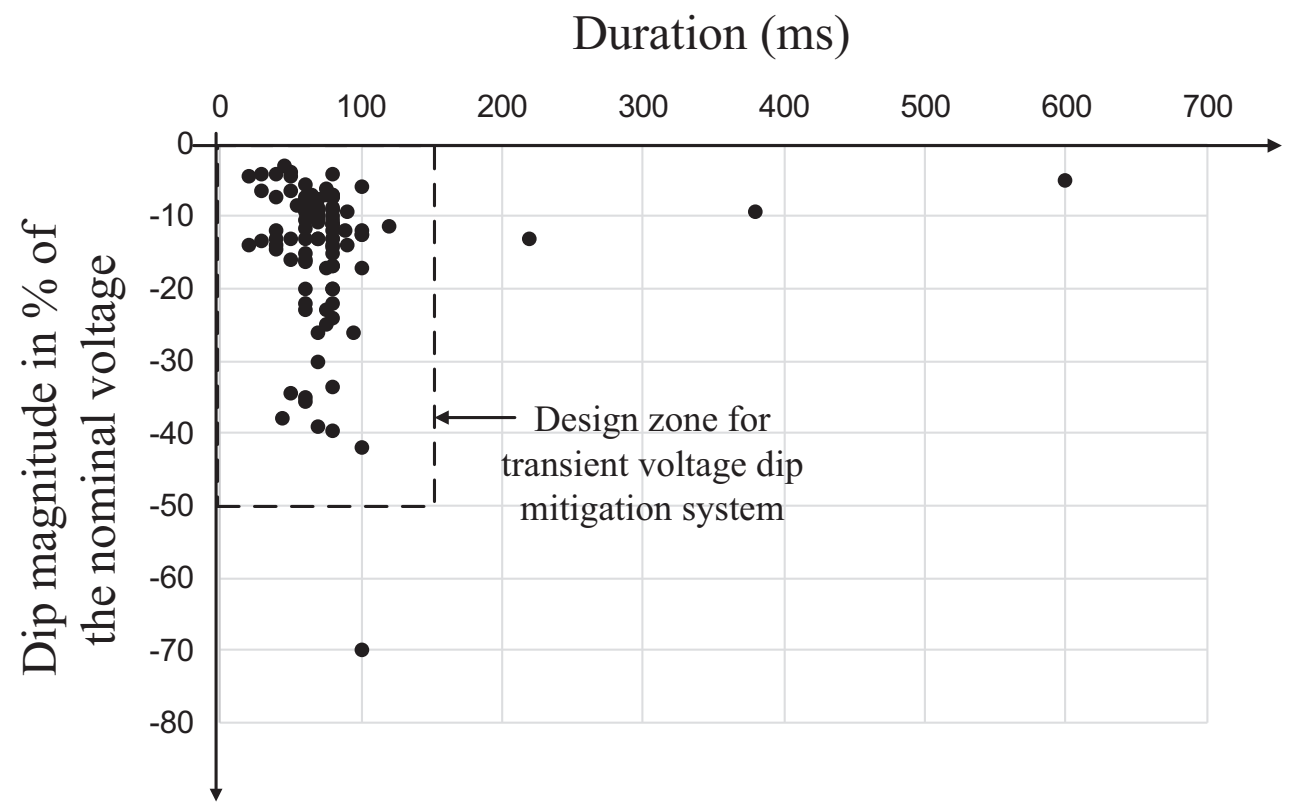

Fig. 5.9. Typical distribution of transient voltage dips recorded within the existing CERN network (collected between 2011 and 2017); the design zone covers most of the transient voltage dips, which are within $0-150 \mathrm{~ms}$ and $0-50 \%$ magnitude.

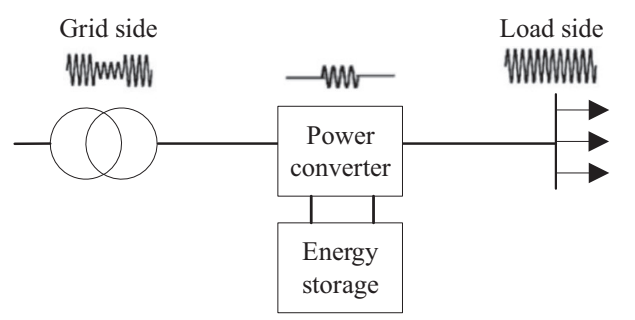

(a)

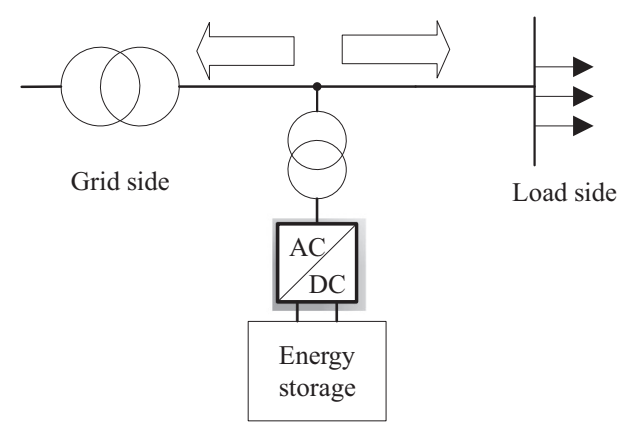

(c)

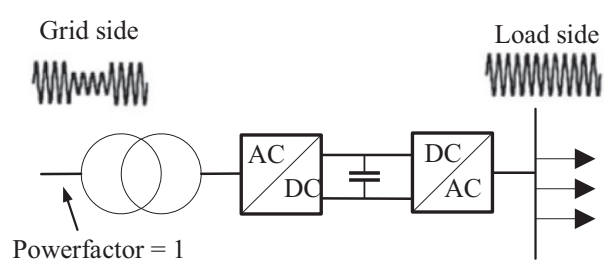

(b)

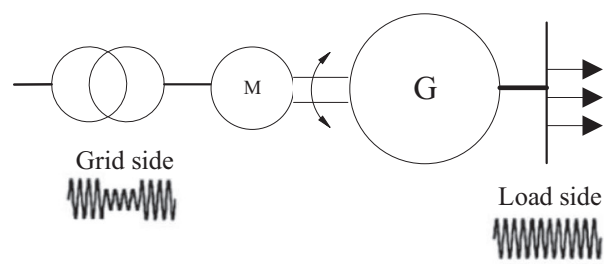

(d)

Fig. 5.10. Simplified layout of various methods for transient voltage dip mitigation. (a) Dynamic Voltage Restorer. (b) HVDC System. (c) STATCOM. (d) Motor-Generator Set. 
Table 5.9. Power quality and transient voltage dip mitigation.

\begin{tabular}{|c|c|c|c|c|c|}
\hline & DVR & $\begin{array}{c}\text { Back-to- } \\
\text { Back }\end{array}$ & DC grid & STATCOM & $\begin{array}{c}\text { Motor- } \\
\text { Generator } \\
\text { Set }\end{array}$ \\
\hline $\begin{array}{c}\text { Transient } \\
\text { voltage dips }\end{array}$ & Covered & Covered & Covered & Covered & Covered \\
\hline $\begin{array}{l}\text { Compensation } \\
\text { of reactive } \\
\text { power on the } \\
\text { load side }\end{array}$ & \begin{tabular}{|c|} 
Not covered, \\
although the \\
resulting \\
voltage \\
deviations on \\
the load side \\
can be \\
compensated
\end{tabular} & Covered & Covered & Covered & Covered \\
\hline $\begin{array}{c}\text { Compensation } \\
\text { of active power } \\
\text { on the load } \\
\text { side }\end{array}$ & Not covered & Covered & Covered & Covered & Covered \\
\hline $\begin{array}{c}\text { AC Harmonic } \\
\text { filtering } \\
\text { capability }\end{array}$ & $\mid \begin{array}{c}\text { Yes (although } \\
\text { additional HF } \\
\text { filter } \\
\text { required) }\end{array}$ & $\begin{array}{c}\text { No } \\
\text { (additional } \\
\text { harmonic } \\
\text { filtering } \\
\text { required) } \\
\end{array}$ & $\begin{array}{c}\text { No (not } \\
\text { necessary) }\end{array}$ & Yes & $\begin{array}{c}\text { No } \\
\text { (additional } \\
\text { harmonic } \\
\text { filtering } \\
\text { required) } \\
\end{array}$ \\
\hline $\begin{array}{l}\text { Steady-state } \\
\text { power losses }\end{array}$ & Very Low & High & Medium & Very Low & Medium \\
\hline $\begin{array}{c}\text { Technology } \\
\text { readiness level }\end{array}$ & $\begin{array}{c}\text { Available in } \\
\text { industry }\end{array}$ & $\begin{array}{l}\text { Available in } \\
\text { industry }\end{array}$ & $\begin{array}{c}\text { Design and } \\
\text { standardisa- } \\
\text { tion } \\
\text { phase }\end{array}$ & $\begin{array}{l}\text { Available in } \\
\text { industry }\end{array}$ & $\begin{array}{l}\text { Available in } \\
\text { industry }\end{array}$ \\
\hline $\begin{array}{l}\text { Protection } \\
\text { aspects }\end{array}$ & $\begin{array}{l}\text { Bypass is } \\
\text { needed }\end{array}$ & $\begin{array}{c}\text { Bypass is } \\
\text { needed }\end{array}$ & $\begin{array}{c}\text { Under } \\
\text { development }\end{array}$ & $\begin{array}{l}\text { Bypass is } \\
\text { needed }\end{array}$ & $\begin{array}{l}\text { Very high } \\
\text { protection }\end{array}$ \\
\hline
\end{tabular}

- Static Synchronous Compensator (STATCOM): this technology is already used for reactive and active power compensation. STATCOM would fully restore the load voltage during transient voltage dips by dynamic shunt (parallel) injection, combined with an integrated energy storage system (Fig. 5.10c).

- Motor-Generator Set: such a system would decouple the network from the load. During transient voltage dips, the load voltage is restored by using the energy stored in a rotating mass (Fig. 5.10d).

- Medium-Voltage DC (MVDC) distribution network: the principle of this approach is the distribution of power using DC. In combination with energy storage, this technology mitigates transient voltage dips, eliminates the reactive power, reduces the distribution losses and, compared to AC distribution, permits a larger spacing between electrical substations in the tunnel. This promising technology is still in its early stage of development and would require considerable R\&D efforts before its use (see Sect. 12.11).

\subsection{Emergency power}

The emergency power concept is based on the requirement to keep essential parts of the accelerator infrastructure operational if the regular power source fails. Par- 
Table 5.10. Load classes and main characteristics.

\begin{tabular}{|c|c|c|}
\hline Load class & $\begin{array}{c}\text { Load type } \\
\text { (non-exhaustive list) }\end{array}$ & $\begin{array}{c}\text { Power unavailability } \\
\text { duration in case of } \\
\text { degraded scenario }\end{array}$ \\
\hline Machine & $\begin{array}{c}\text { Power converters, radio } \\
\text { frequency, cooling } \\
\text { pumps, fan motors }\end{array}$ & $\begin{array}{c}\text { Until return of main } \\
\text { supply }\end{array}$ \\
\hline General Services & $\begin{array}{c}\text { Lighting, pumps, } \\
\text { vacuum, wall plugs }\end{array}$ & $\begin{array}{c}\text { Until return of main or } \\
\text { secondary supply }\end{array}$ \\
\hline Secured & $\begin{array}{c}\text { Personnel safety: } \\
\text { lighting, pumps, wall } \\
\text { plugs, elevators }\end{array}$ & $10-30$ s \\
\hline Uninterruptable & Personnel safety: & Interruptions not \\
& evacuation and & allowed, continuous \\
& anti-panic lighting, & service mandatory \\
& fire-fighting system, & \\
& oxygen deficiency, & \\
& evacuation & \\
& Machine safety: & \\
& sensitive processing & \\
& and monitoring, beam & \\
& loss, beam monitoring, \\
machine protection & \\
\hline
\end{tabular}

ticular emphasis is put on loads related to personnel and machine safety during degraded situations. The various load classes and types are shown in Table 5.10. The main ranking parameters are the acceptable duration of the power interruption and whether the load is part of a personnel or accelerator safety function or not.

Machine loads are energised from the transmission network through the distribution network and do not have a second source of supply. The general services loads typically accept power cuts of several minutes to hours. They can switch to an alternate source or wait until the main source is restored. Secured loads include personnel and machine safety equipment or systems that can only accept short power cuts up to a duration of $30 \mathrm{~s}$. They require three staged supplies. In a degraded situation, the first backup is implemented by a generator power station, which typically starts up within $10 \mathrm{~s}$. If the power station is unavailable, the second backup is implemented by supplying power from the regional grid.

High availability loads require true uninterruptable power supply. The network scheme is composed of two redundant uninterruptable power supply (UPS) systems that are supplied from the distribution network in the two adjacent points. Downstream of the redundant UPS systems, a doubly redundant network delivers two independent sources, each coming from an adjacent point to the end-user plug. Each piece of end-user equipment has two entries and will manage the double source of supply. To meet safety and access requirements, UPS and batteries are located outside the tunnel and above ground. Figure 5.11 shows the functional scheme of the general services loads network and the doubly redundant uninterruptable load network. 


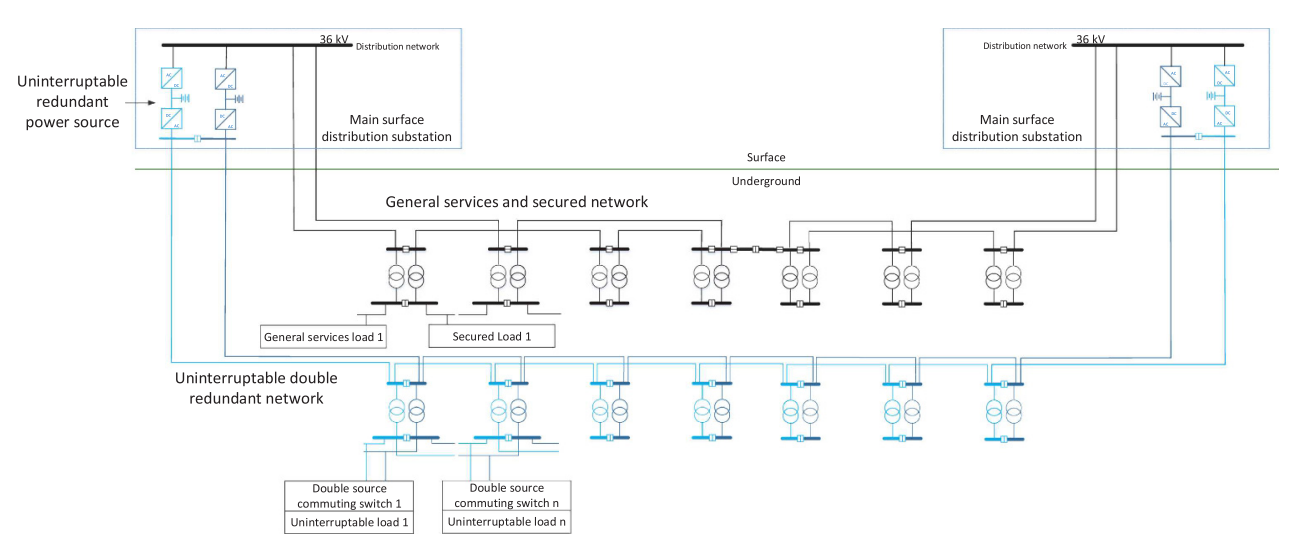

Fig. 5.11. Functional scheme of the general services load network and the doubly redundant uninterruptable load network.

\subsection{Cryogenic system}

\subsubsection{Overview}

The FCC-hh is unique among superconducting synchrotrons because it will be based for the first time on $16 \mathrm{~T}$ superconducting magnets with $\mathrm{Nb}_{3} \mathrm{Sn}$ windings operating at a temperature below $2 \mathrm{~K}$ in order to minimise the amount of superconducting material. In addition, the $50 \mathrm{TeV}$ beam energy will generate a large amount of synchrotron radiation, which for the first time will become the predominant entropic load of the cryogenic system of a superconducting collider [290].

\section{Functions and constraints}

The superconducting magnet windings will be immersed in a pressurised bath of superfluid helium at about $0.13 \mathrm{MPa}(1.3 \mathrm{bar})$ and a maximum temperature of $1.9 \mathrm{~K}$. This provides a sufficient temperature margin for heat transfer across the electrical insulation. As the specific heat of the superconducting material and its copper matrix fall rapidly with decreasing temperature, the very large specific heat of superfluid helium at $1.9 \mathrm{~K}$ (typically $10^{5}$ times that of the conductor per unit mass and $2 \times 10^{3}$ times per unit volume), combined with the heat conductivity at moderate flux (3000 times that of cryogenic grade OFHC copper, peaking at $1.9 \mathrm{~K}$ ) can have a powerful stabilising action on thermal disturbances. This cooling requirement applies during stored beam operation. The temperature excursion during a magnet current ramp-up must remain below $2.1 \mathrm{~K}$. During pre-cycles the temperature excursion may be larger but must still remain below the helium II/helium I phase transition (lambda line).

The cryogenic system must cope with load variations and the large dynamic range induced by operation of the collider. The system must also be able to fill and cool down the cold mass of the machine, $230 \times 10^{6} \mathrm{~kg}$, in less than 20 days, while avoiding thermal gradients higher than $50 \mathrm{~K}$ in the cryomagnet structure. This limit in thermal gradient and time also applies to the forced emptying and warm-up of the machine prior to shutdown periods. The cryogenic system must be able to cope with resistive transitions of the superconducting magnets, which will occasionally occur in the machine, while minimising the loss of cryogen and system perturbations. It must handle the resulting heat release and its consequences, which include fast pressure rises and flow surges. It must limit the propagation to the neighbouring magnets and 


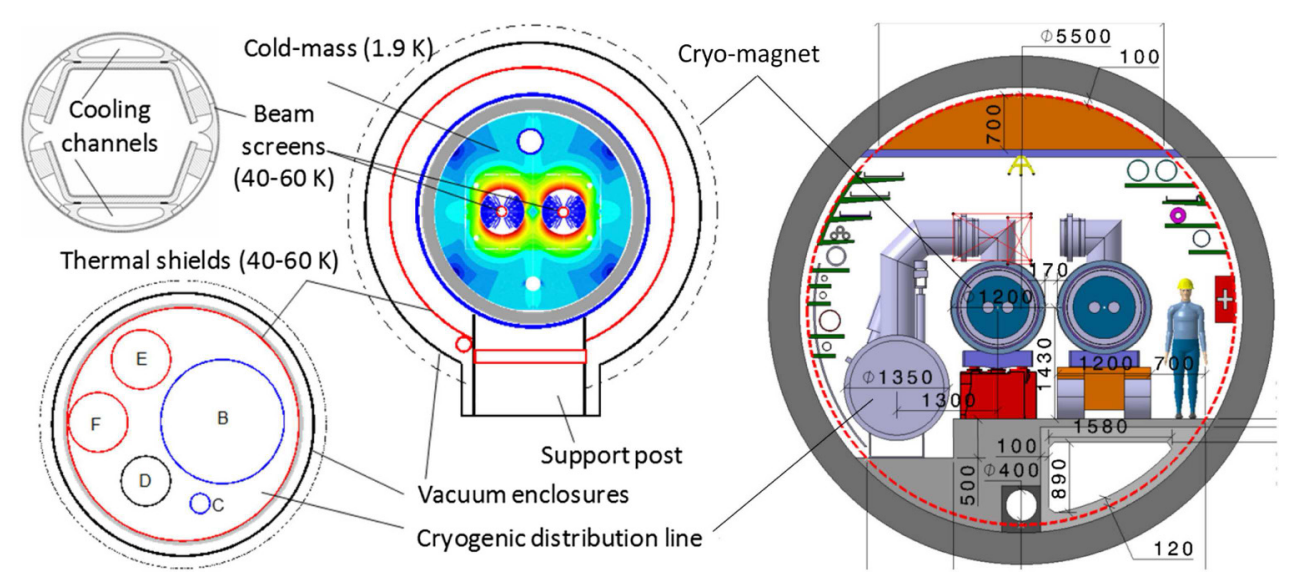

Fig. 5.12. Cross-section of the tunnel and main cryogenic components.

recover in a time that does not seriously affect the operational availability of the facility. A resistive transition extending over one lattice cell should not result in a downtime of more than $8 \mathrm{~h}$. Finally, to ensure reliable operation, the system should have some redundancy among its components and sub-systems.

The cooling power required at each temperature will be produced by 10 refrigeration plants at 6 technical sites and will be distributed to the adjacent sectors over distances of up to $10 \mathrm{~km}$. The number of active cryogenic components distributed around the ring is minimised for reasons of simplicity, reliability and maintenance. To simplify the magnet string design, the cryogenic headers, which distribute the cooling power along a machine sector, and all other active cryogenic components in the tunnel, are contained in a compound cryogenic distribution line (see Fig. 5.12). The cryogenic distribution line runs alongside the magnets in the tunnel and feeds each $107 \mathrm{~m}$ long half-cell in parallel via a jumper connection. Spacing of the jumper connections was optimised to one half-cell. The tunnel is inclined at $0.2 \%$ with respect to the horizontal, thus giving rise to differences in elevation of up to $60 \mathrm{~m}$ across the ring diameter. This will generate hydrostatic heads in the cryogenic headers and could generate instabilities in two-phase, liquid-vapour, flow. All fluids should be transported over large distances in mono-phase state to avoid these harmful instabilities, i.e. in the superheated vapour or supercritical regions of the phase diagram. Local two-phase circulation of saturated liquid, in a controlled direction, can be tolerated over limited distances. Equipment is installed above ground as much as possible to avoid the need for excavation of further large caverns. However, certain components, which must be close to the cryostats, will be installed underground. To limit the effect of the gravity (hydrostatic head and relative enthalpy variation) in the deep areas (up to $274 \mathrm{~m}$ ), the cold part of the helium cycle below $40 \mathrm{~K}$, including cold compressors, must be located in underground caverns.

For safety reasons nitrogen is not used in the tunnel and the discharge of helium is restricted to small quantities. These safety aspects are reflected in specific design features of the system, such as a large acceptance cold recovery header inside the distribution line. The cryogenic system is designed for fully automatic operation. Maintenance will be performed during shutdown periods.

\section{Layout and architecture}

The cryogenic layout of the machine is a direct consequence of the site constraints (see Fig. 5.13). It has 6 cryogenic "islands", where all refrigeration and ancillary 


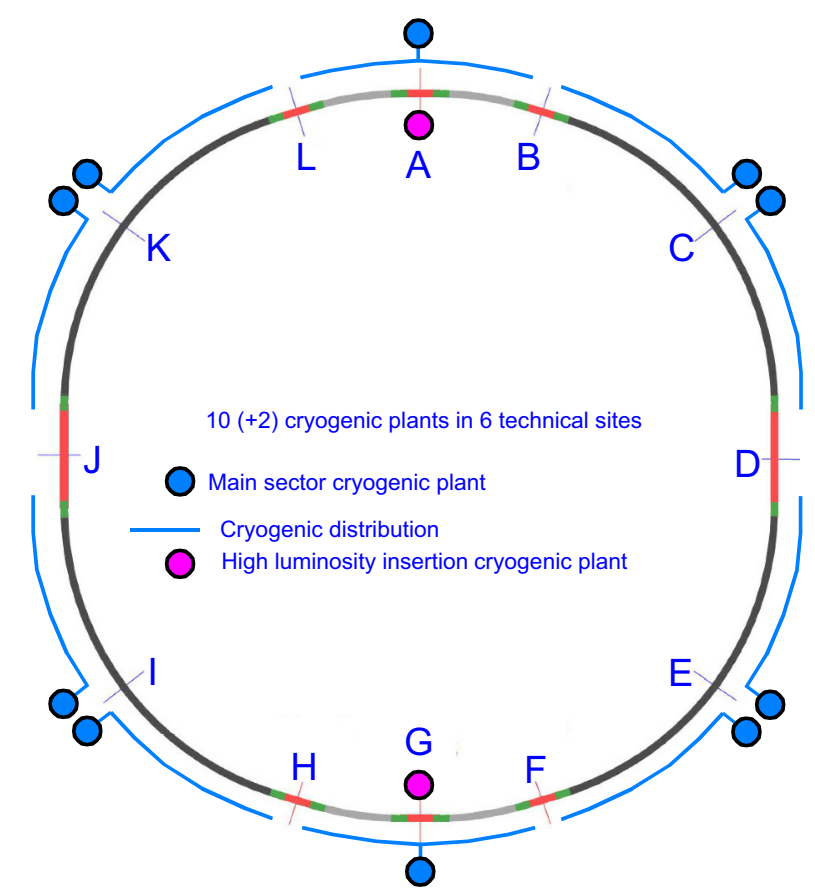

Fig. 5.13. General layout of the cryogenic system.

equipment is concentrated, at points PA, PC, PE, PG, PI and PK. Each site has an electrical substation, a warm compressor station, cryogen storage (helium, neon and liquid nitrogen), cooling towers and cold-boxes. The lower cold-boxes, interconnecting lines, and interconnection boxes are located underground. Each cryogenic island houses one or two refrigeration plants that feed two short $(5 \mathrm{~km})$ or long $(10 \mathrm{~km})$ adjacent tunnel sectors, requiring distribution and recovery of the cooling fluids over distances of $5-10 \mathrm{~km}$ underground. Figure 5.14 shows the general architecture of a cryogenic island. A refrigeration plant comprises one helium refrigerator including a $1.8 \mathrm{~K}$ refrigeration unit and one neon-helium refrigerator for efficient high capacity production above $40 \mathrm{~K}$. At each cryogenic island, an interconnection box couples the various pieces of refrigeration equipment to the cryogenic distribution line. Where possible they also facilitate redundancy of the refrigeration plants. To limit the pressure build-up during helium discharge in case of a sector quench and to mitigate the risks of equipment failures, helium storage is provided at all 12 surface sites.

The cooling of the high-luminosity insertions at Points PA and PG requires additional cryoplants.

\subsubsection{Proximity cryogenics and heat loads}

\section{Temperature levels}

In view of the high thermodynamic cost of refrigeration at $1.8 \mathrm{~K}$, the thermal design aim of the cryogenic components is to intercept the largest fraction of heat loads at higher temperature, hence the multiple, staged temperature levels in the system. The temperature levels are:

- $40 \mathrm{~K}-60 \mathrm{~K}$ for the thermal shield as a first major heat intercept, shielding the cold mass from the bulk of heat in-leaks from the environment; 


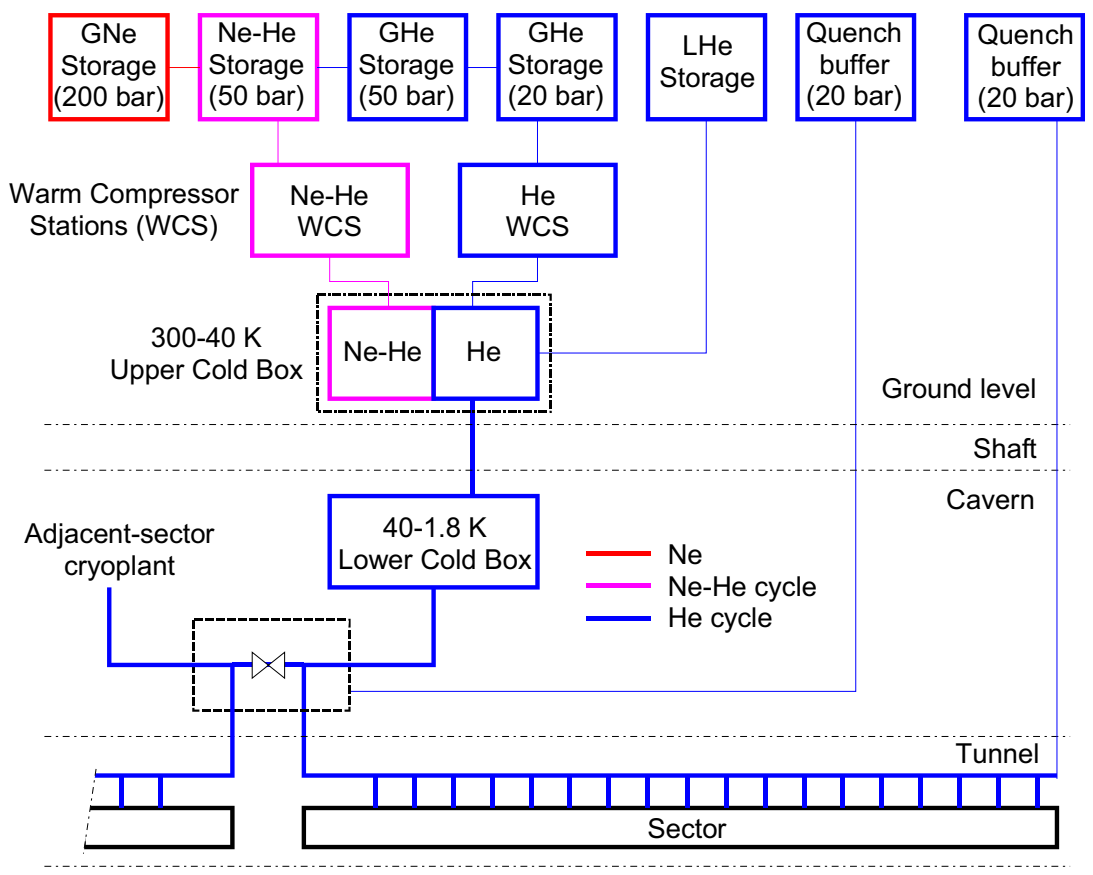

Fig. 5.14. Cryogenic plant architecture.

- $40 \mathrm{~K}-60 \mathrm{~K}$ to cool the beamscreens which protect the magnet cold bore from beam-induced loads;

- $1.9 \mathrm{~K}$ quasi-isothermal superfluid helium to cool the magnet cold mass;

- $4 \mathrm{~K}$ at very low pressure (VLP) to transport the superheated helium coming from the distributed $1.8 \mathrm{~K}$ heat exchanger tubes across the sector to the $1.8 \mathrm{~K}$ refrigeration units;

- 4.5 K normal saturated helium for cooling superconducting radiofrequency cavities and the lower sections of high temperature superconducting (HTS) current leads;

- $40 \mathrm{~K}-300 \mathrm{~K}$ cooling for the resistive upper sections of the HTS current leads.

The cryostats and cryogenic distribution line combine several low temperature insulation and heat interception techniques which will have to be implemented on an industrial scale. These techniques include low-conduction support posts made of nonmetallic fibreglass/epoxy composite, low impedance thermal contacts under vacuum for heat intercepts and multi-layer reflective insulation for wrapping about $1.2 \mathrm{~km}^{2}$ of cold surface below $60 \mathrm{~K}$.

For FCC-hh, the beam-induced heat load is dominated by the $\sim 5 \mathrm{MW}$ of synchrotron radiation in nominal conditions. This beam-induced load must be intercepted at a temperature well above that of the magnet. A thermodynamic optimisation, taking into account the exergetic costs of the beamscreen and cold mass cooling, gives an optimum operating temperature of around $80 \mathrm{~K}$. This temperature is not compatible with the vacuum requirements; the closest operating range compatible with the vacuum requirement is $40-60 \mathrm{~K}$. This operating range reduces the entropic load by a factor of 40 with respect to $1.9 \mathrm{~K}$ isothermal refrigeration and by a factor of 5 with respect to the $4.5-20 \mathrm{~K}$ non-isothermal refrigeration presently used for the LHC beamscreens [291]. Besides their primary function of intercepting beam-induced heat loads at a temperature well above that of the magnets, the beamscreens also act as an intermediate temperature baffle for the cryo-pump resulting 
from the $1.9 \mathrm{~K}$ surface of the magnet bores. In this way it shields the cold surface from synchrotron radiation, preventing desorption of the trapped gas molecules and avoiding breakdown of the beam vacuum.

\section{Heat loads}

Static heat in-leaks are a function of the design of the cryostats and originate in the ambient temperature environment. Thermal calculations for the cryostats and the distribution system are based on LHC thermal performance data with appropriate scaling of the geometry (for radiation terms) and mass (for solid conduction).

Electrical resistive heating is caused by the non-superconducting sections of the magnet excitation circuits, essentially in splices of the superconducting cables and in current leads. The heat load due to magnet splices has to be absorbed by the cold mass helium bath. Resistive heating in current leads has to be absorbed by cooling circuits in the electrical feed boxes.

Beam induced loads are deposited in the cryo-magnets through several processes and by the circulating and colliding proton beams themselves. These loads strongly depend on the energy, the bunch intensity and on the number and length of the circulating bunches. The various beam induced loads are:

- Synchrotron radiation inside the bending magnet, mostly absorbed by the beamscreens;

- Resistive dissipation of beam image currents induced in the resistive walls and geometrical singularities of the beam channel;

- Nuclear inelastic beam-gas scattering corresponding to a continuous distributed loss of particles from the circulating beam, mostly absorbed by the cold mass helium bath;

- Loss of secondary particles, absorbed at $1.9 \mathrm{~K}$ in the magnet cold mass helium bath close to the high-luminosity experimental areas (points PA and PG) and at $40-60 \mathrm{~K}$ in the shielded beamscreens. At a luminosity of $30 \times 10^{34} \mathrm{~cm}^{-2} \mathrm{~s}^{-1}$, secondaries are expected to deposit $6.4 \mathrm{~kW}$ at $1.9 \mathrm{~K}$ and $45 \mathrm{~kW}$ at $40-60 \mathrm{~K}$ per half insertion.

Table 5.11 gives the distributed steady-state heat loads for nominal conditions.

Ramping the magnetic fields up and down generates transient heat loads in the superfluid helium due to eddy currents in the superconducting cables and in the mechanical structure of the magnets. Raising the current to its nominal value in $1600 \mathrm{~s}$ is expected to dissipate $5 \mathrm{~kJ}$ per metre of main dipole. This represents a power of approximately $3 \mathrm{~W} / \mathrm{m}$. This power corresponds to 2 times the total steady-state heat load at $1.9 \mathrm{~K}$ and cannot be extracted in real time. The only practical way to absorb these transient heat loads and to keep the temperature below $2.1 \mathrm{~K}$ during ramping up and below the lambda line during a pre-cycle (ramping up followed by a ramping down: $2 \times 5 \mathrm{~kJ} / \mathrm{m}$ ), is to use the heat capacity of the liquid helium contained in the magnet cold masses. About $33 \mathrm{l}$ of liquid helium per metre length is sufficient to cope with the energy to be buffered. To extract the energy deposited in a time compatible with high-luminosity beam operation $(<2 \mathrm{~h})$ an extra cooling capacity of $3 \mathrm{~kW}$ at $1.8 \mathrm{~K}$ per refrigerator is required.

\section{Cooling scheme and cryogenic distribution}

The cryogenic flow scheme of a half-cell (string of one quadrupole and 6 cryo-dipoles) is shown in Figure 5.15. The pressurised superfluid helium bath at $1.9 \mathrm{~K}$, in which 
Table 5.11. Distributed steady-state heat loads (nominal conditions).

\begin{tabular}{|c|c|c|c|c|}
\hline \multicolumn{2}{|c|}{ Temperature level } & $40-60 \mathrm{~K}$ & $1.9 \mathrm{~K}$ & $4 \mathrm{~K} \mathrm{VLP}$ \\
\hline \multirow{7}{*}{$\begin{array}{l}\text { Static heat } \\
\text { in-leaks } \\
(\mathrm{W} / \mathrm{m})\end{array}$} & Cold mass supporting system & 2.4 & 0.13 & \\
\hline & Radiative insulation & & 0.13 & \\
\hline & Thermal shield & 3.1 & & \\
\hline & Feedthrough and vacuum barrier & 0.2 & 0.1 & \\
\hline & Beamscreen & & 0.12 & \\
\hline & Distribution & 3.6 & 0.1 & 0.24 \\
\hline & Total static & 9.3 & 0.58 & 0.24 \\
\hline \multirow{5}{*}{$\begin{array}{l}\text { Dynamic } \\
\text { heat loads } \\
(\mathrm{W} / \mathrm{m})\end{array}$} & Synchrotron radiation & 57 & 0.08 & \\
\hline & Image current & 3.4 & & \\
\hline & Resistive heating in splices & & 0.3 & \\
\hline & Beam-gas scattering & & 0.45 & \\
\hline & Total dynamic & 60 & 0.83 & \\
\hline \multicolumn{2}{|l|}{ Total } & 70 & 1.4 & 0.24 \\
\hline \multicolumn{2}{|c|}{ Dynamic range } & 8 & 2.5 & 1 \\
\hline
\end{tabular}

the superconducting magnets are immersed, is cooled by saturated two-phase helium flowing in a bayonet heat exchanger (HX) tube extending along the string of magnets and supplied by line $\mathrm{C}$ through expansion valve $\mathrm{V} 1$ and a sub-cooling heat exchanger [292]. The low saturation pressure is maintained by pumping the vapour through line B. Table 5.12 gives the main superfluid helium cooling loop parameters (LHC parameters are also given for comparison).

Cool-down and warm-up are achieved by forced circulation of high pressure gaseous helium supplied at variable temperatures by line E, tapped through valve V2 and returned to the cryogenic plant through valve V3 and line F. Since the operating pressure (50 bar) is not compatible with the cold mass design pressure (20 bar), the cool-down and warm-up is performed indirectly via 4 DN30 tubes equally-distributed in the cold mass yoke. At the end of the cool-down, the liquid helium filling is done through valve $\mathrm{V} 4$.

In case of a magnet quench, the resulting pressure rise is contained below the $2 \mathrm{MPa}$ design pressure by discharging the liquid helium inventory of a half-cell into line D through the V5 and V6 safety relief valves. The low hydraulic impedance of this $200 \mathrm{~mm}$ diameter pipe, normally maintained at $40 \mathrm{~K}$, is very helpful to contain the helium discharge and in buffering the gas storage vessels.

The beamscreens are cooled by forced circulation of high pressure helium, tapped from line $\mathrm{E}$ and returned to line $\mathrm{F}$ through valve V7, after cooling the magnet thermal shields and support post heat intercepts [293]. Table 5.13 gives the main beamscreen cooling loop parameters (LHC parameters also given for comparison).

The weak points of conventional stainless steel tube cryogenic distribution lines are their compensation units based on bellows and their inherent risk of leaks and buckling. The conceptual design is based on Invar tubes [294]. Invar, an iron-nickel alloy, exhibits a very low thermal contraction coefficient and the corresponding cooldown stresses can be handled without compensation units. Moreover, to transport the huge refrigeration capacity between 40 and $60 \mathrm{~K}$ for the beamscreen and thermal shield cooling ( $\sim 600 \mathrm{~kW}$ per sector), an efficient distribution (low pressure drop in narrow beamscreen cooling channels) combined with the need for a limited size of the 


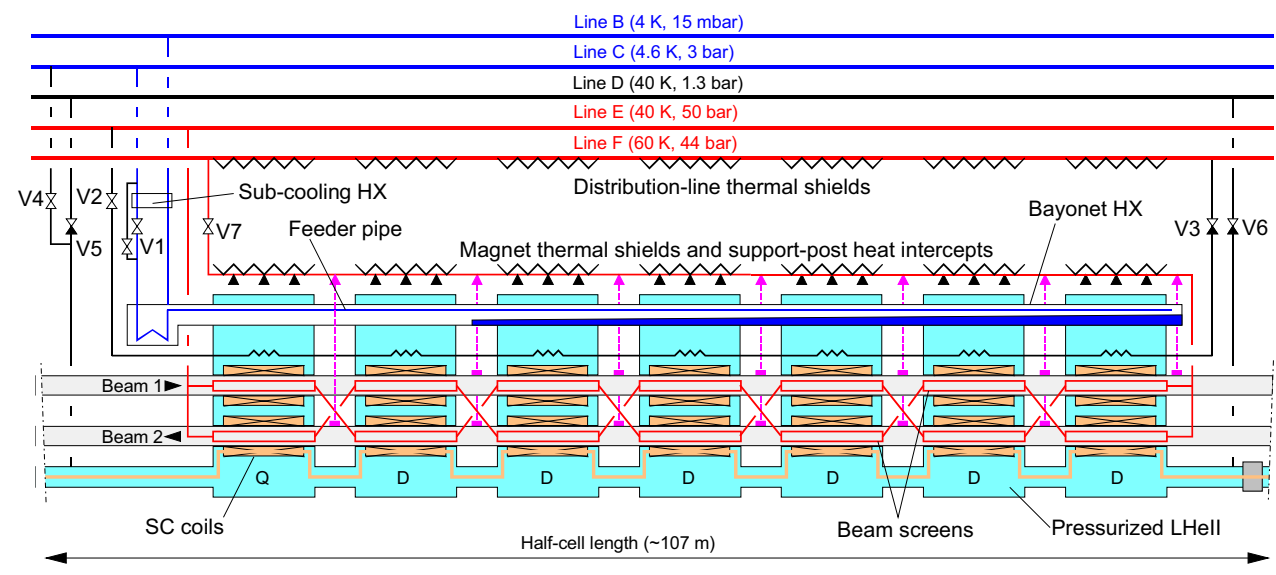

Fig. 5.15. Cryogenic flow scheme of a half-cell.

Table 5.12. Main superfluid helium cooling loop parameters.

\begin{tabular}{|l|c|c|c|}
\hline Parameter & Unit & LHC & FCC-hh \\
\hline Unit cooling length & $\mathrm{m}$ & 106.9 & 107.1 \\
Sector cooling length & $\mathrm{m}$ & 2900 & 8400 \\
Number of parallel cooling loops (total) & - & 216 & 790 \\
Average nominal heat load & $\mathrm{W} / \mathrm{m}$ & 0.40 & 1.4 \\
Bayonet heat exchanger inner diameter & $\mathrm{mm}$ & 53.4 & 83.1 \\
Feeder pipe inner diameter & $\mathrm{mm}$ & 10.0 & 15.0 \\
Wall thickness of bayonet heat exchanger & $\mathrm{mm}$ & 2.3 & 5.0 \\
Joule-Thomson valve inlet temperature & $\mathrm{K}$ & 2.18 & 2.18 \\
Free longitudinal cross-section area & $\mathrm{cm}{ }^{2}$ & 60 & 156 \\
DT max pressurised saturated HeII & $\mathrm{mK}$ & 50 & 50 \\
Cold mass operating pressure & $\mathrm{bar}$ & 1.3 & 1.3 \\
Pumping line (B) diameter & $\mathrm{mm}$ & 270 & 630 \\
Helium supply line (C) diameter & $\mathrm{mm}$ & 100 & 80 \\
Heat load on pumping line & $\mathrm{W} / \mathrm{m}$ & 0.11 & 0.24 \\
Pumping pressure at cryogenic plant interface & $\mathrm{mbar}$ & 15 & 15 \\
Maximum cold mass helium temperature & $\mathrm{K}$ & 1.9 & 1.9 \\
\hline
\end{tabular}

supply and return lines (lines $\mathrm{E}$ and $\mathrm{F}$ ) has led to the choice of an operating pressure of 50 bar. This choice complicates the design of reliable bellows-based compensation units. Table 5.14 gives the dimensions of the main lines and vacuum jackets of the cryogenic distribution line in the tunnel.

It is proposed to have dedicated cooling loops for each inner triplet quadrupole assembly (Q1A_B, Q2A_B_C_D and Q3A_B) in the high-luminosity insertions. The cooling of the shielded beamscreens at $40-60 \mathrm{~K}$ requires four DN15 cooling channels in parallel, which give a maximum pressure drop of 2 bar for a supply pressure of 20 bar. The cooling of the cold masses at $1.9 \mathrm{~K}$ requires four bayonet heat exchangers in parallel (DN125 for Q1 and DN100 for Q2 and Q3).

\subsubsection{Cryogenic plants}

Figure 5.16 shows the design of the proposed refrigeration process cycle for the sector cryogenic plants. For the production of the refrigeration capacity above $40 \mathrm{~K}$, the 
Table 5.13. Main beamscreen cooling loop parameters.

\begin{tabular}{|l|c|c|c|}
\hline Parameter & Unit & LHC & FCC-hh \\
\hline Unit cooling length & $\mathrm{m}$ & 53.4 & 107.1 \\
Sector cooling length & $\mathrm{m}$ & 2900 & 8400 \\
Number of parallel cooling loops (total) & - & 432 & 790 \\
Average beamscreen nominal heat load & $\mathrm{W} / \mathrm{m}$ & 1.6 & 60 \\
Supply pressure & $\mathrm{bar}$ & 3 & 50 \\
Supply helium temperature & $\mathrm{K}$ & 5 & 40 \\
Max. allowed BS temperature & $\mathrm{K}$ & 20 & 60 \\
BS helium outlet temperature (nominal) & $\mathrm{K}$ & 20 & 57 \\
Minimum BS temperature (nominal) & $\mathrm{K}$ & 5 & 43 \\
BS pressure drop (nominal) & $\mathrm{bar}$ & 0.5 & 4.3 \\
DP control valve (nominal) & $\mathrm{bar}$ & 0.8 & 1 \\
$\Delta$ P supply and return line (nominal) & $\mathrm{bar}$ & 0.4 & 0.5 \\
Total cooling loop pressure drop & $\mathrm{bar}$ & 1.7 & 5.8 \\
Supply/return header (E/F) diameter & $\mathrm{mm}$ & $100 / 150$ & $250 / 250$ \\
Exergetic efficiency (distribution only) & $\%$ & 76 & 86 \\
Total exergetic efficiency (with cold circulator) & $\%$ & N/A & 82 \\
Total exergetic efficiency (with warm circulator) & $\%$ & N/A & 71 \\
\hline
\end{tabular}

Table 5.14. Dimensions of the main cryogenic distribution line components.

\begin{tabular}{|l|c|}
\hline Component & Diameter ( mm) \\
\hline Line B & 630 \\
Line C & 80 \\
Line D & 200 \\
Line E & 250 \\
Line F & 250 \\
Vacuum jacket of pipe elements & 1350 \\
Flanges and bellows of pipe elements & 1450 \\
Vacuum jacket of service modules & 1500 \\
\hline
\end{tabular}

design is based on a turbo-Brayton cycle using a mixture of neon and helium, which allows the use of centrifugal compressors in the warm compressor station [295]. These warm centrifugal compressors have oil-free active magnetic bearings and do not need gearboxes and shaft seals.

The Carnot efficiency of such a cycle is expected to be higher than $40 \%$. This cycle will also be used for the pre-cooling of the pure helium cycle at $40 \mathrm{~K}$. This is required for the production of the refrigeration capacity at $1.8 \mathrm{~K}$ to cool the superconducting magnets. Below $40 \mathrm{~K}$, the helium refrigeration cycle is based on a Claude cycle with a Carnot efficiency of $28.8 \%$ (LHC cryogenic plant value). Refrigeration at $1.8 \mathrm{~K}$ is provided by a cold compressor train in series with warm volumetric compressors (C3) allowing efficient turn-down capability. Table 5.15 gives the main heat loads per sector for users in the tunnel. The capacity of a sector cryogenic plant is equivalent to about $110 \mathrm{~kW}$ at $4.5 \mathrm{~K}$. The electrical power required is $24 \mathrm{MW}$ per main sector cryogenic plant, i.e. $240 \mathrm{MW}$ in total for the machine.

For the high-luminosity insertions, the cryogenic plant design is driven by collision debris (secondary particles) power. Per insertion, the cryogenic cooling duties are:

- $90 \mathrm{~kW}$ at $40-60 \mathrm{~K}$ for the beamscreen cooling;

- $1.4 \mathrm{~kW}$ at $40-60 \mathrm{~K}$ for the thermal-shield cooling;

- $12.8 \mathrm{~kW}$ at $1.8 \mathrm{~K}$ for the cold mass cooling;

- $60 \mathrm{~g} / \mathrm{s}$ at $40-300 \mathrm{~K}$ for the current-lead cooling. 


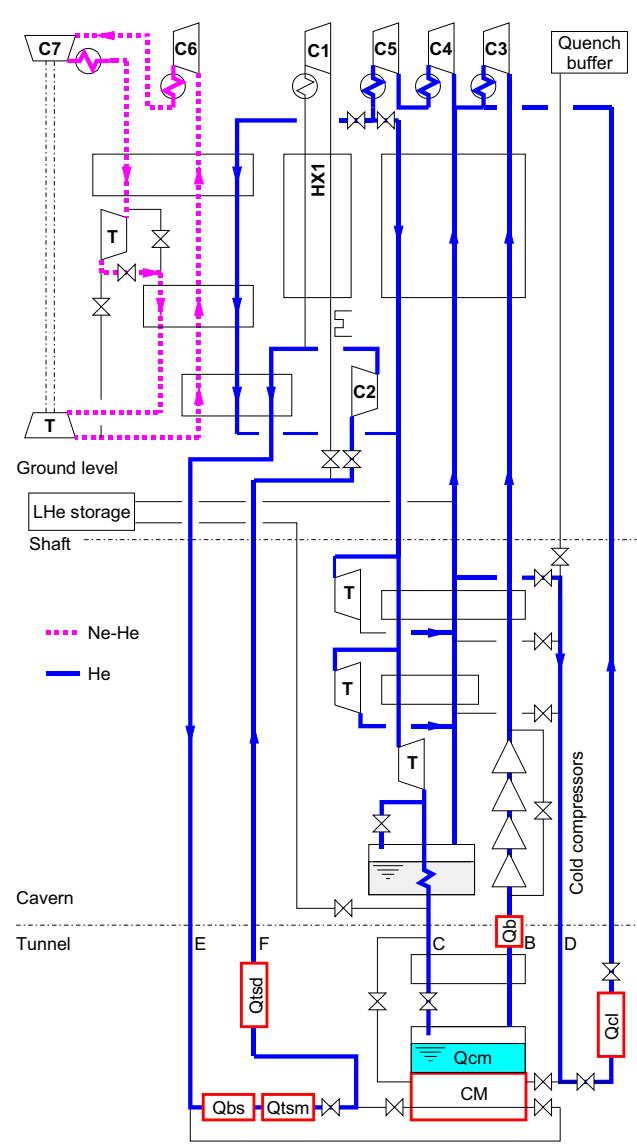

(a)

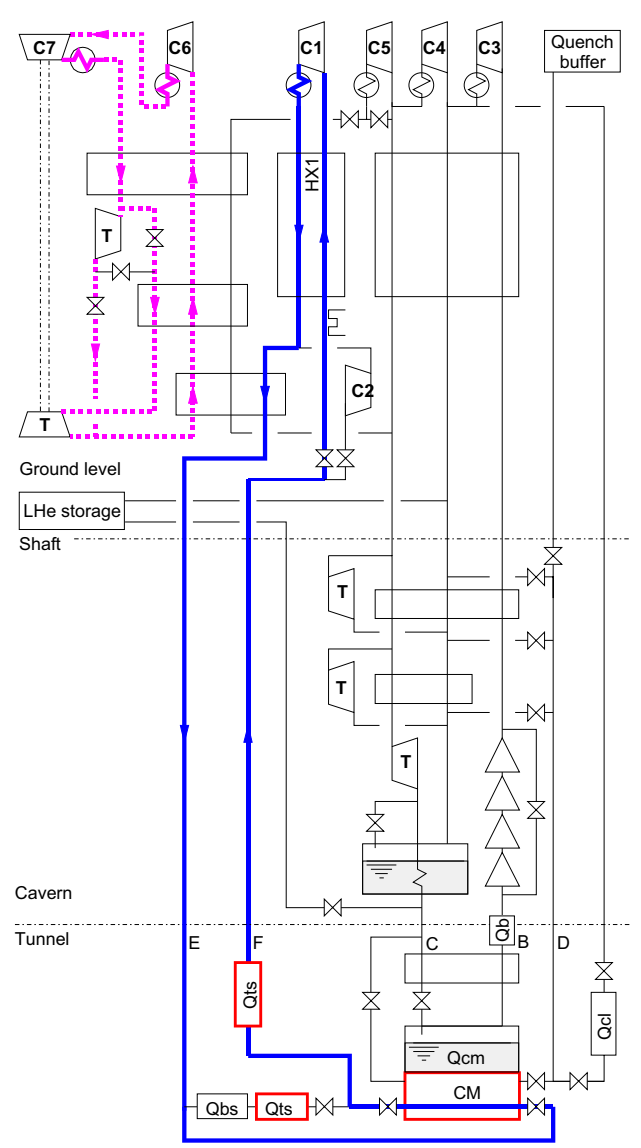

(b)

Fig. 5.16. The design of the proposed refrigeration process cycle. (a) The refrigeration process cycle for nominal. (b) Cool-down modes showing the main tunnel user loads.

One characteristic of a turbo-Brayton cycle is that the refrigeration power is proportional to its operating temperature, i.e. if the cryogenic plant is producing $500 \mathrm{~kW}$ at $50 \mathrm{~K}$, it will be able to produce up to $2.25 \mathrm{MW}$ at $225 \mathrm{~K}$. Consequently, this turbo-Brayton cycle can also be used to produce the cooling capacity to cool down the $23 \mathrm{kt}$ cold mass of a sector. A warm circulator $(\mathrm{C} 1)$ is required to supply the cool-down mass flow. For a cool-down temperature difference of $75 \mathrm{~K}$ per halfcell (giving less than $50 \mathrm{~K}$ temperature difference per magnet), the required mass flow to distribute the cooling capacity is $5.8 \mathrm{~kg} / \mathrm{s}$ and the maximum pressure drop, during the cool-down with a supply pressure of 50 bar, is about 6 bar. The cool-down time from 300 to $40 \mathrm{~K}$ using the turbo-Brayton refrigeration cycle is about 15 days. The cool-down operation cost which corresponds to the electrical consumption of the turbo-Brayton cycle and of the warm circulator is about 2.3 MCHF for the whole machine. This cost has to be compared with a cool-down using liquid nitrogen $\left(\mathrm{LN}_{2}\right)$ pre-coolers which will require $45000 \mathrm{t}$ of liquid nitrogen ( $4 \mathrm{MCHF}$ per cool-down), large $\mathrm{LN}_{2}$ storage reservoirs $\left(56000 \mathrm{~m}^{3}\right.$ equivalent to 10 spherical reservoirs with a diameter of $27 \mathrm{~m}$ ), which will be empty most of the time and challenging delivery logistics to get the $\mathrm{LN}_{2}$ inventory at the right time.

A cold circulator (C2) will supply a mass flow of $5.6 \mathrm{~kg} / \mathrm{s}$ at 50 bar with a pressure ratio of 1.13 to compensate for the 5.8 bar pressure drop in the cooling loop to cool 
Table 5.15. Nominal main tunnel user heat loads per $10 \mathrm{~km}$ long sector.

\begin{tabular}{|c|c|c|c|c|c|}
\hline Temperature level & $\begin{array}{l}40-60 \mathrm{~K} \\
(\mathrm{~kW})\end{array}$ & $\begin{array}{l}1.9 \mathrm{~K} \\
(\mathrm{~kW})\end{array}$ & \begin{tabular}{|l}
$4 \mathrm{~K}$ \\
$\mathrm{VLP}$ \\
$(\mathrm{kW})$
\end{tabular} & $\begin{array}{l}4.5 \mathrm{~K} \\
(\mathrm{~kW})\end{array}$ & $\begin{array}{l}40-290 \mathrm{~K} \\
(\mathrm{~g} / \mathrm{s})\end{array}$ \\
\hline $\begin{array}{l}\text { Cold mass steady state load, } \\
\text { Qcm } \\
\text { Additional load for transient } \\
\text { mode } \\
\text { Beamscreen load, Qbs } \\
\text { Magnet thermal shield load, } \\
\text { Qtsm } \\
\text { Distribution thermal shield } \\
\text { load, Qtsd } \\
\text { Pumping line (line B) load, Qb } \\
\text { Current leads cooling, Qcl } \\
\text { RF cavity modules (Point H left } \\
\text { or right) } \\
\end{array}$ & $\begin{array}{l}504 \\
44.5 \\
33.5\end{array}$ & $\begin{array}{l}12 \\
3\end{array}$ & 2 & 1.5 & 85 \\
\hline Total & 580 & 15 & 2 & 1.5 & 85 \\
\hline
\end{tabular}

the beamscreen and thermal shield. Assuming an isentropic efficiency of $70 \%$, the $130 \mathrm{~kW}$ compression power consumed by this cold circulator, has to be extracted at cryogenic temperature. The total exergetic efficiency of this cooling loop is $82 \%$ (see Tab. 5.13). As the parameters (mass flow, operating pressure and pressure ratio) of the cold circulator $\mathrm{C} 2$ are about the same as the warm circulator $\mathrm{C} 1$, the warm circulator can also be used as a redundant machine for the beamscreen and thermal shield cooling loop. In this case, the total exergetic efficiency will be reduced to $71 \%$ (see Tab. 5.13), assuming a warm circulator isentropic efficiency of $83 \%$ and an NTU of 40 for the heat exchanger HX1. The difference of $11 \%$ in the exergetic efficiency using cold or warm circulators will give an operational cost saving of about $2.8 \mathrm{MCHF}$ per sector over 10 years of operation. This operation cost saving compensates the capital expenditure of a cold circulator.

Another challenge for the cryogenic system is the dynamic range of 8 (see Tab. 5.11) which is mainly due to the synchrotron radiation power deposited on the beamscreen which increases with the 4th power of the beam energy. The corresponding heat load transient is severe, since the synchrotron radiation reaches its maximum value in less than $30 \mathrm{~min}$. This transient has to be handled by the local cooling loops, which do not allow temperature excursions of the beamscreens above $60 \mathrm{~K}$, and by the cryogenic plants, which have an inherent time constant in adapting to the capacity.

The local cooling loop strategy is to keep a constant mass flow corresponding to the expected nominal value in each loop. Without beams, the temperature gradient along the beamscreen is around zero. With beam, when the synchrotron radiation reaches its nominal value, the temperature gradient will obviously appear but only require a small adjustment to the mass flow. Working at constant supply pressure at the outlet of the beamscreen circulator would require that a large quantity of helium was discharged and refilled, when the return line $\mathrm{F}\left(\sim 400 \mathrm{~m}^{3}\right.$ per sector) has its temperature varying alternately between 40 and $60 \mathrm{~K}$. The corresponding density variation of $15 \mathrm{~kg} / \mathrm{m}^{3}$ would yield a mass variation of about $6 \mathrm{t}$ per sector. To avoid this large inventory variation, it is proposed to work with a constant helium inventory in the beamscreen and thermal shield cooling loop. Consequently, the pressures in line $\mathrm{E}$ and $\mathrm{F}$ will be reduced when there is no beam and will progressively increase to their nominal value in steady state with beam. 


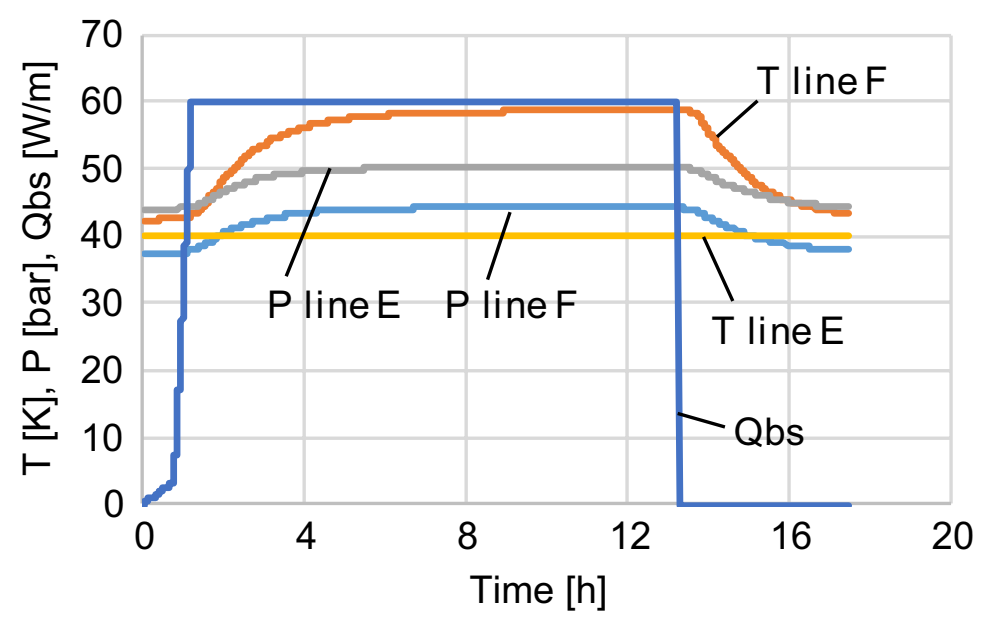

Fig. 5.17. Evolution of the pressure and temperature of lines E and F at the cryogenic plant interface during a typical physics run.

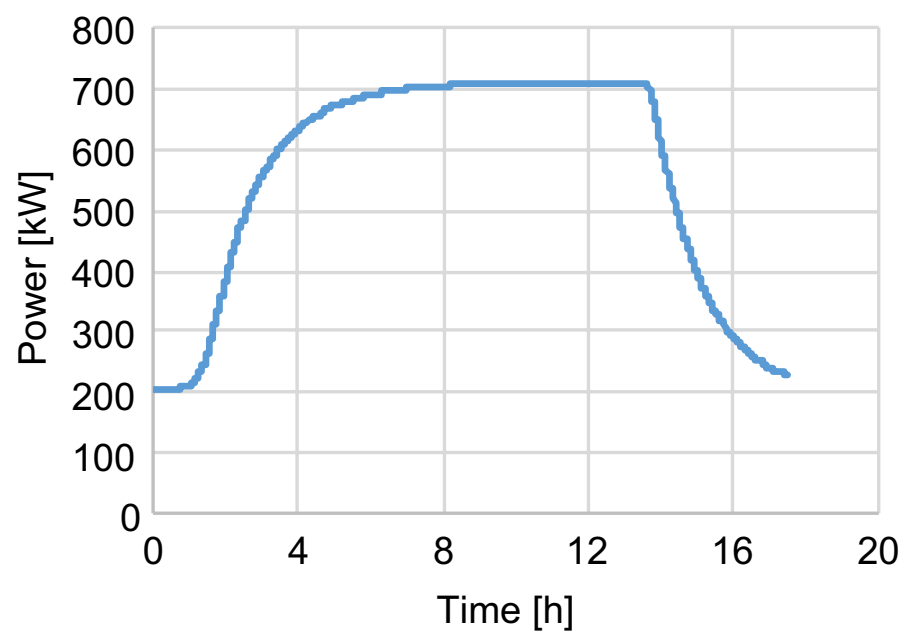

Fig. 5.18. Evolution of the refrigeration capacity during a typical physics run.

In addition, due to the large thermal inertia of the two $250 \mathrm{~mm}$ distribution lines, the capacity at the cryogenic plant interface increases with a time constant of about $4 \mathrm{~h}$, compatible with the ability to adapt to the capacity of such a large cryogenic plant [296]. Figures 5.17 and 5.18 show the evolution of the various parameters during a typical low luminosity physics run with a stable beam plateau of about $10 \mathrm{~h}$. In the case of high luminosity physics runs, the stable beam plateau duration will be about $4 \mathrm{~h}$, i.e. the cryogenic plant will never operate in steady state.

The cooling of the superconducting magnets requires a refrigeration capacity of $15 \mathrm{~kW}$ at $1.8 \mathrm{~K}$ per cryogenic plant, 7 times larger than the state-of-the-art cryogenic plants presently in operation for the LHC. Specific research and development on the design of larger cold compressors and on the concurrent operation of cold compressor trains will be required. The dynamic range of 3 will not be an issue for the proposed mixed compression scheme as demonstrated at LHC [297]. Additional studies are expected to improve the overall efficiency of helium plants. Potential areas for such improvements are the introduction of centrifugal compressors in the warm compres- 


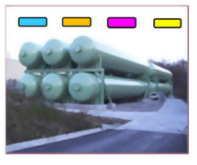

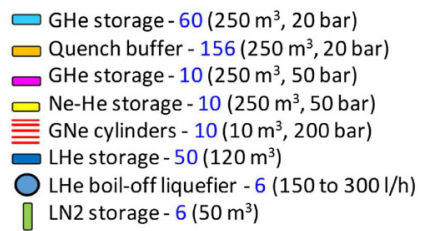

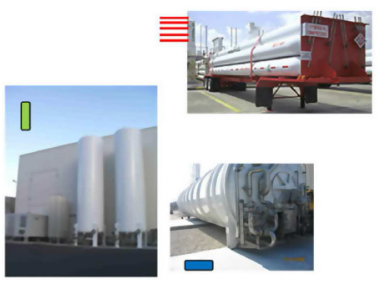

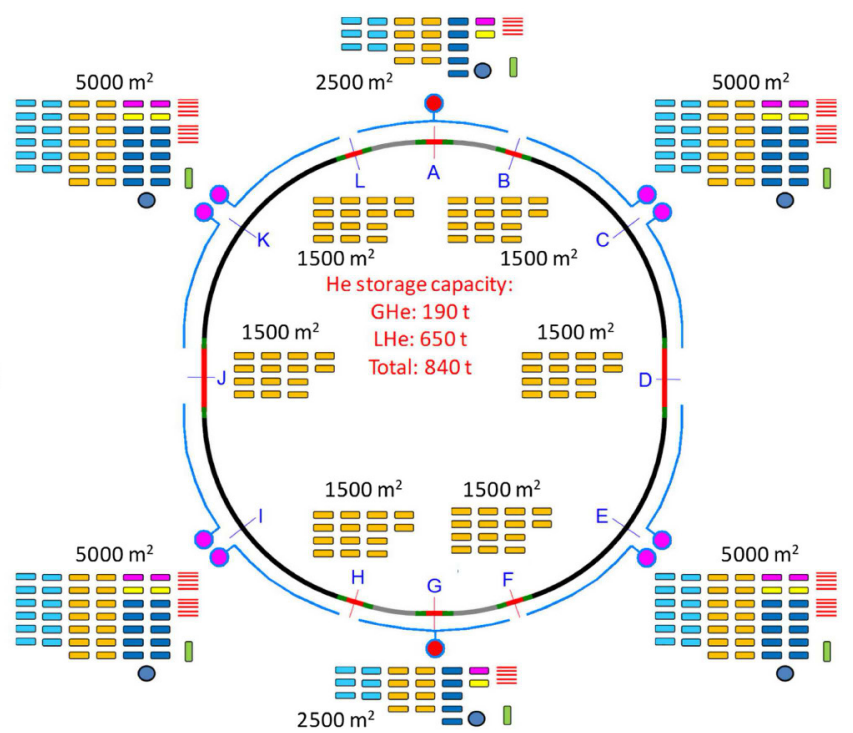

Fig. 5.19. Cryogen storage.

sion station and the pre-cooling capacity of the Claude cycle down to $40 \mathrm{~K}$ usefully provided by the turbo-Brayton cycle as shown in Figure 5.16. Engineering studies to propose alternatives and/or possible improvements are underway with industrial partners specialised in large helium refrigeration.

The present heat loads have to be considered as ultimate conditions, which do not require operational margins. However, to guarantee operation with high availability, the cryogenic system requires a minimum operational margin factor of 1.3 . This factor can be obtained by reducing the beam parameters (beam energy and/or bunch population) which affect the beam-induced heating. This minimum operational margin can be obtained as follows:

- At the nominal bunch population $\left(10^{11} \mathrm{ppb}\right)$ by reducing the nominal beam energy to $92.5 \mathrm{TeV}$;

- At nominal beam energy $(100 \mathrm{TeV})$ by reducing the bunch population to $7.5 \times$ $10^{10} \mathrm{ppb}$

- By reducing the beam energy to $95 \mathrm{TeV}$ and the bunch population to $9 \times 10^{10} \mathrm{ppb}$.

\subsubsection{Cryogen inventory and storage}

The cryogenics system will require neon, helium and nitrogen. Figure 5.19 shows the cryogen storage infrastructure distributed across the various surface sites.

Nitrogen will only be required for the regeneration of absorbers and dryer beds, consequently, one standard $50 \mathrm{~m}^{3} \mathrm{LN} 2$ reservoir is planned for each site housing cryogenic plants ( 6 in total).

The proposed mixing rate of the $\mathrm{Ne}-\mathrm{He}$ cycle is $25 \%$ neon and $75 \%$ helium. As this cycle is localised at ground level, the neon inventory can be kept at about $500 \mathrm{~kg}$ per cryogenic plant. It is proposed to store the neon inventory in $5 \times 2 \mathrm{~m}^{3}$ cylinders at 200 bar per cryogenic plant, corresponding to a storage capacity of about $1.5 \mathrm{t}$ per cryogenic plant. 
The helium inventory is mainly driven by the cold mass void fraction $(33 \mathrm{l} / \mathrm{m}$ scaled from the LHC cold mass inventory) and by the lines E and F (250 mm diameter) which contain high density helium. The total helium inventory is about $880 \mathrm{t}$, equally shared between the cold mass and the distribution system. $20 \%$ of the inventory will be stored in standard $250 \mathrm{~m}^{3}$ medium pressure reservoirs (216 reservoirs in total). The remaining $80 \%$ will be stored in $120 \mathrm{~m}^{3}$ liquid reservoirs (50 reservoirs in total). There must be boil-off liquefiers (one per site, $300-150 \mathrm{l} / \mathrm{h}$ ) to preserve the liquid helium inventory during long shutdown periods, when the main cryogenic plants will be overhauled.

\subsection{Equipment transport and handling}

\subsubsection{Underground vehicles}

The vehicles which will be used in the tunnels will comply with the relevant European Directives (Machinery Directive 2006/42/EC, RoHS Directive 2011/65/EU) as well as CERN safety codes and EN standards. All equipment will have integrated recovery mechanisms and procedures (on-board or separate).

The transport system comprises tractors and trailers which are combined to form convoys tailored to match the dimension and weight of the payload and installation situation. A tractor can tow one or more trailers. The vehicles will be equipped with an automatic guidance system and powered by batteries. The speed and the number of vehicles will be adjusted to meet the installation schedule. All required equipment can be supplied by industry using currently available technologies.

\section{Tractor characteristics}

Each tractor will be equipped with two trailer hitches (one each at the front and rear) to connect the tractor to the trailers. The tractor will be equipped with electric motor drives and therefore run emission-free in the tunnel. An intelligent navigation and control system will drive the tractor autonomously in the tunnels. The general characteristics of the tractors are summarised in Table 5.16.

\section{Trailer characteristics}

The trailer (Fig. 5.20) uses an electronic steering system, a drawbar and a vibrationdamped support for the load. The special wheels are mounted on pendulum axles (swing axle). The general characteristics of the trailers are summarised in Table 5.17.

\section{Magnet transport}

To transport cryo-units (magnets in their cryostats) a convoy will comprise a tractor and two trailers. Figure 5.21 shows the transport and handling of a cryo-unit in the tunnel. It is preferred to have the trailers separate from each other because that way they adapt better to the dimensions of the payload.

Two transfer tables equipped with lifting jacks (Fig. 5.21) will unload the transport vehicle once it has arrived at its destination. The two units will transfer the cryo-unit laterally from the trailers and place it at its final position. The handling units will be externally powered and operated using remote controls. The general characteristics of the magnet transfer tables are summarised in Table 5.18. 
Table 5.16. General characteristics of the transport tractors.

\begin{tabular}{|c|c|c|}
\hline General characteristics & Type/Value & $\begin{array}{l}\text { Complementary } \\
\text { information }\end{array}$ \\
\hline Type & Battery powered & $\begin{array}{l}1 \text { or } 2 \text { tractors per convoy } \\
\text { Front and rear (optional) }\end{array}$ \\
\hline Power & Electric & Equipped with batteries \\
\hline $\begin{array}{c}\text { Operator position (for } \\
\text { non-standard operations) }\end{array}$ & Seated & In longitudinal position \\
\hline Use & Underground & \\
\hline Rated capacity (nominal) & $60 \mathrm{t}$ & \\
\hline Maximum Slope & $2.5 \%$ & \\
\hline Steering & Automatic & Optical guidance device \\
\hline Wheels / Tyres & $\begin{array}{l}\text { Heavy duty traction } \\
\text { wheels }\end{array}$ & \\
\hline $\begin{array}{c}\text { Travel speed: Loaded } \\
\text { Unloaded }\end{array}$ & $\begin{array}{l}\text { Up to } 10 \mathrm{~km} / \mathrm{h} \text { Up to } \\
20 \mathrm{~km} / \mathrm{h}\end{array}$ & $\begin{array}{l}\text { Nominal speed on flat } \\
\text { surface }\end{array}$ \\
\hline $\begin{array}{c}\text { Slope travelling capacity } \\
\text { (loaded) }\end{array}$ & Min. $2.5 \%$ gradient & For min. $1 \mathrm{~km}$ distance \\
\hline Motors/Battery & Type/Value & $\begin{array}{l}\text { Complementary } \\
\text { information }\end{array}$ \\
\hline Motors & Electric & All \\
\hline Battery type & Gel or lead acid & \\
\hline $\begin{array}{c}\text { Autonomy (at nominal } \\
\text { load }-60 \mathrm{t} \text { ) }\end{array}$ & Min. $25 \mathrm{~km}$ & On flat surface \\
\hline
\end{tabular}

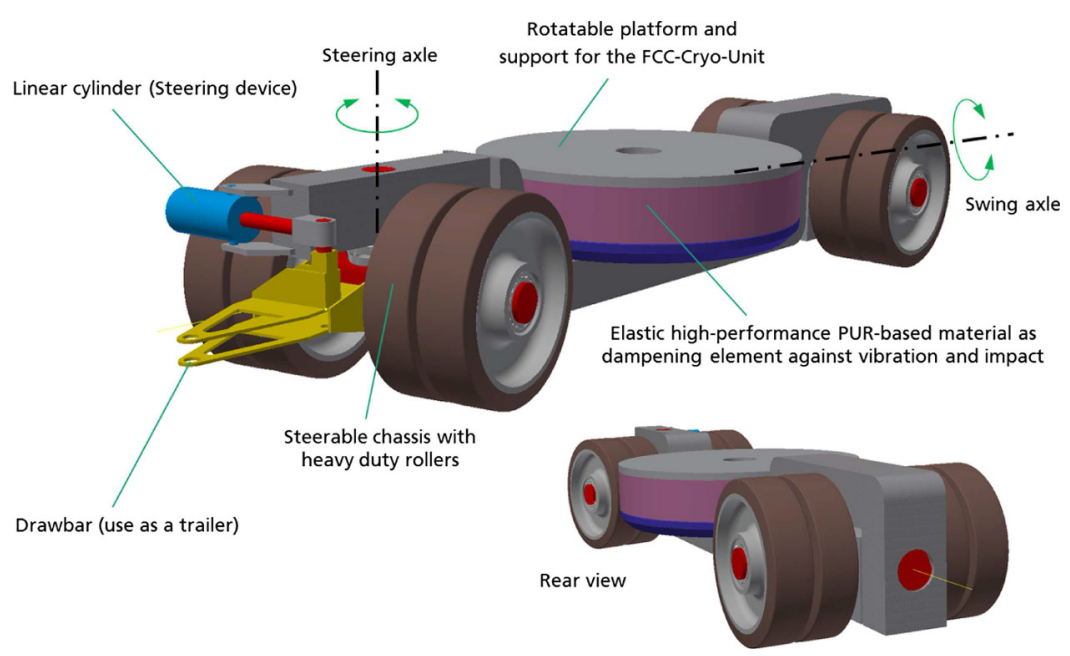

Fig. 5.20. A conceptual view of a trailer.

\subsubsection{Overhead cranes}

The electrical overhead travelling (EOT) cranes for the FCC will comply with relevant European Directives (2006/42/EC, 2014/30/EU and 2014/35/EU) as well as CERN safety codes and EN standards.

They are classified as light/medium duty cranes depending on their frequency of use and load spectra. Their FEM (European Federation of Material Handling) classification is A4/M4. 
Table 5.17. General characteristics of the transport trailers.

\begin{tabular}{|c|c|c|}
\hline General characteristics & Type/Value & $\begin{array}{l}\text { Complementary } \\
\text { information }\end{array}$ \\
\hline Type & Separate trailers & $\begin{array}{c}1,2 \text { or } 3 \text { trailers per } \\
\text { convoy }\end{array}$ \\
\hline Power & Electric/ battery & For steering device \\
\hline Suspension & Damping element & $\begin{array}{l}\text { Elastic high-performance } \\
\text { PUR-based material }\end{array}$ \\
\hline Steering & Steerable chassis & $\begin{array}{c}\text { Automatic guidance } \\
\text { device }\end{array}$ \\
\hline Use & Underground & \\
\hline Rated capacity (nominal) & $30 \mathrm{t}$ & \\
\hline Maximum Slope & $2.5 \%$ & Brakes \\
\hline Wheels/Tyres & Heavy duty rollers & \\
\hline $\begin{array}{c}\text { Travel speed: Loaded } \\
\text { Unloaded }\end{array}$ & $\begin{array}{l}\text { Up to } 10 \mathrm{~km} / \mathrm{h} \\
\text { Up to } 20 \mathrm{~km} / \mathrm{h}\end{array}$ & $\begin{array}{l}\text { Nominal speed on flat } \\
\text { surface }\end{array}$ \\
\hline
\end{tabular}

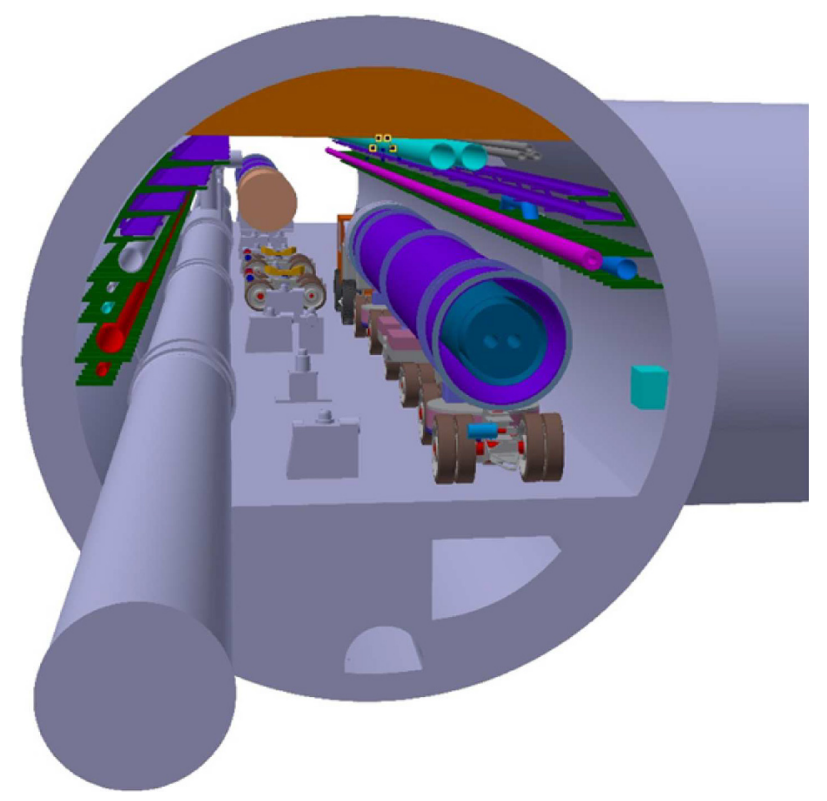

Fig. 5.21. Transport and handling of a cryo-unit in the tunnel.

The hoist design ensures that hook lifting and lowering can be performed without horizontal drift. An emergency brake is installed to avoid dropping the load should any elements of the hoisting drive chain fail. To obtain smooth acceleration, electric motors are driven by inverters. The crane is therefore suited to handle fragile objects. The maximum speeds are chosen in the following ranges:

- Lifting speed: 8-10 m/min;

- Cross-travel speed: $12-15 \mathrm{~m} / \mathrm{min}$;

- Long-travel speed: $15-20 \mathrm{~m} / \mathrm{min}$.

Laser sensors and a programmable logic controller (PLC) control the hook position in order to ensure precise positioning and to define multiple areas where the hook must not access. 
Table 5.18. General characteristics of the magnet transfer tables.

\begin{tabular}{|c|c|c|}
\hline General characteristics & Type/Value & $\begin{array}{c}\text { Complementary } \\
\text { information }\end{array}$ \\
\hline Type & Remotely operated & 2 units per cryo-unit \\
\hline Power & Electric or hydraulic & External supply \\
\hline Operator position & External & $\begin{array}{c}\text { Master/Slave remote } \\
\text { control }\end{array}$ \\
\hline Use & Underground & $\begin{array}{c}\text { Minimum } 2 \text { transfer tables } \\
\text { required for cryo-magnet } \\
\text { positioning }\end{array}$ \\
\hline Rated capacity (nominal) & $30 \mathrm{t}$ & Multidirectional \\
\hline Steering & & On flat surface \\
\hline Wheels/Tyres & Heavy duty wheels & \\
\hline $\begin{array}{c}\text { Slope travelling capacity } \\
\text { (loaded) }\end{array}$ & $1 \mathrm{~m} /$ min & Max. $3 \%$ gradient \\
\hline
\end{tabular}

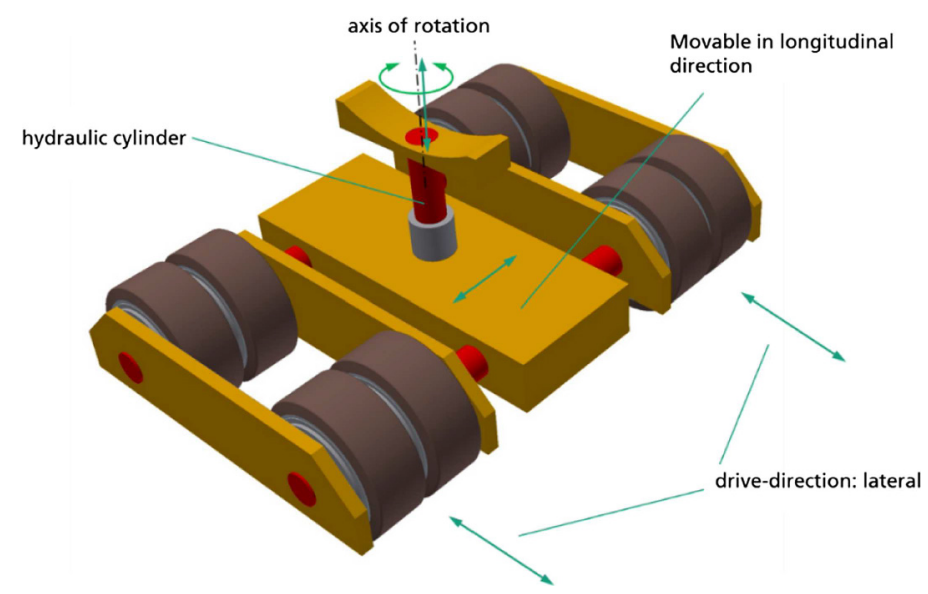

Fig. 5.22. Conceptual view of a handling unit configured with a hydraulic lifting mechanism.

A shaft crane is an overhead crane installed in a surface building, whose hook can reach an underground cavern and the shaft leading to it. Shaft cranes also comply with the following requirements:

- FEM classification of the hoisting gearbox is M6;

- The hoist is fitted with redundant electric motors;

- The hoist runs at twice the nominal speed if no load is attached to the hook;

- A weighing system takes into account the effect of the cable's own weight.

The installation of a crane takes between 3 weeks and 3 months depending on the capacity and lifting height.

\subsubsection{Lifts}

The lifts for the FCC will comply with the relevant European Directives (2006/42/EC and 2014/33/EU) as well as CERN safety codes and EN standards. 
The lifts can use standard technologies like those used in high-rise buildings where they already cover heights of up to $450 \mathrm{~m}$. The FCC-hh has 12 access shafts which will be equipped with two lifts each.

The lifts are the only authorised means for personnel access to and from the underground areas, therefore the lift shaft concrete modules will be over-pressured. The lifts will be powered from the secure power network in order to remain fully operational should the standard electrical network fail. For the same reason, the lift shaft modules were designed with 2 lifts in order to create redundancy (safety) and to ease operational (access) and maintenance (availability) aspects. A view of an access shaft, showing the two lift locations, is given in Figure 4.5.

The lift capacity and speed will determine how many people can simultaneously work in the underground areas. A time of about 4 min has been defined as duration of each lift cycle, including people entering the lift, the time for the lift to travel from underground to the surface, and people exiting the lift (for shaft depths of $300 \mathrm{~m}$ and a lift speed of $4 \mathrm{~m} / \mathrm{s}$ ).

Since both lifts can operate simultaneously and independently, up to 300 people can be evacuated from the underground areas at a single access point within $30 \mathrm{~min}$. This reduces to 150 people if only one lift is operational.

Studies showed that around $80 \%$ of the LHC non-machine components were transported with lifts. Lifts with $3 \mathrm{t}$ capacity have the best cost/capacity ratio; these were thus chosen as baseline solution.

The installation of a lift takes between 3 and 6 months depending on the shaft depth. The main characteristics of a lift are:

- Speed : $4 \mathrm{~m} / \mathrm{s}$

- Capacity : $3000 \mathrm{~kg} / 38$ persons

- Shaft height : (up to) $400 \mathrm{~m}$

- Car (length $\times$ width $\times$ height) $: 2700 \mathrm{~mm} \times 1900 \mathrm{~mm} \times 2700 \mathrm{~mm}$

- Door (width $\times$ height) : $1900 \mathrm{~mm} \times 2700 \mathrm{~mm}$

- Shaft width : $2750 \mathrm{~mm}$

- Shaft length : $3750 \mathrm{~mm}$

- Headroom : $7700 \mathrm{~mm}$

- Pit depth : $5900 \mathrm{~mm}$

\subsection{Personnel transport}

Trailer-towing vehicles can be used for collective or individual transport of personnel (e.g. to and from installation, maintenance and repair locations), including the transport of tools and spare parts. The characteristics and performance requirements correspond to those of the rescue vehicles (see below). The overall width of any vehicle has to be compatible with the aperture of the fire-compartment door.

Since the maximum distance from an access point to an intervention location does normally not exceed $5 \mathrm{~km}$, alternative means of individual transport such as electric bicycles can be used. The bicycles must carry spare batteries and an on-board charger.

\subsubsection{Transport for emergency services}

Transport of emergency teams and their equipment for fire fighting and rescue is a safety-related function. Today, solutions exist for both safety related and normal personnel transport that comply with the relevant regulations and standards. While current CERN vehicles respect European Directives, international standards and CERN safety codes, they will not meet FCC requirements because of the greater distances. 
Table 5.19. Tractor specifications.

\begin{tabular}{|c|c|}
\hline \multicolumn{2}{|c|}{ General conditions } \\
\hline Type & Tow tractor without cabin \\
\hline Driver position & $\begin{array}{c}\text { Standing straight (front) and } \\
\text { seated (back) }\end{array}$ \\
\hline Passenger position & $\begin{array}{c}1 \text { to } 2 \text { seated passengers } \\
\text { (backward) }\end{array}$ \\
\hline Propulsion & Electric/AC drive technology \\
\hline Gears & 1 forward/1 backward \\
\hline \multicolumn{2}{|c|}{ Performance } \\
\hline Speed & Variable up to $25 \mathrm{~km} / \mathrm{h}$ \\
\hline Minimum speed & $\begin{array}{c}4 \mathrm{~km} / \mathrm{h} \text { (fully loaded on a } 12 \% \\
\text { slope) }\end{array}$ \\
\hline Ascending capacity & $\begin{array}{l}\text { Minimum } 12 \% \text { slope ("loaded" for } \\
\text { a min. distance of } 1 \mathrm{~km} \text { ) Minimum } \\
20 \% \text { slope ("unloaded" for a min. } \\
\text { distance of } 50 \mathrm{~km} \text { ) }\end{array}$ \\
\hline Mileage minimum & $\begin{array}{l}50 \mathrm{~km} \text { at } 25 \mathrm{~km} / \mathrm{h} \text { ("loaded" on flat } \\
\text { floor) }\end{array}$ \\
\hline Turning radius & $1800 \mathrm{~mm}$ maximum \\
\hline Payload/Towed load & $300 \mathrm{~kg} / 1000 \mathrm{~kg}$ \\
\hline \multicolumn{2}{|c|}{ Basic dimensions } \\
\hline Overall width & $750 \mathrm{~mm}$ maximum \\
\hline Overall length & $2100 \mathrm{~mm}$ maximum \\
\hline Overall height & $940 \mathrm{~mm}$ maximum \\
\hline $\begin{array}{c}\text { Clearance between the chassis and } \\
\text { the floor }\end{array}$ & $100 \mathrm{~mm}$ minimum \\
\hline Tare weight (including battery) & $800 \mathrm{~kg}$ maximum \\
\hline
\end{tabular}

Furthermore, the need to transport sufficient quantities of breathing apparatus has to be considered.

The concept of trailer-towing tractors satisfies all requirements. One trailer could be equipped with a compressed air foam (CAF) fire fighting system and extinguishers, while a second trailer could carry injured people. The convoy comprises three fire fighters (two on the tractor, one standing on the trailer). The battery powered vehicles will be equipped with an on-board charger. The general specifications for the tractors and trailers are given in Tables 5.19 and 5.20.

\subsection{Geodesy, survey and alignment}

As was already the case for the Large Electron Position (LEP) collider in the 1980's, the FCC-hh will be the most demanding project in terms of positioning accuracy over a circle with nearly $100 \mathrm{~km}$ circumference. Present technical possibilities have to be compared to future physics requirements, to define which developments need to be undertaken.

\subsubsection{Alignment tolerances}

The alignment precision requirements will drive any survey study. The absolute accuracy in the vertical direction is the deviation from the theoretical plane of the collider, whilst in the transverse plane it is the variation of its radius with respect to the theoretical value. The differential variations between several consecutive magnets is the 
Table 5.20. Emergency trailer specifications.

\begin{tabular}{|c|c|}
\hline \multicolumn{2}{|c|}{ General conditions } \\
\hline Assisting fire fighter & $\begin{array}{c}1 \text { standing on a platform facing the } \\
\text { victim }\end{array}$ \\
\hline Passenger & 1 lying on the back on a stretcher \\
\hline \multicolumn{2}{|c|}{ Performance } \\
\hline Minimum turning radius & $1100 \mathrm{~mm}$ \\
\hline Ascending capacity & $12 \%$ slope \\
\hline $\begin{array}{l}\text { Full load tractive force with } \\
\text { acceleration from } 0 \text { to } 15 \mathrm{~km} / \mathrm{h} \text { in } 10 \mathrm{~s}\end{array}$ & $850 \mathrm{~N}$ \\
\hline Storage & Permanently stored with full load \\
\hline \multicolumn{2}{|c|}{ Material transport } \\
\hline Total maximum capacity & $450 \mathrm{~kg}$ \\
\hline \multicolumn{2}{|l|}{ Persons transport } \\
\hline Weight victim on stretcher & $100 \mathrm{~kg}$ \\
\hline Weight fire fighter & $100 \mathrm{~kg}$ \\
\hline Total maximum capacity & $450 \mathrm{~kg}$ \\
\hline \multicolumn{2}{|c|}{ Basic dimensions } \\
\hline Overall width & $750 \mathrm{~mm}$ \\
\hline Overall length & $2300 \mathrm{~mm}$ maximum \\
\hline Overall height & $940 \mathrm{~mm}$ maximum \\
\hline $\begin{array}{c}\text { Clearance between the chassis and the } \\
\text { floor }\end{array}$ & $60 \mathrm{~mm}$ minimum \\
\hline Useful length & $2200 \mathrm{~mm}$ \\
\hline Useful width & $740 \mathrm{~mm}$ \\
\hline Trailer floor height & $340 \mathrm{~mm}$ \\
\hline Void weight & $150 \mathrm{~kg}$ \\
\hline
\end{tabular}

relative accuracy. This latter type of error has a more direct effect on the closed orbit of the particles. A value of several $\mathrm{mm}$ has been assumed as the requirement for the absolute accuracy (this was achieved for LEP and LHC). A relative misalignment of $0.5 \mathrm{~mm}(1 \sigma)$ between consecutive quadrupoles and $1 \mathrm{mrad}(1 \sigma)$ for the roll angle are indications from beam physics simulations [298]. This error budget has to be shared between mechanical errors, due mainly to the assembly process and alignment errors, including misalignments due to ground motion or mechanical constraints.

\subsubsection{Geodesy}

As the area covered by the FCC is ten times larger than that of the LHC, an extension of the mean sea level equipotential surface of gravity (also called the geoid) has to be studied, as well as the installation of a new geodetic network. To achieve the absolute accuracy of the surface geodetic network, Global Navigation Satellite Systems (GNSS) will be used, possibly complemented by electro-optical distance measurements. The transfer of the geodetic network points from the surface to the tunnel, through shafts with a depth of up to nearly $300 \mathrm{~m}$, will require new developments. The underground network will necessitate gyro-theodolite traverses, as well as accurate distance and angle measurements and possibly offsets with respect to a stretched wire.

\subsubsection{Metrological aspects}

Metrological checks and alignments have to be integrated at different times in the manufacturing and assembly processes. This includes the fiducialisation, which 
determines the survey reference points with respect to the reference axes of a component. The techniques proposed are similar to those used for LHC, i.e. laser trackers and photogrammetry. New sensors such as frequency scanning interferometry (FSI) [299] will be used for monitoring elements inside cryomodules and elsewhere when justified by the accuracy required. The positioning of the alignment targets (fiducials) has to take into account the survey needs and the experimental cavern or accelerator tunnel constraints. The equipment supports have to comply with the alignment specifications and constraints.

\subsubsection{Alignment of accelerator components}

The alignment of accelerator components will be done in two steps:

- The first "absolute" alignment from the underground network will be performed using a standard digital level and total station measurements.

- The "relative" alignment or smoothing. For a cell twice as long as an LHC cell, the levelling technique and offset with respect to a stretched wire have to be improved in order to achieve the tolerance. As the magnets will be installed in a new tunnel, the alignment will have to be re-checked and probably adjusted every year, at least during the first ten years. Because of access restrictions, measurements and realignments will have to be performed rapidly. A survey and alignment train will need to be developed; a prototype of such a train using interferometry technology was studied for automatic survey of the ILC [300].

\subsubsection{Interaction regions and collimator areas}

The alignment accuracy for the interaction regions is assumed to be the same as for the LHC, i.e. $0.1 \mathrm{~mm}$ for the triplets located on the same side of the IP, $0.2 \mathrm{~mm}$ from left side of the IP to the right side and $0.5-1.2 \mathrm{~mm}$ from the triplets to the detector (all values given at $1 \sigma$ ). To achieve these specifications, survey galleries will be needed to host part of a permanent monitoring system based on the latest sensor technology available.

Due to the high level of radiation in the collimator areas, a permanent monitoring system, or an automatic measuring solution using a survey train, will be used together with a remotely controlled re-alignment system. They will be based on the latest developments implemented for HL-LHC. A robotic solution must also be developed to maintain, dismantle and re-install the permanent survey systems.

\subsubsection{Experiments}

The alignment accuracy for assembly of the experiment is assumed to be similar to those of Atlas and CMS i.e. $0.5 \mathrm{~mm}$. The positioning of the experiment with respect to the beam line is done using a geodetic network for the experiment derived from the underground network. It is composed of points distributed across the whole cavern volume on the walls and floor. It is used at all stages of the assembly and positioning of the detectors. It is measured once the cavern has been delivered and is still empty, using mainly distances, angles and levelling measurements. The use of 3D laser tracker technology is appropriate for this type of 3D network. From this network, only the outer skin of the experiment is visible and therefore the position of the inner detectors will be reconstructed from the position of the external fiducials and the fiducialisation and assembly measurements. However, the forward trackers are located inside a magnetic field and this has to be studied. 
Data and Computing Services Scope

General IT services and infrastructures

\begin{tabular}{|c|c|c|c|c|}
\hline $\begin{array}{c}\text { Particle } \\
\text { Collider } \\
\text { Experiments }\end{array}$ & $\begin{array}{l}\text { Worldwide } \\
\text { Research } \\
\text { Community }\end{array}$ & $\begin{array}{c}\text { Particle } \\
\text { Accelerators } \\
\text { and Collider }\end{array}$ & $\begin{array}{c}\text { Infrastructure } \\
\text { Services }\end{array}$ & Administration \\
\hline
\end{tabular}

Fig. 5.23. Scope of the IT services found in a high-energy physics research facility.

\subsection{Communications, computing and data services}

During the LHC operation era computing for particle accelerators and the experiments has evolved into a service for a world-wide user community. Adopting products and best practices that emerge from an ever-growing Information and Communication Technologies (ICT) industry has proven to be a cost and performance effective path to serve the community. Large-scale science projects used to be a driver of IT infrastructure developments [301-306]. A future particle collider research facility can again be a case and a driving force for advanced ICT developments in areas that go beyond the hardware and software domains, which dominated the particle accelerator projects of the 1980's and 90's.

A set of general services comprising wired and wireless networks, desktop, mobile and centralised computing for all users, various storage system tiers, software and data provisioning, authentication and authorisation, assistance and consultancy, training and much more, form the backbone of services for individual business units (see Fig. 5.23). Depending on the activity type, different levels of quality of service apply. The particle collider experiments require an elastic data communication and processing infrastructure for data acquisition and high-level event filtering. The data need to be made accessible for a world-wide research community together with data organisation, archiving, retrieval and the management of a dynamically evolving federation of participating organisations. The provision of openly accessible information and the engagement of the public through services to develop citizen-science projects are additional functions for this domain. Computing for the particle accelerator, on the other hand, involves various different networks for data, voice and video communication as well as infrastructures comprising embedded and real-time computing facilities.

Service units of the facility such as the fire brigade and medical services, security and site protection, environmental monitoring, safety-related systems and industrial installations require dedicated IT services. In particular, remote monitoring and intervention has become an important means to reduce service level agreement costs for industrial plants over the last ten years. Finally, administration units ranging from human resources and finance to different types of workflow systems need to be appropriately served whilst taking into account the length of the construction and operation phases which will last for decades. For all domains, resilience, data protection, cyber-security, technology evolution and migration and long-term data accessibility are topics that call for a dedicated organisation to ensure proper coverage with an appropriate mix of in-house personnel, external suppliers and industry/academia partnerships.

The significant geographical extent of the services and long-term sustainability of a new large-scale particle-collider research facility require a shift of activities, traditionally covered by individual detector and accelerator engineering groups, to 
a business oriented scientific IT unit. This approach allows tenders, contracts and operation to be optimised at an organisation-wide scale as well as influencing the value of member state contributions favourably for the member countries, the organisation and the world-wide community. In particular, this approach for the construction and operation of data centres can be beneficial for experiment users, accelerator engineers and users with generic needs.

Computing and interconnect technologies are evolving rapidly and generation changes need to be expected. At the same time the optimum cost effectiveness is continuously swinging between buying and leasing. This ever changing IT environment can best be accommodated by a continuous cost/benefit analysis considering all users in the organisation, carried out by a team which is working closely with industry on one side and with the users on the other.

Embedded and real-time computing including programmable logic controllers are also a concern for a technology infrastructure that is characterised by its longevity and thus dominated by operating costs. Given the significant increase in the number of devices for a future collider, further standardisation, coordinated testing and certification and procurement and maintenance/repair services, available to all users of the organisation, will help to improve the cost effectiveness. A large-scale particle accelerator building on decades of engineers experience presents an ideal case for an openly available architecture and platform for supervisory control to integrate the diverse subsystems. A system that can evolve with emerging "Internet of Things" (IoT) products and yet unanticipated device technologies can create impact far beyond the particle accelerator community.

Cyber-security plays an increasingly important role in IT systems and embedded computing is no exception to this. The use of processors, operating systems and embedded Web servers in the majority of programmable laboratory equipment such as simple digital I/O devices, measurement instruments, oscilloscopes and autonomous robots already require a well organised infrastructure. This is supported by a process that leads to a secure environment on one side and which has the least possible impact on usability on the other side. For example, it should allow the possibility of developing and deploying across network boundaries, create islands and sandboxes to limit potential harm, have transparent virus and malware checking and isolation, have system updates that have little or no impact on work efficiency, provide coordinated rollback and much more. This evolution is expected to continue and the IoT approach will also require an organisation wide vertical integration of services across the horizontal user domains [250].

Cooperation on IT standards, technology developments and organisation with other research facilities which have similar requirements (e.g. DESY, ESRF, ESS, Fermilab) needs to be strengthened. Synergies with other scientific domains (e.g. astronomy and radioastronomy facilities, material sciences with light sources and FELs, astrophysics installations such as neutrino and gravitational wave observatories, particle accelerators for medical applications and nuclear fusion experiments) can be developed to lead to more effective operation of world-wide IT services for research. Activities spawned by DESY on front-end computing hardware [307] and CERN's openlab [308] are good examples for such initiatives.

The geographical extent of a $100 \mathrm{~km}$ long particle collider across the Canton of Geneva and far into the Haute Savoie region suggests the development of cooperation with the host state communication network providers and with telecom operators. Improving the high-tech communication, computing and data service infrastructure in the region is high on the agenda of the host state authorities with an ambition to become a "European Silicon Valley" [309]. It creates further potential for the strengthening of CERN as a focal point of high-technology in a region in which the 
population approaches 2 million (departments Ain and Haute Savoie, canton Geneva) and which also has a high annual growth rate [310].

Possible services do not only include fibre optics and data centres that can be shared with academic partners but can also include mobile communication and the cooperation with emergency and rescue services in a cross-border context. CERN's activities concerning the establishment of the TETRA radio communication system in the region are a first step in this direction [311]. CERN's particular status as carrier-neutral Internet eXchange Point (CIXP) [312] will gain importance in a technology ecosystem that becomes ever more dominated by profit making organisations which are building and operating global communication infrastructures. These collaborations may help to ensure that non-profit making organisations continue to operate independently and become less dependent on infrastructure operator priorities, which may favour financially stronger commercial clients.

Beyond the regional scale, the LHC programme has shown the value of a worldwide computing and communication infrastructure to make the research data available to scientists in all participating countries [313]. The success of a future particle collider programme will rely even more on international participation. Many potential participating countries are not part of CERN's global vision today and some are still technologically underserved. The SESAME light-source under the auspices of UNESCO is a great example of such an initiatives [314]. The capacity to plan, develop and implement a world-wide inclusion policy goes beyond the scope of the high energy physics community and is therefore also a good opportunity to establish a common strategy with other scientific disciplines. A timely development of such an initiative will help raising the interest and acceptance level of large-scale investments in an infrastructure focusing on fundamental scientific research.

Considering the fast evolution of information technologies in all domains [315], the long term cost impact of in-house technology developments and their potentially limited large scale impact, it is prudent to base architectures for the particle accelerator and the experiment data processing environments on industrial hardware, software and service infrastructures. The particular needs of an FCC-scale facility may, however, also represent attractive test-beds for emerging technologies. Co-innovation projects with industrial partners during the early construction phase, permitting precommercial procurement initiatives that can lead to high-performance infrastructure services at a competitive cost are one way to optimise this situation [316].

A preliminary cost-benefit-analysis of the LHC/HL-LHC programme [317] revealed that more than the impact value generated by training coresponds to more than one third of the infrastructure's cost (sum of captital and operation expenditures). The ICT sector represents an ideal case for training at large with ever growing societal and industrial demands. Early stage researchers and engineers are much appreciated participants in CERN's technology programmes, which give them opportunities to acquire skills that are also high on the wish list of industry. It would be possible to extend these training programmes to participating industrial partners, generating value for industry directly. The CERN openlab public-private partnership already demonstrates the validity of this approach. Further industrial cooperation can focus on field testing of pre-commercial products and services, common optimisation, development of standards and best-practices and co-innovation with the research infrastructures as a demonstration case. These activities would be carried out in low risk environment for industrial partners of any size. The scaling up of the open, industrial SCADA platform PVSS around the year 2000 is one example of the success of this approach [318]. Eventually, the company was integrated in the SIEMENS group and the software has been re-branded as WinCC OA to become the SIEMENS flagship SCADA system on the global scale. 
Finally, long-term data availability [319] has become an important aspect of ensuring the lasting impact of the facility [320]. The accessibility of several decades worth of raw LHC data, all metadata and previous analysis results has turned out to be a major topic for the ICT community. With a future particle collider, the time span will extend to the end of the 21st century, calling for evolving data storage and management systems that serve the worldwide particle physics community for more than 100 years. Considering the continuous evolution of data formats, the ever-changing particle detectors and a highly dynamic user community, data quality management is a primary topic. The value of a particle collider research facility depends directly on its quality and long term world-wide accessibility for as large a community of scientists as possible.

\subsection{Safety and access management systems}

A safety management system (SMS) for a future large-scale particle collider will be based on an industry best-practice system which integrates, in a uniform and regulatory-compliant way, everything which contributes to safe operation. This system also includes the procedures associated with the different operating conditions encountered during the lifetime of the collider. A high-level computer-based safety management system integrates underlying safety related functions, including fire detection, oxygen deficiency detection, smoke and helium extraction systems, fire extinction systems, access and authorisation management, door supervision and control, video surveillance, radiation monitoring, conventional environmental monitoring, evacuation signalling, supervision and control of lifts, communication with people in underground zones, emergency lighting and acoustics and communication with emergency services (fire fighting, rescue, healthcare providers, public and private security forces). The sub-systems function autonomously.

There are two complementary systems for personnel protection in the underground areas: the access safety system and the access control system. During machine operation, the access safety system protects personnel from the hazards arising from the operation of the accelerator and from the beams. It acts through interlocks on important safety elements of the accelerator. By interlocking these elements it is possible to establish the right accelerator and equipment conditions in order to allow authorised personnel to access the underground installations or to allow the restart of the accelerator equipment when access is finished. When the accelerator is not operating with beam and is in access mode, the access control system allows the positive identification of any person requesting access and ensures that all pre-requisites and authorisations for that person are valid. For operational and/or safety reasons, the access control system also limits the number of people present simultaneously in the underground areas.

An automatic fire detection system consisting of detectors and air sampling networks to detect the presence of smoke is connected to control and monitoring equipment located in one of the surface buildings at each point. If fire or smoke is detected, the system launches automatic safety functions and alerts the fire and rescue service. Fire detection is installed in all underground areas and will allow accurate location of a fire. An automatic oxygen deficiency detection system warns users of the danger and alerts the fire and rescue service. Underground areas will also be equipped with communication channels to allow a user to contact the fire and rescue service directly.

The SMS launches safety functions if there is a fire or oxygen deficiency is detected. These functions include compartmentalisation, evacuation and smoke extraction. The CERN fire brigade has the possibility to trigger these functions remotely and to broadcast safety instructions in the various areas of the facility. 
Table 5.21. Examples for typical safety management system solutions for large-scale applications.

\begin{tabular}{|l|l|}
\hline Supplier & Product \\
\hline Advancis Software \& Services & PSIM \\
\hline ATS Elektronik & AES5000, DLS4000 \\
\hline Bosch Security Systems & Building Integration System \\
\hline CENARIO solutions & CENARIO \\
\hline digivod & CRISP PSIM \\
\hline ETM/SIEMENS & WinCC OA \\
\hline Genetec & Security Center \\
\hline GEOBYTE & Metropoly BOS \\
\hline Honeywell & $\begin{array}{l}\text { Enterprise Buildings Integrator, WIN- } \\
\text { MAG plus }\end{array}$ \\
\hline KÖTTER Security & LENEL OnGuard \\
\hline PKE & AVASYS \\
\hline Scanvest & ScanVis.Pro \\
\hline Securiton & Universal Management \\
& SecuriLink UMS, IPS \\
\hline SIEMENS & GMA-Manager, Siveillance Vantage \\
\hline Tyco Integrated Fire \& Security \\
CKS Systeme & CELIOS, C-cure 9000 \\
\hline WAGNER Group & VisuLAN X3 \\
\hline
\end{tabular}

The emergency evacuation system is a part of the automatic protection system. It broadcasts audible evacuation signals triggered either automatically by a safety system, such as fire or oxygen deficiency detection, or manually by pushing one of the emergency evacuation buttons installed within the area in question.

The SMS provides prioritised and homogeneous visualisation of the status of all safety relevant parameters, allows the supervisory control of all sub-systems and handles the sub-system interconnections. The SMS communicates with the sub-systems through fail-safe protocols, over a dedicated communication infrastructure. It guarantees that critical alarms are automatically transmitted to the competent services (e.g. fire brigade, radiation protection team) and that all incidents are recorded and suitably documented for potential internal and external examination (auditing). Furthermore, the SMS ensures that any condition which is incompatible with safe beam operation (e.g. intrusion) is detected and the beam is aborted.

Such supervisory systems are in daily operation in most large-scale plants (e.g. particle-accelerator-based ion therapy facilities, oil and gas rigs, manufacturing and processing plants). Examples for typical Safety Management System solutions are listed in Table 5.21.

The future system must be compliant with international norms, be open and extensible and be configurable to the specific application (e.g. GIS and CAD integration, user interface designer). Processing speed is generally not critical, but the system must work extremely reliably, be highly scalable and be open to the integration of a continuously growing set of diverse subsystems from different suppliers. Implementation details (e.g. positioning of a central supervision point, the number and position of decentralised facilities to interact with the system, hard- and software choices, rights management, means to identify people requesting access, or locating people in the machine) will be subject to a requirements specification phase, once the detailed design of the infrastructure and its individual technical systems are well known. 


\section{Injector scenarios}

\subsection{Introduction}

\subsubsection{Requirements}

The FCC-hh injector chain has to fill roughly $80 \%$ of FCC-hh with the nominal $3.3 \mathrm{TeV}$ proton bunches in $\sim 30 \mathrm{~min}$. This corresponds to 10400 bunches spaced by $25 \mathrm{~ns}$ with a bunch intensity of $1 \times 10^{11}$ protons and a normalised emittance of $2.2 \mu \mathrm{m}$. A list of the most relevant beam parameters for the FCC injector complex is given in Table 6.1.

The baseline injection energy of $3.3 \mathrm{TeV}$ requires a High Energy Booster (HEB) in addition to the existing LHC injector chain. The HEB designs explored take into account a range of possible energies, since the optimum transfer energy for FCC-hh may change. A higher energy is favourable in terms of impedance, beam stability, aperture and energy (field) swing, but a lower energy is favourable for the transfer to collider ring as well as for simplicity and cost optimisation of the injector complex. The overall project optimisation needs also to take into account a tradeoff between capital and operation costs.

The injection energy also determines the number of bunches which can be safely transferred to FCC, to avoid damage to the injection protection absorbers. This limit scales non-linearly with beam energy, as the energy deposition in the absorber also depends on the secondary shower development. At $3.3 \mathrm{TeV}, \sim 80$ bunches (compared to $\sim 320$ bunches at $1.3 \mathrm{TeV}$ ) can safely strike the absorber, a staggered transfer is deemed necessary.

Multiple extractions (around 100) are therefore needed to fill the FCC-hh; consequently fast rise-times are required for the HEB extraction and FCC-hh injection kickers. Higher transfer energies need even faster rise-times to maintain a given FCChh filling factor. It is assumed that the HEB extraction kickers will be similar to the FCC-hh injection kickers. Concepts for the collider injection system, using solid-state generators such as the inductive adder [206] or Marx generator [321], for multiple injections of short bunch trains, have been developed for $3.3 \mathrm{TeV}$ injection.

The injector complex has to have a high degree of flexibility in terms of stability and insensitivity to configuration changes. In addition to filling FCC-hh several times per day, it will also be called upon to serve multiple users, for example beams for material test facilities and fixed target experiments using slow extraction.

Other important requirements are that the injector chain including the HEB has high availability, to minimise impact on the FCC-hh collider availability, and the HEB is considerably easier to operate than the collider itself.

New transfer lines from HEB to FCC-hh will also be needed. To reduce the complexity and the operational costs of the injector complex, the transfer line dipole magnet fields have to be as low as possible.

\subsubsection{HEB options}

Reuse of the existing CERN proton and ion chains as an injector complex makes a modified version of the existing $27 \mathrm{~km}$ LHC the obvious candidate as High Energy Booster (HEB) ring, but other options are also being considered.

The other tunnels which are potentially available to house an HEB are the $6.7 \mathrm{~km}$ SPS tunnel, which could reach $1.3 \mathrm{TeV}$ if $6 \mathrm{~T}$ superconducting magnets are used for a superconducting SPS (scSPS), and the $100 \mathrm{~km}$ FCC tunnel itself, which could house a 3.3 TeV HEB with $1 \mathrm{~T}$ iron-dominated or superferric magnets along with the FCC-hh 
Table 6.1. Relevant beam parameters for the FCC injector complex.

\begin{tabular}{|llr|}
\hline Parameter & Unit & Value \\
\hline Injection energy & $\mathrm{TeV}$ & 3.3 \\
Number of bunches & & 10400 \\
Bunch spacing & $\mathrm{ns}$ & 25 \\
Bunch intensity & $\mathrm{p}$ & $1 \mathrm{E} 11$ \\
Normalised emittance & $\mu \mathrm{m}$ & 2.2 \\
Turn around time & $\mathrm{h}$ & 5 \\
FCC filling time & $\mathrm{h}$ & 0.5 \\
\hline
\end{tabular}

LINAC4

PSB

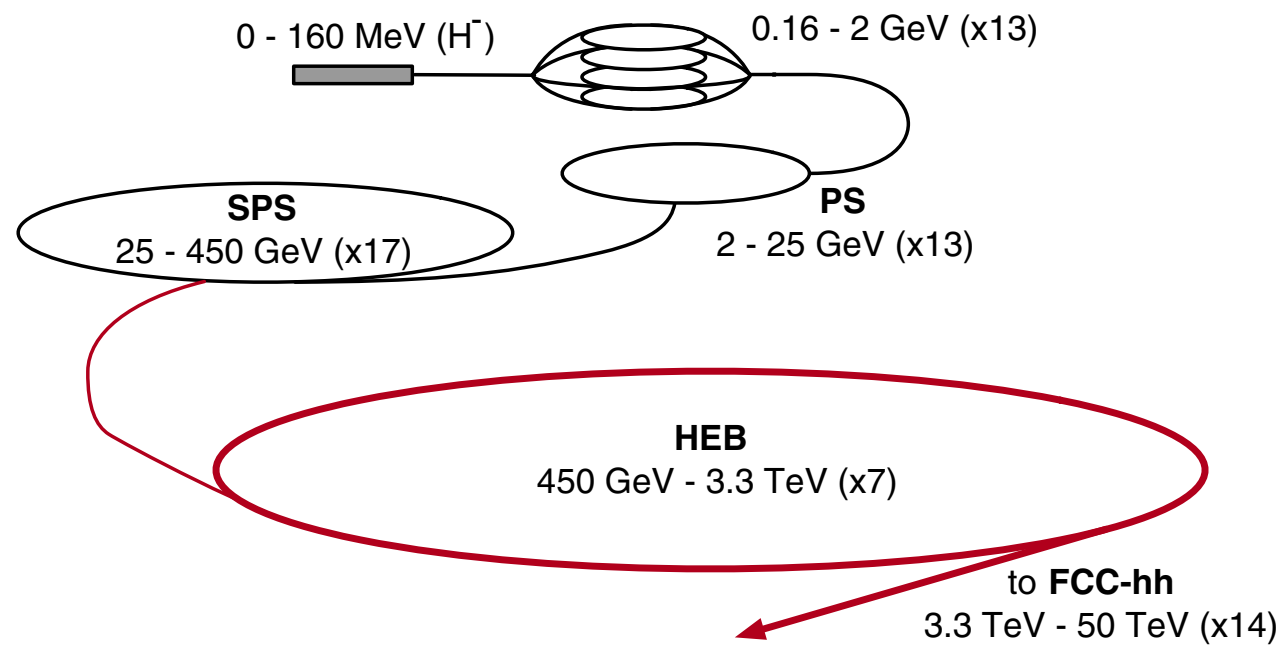

Fig. 6.1. FCC-hh injector chain, based on the existing LHC injector chain and a $3.3 \mathrm{TeV}$ high energy booster - HEB.

Table 6.2. Planned beam parameters at LHC top energy, after the LIU and HL-LHC upgrades. The maximum number of bunches per injection from the SPS is quoted.

\begin{tabular}{|lrrrr|}
\hline Parameter & $\begin{array}{r}\text { Bunch pop. } \\
\left(10^{11} \mathbf{p} / \mathbf{b}\right)\end{array}$ & $\epsilon_{x, y}(\mu \mathbf{m})$ & $\epsilon_{z}(\mathbf{e V s})$ & $\begin{array}{r}\text { Bunches/ } \\
\text { injection }\end{array}$ \\
\hline Standard 25 ns & 2.3 & 2.08 & 0.56 & 288 \\
BCMS 25 ns & 2.3 & 1.6 & 0.56 & 240 \\
\hline
\end{tabular}

collider. The options for HEB have been investigated in terms of maximum energy, magnet technology, transfer line geometry, turnaround time and also the suitability as a fixed-target facility.

\subsubsection{Pre-injector chain}

The pre-injector chain will consist of Linac4, PSB, PS and SPS (the present LHC injector chain), in addition to the HEB, (see Fig. 6.1). The performance is assumed to be that of the existing chain after the LHC Injector Upgrade (LIU) project [37], see Table 6.2 , for which the expected performance at $25 \mathrm{~ns}$ already significantly exceeds that required by FCC. 


\subsection{4 $5 \mathrm{~ns}$ bunch spacing}

For $5 \mathrm{~ns}$ bunch spacing, the total beam current remains the same as for the $25 \mathrm{~ns}$ baseline, with a factor 5 increase in number of bunches, compensated by a factor 5 reduction in bunch intensity. However, the accompanying factor 5 reduction in transverse emittance is more challenging for the injectors.

The present CERN accelerator complex produces the high intensity 5 ns beam with $10^{10} \mathrm{p} / \mathrm{b}$ by modulating the debunched PS beam at $200 \mathrm{MHz}$ for injection into SPS at $14 \mathrm{GeV} / \mathrm{c}$ (below transition) by a multi-turn extraction (MTE) in 5 turns [322]. There is no bunch-to-bucket transfer: beam in the extraction kicker gap is lost in the PS ring. The transverse emittances are $4 \mu \mathrm{m}$ in both planes.

The brightness limits in the PSB and PS, see Figure 6.2, would give about 140 bunches with the required intensity and emittance, filling only about $40 \%$ of the PS circumference. The FCC filling time and filling factor would therefore suffer somewhat. Regarding the production scheme, the PS could be used to produce a 5 ns beam in an adiabatic way at the cost of adding a new RF systems at $50 \mathrm{MHz}(h=105)$ and $100 \mathrm{MHz}(h=210)$. The correct bunch spacing requires a complicated production scheme [323]. Another issue to solve is the challenge to preserve this very small emittance through the injection and ramp of the SPS and HEB - at present low-intensity beams of $1 \mu \mathrm{m}$ transverse emittance are routinely delivered for LHC. An alternative to improve performance would be to replace PSB and PS by the proposed SPL and PS2 [324].

\subsection{Transfer lines}

The geometry and magnet technology needed for the transfer lines from the HEB to FCC-hh depend strongly on the transfer energy and which of the HEB variants is chosen. For the two main options studied, a $3.3 \mathrm{TeV}$ LHC and a $1.3 \mathrm{TeV}$ scSPS, the basic transfer line geometries have been defined using a dipole filling factor of $70 \%$.

For the 3.3 TeV HEB in the LHC tunnel (either LHC reuse or a new $4 \mathrm{~T}$ machine), the transfer line geometry is shown in Figure 6.3. Due to the short distance between LHC LSS1 and FCC PB, the dipole field needs to be $\sim 7 \mathrm{~T}$, which is technically feasible but requires expensive high-field SC magnets.

For the $1.3 \mathrm{~T}$ injector in the SPS tunnel the transfer line layout is shown in Figure 6.4. In this case normal-conducting dipoles of $1.8 \mathrm{~T}$ will be suitable, similar to the MBA magnets already used in the SPS-to-LHC transfer lines.

In addition to the dipole field, the other important figures of merit for the transfer lines are the total dipole length, the number of quadrupoles, the tunnel length, and the maximum vertical slope. These figures are compared for the two options in Table 6.3.

Note that each transfer line has a straight section of at least $424 \mathrm{~m}$ (2 cells), either at the beginning or at the end, to accommodate the injection protection collimation system; however, further studies are needed to define the system together with the optics, and to investigate whether sufficient protection can be provided if the collimation system is at the start of the transfer line. Some optimisation of the magnets required may also be possible, e.g. by using tilted bends.

\subsection{Reuse of LHC as HEB}

Reusing the LHC as the HEB $[325,326]$ is conceptually the most straightforward way to inject into the FCC-hh at $3.3 \mathrm{TeV}$, with well known performance. To make space 


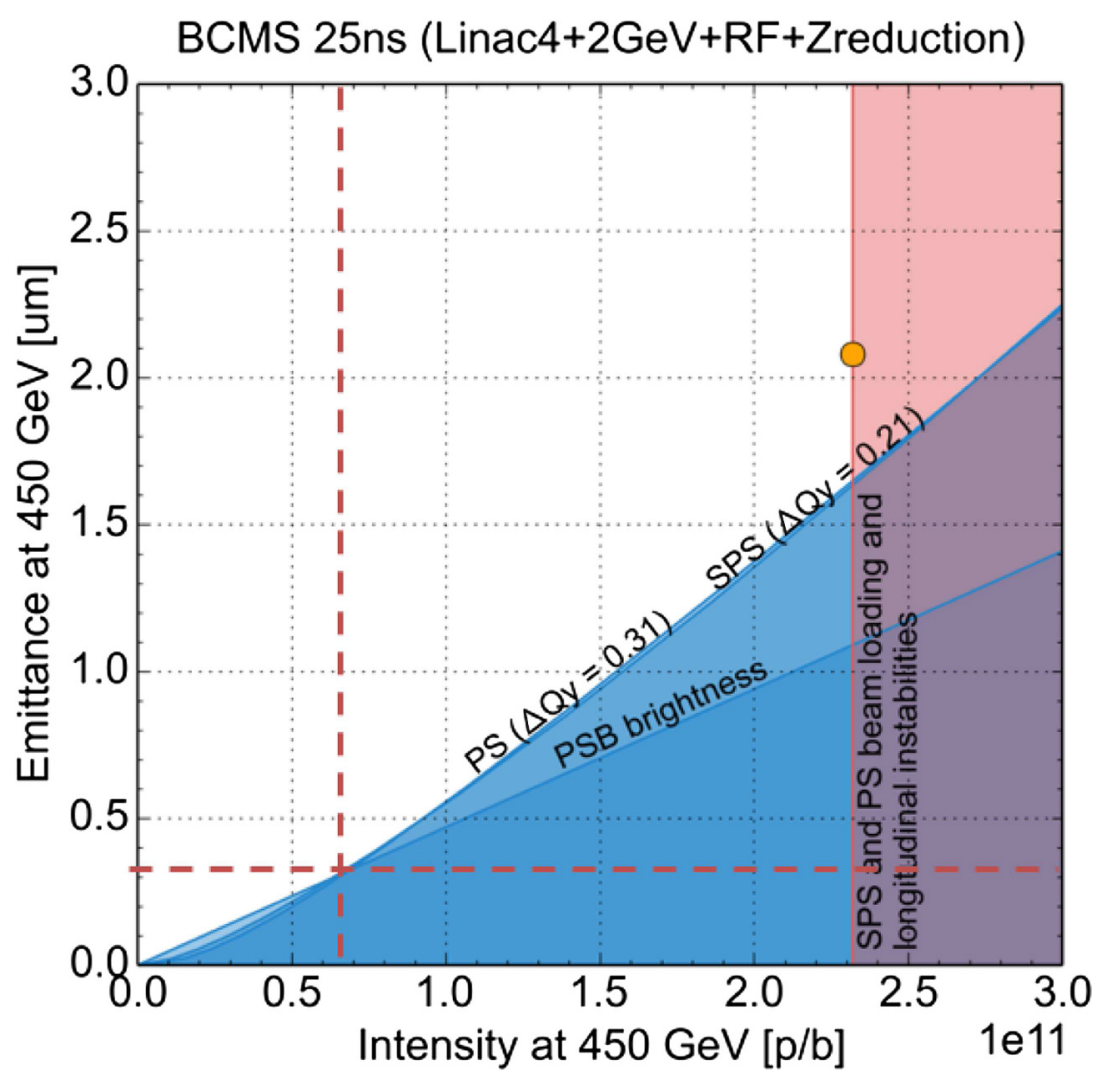

Fig. 6.2. Accessible brightness region for beams injected into the HL-LHC, showing the working point needed for the $5 \mathrm{~ns}$ option for FCC. The preservation of the very small emittances at low intensity through the injector chain and into the FCC needs to be explored.

for the extractions towards FCC, two beam crossings have to be removed from the LHC ring. The existing RF, collimation and beam dump systems will be maintained; however, keeping the orientation of the beam dump while removing two crossings means that the injection will have to be shifted from the outer to the inner rings. The physics experiments and low energy insertions will have to be decommissioned. The changes in the LHC layout are depicted in Figure 6.5.

The other important modification to the LHC is to speed up the ramp rate, which will need to be improved by roughly a factor $5-50 \mathrm{~A} / \mathrm{s}$, to keep the filling time in the $\sim 1$ hour range. This requires new main power converters as well as changes to other systems [327]. These aspects all need to be considered in the overall optimisation. The existing LHC RF system, with a voltage of $16 \mathrm{MV}$ at $400 \mathrm{MHz}$, is able to accelerate an LHC beam to $7 \mathrm{TeV}$ in $2 \mathrm{~min}$. The limiting factor, however, is the ramp in the main dipoles. After the modifications these will be able to ramp to $3.3 \mathrm{TeV}$ in about 3 min [328], hence the RF will not need to be changed.

The early part of the ramp will also need to change from the baseline ParabolicExponential-Parabolic-Linear (PELP) configuration, which otherwise would take over half of the total ramp duration. For this reason a simpler Parabolic-ParabolicLinear-Parabolic (PPLP) ramp was developed in MDs, it was tested successfully in 2017 [329] and it demonstrated a saving of $1.5 \mathrm{~min}$ per ramp with no adverse effect on the beam. This is now being adopted as the operational LHC ramp and should 


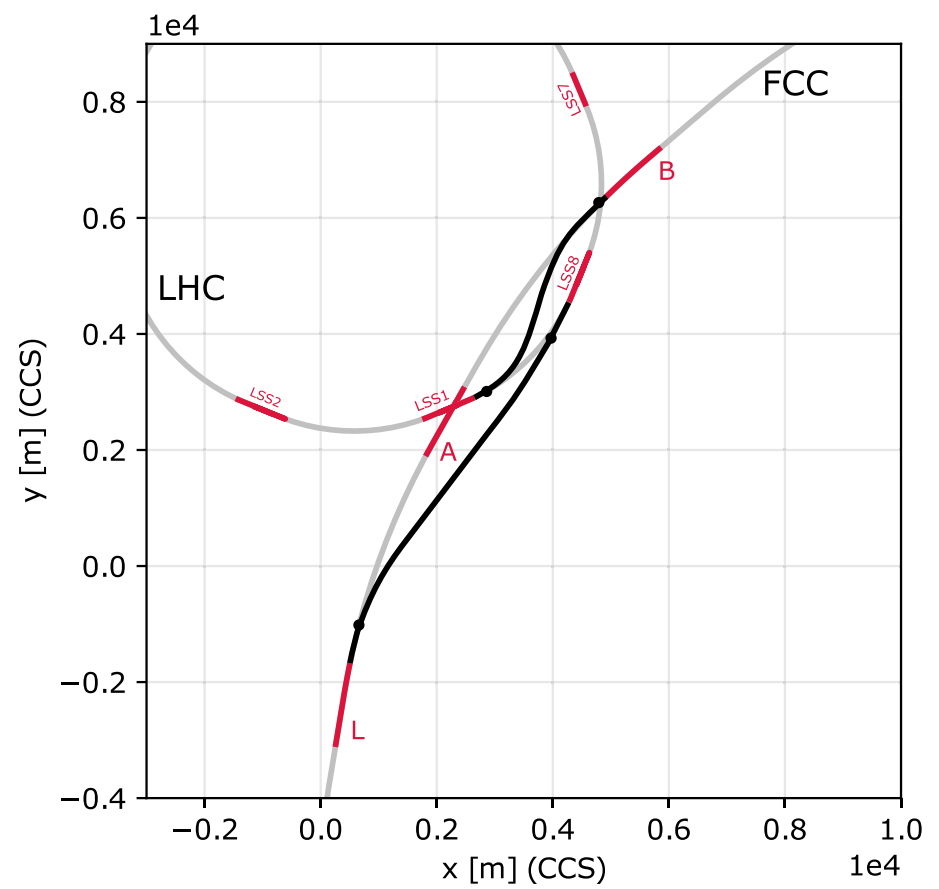

Fig. 6.3. Transfer lines (thick lines) from a $3.3 \mathrm{TeV}$ LHC HEB LSS1 and LSS 8 to the main FCC-hh ring. The proximity of LHC LSS1 and FCC PB mean that $\sim 7 \mathrm{~T}$ superconducting transfer line dipoles will be needed.

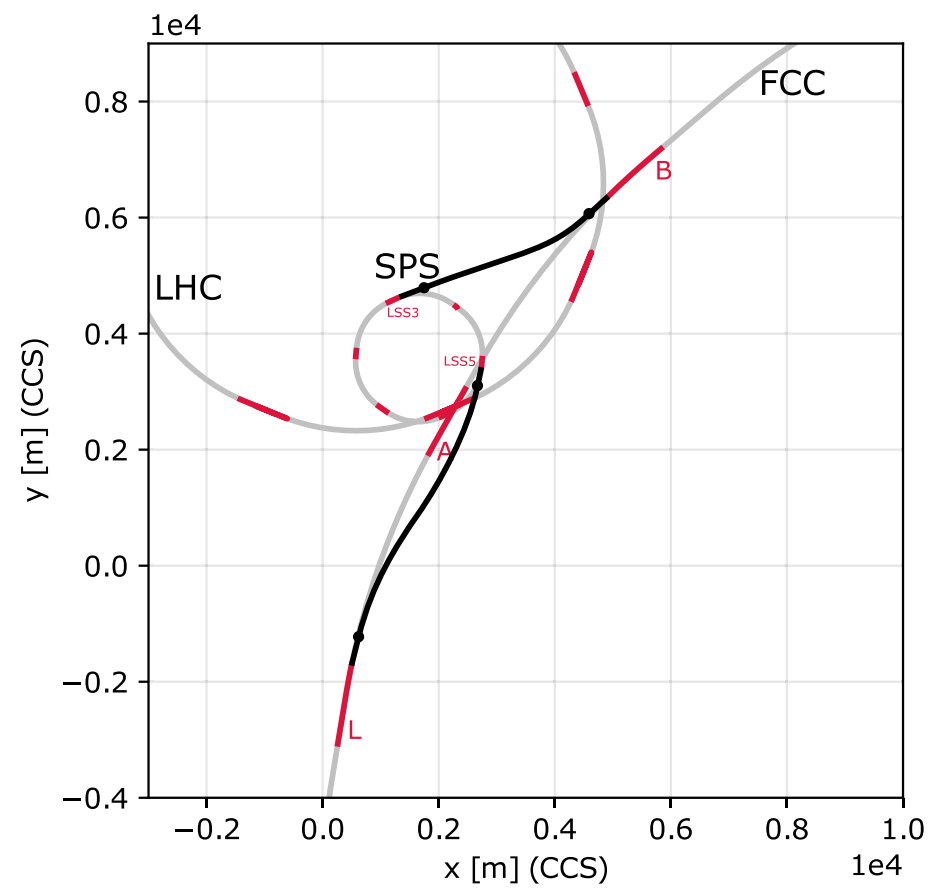

Fig. 6.4. Transfer lines from a $1.3 \mathrm{TeV}$ SPS HEB LSS3 and LSS5 to the FCC-hh main ring. With this layout the transfer lines could be normal-conducting with $1.8 \mathrm{~T}$ dipoles. 
Table 6.3. Comparison of main figures of merit between $3.3 \mathrm{TeV}$ superconducting transfer lines from LHC to FCC and $1.3 \mathrm{TeV}$ normal conducting transfer lines from SPS to FCC.

\begin{tabular}{|llrr|}
\hline Parameter & Unit & LHC to FCC & SPS to FCC \\
\hline Energy & $\mathrm{TeV}$ & 3.3 & 1.3 \\
Bending dipole field & $\mathrm{T}$ & 7.2 & 1.8 \\
Magnet type & & Superconducting & Normal conducting \\
FODO cell length & $\mathrm{m}$ & 106 & 106 \\
Dipoles per bending half-cell & & 2 & 4 \\
Dipole magnet length & $\mathrm{m}$ & 14.3 & 6.2 \\
Dipole filling factor & $\%$ & 72.5 & 72.5 \\
Number of dipoles & & 280 & 736 \\
Number of quadrupoles & & 221 & 189 \\
Total transfer line length & $\mathrm{km}$ & 11.7 & 10.0 \\
Maximum vertical slope & $\%$ & 2.10 & 3.92 \\
\hline
\end{tabular}
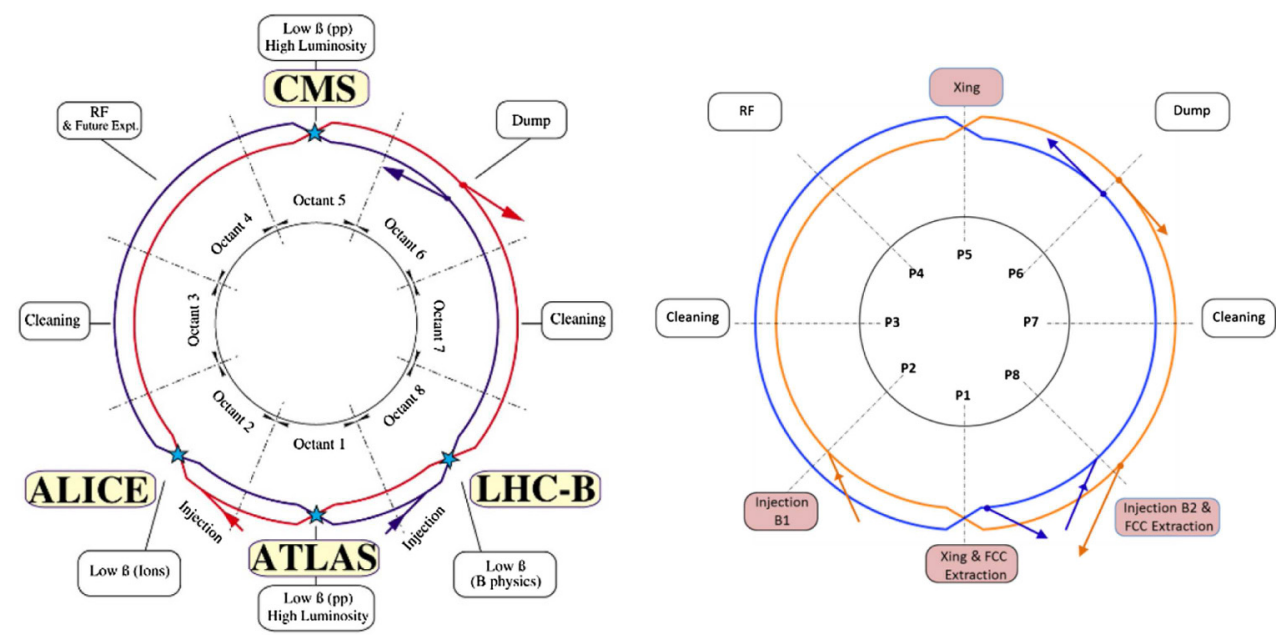

Fig. 6.5. Existing (left) LHC layout, compared to the layout needed for use as HEB (right). Changes are required in four of the eight long straight sections.

save a total of about $10 \mathrm{~h}$ of operation per year. By the way, this clearly demonstrates the benefits of FCC studies for the operating LHC machine.

The remaining concerns for the reuse of LHC will be the high power consumption for the LHC cryogenic system, the operating cost and also the age and related availability of the LHC when FCC-hh will come on line.

The main layout features per LHC straight section are summarised below:

- LSS1: new beam 1 extraction system plus beam crossing;

- LSS2: injection to inside ring plus decommissioning of ALICE;

- LSS3: no changes to momentum collimation;

- LSS4: no changes to RF system;

- LSS5: decommissioning of CMS plus beam crossing;

- LSS6: no changes to beam dump;

- LSS7: no changes to betatron collimation;

- LSS8: injection to inside ring plus new beam 2 extraction system.

The new extraction system in LSS1 can be very similar to the current beam dump extraction system in LSS6, although it will need to open up the space between the 


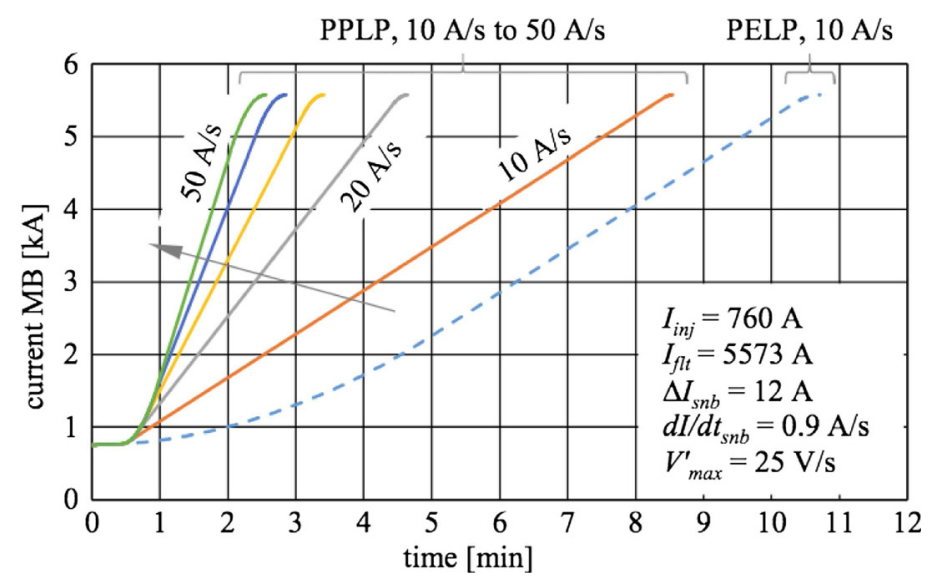

Fig. 6.6. $450 \mathrm{GeV}-3.3 \mathrm{TeV}$ LHC ramps, showing the gain from a PPLP ramp with different linear ramp rates between 10 and $50 \mathrm{~A} / \mathrm{s}$, compared to the present LHC PELP ramp (dashed).

lattice quadrupoles Q4 and Q5 downstream of the septum, to accommodate the new beam crossing. The layout studied will accommodate beam crossing for energies up to $4.5 \mathrm{TeV}$.

Changing the injection from the outer to the inner ring looks feasible in both LSS2 and LSS8, with a $16 \mathrm{~m}$ longitudinal shift of the injection systems and lattice quadrupoles. In LSS8 this needs to be combined with an extraction system; again this is feasible for $3.3 \mathrm{TeV}$.

Decommissioning of the LHC experiments is likely to prove to be one of the cost-drivers for the conversion of LHC to FCC HEB. The residual radiation levels in LSS1 and LSS5 at the end of LHC physics operation are expected to be at around $1 \mathrm{mSv} / \mathrm{h}$ [330]. Work on dose estimates, handling and decommissioning is required, using the detailed dose rate maps as input for the computation of doses for the various tasks, once work scenarios are available.

The possibility of operating LHC as a fixed-target facility, interleaved with filling the FCC-hh, has been studied [331]; here the challenges are the integration of a slowextraction system into the superconducting, small aperture LHC machine, designing the extraction hardware and also the low annual numbers of protons on target that could be achieved due to the long LHC ramp time.

\subsection{Superconducting SPS as $1.3 \mathrm{TeV}$ HEB}

A new superconducting SPS (scSPS) in the existing $6.9 \mathrm{~km}$ tunnel with $1.3 \mathrm{TeV}$ extraction energy has been studied as an alternative HEB option [332], in case the collider can accept such a low injection energy. Such a machine is also of interest for a High-Energy-LHC (HE-LHC [333]) in the LHC tunnel, where an injection energy higher than the $450 \mathrm{GeV}$ available from the existing SPS could be mandatory for field quality, aperture and impedance reasons. For future fixed target programmes in CERN's North Area, the possibility of slow-extracted beams at energies above $1 \mathrm{TeV}$ could open new physics and detector test beam opportunities [331], especially if a relatively fast-ramping concept can deliver large numbers of protons on target per year.

The main parameters of the scSPS can be found in Table 6.4. These values are based on the parameters of a $12 \mathrm{~m}$ long, $6 \mathrm{~T}$ superconducting dipole magnet. The 
Table 6.4. Main parameters of scSPS.

\begin{tabular}{|lll|}
\hline Parameter & Unit & Value \\
\hline Injection energy & $\mathrm{GeV}$ & 25 \\
Extraction energy & $\mathrm{GeV}$ & 1300 \\
Maximum dipole field & $\mathrm{T}$ & 6 \\
Dipole field at injection & $\mathrm{T}$ & 0.12 \\
Dipole magnet length & $\mathrm{m}$ & 12.12 \\
Cold bore inner diameter & $\mathrm{mm}$ & 80 \\
Number of dipoles & & 372 \\
Number of quadrupoles & & 216 \\
Ramp rate & $\mathrm{T} / \mathrm{s}$ & $0.35-0.5$ \\
Cycle length & $\mathrm{min}$ & 1 \\
Number of bunches per cycle & & 640 \\
Number of injections into scSPS & & $8(8 \times 80 \mathrm{~b})$ \\
Number of protons per bunch & & $\leq 2.5 \times 10^{11}$ \\
Number of extractions per cycle & & $2(2 \times 320 \mathrm{~b})$ \\
Number of cycles per FCC filling & & 34 \\
FCC filling time & min & $34-40$ \\
Max stored beam energy & MJ & 33 \\
\hline
\end{tabular}

interconnects between two neighbouring magnets are assumed to have a length of $1.25 \mathrm{~m}$.

The circulating beam would contain $\sim 33$ MJ energy, requiring beam dump and collimation systems which will resemble those of the LHC more than those of the SPS. To respect $\sim 30-40 \mathrm{~min}$ filling time, the average $\operatorname{ramp}$ rate should be $0.35-0.5 \mathrm{~T} / \mathrm{s}$, with 34 ramps to fill both FCC-hh rings.

Using PS as injector, the scSPS injection energy would be $25 \mathrm{GeV}$, giving a very large energy swing of $\times 52$. This is likely to be a challenge for the magnet field quality and dynamic aperture. Injecting at higher energy would help, but poses its own challenges; one would either have to maintain the existing SPS and integrate it with the new scSPS in the same tunnel or one would need a new machine to replace the PS.

In this study, the present SPS tunnel geometry was maintained and locations for the existing long straight section (LSS) functionalities kept as far as possible. The injection was maintained in LSS1 with the beam circulating in a clockwise direction, keeping the slow extraction and transfer lines to the North Area in LSS2. The RF system would have to move from LSS3 to LSS4. The fast extractions towards FCC PB and PL will be located in LSS3 and LSS5. Note that for beam transfer to HE-LHC these extractions should be located in LSS4 and LSS6, respectively. New collimation and external beam dump systems are required in LSS6 and LSS5, respectively. An overview of the layout is shown in Figure 6.7.

The detailed design study of the different straight sections has yet to be made - its basic feasibility has been confirmed, assuming the improved performance from the technological developments in kickers and septa already under study for FCC-hh beam injection and extraction, such as $4 \mathrm{~T}$ septa [213], which are needed to extract beams with three times the rigidity in the same $120 \mathrm{~m}$ long LSS.

A first scSPS lattice has been developed, similar to the present SPS, with a missing-magnet dispersion suppressor. A half-cell of 2 main dipole magnets is assumed, with 372 dipoles in total, each with a bend angle of $16.89 \mathrm{mrad}$. These would need to be curved to avoid a huge sagitta. It should be noted that the current SPS access shafts would need to be rebuilt as they restrict the length of components 


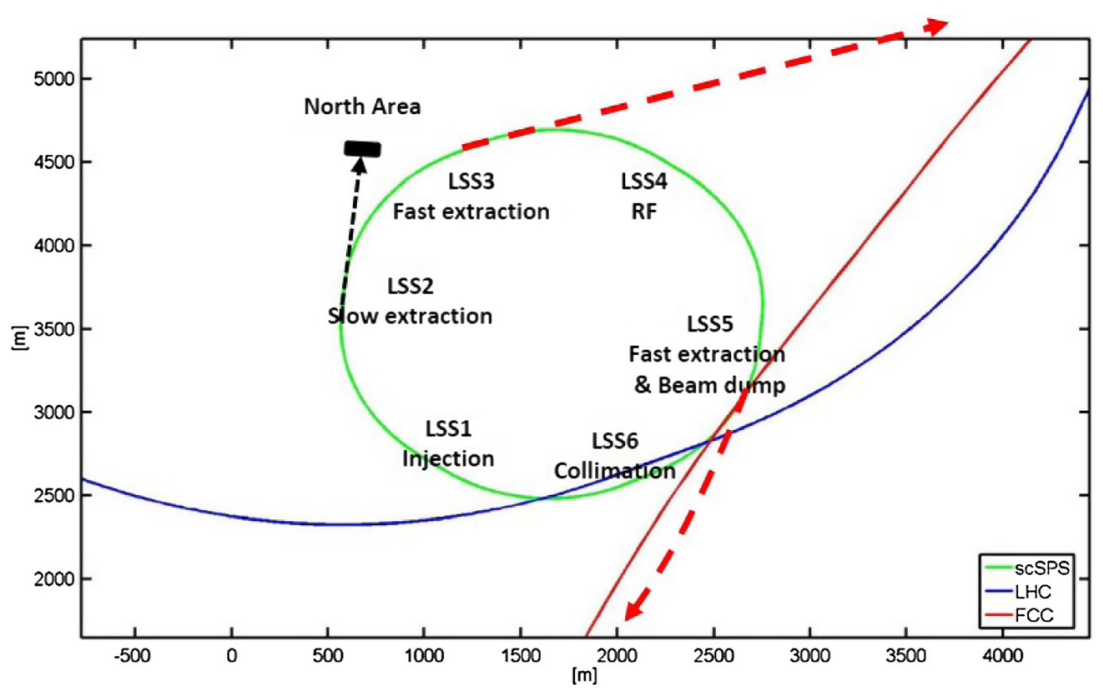

Fig. 6.7. Layout of an scSPS showing the overlap with the LHC and FCC machines. For a single aperture, single polarity scSPS, transfer lines from LSS3 and LSS5 to FCC would be optimal.

which can pass through them horizontally to a maximum of $6.9 \mathrm{~m}$. Peak $\beta$ values are $107 \mathrm{~m}$ in the centres of the quadrupoles, and peak dispersion functions $4.3 \mathrm{~m}$, for an integer tune of 26 in both planes and $89.96^{\circ}$ phase advance per cell. A plot of beta function and dispersion in one sextant is shown in Figure 6.8.

The $6 \mathrm{~T}$ peak field was chosen to have relatively simple, fast-ramping dipoles with single layer NbTi Rutherford-cable. The maximum quadrupole gradient assumed is $67 \mathrm{~T} / \mathrm{m}$, again feasible with $\mathrm{NbTi}$ technology. The magnet aperture was chosen to accommodate both the FCC-hh (and HE-LHC) beams and a low-emittance $(2.2 \mu \mathrm{m})$ fixed target beam. For $26 \mathrm{GeV}$ injection the resulting inner diameter of the circular cold bore is $80 \mathrm{~mm}$, which is rather large - this could be reduced for higher injection energy. The vertical aperture required is slightly smaller because the vertical dispersion is taken as zero and the injection oscillations are assumed to be only in the horizontal plane. The main parameters concerning the aperture are summarised in Table 6.5 .

A superconducting RF system can be placed within one straight section. A detailed study of the optimum frequency (or frequencies) and parameters (also to debunch the beam for slow extraction) is needed. The beam dump has to be combined with one of the two high energy extraction systems towards FCC-hh, due to the limited number of straight sections. TT61 (presently used for HiRadMat) might be modified and used as beam dump line, with dilution kickers and an absorber block similar to the LHC concept [54]. Some civil engineering for a cavern would be needed.

For fixed target physics experiments and test beams for experiments, a slow extraction in the range of one millisecond to several seconds is needed. The machine should be able to deliver around $10^{19}$ protons-on-target (PoT) per year. To avoid increasing the machine aperture dramatically, innovative solutions for slow extraction will need to be developed. At present a crystal-based extraction with low losses is assumed [334], with non-resonant transverse excitation of the beam to avoid large excursions caused by the resonant-extraction separatrices. The impact of high loss levels and additional local protection elements needed to integrate the slow extraction with the collimation system needs to be studied. For other test facilities like 


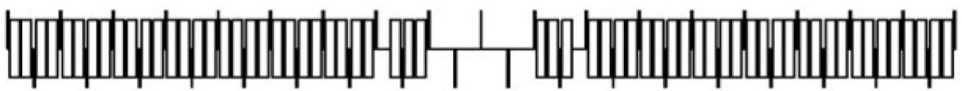

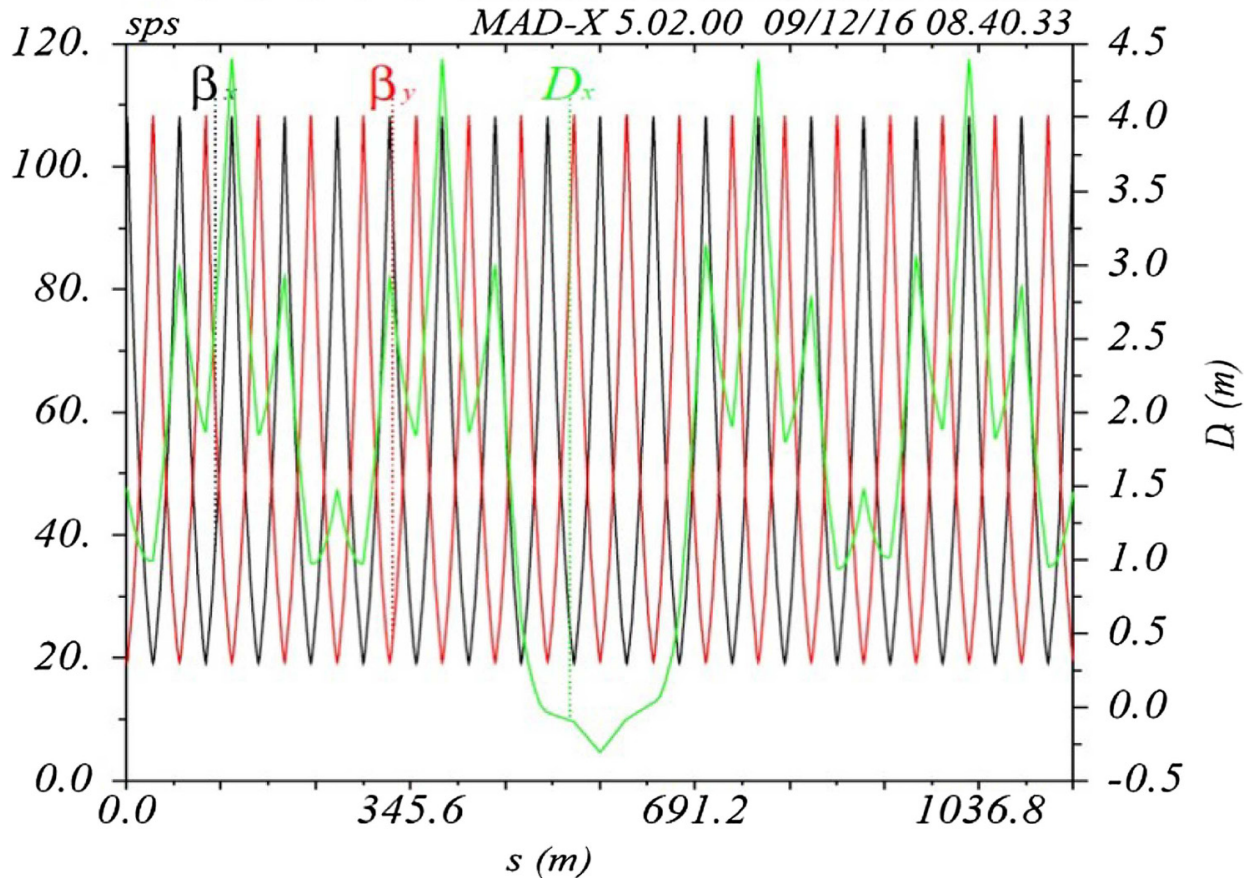

Fig. 6.8. Optics for an scSPS with $32 \mathrm{~m}$ half-cell length and $12 \mathrm{~m}$ long dipoles.

Table 6.5. Main parameters concerning aperture of the scSPS.

\begin{tabular}{|lll|}
\hline Parameter & Unit & Value \\
\hline Maximum beta $\beta_{x, y}$ & $\mathrm{~m}$ & 107 \\
Maximum dispersion $D_{x}$ & $\mathrm{~m}$ & 4.3 \\
Orbit + alignment tolerance $O_{x, y}$ & $\mathrm{~mm}$ & 2.5 \\
Maximum injection oscillation & $\mathrm{mm}$ & 1.5 \\
Emittance $\epsilon_{x, y}(1 \sigma$, norm. $)$ & $\mathrm{m}$ & $2.2 \times 10^{-6}$ \\
$\delta p / p$ & & $5 \times 10^{-4}$ \\
$A_{x} / A_{y} 26 \mathrm{GeV}$ & $\mathrm{mm}$ & $76 / 69$ \\
Cold bore diameter $26 \mathrm{GeV}$ injection & $\mathrm{mm}$ & 80 \\
\hline
\end{tabular}

HiRadMat [218] fast extracted beams will be needed. This type of extraction and beamline does not pose any additional technical challenge.

\subsection{Other HEB options}

Other options for the HEB have been investigated in terms of maximum energy, magnet technology, transfer line geometry, turnaround time and also the suitability as a fixed-target facility. The impact on the overall collider design of a lower injection energy could be considerable; the feasibility of energies lower than $3.3 \mathrm{TeV}$ will be the determining factor in defining which HEB options are even possible. 


\subsection{TeV superconducting (4 T) HEB in the $27 \mathrm{~km} \mathrm{LHC} \mathrm{tunnel}$}

If $3.3 \mathrm{TeV}$ injection energy is mandatory, another option would be to replace the LHC with a fast-ramping, relatively low-cost, superconducting machine, which would need a field of $4 \mathrm{~T}$ to reach $3.3 \mathrm{TeV}$. The machine could either be twin-aperture, although this would substantially increase its cost, both for the magnets and also for powering, instrumentation and other ancillary systems, or it could use polarity reversal to minimise the length of transfer line to FCC-hh, depending on the detailed cost trade-off. Such a machine would follow the existing LHC geometry and layout, reusing the injection and dump transfer tunnels and configured in terms of injection and extraction systems as shown in Figure 6.5.

Although the initial capital cost would (probably) be higher than modifying the LHC, this could be an attractive option in terms of operability and maintainability, compared to reuse of the existing LHC magnet system.

No optics studies have been made, but with the space available, no challenges are expected in developing a lattice. For the provision of beams for fixed-target physics, the integration of a slow-extraction system with the superconducting machine and the collimation system will present similar challenges to the reuse of LHC for this purpose.

\subsection{TeV normal conducting (1 T) $100 \mathrm{~km} \mathrm{HEB}$ in the FCC tunnel}

The final option considered is to house the HEB in the FCC-hh tunnel (as is mandatory for the full-energy top-up injector for FCC-ee). For simplicity and cost this could be a single-aperture machine with polarity reversal, for which magnetic fields of $1.1 \mathrm{~T}$ are proposed, to stay within the range of iron-dominated magnets and powered for energy cost reasons by a superconducting cable [335].

Scaling of impedance and collective effects shows that a half-gap height of about $40 \mathrm{~mm}$ will be needed in the dipoles. When using resistive magnets this would mean a peak dissipated power of about $1 \mathrm{GW}$, so superferric magnets are the only viable option. An optics which alternates focussing and defocussing combined-function dipoles inside the traditional FODO half-cell, which is delimited by pure quadrupoles, has been developed and could be promising from a magnet point of view [335], although limited flexibility and reduction of optics choices also need to be considered.

Due to the large machine circumference, the energy gain per turn delivered by the RF system becomes the limiting factor in the ramp rate. An RF system of similar size to that of the current LHC system would be able to ramp from 450 to $3300 \mathrm{GeV}$ in about $2 \mathrm{~min}$ [326]. To fill the FCC-hh the machine would only have to ramp once per ring. This results in a filling time of $32 \mathrm{~min}$, most of which is due to the SPS cycling time with the current CERN injector chain.

With the HEB in the FCC-hh tunnel, crosstalk of beam losses could be a real challenge, especially near collimation and protection devices and with slow extraction proposed for fixed target beams. Another feature of this option is that, in order to avoid background and radiation damage to the experiments and for integration reasons, the HEB will need bypasses around the experiment insertions for which separate and dedicated bypass tunnels could be required.

\subsection{Summary and comparison of HEB options}

The existing $25 \mathrm{~ns}$ LHC injector chain, supplemented by a new HEB, will provide a firm foundation as the FCC injector chain. Smaller bunch spacings (for lower bunch 
Table 6.6. Main parameters and performance comparison of the different HEB options for FCC-hh.

\begin{tabular}{|c|c|c|c|c|c|}
\hline Parameter & Unit & 6 T scSPS & Reuse LHC & New $4 \mathrm{~T}$ LHC & $1 \mathrm{~T} 100 \mathrm{~km}$ \\
\hline Circumference & $\mathrm{km}$ & 6.9 & 26.7 & 26.7 & 100 \\
\hline Injection energy & $\mathrm{GeV}$ & $25 / 450$ & 450 & 450 & 450 \\
\hline $\begin{array}{l}\text { Extraction } \\
\text { energy }\end{array}$ & $\mathrm{TeV}$ & 1.3 & 3.3 & 3.3 & 3.3 \\
\hline $\begin{array}{l}\text { Energy swing } \\
\text { factor }\end{array}$ & & $50 / 3$ & 7 & 7 & 7 \\
\hline Maximum field & $\mathrm{T}$ & 6 & 4 & 4 & 1 \\
\hline Injection field & $\mathrm{T}$ & $0.12 / 2.0$ & 0.6 & 0.6 & 0.14 \\
\hline Dipole length & $\mathrm{m}$ & 12 & 15 & $15 ?$ & \\
\hline $\begin{array}{l}\text { Number of } \\
\text { dipoles }\end{array}$ & & 372 & 1232 & $1232 ?$ & $\sim 1000$ \\
\hline Number of quads & & 216 & $\sim 480$ & $\sim 480$ & $\sim 1000$ \\
\hline HEB filling time & $\min$ & 0.8 & 7 & 7 & 14 \\
\hline Ramp rate & $\mathrm{T} / \mathrm{s}$ & 0.5 & 0.035 & $0.08^{*}$ & $0.01^{*}$ \\
\hline Total cycle length & $\min$ & 1.2 & 10.8 & 8.0 & 16 \\
\hline Total bunches & & 640 & 2600 & 2600 & 11000 \\
\hline $\begin{array}{l}\text { Cycles per FCC } \\
\text { fill }(25 \mathrm{~ns})\end{array}$ & & 34 & 4 & 8 & 2 \\
\hline FCC filling time & $\min$ & 41 & 43 & 64 & 32 \\
\hline $\begin{array}{l}\text { Stored energy per } \\
\text { beam }\end{array}$ & MJ & 13 & 140 & 140 & 560 \\
\hline
\end{tabular}

Notes. Ramp-rates denoted ${ }^{*}$ are limited by the RF, not by the magnet system. All machines except the existing LHC are considered as single aperture.

intensity and emittance to reduce experimental pile-up) will require new RF systems in the PS and proof that the small emittance beams can be conserved. A modified version of the existing LHC is the present HEB baseline for injecting into FCC-hh at $3.3 \mathrm{TeV}$.

The LHC reuse option has been studied in detail and the concept defined. Other options exist, and the final choice of HEB also depends on the minimum injection energy into the collider. If $3.3 \mathrm{TeV}$ is maintained, then only an LHC or FCC-tunnel based version is possible with the existing CERN infrastructure. The costs of refurbishing and maintaining the present LHC (with its relatively slow ramp time) have to be weighed against the capital costs of a new $4 \mathrm{~T}$ machine in the LHC tunnel or a new $100 \mathrm{~km}$ superferric machine in the FCC-hh tunnel (which gives the fastest FCC-hh filling time). If a $1.3 \mathrm{TeV}$ injection energy is feasible from the collider side, the scSPS will be an attractive option.

The main parameters and performance aspects of the different options are collected together in Table 6.6. The potential integrated number of protons on target for fixed target operation is $\sim 1 \times 10^{19} \mathrm{PoT} / \mathrm{y}$ for all options, if a maximum extracted flux of $10^{13} \mathrm{ps}^{-1}$ is assumed for 180 days of operation at $80 \%$ efficiency.

\section{Experiments and detectors}

\subsection{Physics and detector performance considerations}

Particle physics detectors and experiments have shown significant performance improvements over recent decades. The development of large scale detector struc- 
tures and access to a highly developed silicon industry with exponential performance growth have paved the way to ever more sophisticated detectors. The most recent incarnation of such detectors are e.g. the LHC detectors ALICE [336], ATLAS [337], CMS [338] and LHCb [339]. The present configuration of these detectors, their planned Phase-I upgrades in 2019/2020 and Phase-II upgrades in the middle of the next decade give a good insight into the technology trends which allow an initial projection of performance improvements and $R \& D$ challenges for the design of experiments at a future hadron collider to be made.

The FCC-hh collider layout has two high luminosity interaction points for general purpose detectors and two lower luminosity interaction points, similar to the present LHC configuration. The two general purpose detectors will probably use quite different detector configurations, as is the case for the present ATLAS and CMS experiments. To gain some elementary insight into the scale of a general purpose detector at the FCC-hh collider the general specifications for such a detector are discussed first and then a specific "reference detector" is investigated that serves as a concrete example and allows sub-system and physics studies. This chapter focusses on proton collisions; a brief discussion on heavy ion collisions as well electron-hadron collisions is given at the end of the chapter. The specifications are defined by a set of physics benchmark processes as well as the collision environment which includes parameters like pile-up and radiation load as outlined in Table 7.1.

A $100 \mathrm{TeV}$ proton collider serves as a discovery machine as well as a precision measurement machine. The factor 7 increase in energy over the present LHC increases the mass reach by a similar factor. The much larger cross sections for SM processes together with the higher luminosity lead to a significant increase in measurement precision. As an example when going from 14 to $100 \mathrm{TeV}$, the cross section for $\mathrm{HH}$ production increases by a factor $\approx 40$ and the cross section for $t \bar{t} H$ production increases by a factor of $\approx 55$.

The detector must therefore be able to measure multi-TeV jets, leptons and photons from heavy resonances with masses up to $50 \mathrm{TeV}$, while at the same time measuring the known SM processes with high precision and being sensitive to a broad range of BSM signatures at moderate $p_{T}$. Assuming a two body decay of a particle at rest in the centre of the detector, a geometric acceptance of $|\eta|<2.5$ will catch $90 \%$ of the events. The present LHC detectors were conceived with precision tracking and precision calorimetry in this rapidity range. High mass objects will therefore be measured predominantly in this central detector region. Since, however, the SM particles are at low mass compared to the $100 \mathrm{TeV}$ collision energy, many SM processes show significant forward boost while still having transverse momentum distributions that are comparable to LHC energies. A detector for $100 \mathrm{TeV}$ must therefore increase the acceptance for precision tracking and calorimetry to $|\eta| \approx 4$, while at the same time keeping the $p_{T}$ thresholds for triggering and reconstruction at levels close to the present LHC detectors. The Phase-II upgrades of the ATLAS and CMS detectors are already working in this direction $[342,343]$. It has to be pointed out that the statistics for some SM processes are so large that some measurements might become limited by systematics. Discarding some events by raising the $p_{T}$ thresholds can shift these processes back to the central region, while at the same time significantly improving the signal-to-background ratio. This will also probe these processes at very large $Q^{2}$, which is, in itself, of significant interest.

The detector granularity is mainly defined by two distinct items: first, the occupancy of detector channels due to the high collision rate and second, the spatial separation of tracks from highly boosted objects. The latter is particularly relevant e.g. for highly boosted tau leptons where the tracks from decay particles are extremely close or for the decays of highly boosted top quarks where the $\mathrm{W}$ and b-jets have a very small angular separation. All these aspects require a tracking 
Table 7.1. Key numbers relating the detector challenges at the different accelerators.

\begin{tabular}{|c|c|c|c|c|c|}
\hline Parameter & Unit & $\mathrm{LHC}$ & HL-LHC & HE-LHC & FCC-hh \\
\hline$E_{\mathrm{cm}}$ & $\mathrm{TeV}$ & 14 & 14 & 27 & 100 \\
\hline Circumference & & 26.7 & 26.7 & 26.7 & 97.8 \\
\hline Peak $\mathcal{L}$, nominal (ultimate) & $10^{34} \mathrm{~cm}^{-2} \mathrm{~s}^{-1}$ & $1(2)$ & $5(7.5)$ & 16 & 30 \\
\hline Bunch spacing & ns & 25 & 25 & 25 & 25 \\
\hline Number of bunches & & 2808 & 2760 & 2808 & 10600 \\
\hline Goal $\int \mathcal{L}$ & $a b^{-1}$ & 0.3 & 3 & 10 & 30 \\
\hline$\sigma_{\text {inel }}[340]$ & $\mathrm{mb}$ & 80 & 80 & 86 & 103 \\
\hline$\sigma_{\text {tot }}[340]$ & $\mathrm{mb}$ & 108 & 108 & 120 & 150 \\
\hline BC rate & $\mathrm{MHz}$ & 31.6 & 31.0 & 31.6 & 32.5 \\
\hline Peak pp collision rate & $\mathrm{GHz}$ & 0.8 & 4 & 14 & 31 \\
\hline $\begin{array}{l}\text { Peak av. PU events/BC, nom- } \\
\text { inal (ultimate) }\end{array}$ & & $\begin{array}{l}25 \\
(50)\end{array}$ & $130(200)$ & 435 & 950 \\
\hline RMS luminous region $\sigma_{z}$ & $\mathrm{~mm}$ & 45 & 57 & 57 & 49 \\
\hline Line PU density & $\mathrm{mm}^{-1}$ & 0.2 & 1.0 & 3.2 & 8.1 \\
\hline Time PU density & $\mathrm{ps}^{-1}$ & 0.1 & 0.29 & 0.97 & 2.43 \\
\hline $\mathrm{d} N_{\mathrm{ch}} /\left.\mathrm{d} \eta\right|_{\eta=0}[340]$ & & 6.0 & 6.0 & 7.2 & 10.2 \\
\hline $\begin{array}{l}\text { Charged tracks per collision } \\
N_{\mathrm{ch}}[340]\end{array}$ & & 70 & 70 & 85 & 122 \\
\hline Rate of charged tracks & $\mathrm{GHz}$ & 59 & 297 & 1234 & 3942 \\
\hline$\left\langle p_{T}\right\rangle[340]$ & $\mathrm{GeV} / \mathrm{c}$ & 0.56 & 0.56 & 0.6 & 0.7 \\
\hline $\begin{array}{l}\text { Bending radius for }\left\langle p_{T}\right\rangle \text { at } \\
B=4 \mathrm{~T}\end{array}$ & $\mathrm{~cm}$ & 47 & 47 & 49 & 59 \\
\hline Total number of pp collisions & $10^{16}$ & 2.6 & 26 & 91 & 324 \\
\hline $\begin{array}{l}\text { Charged part. flux at } 2.5 \mathrm{~cm} \text {, } \\
\text { est. (FLUKA) }\end{array}$ & $\mathrm{GHz} \mathrm{cm}^{-2}$ & 0.1 & 0.7 & 2.7 & $8.4(10)$ \\
\hline $\begin{array}{l}1 \mathrm{MeV} \text {-neq fluence at } 2.5 \mathrm{~cm} \text {, } \\
\text { est. (FLUKA) }\end{array}$ & $10^{16} \mathrm{~cm}^{-2}$ & 0.4 & 3.9 & 16.8 & $84.3(60)$ \\
\hline $\begin{array}{l}\text { Total ionising dose at } 2.5 \mathrm{~cm} \text {, } \\
\text { est. (FLUKA) }\end{array}$ & MGy & 1.3 & 13 & 54 & $270(300)$ \\
\hline $\mathrm{d} E /\left.\mathrm{d} \eta\right|_{\eta=5}[340]$ & $\mathrm{GeV}$ & 316 & 316 & 427 & 765 \\
\hline $\mathrm{d} P /\left.\mathrm{d} \eta\right|_{\eta=5}$ & $\mathrm{~kW}$ & 0.04 & 0.2 & 1.0 & 4.0 \\
\hline $90 \% \mathrm{~b} \overline{\mathrm{b}} p_{T}^{\mathrm{b}}>30 \mathrm{GeV} / \mathrm{c}[341]$ & $|\eta|<$ & 3 & 3 & 3.3 & 4.5 \\
\hline VBF jet peak [341] & ||$\eta \mid$ & 3.4 & 3.4 & 3.7 & 4.4 \\
\hline $90 \%$ VBF jets $[341]$ & ||$\eta \mid<$ & 4.5 & 4.5 & 5.0 & 6.0 \\
\hline $90 \% \mathrm{H} \rightarrow 4 l[341]$ & ||$\eta \mid<$ & 3.8 & 3.8 & 4.1 & 4.8 \\
\hline $\mathrm{b} \overline{\mathrm{b}}$ cross-section & $\mathrm{mb}$ & 0.5 & 0.5 & 1 & 2.5 \\
\hline $\mathrm{b} \overline{\mathrm{b}}$ rate & $\mathrm{MHz}$ & 5 & 25 & 250 & 750 \\
\hline $\mid \begin{array}{l}\mathrm{b} \overline{\mathrm{b}} p_{T}^{\mathrm{b}}>30 \mathrm{GeV} / \mathrm{c} \text { cross- } \\
\text { section }\end{array}$ & $\mu \mathrm{b}$ & 1.6 & 1.6 & 4.3 & 28 \\
\hline $\mathrm{b} \overline{\mathrm{b}} p_{T}^{\mathrm{b}}>30 \mathrm{GeV} / \mathrm{c}$ rate & $\mathrm{MHz}$ & 0.02 & 0.08 & 1 & 8 \\
\hline $\begin{array}{l}\text { Jets } p_{T}^{\text {jet }}>50 \mathrm{GeV} / \mathrm{c} \text { cross- } \\
\text { section [340] }\end{array}$ & $\mu \mathrm{b}$ & 21 & 21 & 56 & 300 \\
\hline Jets $p_{T}^{\text {jet }}>50 \mathrm{GeV} / \mathrm{c}$ rate & $\mathrm{MHz}$ & 0.2 & 1.1 & 14 & 90 \\
\hline $\mathrm{W}^{+}+\mathrm{W}^{-}$cross-section [12] & $\mu \mathrm{b}$ & 0.2 & 0.2 & 0.4 & 1.3 \\
\hline $\mathrm{W}^{+}+\mathrm{W}^{-}$rate & $\mathrm{kHz}$ & 2 & 10 & 100 & 390 \\
\hline
\end{tabular}


Table 7.1. (Continued.)

\begin{tabular}{|l|l|l|l|l|l|}
\hline Parameter & Unit & LHC & HL-LHC & HE-LHC & FCC-hh \\
\hline $\mathrm{W}^{+} \rightarrow l+\nu$ cross-section $[12]$ & $\mathrm{nb}$ & 12 & 12 & 23 & 77 \\
$\mathrm{~W}^{+} \rightarrow l+\nu$ rate & $\mathrm{kHz}$ & 0.12 & 0.6 & 5.8 & 23 \\
$\mathrm{~W}^{-} \rightarrow l+\nu$ cross-section $[12]$ & $\mathrm{nb}$ & 9 & 9 & 18 & 63 \\
$\mathrm{~W}^{-} \rightarrow l+\nu$ rate & $\mathrm{kHz}$ & 0.1 & 0.5 & 4.5 & 19 \\
\hline $\mathrm{Z}$ cross-section $[12]$ & $\mathrm{nb}$ & 60 & 60 & 100 & 400 \\
$\mathrm{Z}$ rate & $\mathrm{kHz}$ & 0.6 & 3 & 25 & 120 \\
$\mathrm{Z} \rightarrow l l$ cross-section $[12]$ & $\mathrm{nb}$ & 2 & 2 & 4 & 14 \\
$\mathrm{Z} \rightarrow l l$ rate & $\mathrm{kHz}$ & 0.02 & 0.1 & 1 & 4.2 \\
\hline t-t cross-section $[12]$ & $\mathrm{nb}$ & 1 & 1 & 4 & 35 \\
t-t rate & $\mathrm{kHz}$ & 0.01 & 0.05 & 1 & 11 \\
\hline
\end{tabular}

precision of $<5 \mu \mathrm{m}$ in $r-\phi$ direction for the vertex tracker as well as an increase of the transverse calorimeter granularity over the present ATLAS and CMS values by at least a factor 4 .

The measurement of jets from Vector Boson Fusion (VBF) represents another key task that puts stringent demands on the detector acceptance. At LHC energies, the $\eta$-distribution of these jets peaks at $|\eta| \approx 3.4$ and they are measured in the forward calorimeters that extend up to $|\eta| \approx 5$. For the Phase-II upgrades of ATLAS and CMS, the increase of tracking acceptance from $|\eta|=2.5$ to $|\eta|=4$ relates to the challenge of measuring these jets in an environment of up to $200 \mathrm{p}-\mathrm{p}$ collisions per bunch-crossing [344-346]. At $100 \mathrm{TeV}$ the VBF jet distribution peaks around $|\eta|=4.4$ with a significant tail towards larger values, therefore calorimeter acceptance of up to $|\eta|=6$ is required.

The large number of $\mathrm{p}-\mathrm{p}$ collisions per bunch-crossing, the so called pile-up, imposes stringent criteria on the detector design. While the present LHC detectors have to cope with pile-up numbers up to 60 , the HL-LHC will produce values of up to 200. The projected value of 1000 for the FCC-hh creates a challenge that needs to be addressed. Continuing technology advancements, specifically in the context of high precision timing detectors, will likely allow such numbers to be handled. While the overall impact of the pile-up on detector performance and resolution can be quantified, the impact on the physics performance cannot be assessed in a general manner. For the search of high mass objects the impact of pile-up will be less relevant. For signatures related to e.g. displaced vertices, only a full cycle of simulation, reconstruction and analysis can quantify it's impact. Since these detailed studies will only be conducted in the future, it is important that the accelerator design can accommodate scenarios with smaller bunch-crossing intervals (12.5 or $5 \mathrm{~ns}$ ) or higher harmonic cavities that allow stretching the luminous region to reduce this effect.

\subsection{Detector reference design}

Figures 7.1 and 7.2 show the layout of the FCC-hh reference detector. This detector concept does not represent a specific choice for the final implementation, but it serves as a concrete example for sub-system and physics studies and allows identification of topics where dedicated $\mathrm{R} \& \mathrm{D}$ efforts are needed. The detector has a diameter of $20 \mathrm{~m}$ and a length of $50 \mathrm{~m}$, comparable to the dimensions of the ATLAS detector. The central detector with coverage of $|\eta|<2.5$ houses the tracking, electromagnetic calorimetry and hadron calorimetry inside a $4 \mathrm{~T}$ solenoid with a free bore diameter of $10 \mathrm{~m}$. In order to reach the required performance for $|\eta|>2.5$, the forward parts of 


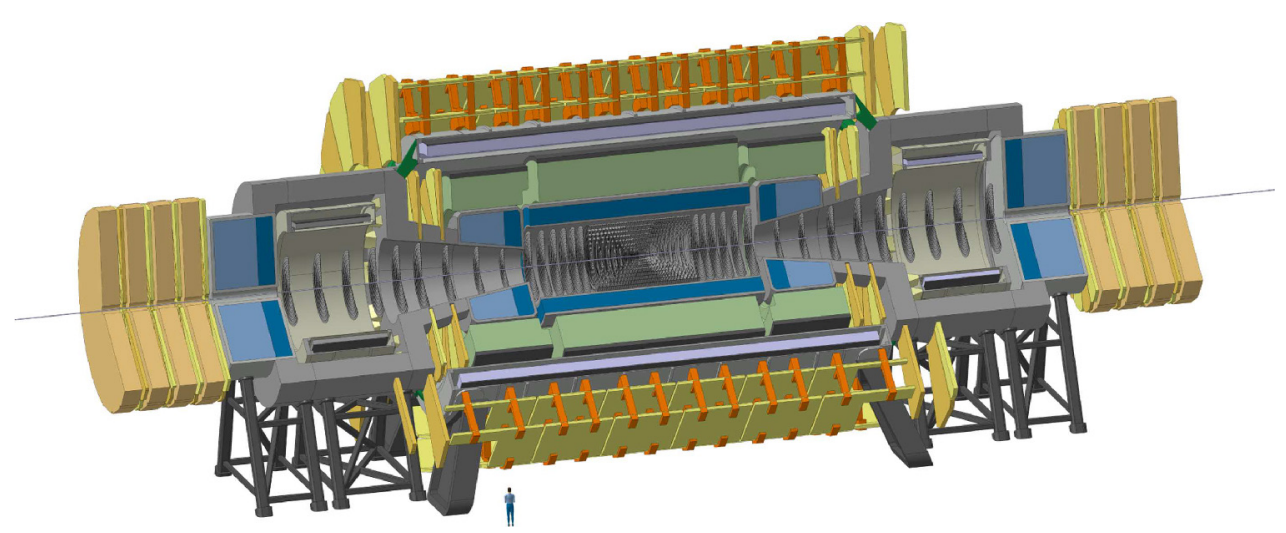

Fig. 7.1. The FCC-hh reference detector with an overall length of $50 \mathrm{~m}$ and a diameter of $20 \mathrm{~m}$. A central solenoid with $10 \mathrm{~m}$ diameter bore and two forward solenoids with $5 \mathrm{~m}$ diameter bores provide a $4 \mathrm{~T}$ field for momentum spectroscopy in the entire tracking volume.

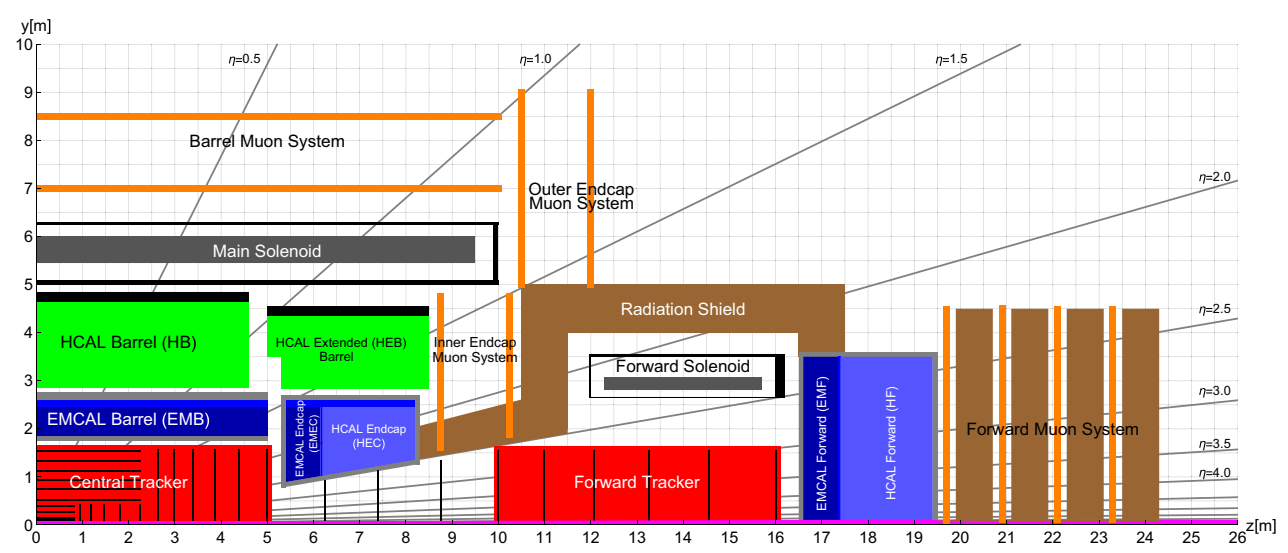

Fig. 7.2. Longitudinal cross-section of the FCC-hh reference detector. The installation and opening scenario for the detector requires a cavern length of $66 \mathrm{~m}$, which is compatible with the baseline assumption of $L^{*}=40 \mathrm{~m}$ for the FCC-hh machine.

the detector are displaced from the IP along the beam axis. Two forward magnet coils with an inner bore of $5 \mathrm{~m}$ provide the required bending power. These forward magnets are also solenoids with a $4 \mathrm{~T}$ field, essentially providing a total solenoid volume of $32 \mathrm{~m}$ length for high precision momentum spectroscopy up to rapidity values of $|\eta| \approx 4$ and tracking up to $|\eta \approx| 6$. An alternative consisting of 2 dipole magnets placed in the forward regions, with a field integral along the $z$-axis of about $4 \mathrm{Tm}$, similar to the value for the ALICE and LHCb dipoles, is also studied. Although such a magnet system shows better tracking performance in the very forward region, the fact of losing the rotational symmetry and the requirement for a compensation system for the hadron beam are seen as a drawback. The tracker is specified to provide better than $20 \%$ momentum resolution for $p_{T}=10 \mathrm{TeV} / \mathrm{c}$ for heavy $\mathrm{Z}^{\prime}$ type particles and better than $0.5 \%$ momentum resolution at the multiple scattering limit at least up to $|\eta|=3$.

Figure 7.2 shows a longitudinal cross section of the detector. The tracker cavity has a radius of $1.7 \mathrm{~m}$ with the outermost layer at around $1.6 \mathrm{~m}$ from the beam in the central and the forward regions, providing the full spectrometer arm up to $|\eta|=3$. The Electromagnetic CALorimeter (ECAL) uses a thickness of around 30 radiation 
lengths $\left(X_{0}\right)$ and provides together with the Hadron CALorimeter (HCAL) an overall calorimeter thickness of more than 10.5 nuclear interaction lengths $(\lambda)$, to ensure $98 \%$ containment of high energy showers and to limit punch-through to the muon system.

The ECAL is based on Liquid Argon (LAr) due to its intrinsic radiation hardness. The barrel HCAL is a scintillating tile calorimeter with steel and $\mathrm{Pb}$ absorbers, divided into a central barrel and two extended barrels. The HCALs for the endcap and forward regions are also based on LAr. The requirement of calorimetry acceptance up to $|\eta| \approx 6$ translates into an inner active radius of only $8 \mathrm{~cm}$ at a $z$-distance of $16.6 \mathrm{~m}$ from the IP. The ECAL is specified to have an energy resolution around $10 \% / \sqrt{E}$ and the hadron calorimetry around $50 \% / \sqrt{E}$ for single particles. The central beam pipe is assumed to be cylindrical with a radius of $20 \mathrm{~mm}$ up to a $z$-distance of $8 \mathrm{~m}$. Between this point and the forward calorimeters there will be a conical beam pipe or straight beam pipe sections around a rapidity of $|\eta|=6.7$, projecting towards the IP, which represents an angle of $2.5 \mathrm{mrad}$.

The considerations for the muon system have a significant impact on the overall detector design. When the LHC detectors were designed, it was still unclear whether precision tracking close to the IP would be possible, so significant emphasis was put on the stand-alone performance of the muon systems. The ATLAS detector therefore uses a large air core toroid magnet system for excellent stand-alone muon momentum spectroscopy up to $|\eta|=2.5$ with a multiple scattering resolution limit of about $2.5 \%$ and around $10 \%$ resolution at $p_{T}=1 \mathrm{TeV} / \mathrm{c}$. The stand-alone muon performance of CMS shows about $8 \%$ resolution at the multiple scattering limit and $20 \%$ resolution at $p_{T}=1 \mathrm{TeV} / \mathrm{c}$ in the central region, with a significant performance decrease at large $p_{T}$ in the endcaps. However, the CMS detector shows better performance than ATLAS for the combined muon momentum resolution, by also using the tracker information (except for very high $p_{T}$ muons in the end-cap region). Since today there is little doubt that large scale silicon trackers will be core parts of future detectors, the emphasis on stand-alone muon performance is less pronounced and the focus should be on the aspects of muon trigger and muon identification.

The reference detector does not assume any shielding of the magnetic field. A massive iron yoke, in this case much heavier than the one used for CMS, has significant cost and weight for such a large magnet system. Alternative solutions with active shielding coils increase the system size to dimensions that require access shafts in excess of $25 \mathrm{~m}$ diameter to lower these coils to the detector cavern. Figure 7.3 shows the FCC-hh and FCC-ee reference detectors inside the experiment cavern and the layout of the service cavern. The cavern height of $37 \mathrm{~m}$ and the width of $35 \mathrm{~m}$ are similar to the present ATLAS cavern. The stray magnetic field reaches $5 \mathrm{mT}$ at a radial distance of $50 \mathrm{~m}$ from the beamline. Therefore there is no relevant stray field in the service cavern, which is placed at a distance of $50 \mathrm{~m}$ from the experiment cavern and separated by rock. Equipment in the experiment cavern will be exposed to stray fields ranging from $300 \mathrm{mT}$ at the outer radius of the muon system to $150 \mathrm{mT}$ at the cavern walls. This requires all structures and devices, including LV supplies, motors and ventilation systems, to be adapted for this environment. The additional signal latency of about $0.5 \mu$ s due to the increased bi-directional distance of $100 \mathrm{~m}$ is small compared to e.g. the overall first level trigger latency, which is already at the level of $10 \mu$ s for the LHC Phase-II detectors.

As indicated in Table 7.1, the energy deposited in the calorimeters increases strongly in the forward direction and reaches a value of $4 \mathrm{~kW}$ per unit of rapidity around $|\eta|=5$, which poses a significant challenge to the cryogenics of the LAr calorimeters. The shower and absorption processes inside the forward calorimeter produce a large quantity of low energy neutrons of which a significant fraction enters the tracker volume. In order to keep these neutrons from entering the muon system and the detector cavern, a heavy radiation shield is placed around the forward solenoid 


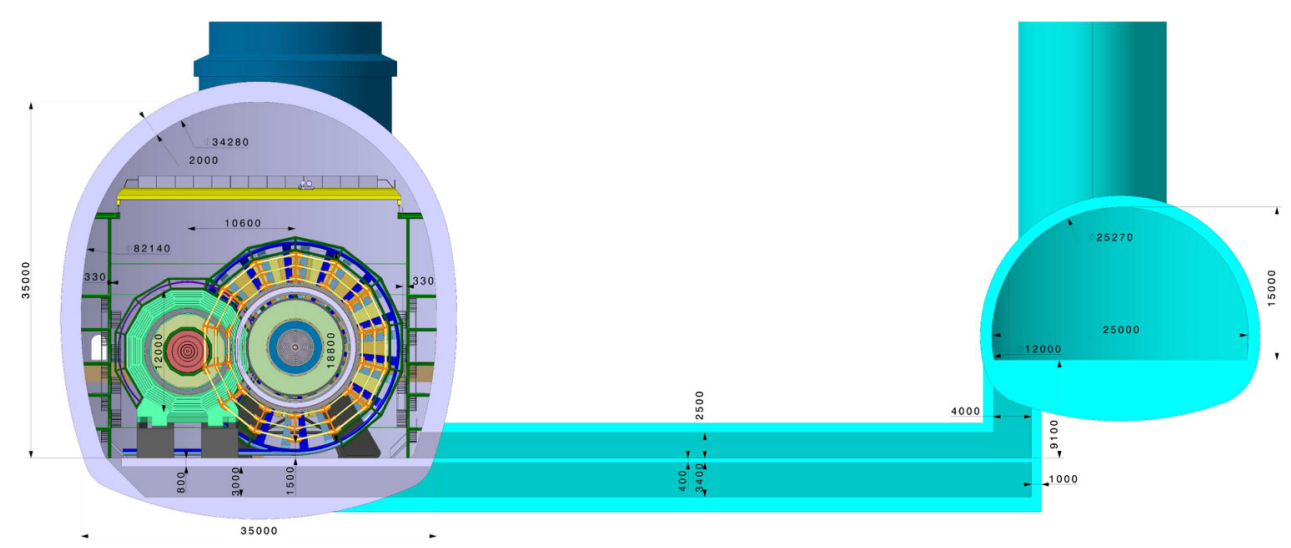

Fig. 7.3. Detector cavern and service cavern for the FCC-hh and FCC-ee detectors. The distance of $50 \mathrm{~m}$ between these caverns reduces the civil engineering risk and cost and ensures sufficient radiation shielding as well as low stray magnetic field in the service cavern.

magnets to close the gap between the endcap and forward calorimeters. In addition, a lithiated polyethylene moderator is placed in front of the forward calorimeter to attenuate this neutron fluence by a factor 2-3.

Future detector advances might allow resolution and granularity improvements beyond current expectations. Therefore, a smaller magnet system without the need for an extended forward part, could still be feasible. These considerations will evolve from future R\&D and more detailed detector simulations studies.

\subsection{Radiation environment}

The significant radiation levels create strong constraints on the technologies for the various sub-systems. While Table 7.1 shows that $\mathrm{d} N / \mathrm{d} \eta$ and the total track number per collision do not change significantly when going from $14 \mathrm{TeV}$ to $100 \mathrm{TeV}$, the increased collision rates, increased integrated luminosity goals, having silicon layers very close to the beam pipe as well as increased forward acceptance add one to two orders of magnitude of radiation load in some regions of the detector, compared to HL-LHC. Assuming that only primary hadrons pass through the first silicon layer and neglecting the bending in the magnetic field, the (track length weighted) charged particle rate per unit area at a given radius $r$ is $\mathcal{L} \times \sigma_{\text {inel }} \times \mathrm{d} N / \mathrm{d} \eta \times 1 /\left(2 r^{2} \pi\right)$. This number is indicated in Table 7.1 and does not depend on $\eta$ as long as $d N / d \eta$ is constant. This fact can also be seen in Figure 7.4a where the lines of equal charged particle rate in the tracker are parallel to the beamline. At $r=2.5 \mathrm{~cm}$ this evaluates to around $0.8 \mathrm{GHz} / \mathrm{cm}^{2}$ for HL-LHC and $8 \mathrm{GHz} / \mathrm{cm}^{2}$ for FCC-hh. The detailed FLUKA [69] simulation of the reference detector gives $10 \mathrm{GHz} / \mathrm{cm}^{2}$ at $r=2.5 \mathrm{~cm}$, which is close to this basic estimate. The number drops to about $3 \mathrm{MHz} / \mathrm{cm}^{2}$ at the outer radii of the tracker. In the forward ECAL the number rises to levels of $100 \mathrm{GHz} / \mathrm{cm}^{2}$.

The charged particle rate in the muon system is dominated by electrons, created from high energy photons in the $\mathrm{MeV}$ range. These photons are created by processes related to thermalisation and capture of neutrons that are produced in hadron showers, mainly in the forward region. These processes immerse the muon system and the cavern in a "gas" of high energy photons that convert to electrons in the muon system material. Because of their relatively low energy, these electrons curl around the magnetic field lines and reveal the B-field as seen in Figure 7.4a. This charged particle 
a)

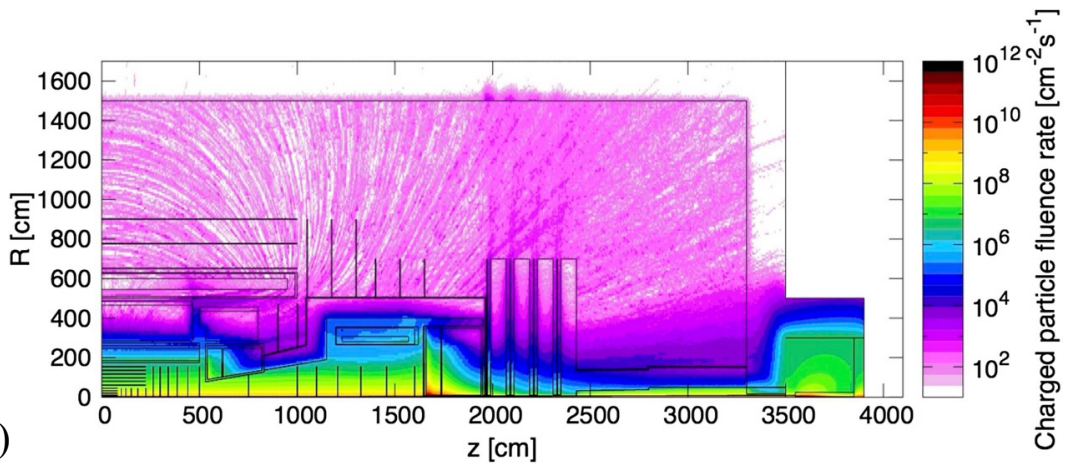

b)

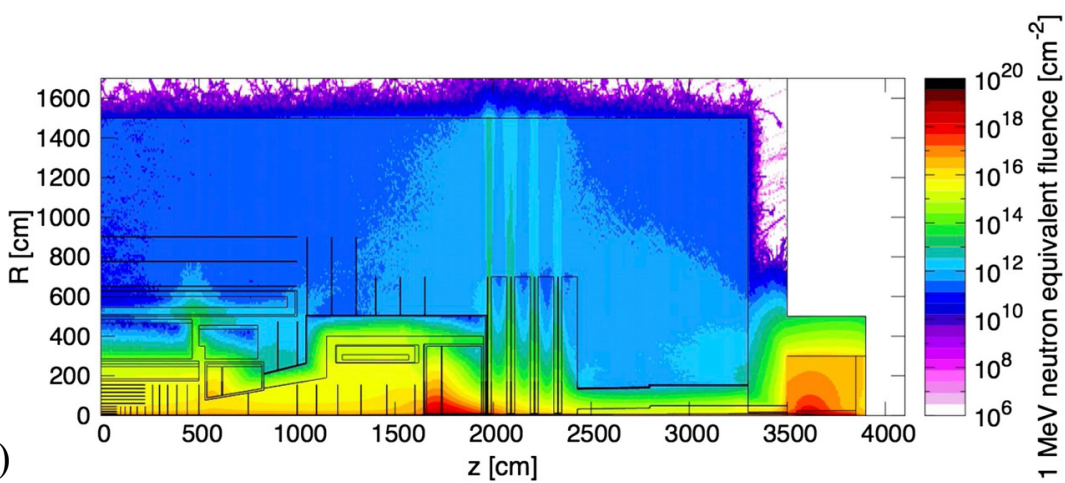

Fig. 7.4. (a) Charged particle rate for $\mathcal{L}=30 \times 10^{34} \mathrm{~cm}^{-2} \mathrm{~s}^{-1}$. (b) $1 \mathrm{MeV}$ neutron equivalent fluence for $30 \mathrm{ab}^{-1}$.

rate is the key number for the choice of muon system technology and granularity. In the barrel muon system and the outer endcap muon system the rate does not exceed $500 \mathrm{~Hz} / \mathrm{cm}^{2}$, the rate in the inner endcap muon system goes up to $10 \mathrm{kHz} / \mathrm{cm}^{2}$ and in the forward muon system the rate increases to $500 \mathrm{kHz} / \mathrm{cm}^{2}$ at a distance of $1 \mathrm{~m}$ from the beam. These rates are comparable to those in the ATLAS, CMS and LHCb muon systems [9-11] and gaseous detectors employed in these experiments can therefore be adopted in these regions. For radii smaller than $1 \mathrm{~m}$ in the forward muon system, the rates increase strongly and approach levels similar to those in the central tracker. Silicon sensor technology could be applied in these regions.

Figure $7.4 \mathrm{~b}$ shows the so called $1 \mathrm{MeV}$ neutron equivalent fluence ( $1 \mathrm{MeV}$-neq) which is a key number for long term damage of silicon sensors and electronics in general. Assuming, as before, only the primary hadrons for the first silicon layer, the value is $N_{0} \times \mathrm{d} N / \mathrm{d} \eta \times 1 /\left(2 r^{2} \pi\right)$, where $N_{0}$ is the total number of collisions amounting to $3.2 \times 10^{18}$ for $30 \mathrm{ab}^{-1}$. For a silicon detector at $2.5 \mathrm{~cm}$ radius this evaluates to a fluence of $8 \times 10^{17} / \mathrm{cm}^{2}$, which compares well with the value of $6 \times 10^{17} / \mathrm{cm}^{2}$ from the detailed FLUKA calculations. Beyond a radius of $40 \mathrm{~cm}$ the number drops below $10^{16} / \mathrm{cm}^{2}$ and in the outer parts of the tracker this number is around $5 \times 10^{15} / \mathrm{cm}^{2}$. Technologies used for the HL-LHC detectors are therefore applicable to radii $>40 \mathrm{~cm}$. Novel sensors and readout electronics have to be developed for the innermost parts of the tracker. In the gap between barrel and endcap calorimeters the values range from $10^{14}-10^{16} / \mathrm{cm}^{2}$. In the inner part of the forward calorimeters the radiation levels rise to extreme values of of $5 \times 10^{18} / \mathrm{cm}^{2}$.

The total ionising dose for an integrated luminosity of $30 \mathrm{ab}^{-1}$ is shown in Figure 7.5a. This is also a key number for long term damage of electronics and detector elements. Assuming an energy loss of $2 \mathrm{MeV} \mathrm{cm}^{2} / \mathrm{g}$ for each charged hadron, 
a)
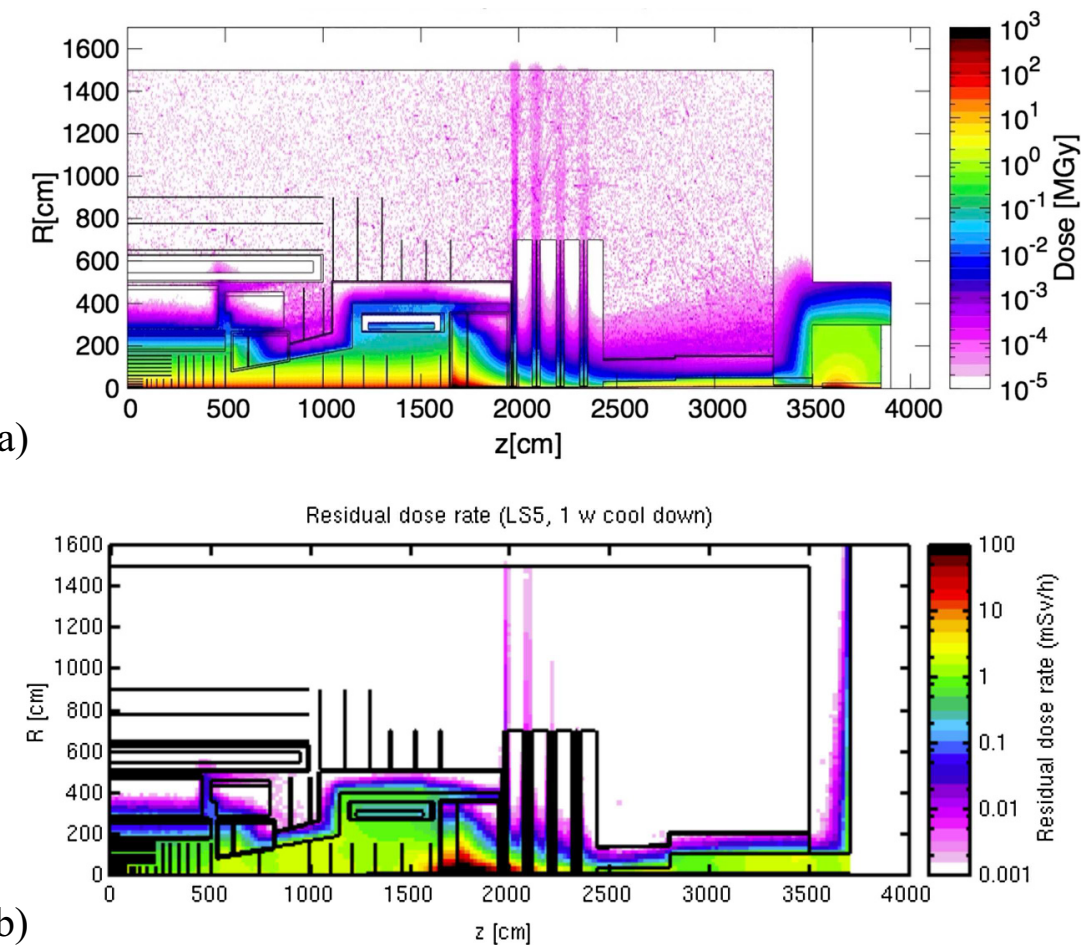

Fig. 7.5. (a) Total ionising dose for $30 \mathrm{ab}^{-1}$ of integrated luminosity. (b) Radiation dose rate after 1 week of cool-down towards the end of the FCC-hh operation.

the charged hadron fluence $/ \mathrm{cm}^{2}$ has to be multiplied by $2 \times 10^{6} e_{0} \times 10^{3}=3.2 \times 10^{-10}$ to get an estimate of the total ionising dose in units of Gy for the innermost parts of the tracker. This evaluates to about 270 MGy in the first silicon layer, compared to the value of 300 MGy from the detailed FLUKA simulations. In the central region of the hadron calorimeter the values are around $6 \mathrm{kGy}$ in the HCAL Barrel and $8 \mathrm{kGy}$ in the HCAL Extended Barrel. They stay below $10 \mathrm{kGy}$, which is a limiting number for the use of organic scintillators.

A key radiation aspect related to detector maintenance is the activation of the detector material and the related cool-down time, as indicated in Figure 7.5b. Towards the end of FCC-hh operation, having already collected a large fraction of the luminosity, the dose rate levels are around $1 \mathrm{mSv} / \mathrm{h}$ in the entire tracker volume after about 1 week of cooling time, and the values do not decrease significantly for 1 month or 1 year of cooling time. This radiation comes mainly from the highly activated calorimeters. Consequently the opening of the detector and placing of shielding elements must be automated to a large extent in order to allow access for maintenance and to limit the dose to personnel.

\subsection{Magnet system}

The magnet system of the reference detector has been designed to provide an axial magnetic field of $4 \mathrm{~T}$ in the entire tracker volume of the $30 \mathrm{~m}$ long detector. It comprises a central solenoid and two forward solenoids. An alternative, technically much more complex, design uses two dipoles with a magnetic field integral of about $4 \mathrm{Tm}$ in both forward regions to improve momentum spectroscopy for very high $\eta$ particles. 
a)

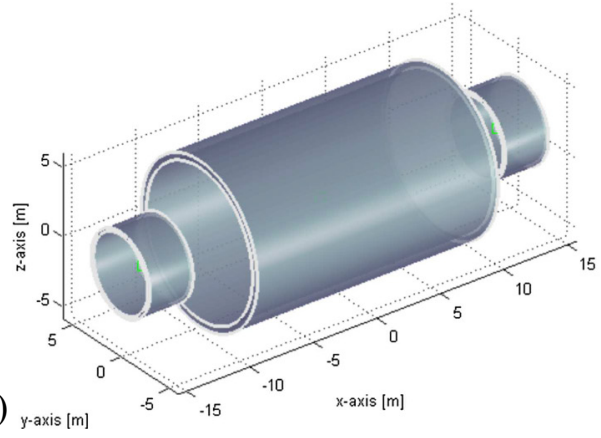

b)

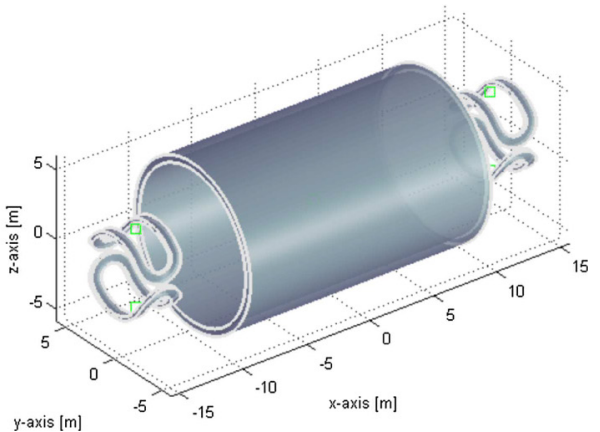

Fig. 7.6. (a) Cold mass for a central solenoid of $4 \mathrm{~T}$ with two forward solenoids and (b) a central solenoid of $4 \mathrm{~T}$ and two forward dipole magnets with field integral of $4 \mathrm{Tm}$.

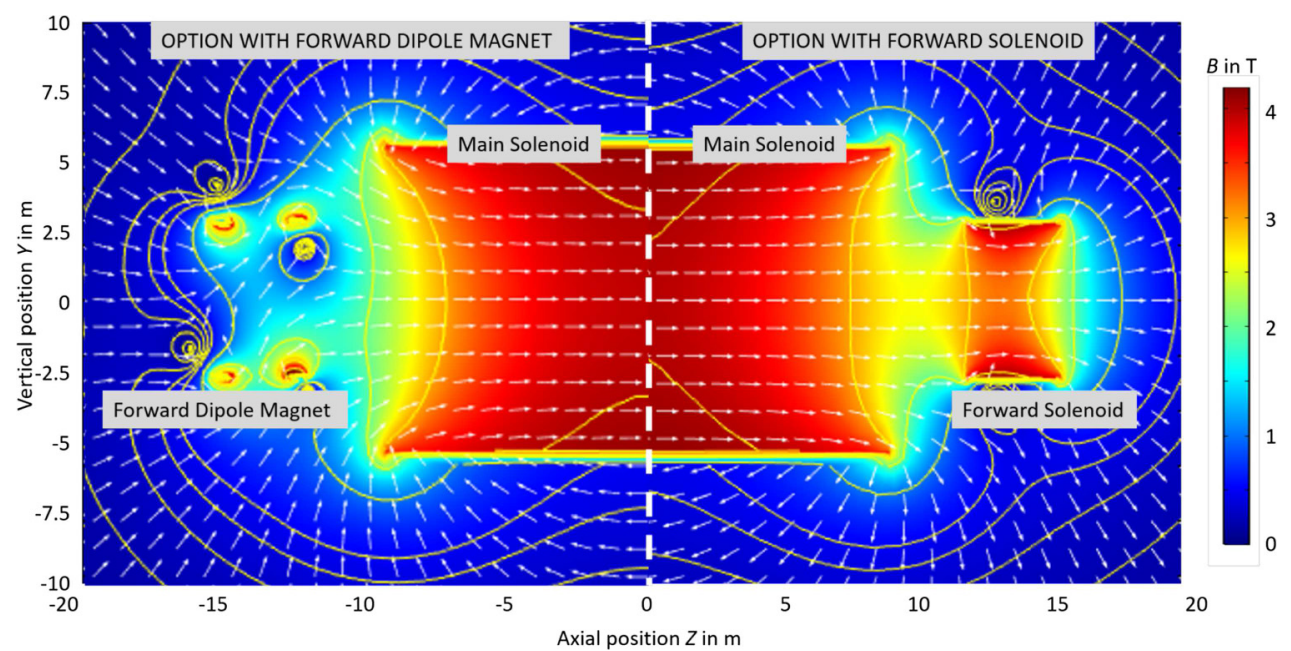

Fig. 7.7. Longitudinal half-sections of the two versions of the magnet system. Magnetic fieldmap for a central solenoid of $4 \mathrm{~T}$ with a forward dipole (left) and a forward solenoid (right).

The cold mass and field map of the baseline design and the system with forward dipole magnets are shown in Figures 7.6 and 7.7.

The main characteristics of the magnet systems are presented in Table 7.2. The stored magnetic energy of the main solenoid is 12.5 GJ, which can be compared to 1.6 and 2.7 GJ for the ATLAS and CMS magnet systems, respectively. The mass of the main solenoid and its cryostat is about $2 \mathrm{kt}$, so lifting equipment with a $2.5 \mathrm{kt}$ capacity has to be provided to lower this solenoid into the experiment cavern. This capacity is similar to that used for lowering the central part of the CMS detector to the cavern. The proposed magnet system is unshielded, i.e. there is no return yoke or active shielding for the magnetic field inside the detector cavern. Shielded options were studied [347], but size and cost of such magnets are considered excessive and not justified. The services inside the experimental cavern must therefore be adapted to the significant stray fields. The magnitude of the magnetic field along the $z$-axis of the system is shown in Figure 7.8a. It can be seen that the $5 \mathrm{mT}$ level is reached at a distance of about $70 \mathrm{~m}$ from the IP. The magnitude of the magnetic field in radial direction at $z=0,8$ and $16 \mathrm{~m}$ is shown in Figure $7.8 \mathrm{~b}$, confirming that the stray field 
Table 7.2. Main characteristics of the central solenoid, a forward solenoid and a forward dipole magnet.

\begin{tabular}{|l|c|c|c|c|}
\hline & Unit & $\begin{array}{c}\text { Main } \\
\text { solenoid }\end{array}$ & $\begin{array}{c}\text { Forward } \\
\text { solenoid }\end{array}$ & $\begin{array}{c}\text { Forward } \\
\text { dipole }\end{array}$ \\
\hline Operating current & $\mathrm{kA}$ & 30 & 30 & 16 \\
Stored energy & $\mathrm{GJ}$ & 12.5 & 0.43 & 0.20 \\
Self-inductance & $\mathrm{H}$ & 27.9 & 0.96 & 1.54 \\
Current density & $\mathrm{A} / \mathrm{mm}^{2}$ & 7.3 & 16.1 & 25.6 \\
Peak field on conductor & $\mathrm{T}$ & 4.5 & 4.5 & 5.9 \\
Operating temperature & $\mathrm{K}$ & 4.5 & 4.5 & 4.5 \\
Current sharing temp. & $\mathrm{K}$ & 6.5 & 6.5 & 6.2 \\
Temperature margin & $\mathrm{K}$ & 2.0 & 2.0 & 1.7 \\
Heat load cold mass & $\mathrm{W}$ & 286 & 37 & 50 \\
Heat load thermal & $\mathrm{W}$ & 5140 & 843 & 1500 \\
shield & & & & \\
Cold mass & $\mathrm{t}$ & 1070 & 48 & 114 \\
Vacuum vessel & $\mathrm{t}$ & 875 & 32 & 48 \\
Conductor length & $\mathrm{km}$ & 84 & 16 & 23 \\
\hline
\end{tabular}
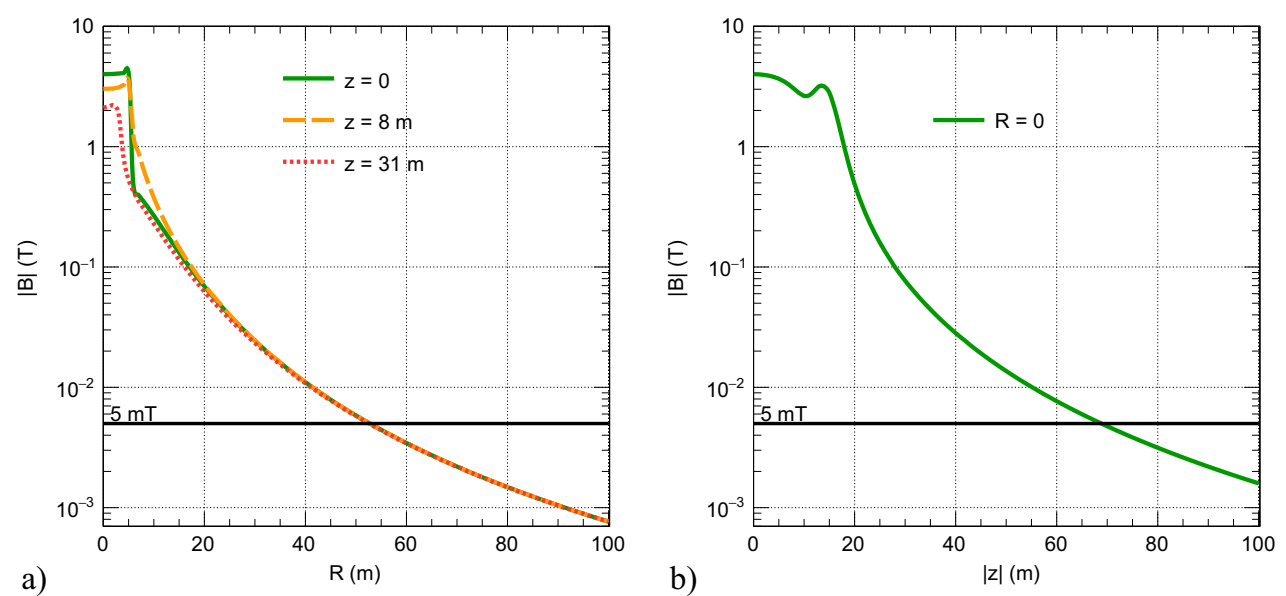

Fig. 7.8. (a) Magnitude of the B field as a function of radial distance from the beamline. (b) Magnitude of the B field along the $z$-axis as a function distance from the IP.

is below $5 \mathrm{mT}$ as from $52 \mathrm{~m}$, and thus also in the service cavern, which is positioned at a radial distance of $66-96 \mathrm{~m}$ from the beamline.

The proposed conductors for the solenoids are $\mathrm{Al}$ stabilised $\mathrm{Nb}-\mathrm{Ti} / \mathrm{Cu}$ Rutherford cables following the experience with ATLAS and CMS. For the optional forward dipole magnets internally cooled conductors are proposed following the Morpurgo magnet design [348]. Figure 7.9a shows the dimensions and the materials proposed for these conductors. The electrical circuit of the reference magnet with three solenoids is shown in Figure 7.9b. The cryogenic plant for the magnet system comprises a shield refrigerator to cool down the system and maintain it permanently cold, and a main refrigerator to supply liquid helium are installed on the surface. It supplies $20 \mathrm{~K}$ helium gas at 20 bar pressure to the cavern for liquefaction close to the detector. Only the liquefaction stage and the helium distribution lines to the main and forward magnets are installed in the main cavern. The cooling of the magnets is based on a thermosiphon that circulates helium through the cold masses. The magnet current 

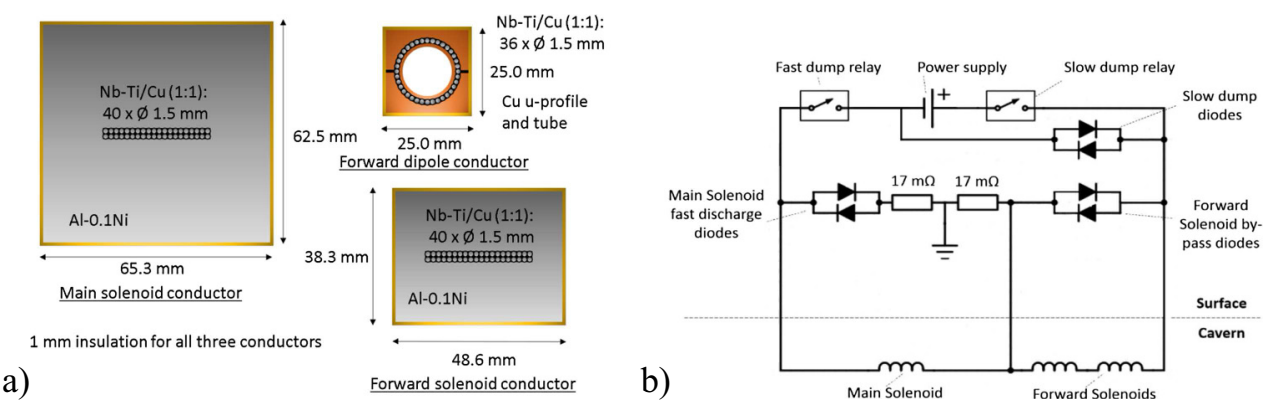

Fig. 7.9. (a) Conductors for the main solenoid, forward solenoid and forward dipole. (b) Electrical circuit of the magnet system showing the series connection of the three solenoids and the powering and protection devices.
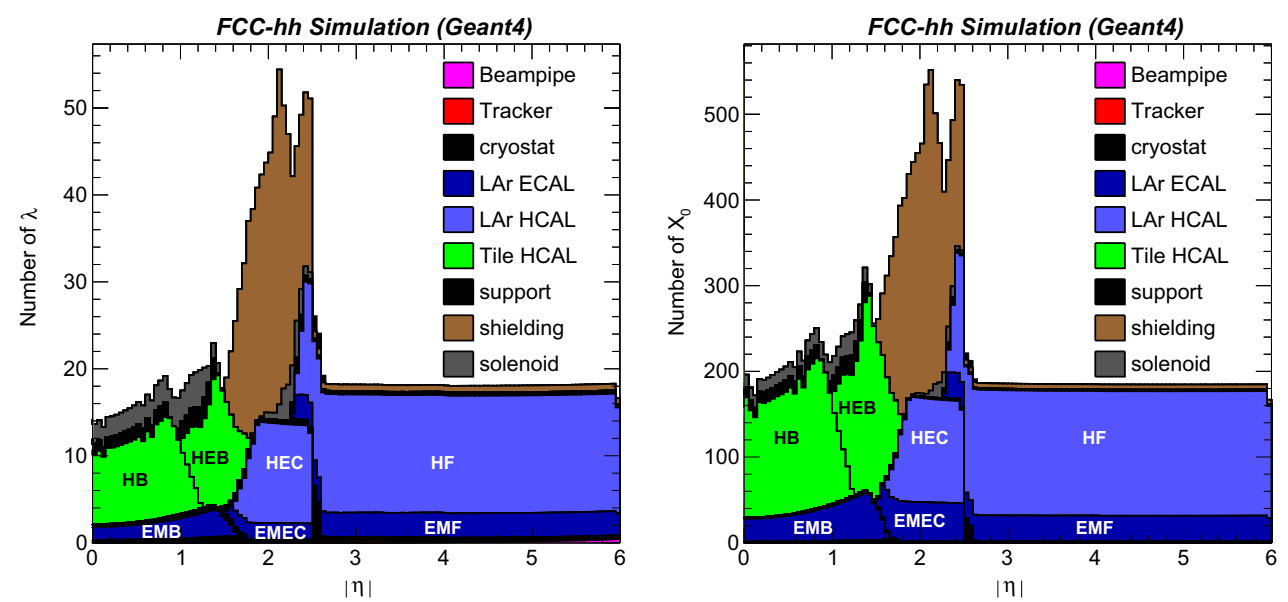

Fig. 7.10. Material budget of the different sub-systems. The calorimetry provides $\geq 10.5 \lambda$ nuclear interaction lengths to maximise shower containment and the total detector material represents between 180 and $280 X_{0}$ radiation lengths.

is supplied via high-temperature superconducting current leads that are cooled with pressurised helium gas. Magnet controls and safety systems as well as diodes, resistors dump unit, breakers and power converter are installed on the surface, which allows direct access for interventions.

\subsection{Detector sub-system concepts}

The concepts for the individual detector sub-systems are based on extrapolations of existing detectors and allow the formulation of the design challenges. Expected improvements in technology may lead to more ambitious and non-conventional detector concepts in the near future. The material budget of the reference detector is shown in Figure 7.10. The calorimetry provides $\geq 10.5$ interaction lengths $(\lambda)$ to ensure high energy shower containment and sufficient radiation shielding of the muon system. The calorimeters and magnet coils represent between 180 and $280 X_{0}$ radiation lengths, which defines the multiple scattering performance limit of the muon system. 


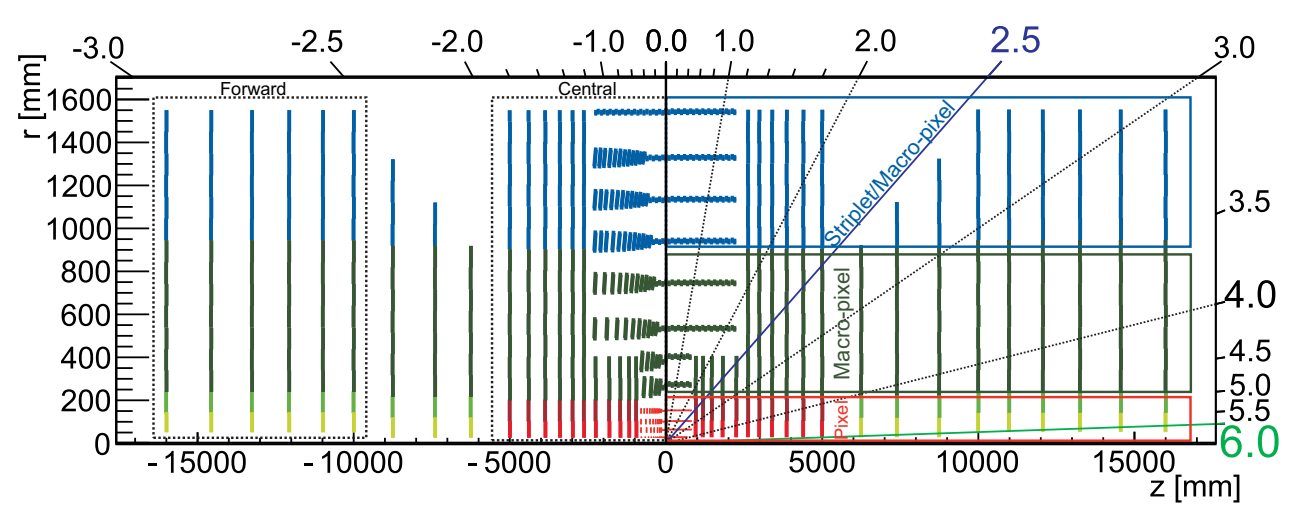

Fig. 7.11. Tracker layout using the so called "tilted geometry" (left) and "flat geometry" (right).

\subsubsection{Tracking}

The central tracker provides precision momentum spectroscopy in the region $|\eta|<2.5$ and is modelled according to the Phase-II trackers of the ATLAS and CMS detectors [344-346]. The forward tracker for $|\eta|>2.5$, which uses a forward solenoid, is unusual, but does not introduce any novel principles, except for the task of precise tracker alignment over a very large distance. The option of a dipole in the forward region is closely related to the spectrometers of the present ALICE and LHCb detectors.

The layout of the tracker for a "flat geometry" and a "tilted geometry" is shown in Figure 7.11. The barrel ECAL cryostat has an inner radius of $1.7 \mathrm{~m}$, and the outermost tracking layer is placed at $r=1.55 \mathrm{~m}$ to leave space for services. The central tracker extends up to $5 \mathrm{~m}$ from the IP and the forward tracker extends up to a distance of $16 \mathrm{~m}$ from the IP. The total silicon surface amounts to $430 \mathrm{~m}^{2}$ for the flat geometry and $391 \mathrm{~m}^{2}$ for the tilted geometry. This has to be compared to $\approx 250 \mathrm{~m}^{2}$ for the Phase-II trackers of ATLAS and CMS. The tilted geometry as seen in Figure $7.14 \mathrm{a}$, represents up to $50 \%$ less material budget (thickness of material in units of radiation length $X_{0}$ ) for the sensor modules in the critical transition region between barrel and endcap, resulting in a significant improvement of tracking performance. The final advantage of the tilted over the flat layout will however depend very much on the mechanical structure and specific service routing, which is not part of this study. The performance of the Phase-II trackers of ATLAS and CMS will give important insight into such layouts.

The tracker granularity is determined by the specification of $20 \%$ momentum resolution at $p_{T}=10 \mathrm{TeV} / \mathrm{c}$, the requirement of $<1 \%$ occupancy at the highest luminosity and specific requirements on double track separation for highly boosted objects. The innermost tracking layer is placed at a radius of $2.5 \mathrm{~cm}$ and the tracker assumes sensors with binary readout: pixels of $25-33.3 \mu \mathrm{m} \times 50-400 \mu \mathrm{m}$ inside a radius of $r<200 \mathrm{~mm}$ from the beam, macro-pixels of $33.3 \mu \mathrm{m} \times 400 \mu \mathrm{m}$ for $200<r<900 \mathrm{~mm}$ and striplets or macro-pixels of $33.3 \mu \mathrm{m} \times 2-50 \mathrm{~mm}$ for $900<r<1600 \mathrm{~mm}$. This represents an $r-\phi$ resolution of $7.5-9.5 \mu \mathrm{m}$ per detector layer. The channel count is $5.5 \times 10^{9}, 10 \times 10^{9}$ and $0.5 \times 10^{9}$ in these three regions, adding up to a total of around $16 \times 10^{9}$ readout channels, to be compared to $6 \times 10^{9}$ and $2.2 \times 10^{9}$ readout channels for the Phase-II trackers of ATLAS and CMS. The material budget per layer, including services, is assumed as $1.5 / 2 / 2.5 \%$ of $x / X_{0}$ in these three regions, only the innermost vertexing layers are assumed to have $1 \%$. These numbers are supposed to include the services, which can of course be considered optimistic. 


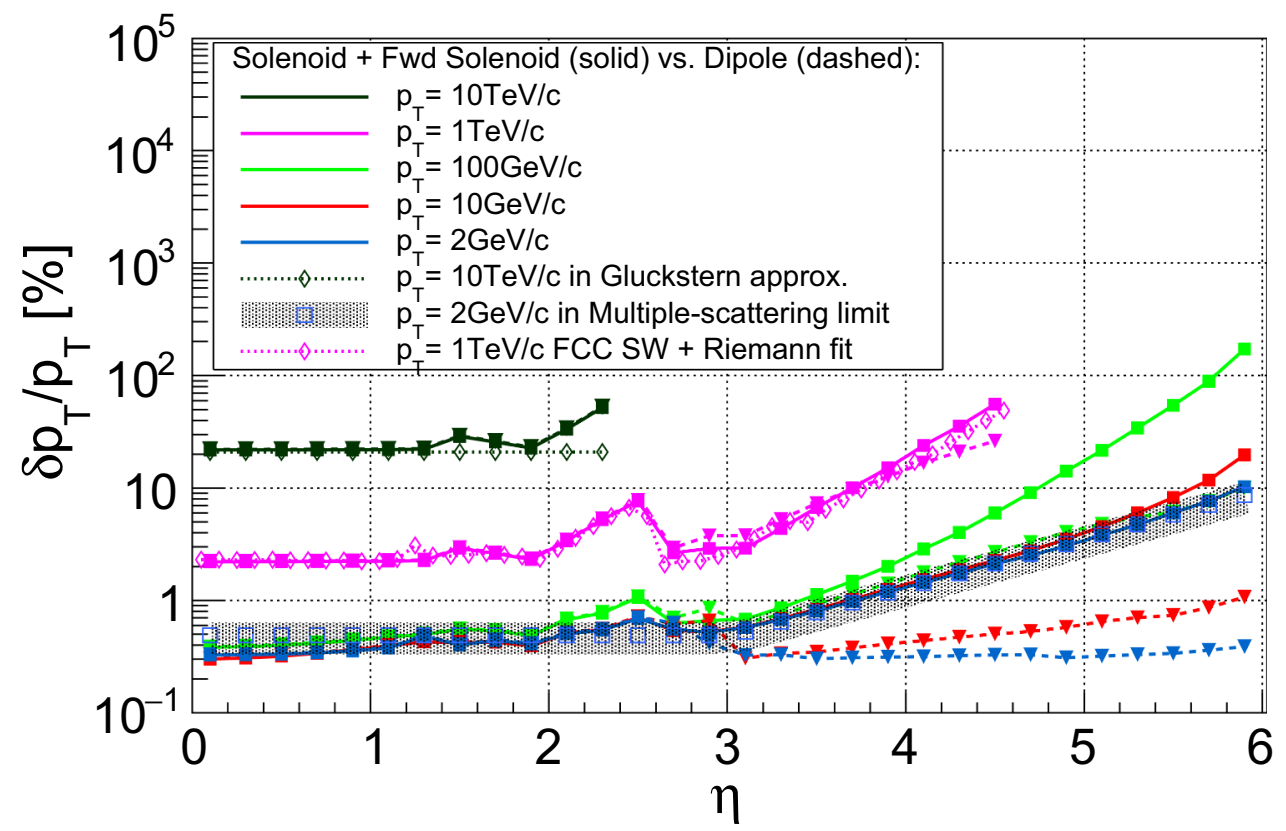

Fig. 7.12. Momentum resolution for the option with forward solenoids (solid lines) and the option with forward dipoles (dashed lines). The results from the analytic approximations are superimposed for $10 \mathrm{TeV} / \mathrm{c}$ and at the multiple scattering limit for $x_{\text {tot }} / X_{0}=0.2-0.7$. For $p_{T}=1 \mathrm{TeV} / \mathrm{c}$ the result from a full simulation is superimposed.

Figure 7.12 shows the momentum resolution for both the forward solenoid and forward dipole options. The track covariance matrix has been obtained by calculating the template track shape using numeric integration in the non uniform B field and then performing a global $\chi^{2}$ minimisation. The momentum resolution can be approximated by the square sum of the contribution due to detector resolution that dominates at large $p_{T}$ and the contribution due to multiple scattering in the detector material that dominates at low $p_{T}$. To relate these results to elementary expressions, the Gluckstern formulae are used, assuming a uniform solenoid field, $N$ equidistant detector planes, a resolution $\sigma$ per layer, a tracker radius of $L$ and total tracker material budget of $x_{\text {tot }} / X_{0}[349,350]$ :

$$
\begin{aligned}
\left.\frac{\Delta p_{T}}{p_{T}}\right|_{\text {res. }} & \approx \frac{\sigma[\mathrm{m}] p_{T}[\mathrm{GeV} / \mathrm{c}]}{0.3 B[\mathrm{~T}] L[\mathrm{~m}]^{2}} \sqrt{\frac{720}{N+4}} \\
\left.\frac{\Delta p_{T}}{p_{T}}\right|_{m . s .} & \approx \frac{0.014}{\beta 0.3 B[\mathrm{~T}] L[\mathrm{~m}]} \sqrt{\frac{x_{\mathrm{tot}}}{X_{0}}}\left(1+0.038 \ln \frac{x_{\mathrm{tot}}}{N X_{0}}\right) .
\end{aligned}
$$

For the $10 \mathrm{TeV} / \mathrm{c}$ curve, the resolution formula above is superimposed assuming $N=12, \sigma=9 \mu \mathrm{m}, B=4 \mathrm{~T}$ and $L=1.55 \mathrm{~m}$. For the low $p_{T}$ resolution limit, the multiple scattering expression above is shown as a band with values for $x_{\text {tot }} / X_{0}=$ 0.2-0.7 (see Fig. 7.14) and $\mathrm{L}$ as above. It is important to realise that, for this level of material budget, the multiple scattering dominates the momentum resolution up to transverse momenta of $250 \mathrm{GeV} / \mathrm{c}$, which is a momentum range where a significant fraction of the SM processes takes place.

In the region $0<|\eta|<1.9$ the tracks see the full spectrometer arm of $1.6 \mathrm{~m}$ and the momentum resolution only shows very weak dependence on $\eta$ from material variations and geometric variations of the lever arm. Beyond $|\eta|=1.9$ the resolution 

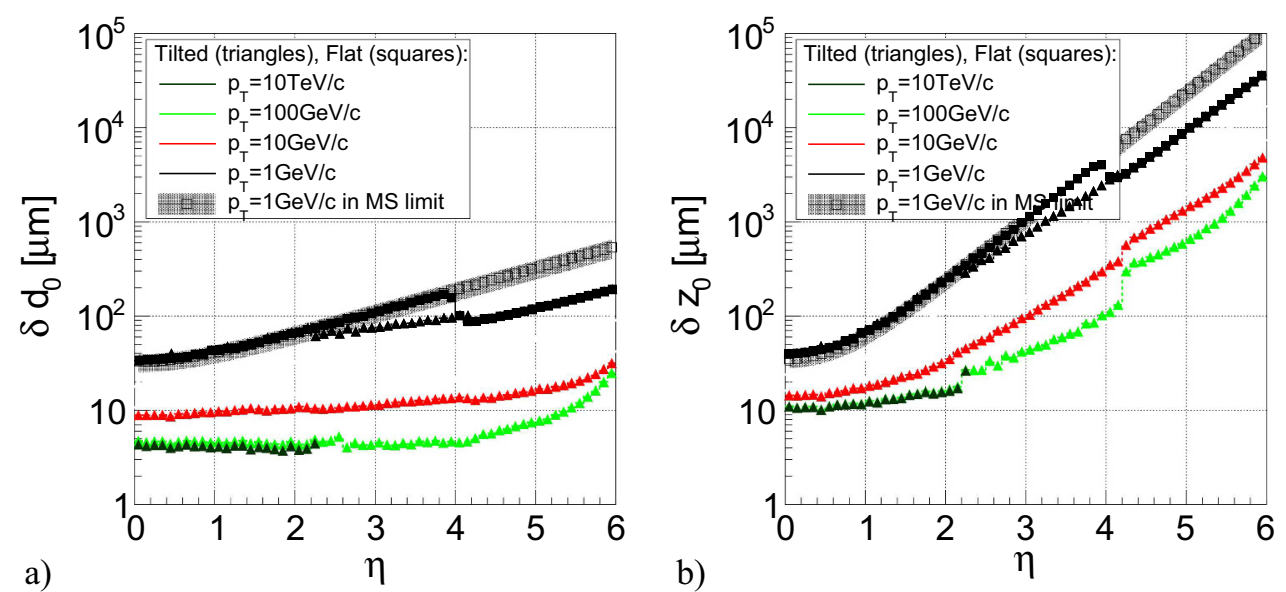

Fig. 7.13. (a) Transverse impact parameter resolution. (b) Longitudinal impact parameter resolution. The open symbols in both figures represent elementary formulae for high $p_{T}$ and multiple scattering limits.

degrades up to a factor 4 at $|\eta|=2.5$ where the spectrometer arm has decreased by a factor 2. For larger values of $\eta$ the forward tracker restores the full spectrometer arm up to $|\eta|=3$. Beyond this value the resolution degrades due to a continuous loss of lever arm. The forward solenoid therefore increases the acceptance for precision momentum spectroscopy by about one unit of rapidity. Figure 7.12 also shows that, by using a dipole magnet in the forward region, one can significantly improve the momentum resolution beyond $|\eta|=3.1$, staying below $1 \%$ at the multiple scattering limit in the entire acceptance. This improvement has of course to be contrasted with the loss of rotational symmetry and the increased complexity of the magnet system.

The impact parameter resolution and related capability to identify interaction vertices is dominated by the position resolution of the tracker at high $p_{T}$ and by the material budget of the first tracker layer together with the beam pipe at low $p_{T}$. The transverse and longitudinal impact parameter resolution for various momenta is shown in Figure 7.13. The transverse impact parameter resolution $\delta d_{0}$ (Fig. 7.13a) is the key number for secondary vertex resolution i.e. b, $\tau$ and c tagging. The limit of $5 \mu \mathrm{m}$ at high $p_{T}$ is due to the tracker position resolution. Below $50 \mathrm{GeV}$ the resolution is dominated by multiple scattering in the beam pipe and the first tracker layer.

In Figure 7.14b, a study of the b-tagging capability in the central part of the detector for different jet energies is summarised. For energies of tens to hundreds of $\mathrm{GeV}$, good performance is achieved for the reference geometry with multivariate classifiers using information from secondary vertices and track impact parameters as input [351]. In $100 \mathrm{TeV}$ proton-proton collisions, multi-TeV b-hadrons and tau leptons can be produced in various new physics scenarios. The extremely boosted b-hadrons with $p_{T}$ of several $\mathrm{TeV}$ can travel several tens of $\mathrm{cm}$ inside the detector before decaying. In addition, the separation of their highly collimated decay products is limited by the detector granularity [352]. Traditional taggers require the reconstruction of individual tracks from secondary decay vertices and hence have a significantly worse performance in the multi- $\mathrm{TeV}$ regime. A so-called hit multiplicity jump tagger exploits the change in hit multiplicity for b-hadrons decaying between two consecutive tracker layers. It is found that this approach can be superior in performance for jets of very high energy [353].

The longitudinal impact parameter resolution $\delta z_{0}$ (Fig. 7.13b) is crucial for pileup rejection. The high $p_{T}$ resolution limit is $10 \mu \mathrm{m}$. Due to multiple scattering, mainly 

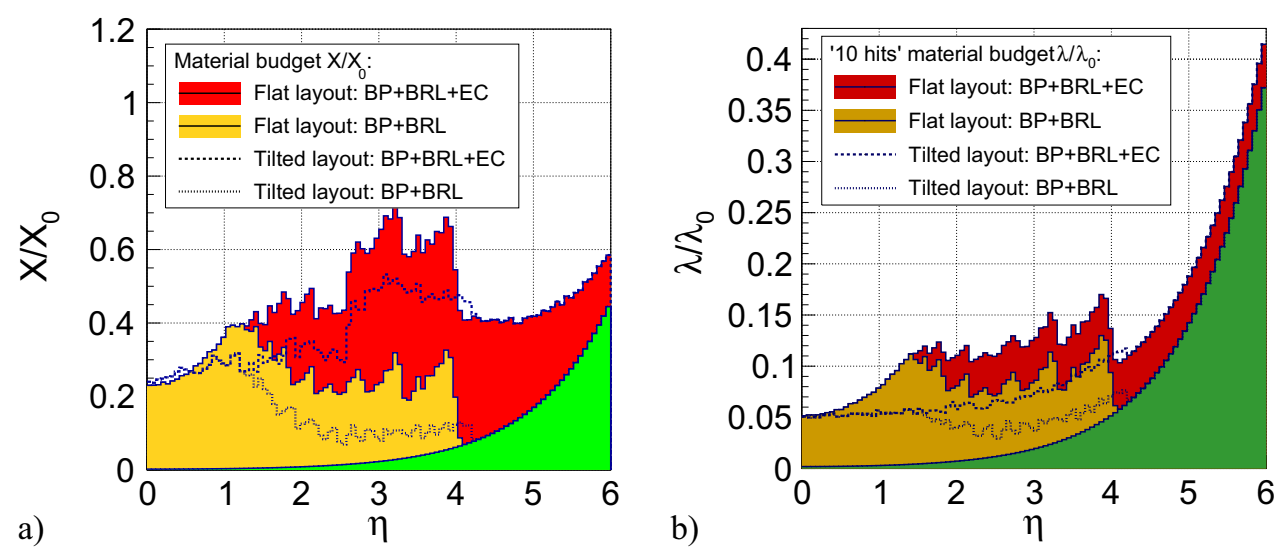

Fig. 7.14. (a) Material budget in units of radiation length for the flat and tilted tracker geometries. (b) Material budget in units of nuclear interaction length for the flat and tilted tracker geometries, assuming a limit of 10 hits on the track.

in the beam pipe, a serious degradation of this resolution with increasing rapidity is observed. Considering that the average distance between vertices is $125 \mu \mathrm{m}$ it can be seen that the $\delta z_{0}$ resolution is larger than this value for $|\eta|=1.5,3$ for $p_{T}=1,10 \mathrm{GeV} / \mathrm{c}$. Hence one needs additional information such as precision timing to correctly assign the tracks to vertices.

In addition to the tracking resolution, the track reconstruction performance can be quantified by two complementary variables: track reconstruction efficiency and track fake rate. Current experience from ATLAS and CMS Phase-II upgrade studies, that foresee tracking systems that are similar in terms of material budget and position resolution, suggests that requiring 10 hits per track is sufficient to suppress fake tracks to an acceptable level. Fake tracks are mainly caused by the fact that multiple scattering effects render random combinations of hits as compatible with a track hypothesis. This effect is dominated by the amount of material in the tracker (see Fig. 7.14), combined with the propagation distance between the tracker layers. Assuming optimal pattern recognition performance the effect of multiple scattering noise is regulated within the track fit, and the single remaining source for reconstruction inefficiency is attributed to the nuclear interactions and related production of secondary particles. This was demonstrated by the close to $100 \%$ reconstruction efficiency for muons over a large momentum spectrum, independently of the pile-up for the Phase-II tracker studies for ATLAS and CMS. In such an ideal scenario only the hadron reconstruction efficiency is expected to be affected. The material budget in units of nuclear interaction length $x / \lambda_{0}$ is therefore shown in Figure 7.14b with a requirement of finding 10 hits along the track. The result demonstrates that the tilted layout is clearly superior to the flat layout, specifically in the barrel-endcap transition region. The tilted layout presented in this report has been optimised in terms of all these effects.

To quantify the pile-up problem the so-called effective pile-up is defined, referring to the number of primary vertices that a track of a given $p_{T}$ is compatible with, at $95 \%$ confidence level. Figure 7.15a shows this quantity for a pile-up of 1000 and compares the FCC-hh tracker to a design similar to the CMS Phase-II tracker with a pile-up of 140 . While a time resolution of $25 \mathrm{ps}$ for the HL-LHC environment can already reduce the effective pile-up for $1 \mathrm{GeV}$ tracks to the level of 1 in the entire tracking acceptance, such a time resolution only brings the effective pile-up to levels of 10 at $|\eta|=4$ and around 100 at $|\eta|=6$ for the FCC-hh environment. Very 

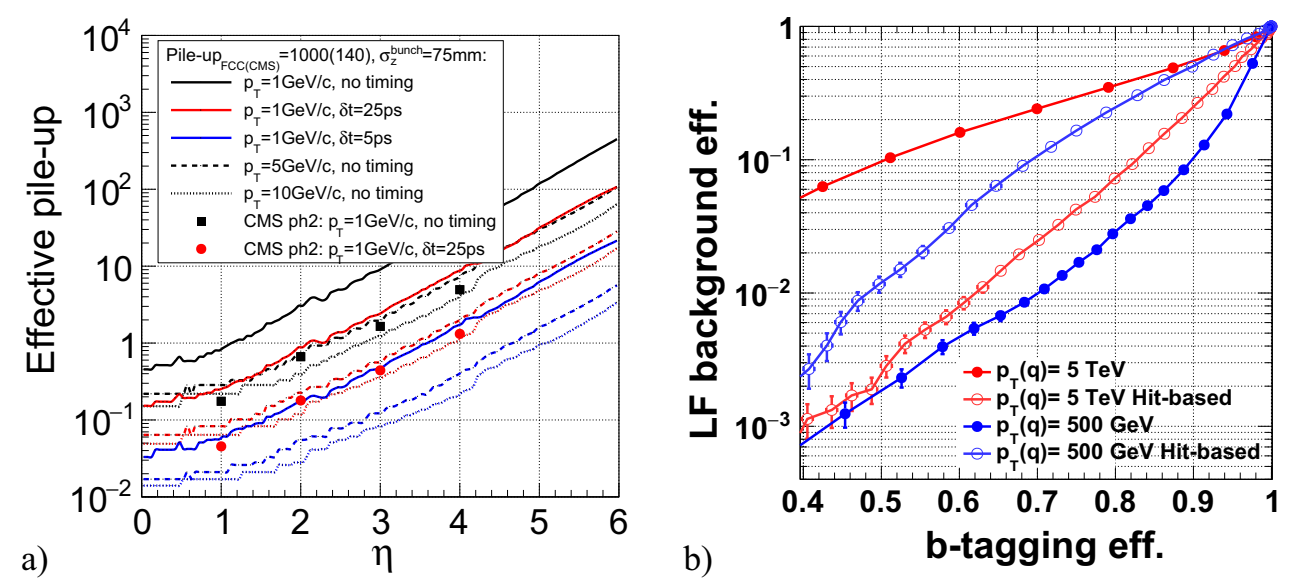

Fig. 7.15. (a) Effective pile-up for different particle momenta. (b) Examples of b-tagging performance at very large $p_{T}$.

challenging time resolutions of 5 ps per track bring these numbers down by a factor of 5 , keeping the effective pile-up below 1 within the central region. A similar effect can be achieved by stretching the luminous region by a factor of 2 .

The level at which this pile-up becomes a problem depends very much on the specific physics topic under study and only a detailed simulation including reconstruction and analysis can give a definite answer to this question.

\subsubsection{Calorimetry}

The energy resolution of a calorimeter can be parameterised according to

$$
\frac{\sigma_{E}}{E} \approx \frac{a}{\sqrt{E}} \oplus \frac{b}{E} \oplus c
$$

where $a$ is the "stochastic term" due to shower fluctuations and sampling, $b$ the "noise term" due to electronic noise and pile-up and $c$ the "constant term" due to numerous effects like shower leakage, construction uniformity and cell-to-cell calibration variations. Table 7.3 shows the performance goals, granularity, radiation levels and technologies chosen for the calorimeter system of the FCC-hh reference detector. It has to be pointed out that the longitudinal segmentation is still preliminary and must be optimised with detailed performance studies.

LAr calorimetry is used as reference technology for the ECAL because of its intrinsic radiation hardness and stability as well as the successful application in the ATLAS experiment [354]. Another technology that can cope with a 1-MeV-neq fluence of $10^{16} / \mathrm{cm}^{2}$ is the $\mathrm{Si} / \mathrm{Pb}$ or $\mathrm{Si} / \mathrm{W}$ calorimetry that will be used for the endcap calorimetry of the CMS Phase-II upgrade [355] and could be a possible choice for an FCC-hh barrel detector. The $\eta-\phi$ granularity of the barrel ECAL (EMB) is chosen as $\Delta \eta \times \Delta \phi=0.01 \times 0.009$ except for $\Delta \eta=0.0025$ in the second layer to optimise $\eta$-resolution and $\pi^{0}$ rejection. This corresponds to cells of $20 \times 20 \mathrm{~mm}$ at rapidity $\eta=0,8 \times 8 \mathrm{~mm}$ at $|\eta|=2$ and $6 \times 6 \mathrm{~mm}$ at $|\eta|=4$. This high granularity, together with the importance of longitudinal segmentation, demands a geometry that is different from the "accordion" geometry employed in the barrel ECAL of the ATLAS experiment. Figure 7.16a shows a geometry using straight inclined plates as assumed for this study. It allows highly granular readout by use of multilayer printed circuit 
Table 7.3. Calorimeter system for the reference detector.

\begin{tabular}{|l|c|c|c|c|c|c|c|c|c|c|c|}
\hline & $\eta_{\min }$ & $\eta_{\max }$ & $\mathrm{a}$ & $\mathrm{c}$ & $\Delta \eta$ & $\Delta \phi$ & Fluence & Dose & Material & Mix & Seg. \\
\hline Unit & & & $\% \sqrt{\mathrm{GeV}}$ & $\%$ & & & $\mathrm{~cm}^{-2}$ & $\mathrm{MGy}$ & & & \\
\hline EMB & 0 & 1.5 & 10 & 0.7 & 0.01 & 0.009 & $5 \times 10^{15}$ & 0.2 & $\mathrm{LAr} / \mathrm{Pb} / \mathrm{PCB}$ & $1 / 0.47 / 0.28$ & 8 \\
EMEC & 1.5 & 2.5 & 10 & 0.7 & 0.01 & 0.009 & $3 \times 10^{16}$ & 4 & $\mathrm{LAr} / \mathrm{Pb} / \mathrm{PCB}$ & $1 / 0.75 / 0.6$ & 6 \\
EMF & 2.5 & 4 & 10 & 0.7 & 0.025 & 0.025 & & & $\mathrm{LAr} / \mathrm{Cu} / \mathrm{PCB}$ & $1 / 50 / 6$ & 6 \\
& 4 & 6 & 30 & 1 & 0.025 & 0.025 & $5 \times 10^{18}$ & 5000 & $\mathrm{LAr} / \mathrm{Cu} / \mathrm{PCB}$ & $1 / 50 / 6$ & 6 \\
\hline $\mathrm{HB}$ & 0 & 1.26 & 50 & 3 & 0.025 & 0.025 & $3 \times 10^{14}$ & 0.006 & $\mathrm{Sci} / \mathrm{Pb} / \mathrm{Fe}$ & $1 / 1.3 / 3.3$ & 10 \\
$\mathrm{HEB}$ & 0.94 & 1.81 & 50 & 3 & 0.025 & 0.025 & $3 \times 10^{14}$ & 0.008 & $\mathrm{Sci} / \mathrm{Pb} / \mathrm{Fe}$ & $1 / 1.3 / 3.3$ & 8 \\
$\mathrm{HEC}$ & 1.5 & 2.5 & 60 & 3 & 0.025 & 0.025 & $2 \times 10^{16}$ & 1 & $\mathrm{LAr} / \mathrm{Cu} / \mathrm{PCB}$ & $1 / 5 / 0.3$ & 6 \\
$\mathrm{HF}$ & 2.5 & 4 & 60 & 3 & 0.05 & 0.05 & $5 \times 10^{18}$ & 1000 & $\mathrm{LAr} / \mathrm{Cu} / \mathrm{PCB}$ & $1 / 200 / 6$ & 6 \\
& 4 & 6 & 100 & 10 & 0.05 & 0.05 & $5 \times 10^{18}$ & 1000 & $\mathrm{LAr} / \mathrm{Cu} / \mathrm{PCB}$ & $1 / 200 / 6$ & 6 \\
\hline
\end{tabular}

Notes. Acceptance, performance goals (single electron for ECAL and single pion for ECAL+HCAL), granularity, radiation levels for $\mathcal{L}_{\text {int }}=30 \mathrm{ab}^{-1}$ and technologies chosen.
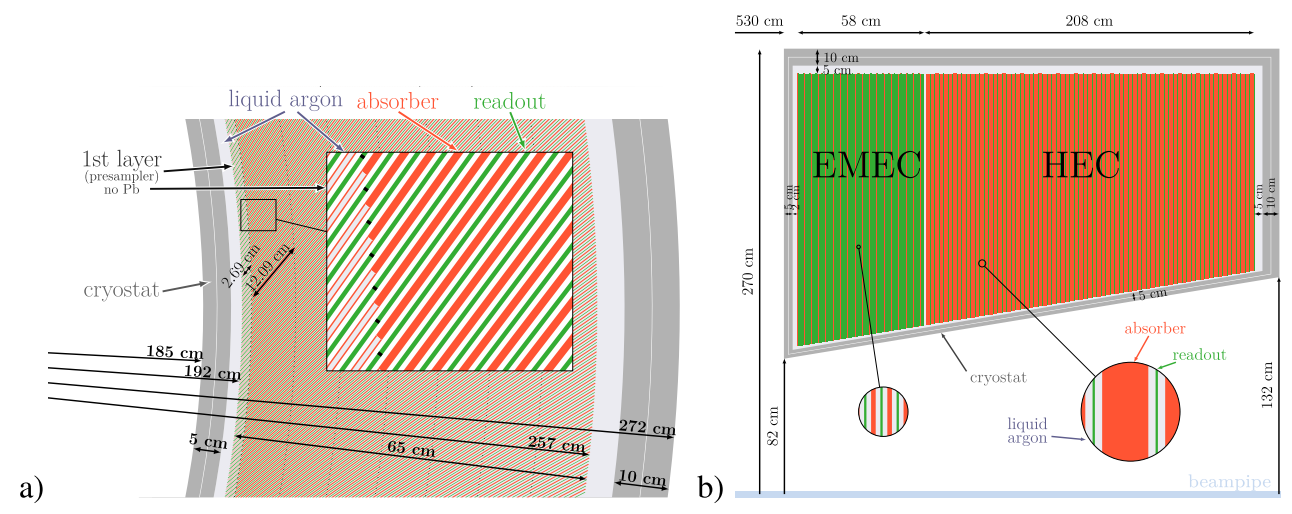

Fig. 7.16. (a) LAr barrel ECAL geometry and (b) LAr endcap calorimeter geometry.

boards. The $2 \mathrm{~mm}$ steel plated lead absorber plates are inclined at $50^{\circ}$ and 8 layers are assumed in the radial direction. The LAr gap increases from $1.15 \mathrm{~mm}$ at the inner radius to $3.09 \mathrm{~mm}$ at the outer radius, which results in a variation of the sampling fraction in radial direction. The ECAL therefore has to be calibrated separately for each of the 8 layers.

The aluminium cryostat is $5 \mathrm{~cm}$ thick, representing $56 \%$ of $X_{0}$ in front of EM calorimeter at $\eta=0$. In order to correct for the material in front of the ECAL, the fact that there is a linear relation between the upstream energy loss and the energy deposited in the first layer is exploited. The full simulation of single electrons shows a stochastic term of $a=8.2 \% \sqrt{\mathrm{GeV}}$ and a constant term of $c=0.15 \%$ at $\eta=0$.

The contribution from electronics noise is estimated by calculating the capacitances of the readout electrodes and scaling to the ATLAS numbers, which results in values ranging from 1 to $35 \mathrm{MeV}$ per cell. For a cluster of size $\Delta \eta \times \Delta \phi=0.07 \times 0.17$ this gives a value for the noise term of $b \approx 0.3 \mathrm{GeV}$. A significant contribution to the noise term does however, come from the pile-up, therefore calling for an optimisation of the cluster size $(\Delta \eta \times \Delta \phi=0.03 \times 0.08$ was found to be optimal). A simulation of 1000 superimposed minimum bias events yields a pile-up noise contribution of $b=1.3-2.7 \mathrm{GeV}$ in the region of $0<|\eta|<1.5$ for the above mentioned optimised cluster size. Figure 7.17a shows the energy resolution for electrons for three different levels of pile-up. It is assumed that the out-of-time pile-up due to the electron drift in the liquid argon gaps can been corrected by use of the full event history. The 

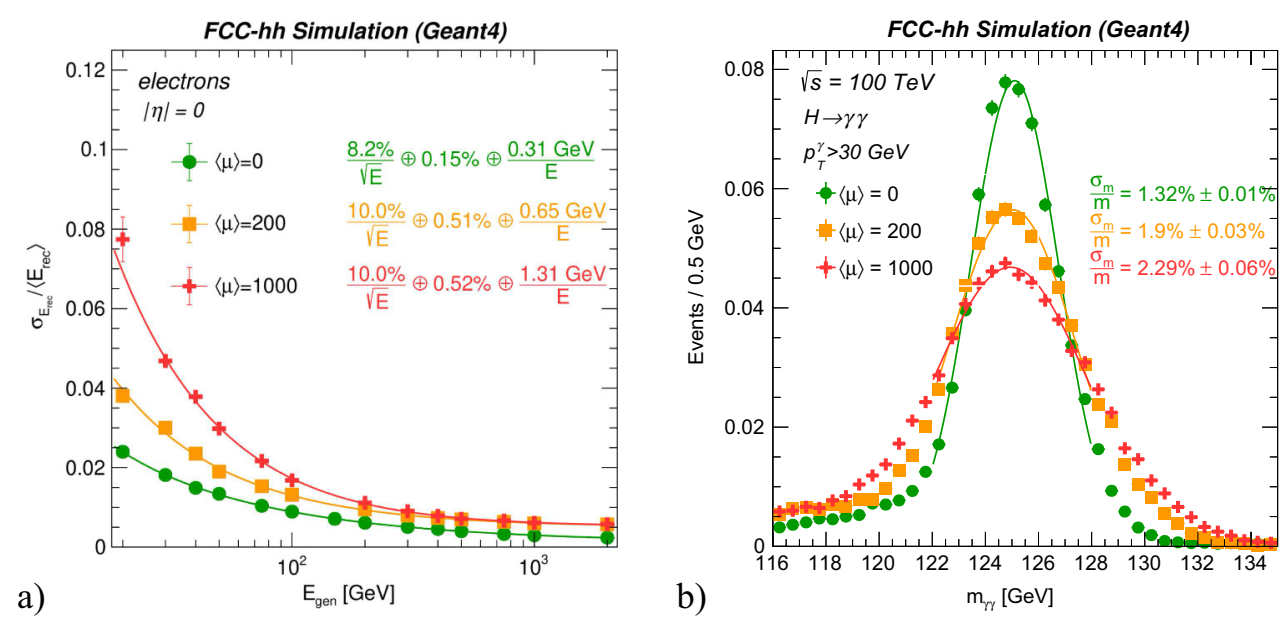

Fig. 7.17. (a) ECAL energy resolution for different levels of pile-up at $|\eta|=0$. The no pile-up configuration uses a cluster size of $\Delta \eta \times \Delta \phi=0.07 \times 0.17$ while with pile-up the optimised cluster size is $\Delta \eta \times \Delta \phi=0.03 \times 0.08$. (b) Effect of pile-up on the Higgs invariant mass distribution by selecting two photons with $p_{T}^{\gamma}>30 \mathrm{GeV}$.

remaining in-time pile-up represents a noise contribution that is independent of the chosen calorimeter technology. The effect of pile-up on the invariant mass peak of the Higgs is shown in Figure 7.17b. To what extent this pile-up noise can be mitigated by estimating the pile-up energy in a cell using tracker information must still be studied.

The endcap ECAL (EMEC) shown in Figure 7.16b assumes $1.5 \mathrm{~mm}$ steel plated lead plates with $0.5 \mathrm{~mm}$ LAr gaps. The forward ECAL (EMF) uses same geometry layout with $0.1 \mathrm{~mm}$ LAr gaps and $1 \mathrm{~cm}$ copper plates. A full simulation of the single electron resolution in the endcap ECAL at $|\eta|=2$ gives $a=7.6 \% \sqrt{\mathrm{GeV}}$, very similar to the number for $\eta=0$.

The barrel HCAL is divided into three sections, a central barrel (HB) and two extended barrels (HEB) allowing for a pseudorapidity coverage of up to $|\eta|=1.81$. The radiation levels of $<8 \mathrm{kGy}$ allow the use of an organic scintillating tile based calorimeter (TileCal). This calorimeter, shown in Figure 7.18a, uses tile scintillators oriented perpendicular to the beam axis, which are read out by wavelength shifting (WLS) fibres, similar to the technology employed in ATLAS. These WLS fibres are then read out by silicon photomultipliers (SiPM). The $\eta-\phi$ granularity of the Tile HCAL is chosen as $\Delta \eta \times \Delta \phi=0.025 \times 0.025$ with 10 longitudinal layers for the barrel and 8 layers for the extended barrel, as summarised in Table 7.3. This corresponds to cells of $72 \mathrm{~mm} \times 72 \mathrm{~mm} \times 100 \mathrm{~mm}$ in $\eta-\phi-r$ in the first layer at $\eta=0$. The SiPM readout allows for an even finer $\eta$ granularity, if needed, since the distance between two adjacent tiles is only $18 \mathrm{~mm}$. The performance for a mix of scintillator/steel 1/4.7, as used in ATLAS, has been studied in detailed standalone simulations [356] and provides a stochastic term of $a=43 \% \sqrt{\mathrm{GeV}}$ and a constant term of $c=4 \%$ for an effective calorimeter depth of $9 \lambda$. The proposed mix of scintillator/lead/steel $1 / 1.3 / 3.3$ provides an improved resolution, with a similar sampling term, but significantly smaller constant term despite the decreased effective depth to $8.6 \lambda$ (dimensions in $\mathrm{cm}$ kept constant). This is due to an e/h ratio closer to unity which also improves the linearity.

The single pion resolution for the combined barrel EMB $+\mathrm{HB}$ system using a simple hadronic calibration, which corrects for the different e/h ratios of the EMB and HB (benchmark method [357]), is shown in Figure 7.18b for a pseudorapidity 


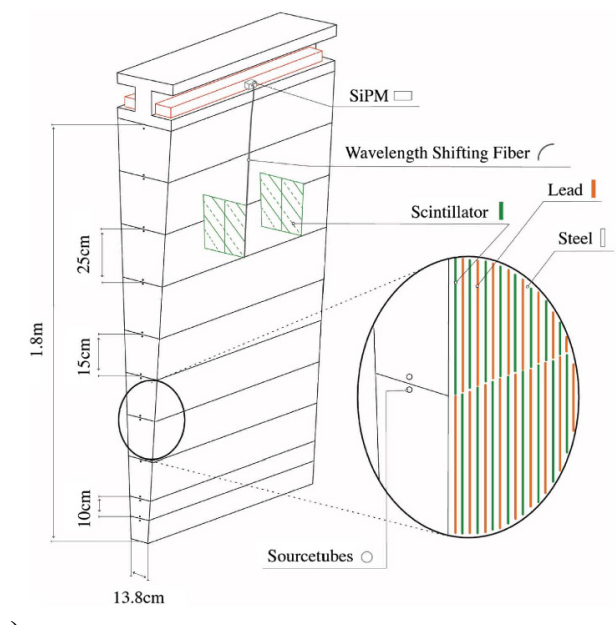

a)

Fig. 7.18. (a) Barrel HCAL technology based on scintillators similar to the ATLAS TileCal. (b) Combined single pion resolution of the barrel EMB + HB using a benchmark hadronic calibration method [357].

of $\eta=0.36$ (corresponding to a total effective depth of $10.5 \lambda$ ). These results are well within the target goal of a constant term below 3\%. The small degradation observed on the combined pion resolution with magnetic field, from 44 to $48 \%$ in the stochastic and from 1.7 to $2.2 \%$ in the constant term, could be partially explained by the increased energy loss in the cryostat walls.

A reconstruction using so called "topological clusters" has been studied for single pions and jets including electronics noise in the EMB as described above and on average $10 \mathrm{MeV}$ per cell in the HB and HEB. In addition, the impact of pile-up for $\langle\mu\rangle=200$ was evaluated for single pions. Figure 7.19 a shows the single pion resolution using the cluster algorithm as described in [358]. Compared to the benchmark calibration the resolution without pile-up degrades due to the non-optimised thresholds and calibration of the clusters. When pile-up of $\langle\mu\rangle=200$ is added, the stochastic term of the single pion resolution further deteriorates. It should be noted that these results should be understood as a demonstration that the effect of pile-up can be correctly simulated and its impact on the resolution estimated. However, it also reveals the need for more sophisticated calorimeter reconstruction and calibration algorithms and the combination with tracking information (particle flow algorithms), to achieve the best possible performance in the medium and low energy range [359].

The jet momentum resolution, taking into account realistic electronics noise in the absence of pile-up and at $B=0 \mathrm{~T}$, is shown in Figure $7.19 \mathrm{~b}$. The jet momentum resolution, obtained using the anti-kt jet cone algorithm [360] on top of the cluster reconstruction, is better than $2 \%$ for jets with $p_{T}>1 \mathrm{TeV}$, which reflects the performance goals. When introducing the $4 \mathrm{~T}$ magnetic field, approximately $15 \%$ of the charged particles within jets of momenta $<100 \mathrm{GeV}$ are not reaching the calorimeters and are thus lost for reconstruction. In contrast to that, the resolution of jets with $p_{T}>1 \mathrm{TeV}$ is not degraded by the magnetic field. Further studies including the tracking information are needed to access the jet performance in the presence of magnetic field and realistic pile-up.

The hadron calorimetry in the endcap (HEC) and forward region (HF) is based on LAr technology and shares the cryostats with the ECAL. The proposed geometry of the HEC is shown in Figure 7.16b and parameters are given in Table 7.3. 

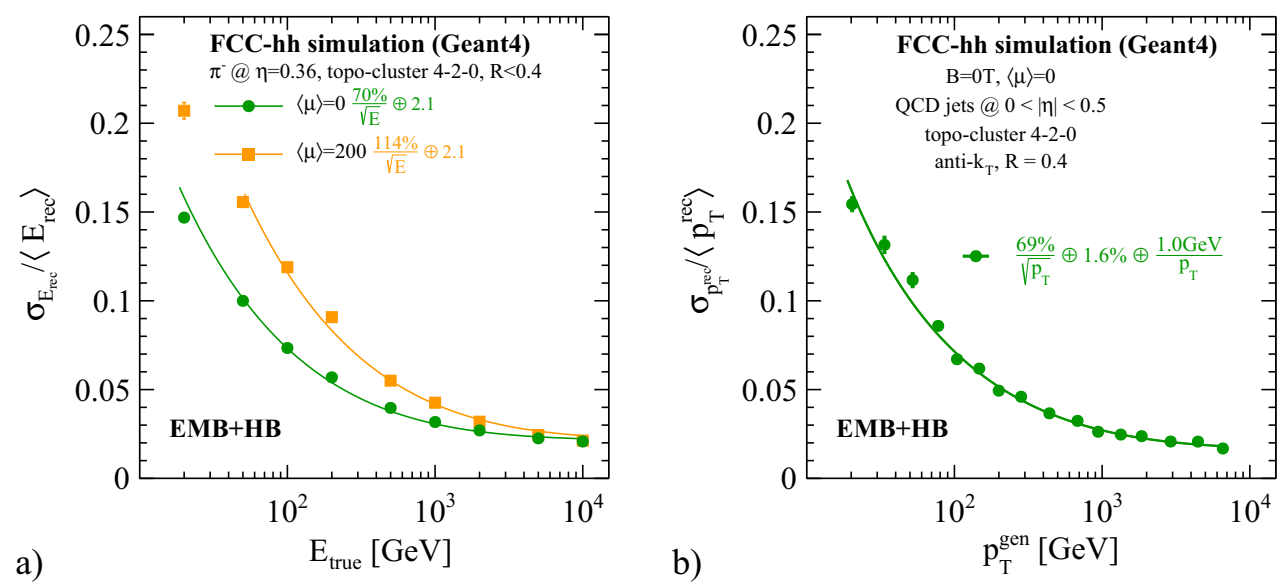

Fig. 7.19. (a) Single pion resolution using for barrel $\mathrm{EMB}+\mathrm{HB}$ at $\eta=0.36$ and $B=4 \mathrm{~T}$ with electronics noise only (green circles) and for a pile-up of $\langle\mu\rangle=200$ (orange squares). Only clusters with $\Delta R<0.4$ from the generated particle direction are included. (b) Momentum resolution for jets in the barrel $\mathrm{EMB}+\mathrm{HB}$ at $B=0 \mathrm{~T}$ and without pile-up using the anti-kt jet cone algorithm on top of the cluster reconstruction.

The total number of ECAL and HCAL channels is about $2.5 \times 10^{6}$. Sampling all calorimeter cells at $40 \mathrm{MHz}$ with 16 bits per sample corresponds to a total data rate of 200 TByte per second which has to be sent via optical fibres to the first level trigger.

An alternative ECAL technology uses silicon as the active material together with $\mathrm{Pb}$ or $\mathrm{W}$ absorbers. The simulation of 50 layers of $2.1 \mathrm{~mm} \mathrm{~W}$ sheets, interleaved with $300 \mu \mathrm{m}$ silicon sensors with $5 \times 5 \mathrm{~mm}$ analogue pads, shows a stochastic term of $a=16 \% \sqrt{\mathrm{GeV}}$. The idea of a digital ECAL (DCAL) with monolithic active pixel sensors (MAPS) uses the number of pixels fired as a measure of the energy. A simulation with $50 \times 50 \mu \mathrm{m}$ pixels and a sensitive layer thickness of $18 \mu \mathrm{m}$ and a threshold of 480 electrons shows a stochastic term of $a=12.6 \% \sqrt{\mathrm{GeV}}$. Linearity is good up to energies of $\approx 300 \mathrm{GeV}$. Beyond this energy the effect of multiple electrons passing a single pixel leads to non-linearities and efforts to apply corrections in this range are underway. Applying this technology to the reference detector would result in $3500-6000 \mathrm{~m}^{2}$ of silicon sensors representing a total of around $10^{12}$ pixels fed into $10^{8}$ readout pads.

The geometries of the barrel HCAL scintillator calorimeter as well as the endcap and forward LAr calorimeters are inspired by those of the ATLAS experiment but provide a much higher granularity and improved performance. The barrel ECAL with inclined plates represents a new concept that needs $R \& D$ and proof of principle. The extreme particle rates in the forward calorimeter, together with the total deposited power of $3.5 \mathrm{~kW}$ per unit of rapidity, also pose significant engineering challenges. The application of silicon sensors for the forward calorimeter of the CMS PhaseII detector will show whether this technology can be successfully applied for an FCC-hh detector. The ideas of digital electromagnetic calorimetry are quite recent and still need a significant R\&D effort. In addition, a considerable effort is needed to develop novel reconstruction algorithms building on the full event information, including tracker, calorimeters and muon system.

\subsubsection{Muon system}

Neglecting energy loss, muons of $p_{T}=4 \mathrm{GeV} / \mathrm{c}$ would just make it into the muon system and muons of $p_{T}=5.5 \mathrm{GeV} / \mathrm{c}$ would exit the solenoid coil at $45^{\circ}$. The energy 


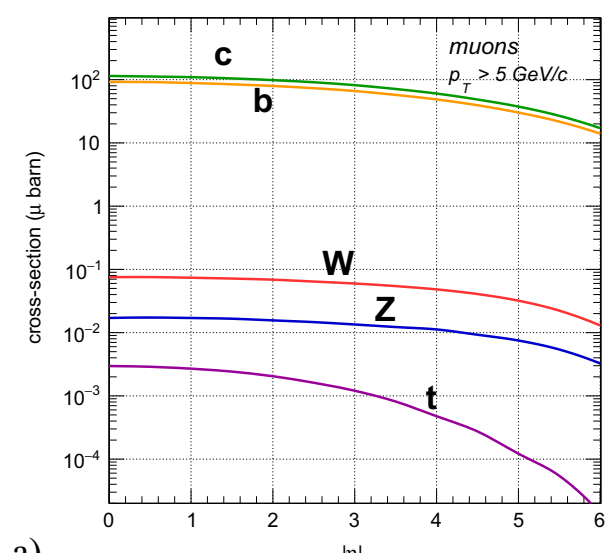

a)

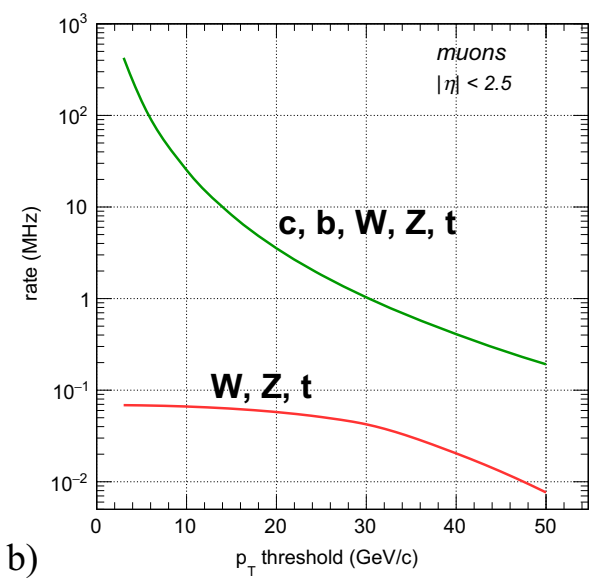

b)

Fig. 7.20. (a) Differential cross-section for muons with $p_{T}>5 \mathrm{GeV} / \mathrm{c}$ [361]. (b) Rate of muons inside $|\eta|<2.5$ including all muons and only the ones from $W, Z, t$.

loss of $3-4 \mathrm{GeV}$ in the calorimeters will result in a $p_{T}$ cutoff around $6-7 \mathrm{GeV} / \mathrm{c}$. Figure 7.20a shows the differential cross-sections for muons from c, b, W, Z and t decays assuming a lower $p_{T}$ cutoff of $5 \mathrm{GeV} / \mathrm{c}$. The muon rate is dominated by $\mathrm{c}$ and $\mathrm{b}$ decays with cross-sections of $\approx 100 \mu \mathrm{b}$ each. The total differential cross-section for muons of $p_{T}>5 \mathrm{GeV} / \mathrm{c}, \sigma_{\mu 5} \approx 200 \mu \mathrm{b}$, therefore results in a muon flux of $\mathcal{L} \times \sigma_{\mu 5} /\left(2 \pi r^{2}\right)$ in the muon system, which evaluates to about $20 \mathrm{~Hz} / \mathrm{cm}^{2}$ in the first muon stations at $r=650 \mathrm{~cm}$ for the peak luminosity. The additional rate from punch-through still has to be evaluated, but it is clear that the charged particle count rate of about $500 \mathrm{~Hz} / \mathrm{cm}^{2}$ due to high energy photons will dominate the rate in the muon system. The total rate of muons above a given threshold inside an acceptance of $|\eta|<2.5$ is shown in Figure 7.20b, together with the muon rate from W, Z, t decays only. The total muon rate is above $20 \mathrm{MHz}$ for a $p_{T}$ threshold of $10 \mathrm{GeV} / \mathrm{c}$ and a single muon trigger with this threshold will therefore not provide any selectivity. However, the rate of muons from W, Z, t always stays below $100 \mathrm{kHz}$ for any threshold. Since muons from $\mathrm{c}$ and $\mathrm{b}$ decays are always accompanied by jets, the determination of muon isolation at the first trigger level is a key item for useful selectivity.

Figure 7.21a shows the stand-alone muon momentum resolution, the tracker momentum resolution and the combined momentum resolution for different levels of position resolution in the muon system. Stand-alone performance is provided by the angular difference between track angle in the muon system and the radial line connection to the FCC beam axis. The limit of the stand-alone resolution due to multiple scattering in the calorimeters is around 4\%. Assuming a position resolution of $50 \mu \mathrm{m}$ in the muon system a combined muon momentum resolution of $6 \%$ is found even for momenta as high as $10 \mathrm{TeV} / \mathrm{c}$. Figure $7.21 \mathrm{~b}$ shows the stand-alone muon momentum resolution as a function of $\eta$. It can be seen that at $\eta=2.5$ the multiple scattering limits the momentum resolution to about $28 \%$. Beyond $\eta=2.5$ in the forward region, the momentum resolution is beyond $100 \%$, so the forward muon system using the forward solenoid can only provide muon identification but no muon trigger capabilities. If a muon trigger for $\eta>2.5$ were needed, as applied in the LHCb and ALICE experiments, one has to use dipole magnets in the forward region.

Including a safety factor of 2.5 , the charged particle rates amount to $1.25 \mathrm{kHz} / \mathrm{cm}^{2}$ in the barrel and outer endcap muon system and rise up to $25 \mathrm{kHz} / \mathrm{cm}^{2}$ in the inner part of the inner endcap muon system. In the forward muon system the rates range from 25 to $250 \mathrm{kHz} / \mathrm{cm}^{2}$. It is therefore concluded that for the barrel and endcap 

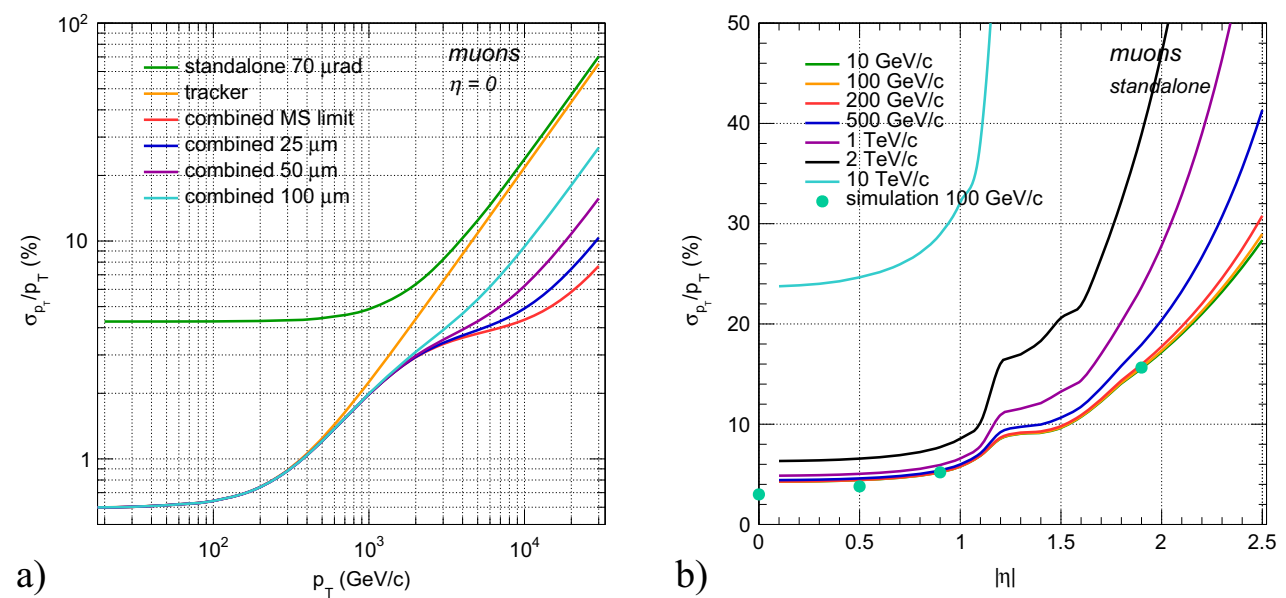

Fig. 7.21. (a) Muon momentum resolution at $\eta=0$. (b) Muon stand-alone momentum resolution as a function $\eta$ for different muon momenta.
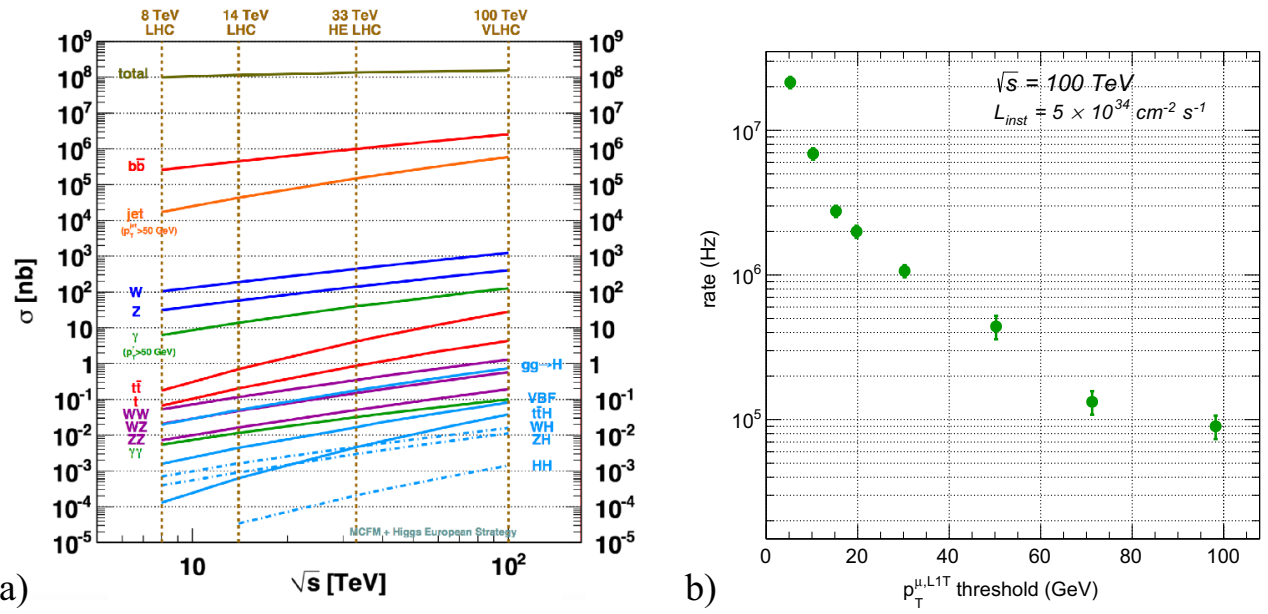

Fig. 7.22. (a) Cross-sections for key SM processes. (b) Muon trigger rates at $100 \mathrm{TeV}$ for $\mathcal{L}=5 \times 10^{34}$ assuming the CMS Phase-I calorimeter and muon triggers.

regions, technologies planned to be used at the HL-LHC, like the sMDTs from the ATLAS experiment with a diameter of $15 \mathrm{~mm}$, are suitable. An arrangement of $2 \times 4$ layers of sMDTs at a distance of $1.4 \mathrm{~m}$, constructed as one mechanical object, can provide an angular resolution of $60 \mu \mathrm{m}$ and spatial resolution of $40 \mu \mathrm{m}$ and therefore fulfil the requirements. A total area of $1150 \mathrm{~m}^{2}$ with about 250 MDT chambers would consist of $260 \mathrm{k}$ tubes and would therefore be an efficient implementation of the muon system. The points in Figure 7.21b show a GEANT simulation of the reference detector with such an arrangement. An additional layer of thin-gap RPCs with $1 \mathrm{~mm}$ gas gap could provide 0.5 ns time resolution if needed.

\subsubsection{Trigger and data acquisition}

Figure 7.22a shows how the cross-sections for typical SM processes increase from $14 \mathrm{TeV}$ at the LHC to $100 \mathrm{TeV}$ at the FCC-hh [362]. In addition the factor 6 increase 
in peak luminosity from HL-LHC to FCC-hh results in an average pile-up increase from 130 to 1000 that has to be taken into account. Since the b $\bar{b}$ cross-section is only 50 times smaller than the inelastic pp cross-section, there will be on average $20 \mathrm{~b} \bar{b}$ pairs in a single bunch crossing event. One will also find 3 jets with $p_{T}>50 \mathrm{GeV}$ in a single bunch crossing. The rates for a few SM processes are shown in Table 7.1, where around $400 \mathrm{kHz}$ of Ws, $120 \mathrm{kHz}$ of Zs, $40 \mathrm{kHz}$ of W to leptons and $4 \mathrm{kHz}$ of Z to leptons are found. The ratio of photons with $p_{T}>50 \mathrm{GeV}$ to jets with $p_{T}>50 \mathrm{GeV}$ is of the order of $10^{-4}$, similar to LHC energies, so to avoid increasing the rate of fake photon triggers due to jets, the discrimination between jets and photons on the trigger must reach levels of $10^{-5}$.

To understand the order of magnitude of the challenge, the CMS Phase-I calorimeter and muon triggers are simulated, and the rates at $14 \mathrm{TeV}$ and $100 \mathrm{TeV}$ are compared, both at a pile-up of 140 and a luminosity of $\mathcal{L}=5 \times 10^{34} \mathrm{~cm}^{-2} \mathrm{~s}^{-1}$. This setup essentially gives the performance of the CMS Phase-II detector, in the absence of the track-trigger that is planned to reduce trigger rates. While the $100 \mathrm{kHz}$ rate limits for single muon, photon/electron and jet triggers are at thresholds of $25 / 30 / 120 \mathrm{GeV}$ at $14 \mathrm{TeV}$, the thresholds become $78 / 150 / 300 \mathrm{GeV}$ at $100 \mathrm{TeV}$ due to the increased cross-sections alone. Figure $7.22 \mathrm{~b}$ shows the trigger rate of single muons at $100 \mathrm{TeV}$. Clearly, more sophisticated trigger algorithms, like isolation for muons or longitudinal segmentation for the calorimetry are needed to keep the trigger rates at acceptable levels. This essentially means that today's offline algorithms have to be migrated to the trigger.

The detectors for the Phase-II upgrades of the ATLAS and CMS experiments will generate data volumes of 6 and 7.5 MByte per event, assuming the ultimate average pile-up of 200 [363,364]. This results in a total data production of about $200 \mathrm{~TB} / \mathrm{s}$ at $40 \mathrm{MHz}$ bunch crossing frequency. Moving this amount of data from the detector directly into a High-Level Trigger (HLT) system is technically possible. However, the data rates provided by the radiation-hard optical links designed for the LHC experiments would still call for 150-300 k optical links, a non-negligible cost factor. In addition, the electrical links providing the power and necessary cooling infrastructure would significantly impact the tracker performance. A first level trigger will therefore reduce the tracker readout rate to about $0.5-1 \mathrm{MHz}$ for the upgraded ATLAS and CMS detectors. The data from calorimeters and muon system will be sent to the central trigger processors in the underground service cavern at the full bunch-crossing frequency of $40 \mathrm{MHz}$. In addition, CMS will read reduced tracker information at the same rate. This amounts to a total data rate of $30 \mathrm{~TB} / \mathrm{s}$ for ATLAS and $40 \mathrm{~TB} / \mathrm{s}$ for CMS. Trigger decisions are taken with a latency of $10-12 \mu \mathrm{s}$ and the tracker information is then read out at a rate of $0.5-1 \mathrm{MHz}$. The HLT system uses the entire detector information in the form of fully built events to perform further event selection, targeting a storage rate of about $10 \mathrm{kHz}$ at $60 \mathrm{~GB} / \mathrm{s}$.

The increased granularity and acceptance of the FCC-hh reference detector will result in about $250 \mathrm{TByte} / \mathrm{s}$ of data for calorimetry and muons systems, about 10 times more than in the ATLAS and CMS Phase-II scenarios. It is likely that it will be possible to cope with these amounts of data by the time of detector construction. The question remains, whether the data rate of $1-2 \mathrm{~PB} / \mathrm{s}$ from the tracker can also be read out at the full bunch crossing rate or whether calorimeter, muon and possible coarse tracker information allow a first level trigger to be formed, reducing the tracker readout rate to the few $\mathrm{MHz}$ level, without the loss of important physics. Even if the optical link technology for full tracker readout were available and affordable, sufficient radiation hardness of devices and infrastructure constraints from power and cooling might be prohibitive. It was shown that a triplet of tracking layers at a radius of $600 \mathrm{~mm}$ with layer separation of $30 \mathrm{~mm}$ can provide a track trigger that retains $50 \%$ of the $\mathrm{HH}$ and $\mathrm{t} \overline{\mathrm{t}} \mathrm{H}$ signals at a trigger rate of only $100 \mathrm{kHz}$ at the highest luminosity with 
pile-up of 1000 [365]. This is evidence that tracking can provide enough selectivity if calorimetry and muon measurements are not sufficient. About half of the tracker data originate from the region of $<200 \mathrm{~mm}$ from the beam pipe, where hit densities are enormous, radiation levels very high and material budget has to be very low for excellent vertex tracking. It is therefore conceivable to assume $40 \mathrm{MHz}$ readout only for the tracking layers at radii $>200 \mathrm{~mm}$ for a high performance track trigger and to read out the vertex tracker at a lower rate.

\subsection{Detector performance and physics benchmark studies}

Physics at the FCC-hh will involve final state particles with momenta ranging from a few tens of $\mathrm{GeV}$ to tens of $\mathrm{TeV}$. The high end of the spectrum features searches for resonances at the highest possible mass (up to $40 \mathrm{TeV}$ ), whose decay products are $\mathrm{SM}$ multi-TeV particles. High momentum decay products such as muons, electrons, photons or charged hadrons can be used to define minimal requirements on tracking and calorimetry momentum/energy resolution. Multi-TeV unstable heavy objects such as gauge bosons, top or bottom quarks and tau leptons that decay into highly boosted and collimated stable particles are useful benchmarks for constraining the angular separation power of the detector, in particular of the tracker and of the calorimeters. At such high $p_{T}$ the relative impact of pile-up can be neglected to first approximation, as shown in Figures 7.17a and 7.19a. The detector performance at low $p_{T}$ is instead fixed by targeting maximum precision and by requiring the best sensitivity to searches for elusive final states, such as those involving compressed or low-mass spectra. The goal is to define targets for object reconstruction efficiencies and fake-rates, as well as energy/momentum resolution at low momentum, in a regime where the relative impact of the pile-up is large.

A complete discussion of the physics opportunities at the FCC-hh can be found in Volume 1. This section focusses on a small selection of physics studies, which played a key role in defining the target performance for the FCC-hh reference detector presented in the previous sections and in shaping its design. First the measurement of the Higgs self-coupling, and the impact of pile-up is presented. Next, the search for a $\mathrm{Z}^{\prime}$ resonance decaying to leptons and for a pair of stop squarks decaying to fully hadronic final states is covered. These searches define the momentum resolution and calorimeter granularity requirements for multi-TeV leptons and boosted hadronic jets, respectively. Finally, a search for disappearing tracks, with a focus on the inner tracking detector design is presented. A more complete description of these analyses can be found in $[15-17,23]$.

For the studies presented in this section, most of the Monte Carlo samples have been generated with the MADGRAPH5_AMC@NLO [341] v2.5.2 package, showered and hadronised with PYTHIA 8 [366] v8.230. The signals for the heavy resonances are directly generated using PYTHIA 8 and for the disappearing tracks with SOFTSUSY v3.7.3 [367] and SUSYHIT [368]. With the exception of the disappearing track study, the detector simulation was performed with the fast simulation framework DeLPHES [369] v3.4.2, parameterising the sub-detector specifications defined in the previous sections. Rather than simulating the pile-up directly in fast simulation, its possible impact on the final sensitivities through its indirect effects on the performance of specific objects, has been studied. The disappearing track study uses a custom pile-up simulation and track clustering algorithm.

\subsubsection{The Higgs self-coupling}

A precise measurement of the Higgs self-coupling, $\lambda$, is crucial for constraining the shape of the Higgs potential near its ground state and can be determined from 

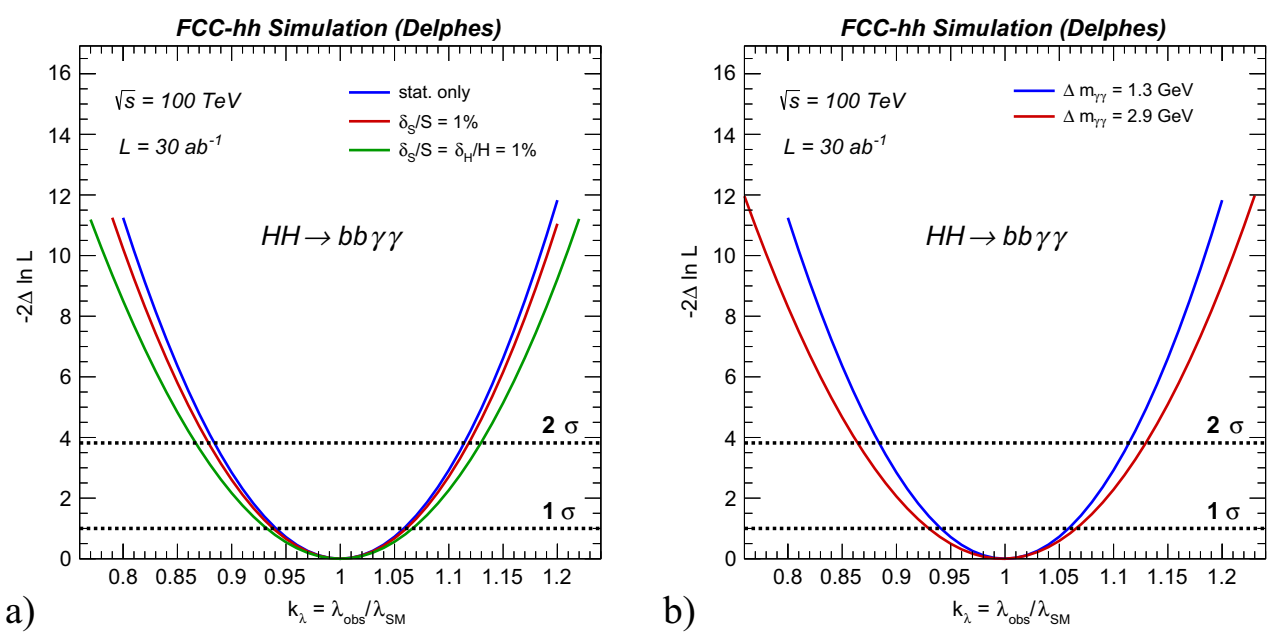

Fig. 7.23. (a) Expected precision on the Higgs self-coupling modifier $\kappa_{\lambda}=\lambda_{\text {obs }} / \lambda_{\mathrm{SM}}$ with no systematic uncertainties (blue), $1 \%$ signal uncertainty (red) and with $1 \%$ uncertainty on the ttH background (green). (b) Comparison of the precision on $\kappa_{\lambda}=\lambda_{\text {obs }} / \lambda_{\mathrm{SM}}$ obtained by assuming either nominal $\delta m \gamma \gamma=1.3 \mathrm{GeV}$ (pile-up=0) or degraded $\delta m \gamma \gamma=2.9 \mathrm{GeV}$ (pile-up $=1000)$ di-photon invariant mass resolution.

non-resonant di-Higgs production [370]. Because of the small di-Higgs production cross-section (at $\sqrt{s}=100 \mathrm{TeV}, \sigma_{g g \rightarrow \mathrm{HH}} \approx 1.5 \mathrm{pb}$ [371]) it is an extremely challenging measurement. The $\mathrm{b} \bar{b} \gamma \gamma$ decay mode is the most promising channel despite the small branching ratio of $0.25 \%$. The main backgrounds for this measurement are single Higgs production, $\gamma \gamma+$ jets, and $\gamma+$ jets.

The photon identification efficiency is assumed to be $\epsilon_{\gamma}=95 \%$ for $|\eta|<2.5$. The light jet-to-photon mis-identification probability is parameterised by the function $\epsilon_{j \rightarrow \gamma}=0.002 \exp \left(-p_{T}[\mathrm{GeV}] / 30\right)$. The b-tagging efficiency $\epsilon_{b}$ and the light (charm) mistag rates $\epsilon_{l(c) \rightarrow b}$ are assumed to be $\epsilon_{b}=85 \%$ and $\epsilon_{l(c) \rightarrow b}=1(5) \%$.

The event selection requires two isolated photons and two b-tagged jets. Additional selection criteria are applied to suppress backgrounds, such as requiring the di-photon and di-jet pairs $p_{T}(\gamma \gamma, \mathrm{bb})>100 \mathrm{GeV}$. The invariant mass of the bb pair is required to be within $100<m_{\mathrm{bb}}<130 \mathrm{GeV}$.

The signal is extracted via a $2 \mathrm{D}$ fit on the photon pair and the Higgs pair invariant masses, $m_{\gamma \gamma}$ and $m_{b b \gamma \gamma}$ using analytical parameterisations for the signal and background shapes. Figure 7.23a shows that, assuming a $1 \%$ systematic uncertainty on the signal cross-section and the single Higgs background normalisation, $\lambda$ can be measured with a precision of $6.5 \%$ (at $68 \%$ confidence level) with an integrated luminosity of $30 \mathrm{ab}^{-1}$. The single most important variable that controls the sensitivity of this measurement is the di-photon pair invariant mass. In accordance with Figure $7.17 \mathrm{~b}$, the di-photon invariant mass resolution is found to be $\delta m_{\gamma \gamma} \approx 1.3 \mathrm{GeV}$ with pile-up=0 and $\delta m_{\gamma \gamma} \approx 2.9 \mathrm{GeV}$ with pile-up=1000. The resulting impact on the Higgs self-coupling precision, shown in Figure 7.23b, is found to be of the order of $1 \%$. The assessment of the impact of pile-up on $\delta m_{\gamma \gamma}$ has to be considered as conservative. Indeed, the use of timing information as well as the optimisation of the photon clustering using deep learning techniques is expected to bring large improvements in terms of pile-up rejection. It should be noted that the precision of the Higgs self-coupling measurement will benefit from the combination with other Higgs decay

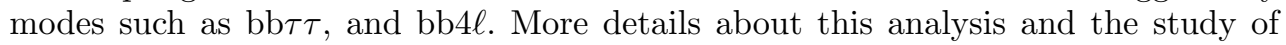
alternative final states are given in [17]. 


\subsubsection{High mass resonances decaying to leptons}

New Physics models with extended gauge groups often feature additional U(1) symmetries with corresponding heavy spin- 1 bosons. These bosons, generally referred to as $\mathrm{Z}^{\prime}$, appear as a narrow resonance $(\Gamma / m \sim 2-3 \%)$ through their decay to two high energy SM particles [372-375]. Searching for these heavy objects typically involves reconstructing an invariant mass peak as a proxy for the $\mathrm{Z}^{\prime}$ resonance. An excellent momentum resolution is needed in order to achieve the highest possible discovery reach.

The decay products of heavy resonances accessible to FCC-hh could be in the multi- $\mathrm{TeV}$ regime and the capability to reconstruct their momentum imposes stringent requirement on the detector design. For simplicity, only the $\mathrm{Z}^{\prime} \rightarrow \ell \ell$ decays are considered here, where $\ell=e, \mu$ (decays to tau leptons, top quarks or EW gauge bosons are discussed in Ref. [15]). Reconstructing the track curvature of multi-TeV muons requires excellent position resolution and a large lever arm. Figure 7.21a shows that the current tracking plus muon design allows for the outstanding $p_{T}$ resolution of $\sigma\left(p_{T}\right) / p_{T} \approx 10 \%$ at $p_{T}=20 \mathrm{TeV}$ at $\eta=0$. Reconstructing multi-TeV electrons requires an ECAL design with a small constant term, which is ensured with a highly uniform calorimeter with no leakage. The present ECAL design ensures an excellent $\sigma(E) / E \approx 0.2 \%$ at multi-TeV energies, as shown in Figure 7.17a.

The Sequential SM (SSM) [374] Z', whose couplings are identical to those of the $\mathrm{SM} \mathrm{Z}$ boson, is taken as a benchmark model. Events are selected by requiring two high- $p_{T}$ isolated leptons with $p_{T}(\ell)>6 \mathrm{TeV}$ and $|\eta(\ell)|<3$. The only significant background is the di-lepton continuum Drell-Yan process. A $50 \%$ systematic uncertainty on the background rate is assumed (extremely conservative, but with little impact on the final result). The signal extraction is performed via a profile likelihood fit on the di-lepton invariant mass. The di-muon spectrum for a $m_{\mathrm{Z}^{\prime}}=35 \mathrm{TeV}$ is illustrated in Figure 7.24a. Figure 7.24b shows the discovery reach for the separate and combined di-lepton channels. Despite the worse resolution at high momenta, the reach in the di-muon channel is similar to that in the di-electron channel, because of the rapidly falling background yields at very large invariant masses. It can thus be concluded that the present muon performance is close to optimum and satisfies the physics potential of the FCC-hh at high $p_{T}$. With the current detector design, the combination of the $\mathrm{e}^{+} \mathrm{e}^{-}$and $\mu^{+} \mu^{-}$channels allows for a $\mathrm{Z}^{\prime}$ discovery with a mass up to $m_{\mathrm{Z}^{\prime}}=42 \mathrm{TeV}$.

\subsubsection{Top squarks}

Supersymmetry is a theoretical framework that can address the hierarchy problem, allows for the unification of couplings at high energies and provides a natural dark matter candidate, often the lightest neutralino. The scalar partner of the top quark, known as the top squark or stop, can be produced through $\mathrm{pp} \rightarrow \tilde{\mathrm{t}} \tilde{\mathrm{t}}$, and detected via the $\tilde{\mathrm{t}} \rightarrow \mathrm{t}+\tilde{\chi}_{1}^{0}$ decay mode, where $\tilde{\mathrm{t}}_{1}$ is the stop's lightest mass eigenstate and $\tilde{\chi}_{1}^{0}$ is the lightest neutralino. Here the focus is on fully hadronic final states which have a striking signature: the presence of two on-shell top quarks, decaying to multiple jets and b-jets, and large missing transverse momentum $p_{T}^{\text {miss }}$. At the FCChh, the production rate of stop pairs is sizeable up to $m_{\tilde{t}} \approx 10 \mathrm{TeV}$. For such extreme kinematics, this search is very challenging, since highly boosted top quarks will be produced in the decay of massive stop quarks, leading to jets that exhibit three extremely collimated prongs. Resolving the sub-structure inside such "top" jets is key to reject the background from QCD jets and requires excellent granularities in the tracking detectors and the calorimeters. For example, the decay products of a 

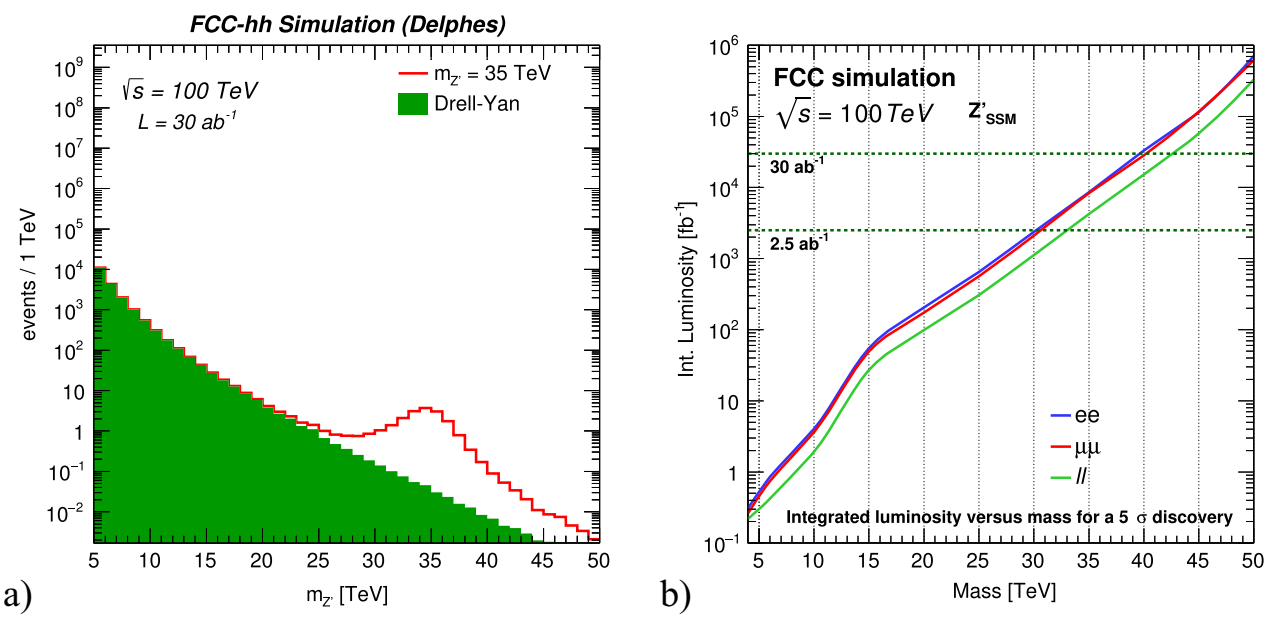

Fig. 7.24. (a) The di-muon invariant mass spectrum for a signal of $m_{\mathrm{Z}^{\prime}}=40 \mathrm{TeV}$ and background. The background is displayed with a solid histogram and the distribution of the signal model is shown by the solid red line. The expected yields are scaled to $30 \mathrm{ab}^{-1}$. (b) Integrated luminosity as a function of the $5 \sigma$ discovery reach for the $\mathrm{e}^{+} \mathrm{e}^{-}, \mu^{+} \mu^{-}$channels and their combination.

$5 \mathrm{TeV}$ top quark are separated by $\Delta R=\Delta \eta \times \Delta \phi \sim 0.05$. This number has to be compared to the angular resolution estimated at the first tracker pixel layer, given by $\sigma_{\eta \times \phi} \approx 0.005$. The ECAL (HCAL) design shown in Table 7.3 ensures a granularity in $\sigma_{\eta \times \phi} \approx 0.01(0.025)$ in the barrel. As a result, the present detector design should provide the necessary angular separation power to resolve such boosted signatures.

Events are selected by vetoing electrons or muons and requiring high missing transverse energy $\left(p_{T}^{\text {miss }}>2 \mathrm{TeV}\right)$. In addition, at least two high- $p_{T}$ jets $\left(p_{T}>1 \mathrm{TeV}\right)$ are required, of which at least one is b-tagged $\left(N_{\mathrm{b}} \geq 1\right)$, and at least one top-tagged $\left(N_{\mathrm{t}} \geq 1\right)$. A top-tagging multi-variate discriminant for QCD background discrimination based on jet substructure observables has been developed specifically for this search, by using a combination of track and calorimeter-based jet variables. It provides a working point with $\sim 5 \%$ mistag rate from $\mathrm{QCD}$ and $\mathrm{a} \sim 60 \%$ top identification efficiency. The dominant SM backgrounds with intrinsic $p_{T}^{\text {miss }}$ arise from $\mathrm{t} \overline{\mathrm{t}}$ and $\mathrm{t} \overline{\mathrm{t}} \mathrm{V}$ $(\mathrm{V}=\mathrm{W}, \mathrm{Z}, \mathrm{H})$ production. These backgrounds are reduced by selecting events with $\left|\Delta \phi\left(\mathrm{t}, p_{T}^{\mathrm{miss}}\right)\right|>0.5$. Their contributions are estimated in dedicated control regions extrapolated to the search regions with simulation. Contributions arising from EW (e.g. $V+$ jets), singlet quark, $t \bar{t} V V$ and $t \bar{t} t \bar{t}$ processes represent much smaller backgrounds and were estimated directly from simulation.

Events are divided into categories based on $N_{\mathrm{t}}$ and $N_{\mathrm{b}}$ and the shape of $p_{T}^{\text {miss }}$ is used as the discriminating variable. Figure 7.25 shows the expected signal and background contributions in the $N_{t} \geq 2$ and $N_{\mathrm{b}}=1$ category. A simultaneous fit to signal and control regions is used to determine the exclusion potential. Figure 7.25b shows that, with $30 \mathrm{ab}^{-1}$ of integrated luminosity, top squarks with mass up to $\sim 10 \mathrm{TeV}$ can be excluded if the neutralino mass is below $4 \mathrm{TeV}$. It was verified that this limit is robust against the choice of systematics scenario. A more detailed discussion of this analysis can be found in [16].

\subsubsection{Disappearing tracks}

The simple and minimal way to add a Dark Matter candidate to the SM consists of adding an SU(2) multiplet that contains a neutral state. In Supersymmetry, Higgsinos and winos represent a doublet and a triplet, respectively. If DM is the neutral 

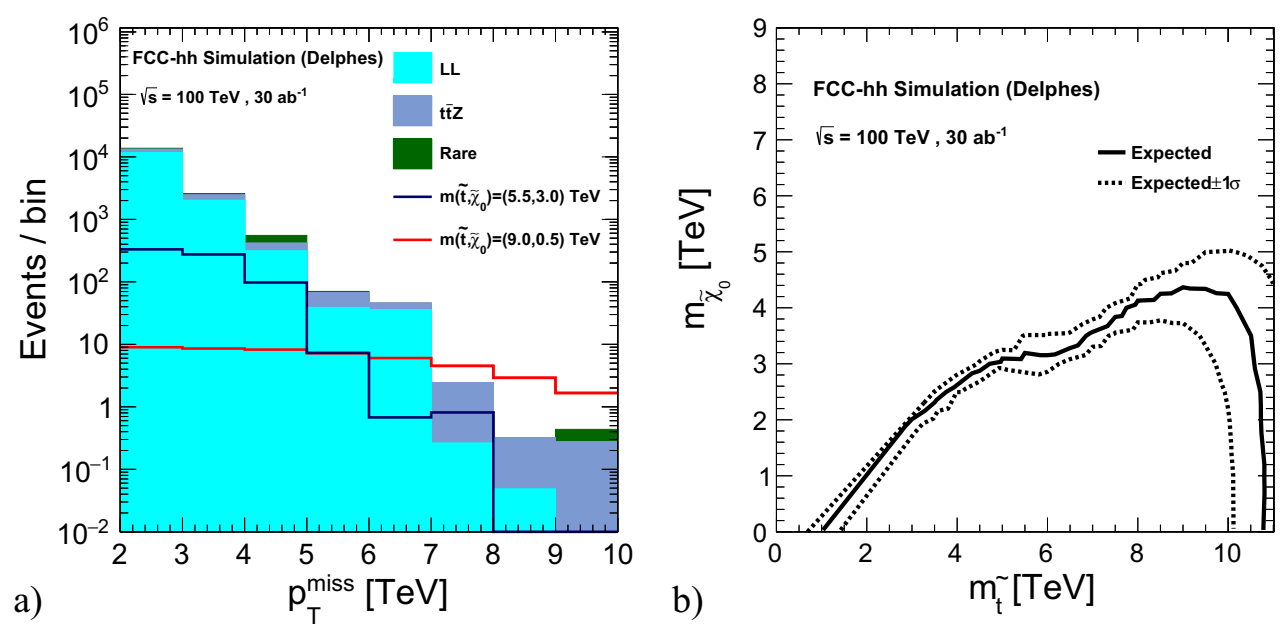

Fig. 7.25. (a) The $p_{T}^{\text {miss }}$ distribution in background and signal events with $N_{\mathrm{t}} \geq 2$ and $N_{\mathrm{b}}=1$. The background processes are displayed with solid histograms and the distribution of one signal model is shown by the solid red line. "LL" stands for "Lost Lepton" and collects all backgrounds where a lepton was not reconstructed due to inefficiency or limited detector acceptance. The expected yields are scaled to $30 \mathrm{ab}^{-1}$. (b) Exclusion potential for $30 \mathrm{ab}^{-1}$, and the $\pm 1 \sigma$ contours for the nominal systematic uncertainties.

wino (Higgsino) and was produced in thermal equilibrium, the upper limit on its mass is determined by the observed DM relic abundance: $3 \mathrm{TeV}$ for the wino LSP scenario and $1 \mathrm{TeV}$ for the Higgsino LSP scenario. The FCC-hh therefore provides a unique opportunity to probe wino and Higgsino DM.

When a pure wino/Higgsino is the LSP, the mass of the $\tilde{\chi}_{1}^{0}$ is highly degenerate with the lightest chargino $\left(\tilde{\chi}_{1}^{ \pm}\right)[376,377]$. Therefore, $\tilde{\chi}_{1}^{ \pm}$is a long-lived state with a lifetime $\tau_{\chi} \approx 0.2(0.023)$ ns for a $3 \mathrm{TeV}$ wino (1 TeV Higgsino) [378]. A $\tilde{\chi}_{1}^{ \pm}$decays into a $\tilde{\chi}_{1}^{0}$ and a soft charged pion. The neutralino does not interact with the detector and the pion is too soft to be reconstructed. Therefore, the long-lived chargino can be detected as a short-distance disappearing track with a typical length scale of $\mathcal{O}(1-10) \mathrm{cm}[379-381]$.

The backgrounds for this search can be categorised into two components. Physical backgrounds arise from charged particles (electrons or charged pions) scattered by the material in the inner tracker. These are mainly $\mathrm{W}(\rightarrow \ell \nu)+$ jets events, with large $E_{T}^{\text {miss }}$ and a high $p_{T}$ track from a $\mathrm{W}$ decay product. The normalisation of the physical background is derived by taking the background rate estimated at ATLAS, and rescaling it by the ratio of the amount of the material in the FCC tracker to that in the ATLAS inner tracker. The unphysical background consists of fake tracks; they arise from a random combination of hits that form a short track. The fake-track rate is evaluated from simulated minimum bias events by counting the number of fake tracks passing the quality requirements. The final estimate is obtained by scaling by a pile-up dependent fake rate probability. Good coverage of the volume within $r<10 \mathrm{~cm}$ is crucial for optimum fake-track rejection and thus for the success of this measurement.

Candidate events are required to contain at least one high $p_{T}$ jet, large $p_{T}^{\text {miss }}$ and no lepton (electron or muon) with $p_{T}$ above $10 \mathrm{GeV}$. The jet $p_{T}$ and $p_{T}^{\text {miss }}$ thresholds are determined by maximising the sensitivity at each $\tilde{\chi}_{1}^{ \pm}$mass point based on the signal acceptance and background rate. Additionally, at least one short track with $N_{\text {layer }}^{\text {hit }} \geq 5$ is required. 

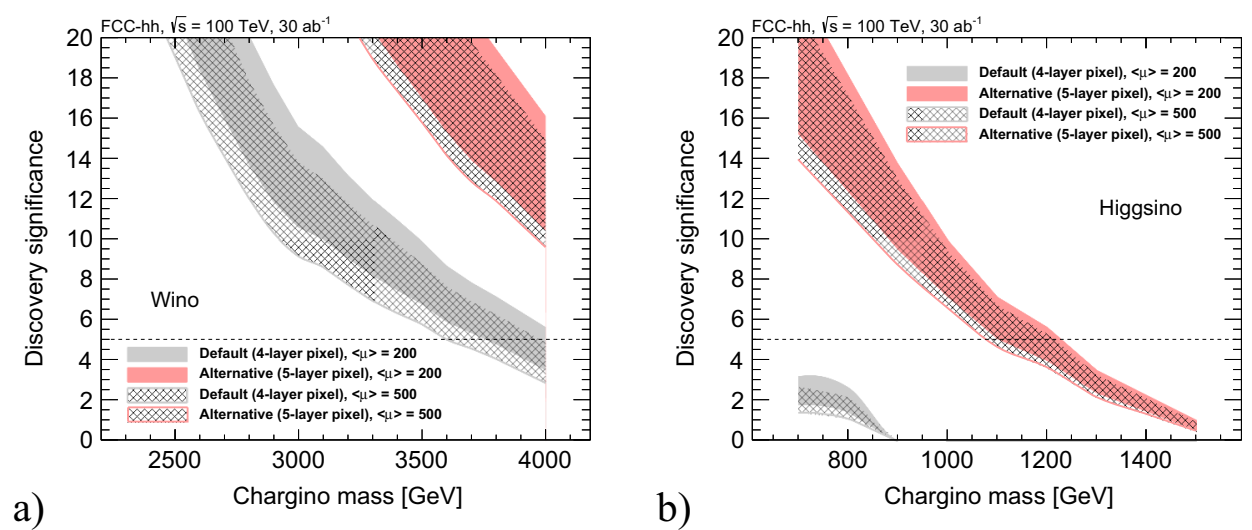

Fig. 7.26. (a) Wino, $\langle\mu\rangle=500$. (b) Higgsino, $\langle\mu\rangle=200$. Expected discovery significance at $30 \mathrm{ab}^{-1}$ with requirements of $N_{\text {layer }}^{\text {hit }} \geq 5$ with 200 (solid) or 500 (hatched) pile-up collisions and $|\eta|<1$ with the default (grey) and alternative (red) layouts. The band width corresponds to the difference of the two configurations of the soft QCD processes.

The expected significance obtained with the default tracker layout from Figure 7.11 is shown in Figure 7.26 as the grey band. The area represents an estimate of the uncertainty obtained from different QCD models of pile-up simulation. The impact of a layout with 5 pixels tracking layers instead of 4 is shown in red. Two pile-up conditions, $\langle\mu\rangle=200$ and 500 , have been considered to evaluate the faketracks background. A significance well above $5 \sigma$ for the $3 \mathrm{TeV}$ wino can be reached. However, a $5 \sigma$ significance for the $1 \mathrm{TeV}$ higgsino can only be reached using the alternative layout with 5 pixel layers. The sensitivity could be further improved by using the hit-timing information to suppress fake tracks or using $\mathrm{dE} / \mathrm{dx}$ information to identify the velocity of the disappearing track.

\subsection{Special purpose experiments: ions}

The physics opportunities of heavy ion collisions at the FCC-hh allow one to identify some general prerequisites for the detector design. To fully exploit the opportunities for physics with soft probes, one requires a detector with excellent charged-hadron identification to measure low- $p_{T}$ pions, kaons, protons and light nuclei, their abundance, spectra, flow and correlations, as well as low- $p_{T}$ charm and beauty mesons and baryons. Such identification capability could be provided by measurements of specific energy deposition in silicon trackers, time-of-flight, Čerenkov radiation, or a combination of these. Track reconstruction capability down to low $p_{T}$, ideally starting from few hundred $\mathrm{MeV} / \mathrm{c}$, is mandatory for all these measurements. To fully exploit the opportunities for physics with hard probes, the basic requirements should match those for the pp programme at the FCC, namely hadronic and electromagnetic large acceptance calorimeters with excellent energy resolution at high- $p_{T}$ and excellent detection capabilities for the leptonic decay products of hard processes. For saturation physics, one requires a detector with excellent forward coverage for charged particles, photons and jets, ideally up to $|\eta| \approx 6$.

Whether the general purpose pp detectors will be able to fulfil all these requirements or whether a dedicated detector should be considered is subject to further studies. The bandwidth required for pp collisions will definitely allow all $\mathrm{PbPb}$ collisions to be read into the high level trigger for detailed inspection. The timing detectors planned for pile-up rejection in high luminosity pp collisions can allow for significant 
Table 7.4. Key parameters defining the detector requirements for $\mathrm{PbPb}$ collisions.

\begin{tabular}{|l|c|c|c|c|c|}
\hline Parameter & Unit & LHC & HL-LHC & HE-LHC & FCC-hh \\
\hline$E_{\text {cm per nucleon }}$ & $\mathrm{TeV}$ & 5.5 & 5.5 & 10.6 & 39.4 \\
Circumference & $\mathrm{km}$ & 26.7 & 26.7 & 26.7 & 97.8 \\
Peak $\mathcal{L}$ & $10^{27} \mathrm{~cm}^{-2} \mathrm{~s}^{-1}$ & 1 & 6.5 & $15-50$ & 320 \\
Bunch spacing & $\mathrm{ns}$ & 100 & 50 & 50 & 50 \\
Number of bunches & & 1232 & 1232 & 1232 & 5400 \\
Goal $\int \mathcal{L}$ & $\mathrm{nb}^{-1}$ & 1 & 10 & $10 /$ month & $110 /$ month \\
\hline$\sigma_{\text {inel }}$ & $\mathrm{b}$ & 7.8 & 7.8 & 8 & 9 \\
$\sigma_{\text {tot }}$ & $\mathrm{b}$ & 515 & 515 & 530 & 597 \\
BC rate & $\mathrm{MHz}$ & 13.8 & 13.8 & 13.8 & 16.6 \\
Peak PbPb collision rate & $\mathrm{kHz}$ & 7.8 & 50.7 & 400 & 2880 \\
\hline RMS luminous region $\sigma_{z}$ & $\mathrm{~mm}$ & $50-70$ & $50-70$ & $35-70$ & $30-60$ \\
$\mathrm{~d} N_{\text {ch }} /\left.\mathrm{d} \eta\right|_{\eta=0}$ & & 500 & 500 & 610 & 900 \\
Charged tracks per collision $N_{\mathrm{ch}}$ & & 5800 & 5800 & 7500 & 12500 \\
Rate of charged tracks & $\mathrm{GHz}$ & 0.05 & 0.3 & 3 & 36 \\
$\left\langle p_{T}\right\rangle$ & $\mathrm{GeV} / \mathrm{c}$ & 0.47 & 0.47 & 0.49 & 0.55 \\
Bending radius for $\left\langle p_{T}\right\rangle$ at $B=4 \mathrm{~T}$ & $\mathrm{~cm}$ & 39 & 39 & 41 & 46 \\
\hline \multicolumn{4}{|r|}{}
\end{tabular}

particle identification performance for $\mathrm{PbPb}$ collisions using time-of-flight measurements. Excellent $p_{T}$ resolution down to low momenta is also an important goal for the general purpose detectors, the reduced demand on radiation hardness might however allow for different, highly optimised detector technologies. To allow acceptance of very low momentum particles, the possibility of operating the magnet system at a lower magnetic field of around $1 \mathrm{~T}$ should be considered. Table 7.4 lists a few key parameters of heavy ion collisions that define some of the detector requirements.

\subsection{Special purpose experiments: Lepton-Hadron}

The FCC hadron beams provide a unique opportunity to create luminous electronproton and electron-ion scattering at the energy frontier. The default configuration considered for FCC-eh combines the proton beam of energy $E_{\mathrm{p}}=50 \mathrm{TeV}$ with an electron beam of $E_{\mathrm{e}}=60 \mathrm{GeV}$, generated by an energy recovery linac (ERL). In this design, ep operates concurrently with pp: the FCC in its hadronic configuration becomes a twin pp and ep collider with greatly enhanced physics reach.

The FCC-eh reaches a maximum value of negative 4-momentum transfer squared, $Q^{2}$, of $s=4 E_{\mathrm{e}} E_{\mathrm{p}}=1.2 \times 10^{7} \mathrm{GeV}^{2}$. The default cms energy thus is $\sqrt{s}=3.46 \mathrm{TeV}$, a tenfold increase compared to HERA and threefold compared to the LHeC. The corresponding coverage for the parton's momentum fraction of the proton, Bjorken $x$, for $Q^{2}$ between $1 \mathrm{GeV}^{2}$ and $s$, ranges from $x_{\text {min }}=1 / s=8 \times 10^{-8}$ to 1 . Although this is a very asymmetric e-p energy configuration, the inclusive scattering kinematics can be well reconstructed in the complete $x, Q^{2}$ plane, owing to the mostly redundant determination of $x, Q^{2}$ from the angles $\left(\theta_{\mathrm{e}}, \theta_{h}\right)$ and energies $\left(E_{\mathrm{e}}^{\prime}, E_{h}\right)$ of the scattered electron and the hadronic final state, respectively. The asymmetry leads to very strong variations of $E_{\mathrm{e}}^{\prime}$ and $E_{h}$ in different regions of the detector as is illustrated in Figure 7.27. This has a large influence on the choice of detector technology and determines the geometric placements.

A design concept for the FCC-eh detector is presented in Figure 7.28. It is a classic $4 \pi$ acceptance collider detector using a solenoid magnetic field of $3.5 \mathrm{~T}$. Its outer dimensions of $6 \mathrm{~m}$ radius and $19 \mathrm{~m}$ length are comparable to those of the CMS 


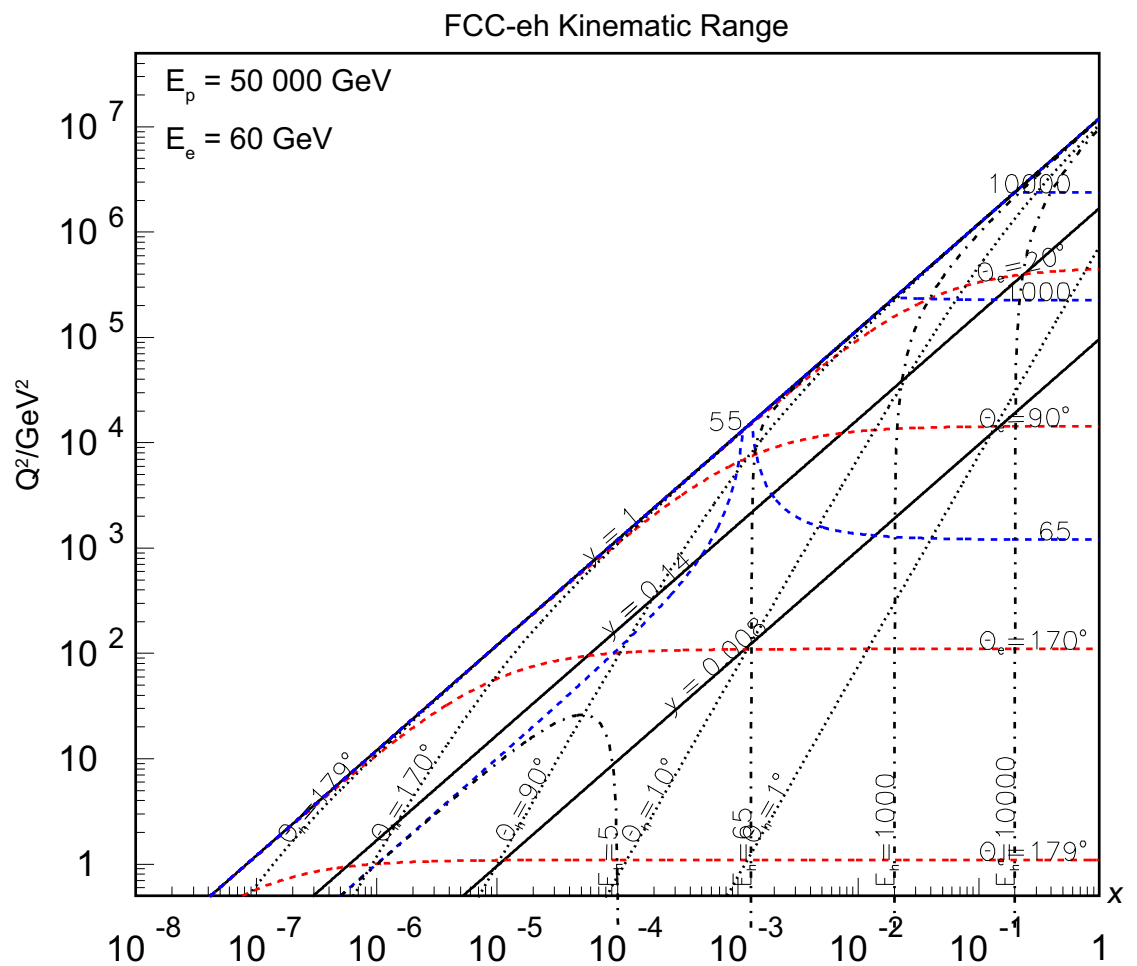

Fig. 7.27. Kinematics of the FCC-eh for $E_{\mathrm{p}}=50 \mathrm{TeV}$ and $E_{\mathrm{e}}=60 \mathrm{GeV}$. Blue dashed lines: constant scattered electron energy, which for $Q^{2}$ below $1000 \mathrm{GeV}^{2}$ never exceeds $65 \mathrm{GeV}$. Red dashed lines: constant electron polar angle. One observes that the low $x$ region is very accessible with a detector acceptance down to one degree for backward electrons. Black dashed-dotted lines: constant hadronic final state energy. At large Bjorken $x$, energies of up to tens of $\mathrm{TeV}$ are scattered in the forward detector region; black dotted lines: constant polar angle of the hadronic final state.

detector. The concept has been derived from the extended study of the LHeC detector, described in [28]. As in the LHeC, head-on ep collisions are essential and this requires that a dipole with a field of the order of $0.1 \mathrm{~T}$ is integrated in the FCC-eh detector in order to bend the electron beam in and out of the interaction region. Compared to the LHC, the increased proton beam energy leads to a more demanding forward region into which electrons and hadrons can be scattered with energies of tens of $\mathrm{TeV}$. The radial and forward calorimeter extensions are thus scaled by factors of $\ln (50 / 7) \simeq 2$ compared to the LHeC. High precision Higgs physics and BSM searches introduce specific constraints on the design and heavy neutrinos and dark matter demand high resolution track impact parameters, of the order of $5 \mu \mathrm{m}$, optimum acceptance and resolution for the hadronic final state as well as missing energy. Salient features of measurements of the ep processes are the estimated pile-up of 1 , compared to $\sim 1000$ in $\mathrm{pp}$ and the clear distinction between neutral and charged current scattering as opposed to VBF in pp. In the same way, the FCC-eh radiation level is much more tolerable than that for hadron-hadron collisions. Characteristic numbers for experimentation with FCC-eh are summarised in Table 7.5.

The backward region (that of the electron beam) is guarded by the much lower electron beam energy, and thus quite different from the forward region, as can be 


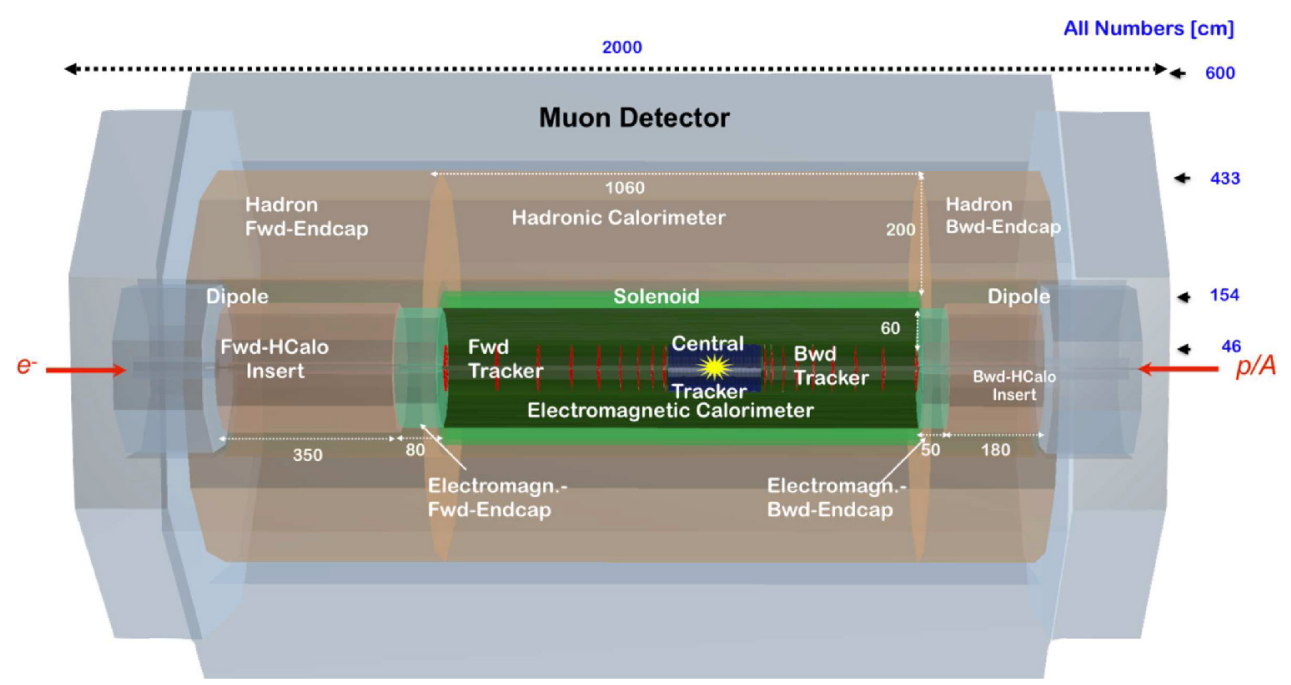

Fig. 7.28. A sketch of the FCC-eh detector layout with a single solenoid and an extended muon detector. Novel technology designs for combined tracking and calorimetry allow muon detection without dedicated large scale chambers. The size of the detector is $19.3 \mathrm{~m}$ in length and $6 \mathrm{~m}$ radius which is comparable to the LHC CMS detector of $21 \times 7.5 \mathrm{~m}^{2}$. There are different versions for the barrel calorimeters based on crystal or liquid argon calorimeter technology.

Table 7.5. Parameters and cross-sections, for deep inelastic ep and eA scattering, single top and Higgs production.

\begin{tabular}{|l|c|c|c|c|}
\hline Parameters & Unit & LHeC & HE-LHeC & FCC-eh \\
\hline$E_{\mathrm{p}}$ & $\mathrm{TeV}$ & 7 & 13.5 & 50 \\
$\sqrt{s}$ for $E_{\mathrm{e}}=60 \mathrm{GeV}$ & $\mathrm{TeV}$ & 1.3 & 1.7 & 3.5 \\
Peak $\mathcal{L}$ & $10^{33} \mathrm{~cm}^{-2} \mathrm{~s}^{-1}$ & 8 & 12 & 15 \\
Bunch spacing & $\mathrm{ns}$ & 25 & 25 & 25 \\
Goal $\mathcal{L}$ electron-proton & $\mathrm{ab}^{-1}$ & 1 & 2 & 2 \\
Goal $\mathcal{L}$ electron-ion & $\mathrm{fb}^{-1}$ & 10 & 20 & 20 \\
\hline Events per bunch crossing (pile-up) & & 0.1 & 0.2 & 1 \\
$1 \mathrm{MeV}-$ neq fluence at $r=2.5 \mathrm{~cm}$ & $10^{15} \mathrm{~cm}^{-2}$ & 1 & 2 & 5 \\
\hline DIS ep: $\sigma_{\mathrm{e} \rightarrow \mathrm{e} X} Q^{2} \geq 1 \mathrm{GeV}^{2}$ & $\mu \mathrm{b}$ & 3.0 & 3.4 & 4.6 \\
DIS $\mathrm{Pb}: \sigma_{\mathrm{e} N \rightarrow \mathrm{e} X} /\left[\right.$ nucleon,Pb] $Q^{2} \geq 1 \mathrm{GeV}^{2}$ & $\mu \mathrm{b}$ & 1.8 & 2.1 & 2.7 \\
Top: $\sigma(\mathrm{ep} \rightarrow \nu \overline{\mathrm{t} X}), p_{T}^{j}>10 \mathrm{GeV},|\eta|<6$ & $\mathrm{pb}$ & 0.9 & 2.4 & 11 \\
Higgs: $\sigma(\mathrm{ep} \rightarrow \nu \mathrm{H} X), P=-0.8$ & $\mathrm{pb}$ & 0.2 & 0.4 & 1 \\
\hline
\end{tabular}

deduced from Figure 7.27. The overall detector length is determined by the requirements to observe both very low $Q^{2}$ and large $x$ events in the main detector.

As at HERA, an ep collider detector requires a set of backward photon and electron taggers, to tag the low $Q^{2}$ photoproduction background and to measure the luminosity through Bethe Heitler scattering, ep $\rightarrow$ ep $\gamma$. In the forward direction, proton and neutron taggers are necessary to measure deep inelastic diffraction and to study the neutron structure. Such taggers were studied in detail for the LHeC [28] but, given its early stage of development, not for the FCC-eh.

The tracking detector, shown in Figure 7.29, has been optimised for compactness, high precision and large acceptance vertexing and also, in conjunction with the 


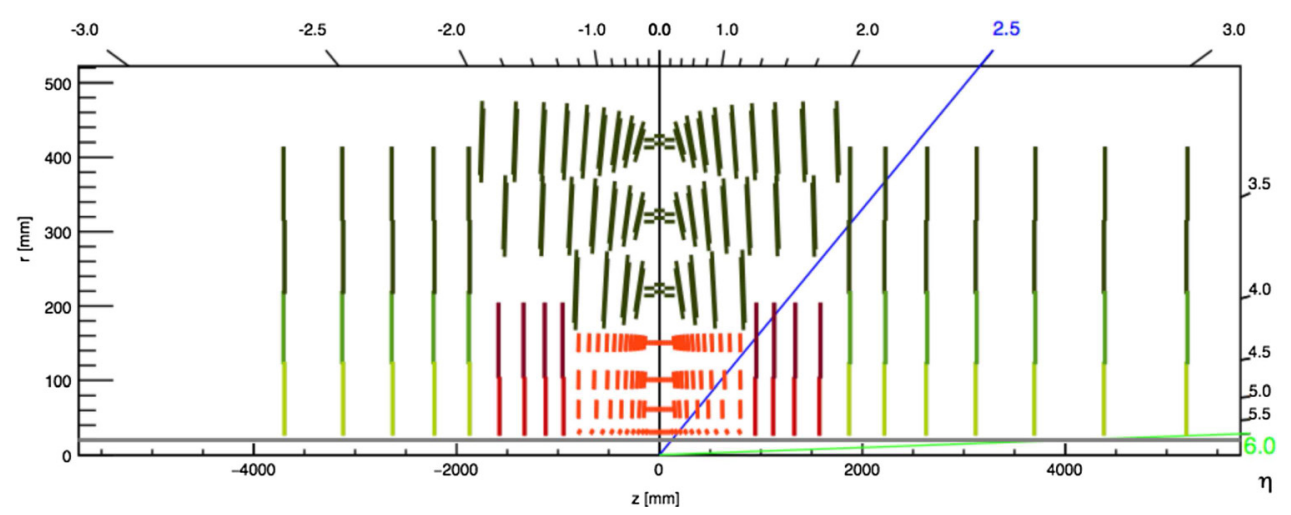

Fig. 7.29. $r-z$ view of the all-silicon FCC-eh tracking detector; left: direction of the electron beam (backward), right: direction of the hadron beam (forward). The radius extension is $0.5 \mathrm{~m}$ to facilitate a dipole radius that is not too large and to limit the area of silicon required.

Table 7.6. Parameters of the FCC-eh silicon tracking detector shown in Figure 7.29.

\begin{tabular}{|c|c|c|c|c|c|c|c|}
\hline Tracker & \multicolumn{2}{|c|}{ Inner Barrel } & \multirow{2}{*}{$\begin{array}{c}\text { ECAP Barrel } \\
\text { Pix }\end{array}$} & \multicolumn{2}{|c|}{ Fwd Tracker } & \multicolumn{2}{|c|}{ Bwd Tracker } \\
\hline Type & Pix & Strix & & Pix & Strix & Pix & Strix \\
\hline \#Layers/Wheels & $4_{\text {inner }}$ & $3_{\text {outer }}$ & 4 & \multicolumn{2}{|r|}{7} & \multicolumn{2}{|r|}{5} \\
\hline \#Mods/Ring/Wheel & $\max 12^{\text {flat }}$ & $\max 24^{\text {tilt }}$ & 2 & $2_{\text {inner }}$ & $2_{\text {outer }}$ & $2_{\text {inner }}$ & $2_{\text {outer }}$ \\
\hline \#Modules & \multicolumn{3}{|c|}{5538} & \multicolumn{2}{|c|}{1512} & \multicolumn{2}{|c|}{1080} \\
\hline$\eta_{\max / \min }$ & \multicolumn{2}{|c|}{ \pm 1.5} & \pm 2.5 & \multicolumn{2}{|c|}{$2.5-5.6$} & \multicolumn{2}{|c|}{$-2.5--5.4$} \\
\hline$\sigma^{r-\phi}$ & $5-7.5$ & $7-9.5$ & $5-7.5$ & $5-7.5$ & $7-9.5$ & $5-7.5$ & $7-9.5$ \\
\hline$\sigma^{z}$ & 15 & 15 & 15 & 15 & 30 & 15 & 30 \\
\hline$X_{0} / \Lambda_{I}$ & \multicolumn{3}{|c|}{$9.51 / 2.95$} & \multicolumn{2}{|c|}{$5.31 / 1.65$} & \multicolumn{2}{|c|}{$3.79 / 1.18$} \\
\hline Total silicon area $\left(\mathrm{m}^{2}\right)$ & \multicolumn{3}{|c|}{15.8} & \multicolumn{2}{|c|}{7.7} & \multicolumn{2}{|c|}{5.5} \\
\hline
\end{tabular}

Notes. High resolution requires the use of precise pixel and macro-pixel (strixel) silicon detectors. The overall area of the compact design is $29 \mathrm{~m}^{2}$.

subsequent calorimeters, to permit particle flow measurements. Redundancy, optimum pattern recognition and minimisation of passive material lead to a geometrical concept, which combines parallel inner barrel, perpendicular end cap ring and inclined wafer arrangements in between. Characteristic parameters of the FCC-eh tracker design are summarised in Table 7.6. Given the dynamic development of silicon wafer and mechanical support technology a design made at a later stage in the project will certainly differ from that presented here.

The FCC-eh detector requires high precision calorimetry which is particularly demanding in the forward plug region, where dense hadronic and electromagnetic energy jets of up to multi-TeV energies ought to be reconstructed. This dictates the various choices of technology indicated in the calorimeter summary table, Table 7.7. The resolutions quoted were obtained in initial simulations of all calorimeters. Future studies will combine physics requirements, such as from Higgs final state measurements, with technology choices, channel segmentation and the reduction of passive material. Light weight designs are being studied for the dipole and solenoid magnets which are currently placed in a common volume in front of the hadronic calorimeters. This design of the dipole is dictated by the bend but for the solenoid it is driven by cost. Liquid argon technology can be considered for the barrel electromagnetic calorimeters, as done for the $\mathrm{LHeC}$ reference detector [28]. It is worth emphasising 
Table 7.7. Summary of electromagnetic and hadronic calorimeters of the FCC-eh detector showing technology, rapidity coverage, resolution parameters $A$ and $B$ from fits, $\sigma / E \simeq$ $A / \sqrt{E} \oplus B$, energy flow resolution, interaction length $\Lambda$ and radiation length $X_{0}$ and volumes.

\begin{tabular}{|l|c|c|c|c|c|c|}
\hline $\begin{array}{l}\text { Calorimeters } \\
\text { Location }\end{array}$ & $\begin{array}{c}\text { FHC[SiW] } \\
\text { Plug }\end{array}$ & $\begin{array}{c}\text { FEC }[\mathbf{S i W}] \\
\text { Plug }\end{array}$ & $\begin{array}{c}\text { EMC[SciPb] } \\
\text { Barrel }\end{array}$ & $\begin{array}{c}\text { HC[SciFe] } \\
\text { Barrel/Endcap }\end{array}$ & $\begin{array}{c}\text { BEC[SiPb] } \\
\text { Plug }\end{array}$ & $\begin{array}{c}\text { BHC[SiCu] } \\
\text { Plug }\end{array}$ \\
\hline$\eta_{\text {max } / \text { min }}$ & 6.0 & 5.6 & $3.0 /-2.7$ & $2.5 /-2.2$ & -5.3 & -5.6 \\
\hline A, B in \% & $57.8,2.2$ & $18.6,0.3$ & $17.2,1$ & $35.9,2$ & $14.3,0.7$ & $47.8,3.3$ \\
\hline E-Flow & $\sigma_{E_{\text {jet }}} / E_{\text {jet }}=0.03$ (at lower energies $25 \% / \sqrt{E} ;$ sampling $\left.\sim 55 ; \sigma_{\text {jet }} \sim 3 \%\right)$ \\
\hline$\Lambda_{I} / X_{0}$ & $\Lambda_{I} \geq 12$ & $X_{0} \geq 28$ & $X_{0} \geq 28$ & $\Lambda_{I} \geq 12$ & $X_{0} \geq 25$ & $\Lambda_{I} \geq 10$ \\
\hline Volume $\left(\mathrm{m}^{3}\right)$ & 13.2 & 3.1 & 28.8 & 407 & 1.98 & 7.0 \\
\hline
\end{tabular}

that the ep configuration has the appealing feature of redundant reconstruction of the secondary energies and scattering angles which leads to their precise cross calibration [382], especially in the ultra-high luminosity environment for the LHeC and the FCC-eh. New and updated ep detector concepts, as in FCC-eh, have been designed for the LHeC and the HE-LHeC [383].

\section{Safety}

\subsection{Safety policy and regulatory framework}

The concept study aims at demonstrating that hazard and risk control for a future collider is possible with best practices and industrial standards, which are complemented, where necessary, with techniques specific for a particle accelerator facility. The approach is introduced below by first presenting CERN's legal context and then the hazard and risk management in place for the existing accelerator complex. The subsequent sections show how occupational health and safety topics are addressed, which are specific for a particle collider, and how the risks associated with ionising radiation are managed. For the conceptual design, a two-stage process has been chosen: first, a hazard register lists the safety hazards that will be present at the facility and identifies those, for which standard best practices can be applied to control the associated risks. Second, a detailed analysis will be carried out, for the remaining safety risks, to assess the risk levels and to identify mitigation measures.

\subsubsection{Legal context of CERN}

CERN's status as international organisation requires that it defines a safety policy in a pro-active and consensus-based process with the host states (see Art. II 2 de l'accord de statut de 1973 entre le CERN et la France [384]). This approach is applicable for non-standard installations like the accelerators, the experiments and the technical infrastructure needed to operate these facilities. Where standard infrastructure dominates (e.g. offices, car parks and workshops), CERN ensures uniformity in the safety conditions across its sites, taking into account the laws and regulations of the host states and EU regulations and directives. Where uniformity cannot be achieved or where compliance is not required to ensure the proper functioning of the organisation, a dedicated risk management process is applied, including the planning, implementation and verification of risk mitigation measures. 


\subsubsection{Hazard register and safety performance based design}

A systematic collection of safety hazards associated with the construction and operation of the accelerator complex is the starting point of the safety assessment. Hazard registers are an established technique in industry. To compile the register, a processcentred approach has been used [385]. In a first step, a systematic description of the processes during the life cycle of the accelerator facility has been established, based on the Project Breakdown Structure (PBS). Each process is characterised by activities, equipment and substances used or released. Hazards may emerge from these activities, equipments and substances at different locations.

As an example, the process of providing electrical power to accelerator magnets is active during commissioning and operation of the accelerator. It relies on transformers and power converters (equipment) at the surface and in underground locations. This equipment is at the origin of electrical hazards, noise and potential environmental pollution in case of dispersion of insulating fluids.

It is assumed that the relevant risks associated with the hazards will be mitigated by standard best practices, implementing compliance with laws and regulations of the host states, EC regulations and directives, international regulations, standards and directives and recommendations from technical or prevention organisms.

Where standard best practices cannot be applied or would affect the proper operation of the organisation, a performance-based design approach is used [386]. It is proposed to use a standard method like "Failure Mode, Effects and Criticality Analysis" (FMECA) [387] to identify those risks which remain unacceptable. From this starting point, the performance based approach is used. In this process, essential safety objectives, such as preservation of human lives or prevention of environmental damage, are defined. The safety performance of design choices is evaluated for different incident scenarios. If the objectives are met, the design can be retained as mitigation measure.

\subsection{Occupational health and safety}

The study has initiated a methodological approach in tackling occupational health and safety aspects. The hazard registry classifies relevant sources of risks to permit identifying those, which can be addressed by standard approaches and those, for which project-specific assessments need to be performed, followed by a definition of mitigation measures against residual risks. This preliminary activity has identified that two main hazards are present in underground areas: fire and oxygen deficiency $(\mathrm{ODH})$. The latter is a residual risk that emerges after applying "safety-by-design" to cryogenic hazards, such as the avoidance of cold surfaces and functional measures such as combined vacuum and superinsulation blankets. This report, focusing on the feasibility and conceptual elements of a future particle accelerator research infrastructure does not allow the presentation of the technical risk management files in a comprehensive manner. It therefore only focuses on the presentation of the approach for the two main hazards (fire and oxygen deficiency). The results of the studies [388-391] are summarised in the following sections. The agreed safety objectives for these two hazards are listed in Table 8.1.

To create safe zones along the entire perimeter of the particle collider it is recommended that smoke and fire resistant compartment walls should be installed every two accelerator lattice cells (every $440 \mathrm{~m}$ ). Under normal conditions, the compartment doors are open. Each compartment receives fresh air from the underfloor air duct; the used air is evacuated along the tunnel. Smoke and oxygen deficiency hazard $(\mathrm{ODH})$ detectors trigger the closure of the doors. An extraction duct traverses 
Table 8.1. Safety objectives in the design-oriented study.

\begin{tabular}{|c|c|c|l|}
\hline Life safety & $\begin{array}{c}\text { Environmental } \\
\text { Protection }\end{array}$ & $\begin{array}{c}\text { Property } \\
\text { Protection }\end{array}$ & $\begin{array}{l}\text { Continuity of } \\
\text { Operation }\end{array}$ \\
\hline $\begin{array}{c}\text { Safety of } \\
\text { authorised } \\
\text { occupants (O1) }\end{array}$ & $\begin{array}{c}\text { Limited release of } \\
\text { pollutants to air } \\
\text { (O4) }\end{array}$ & $\begin{array}{c}\text { Continuity of } \\
\text { essential services } \\
(\text { O6) }\end{array}$ & $\begin{array}{l}\text { Limited down- } \\
\text { time (O9) }\end{array}$ \\
\hline $\begin{array}{c}\text { Safe evacuation } \\
\text { or staging of } \\
\text { injured occupants } \\
\text { (O2) }\end{array}$ & $\begin{array}{c}\text { Limited release of } \\
\text { pollutants to } \\
\text { water (O5) }\end{array}$ & $\begin{array}{c}\text { Incident must not } \\
\text { cause further } \\
\text { incidents (O7) }\end{array}$ & \\
\hline $\begin{array}{c}\text { Safe intervention } \\
\text { of rescue teams } \\
\text { (O3) }\end{array}$ & & $\begin{array}{c}\text { Limited property } \\
\text { loss (O8) }\end{array}$ & \\
\hline
\end{tabular}

Table 8.2. Fire scenarios in the design-oriented safety study.

\begin{tabular}{|l|l|l|}
\hline Scenario & Description & Ignition source \\
\hline Fire 1 & Cable tray fire & Electrical fire \\
\hline Fire 2 & Cable drum fire & Hot work \\
\hline Fire 3 & Transport vehicle fire & Battery malfunction \\
\hline
\end{tabular}

all compartments and can extract smoke or helium. The fresh air admission and extraction for each compartment can be controlled individually.

\subsubsection{Fire hazard}

The most critical phases for a fire hazard are (1) the operation with beam, (2) a long shutdown and (3) a technical stop. During operation, all electrical systems are powered and are potential ignition sources. During other periods, personnel who may inadvertently cause a fire are present, e.g. during welding. Table 8.2 shows the three fire scenarios studied.

The fire compartment size is sufficiently small to ensure fire fighter safety during an intervention, in conjunction with secure communications and structural stability of the tunnel. The size has been chosen as a trade-off between keeping evacuation times low, to provide sufficient margins for the intervention teams, to limit the damage of the equipment and to keep cost at reasonable levels. Smoke would still spread in at least one neighbouring compartment and might affect the equipment there. Worstcase scenarios for incidents with equipment loss point to a downtime of about one year. This compartment size has also been adopted by another, recently commissioned accelerator project, the European XFEL. It is based on a safety concept that has been developed by an independent underground engineering society, which has been accepted by the relevant authorities [392] as part of the authorisation to construct the facility. The FCC study gained valuable insight from this project in the framework of a multi-year collaboration with the project designers.

Life safety, safety of occupants and rescue teams were quantified by fractional effective dose (FED), a measure of the harm from toxic fire products as a function of temperature conditions, and visibility through the developing smoke. These parameters were estimated with the industry-standard computational fluid dynamics (CFD) program for fire and smoke propagation, Fire Dynamics Simulator 6.5 [393] from the National Institute of Science and Technology. The studies revealed that uninjured 
Table 8.3. Cryogenic accident scenarios in the design-oriented safety study for FCC-hh.

\begin{tabular}{|l|l|c|}
\hline Scenario & Description & Helium mass flow \\
\hline Cryo 1 & Occupants $5 \mathrm{~m}$ from release point & $1 \mathrm{~kg} / \mathrm{s}$ \\
\hline Cryo 2 & $\begin{array}{l}\text { Occupants } 5 \mathrm{~m} \text { from release point, } \\
\text { emergency extraction on }\end{array}$ & $1 \mathrm{~kg} / \mathrm{s}$ \\
\hline Cryo 3 & Occupants $200 \mathrm{~m}$ from release point & $1 \mathrm{~kg} / \mathrm{s}$ \\
\hline Cryo 4 & Occupants $150 \mathrm{~m}$ from release point & $0.3 \mathrm{~kg} / \mathrm{s}$ \\
\hline Cryo 5 & Occupants $5 \mathrm{~m}$ from release point & $0.1 \mathrm{~kg} / \mathrm{s}$ \\
\hline Cryo 6 & magnets powered, no occupants & $32 \mathrm{~kg} / \mathrm{s}$ \\
\hline
\end{tabular}

occupants can evacuate the compartment concerned in all scenarios. A smoke and fire detection system with a response time of under two minutes is needed and it musst be combined with an autonomous fire extinguishing system with an intervention time of less than $15 \mathrm{~min}$, to ensure also the safety of injured persons with restricted mobility. Standby rescue teams in the vicinity of the access sites, which can be established with the assistance of host state emergency services, also in remote locations, are a viable approach to establish a working intervention programme. Fire fighting and rescue equipment will be kept available underground in a ready-to-use state. The latter approach will already be put in place for the HL-LHC upgrade project.

Protection of the environment calls for a careful selection of fire-fighting agents (e.g. water, foam) which will be done at a later, detailed design stage. The system configuration will help keeping the release of smoke, chemical and radioactive contaminants to quantities as low as reasonably possible.

Main conclusions. The proposed safety concept fulfils the

- life safety objective for physically fit occupants and the safety of rescue forces in all scenarios,

- life safety objective for injured occupants with an early fire detection and early intervention system,

- continuity of operation goal by limiting the high impact downtimes to the unlikely worst case scenarios and

- environmental impact management goal with standard, best-practice measures.

\subsubsection{Oxygen deficiency}

An oxygen deficiency hazard arises from the release of asphyxiating cryogenic liquids $\left(\mathrm{He}, \mathrm{Ar}, \mathrm{N}_{2}\right.$ ) in closed environments, where they may displace oxygen during a sudden evaporation of the fluids. Commissioning and maintenance are the most critical phases concerning this hazard because of the presence of personnel in the underground areas. Six accidental and uncontrolled helium release scenarios were identified and analysed (see Tab. 8.3).

A qualitative consequence analysis of the scenarios has been carried out with simplified models [391], which is adequate for a conceptual study. The impact of the various scenarios has been compared to the safety objectives (see Tab. 8.1). The results are summarised in Table 8.4.

Table 8.4 shows that some safety objectives cannot be met upfront with the current concept. However, standard organisational measures, as already implemented today in the LHC [394], such as further access restrictions under particular conditions (e.g. operation when accidental helium release with a mass flow of above $1 \mathrm{~kg} / \mathrm{s}$ is 
Table 8.4. Preliminary results of the ongoing analysis of cryogenic accident scenarios in FCC-hh as an example of the approach.

\begin{tabular}{|c|c|c|c|l|l|l|l|l|c|c|}
\hline & \multicolumn{2}{|l|}{ Occupational health \& safety } & \multicolumn{2}{|l|}{ Environment } & Property protection & Continuity \\
\hline Objectives & O1 & O2 & O3 & O4 & O5 & O6 & O7 & O8 & O9 \\
\hline Cryo 1 & not met & not met & $\begin{array}{l}\text { under } \\
\text { study }\end{array}$ & met & met & met & met & met & met \\
\hline Cryo 2 & not met & not met & $\begin{array}{l}\text { met with } \\
\text { measures }\end{array}$ & met & met & met & met & met & met \\
\hline Cryo 3 & met & not met & $\begin{array}{l}\text { under } \\
\text { study }\end{array}$ & met & met & met & met & met & met \\
\hline Cryo 4 & understudy & understudy & met & met & met & met & met & met & met \\
\hline Cryo 5 & met & OK & $\begin{array}{l}\text { under } \\
\text { study }\end{array}$ & met & met & met & met & met & met \\
\hline Cryo 6 & not met & not met & not met & met & met & met & not met & not met & not met \\
\hline
\end{tabular}

Notes. The safety objectives indicated correspond to those in Table 8.1; the scenarios correspond to those indicated in Table 8.3. A comprehensive assessment needs to be performed for a specific particle collider scenario in a subsequent technical design phase.

possible) would also permit mitigating those situations. Further detailed assessments for other objectives, with definitive mitigation proposals, can only be performed during a technical design phase for a specific particle collider scenario.

Main conclusions. [395]:

- The safety objectives that are not fulfilled in this preliminary analysis can be mitigated by standard organisational measures; the concept of the underground facility is therefore acceptable at this stage.

- An exhaustive assessment is mandatory during a technical design phase, for a specific particle collider scenario.

\subsection{Radiation protection}

For the mitigation of risks associated with ionising radiation, the standard prescriptive methods of the existing CERN radiation protection rules and procedures have been used. Risks resulting from ionising radiation are analysed from a very early design phase, to develop mitigation approaches throughout the entire design process. Design constraints will ensure that the doses received by personnel working on the sites as well as the public will remain below regulatory limits [396,397] under all operation conditions. A reliable radiation monitoring system coupled with an effective early warning and emergency stop system will therefore be an essential part of the system implementing risk control measures.

The FCC-hh will be comparable in terms of radiological hazards to LHC and other high-energy accelerator facilities. These are reliable sources of experience to take into account when planning the radiation protection measures during the design phase. The main differences for FCC-hh are the higher beam energy and luminosity which lead to higher activation levels in some sections of the accelerator and experiments.

Radiation protection is concerned with two aspects: protection of personnel operating and maintaining the installations and the potential radiological environmental impact. The second topic is addressed in Section 10.2.1. To enable the assessment of the potential radiological risks to the personnel working on the sites, the radiological hazards are classified by their sources: (1) particle beam operation, (2) activated solids, liquids and gases. 


\subsubsection{Particle beam operation}

Radiation hazards from high energy particle beams arise from their interaction with matter and other particles. Shielding protects personnel by absorbing the primary radiation and the subsequently generated stray radiation. An access control system prevents people from accessing hazardous areas. An interlock system prevents beam operation in the case of an unauthorised access. An emergency stop system ensures that the operation with beam is terminated quickly and in a controlled way if needed. Areas accessible during beam operation will be designated as non-radiation areas to avoid the need for specific restrictions for radiation protection reasons.

Depending on the position in the accelerator, about $15 \mathrm{~m}$ of rock or concrete will sufficiently shield from the radiation resulting from a total beam loss. Chicanes will effectively reduce radiation streaming through feed-throughs and passages. Access to the accelerator tunnel and the experimental caverns will not be permitted during operation.

Underground facilities which remain accessible during beam operation will be located on the inside of the accelerator ring, to avoid exposure to forward stray radiation such as muons which penetrate through extended distances of soil and concrete.

The shafts above the experiments connect directly to the surface. With the shielding effect of the detectors around the interaction points, more than $100 \mathrm{~m}$ distance to the surface and a concrete shielding cap on top of the shaft, the radiation levels on the surface will be low enough to prevent any relevant direct exposure to stray radiation or sky-shine effects on the surface sites or beyond.

\subsubsection{Activation of solids}

Activation of solids represents a potential hazard to persons through exposure to gamma radiation during interventions inside the accelerator tunnel such as in-situ maintenance or handling of radioactive parts. The radiation levels differ considerably amongst the various sectors of the accelerator and depend on the type of beam operation and on the time that has passed since the machine was stopped. Locations close to the interaction points, the beam cleaning insertions as well as the absorbers of the beam dumping system will exhibit the highest radiation levels from activation, exceeding those at the LHC [398].

The first means to mitigate risks from this hazard will be to optimise installation, maintenance and repair processes: automated and remotely controlled interventions are one way to reduce the exposure of personnel. Bypass tunnels for high radiation areas will also help to keep exposure low.

Activated materials are routinely removed from the accelerator tunnel and experimental caverns for maintenance or disposal. Dedicated and specially equipped areas for handling and storage of this equipment [399] will be planned. Corrosion and machining of activated materials can produce dispersed activated solids in the accelerator and workshop areas. Experience from high energy accelerators at CERN shows that this does not lead to relevant radiation risks; therefore, standard procedures apply.

\subsubsection{Activated or contaminated liquids}

Infiltration water and leakage water from closed water circuits will be collected by the tunnel drainage system. The water will be pumped to the surface sites for collection 
and further treatment before being cleared and released. Continuous monitoring and confinement will ensure that no activated and/or contaminated water will be released. The demineralised water filtering units collect and concentrate radioactive particles and will be treated through standard procedures.

Ventilation cooling units for the tunnel and experimental areas can, under certain operational conditions, concentrate airborne radioactivity in their condensates, mainly in the form of tritiated water. This liquid waste water will be collected and treated according to standard procedures. The activation of liquid helium in the superconducting circuits results in the production of tritium in quantities which may be above the clearance levels. Sufficient storage capacity for slightly activated helium will be installed at the various sites.

\subsubsection{Activated or radioactive gases and radioactive aerosols}

Air in the accelerator and experimental areas will become radioactive during beam operation. Ventilation systems will be fault-tolerant and either be partially or fully recycling, depending on the assessments to be done at a later design stage for a specific particle collider scenario. Areas will be ventilated with fresh air before allowing access to avoid undue exposure of personnel. Areas with different activation potentials will be separated, such that only those areas are vented where access is required, thus avoiding unnecessary releases of radioactive air. Experience shows that outgassing from activated concrete or radon decay products will only be present in small concentrations in the tunnel air because they are continuously removed by the ventilation system. Dust activation and airborne corrosion products are not relevant sources of exposure to intervening personnel, since aerosols are continuously removed by the air treatment systems.

\section{Energy efficiency}

\subsection{Requirements and design considerations}

Energy efficiency is a topic which is high on the societal agenda in general and must consequently also be a core value of any future research facility, especially energy-intensive facilities like particle accelerators. It concerns the availability and cost of electricity, the reduction of climate-relevant emissions, and other side-effects linked to energy production, transmission and conversion. Operating the FCC in an energy efficient way will enhance its public acceptance. Moreover, the FCC is an opportunity to drive the evolution of energy efficient technologies leading to socioeconomic benefits. A sign of the importance of the topic is the "Energy for Sustainable Science" workshop series [400] established several years ago. Concerted efforts are being made in the framework of the EuCard2 Project [401] (Work Package 3 - Energy efficiency of particle accelerators [402]) and the ARIES Project [403] in the EC Horizon 2020 Research and Innovation Programme. An overview of activities in view of greater energy efficiency of particle accelerators can be found in [404].

Energy-efficient approaches can be categorised in three levels. The first level, with the highest priority, is to consume less energy by making appropriate design choices. The proposed superconducting magnets not only provide a very high field of $16 \mathrm{~T}$, but their combined energy consumption (magnet powering, cryogenics) is also dramatically lower than it would be for a hypothetical collider that was to deliver the same performance based on the superconducting technology used for the LHC. The second level aims to use energy more efficiently while providing the same level 
of service or performance. An example is the use of high-efficiency klystrons, such as those presently under development. Recovering and reusing energy for other purposes, ranks third. A typical example is to recuperate waste heat from cooling circuits, using it within the research infrastructure and feeding it into an energy storage system from which it can be used later for district heating purposes.

Applying fine-grained monitoring of energy use will help to raise energyawareness, control the peak power and energy losses and allow better predictions of energy use to be made. All equipment or facilities not currently in use will be switched off or ramped down. Surface buildings will be constructed to follow high environmental standards, bearing in mind that by far the dominant share of the energy consumption comes from the operation of the accelerator. As far as possible, electrical energy will be purchased from renewable sources. Generally, it should be noted that reconciling the demand for highest performance of the facility over extended running periods with sustainability and reasonable/acceptable energy use is not a simple task. Experts specialising in matters of energy efficiency or energy saving will be consulted to devise a comprehensive and coherent concept from the outset.

The collider will be operated like the injector complex in a cyclic way, creating a cyclic peak power demand from the power grid. A combination of different ways of supplying power are considered:

- Supply of peak power from external network: the peak power is provided by the external network. This is the simplest solution; it might, however, require partial reinforcements of the external network, which is operated by Réseau de transport d'électricité (RTE).

- Use of energy storage systems: this concept uses a combination of switchmode power converters (Fig. 9.1) and local energy storage systems to power magnet circuits. Peak demands during the ramp-up are fully or partially provided by the storage system. During the ramp-down the energy is recovered and fed back into the storage system. This principle is already being used to power the PS magnets (POPS system) and will also be used to power the PS Booster (PSB) at CERN after its upgrade to $2 \mathrm{GeV}$ (POPS-B). The energy storage system for the injector can be based on a set of different energy buffering technologies such as high voltage DC capacitors and batteries. This concept eliminates the positive power peak during ramp-up and the negative during ramp-down, thus resulting in a flat power profile seen by the grid. As a consequence, the external power transmission and the machine internal distribution networks can have lower component ratings for substations, cables and transformers, and will also cause less distribution losses.

\subsection{Power requirements}

The FCC-hh collider will be operated according to the operational model discussed in Section 2.5. The electrical power requirements of the various systems during physics operation at top energy are listed in Table 9.1.

The overall peak power need of $580 \mathrm{MW}$ of this particle collider during the flat top phase of each cycle has to be compared to a simple, size-based scaling of the LHC pointing to about $450 \mathrm{MW}$, which delivers beams at seven times lower collision energies and 10 times lower peak luminosities. This electrical consumption requirement is the result of a combination of energy-efficient designs and the use of novel, energy saving technologies in numerous accelerator subsystems. 


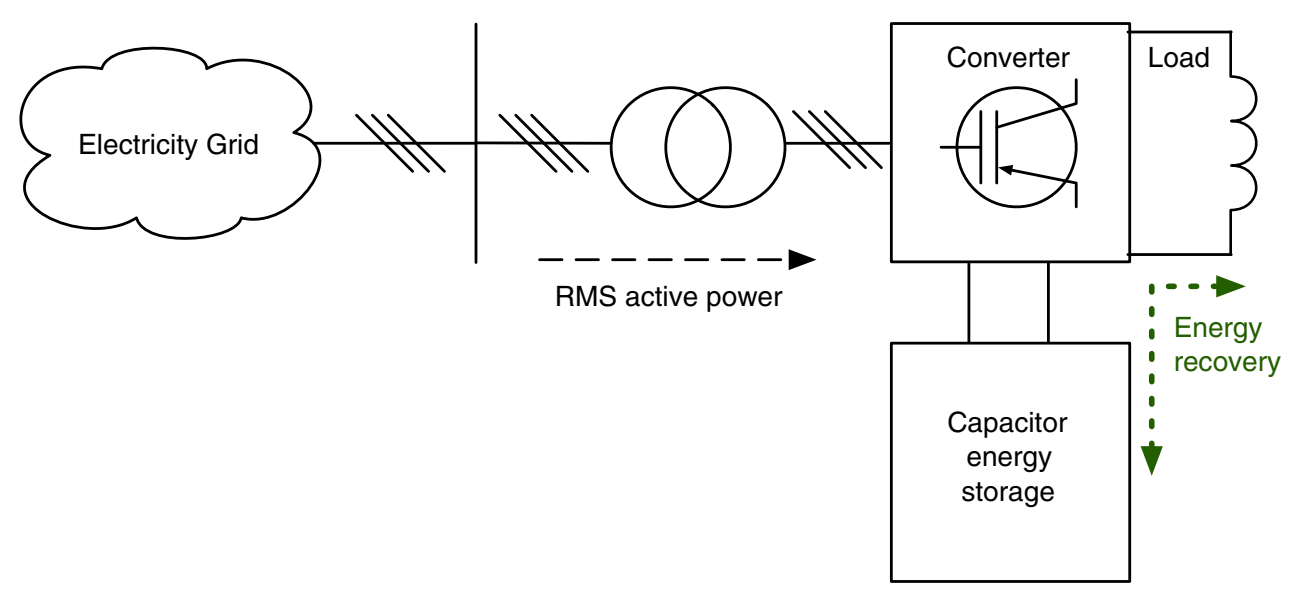

Fig. 9.1. Simplified schematic of the principle of combining switch-mode converters with energy storage.

Table 9.1. Power requirements of accelerator subsystems for the highest performance operation period during the luminosity production phase at the flat top of the cycle.

\begin{tabular}{|l|c|}
\hline Subsystem & Electrical needs (approx. MW) \\
\hline Cryogenics & 276 (ca. 250 with further optimisations) \\
Radiofrequency & 26 \\
Magnets & 80 \\
Cooling and & 30 \\
ventilation & \\
General services & 40 \\
Four experiments & 42 \\
Data centres for & 18 \\
four experiments & \\
Injector complex & 68 \\
\hline Total & $\mathbf{5 8 0}$ (ca. 550 with further optimisations) \\
\hline
\end{tabular}

The power requirement figure for the cryogenic system includes the cooling of the main ring magnets, the beamscreen, the insertion-region quadrupoles, the magnets of the superconducting transfer lines from LHC Point 1 to FCC PB and from LHC Point 8 to FCC PL, the superconducting link to distribute the power for the magnet system and the RF system. To minimise the overall cryogenic power required for the main-ring magnets the temperature of the beamscreen was optimised to about $50 \mathrm{~K}$, as compared with 5-20 K used for the LHC beamscreen, in view of the much higher synchrotron radiation heat load (see Sect. 3.3.2). This allows the synchrotron radiation heat load of $5 \mathrm{MW}$ to be extracted with a total electric power of $100 \mathrm{MW}$.

Losses of the order of $5-7 \%$ in the electrical distribution chain (e.g. magnetisation losses in transformers) have to be added to calculate the power to be supplied by the grid.

Using similar data for other significant phases in the operation cycle (injection, ramping) and incorporating the projected yearly operation programme (see Fig. 2.10), one obtains an energy consumption during a typical year of operation of $\sim 4$ TWh. For comparison, CERN's energy consumption during 2016 was close to 1.2 TWh. 


\subsection{Energy management and saving}

One of the principal challenges of the 21st century will be to develop solutions for the sustainable use of energy. In this context, one of the key design aspects must be a strict focus on energy efficiency, energy storage and energy recovery. This project can and must be used as a technology driver, pushing towards more efficient ways to use electrical and thermal energy.

The foundation for sustainable energy management is real-time energy monitoring, for example using smart meters. This opens the possibility to precisely predict and optimise the overall electricity consumption profile, with the objective of reducing the peak power as well as the electrical losses. For the reduction of the peak power demand, cyclic loads of the injector chain also need to be taken into consideration.

By systematically applying the concept of energy storage for the powering of the magnet circuits, it will be possible to recover a significant part of the energy stored in the magnets. When combining energy storage with complementary measures such as optimisation of the power cycles, the costs for the electrical infrastructure as well as for electrical losses can be greatly reduced.

Electricity transmission over long distances and voltage step-down or step-up leads to power losses. The overall losses of a conventional transmission and distribution network range between $5 \%$ and $7 \%$. Power line losses are resistive losses due to the Joule effect. Transformers contribute load losses which vary according to the load on transformer and no-load losses which are caused by the magnetising current needed to energise the transformers and which are steady losses. The proposed baseline transmission and distribution scheme aims to provide the required level of availability, maintainability and operability from early in the conceptual phase. As an example, where needed, two $135 / 3 \mathrm{kV}$ transformers are operated on access points in parallel, each at a nominal load level of $50 \%$. Such a configuration responds to the above mentioned, non-functional requirements, but it is not optimised for energy efficiency. Alternative schemes aiming to improve the overall efficiency of the transmission and distribution network, while maintaining the required level of availability, maintainability and operability therefore need to be studied. Industrial partners will lead the development, potentially demonstrating the technology in a pilot scheme at CERN's LHC so that a system at acceptable cost and with the required level of reliability can be obtained from market suppliers when needed.

The power demand is particularly high during ramping-up of magnets. With the proposed powering layout (see Sect. 3.8) and the baseline ramp-up time of $20 \mathrm{~min}$, the main dipoles would require a peak power of $270 \mathrm{MW}$. This value can be reduced by having a different ramp shape, ramping the circuits up with constant power instead of constant voltage. Extending the time of the ramp is another way to reduce the peak power. For instance, using a constant-power ramp over $30 \mathrm{~min}$, the peak power for the main dipole circuits will be reduced to $98 \mathrm{MW}$.

If the power for ramping up the collider were to be drawn from the external supply, the power grid would need to be significantly reinforced. Despite this additional equipment, impacts on the grid would still occur. Instead of drawing the power directly from the grid, it could also be taken from local storage, typically in the form of batteries, high-voltage DC capacitors, or a combination of different technologies. Examples of this have already demonstrated and are being gradually implemented in the new power converter systems of PS and PSB at CERN. These have the advantage that the energy stored in the magnets can be recovered and fed back into the storage media, from where it can be drawn again during the next ramp. 4668 magnets each returning $37 \mathrm{MJ}$ per cycle can provide additional power of $\sim 48 \mathrm{MWh}$ to significantly reduce the peak power needed during the next FCC acceleration cycle. Depending on where the local energy storage is located, this approach would also reduce the 
cross section of the cables supplying the power converters and the dimensions of the sub-stations and transformers.

The RF system energy efficiency can be improved by optimising the electrical to $\mathrm{RF}$ power conversion efficiency and the cryogenic refrigeration system energy consumption. With new bunching technologies for high-efficiency klystrons, efficiencies are expected to rise from $65 \%$ to above $80 \%$. These improvements reduce the water cooling requirements by the same amount. The cryogenic losses can be reduced by improving the static and dynamic losses of the cavity cryomodule. The static losses in an optimised cryomodule are expected to be up to $40 \%$ lower than in the LHC. Recent findings on alternative cavity materials promise real progress in cutting the dynamic losses [185].

A future particle collider will use a cryogenics system based on a neon-helium mixture (nelium), which leads to electrical energy savings of about $16 \%$ with respect to the LHC-type infrastructure. Further electrical power savings leading to about $20 \%$ savings with respect to the LHC can be achieved by improving the efficiency of the 40$60 \mathrm{~K}$ cooling stage. This includes improvements in the technology of turbo-expanders and recovering energy from the system. Additional improvements may be achieved with centrifugal compressors on the helium cycle. However, such developments are on a long time-scale and are, therefore, not considered at this stage. The savings outlined above of $20 \%$ of the FCC-hh cryogenics system consumption correspond to about one third of CERN's electricity expenditure today.

\subsection{Waste heat recovery}

Currently, a project is being implemented to connect the cooling system of LHC at Point 8 to a "thermal energy exchange" loop which will supply a new industrial zone near Ferney-Voltaire (France) with warm and cold water circuits for both heating and refrigeration purposes (see Fig. 9.2) [405]. This is a hybrid system, consisting of waste-heat recovered from cryogenic machinery that is stored by heating the ground and which is made available via geothermal probes. Similarly, plans have been developed to feed waste heat from LHC Point 1 into CERN's distributed heating system. Installations of this type can also be envisaged for the infrastructure sites. However, the waste heat can also be supplied directly or through storage in dedicated buffers rather than in the ground. Although a significant potential for waste heat recovery from FCC exists, the needs and opportunities and the infrastructure conditions need to be evaluated for the specific collider and for each specific technical system (e.g. whether a distributed heating system exists already, or whether it could be implemented for a new industrial or housing area close to a surface site). Points PA, $\mathrm{PB}$ and $\mathrm{PC}$ are currently located in areas with a high population density, whereas most of the other points are located in rather rural areas. Potential applications of such recuperated heat are being envisaged. The definitive energy recovery potential depends on the developments planned in the vicinity of the surface sites and must be studied in collaboration with the host states.

The amount of recoverable energy increases with the proximity to the consumer and also with the temperature of the medium, typically water which is at $10-15^{\circ} \mathrm{C}$ above ambient temperature. How much this can be increased, by e.g. operating specific equipment at higher temperatures, will be studied. The most promising candidates for waste heat recovery are the cryogenic plants, which are the top electrical energy consumers, and the conventional cooling systems. The host states have requested that an effort is made to reduce the water consumption with respect to today's operating installations.

It should be noted that the host state representatives expect that project owners develop a plan in cooperation with industrial and public partners to ensure that 


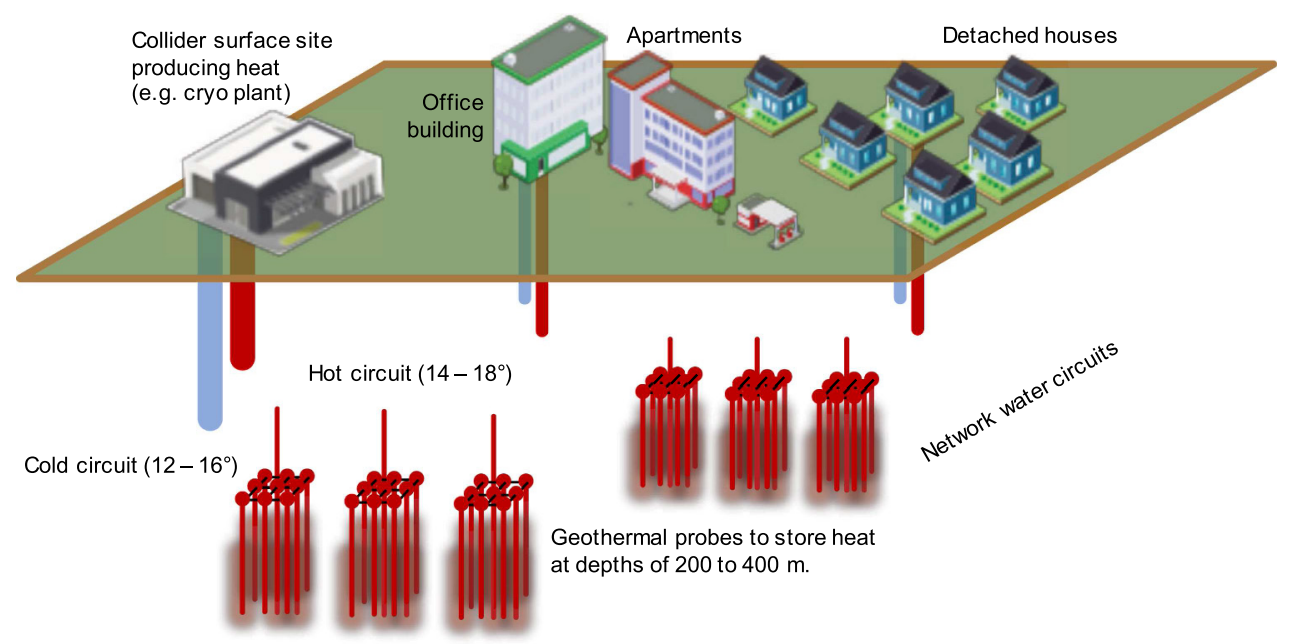

Fig. 9.2. Concept of a heat recovery facility based on a thermal network. The concept is similar to a project, which is currently under construction in the vicinity of CERN. In that project, waste heat is supplied from LHC point 8 to consumers in an industrial zone in Ferney-Voltaire, France. The distance between the LHC surface point and the industrial zone is $2 \mathrm{~km}$.

ongoing efforts on a transition towards a sustainable and circular society are given priority. This is comparable to approaches which are currently being developed in the frame of smart-city projects.

Another potential area of interest is the return to the supplier of cooling water after use for subsequent heating purposes. GeniLac ${ }^{2}$, a $280 \mathrm{MCHF}$ investment project close to the United Nations district (Geneva, Switzerland) with a total cooling capacity of $280 \mathrm{MW}$, can be further investigated with a view to optimising the overall energy balance. This concept is relevant for point PB in particular. The cooling water could also be transported to remote points through the tunnel, thus opening the possibility of extending the concept to points PC and PD. The access sites in France are mainly in rural areas, therefore a dedicated study needs to be carried out to assess the potential for this technology. This study should take the distances from potential consumers and the evolution of urban areas up to the middle of the century into consideration. Potential consumers include, first of all, public institutions such as healthcare providers, schools, public buildings and industrial zones. Next, innovation potential such as indoor farming, health care centres and support for private district heating can be considered.

A third concept involves a step increase of the water temperature in the outer circuits such that the heat supplied becomes an interesting resource for industries such as chemical and food processing. Systems for vapour generation stepping up from $25^{\circ} \mathrm{C}$ quickly become economically feasible [406], but a client needs to be close to the heat production source and willing to engage in a project that does not require the research infrastructure to function as a reliable energy supplier.

Eventually, direct power generation from waste heat can also be envisaged. Today, the effectiveness of such systems is still low, in the few percent range, but industrial interest [407] in increasing the efficiency of industrial plants is driving R\&D activities, for instance via ORC [408] devices. For a large-scale research facility with a system

\footnotetext{
${ }^{2}$ https://www.genie.ch/project/h/genilac-une-innovation-energetique-majeureet-durable-pour-geneve.html
} 
that generates of the order of $100 \mathrm{MW}$ of heat, even modest gains from converting heat into electrical or mechanical energy can be beneficial, particularly considering technological advances on a time scale of twenty years.

\section{Environment}

\subsection{Requirements and approach considerations}

\subsubsection{Legal context}

For the correct operation of CERN's facilities, its status as an international organisation requires that it establishes the requirements and constraints concerning the management of its environmental impact in a pro-active and consensus-based process with the host state on whose territory the installation lies (see Art. II 2 of "L'accord de statut de 1972 entre le CERN et la France" [409]). Where there is standard infrastructure on the surface sites (e.g. office buildings, car parks, ordinary workshops), CERN implements the national laws and regulations that apply at the location where the facility is located (see also "Art. II Convention entre la France et la Suisse de 1965" [410]). A specific process is necessary for the non-standard installations like the accelerators, the experiments and the technical infrastructure needed to operate these facilities. Different rules apply to a project with underground infrastructure which crosses the international border and which has surface sites in both Switzerland and France:

Underground infrastructure. In Switzerland, underground volumes below a depth that is considered useful for the land owner is not subject to the acquisition of rights-of-way and the law applying to private property. A communication from the Département Fédéral des Affaires Étrangères (DFAE) on 16 July 1982, informs CERN that it is exempt from right-of-way acquisition regulations for the LEP/LHC underground structures. In France, land ownership extends to the centre of the earth. Therefore either a process to acquire the underground volumes or to acquire the rights-of-way needs to take place. For both host states, CERN remains liable for any potential impact on the population and the environment resulting from the construction and operation of underground and surface installations.

Surface sites. The land plots for surface sites need to be acquired or leased in both host countries. According to a preliminary study carried out with an environmental impact assessment contractor [411], in Switzerland, an environmental impact assessment needs to be performed when new car parks with more than 500 places are constructed or if per year more than $10000 \mathrm{t}$ of excavation material are processed on Swiss territory with the purpose to reuse that material [412]. The "Ordonnance relative á l'étude de l'impact sur l'environnement (OEIE, Oct. 1988 and 2016)" and the manual "L'étude de l'impact sur l'environnement (EIE) (2009)" [413] define the scope and contents of the assessment. In France, a recent law revision ${ }^{3}$ introduced a new environmental impact management process [414]. This new process explicitly requires a public consultation process on the scope, objectives, socio-economic potential and the impact on the development of the territory that needs to take place well before a specific technical design is developed and before a decision to construct is taken $^{4}$. The host countries require an early and continuous involvement of the population in the project development and construction preparation phases that goes

\footnotetext{
3 https://www.ecologique-solidaire.gouv.fr/levaluation-environnementale

4 See also Art. L121-15-1 of law 2018-148 of 2 March 2018.
} 
beyond information exchange. It calls for an active participation, giving people the possibility to contribute in well-defined and limited ways in shaping the project and in particular in developing the potential for added value. Consequently, France and Switzerland require that the initial assessment process is carried out from the early design phase onwards, followed by regular reviews of the effectiveness of the mitigation measures and assessment of residual or new impacts which become apparent during the construction and operation phases.

Both host states have regulations and laws concerning the continuous assessment and limitation of environmental impact for a variety of different topics. While the processes comprise very similar topics, the organisation of the information and the reporting templates are different for the two host states. In Switzerland the impact study may be limited to certain topics depending on the project needs, whereas in France all topics need to be discussed. In Switzerland the reporting is topically structured and in France the reporting is chronological across the entire project life cycle.

Since the project is international, the Espoo agreement applies [415]. CERN has to ensure that both host states are informed about the effects of any new infrastructure project in their country and the effects on the neighbouring countries. This includes for instance the use of energy, consumption of water, traffic and the management of waste across the borders.

\subsubsection{Environmental compatibility management concept}

The international nature of the project and the similarity of the surface points suggest a uniform and streamlined framework to carry out an environmental impact assessment across both host states. This approach splits the project into locations (e.g. underground structure, individual surface points, associated infrastructures), topics relevant for the impact assessment (e.g. water, air, noise) and the life cycle phases of the project (e.g. construction, operation, maintenance and retirement). Different requirements and constraints apply to the various locations and phases. For some it may be necessary to meet the standard national guidelines of the relevant host state or, for some particular installations, the guidelines need to be agreed between CERN and the host state on a case-by-case basis.

It is planned to have a central, uniform platform to manage the analysis, the assessment of proposed mitigation measures, the follow up of the effectiveness of mitigation measures and the analysis of the residual impact. This platform will permit the extraction of information according to the specific needs of the individual host states. Specialised companies and software solutions exist and should be used whenever possible (e.g. Envigo by eon+, see Fig. 10.1). A market survey and competitive selection process should be performed in cooperation with the host state partners in order to ensure that a suitable set of experts and tools are selected for this process. It is considered good practice in Switzerland that the owner of a largescale project delivers a "Notice d'Impact sur l'Environnement (NIE)", which is more comprehensive than the minimum required environmental impact assessment. The uniform framework mentioned here permits this approach.

The need to perform the environmental impact assessment and management process before a decision to construct is made, calls for the preparation of the assessment framework with the help of experienced consultants and the host state authorities in the years 2019-2021. Given some basic infrastructure, consultants and authority partners who know the project vision and goals can work with the scientific and engineering team until the design has reached maturity by 2023. By this time, CERN must have reached consensus with the authorities and the population that permits the formal initiation of a public consultation process as required in both host states. 


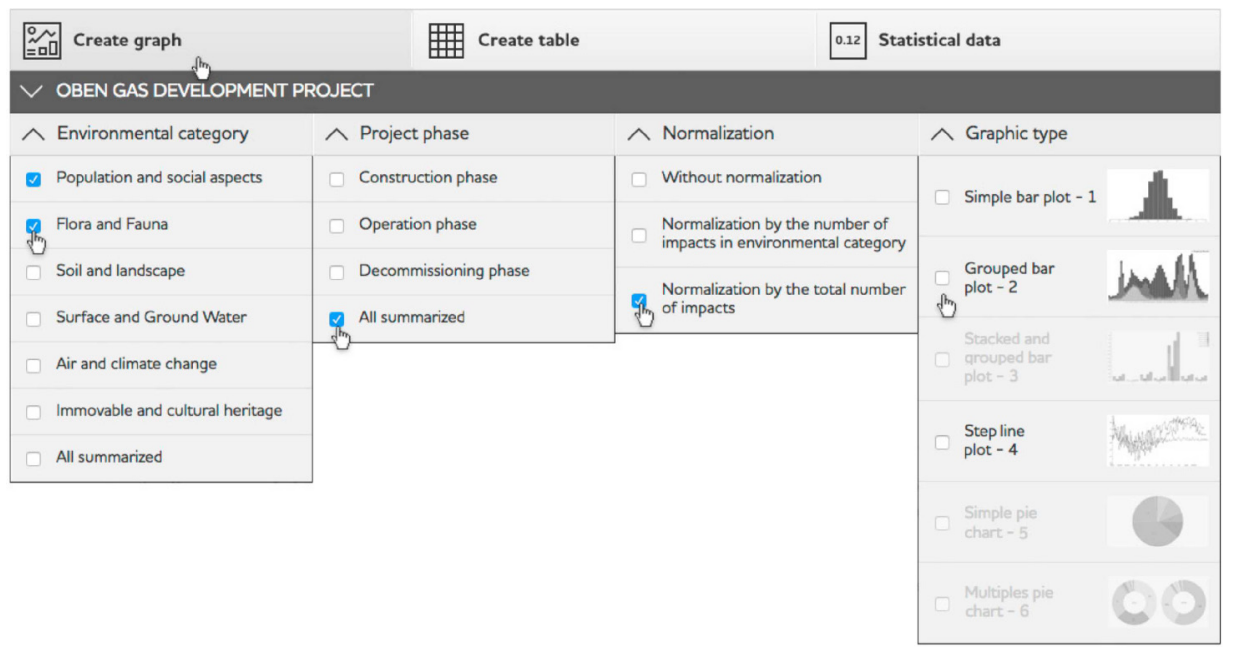

Fig. 10.1. Example of a continuous environmental impact assessment system supported by an information system.

The process is considered lengthy in both countries and is expected to require a few iterations. The goal is to obtain clearance to submit a request for construction permits by 2026, i.e. before an international consortium (e.g. the CERN member states or an international consortium bound by a memorandum, a letter of intent or a similar type of collaboration agreement) takes a formal decision to build the facility.

\subsection{Environmental impact}

\subsubsection{Radiological impact}

The hadron collider will operate at seven times higher beam energy than the LHC, causing higher radiation and activation levels in some parts of the accelerator and experiments. The potential radiological environmental impact comprises (i) dose from stray radiation emitted during beam operation, (ii) dose from radiation emitted by radioactive materials and waste, (iii) operation of ion sources and $\mathrm{X}$ ray emitting devices and (iv) the dose from release of activated water and air. Safeguards will be included in the design of the accelerator infrastructure to control the impact on the environment. Dedicated monitoring systems and procedures will ensure continuous parameter recording and auditing throughout the entire operational phase of the facility and will facilitate control of the impact. LHC operational experience shows that the radiological impact on the environment and population are well below the legal limits. Since the beginning of the operation of the LHC, levels of stray radiation measured on surface sites remain negligible. The effective dose received by the public exposed to atmospheric and effluent releases of the existing particle collider remain below $10 \mu \mathrm{Sv}$ /year. Release levels and dose values are regularly reported to the host states [416]. This experience provides confidence that the particle collider described in this report can indeed be operated in compliance with the host-state laws and regulations.

The accelerator will be located at least $50 \mathrm{~m}$ below the surface and experiment interaction points will be at least $100 \mathrm{~m}$ below ground level. There will be no publicly accessible underground infrastructure. Therefore sufficient shielding against stray 
radiation from beam operation exists at all times. Two scenarios need to be considered to estimate the environmental impact: continuous beam losses during the operation and the effect of a total loss of the stored, high energy particles. In both cases, $15 \mathrm{~m}$ of lateral shielding by rock is sufficient to ensure a negligible impact on the environment and population $[398,417]$. Muon radiation emitted from losses in the plane of the accelerator will be attenuated by hundreds of meters of rock. The shafts are the only direct connections to the surface. At the interaction points they are sufficiently deep $(100 \mathrm{~m}-500 \mathrm{~m})$ to exclude radiological impact from stray radiation [418]. Additional concrete slabs could be placed on top of the shafts to exclude residual impact from scattered radiation.

Activities involving handling, transport and storage of radioactive materials and the operation of X-ray emitting equipment on the surface sites are well regulated and are no different from current operations at CERN. The standard procedures in place within the current framework of radiation protection at CERN are well developed and proven to effectively control the radiological impact.

Beam operation activates air and potentially water close to the machine. The potential environmental impact originating from these sources is addressed as follows:

- Air activation:

Redundant, partially or fully recycling ventilation systems will limit the release of gaseous isotopes (mainly short-lived) during beam operation. This operation scheme is different from the LHC and has the potential to help achieve annual doses to members of the public lower than those with LHC [416]. Aerosol releases are expected to be insignificant due to the low activity content and efficient air filtration at the release points, similar to the LHC. Long term experience at many accelerator installations confirms this estimation [416].

- Water activation:

Drain water, raw water and demineralised water in the accelerator tunnel can become activated during beam operation and can carry trace amounts of radioactive corrosion products. Demineralised water circuits will be operated in a filtered, closed circuit. Leakage and infiltration water will be collected in the tunnel and will be pumped to retention and treatment basins at the surface. The water will be continuously monitored so that release will only occur after clearance. Experience shows that radioactivity in water is not a relevant source of radiological impact on the environment at the LHC [416]. The production rate of radioactivity in water at the future collider is expected to be lower or equal to the LHC, given the possibility to optimise pipe routing and avoid high activation areas [417].

- Ground activation and migration of radioactivity towards the biosphere:

A limited amount of rock around the tunnel will be activated. Along the arcs, the largest part of the collider ring, activation remains at very low levels, well within the set limits [417]. Sections with higher activation potential (e.g. collimation regions, regions close to the high luminosity interaction points) will be located in rock with negligible water migration risk so that transfer to the biosphere can be avoided. Detailed ground investigations at an early design stage phase must be carried out to optimise the tunnel placement. Considering the low levels of concentrations produced [417] and the small residual risk, no radiological impact is expected. Effective mitigation measures to limit the rock activation, such as additional wall shielding can be implemented, if necessary.

- Solid materials:

Equipment and solid materials removed from the accelerator area can be radioactive. Their handling, transport, storage and elimination is subject to regulations and processes already in place for the operating installations at CERN. No radiological exposure is expected in the environment from these tasks. 
The impact of ionising radiation on personnel during operation and maintenance phases, as well as the management of radioactive waste are described in Chapter 8 Safety and Section 10.3 Waste Management, respectively.

\subsubsection{Conventional impact}

A preliminary review of underground and surface sites has been performed with expert organisations in France and Switzerland [411,419]. The studies established a working framework for the subsequent optimisation of the placement of the particle collider which is compatible with the existing requirements and constraints of both host states. The first investigation revealed that a placement of the collider, which is compatible with the legal and regulatory boundary conditions in both countries can be developed. No conflicts with geothermal boreholes, seismic activities, underground technical features such as pipelines, critical power and communication lines could be found so far. Also, no relevant conflicts with underground water layers or hydrocarbons could be identified and puncture of protected water reservoirs can be avoided with an optimised layout and placement. However, the current, first preliminary placement does not meet all requirements and constraints at the same time (technical requirements, lepton and hadron collider optimised layout, environmental exclusion zones, socio-urbanistic constraints). Therefore, further iterations need to be done on the layout and placement optimisation in order to come to a coherent overall proposal that meets all the criteria. In addition, dedicated underground investigations need to be carried out soon in order to validate the preliminary findings with more accurate data and first surface site field studies need to be carried out in order to validate those locations, which need to be considered for an optimised layout and placement. The entire Geneva basin features water-saturated ground, but the water remains locally confined. Consequently, it is unlikely that water, which is for human consumption and which reaches the surface or rivers would be activated by ionising radiation.

Compatibility with protection of flora and fauna as well as agricultural activities has been considered from the beginning by taking into account a number of national and European conservation laws and guidelines. In this context, preliminary surface site candidates have been identified. Potential sites for a next iteration of the surface sites to be considered for a layout and placement proposal for a specific particle collider scenario have been identified.

Some surface sites require further optimisation in the design phase in order to simplify potential landscaping or indemnity processes and to ease accessibility by road. Swiss law requires the reservation of a certain surface area for agricultural activities in order to remain self-sufficient in case of crisis [420]. This constraint imposes the launch of a declassification and ground/right-of way acquisition process in the subsequent design phase. Dedicated working groups are currently being established with representatives of the Swiss confederation and the relevant offices of the Canton and State of Geneva in order to facilitate this multi-year process. The legal framework in both countries requires further detailed information in order to jointly develop an optimised placement. These data can only be obtained by dedicated ground investigations which need to occur before the relevant environmental impact analysis can take place. Confirmation that inadvertent activation of water due to infiltration can be avoided may need to be verified by targeted surveys in a limited number of locations. The environmental impact during the construction phase, which extends over many years, needs to be studied. The reuse of the excavated material (in order of priority: on-site use, processing and reuse, landscaping, storage), construction site traffic, noise and dust are all elements which also need to be considered. 
Official bodies of both host countries (Secrétariat Generale de la Région AuvergneRhône-Alpes and Département de l'aménagement, du logement et de l'énergie de la République et canton de Genève) have informed the study management that for emerging urban areas and where there is a region with high-value natural assets, early participation of the authorities and representatives of the population in the further development of the project plans is required. Surface sites need to blend into the landscape. Synergies with local and regional activities that profit from the infrastructure in the host countries need to be developed. Examples include cooling via the GeniLac [421] water project, waste-heat recovery for residential districts and healthcare providers, possibilities for temporary energy storage and release in cooperation with neighbouring industries. For the construction phase, particular attention needs to be given to noise, dust and traffic. For the operation phase, topics include the consumption of water, electricity, the emission of noise and the increased need to provide all kinds of infrastructure for an ever growing community of scientists, engineers and visitors.

The immediate subsequent design phase of the project will focus on the further optimisation of the collider and surface site placement, based on the findings already obtained in cooperation with the host state authorities and their technical advisory bodies nominated for the concept phase. This work will, in compliance with the regulations of both host countries, involve representatives of the local population in order to ensure a seamless evolution of the project design towards a later construction decision.

\subsection{Waste management}

\subsubsection{Radioactive waste management}

CERN has a radioactive waste management system which has been implemented in agreement with its host states [399]. The production, temporary storage, processing and elimination of radioactive waste is performed according to the processes defined in the radioactive waste management system. During the technical design phase of the FCC-hh, the production of waste and its provisional classification will be addressed and quantified and the temporary storage and treatment facilities required will be evaluated in detail. The choice of materials to be used in terms of their isotope production and known elimination pathways will be optimised during the design phase.

The FCC-hh will produce similar types of radioactive waste to LHC. Given the similarity in materials used, the radionuclide inventory is not expected to change significantly but due to the longer tunnel, the volume of waste will increase accordingly. The expected beam loss rate per metre is comparable to the LHC. The increase in beam energy will account for an increase in activity concentration, and it is assumed that the fraction of medium level radioactive waste (classified in the French system as "faible et moyenne activité", FMA, and "très faible activité", TFA) with respect to the total volume, will not change significantly compared to LHC. The higher luminosity in FCC-hh will lead to higher activation levels in the detectors of the high-luminosity experiments at $\mathrm{PA}$ and $\mathrm{PG}$, in the final focusing regions next to the interaction points, as well as in the regions with beam cleaning, collimation system and dumps. The dose rates are expected to increase compared to the levels in the LHC. In terms of handling and waste processing, technical developments will be required. These are already planned and will be implemented for the High Luminosity LHC (HL-LHC) project.

The construction and operation of a high-energy particle collider in a new tunnel will reuse the existing LHC injector chain, with the LHC becoming an injector to 
Table 10.1. Estimates of radioactive waste for the high-energy hadron collider.

\begin{tabular}{|lcc|}
\hline & TFA & FMA \\
\hline Construction phase & & \\
New SPS as injector & $5000 \mathrm{~m}^{3}$ & $200 \mathrm{~m}^{3}$ \\
LHC as injector & $300 \mathrm{~m}^{3}$ & $900 \mathrm{~m}^{3}$ \\
\hline Operation phase & & \\
Injectors (including LHC) & $650 \mathrm{~m}^{3} /$ year & $30 \mathrm{~m}^{3} /$ year \\
Collider & $1450 \mathrm{~m}^{3} /$ year & $70 \mathrm{~m}^{3} /$ year \\
\hline
\end{tabular}

the main ring. As an alternative, a superconducting SPS could serve as an injector. During the construction, parts of the existing accelerator chain need to be upgraded. As the components of these accelerators are already activated, the material to be removed from those areas will be radioactive. Two scenarios are considered for the estimation of radioactive waste production during the construction phase of the FCChh: (i) The modification of the current LHC to operate as an injector to the FCC-hh. (ii) The full replacement of the current SPS by a new superconducting accelerator.

The conversion of the LHC into an injector requires the dismantling of all LHC experiments and the removal of the matching and inner triplet elements at the four interaction points. The estimated volumes are $300 \mathrm{~m}^{3}$ (TFA) and $900 \mathrm{~m}^{3}$ (FMA). The full replacement of the SPS by a superconducting accelerator would generate an estimated total volume of $5000 \mathrm{~m}^{3}$ (TFA) and $200 \mathrm{~m}^{3}$ (FMA) [422]. In the case of a complete decommissioning of SPS, the temporary storage space required for the radioactive waste during the decommissioning remains to be evaluated in terms of the disposal capacity per elimination pathway.

During the operation phase of the accelerator complex, radioactive waste will be produced from the collider and the injectors, mostly during the long shutdown periods. As for the LHC, about $87 \%$ of the waste generated will be TFA type and $4 \%$ FMA. The remaining $9 \%$ of the volume could be considered as a candidate for release from regulatory control via the clearance procedure. The average volume produced per year is estimated to be $2100 \mathrm{~m}^{3}$ (TFA) and $100 \mathrm{~m}^{3}$ (FMA) [422], which corresponds to a considerable increase with respect to the current capacities of reception, treatment and disposal of radioactive waste (average $400 \mathrm{~m}^{3}$ per year). The volumes given above take into account the accelerator components themselves, but not the associated infrastructure like cables and cable trays inside the tunnel. It is expected that these other elements would add less than $20 \%$ to the total volume.

\subsubsection{Conventional waste management}

A large-scale research facility produces conventional waste during the construction and operation phases. Typical classes of waste produced during construction are shown in Table 10.2 .

While plans for the management of waste during both phases need to be established, the regulatory frameworks of both host countries require the development of waste prevention plans for the construction phase as part of a preparatory phase. In France, the "Plan regional de prevention et de gestion des dechets" [423] requires the project owner to include approaches that work towards a circular economy, taking into account the possibilities offered by the regional infrastructures. Waste reduction targets are documented in the "Loi relative à la transition énérgetique et a la croissance verte" [424]. In Switzerland, the "Ordonnance relative à l'étude d'impact sur l'environnement (OEIE)" [412] specifies the activities related to the planning of 
Table 10.2. Examples for conventional waste.

\begin{tabular}{|c|c|c|}
\hline Class & Description & Subsequent reuse \\
\hline Inert waste & Excavation materials & $\begin{array}{l}\text { Use on construction site, } \\
\text { processing for create raw } \\
\text { materials for industry and } \\
\text { construction and isolation } \\
\text { materials, landscaping, } \\
\text { environmental protection } \\
\text { measures, addition to } \\
\text { construction material, final } \\
\text { disposal (e.g. quarries) }\end{array}$ \\
\hline Inert waste & Construction materials & $\begin{array}{l}\text { Recycling, final disposal (e.g. } \\
\text { quarries) }\end{array}$ \\
\hline Inert waste & $\begin{array}{l}\text { Polluted excavation materials } \\
\text { (e.g. from drill \& blast) }\end{array}$ & $\begin{array}{l}\text { Final disposal in dedicated } \\
\text { sites }\end{array}$ \\
\hline Wood (treated) & $\begin{array}{l}\text { Cable drums, palettes, con- } \\
\text { struction wood, packaging } \\
\text { materials }\end{array}$ & Taken back by supplier \\
\hline Wood (untreated) & $\begin{array}{l}\text { Cable drums, palettes, con- } \\
\text { struction wood, packaging } \\
\text { materials }\end{array}$ & Recycling, incineration \\
\hline Plastics & Packaging materials & $\begin{array}{l}\text { Taken back by supplier, } \\
\text { recycling }\end{array}$ \\
\hline Metals (low quality) & Construction & Recycling \\
\hline Metals (high quality) & $\begin{array}{l}\text { Wires, cables, machinery, } \\
\text { sheets }\end{array}$ & $\begin{array}{l}\text { Taken back by supplier for } \\
\text { reintegration into production } \\
\text { cycle }\end{array}$ \\
\hline Electronics & $\begin{array}{l}\text { Cards, computers, outdated } \\
\text { devices }\end{array}$ & Recycling \\
\hline Insulation materials & $\begin{array}{l}\text { Construction, machine con- } \\
\text { struction }\end{array}$ & Taken back by supplier, reuse \\
\hline Paint, solvents, glue & Construction, maintenance & $\begin{array}{l}\text { Residuals from construction } \\
\text { taken back by suppliers, } \\
\text { recycling after stabilisation }\end{array}$ \\
\hline Chemicals & $\begin{array}{l}\text { De-mineralised water produc- } \\
\text { tion, cooling tower cleaning } \\
\text { and anti-legionella treatment }\end{array}$ & $\begin{array}{l}\text { Favour bio-degradable } \\
\text { products and processes } \\
\text { involving less or no chemicals }\end{array}$ \\
\hline $\begin{array}{l}\text { Paper and } \begin{array}{r}\text { card- } \\
\text { board, } \\
\text { fextile, }\end{array} \\
\text { filters, glass }\end{array}$ & Packaging & Recycling \\
\hline $\begin{array}{l}\text { Plaster, coating, } \\
\text { gypsum, tiles }\end{array}$ & $\begin{array}{l}\text { Residual construction materi- } \\
\text { als }\end{array}$ & Taken back by contractors \\
\hline Oil & $\begin{array}{l}\text { Hydraulics, cooling, insula- } \\
\text { tion, lubrication }\end{array}$ & $\begin{array}{l}\text { Taken back by contractors } \\
\text { with obligation to } \\
\text { decontaminate and recycle }\end{array}$ \\
\hline
\end{tabular}

how conventional waste is managed. In the canton of Geneva, the "Plan directeur cantonal 2030" documents the specific waste reduction targets [425].

The management of waste follows three priorities: (i) keeping the amount of conventional waste as low as reasonably possible, (ii) develop a plan to reuse the waste locally and regionally and (iii) keep the impact of residual waste transport low.

Today, excavation material is still considered waste by law, but the legislation is evolving in many European countries. Millions of cubic metres of excavation material 
can be a valuable primary resource. Large-scale processes to transform and make the materials available on the market remain to be developed. The FCC study has launched a research activity with the French tunnel design centre (CETU), the Geneva based "Service de géologie, sols et déchets" (GESDEC) and Montanuniversität Leoben (Austria) on this subject. The study will serve as an input to the environmental impact assessment. Furthermore, detailed plans for the construction phase, including the identification of waste, its collection, treatment, reuse and disposal in compliance with the individual national laws have to be developed as part of the environmental impact assessment process. This plan should include measures that can be included in procurement procedures and in the agreements concerning inkind contributions from collaboration members. The procedures should address the sharing of the responsibility between project owner and suppliers for dealing with conventional waste.

\section{Education, economy and society}

\subsection{Implementation with the host states}

\subsubsection{Overview}

Assuming 2039 for the start of physics operation, a decision point for a construction project in 2026 and start of construction around the end of 2028, a number of administrative processes need to be set up with the two host states and to be carried out during a preparatory phase. Therefore, work with the host state authorities has been launched in order to develop a workable schedule for the available time window. The results of this work so far show that an eight-year preparatory process is required, in cooperation with both host states and involving a diverse set of administrative processes with public engagement. A large portion of this schedule has to be completed before a decision for construction is taken. Only the activities concerning the preparation of the construction sites can be postponed until after a decision to build.

The preparatory phase schedule, which has been developed jointly by CERN, government representatives of France and Switzerland is shown in Figure 11.1. The plan represents a minimum-duration process, owned and steered by the project owner. This is an international consortium, represented by CERN, including its member states and, potentially, additional project partners from non-member states. Additional time may be needed to accommodate further design iterations, in order to integrate feedback and additional requirements by authorities and to adapt to potentially evolving regulations in the host states.

The host state authorities recommend working with locally experienced contractors for tasks that relate to the development of an implementation scenario in the region, to benefit from their knowledge concerning territorial specificities and to set up a working environment that permits putting workforce and contracts quickly in place and to be able to adjust to the evolving requirements in a timely manner. Both host states have demonstrated their will to accompany CERN throughout the preparatory phase in order to ensure a coherent development of the project, thus increasing the likelihood of success when a decision to move forward with construction is taken by the research community. Although the legal frameworks and government structures in the two host states differ and different types of actors need to be involved on each side of the border, the goals and scope of the individual processes are similar. Some specific elements have to be planned and carried out in an international framework, e.g. the environmental impact assessments according to the Espoo "Convention on Environmental Impact Assessment in a Transboundary Context" [415] and the establishment of an international agreement between France and 


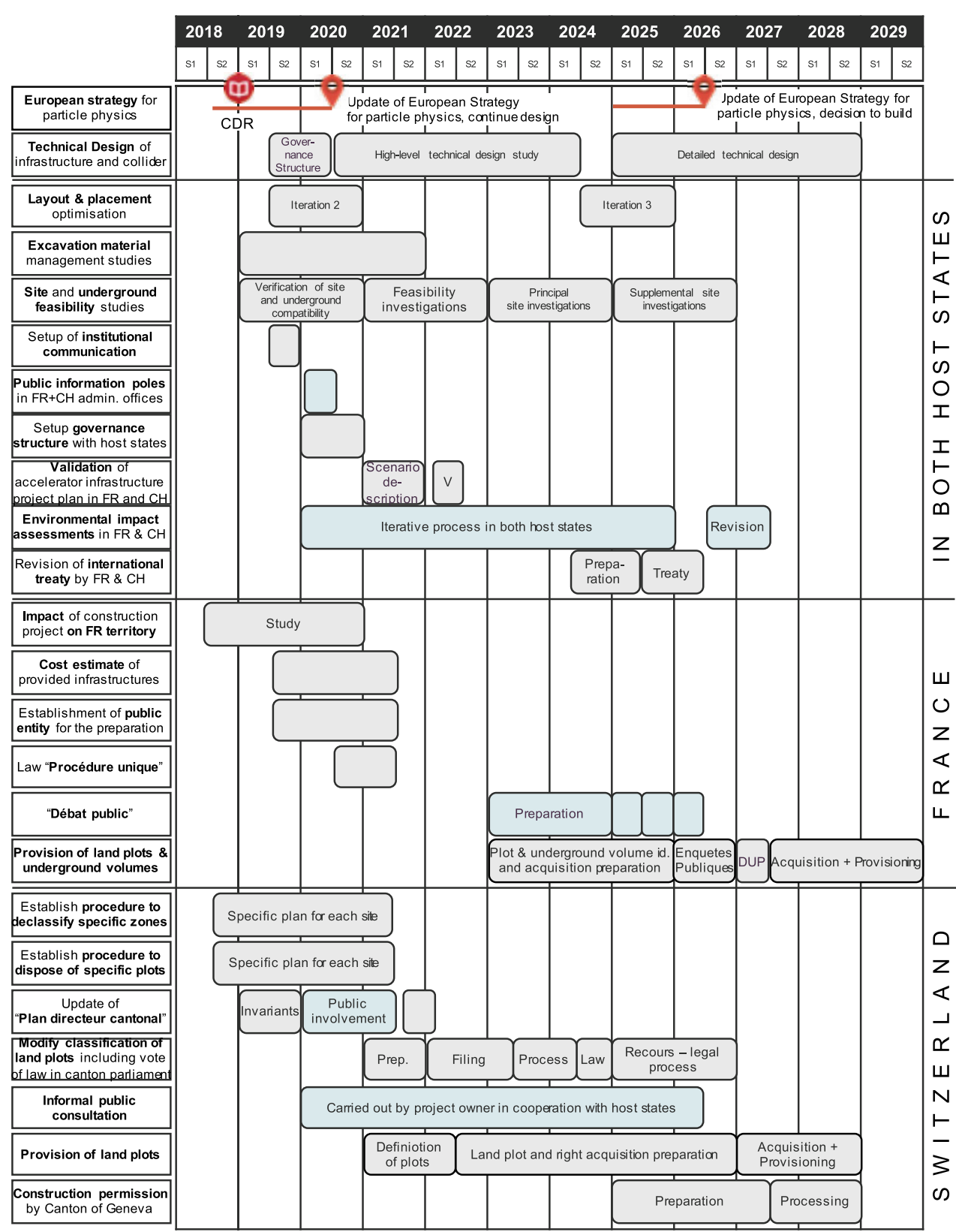

Fig. 11.1. Schema for the administrative processes required in both host states including those needed during a preparatory phase, before a decision to construct is taken.

Switzerland for the purpose of preparing, implementing and operating an extension of CERN's current particle accelerator infrastructure on the two territories.

\subsubsection{France}

The Auvergne-Rhône-Alpes regional government is headed by a prefect who is supported by an office (Secrétariat Général de la Région Auvergne-Rhône-Alpes, SGAR) 
located in Lyon. Within this administration, the prefect is the representative of the French Republic. The SGAR and the prefect's office work together on all development activities in the area. In 2016 the SGAR launched an analysis so that the prefect, and in turn the French national administration, could be informed about a significant extension of CERN's infrastructure and to estimate the associated administrative and financial impacts for France. This activity led to the establishment of a series of regular meetings with CERN for the development of the preparatory phase. France promotes a "participative democracy" [426-428], calling for a process to find a consensus through open discussions amongst all stakeholders in the project from the conceptual stage onwards. The process starts with the development of the basic concept, a feasibility study concerning the infrastructural challenges, a proposal for the governance and funding and a socio-economic cost-benefit analysis. The infrastructural changes concern a wide range of topics: the use of excavation material, a concept for managing conventional waste during all project phases, an energy management plan that can be integrated with the territorial energy balance plan, plans for the consumption of resources such as water, proposals for synergies with the host state to contribute to the territorial development, the development of traffic and mobility concepts and further topics. CEREMA ${ }^{5}$, a public entity associated with the ministry of ecological transition and solidarity and the ministry of territorial cohesion, has produced a report for the SGAR and the prefect on the impact of an FCC for the host state concerning administrative processes and the associated financial engagements [429].

Initially, a specific project scenario needs to be produced since government offices cannot work on concrete preparatory tasks based on generic processes, but need to base their actions on so called project invariants that need to be considered in the update of regional development plans. This scenario description will cover a wide range of subjects including for instance, the purpose of the research facility, an initial proposal for the placement of the underground and surface sites, known technical infrastructure requirements including the resources and services (e.g. electricity, water, communication, transport, emergency and security services), the high-level construction and operation project plans, a catalogue of initial risks and benefits, a list of known uncertainties, a construction and operation cost estimate, a cost-benefit analysis, a first realisation plan, a governance and management structure and a preliminary analysis of the administrative impact on the host states. Once the scenario is validated by the office of the President of the Republic, which is where the regional and national stakeholders come together, the preparation of the legally required process for the public to participate in the project ("débat public") [430] can begin. The goal of this process is to develop an acceptance and appreciation of the project amongst the population, which forms the basis for a governmental clearance for the project owner to continue with the preparation. A commission defines which topics are relevant for the population and where there can be reasonable involvement in this process. The formal procedure lasts six months, but it requires about two years of preparation and is concluded with a one-year phase for its implementation.

After this internal preparatory phase, the project owner in close cooperation with the public debate commission, prepares information material (documents, databases, Web contents, booklets, videos, travelling information stands, town meetings), organises the nation-wide process, employs consultants and companies experienced with this process, sets up the required technical infrastructure and finally, defines a schedule that will allow all relevant public representatives to be involved. This takes about one year. According to the law revised in 2017 [431], the three-year period also involves the publication of summaries of the environmental impact assessment. At

${ }^{5}$ http://www.cerema.fr 
the end of the formal "débat public" phase, a follow-up consultation process, which continues until the end of the construction phase, must be put in place. Its findings need to be integrated in an iteration of the technical design and the project documents.

Once the national committee of the public enquiry (CNDP) [430] agrees that the documentation is mature, the formal open discussion can be launched. This milestone is associated with a certain risk. If the process is insufficiently mature, an extension of the preparatory period will be required. During this consultation phase, the plan for the project is presented and discussed with representatives of the population at local, regional and national levels during formal, recorded and audited sessions [432]. The project owner is asked to collect all questions and provide answers in a secure and auditable way. The findings lead to another iteration of the required project documentation (e.g. environmental impact assessment, plan for the management of excavation material and waste, energy management plan, traffic and mobility concept, land and rights-of-way acquisition plan, final land plot valuation). After the successful completion of this phase, the acquisition of rights-of-way of underground volumes and surface plots can start.

A public enquiry ("Enquête publique") concludes the process by confirming the agreed infrastructure design which emerged from the public debate phase. If the tribunal has no objections, the French government will issue the approval of the infrastructure project's utility for the nation ("Déclaration d'utilité publique") [433] and the project owner can proceed with the preparation of a construction project. The acquisition of the rights-of-way and land plots can take place concurrently with the civil engineering calls for tender.

The entire process relies on the parliamentary submission and acceptance of a law that needs to be prepared for defining a unique process for the environmental authorisation [434] of a new research infrastructure project. Such a law includes a list of national laws and regulations that apply to the specific project, adjustments of existing laws, indications of which laws do not apply and specific regulations concerning particularly relevant topics such as the management of excavation material. This instrument, which appeared in 2016, specifically targets the timely preparation of large-scale projects of public interest such as the Grand-Paris [435] project.

\subsubsection{Switzerland}

Switzerland is a republic, federating significantly autonomous "Cantons"; this system of semi-direct democracy which has been in place since 1848, is unique. The country has a long standing tradition of involving the population at all levels (municipal, cantonal and federal) and at all phases of infrastructure projects. The procedures differ substantially in the different Cantons. Therefore, a workable process has been developed with the assistance of the Canton and State of Geneva, the Swiss permanent mission for international organisations in Geneva, the representatives of the Swiss Confederation and a consultant for public construction project administrative procedures.

Although CERN's primary communication partner for administrative matters in Switzerland is the permanent mission and the ministry of foreign affairs (DFAE), the common work so far revealed that for the preparatory phase of a large scale particle collider project located in the Canton of Geneva the majority of processes will be carried out together with the State Office of Geneva. The federal government will be involved in the entire process with respect to the approval of applicable federal laws, regulations and financial contributions. In 2017, the "Structure de Concertation Permanente" (SCP) was established for this purpose. It includes Swiss government 
representatives at federal and cantonal level, experts from different administrative domains on an "as-needed" basis and representatives of CERN for various development areas, including future projects.

The schedule, which has been developed in the frame of the SCP takes note of the status of CERN as an international organisation developing research instruments on a global scale, which are technically and organisationally unique. Several years before initiating the cantonal formal processes to obtain permission to construct, the acquisition of rights of way on land plots and the declassification of those agricultural zones needed for construction have to be launched and successfully completed. The authorities assume that CERN also carries out a set of informal processes which include a public consultation process, which is similar to the French "débat public", with a preparatory period to create public information booths, information materials and events, public hearings, the establishment of an office to collect questions and to develop responses. In addition, the engagement of a diverse set of stakeholder groups at municipal, cantonal and federal levels is required, with an initial institutional communication strategy and plan which will be developed in close cooperation with the cantonal and federal authorities.

In order to be able to meet the envisaged overall schedule, which aims to start construction towards the end of 2028, two major preparatory actions have been identified by the representatives of the Swiss Federation and the Canton of Geneva that need to be initiated as soon as possible: a working group needs to develop a procedure in cooperation with the representatives of the Swiss Federation and the Canton of Geneva that permits CERN to request the Canton of Geneva to process the re-classification of agriculturally reserved, green-belt zones, called "Surface d'assolement" (SDA) [436,437]. The working group needs to tackle the process based on concrete, yet preliminary and non-binding assumptions of surface sites in order to develop concrete plans for the de-classification process, which will include mitigation and compensation measures. This process goes along with the development of a re-classification plan for a concrete set of potential plots of land that need to be acquired or rented long-term, for the construction project at a later stage. At the same time, another working group needs to develop the procedures to obtain the rights of way or the acquisition of those specific plots of land on behalf of CERN. The procedures are expected to differ for the different specific plots and involve the development of negotiation and acquisition strategies and the drawing up of plans to provide the sites to the international organisation. Some areas may be provided only for limited periods of time, for instance during the construction and commissioning periods. Expropriation of land in the interest of the public is generally avoided in Switzerland and therefore negotiation with the owners of potential surface sites must be started early in the preparatory phase, to allow sufficient time to adjust the placement if needed. This work goes along with an evaluation of the market value of the land plots, notification of the cantonal authorities of the intent to use the plots for an infrastructure project and pre-agreements with land owners for obtaining the rights of way. These processes have to be managed by CERN or a mandated project consultant and rely on the active participation of a number of federal and cantonal offices.

Although an environmental impact assessment is formally only required for the creation of parking spaces for more than 500 cars and for the processing of excavation materials of more than $10000 \mathrm{t}$ per year on a site [412], it is seen as good practice and in agreement with the Espoo Convention that the project owner pro-actively carries out this process. A specific framework needs to be developed to be able to perform one single integrated environmental impact assessment of the project across the two host states with the possibility to provide information in a way that is accepted by the relevant administration offices in the two nations. 
Referenda and federal approval processes may be applicable. They need to be identified during the preparatory phase as soon as specific topics are identified by the authorities that may require public approval. In such cases, dates need to be agreed with the federal and cantonal authorities to limit public initiatives to a certain period so that appropriate reaction to enquiries from citizens and interest groups can be made without jeopardizing the overall project schedule.

In order to get started with a number of different federal and cantonal offices efficiently, CERN is asked to initially put emphasis on the establishment of an institutional communication plan and its implementation. This will inform all officials who have to support the work of CERN about the project scope, goals, scale and schedule. Regular informal meetings with the regional population, their representatives and various interest groups are welcomed from the initial design phase, since transparency is considered the most efficient aspect of activities for the preparation of a potential project.

Authorisation for construction can be requested after successful completion of the necessary steps and before a decision to build takes place. The French authorities ask for a Cost-Benefit-Analysis (CBA). The Swiss authorities encourage the idea of aiming at creating added value from training, tourist development, creation of a technology pole and synergies concerning transport and energy recovery in the Geneva region.

\subsection{Socio-Economic opportunities}

\subsubsection{Introduction and motivation}

Cost-Benefit-Analysis (CBA) [438] for conventional infrastructures such as roads, power plants, energy distribution networks and public transport systems is a useful tool to identify, quantify and communicate the potential value, in addition to the gain of scientific knowledge, that a new research facility can bring to society.

A preliminary, quantitative study of the LHC/HL-LHC project using this method has been carried out [317]. This work serves as a foundation for a dedicated CostBenefit-Analysis of one particular future collider scenario that needs to be carried out at an early stage of the preparatory phase. The study has been performed by three independent organisations: University of Milan, University Roma Tre and the Centre for Industrial Studies (CSIL), Italy. The result reveals that the estimated Net Present Value (NPV) for the entire, combined LHC/HL-LHC programme over a period of 45 years (from 1993 to 2038) is positive, taking into account an annual social discounting rate of $3 \%$. The likelihood of generating benefits of up to 5 billion CHF over the life of the programme is $80 \%$ [317]. This shows that a research facility like the FCC can generate added value for both industry and society. Despite the significant investment for constructing a new particle-collider infrastructure (CAPEX), the overall costs are dominated by operational expenditure (OPEX).

CBA has become a requirement for the preparatory phase of research facilities. The upcoming ESFRI roadmap includes a specific request [439] for it and the EC staff working document on sustainability of research facilities asks for it. In 2018 an EC supported H2020 project, RI Impact Pathways [440], was launched with CERN's participation to develop guidelines that help homogenising benefit and impact assessment for research facilities in the European Research Era (ERA). Here, the results from the study on the combined LHC/HL-LHC programme are presented. The results of the investigations so far triggered a set of complementary surveys and studies by the university of Milan in cooperation with CSIL in order to establish a set of solid input parameters and assumptions for a CBA of a post-LHC particle collider scenario. 
So far, the studies of the LHC/HL-LHC programme revealed six economically relevant benefits: (1) the value of scientific publications, (2) technological spillover, (3) training and education, (4) cultural effects, (5) services for industries and consumers and (6) the value of knowledge as a public good. There are two direct socio-economic benefits that stem from the dissemination of scientific information [441-443] (publications, reports, conference presentations): first, the direct value of scientific products is conservatively estimated to be equivalent to their production costs [444,445]. Second, the value of publications produced by an additional tier of scientists which cite the scientific products directly emerging from the research infrastructure is equivalent to the cost of their production. For the LHC/HL-LHC project, this added value is around $2 \%$ of the total benefits [442]. This report focusses on the findings concerning training, impact on industry and cultural effects in the following sections.

\subsubsection{The value of training}

The value of education and training is the single largest socio-economic benefit of a large-scale, hi-tech research facility. The impact is high, if the project is carried out in an open, international environment with strong cooperation between educational institutes, research centres and industrial partners (the "knowledge triangle") [446]. A conservative estimate of the net present value added to the lifetime salary of early stage researchers (master's degree, doctoral degree and post-doctoral researcher) after leaving the LHC/HL-LHC project has been estimated. The lifetime salary premium for people who perform research and development in the scope of the LHC/HL-LHC project at CERN ranges between $5 \%$ and $13 \%$ in excess of the salary premium of the acquired academic degree. This translates into an absolute lifetime salary premium of about 90000 Euro to 230000 Euro per person with an average added value of ca. 160000 Euro for a doctorate degree obtained during a research period at CERN (2018 currency value) $[447,448]$. Based on CERN's current training programme capacity, during a 15 year construction phase and an initial operational phase of 20 years. 40000 individuals would generate more than 6 billion Euros for the economies of the participating nations. The training value potential depends significantly on the possibility to acquire intersectoral and transferable skills [449] and the number of supervisors that are available to ensure the quality of the training provided.

These results triggered a process with the two host states [450] to find how the value of training can be further increased. Proposals include extending the training to non-academic teaching levels (apprentices, technical schools), continuous professional training (partnerships with companies in the frame of maintenance and upgrades as well as the increasing exploitation of under-used equipment), partnerships with public services (healthcare providers, public security and emergency services) and the continued enlargement of the training opportunities for non-technical domains (e.g. management, finance, economy, law, business administration). Also, joint academic education through partnerships with universities and industry should be further strengthened. The EASITrain H2020 Marie Skłodovska Curie Action Innovative Training Network project [451], launched by 11 institutes collaborating in the FCC study and 13 industry partners is a first step in this direction. It includes as goal the development of a European joint doctorate in applied superconductivity.

\subsubsection{Opportunities for industries and technological spillover}

The industrial activities that emerge from physics research projects are important for the European economy [452]. The "utility factor" is a key figure on which all 
estimates of industrial impacts are based. It indicates that every Swiss franc spent on co-development and customisation projects with industrial partners generates, on average, three Swiss francs worth of follow-up sales revenues for the industrial partner [453]. The LHC/HL-LHC CBA study provides quantitative evidence that companies participating in the project and exhibiting medium to high levels of innovation also benefit from a measurable increase of their earnings before interest and taxes (EBITDA) [454,455]. The relevance of this effect has been tested using the accounting figures of 669 companies involved in CERN procurements worth more than 10,000 CHF and a control group [456]. The majority of companies report that the main benefit stems from the learning experience in such a project.

The LHC/HL-LHC CBA also reveals that there is added economic value due to improved products, services and operation models including information and communication technologies (ICT) of 10 billion CHF: more than $40 \%$ of the total cost (capital and operation expenditures). Further intensification of industrial involvement in core R\&D activities in the framework of a well-planned industrialisation strategy can increase this share, so that a payback of $50 \%$ of the total costs can become realistic. The most prominent example for such research-induced industrialisation is the development of $\mathrm{Nb}-\mathrm{Ti}$ superconducting wires for the Tevatron collider which triggered the mass production of medical magnetic resonance imaging (MRI) devices [457]. Another noteworthy example is the development of large-scale cryogenic refrigeration plants - the architecture and machinery developed for helium liquefaction for use in particle accelerators has been the industry standard for almost 40 years [458].

For a future particle collider, the benefits emerging from procurement and coinnovation depend on (a) the investment volume, (b) the potential for innovation related to the individual subsystems and (c) on the value of the utility factor. It is not yet possible to reliably quantify the overall impact for industry. A dedicated study for a specific accelerator scenario needs to be carried out, once the detailed technical design starts. Technologies that lead to innovation on a broader scale (see Fig. 11.2) for the different particle collider scenarios include superconducting radiofrequency systems for a lepton collider (estimated benefit for industries about $150 \mathrm{MCHF}$ ), superconducting wires for a hadron collider (estimated benefit for industries around 1,200 MCHF depending on the revenue margin that the supplier is able to generate for non-research customers), construction and testing of superconducting magnets for a hadron collider (estimated benefit for industries from 150 to $200 \mathrm{MCHF}$, depending on the potentials of the different technologies along the magnet manufacturing value chain) and large-scale cryogenic refrigeration infrastructure for a hadron collider (estimated benefit for industries from 250 to $300 \mathrm{MCHF}$ ).

An earlier study [459] has shown that hi-tech companies can transfer the knowledge and experience acquired during work with CERN to new products and services easier than companies who offer off-the-shelf products and services. Therefore, it is crucial to make an effort to cooperate with industry on increasing the potentials for other fields, such as civil engineering. As Figure 11.3 shows, the potential industrial spillover for a $100 \mathrm{~km}$ civil engineering project with tunnel and caverns is quite different, if the project is carried out purely as contractor work or if co-innovation is introduced for advanced excavation material analysis, separation, processing and use.

Treating the excavation material as a primary resource rather than waste that needs to be disposed of can lead to significant additional economic benefits of the order of several hundred million $\mathrm{CHF}^{6}$. Today, excavation material is still considered waste, but the necessity to revise CERN's existing international agreement with

\footnotetext{
${ }^{6} 8.5$ million tons of molasse at a value of ca. $35 \mathrm{CHF} / \mathrm{t}$ and 260,000 tons of limestone at ca. $85 \mathrm{CHF} / \mathrm{t}$.
} 

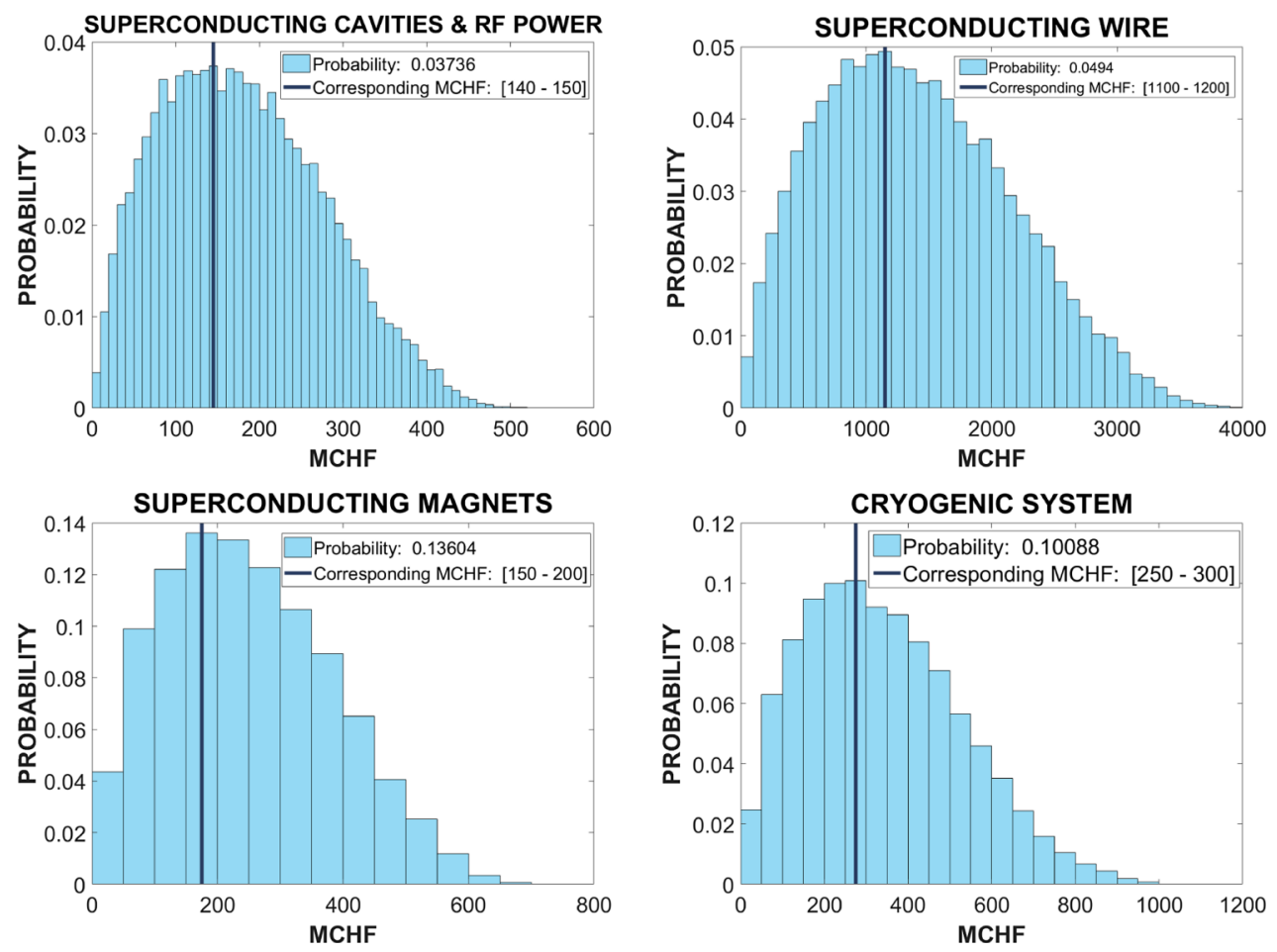

Fig. 11.2. Initial estimates for industrial spillovers of individual technology projects carried out as co-developments with industrial partners for different particle collider scenarios (selected FCC-ee and FCC-hh related technologies). The left upper figure indicates the industrial impact potentials stemming from the construction of superconducting RF and associated powering systems, the right upper figure highlights the potentials that superconducting wire manufacturing can create. The lower left image depicts the potentials stemming from the superconducting magnet project and the lower right image highlights the potentials that a new cryogenic refrigeration system can generate. The $\mathrm{x}$-axis indicates estimates for expected benefits and the y-axis denotes the probabilities of the expected benefits as resulting from 25000 Monte Carlo simulation rounds. The vertical line denotes the overall average expected benefit. Estimates include social discounting and are reported in 2018 currency values.

France and Switzerland and the drafting of the law in France that is needed for the preparatory phase, renders this opportunity a realistic scenario. The GrandParis [460] and the Lyon-Turin railway projects [461] serve as pre-cursors of this approach.

The introduction of Information and Communication Technologies (ICT) [462] are also important levers. One topic is the development of open source software and open ICT specifications that can be used in other scientific domains or for business. The HTML and HTTP specifications that form the basis of the World Wide Web are prime examples [463]. However, since its value for society cannot be reliably quantified, this invention was not considered in the LHC/HL-LHC CBA study. Selected cases for which the impact is currently being estimated include: software tools used in medical imaging $\left(\mathrm{GEANT}^{7}\right)$, collaborative tools $\left(\right.$ Indico $\left.^{8}\right)$, library

\footnotetext{
7 https://geant4.web.cern.ch/applications

8 https://getindico.io
} 

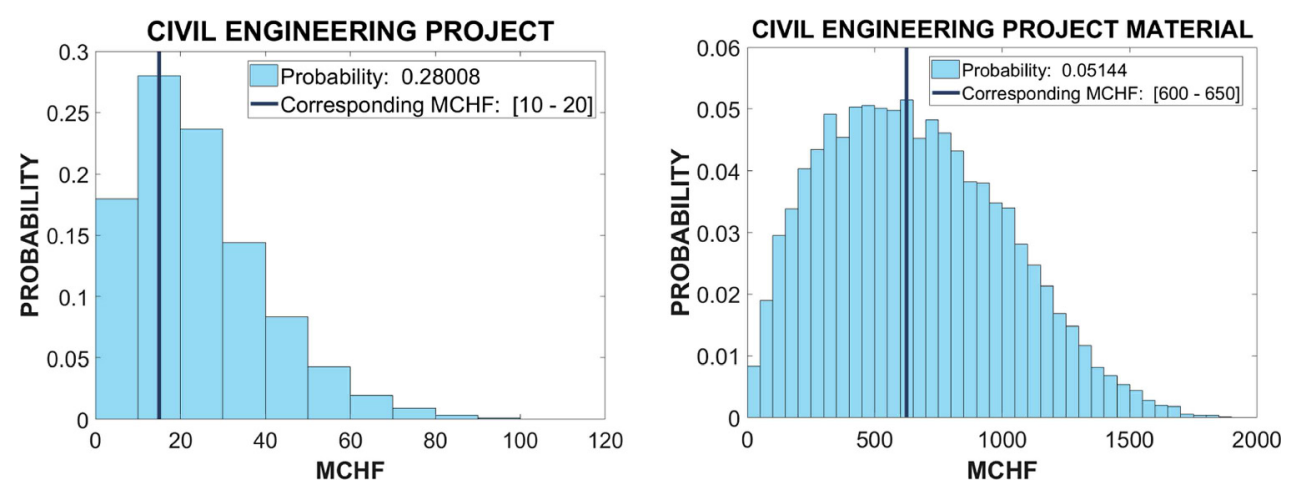

Fig. 11.3. Initial estimates for industrial spillover of the civil engineering project, which is common to both $100 \mathrm{~km}$ particle collider scenarios (FCC-ee and FCC-hh related technologies). The left analysis reports on the industrial spillover opportunities for a classic civil engineering project and the right analysis reports the spillover potentials if innovations such as the use of the excavation materials are introduced during the project phase. The $\mathrm{x}$-axis indicates estimates for expected benefits and the $\mathrm{y}$-axis denotes the probabilities of the expected benefits resulting from 25000 Monte Carlo simulation rounds. The vertical line indicates the overall average expected benefit. Estimates include social discounting and are reported in 2018 currency values.

software $\left(\right.$ INVENIO $^{9}$ ), scalable storage systems (EOS, cernbox [464]), scalable modelling and simulation tools needed to devise reliable and energy efficient industrial systems (ELMAS $[140,141]^{10}$ ), measurement and control systems (for instance addon components and extensions for SIEMENS WinCC OA and National Instruments Labview).

\subsubsection{Cultural effects}

The creation of cultural activities generates economic value and helps to involve the public in research. With 130000 visitors $^{11}$ per year [442], CERN is Geneva's leading tourist attraction. The travel-cost method [465-467] estimates the value of this activity to be $\sim 120$ million CHF per year [468]. Designing future surface sites with this in mind and gradually transferring the responsibility for public involvement to a dedicated operation and marketing organisation leads to a sustainable concept, which can increase this figure. A preliminary investigation of such a concept by one of the FCC collaboration members led already to the award of a prestigious architecture prize [469] (see also Fig. 11.4). First estimates for the net benefits from 180000 visitors (to a dedicated visitor centre at the main site) and 280000 visitors (with an additional visitor centre on French territory) per year suggest additional annual societal benefits of 25-80 million $\mathrm{CH}$ [470]. This initial estimate includes annually assumed costs of 1 million CHF for the operation of these services. NASA's approach for a self-sustaining and independently operated visitor centre, which has been operating since 1967, is evidence for the success of this model [471].

Web and social media have become key communication platforms and their importance is expected to grow quickly with the introduction of new features and full coverage, high-speed mobile networks. The value of the contents directly produced by

\footnotetext{
9 https://invenio-software.org

10 http://www.ramentor.com/products/elmas/

11 See also at https://visit.cern/tours/guided-tours-individuals
} 


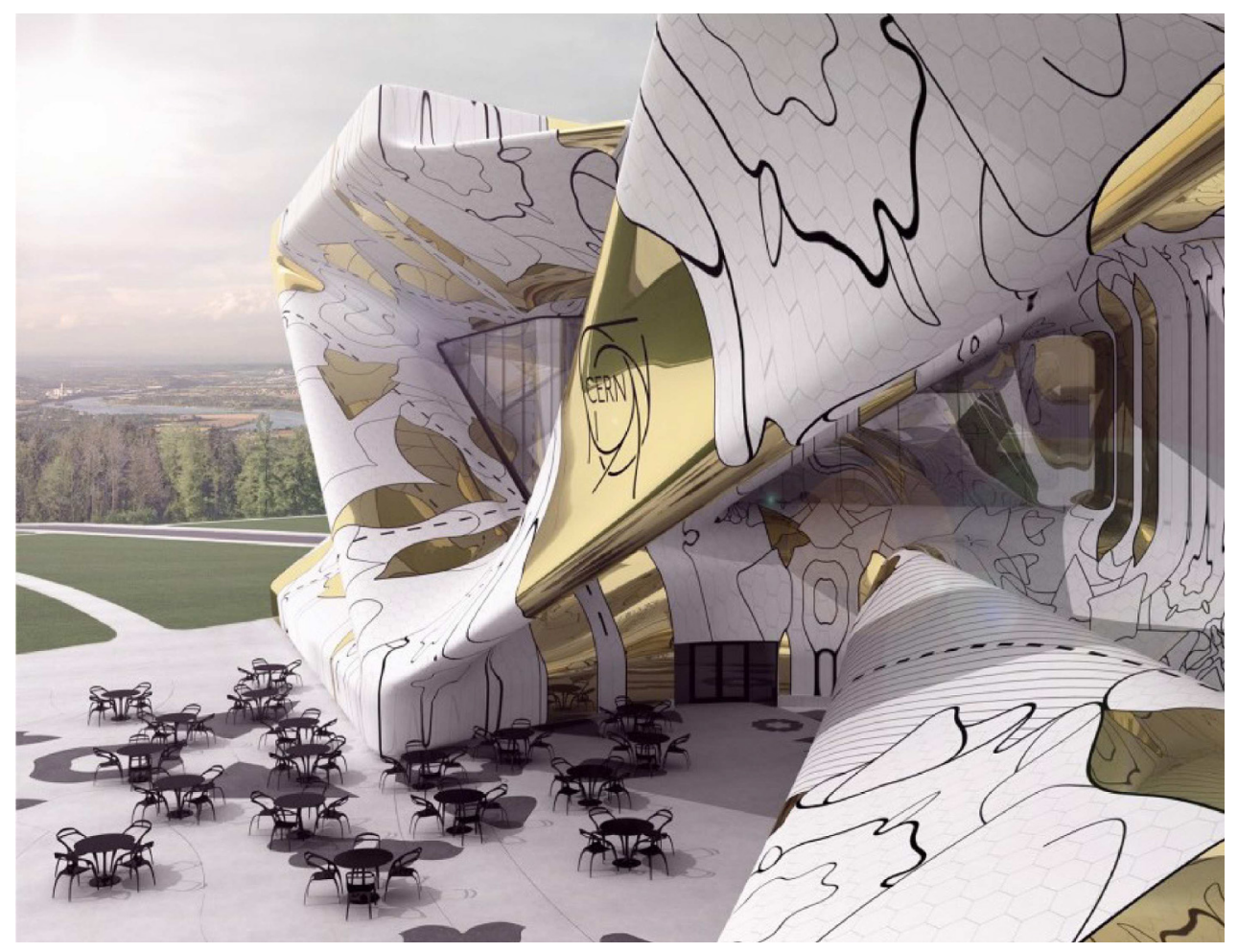

Fig. 11.4. One of many concepts for a visitor centre at a new surface site that FCC collaboration member institute, the University of Michigan, is probing in order to identify paths for working with novel materials, to conceive energy efficient and environmentally friendly buildings, which blend into the region and which can be implemented in cost effective ways.

CERN and the LHC experiment collaborations, measured by the time people spend on average consuming the contents, is estimated to be in the order 120 million CHF per year [470], with videos being the largest contributor in the order of 90 million CHF per year. Channels combining different types of media have the highest impact (text interleaved with short videos, interactive info-graphics, apps, functions that encourage the reader to provide interactive feedback and to participate in collective tasks to shape opinion and to create new contents). The documented impact suggests that investing further in the provision of complementary, media-rich contents permits multiplying today's measured impacts many times. If technology evolutions such as augmented reality, tailored contents for different audiences, multi-language support and high-resolution virtual reality are adopted quickly, the research facility can significantly leverage the cultural effects.

In all cases, the research infrastructure operator should ensure that the content is made available by independent Web and social media content producers via well-working communication networks to achieve high technical quality and user satisfaction. Other powerful cultural products are computer programs ("apps") which engage their users in the research. Higgs Hunters [472] is a good example of such a citizen science project - it attracted 32000 volunteers in a single year. The strong use of this technology by other research communities [473] motivates the development of further cases in the particle accelerator and high-energy physics domains. Computational physics applications as needed for the lepton collider FCC-ee and data analysis challenges as created by the hadron collider are ideal topics to create 
citizen science and public engagement programmes that also help disseminating the concepts of modern physics ("the Standard Model of Particle Physics"), which have so far not reached a wide audience.

Exhibitions such as Extreme [474] in Italy and Begin [475] in Austria, combined with interactive sessions and public events are an effective way to introduce the public to the research carried out in their countries in their own language. Their value, measured by the number of visitors and the time visitors dedicate, has been estimated to be in the order of 4.4 million CHF per year. The most successful item is the travelling LHC interactive tunnel, which alone creates about 1.1 million CHF of societal benefit per year, because it engages a large number of people for substantial amounts of time. When designed and created in cooperation with experienced museums and science exhibition centres in the member states and when accompanied by Web and social media contents that help to amplify the impact, the concept is a powerful vehicle to create a lasting awareness and promote the vision of a future particle collider facility.

The value of numerous books, TV shows, films and music produced by people not directly participating in the research programme undoubtedly has a further economic impact. It is a challenge to provide evidence for a causal relationship between a particular communication product and the research facility's activity. Therefore it is difficult to quantify the impact and the study is ongoing, focussing on video productions, TV and journal coverage and books.

CERN builds an image amongst the population of all member states through its communication activities. Because it exists and deals with questions that touch everybody, CERN has a value for everybody. This value has been estimated with "Willingness To Pay" (WTP) [438] surveys in CERN's member states specifically targeting the topic of a future particle collider. An initial LHC/HL-LHC CBA study suggested a total discounted value of 3.1 billion CHF for the total project period from 1993 to 2038. It is based on the assumption that the average annual voluntary contribution per year and person is 1.5 Euro. For the LHC/HL-LHC programme this very conservative, lower-bound discounted estimate represents about $\sim 12 \%$ of the total forecast impact [317]. However, a recent survey among French taxpayers [476], that has been performed to establish solid assumptions for a CBA of a post-LHC particle collider infrastructure, suggests a much higher WTP of around 4.7 CHF per taxpayer and year. In comparison, French taxpayers contribute to space research through funding of CNES with 35 Euro per year [477]. A number of eligible contributors in CERN member states of the order of 350 million people, needs to be considered for future WTP estimates.

\subsubsection{Impact potential}

An initial set of recommendations to increase the impact of a post-LHC particle collider beyond the science community was developed in the frame of the CBA work carried out during the FCC study. It involved the University of Milano, the Centre for Industrial Studies and the University Roma Tre in Italy, a field study carried out by the company "iddest", which specialises in public consultation processes [478] and finally the common working groups with French [450] and Swiss [479] host state representatives. Some impact pathways are outlined in Table 11.1.

All consultants for relations with the public recommended the creation of a future collider ambassador programme. A dedicated communication plan, regular training and support material for target groups at different levels (institutional, local, regional, national, international) should make project members at all levels and from all sectors effective advocates of a future project. In the member states, this source of 
Table 11.1. Opportunities to generate societal impact with a large-scale particle collider infrastructure.

\begin{tabular}{|c|c|}
\hline Domain & Actions \\
\hline Training & $\begin{array}{l}\text { Common intersectorial education programmes with universities world- } \\
\text { wide, extension of trainee programmes with industry, extension of adult } \\
\text { training in the scope of development and service contracts, strengthening } \\
\text { of the involvement of non-STEM domains in the training programmes. } \\
\text { Reliable reporting of the research infrastructure induced salary premium } \\
\text { will require periodic surveys via the CERN Alumni network, HR offices } \\
\text { of collaborating universities and research centres as well as cooperation } \\
\text { with national and international statistics institutes and selected indus- } \\
\text { trial partners. }\end{array}$ \\
\hline Industry & $\begin{array}{l}\text { Technological competence leveraging projects to increase the probability } \\
\text { of creating products and services from leading-edge technologies devel- } \\
\text { oped in the framework of the new particle accelerator, strengthening } \\
\text { of the participation in the development of standards and specifications, } \\
\text { develop technological spillovers for conventional project domains such } \\
\text { as civil engineering, increase the focus on the development of informa- } \\
\text { tion technologies, open specifications and open source software with use } \\
\text { cases outside the particle accelerator and high-energy physics domain, } \\
\text { closer work with national ministries of economy and industry interest } \\
\text { groups to create denser networks with industrial partners. }\end{array}$ \\
\hline $\begin{array}{l}\text { Cultural } \\
\text { goods }\end{array}$ & $\begin{array}{l}\text { The channels with the highest impact potentials feature multi-media and } \\
\text { on-line contents. It makes sense to further strengthen the presence in this } \\
\text { domain, to complement it with media-rich contents (videos, interactive } \\
\text { applications, augmented reality, citizen engagement). Creation and oper- } \\
\text { ation of high-quality and high-capacity visitor centres by an independent } \\
\text { profit-making organisation that continuously involves the scientists, citi- } \\
\text { zen science projects in collaboration with academic partners worldwide, } \\
\text { promoting exhibitions developed by professional organisations in the } \\
\text { member states, particular projects in other areas of the world (Africa, } \\
\text { Asia, South America). }\end{array}$ \\
\hline
\end{tabular}

information will be the first and most effective communication channel to promote future research, to explain its benefits and challenges and to explain its purpose in simple terms. It is important that this programme also addresses common concerns. It should include a balanced set of ambassadors with different professional, cultural and personal backgrounds. The narratives should focus on what stimulates people's imagination like the origin of the universe, the nature of the matter which we are made of and where we come from.

\section{Strategic research and development}

\subsection{Introduction}

The information presented in this volume presents a feasible concept for a future circular collider. The level of detail corresponds to the conceptual development stage requirements as defined in the European Strategy for Research Infrastructures (ESFRI) methodology roadmap [480,481]. At this level, a number of concepts are presented that can be screened by experts in the domain, funding agencies and other stakeholders. It forms the basis for an implementation project of one of the scenarios and prepares the ground for developing a funding concept. The subsequent 
preparatory phase will focus on the development of the implementation plans, relying on credible designs that need to be based on a set of technologies that enable the research infrastructure to be built.

At this stage, any project of such scale, ambition and with a long-term vision spanning many decades, comprises a number of uncertainties with different probabilities and potential impact. Appendix B compiles the most relevant uncertainties for the specific collider scenario presented in this volume. Before a decision to build is taken, the most relevant technical uncertainties require investments in research and development to bring the technologies to readiness levels that permit the construction and subsequent operation with acceptable project risks. Therefore, the immediate next step, the design phase, will include the development of a technically achievable and coherent blueprint, which successfully responds to the requirements and concepts presented at this first stage.

The R\&D topics presented in this section are considered "strategic", since they represent those key elements, which are considered necessary prerequisites to come to a technical design that can actually be implemented within acceptable time and cost envelopes. The topics are not ranked in any way and many more research topics need to be addressed before arriving at a particle collider technical design, which can meet the physics goals and which can be operated in a sustainable fashion throughout the planned operational period. However, additional topics which are not considered decisive in terms of technical feasibility or operational sustainability are not presented in this volume. This allows the scenario to be screened whilst remaining focused on the physics opportunities, the long-term importance for the worldwide particle and high energy physics community and on the most essential technical feasibility questions. It is assumed that also the existing diverse and vibrant set of $R \& D$ activities in the field of particle accelerator technology will continue and will lead to a converging programme for a future particle collider, nourished by cross-fertilisation of different particle acceleration technologies, design studies and the continuous optimisation of facilities in operation. Some of the enabling technologies are equally important for all three particle collider scenarios (FCC-hh, FCC-ee and HE-LHC).

The following subsections present R\&D plans for these topics:

- 16 Tesla superconducting high-field, dual aperture accelerator magnet.

- Cost-effective and high-performance $\mathrm{Nb}_{3} \mathrm{Sn}$ superconducting wire at industrial scale.

- High-temperature superconductors.

- Energy efficient, large-scale cryogenic refrigeration plants for temperatures down to $40 \mathrm{~K}$.

- Invar based cryogenic distribution line.

- Superconducting septum magnet (to be merged with high-power switching element).

- High-speed, high-power switching system for beam transfer elements.

- Decentralised, high-capacity energy storage and release.

- Particle detector technologies.

- Efficient and cost-effective DC power distribution.

- Efficient treatment and use of excavation material.

\subsection{6 tesla superconducting magnet}

\section{Motivation}

At the design stage of the LHC, the dipole field level was chosen to give the highest collision energy possible with $\mathrm{Nb}-\mathrm{Ti}$ technology, cooling the magnets to $1.9 \mathrm{~K}$ and 
operating them with a $14 \%$ margin on the load line. For the future hadron collider a similar approach is used. The goal is to fill the $100 \mathrm{~km}$ long tunnel with magnets that can provide the highest achievable field using the technology which provides the best cost/performance ratio. With $\mathrm{Nb}_{3} \mathrm{Sn}$ superconductors, hadron beams of $50 \mathrm{TeV}$ can delivered in a $100 \mathrm{~km}$ long circular accelerator with bending magnets with a nominal field of $16 \mathrm{~T}$. This is about twice the $8.3 \mathrm{~T}$ generated by the $\mathrm{Nb}-\mathrm{Ti}$ magnets in the LHC and about $5 \mathrm{~T}$ higher than the $11 \mathrm{~T}$ of the $\mathrm{Nb}_{3} \mathrm{Sn}$-based dipole magnet of the high-luminosity LHC upgrade. The cost of such superconducting magnets is dominated by the amount of conductor. The cost increases rapidly when the operating parameters approach the conductor's critical surface. Consequently, to be able to exploit the full potential of the conductor in order to achieve the required field and quality, the smallest possible margin on the load line needs to be chosen.

\section{Objectives}

The goal is to develop a viable design for a superconducting $16 \mathrm{~T}$, dual-aperture magnet. The design needs to be verified using simulations first and then with short $(\sim 1.5 \mathrm{~m})$ models. Eventually, full-scale models need to be built to validate the design. This programme depends on a separate $R \& D$ programme for the development of a high-performance, cost-effective $\mathrm{Nb}_{3} \mathrm{Sn}$ superconducting wire. For the R\&D phase, it is assumed the FCC-hh will work with the same 14\% margin on the load line as was adopted for the LHC. The programme aims to achieve a margin which is lower than for today's state-of-the-art $\mathrm{Nb}_{3} \mathrm{Sn} \mathrm{HL}-\mathrm{LHC}$ magnets, where the it ranges between 20 and $22 \%$. A main concern is to achieve reliable operation with a margin of $14 \%$ and to reduce it further to $10 \%$. It is also necessary to reduce the amount of superconducting magnet training needed, so that initial test times are shorter and consequently fewer resources are needed. Eventually, operation during the nominal thermal cycle must be demonstrated.

\section{Description of the work}

The programme spans three distinct domains:

1. The development of magnets based on different designs and manufacturing technologies.

2. The design, construction and verification of magnet components and partial magnets for R\&D purposes.

3. The verification and validation of model magnets.

The design studies aim to develop various feasible concepts for a high-field accelerator magnet. The designs will be verified and compared using electromechanical performance simulations including their behaviour under mechanical stress. The design process includes the identification and specification of new structural components for the ferromagnetic yoke and the coil end spacers and wedges. It also includes the development and specification of new resin impregnation systems and the development of advanced internal splicing techniques for the superconducting cables. The design process will also lead to the development of an in-depth understanding of the mechanical conductor and coil stack properties corresponding to the various manufacturing processes (insulation materials and layout, heat treatment and impregnation).

The construction of magnets for R\&D purposes is based on the study of short model coil (SMC), enhanced racetrack model coil (ERMC) and racetrack model magnet (RMM) assemblies. The SMC is used as an intermediate stage to determine the 
effectiveness of the technologies developed for training improvements, conductor insulation and impregnation resins, as well as specific production steps, in particular the adhesion conditions between coil and poles. ERMCs are developed in two variants with non-graded and with graded coils. They facilitate the assessment of strategies for coil manufacture and assembly, namely the interface between conductor and end spacers or conductor and pole, heat treatment and impregnation conditions (volume, stress), internal splices for the graded versions, loading conditions to minimise degradation and training and finally, the management of transitions (layer jump, ends). Eventually, ERMCs will demonstrate that the field level of $16 \mathrm{~T}$ can be achieved with some margin and with limited or no training. The RMM and the ERMC share the same structure but the insertion of an additional coil between the two pancakes of the ERMC creates an aperture of $50 \mathrm{~mm}$ over a straight section of $470 \mathrm{~mm}$ in addition to a $250 \mathrm{~mm}$ layer jump. The structure will be designed to contain large longitudinal rods made of stainless steel or aluminium, tightened in different ways to explore various conditions of longitudinal loading, including extreme situations of nearly rigid boundaries. These magnets will enable the training performance for a straight section of a real accelerator magnet to be determined. In addition, the measurement of the magnetic field in the aperture will be possible, even in the absence of free access from the ends. Finally, the magnetisation effects over the powering cycle, including reproducibility from cycle to cycle as a function of the injection field will be recorded.

By building short models of all the viable options explored by the EuroCirCol H2020 project, the cosine-theta, block coils, common coils and canted cosine-theta designs can be verified under realistic conditions. Eventually, full-scale models of the most promising designs have to be constructed to validate the approach before a decision to launch a construction project for a high-energy hadron collider can be taken.

\section{Collaboration with universities and research institutes}

From the beginning this R\&D programme has been a collaborative endeavour which builds on the committed involvement of universities and research centres from around the world. The EuroCirCol H2020 project initiated a worldwide consortium to develop and explore designs of different high-field accelerator magnets. Research institutes with established track records in the design and development of superconducting high-field magnets need to intensify their contributions further. Model magnets are already planned to be constructed by CERN (international organisation), CEA (France), CIEMAT (Spain), INFN (Italy), PSI (Switzerland) and Fermilab (USA). The design, construction and testing activities include the cooperation of well-known institutes and universities such as EPFL (Switzerland), KEK (Japan), Technical University of Tampere (Finland), Karlsruhe Institute of Technology (Germany), University of Patras (Greece), University of Twente (The Netherlands), University of Geneva (Switzerland), National High Magnetic Field Laboratory (USA), Brookhaven National Laboratory (USA) and Berkeley Lab (USA). In particular, the high-field magnet development programme led by the Department of Energy (USA) will be a crucial factor on the way to determine a viable design for a $16 \mathrm{~T}$ magnet. In the near future, the consortium needs to be enlarged with additional academic partners, in particular universities and institutes in Russia with a track record in the development of high-field superconducting magnets. The consortium also needs the involvement of academic partners in Asia and more regional partners in North America, including Canada. 


\section{Collaboration with industrial partners}

The design and development of the $16 \mathrm{~T}$ magnet relies strongly on the capability to manufacture, install and operate a large number of devices. Therefore, industrial partners will be included at all stages of the R\&D programme, from the development of fundamental technologies from assembly and process-related activities to quality management, installation, maintenance and repair concepts. Codevelopment with industrial partners during the R\&D phase prioritises the following key topics: automated winding systems with integrated quality control, quality improvements and resource optimisation of impregnation and heat treatment, the optimisation of assembly, the optimisation of internal and external interfaces to improve assembly, testing, installation, maintenance and repair. These activities will include the definition of collaborative EC co-funded projects to increase the impact of transferring technological developments from research to industry.

\section{Milestones and deliverables}

Table 12.1. Milestones and deliverables for $16 \mathrm{~T}$ magnets.

\begin{tabular}{|l|l|}
\hline Title and description & Year \\
\hline 4 different electro-mechanical magnet designs studied & 2019 \\
\hline SMC tested & 2019 \\
\hline ERMC tested & 2019 \\
\hline 15 T cos-theta model (US) tested & 2020 \\
\hline RMM tested & 2021 \\
\hline SMC, ERMC, RMM results & 2022 \\
\hline 3 short 16 T models validated & 2023 \\
\hline 1 long 16 T model validated & 2025 \\
\hline
\end{tabular}

\section{3 $\mathrm{Nb}_{3} \mathrm{Sn}$ wire}

\section{Motivation}

The feasibility of a $16 \mathrm{~T}$ superconducting accelerator magnet with two apertures of $50 \mathrm{~mm}$ each depends on the availability of an affordable conductor that can deliver a current density $\left(\mathrm{J}_{\mathrm{c}}\right)$ of at least $1500 \mathrm{~A} / \mathrm{mm}^{2}$ at $4.2 \mathrm{~K}$ and 16 Tesla [482]. If a highenergy hadron collider were to be built as an immediate next step after the LHC/HLLHC programme, $\mathrm{Nb}_{3} \mathrm{Sn}$ is seen today as the only superconducting material that can be produced at the rate and in the quantity needed for a series production of thousands of magnets by 2030 [483]. This material is being produced for use in the magnets of the HL-LHC upgrade project, the first application of $\mathrm{Nb}_{3} \mathrm{Sn}$ technology in a particle accelerator. Industrial production of such wire is presently contracted to one leading company in the field (Bruker) that masters two processes: the Rod Restack Process (RRP(C), a variant of internal tin and powder-in-tube (PIT). A $\mathrm{J}_{\mathrm{c}}$ performance of up to $1200 \mathrm{~A} / \mathrm{mm}^{2}$ at $4.2 \mathrm{~K}$ in a field of $16 \mathrm{~T}$ is achievable at the time of writing this report and is therefore considered to be the current state-of-theart. The push to reach higher critical current density to meet the target requires extensive material science research effort and the development of new manufacturing technologies. 
Table 12.2. Target parameters for the conductor of $16 \mathrm{~T}$ magnet series production.

\begin{tabular}{|l|c|c|}
\hline Parameter & Value & Unit \\
\hline Wire diameter & ca. 1 & $\mathrm{~mm}$ \\
\hline Non-copper $\mathrm{J}_{\mathrm{c}}(4.2 \mathrm{~K}, 16 \mathrm{~T})$ & $\geq 1500$ & $\mathrm{~A} / \mathrm{mm}^{2}$ \\
\hline Copper to non-copper ratio & $0.8: 1$ & \\
\hline Sub-elements effective diameter & 20 & $\mu \mathrm{m}$ \\
\hline Magnetisation $-\mu \mathrm{o} \Delta \mathrm{M}(4.2 \mathrm{~K}, 1 \mathrm{~T})$ & $\leq 150$ & \\
\hline Residual resistivity ration & $\geq 150$ & \\
\hline Wire unit length & $\geq 5$ & $\mathrm{~km}$ \\
\hline Cost $(4.2 \mathrm{~K}, 16 \mathrm{~T})$ & $\leq 5$ & Euro $/ \mathrm{kAm}$ \\
\hline
\end{tabular}

High critical current density is necessary, but not sufficient. Dynamic and adiabatic stability, field quality and protection considerations as well as a need for highcurrent in compact Rutherford cables call for small superconducting sub-elements, low resistivity of the matrix stabiliser and good mechanical properties. The conductor research and development programme launched by the FCC study aims to develop a $\mathrm{Nb}_{3} \mathrm{Sn}$ conductor that can meet all those needs. This programme is based on a network of academic institutes and industrial partners worldwide that are tied together by an FCC R\&D agreement. This network has started to investigate routes to reach the performance goals [169], including the production of R\&D wires by four industrial partners, complemented by advanced characterisation and the investigation of novel approaches by academic partners. The programme aims first to verify different approaches and then to validate a viable technology in a full-scale magnet. For the series production of the particle collider magnets, about $8000 \mathrm{t}$ of superconducting material will be needed. Therefore, the initiative is also a first step in the direction of preparing the ground for a credible large-scale supply chain on a global scale. Affordable wire cost is the ultimate goal of the conductor programme. Since the early research and development phase, effort has been focused on production processes that appear promising in terms of scalability and that show potential for industrial low-cost production. The target maximum cost, derived from magnet design and conductor analysis, is 5 Euro $/ \mathrm{kA}$ at $4.2 \mathrm{~K}$ and $16 \mathrm{~T}$.

\section{Objectives}

The main objective is to achieve a performance of $1500 \mathrm{~A} / \mathrm{mm}^{2}$ at $4.2 \mathrm{~K}$ and $16 \mathrm{~T}$ in order to achieve a $\mathrm{J}_{\mathrm{c}}$ of at least to $2300 \mathrm{~A} / \mathrm{mm}^{2}$ at $1.9 \mathrm{~K}$ and $16 \mathrm{~T}$ - the latter are the operating conditions set for the magnet. The full set of objectives are outlined in Table 12.2.

Smaller sub-element diameters are important to limit losses in coils during transients (acceleration ramp), to ensure dynamic and adiabatic stability and to achieve the required field quality. State-of-the-art $\mathrm{Nb}_{3} \mathrm{Sn}$ wires have sub-elements diameters of about $50 \mu \mathrm{m}$. Obtaining smaller sub-element size together with higher $\mathrm{J}_{\mathrm{c}}$ and high $\mathrm{RRR}$ are requirements are conflicting requirements. This is the challenge that the conductor development programme faces (Tab. 12.3).

\section{Description of the work}

The main focus of the work is the development of a high-performance $\mathrm{Nb}_{3} \mathrm{Sn}$ wire using a variant of the internal tin process. Different wire layouts have to be developed 
and studied in collaboration with industry, including both common and separated (distributed) barrier designs. Industrial partners will produce R\&D billets in order to prototype new methods and layouts, validate industrial scalability and produce wire that industry can also assess for applications beyond particle physics applications.

The conductor programme needs to be pursued concurrently with the magnet development programme, accompanying the development and verification of short and long model magnets. The programme progresses through three stages:

1. Development of novel wire layouts and compositions;

2. Industrial production of medium size billets;

3. Development of cost-efficient, large-scale industrial production of large billets.

Task (1) focuses on studying and developing novel wire layouts and compositions with improved critical current and high RRR properties. The $\mathrm{Nb}_{3} \mathrm{Sn}$ wire successfully produced for the HL-LHC upgrade project does not indicate a way to achieve the target performance through further, gradual optimisation. Therefore, the development a novel process is highlighted in this activity, prioritising improved $\mathrm{J}_{\mathrm{c}}$ and RRR over properties such as the sub-element size. "Internal oxidation" is considered a potential route towards a substantial improvement of the conductor in-field performance. Only limited work has been performed by the community to date, but preliminary results are promising. Both industry and research laboratories have taken up this challenge, but further fundamental research is required.

Task (2) extends from understanding the fundamental material properties in model system configurations to the production of small amounts of wire for research purposes. The development needs to also include wire performance indicators such as the effective filament diameter. Test samples will be produced in simplified configurations (e.g. material layers, mono-filaments) up to small-size billets of a few kilometres for selected routes. Independent academic partners will perform extensive analysis of electro-magnetic, mechanical and thermal properties, as well as advanced analysis such as SEM, TEM and XRD. This research is an iterative process, during which results from wire manufacturing and testing provide feedback for improved designs. Experimental work, materials analysis and testing are at the core of this activity, including optimisation of heat treatments, study of tin diffusion and wire composition, in-depth micro-structural analysis, study of phase transformations, preliminary evaluation of mechanical properties and measurement of in-field electrical and magnetic properties are typical investigations that need to be performed. Experimental work, material analysis and testing/analysis are at the core of this activity.

Task (3), the production of medium-sized billets by industrial partners aims to validate the most promising $R \& D$ layouts in billets of industrial scale, increasing the technological readiness of the most promising designs that emerge from the wire R\&D activity. The goal is to produce a few tens of kilometres of wire. This up-scaling and industrialisation will be accompanied by further wire design improvements in order to meet all additional requirements: stable and controlled magnetisation, filament diameter, mechanical properties. Achieving this objective will require further progress in wire design and layout. It is therefore an iterative development loop. At this stage, in addition to wire measurements and testing, the qualification of wires in Rutherford cables will begin. Short cable samples will be tested and, according to the results, will be used for short model coils. For each wire layout, production of Rutherford cables requires specific developments with iterative studies, production runs, optimisation of cable geometries and heat treatments and electro-mechanical and magnetic measurements.

The time necessary to perform tasks (1) and (2) is expected to cover a period of about ten years. During these phases, the production of about $1.5 \mathrm{t}$ of conductor per year is needed to be able to meet the requirements for testing, development 
of cables and short model coils and to build short magnet models. The production and measurement of model coils is essential to provide feedback to the conductor programme. Conductor and magnet $\mathrm{R} \& \mathrm{D}$ therefore proceed in a synchronised manner and are closely interlinked.

Task (4), covers the implementation of cost-efficient, large-scale production of large billets. The industrial production processes need to be developed and validated for wire architectures that meet all objectives, including the full target performance. This part of the programme aims to show the feasibility of the production of long conductor lengths of up to $5 \mathrm{~km}$ in a cost-effective manner. Ultimately, large quantities, in the order of hundreds of kilometres of wire with the target characteristics will be required to feed the optimised Rutherford cable production for the long model magnets and prototype magnets.

\section{Cooperation with universities and research institutes}

From the beginning, this R\&D programme has been a collaborative endeavour requiring the committed involvement of universities and research centres world-wide. The EuroCirCol and EASITrain H2020 projects catalysed the establishment of a solid set of committed academic partners to make progress in fundamental materials research.

The Technical University of Vienna (Austria) is leading the microstructural analysis of superconducting wires by electron microscopy, including analysis of tin concentration gradients, artificial pinning and correlations of superconducting properties, to support wire optimisation. Technical University Bergakademie Freiberg (Germany) has launched a project to investigate phase formation in the ternary $\mathrm{Cu}-$ $\mathrm{Nb-Sn}$ system. In its fundamental material research programme, the University of Geneva (Switzerland) is exploring ways of reducing the grain size using an internal oxidation method, thus advancing wire performance. This is complemented by a project with the Applied Superconductivity Center at Florida State University in the US, which is investigating novel alloys and internal oxidation methods, and will propose routes for industrial scale-up. Wire development and analysis in Japan is coordinated by KEK, the high energy accelerator research organization, as part of a collaboration agreement with CERN. This programme includes the participation of manufacturers (JASTEC and Furukawa) and academic partners, including the University of Tohoku. The Bochvar Institute (Russia) is experimenting with various layouts of internal tin wires which show a significant performance increase, currently reaching $1200 \mathrm{~A} / \mathrm{mm}^{2}$ at $16 \mathrm{~T}$ and $4.2 \mathrm{~K}$.

\section{Cooperation with industrial partners}

The establishment of a representative set of industrial partners who participate in the improvement of $\mathrm{Nb}_{3} \mathrm{Sn}$ wire performance is vital for the preparatory phase of a highenergy hadron collider project. Therefore, in 2016 the FCC collaboration launched a conductor R\&D programme involving industry. To date JASTEC and Furukawa in Japan, KAT in South Korea, TVEL, including the Bochvar Institute in Russia and Bruker EAS in Germany have joined the effort. The participation of Luvata Pori in Finland is planned. Three of these partners have already produced R\&D billets achieving the $\mathrm{J}_{\mathrm{c}}$ performance specified for HL-LHC with wire layouts not previously explored for this application, establishing a good baseline for further development towards the FCC performance target. 
Table 12.3. Milestones and deliverables for $\mathrm{Nb}_{3} \mathrm{Sn}$ wire.

\begin{tabular}{|l|l|}
\hline Title and description & Year \\
\hline Multi-phase R\&D initiative set up on an international scale & 2019 \\
\hline Routes to develop performance improvements selected & 2021 \\
\hline $\begin{array}{l}\text { Methods and processes to increase the current density towards the tar- } \\
\text { get performance validated by industry }\end{array}$ & 2024 \\
\hline Target performance demonstrated & 2025 \\
\hline Industrial process for large scale production developed & 2028 \\
\hline Scale-up and preparation of series production achieved & 2030 \\
\hline Procurement start & 2030 \\
\hline
\end{tabular}

\section{Milestones and deliverables}

The joint research initiative with the HL-LHC project and the progress in conductor development during the FCC study period has created confidence in the strategy and schedule for developing $\mathrm{Nb}_{3} \mathrm{Sn}$ wire meeting the performance requirements. A workshop at CERN in March $2018^{12}$, hosted a large and active group of participants from academia and industry. During this workshop, encouraging initial results were presented and reviewed. The commitment of industrial partners, and the confidence of participants that the FCC performance and cost targets are ultimately achievable, supported detailed discussion in closed sessions and the definition of a focused plan for the continuation of this R\&D initiative on an international scale.

\subsection{High temperature superconductors}

\section{Motivation}

The accelerator dipole magnet under design for the FCC-hh which is based on $\mathrm{Nb}_{3} \mathrm{Sn}$ technology would generate the highest possible field that can be reached by low-temperature superconductor (LTS) materials. High-temperature superconductor (HTS) materials are the only technology that can lead to accelerator magnets with fields beyond the $16 \mathrm{~T}$ limit. In addition, HTS materials come with the benefit of a high temperature margin during operation, making them compatible with scenarios that are subject to high heat load and high radiation levels. If the LHC/HL-LHC research programme is followed by an intensity-frontier lepton collider (FCC-ee) with a physics programme duration of about 15 years, a new time window appears for the development of high-field accelerator magnets based on HTS for an energy-frontier hadron collider at a subsequent stage.

Besides research, industry is also considering HTS materials such as ReBCO and BSCCO for diverse applications. The main motivation is the potential of operation at higher temperatures than those that are used today in industrial and medical devices. Although refrigeration based on liquid helium is feasible even for a large research facility such as the FCC-hh, a cryogenic plant for industrial applications imposes cost and maintenance constraints that limit the application of superconducting technology. Higher operating temperature simplifies the system and eases the diffusion into commodity markets.

A higher operating temperature is also relevant for a large-scale particle collider. The cryogenic efficiency of the refrigerator would be improved, though this effect is

12 See https://indico. cern.ch/event/698917 
not directly proportional to the temperature increase. A high cryogenic temperature cooling scheme (e.g. supercritical helium flow) may be less efficient than the current scheme based on superfluid bath cooling. The interest comes from the potential to intercept synchrotron radiation, the major source of heat load in the hadron collider, at a higher temperature.

The higher field reach opens routes to energy upgrades of particle colliders with magnets reaching fields of $20 \mathrm{~T}$ and beyond. The peculiar properties of HTS materials lead to a situation in which the challenge of designing the magnet system will shift from field reach and current limitations to mechanical aspects, protection systems and cost-effective design. The latter are topics which are easier to manage with modern design tools and manufacturing approaches.

HTS materials are still prohibitively expensive with volumetric costs that are at least an order of magnitude higher than state-of-the-art low temperature superconductors. However, with larger quantities being manufactured, with materials beginning to mature and considering the Total Cost of Operation (TCO), break-even is expected to occur within the horizon of a future hadron collider even at price levels slightly above those of the $\mathrm{Nb}_{3} \mathrm{Sn}$ target.

\section{Objectives}

The first objective of an R\&D programme on alternative superconductors is to identify those materials which have the highest probability of meeting the target performance for the high-field accelerator magnets. At the same time they must be produced at a cost which leads to an overall TCO (combined CAPEX and OPEX) that is much lower than a $\mathrm{Nb}_{3} \mathrm{Sn}$ based machine. Current potential candidates for different use-cases in the particle accelerators include rare-earth barium-copper oxide ReBCO (YBCO) and Bi-2212, and possibly iron-based superconductors (IBS) if their high-field performance can be demonstrated within a reasonable time scale. These materials all pose major technological challenges for their use in accelerators. The main challenges for ReBCO are cable assembly, protection and field quality, while for BSCCO the magnet manufacturing with a high-temperature step and mechanics are the main concerns.

Today, work is focusing on developing HTS wires that can be produced in quantities that are sufficient to build accelerator-quality magnets in the $3-5 \mathrm{~T}$ range. The aim is to approach $8 \mathrm{~T}$ before the end of the decade. The levels of technological readiness are heterogeneous for the different materials: while for some coated conductor tapes the investigations already aim at larger-scale quality production and achieving usable fields and adequate field qualities for particle accelerator magnets, novel conductors such as IBS are still to be considered laboratory developments for which research focuses on fundamental material properties. Once the candidates are defined, a well-focused R\&D programme has to be established to drive the development of conductors to achieve the required high fields and towards production processes for wires or tapes in sufficient quantities of adequate quality.

\section{Description of the work}

This research, which today still needs to be classified as basic, for the validation of basic components (e.g. the coils) in laboratory environments will need to work in the following directions in order to demonstrate high-fields at accelerator-magnet grade field quality at acceptable total cost of ownership: 
- Identify the most promising materials and the main challenges associated with them in terms of performance, production and cost. Set long-term development targets for overall performance (electrical, mechanical) and realistic cost for each variant;

- Define performance targets and develop a suitable conductor for magnets, starting either from tape or wire, depending on the material. A specific challenge is finding a suitable cable topology for material that comes in tapes (ReBCO, IBS, Bi2223). Specifically for Bi-2212, develop manufacturing, insulation technologies and industrial reaction processes for large coils that suit the highly brittle material;

- Develop a design for a magnet system with the required field quality from injection (low field) to collision (high field) and which manages the mechanical and protection aspects. Study several HTS material options and identify the leading technology questions for each of the variants;

- Understand how materials, cables and coils behave under high stress and strain conditions;

- Acquire an in-depth understanding of the quench behaviour in order to identify suitable approaches for quench detection and quench protection. HTS materials behave differently to LTS and today's methods do not seem appropriate for these materials. In particular, the larger temperature margin may avoid the training phase and by developing early detection of quench onset it would be possible to avoid a fully developed quench. This would shorten commissioning and significantly improve accelerator availability;

- In the long-term, develop suitable high-volume and low-cost manufacturing routes and techniques for HTS materials. Demonstrate the manufacture of tens of kilometres of conductor at acceptable quality and sufficient homogeneity.

\section{Cooperation with universities and research institutes}

The ongoing R\&D on $\mathrm{Nb}_{3} \mathrm{Sn}$ and the investigations of HTS for beamscreen coatings has already created a core of collaborating academic partners, which can also serve as the starting point for a well-focused, long-term high temperature superconductor R\&D programme. It federates universities with knowledge in fundamental material sciences and research centres worldwide with the possibility to build up laboratories and infrastructures to perform such a long-term research programme. However, a coordination body is yet to be defined and set-up. This body will ensure that the research and development is carried out in a topically complementary fashion with a clear convergence towards the objective and with clearly defined knowledge ownership and access policies.

The set of loosely-associated academic partners today includes Bochvar Institute (Russia), CERN, CNR-SPIN (Italy), EPFL (Switzerland), ETH Zurich (Switzerland), Florida State University (USA), FNAL (USA), KEK (Japan), KIT (Germany), LBNL (USA), NHMFL (USA), PSI (Switzerland), TU Bergakademie Freiberg (Germany), TU Wien (Austria), CEA (France), cooperative efforts of various INFN institutes (Italy), University of Geneva (Switzerland) and University of Genoa (Italy).

\section{Cooperation with industrial partners}

The creation of a long-term, forward looking technology advancement programme also permits SMEs to play a leading role at the forefront of a technology and provides opportunities to grow with the technology in a risk-controlled environment. In addition to established players such as Bruker, Fujikura, Nexans, Sunam, SuperPower, 
SuperOx and Theva companies like nGimat and MetaMateria and other companies worldwide have started to participate in the investigations. As with the academic partners, a defined governing structure and the definition of a well-scoped R\&D consortium that coordinates the activities on a global scale and which works towards openly accessible technologies for the research and industrial product market remains to be established. As with the academic partners, the governing structure should be centred around strong technical involvement of CERN, profiting from existing national and international funding mechanisms and opportunities.

\subsection{Efficient and cost-effective cryogenic refrigeration}

\section{Motivation}

Satisfying the cryogenic refrigeration requirements of a future hadron collider with the traditional technologies in use today at the LHC is not possible. The FCC study therefore launched a long-range $R \& D$ programme at an early stage to develop an alternative approach. The current machinery for helium refrigeration is based on warm screw compressors which have a unit capacity more than 10 times smaller than that required for the FCC-hh and they exhibit a poor isothermal efficiency of about $50 \%$. Since pure helium cannot be compressed easily, a novel concept based on a mixture of about $75 \%$ light helium and $25 \%$ heavier neon (known as "nelium") is being developed for cooling down to $40 \mathrm{~K}$ in a scalable and cost-effective way. The light gas mixture together with a high isothermal efficiency centrifugal compressor reduces the number of cooling stages required from 20 to 14, leading to power and cost savings of $\sim 16 \%$. This translates into savings in operating expenditure of almost $20 \mathrm{MCHF}$ per year. Building on recent advances, a novel turbo compressor with fewer moving parts, less lubrication and a sealed design for light gas mixtures will boost reliability and efficiency.

\section{Objectives}

The primary objective is to showcase a full scale nelium cryogenic refrigeration system. The nelium cycle has been studied conceptually but the practical feasibility remains to be demonstrated. In order to do this, the core components, specific centrifugal compressors for light gases with up to $80 \%$ of isoentropic efficiency and with larger unit capacities, need to be designed as an alternative to the screw-based compressors.

\section{Description of the work}

In parallel with the simulations of a nelium cycle-based refrigeration architecture, design studies and a limited-scale test bed for turbo-compressor machinery is currently being established in the framework of the EASITrain H2020, EU funded, project. To demonstrate that the nelium cycle is viable, a dedicated test bed must be built with the smallest centrifugal compressor available today. Such a machine would be about one third of the size of the full-scale system required for FCC-hh and will consume about $5 \mathrm{MW}$ of electrical power. The project goals are:

- validating the concept,

- determining the optimum helium/neon mixture,

- optimising the overall efficiency,

- determining the availability of such a system,

- identifying the turndown capacity of the machinery. 


\section{Cooperation with universities and research institutes}

In the last few years a set of academic partners including the Technical University of Dresden (Germany), the Technical University Stuttgart (Germany) and CEA Service Basses Temperatures Grenoble (France) has been formed to push this technology forward. As the R\&D progresses, further academic partners will be attracted and they can become involved in additional activities such as materials for machinery, dedicated system simulation including fluid dynamics, reliability and availability engineering, business case development, automation technologies, mechanical engineering and much more.

\section{Cooperation with industrial partners}

As the development of the technology advances towards general implementation, it becomes an interesting test bed for the industrial partners who drive compressor machinery technology forward (e.g. companies MAN Turbo and Atlas Copco). Eventually, when the programme approaches the practical demonstration of the nelium cycle, companies who are leaders in cryogenics such as Linde and Air Liquide will be interested in the developments for their own use.

\section{Impact potential}

The main impact of this technology is the production of a large-scale cryogenic refrigeration system, which will make the FCC-hh feasible. Beyond that goal, the development of an entirely novel refrigeration technology can have a substantial economic impact. The potential extends to hydrogen liquefaction, considered the most difficult liquefaction process but a key technology to facilitate a shift to clean energy production. In general, any application that requires highly efficient and reliable compressors will directly profit from this R\&D. Examples include the production of liquid oxygen for space transport systems, industrial production of metal, natural gas production and transport.

\section{Milestones and deliverables}

The milestones and deliverables are presented in Table 12.4.

Table 12.4. Milestones and deliverables for cryogenic refrigeration.

\begin{tabular}{|l|l|}
\hline Title and description & Year \\
\hline Nelium cycle modelling and simulation results available & 2019 \\
\hline $\begin{array}{l}\text { Test station and compressor prototype design and specifications avail- } \\
\text { able }\end{array}$ & 2020 \\
\hline Test station operational & 2021 \\
\hline Results from experience with test station available & 2023 \\
\hline Architecture for a demonstrator with optimised machinery available & 2024 \\
\hline Designs of the machinery for a demonstrator available & 2025 \\
\hline Demonstrator operational & 2026 \\
\hline $\begin{array}{l}\text { Results available for co-design of full-scale nelium plant with leading } \\
\text { industries }\end{array}$ & 2027 \\
\hline
\end{tabular}




\subsection{Cryogenic distribution line}

\section{Motivation}

The state-of-the-art large cryogen distribution systems deploy stainless steel headers equipped with compensation units and fixed/sliding points in order to accommodate the $3 \mathrm{~mm} / \mathrm{m}$ contraction during the cool-down phase. The $100 \mathrm{~km}$ long cryogen distribution line requires the compensation of a total thermal contraction of $300 \mathrm{~m}$ (c.f. $90 \mathrm{~m}$ for the HL-LHC collider). In the conventional design, the compensation units and the sliding points are the main source of failure (buckling, leaks). In order to achieve a feasible technical design and good reliability, R\&D for a new approach is required.

\section{Objectives}

Designing the internal cold headers based on invar is one way to have a system that is technically feasible and is sufficiently robust. Invar is a nickel-iron alloy, invented in 1896, notable for its uniquely low coefficient of thermal expansion. Its inventor, physicist Charles Edouard Guillaume received the Nobel prize for his discovery in 1920. Using this material, compensation units and sliding points are not required and consequently, the overall reliability of the system increases.

\section{Description of the work}

In order to validate the approach, a $100 \mathrm{~m}$ long demonstration example of a compound cryogenic distribution line needs to be designed and studied under realistic operating conditions. This project comprises the proof of feasibility of production (e.g. definition of welding procedures), the development of a mechanical and thermal design that suits the various operation modes (cool-down, steady-state, warm-up) and the gathering of detailed information about the reliability of the system over long periods of operation.

\section{Cooperation with universities and research institutes}

Several universities who are active in the field of low temperature engineering are candidates for this project. In particular, Wroclaw University of Technology (Poland) has a background in this domain. The possibility to set up a large-scale test facility is also an ideal opportunity for universities of applied sciences, higher technical schools and apprentices to participate in an international technological research project which has industrial collaboration.

\section{Cooperation with industrial partners}

The nature of this project with its close links to industry creates a favourable environment for a collaborative project with industry. In addition to the leaders in cryogenic systems, Air Liquide (France) and Linde (Switzerland), other companies such as Alstom (Switzerland), Kraftanlagen (Germany), Messer-Griesheim (Germany), Nexans (Germany), Nordon (France), as well as ancillary equipment providers such as Criotec (Italy) can all contribute to the project. 


\section{Impact potential}

The large-scale demonstration of the Invar based approach can translate into direct benefits for those industries which deliver cryogenic refrigeration plants and infrastructure. In addition, the knowledge gained by participating universities, schools, apprentices and industrial engineers is a notable facet of this $R \& D$. The outcome should be an expansion of the market and an increase of competitiveness through the adoption of this validated advanced technology.

\section{Milestones and deliverables}

A set of milestones and deliverables are presented in Table 12.5.

Table 12.5. Milestones and deliverables for the cryogenic distribution line.

\begin{tabular}{|l|l|}
\hline Title and description & Year \\
\hline Requirements and design & 2019 \\
\hline Prototype specification and test-bed definition & 2020 \\
\hline Test bed operational & 2022 \\
\hline Prototype built and installed & 2023 \\
\hline Tests complete & 2024 \\
\hline Concept validated and recommendations formulated & 2025 \\
\hline
\end{tabular}

\subsection{Superconducting septum magnets}

\section{Motivation}

The extraction system is a key component of the collider which ensures safety of operation. Conventional normal conducting septa are limited to magnetic fields of around $1.5 \mathrm{~T}$. To transfer beams with very high energies at the FCC-hh or HE-LHC this limitation would lead to power-intensive systems which occupy a large amount of space. Superconducting septa reaching field levels of 3-4 T will allow significantly more compact injection and extraction systems, will simplify straight section and overall system design and will also greatly reduce the operating cost by having a lower electrical power consumption. The development of such an accelerator device is therefore considered a key research topic in enabling technologies.

\section{Objectives}

This specific R\&D project aims to design, build and validate a short $(\sim 0.5 \mathrm{~m})$, accelerator-quality prototype magnet based on the flux-shield principle. It can be used to demonstrate the validity of the concept and will have the following target parameters: $20 \mathrm{~mm}$ aperture for the circulating beam, $30 \mathrm{~mm}$ aperture for the extracted beam, $3.2 \mathrm{~T}$ field, $1 \%$ field homogeneity and an apparent septum thickness of $25 \mathrm{~mm}$.

\section{Description of the work}

The project consists of the following steps:

- Design of the shield, including simulation of the element. 
- Investigation of quench protection aspects and design of a viable quench protection system.

- Construction of a $700 \mathrm{~mm}$ long, $\mathrm{MgB}_{2}$-based, superconducting half-moon shield.

- Test of shield performance in an HL-LHC CCC corrector prototype.

- Design of a dedicated CCT septum magnet incorporating the half-moon shield.

- Construction and test of a CCT magnet.

- Construction of a Ni-Ti/Cu multilayer shield (optional).

- Test of shields in the CCT septum magnet.

- Assessment of the septum system performance.

\section{Cooperation with universities and research institutes}

This well-defined and self-contained R\&D package is an ideal candidate for a collaborative research activity. Wigner Institute (Hungary), having already accumulated experience in this field of superconducting technologies, would lead the design and simulation activities as well as the manufacture of the CCT magnet. CERN would provide essential components, expertise in superconducting magnet design and quench protection systems as well as a cryogenic test facility (SM18). The project is open to further academic partnerships in the fields of superconducting magnets, instrumentation and field testing, electronics and software development.

\section{Cooperation with industrial partners}

Potential industrial partners for this topic are in the process of being identified. Engious Ltd. (Hungary) for instance, is an advanced manufacturing company that could develop the precision tooling and mechanical assembly methods for the shield and magnet. Additional high-tech companies could be partners for the provision of services for ancillary equipment components and the testing facility.

\section{Impact potential}

In addition to the design of a viable extraction system of the FCC-hh or the HELHC, applications include the FAIR facility at GSI (Germany) and a new extraction system at the potential new superconducting SPS accelerator at CERN. The codevelopment with a fast growing high-tech manufacturing company would provide the partner with the possibility of gaining experience in an international project and thus building its reputation and allowing it to enter new markets. In particular the acquisition of expertise in the handling of the superconducting material $\mathrm{MgB}_{2}$, which is increasingly used in medical imaging devices and in the domain of lossless power transmission will help to improve the competitiveness of the R\&D partners.

\section{Milestones and deliverables}

A preliminary set of milestones and deliverables is shown in Table 12.6. 
Table 12.6. Milestones and deliverables for superconducting septum magnets.

\begin{tabular}{|l|l|}
\hline Title and description & Year \\
\hline $700 \mathrm{~mm} \mathrm{MgB}_{2}$ shield manufactured & 2019 \\
\hline Shield tested in HL-LHC CCT corrector in SM18 at CERN & 2019 \\
\hline Design of CCT septum dipole available & 2019 \\
\hline CCT septum dipole constructed & 2020 \\
\hline Septum assembly tested in SM18 at CERN & 2020 \\
\hline Ni-Ti/Cu multilayer shield manufactured & 2020 \\
\hline Ni-Ti/Cu septum assembly tested in SM18 at CERN & 2021 \\
\hline Assessment of superconducting septum documented & 2022 \\
\hline
\end{tabular}

\subsection{Solid state generators}

\section{Motivation}

Modular, scalable, fast and affordable high-power switching systems are key components of beam transfer systems. The current gas-filled thyratron switches are becoming increasingly outdated and their future commercial availability is already uncertain. In addition, they suffer drifts in performance and have high operational complexity. Solid-state switches are the ideal technology for large systems and for systems with high operational availability requirements. However, such highperformance systems are not yet commercially available.

\section{Objectives}

This R\&D programme progresses along three well-aligned paths:

1. Fundamental research on switches aims to increase the performance of individual switching SiC, dynistor and GTO modules. This activity includes the development of the associated high-speed, reliable triggering methods such as impact ionisation, laser and potentially other optical methods, which are robust against spurious activation. The developments aim to operate the switches reliably in radiation environments, therefore one part of the work will deal with understanding and increasing the radiation-related performance limits. Finally, this package needs to carefully cover all protection-related issues.

2. A novel inductive adder generator with fast rise-time and high-voltage for shortpulses for use with terminated and short-circuit loads needs to be developed.

3. A Marx generator for slower rise time, lower voltages and longer pulses for use with terminated and short-circuit loads, needs to be developed.

Description of the work

The project consists of the three steps described below.

For fundamental research and development on switches, the following activities are needed:

- Evaluate the basic performance of single devices such as MOSFET, SiC, dynistor and GTO.

- Improve the performance of stacked arrays and single-switch applications. Verify the switching time, voltage, current and resistance.

- Establish the potential performance reach of the individual technologies from the technology assessment and improvement activities. 
- Develop appropriate triggering methods that match the individual device characteristics, stacking topologies, EMC and performance needs. In particular, drive the development of laser triggering and investigate the potential of impact ionisation triggering for GTOs.

- Determine the characteristics of the different switching modules for use in radiation environments. To achieve this, advances in the measurement techniques for radiation-related performance analysis are required.

- Develop fast, scalable, modular systems that meet the new protection requirements of single and stacked switches, especially in view of adaptation to shortcircuit loads.

For the inductive adder generator development, the following activities are needed:

- Design and build an inductive adder generator with a target pulse length of $1 \mu \mathrm{s}$ to be evaluated in the PS complex at CERN (e.g. test on the ion injection kicker).

- Set up a demonstration device consisting of a series of generators to gain experience with the operational behaviour of a large distributed system.

- Based on the findings, develop the detailed specifications for a complete system including non-functional requirements such as operation, maintenance and repair, safety and industrial supply.

For the Marx generator, the following activities are needed:

- Identify institutes for a collaborative project for the construction of a Marx generator with a target pulse length of $10 \mu \mathrm{s}$.

- Design and build the generator. Deploy it in the PS complex at CERN to gain experience in operating such a system.

- Based on the findings, develop the detailed specifications for a complete system including non-functional requirements such as operation, maintenance and repair, safety and industrial supply.

\section{Cooperation with universities and research institutes}

The individual, well-defined and self-contained parts of this R\&D package are ideal candidates for collaborative research activities. Several institutes such as ETH Zürich (Switzerland), various Helmholtz Gesellschaft institutes (Germany), the Karlsruhe Institute of Technology (Germany), the Institute of Electrophysics (Russia), the University of Manchester (UK) and Lisbon University (Portugal) have already been identified as partners in this programme. The project is open to further academic partners in the fields of high-power semiconductor technology, electronics and embedded systems development.

\section{Cooperation with industrial partners}

Potential industrial partners are in the process of being identified. ABB (multinational with headquarters in Switzerland) is a potential co-innovation partner for switches and EnergyPulseSystems (Portugal) for modulators. Additional high-tech companies could be partners to provide services for ancillary equipment and for system integration.

\section{Impact potential}

In addition to ensuring the long term future of kicker systems for particle accelerator laboratories (e.g. ALBA-CELLS, ESRF, INFN labs, ISIS, KIT, SOLEIL, GSI, PSI, FNAL, BNL, JPARC to name but a few), developing this innovative technology with industrial partners has many potential applications in industry: wherever 
high-energy (voltage and current) pulses are needed, the technology can be deployed. Examples include high-speed forming systems, such as electro-hydraulic magnetic forming and magnetic pulse forming, which has become wide-spread in the automotive and aerospace industries, and for metallic forming processes of commodity goods, radar power supplies and medical x-ray devices.

\section{Milestones and deliverables}

A preliminary set of milestones and deliverables is outlined in Table 12.7.

Table 12.7. Milestones and deliverables for solid state generators.

\begin{tabular}{|l|l|}
\hline Title and description & Year \\
\hline $\begin{array}{l}\text { Establishment of a consortium comprising academic and industrial } \\
\text { partners }\end{array}$ & 2019 \\
\hline Technology survey available & 2020 \\
\hline Procurement of components for test systems completed & 2020 \\
\hline Laboratory test benches operational & 2021 \\
\hline Radiation tolerance assessment complete & 2022 \\
\hline Performance limitations for device triggering evaluated & 2022 \\
\hline Switch and adder system designs and test procedures complete & 2023 \\
\hline Prototype systems ready for deployment in a particle accelerator & 2023 \\
\hline Experience from practical operation documented & 2025 \\
\hline System specifications available (requirements, architecture, design) & 2026 \\
\hline
\end{tabular}

\subsection{Particle detector technologies}

\section{Motivation}

The particle detectors at future circular lepton and hadron colliders will require significant performance advances and the development of cost-effective and commercially available devices using new detection technologies. Since this is an ongoing process of improvements that have to be validated in operating environments with a continuous effort to develop ever more precise and faster particle detection, a significant amount of preparative R\&D will already be performed in the frame of the LHC/HL-LHC experiment upgrade projects.

A targeted detector $\mathrm{R} \& \mathrm{D}$ programme needs to be defined once a particular particle collider scenario is selected for in depth design and when the corresponding experiment projects have been defined.

Therefore, the focus here is on a brief description of the ongoing effort and initiatives which underline the continuing need to support collaborative particle detection research in programmes at academic institutes worldwide. Such initiatives will lead to the development of technologies which will be used in the LHC/HL-LHC experiments and will therefore create lasting impacts beyond individual efforts. Proof-of-concept and fundamental technology research will accompany these developments and the results will flow into the development of technical proposals for specific projects. These proposals will become part of a focused particle detector research programme following the selection of a specific particle collider scenario. 


\section{Objectives}

At CERN, a strategic R\&D initiative, to define and organise the detector technology related research activities for the coming years, was launched in 2017 [484]. It covers the following topics:

1. Silicon sensors;

2. Gas detectors;

3. Calorimetry and light based detectors;

4. Mechanical supports for detectors;

5. Integrated circuits;

6. High-speed data links;

7. Simulation and analysis software;

8. Detector magnets.

These technological aspects will be accompanied by detector performance studies in order to have a clear understanding of the improvement potential for various detection technologies and to be able to quantitatively document the further improvement potential and to identify potential limits of the various technologies. Only at that stage can the detector concepts developed for the hadron and lepton particle colliders be simulated with a sufficient degree of fidelity. The results will give a better understanding of the physics performance that can be expected and will identify the technological gaps and how they can be overcome. This activity will eventually lead to the specification of the function, performance, reliability and other qualitative requirements that form the foundation of the technical design of the experiments.

\section{Description of the work}

The CERN coordinated detector technology research initiative spans a five-year period. Regular workshops are already taking place (e.g. [485]) to develop the detailed research programme and to prioritise the work together with an open, international consortium of academic institutes. The programme also aims to identify possible involvement of industrial partners from a very early stage to understand which technologies can eventually be produced on an industrial scale and which technologies industry is particularly interested in.

The initiative is structured in several working groups, each one focusing on a particular technological domain. This setup forms the nucleus of a converging activity of international research institutions and universities which provides the opportunity to involve partners from all over the world.

The depleted CMOS technology attracts a lot of interest in the domain of silicon detectors, as it allows low-mass and high-resolution sensors, which have the potential to cover large areas at affordable cost, to be built. In recent years, substantial progress was achieved in terms of radiation hardness, a key requirement for all future experiments. Another trend is in low gain avalanche detectors (LGAD), which have achieved timing precision in the range of a few tens of picoseconds. Finally, there is also a strong need to continue the development of classic silicon detectors (pixels, strips and pads) and the associated electronics.

Gas detectors will continue to play an important role in future experiments as they can cover large areas at moderate cost. Micro pattern technologies have boosted their performance and a wealth of new ideas in terms of materials, production techniques and readout methods, show how this success story can be pursued. An important question concerns the choice of the gases and their compatibility with environmental standards. 
Calorimetry and light based detectors are two topics that are often closely linked. One focus is on highly granular calorimeter concepts which allow the decomposition of jets into their individual particles ("particle flow calorimetry"). The requirements are manifold as are the options in terms of active media (e.g. scintillators, noble liquids, silicon), absorbers and readout concepts. Accurate and high-precision timing is a key topic, too. In recent years, photosensors have been evolving rapidly. The silicon photomultiplier ( $\mathrm{SiPM}$ ) has matured and has become a standard tool for many applications. However, its high dark count rate and moderate radiation hardness limit its use when it comes to the detection of low light levels, e.g. in Cerenkov detectors. This is still the domain of vacuum tubes, where emerging, finely segmented multianode devices, micro-channel plate tubes (for ultra-fast timing) and completely new concepts go far beyond the classic PMT.

One research topic also covers detector mechanics, exploring the use of advanced materials, design tools and production technologies, which have the potential to change the way detectors are built and help to boost their performance. Ultra-light, precise and large size carbon-fibre components have become accessible and permit the development of solutions which were inconceivable ten years ago. Environmentally friendly cooling technologies, combined with 3D printing and micro-fabrication technologies reduce the amount of material in vertex and tracking detectors. Close cooperation with industrial partners is seen as the best way forward.

Another interest is the development of application-specific electronics and highrate, high throughput, data transmission for applications with severe constraints (radiation, power consumption, heat dissipation and space limits, accessibility, cable path limitations and more). The numbers of custom made chips in the LHC experiments is counted in millions and the high energy physics community is considered to be an expert customer with very special requirements. The ever-decreasing size feature in the CMOS manufacturing processes leads to benefits (higher functionality), but also to emerging behaviour (higher sensitivity to radiation, challenging cooling needs) and much higher costs (the number of transistors that can be obtained levelled out in around 2012 and since then it has started decreasing again. As a result of consolidation in industry fewer suppliers of very high density integration devices exist and the competition across the market has decreased. The main challenges remain the strongly increasing ionising doses and the necessity to read and transfer continuously increasing amounts of data.

High-capacity and throughout data processing infrastructures are needed for event filtering. The software run on these systems calls for advanced software for pattern recognition, track reconstruction and other event selection processes. New approaches to cope with the track reconstruction challenge at event pile-up rates of 1000 and more (FCC-hh scenario) need to be developed. Machine learning is one topic that needs to be investigated as a possible support technology for such challenges. Already more than ten thousand physicists world-wide use the software frameworks and algorithm toolkits produced by such software development initiatives. The Exabyte era, which is appearing on the horizon, calls for new concepts to manage such amounts of data on a global scale throughout several decades of research.

Last but not least, one working group also deals with the development of sustainable concepts for large high-field magnets for the experiments. There is a study for FCC-ee of an ultra-thin $2 \mathrm{~T}$ magnet with a free bore of $4.4 \mathrm{~m}$ and $6 \mathrm{~m}$ lengths and a target material budget below 1 radiation length. The FCC-hh baseline design has a main solenoid with a free bore of $10 \mathrm{~m}$ and a length of $20 \mathrm{~m}$ and forward solenoids at both ends. All of these magnet developments require progress in superconducting wire technology. 


\section{Milestones and deliverables}

A second workshop took place in autumn 2018 [486], dedicated to prepare the writing of a report that summarises the focus and the priorities of the R\&D programme. This roadmap will include the milestones and deliverables for the initial five-year period.

\subsection{Energy storage and release $R \& D$}

\section{Motivation}

The development of novel energy storage systems has seen impressive progress over recent years, mainly driven by the automotive sector and the increasing use of renewable energies. Batteries appear to be the most promising solution to store the energy recovered from superconducting magnets at the end of a cycle to support the powering of the accelerator during the subsequent ramp phase. This approach could significantly reduce the requirements for peak-power and would keep the overall energy consumption and cost of the electrical infrastructure within the limits that have been established based on average power consumption estimates. Batteries are the focus of the $R \& D$ programme due to the continuing developments towards ever higher energy storage densities. Today, the most suitable battery technology for this application are Lithium batteries, in particular lithium titanium oxide (LTO). On a longer time scale, other technologies can also be considered and a targeted R\&D initiative would feature an ongoing assessment of technology options in cooperation with academic and industrial partners.

\section{Objectives}

This R\&D initiative aims to develop a suitable battery-based energy storage and release system to recover the energy stored in superconducting magnets, to temporarily buffer the energy and to provide the energy to magnet power converters to support the accelerating phase of the cycle. Such a system would require batteries capable of at least 20000 charge-discharge cycles suitable for the power profile of the magnet cycles. Due to the underground space limitations, energy density and volume reduction are additional requirements to be considered. The system must comply with the safety requirements for underground installation and operation. Maintenance, reliability and total-cost-of-ownership (TCO) need to be part of the investigation from the beginning.

\section{Description of the work}

The first stage of this programme consists of drawing up a battery-supported powering system concept for a particular particle collider. This scheme will be used to develop requirements specifications for a battery-supported energy storage and release system that can be used with a superconducting magnet/power converter circuit. An essential part of this work is the definition of the interface and the interplay between the battery storage system and the power converter, which is part of the magnet circuit. The second stage focuses on the technical design studies for the energy storage systems, including the co-development activities with industrial partners for all major system components. A third stage would focus on a demonstration of a battery-based energy storage system at an existing particle accelerator, such as the HL-LHC. 


\section{Collaboration with universities and research institutes}

The cooperation with universities and research institutes focuses on the requirements finding process and on the development of a concept for energy recovery, buffering and release in the particle accelerator domain. The work on developing a concept for a future circular collider will also be carried out as a cooperative effort with universities and research centres. The construction of the HL-LHC upgrade, in particular an energy recovery system for the inner triplet magnets, opens an ideal opportunity and time window for this activity.

\section{Collaboration with industrial partners}

The cooperation with industrial partners focuses on the design, development, testing and co-innovation of major system components to build an energy recovery system for a particle accelerator. The following equipment components would be candidates for such an activity: battery-cells and modules that can meet the operation and deployment conditions and the reliability requirements and battery management and protection systems. Finally, cooperation on assessing the total cost of ownership, that permits a cost-benefit analysis to be carried out, will be included in the work with industry.

\section{Milestones and deliverables}

A preliminary set of milestones and deliverables is outlined in Table 12.8.

Table 12.8. Milestones and deliverables for an energy recovery demonstrator project.

\begin{tabular}{|l|l|}
\hline Title and description & Year \\
\hline Definition of a demonstration case for an existing particle accelerator & 2019 \\
\hline $\begin{array}{l}\text { Documentation of requirements and constraints for an energy recovery } \\
\text { system }\end{array}$ & 2020 \\
\hline Creation of a consortium for the research project & 2021 \\
\hline System concept specification and architecture definition & 2022 \\
\hline Key component definition and start of research work with industry & 2023 \\
\hline Development of test bed complete & 2024 \\
\hline Testing of key components performed & 2026 \\
\hline Demonstrator operational & 2028 \\
\hline
\end{tabular}

\subsection{Efficient power distribution infrastructure}

\section{Motivation}

Power quality is a primary concern for all three of the colliders (FCC-hh, FCCee and HE-LHC). Achieving adequate availability to ensure efficient use of the new infrastructure, to avoid costly downtimes and to achieve energy-efficient operation by reducing the need for recovery and restore actions, is closely linked to the parameters that determine power quality: transient voltage dip mitigation, reactive power control, harmonic filtering and voltage stability. Switching from an alternating current (AC) distribution network to a direct current (DC) power distribution in combination with local energy buffering addresses several of the key impact factors. Frequent 
transient power dips can be tolerated, active and reactive power can be compensated on the load side, AC harmonic filtering is not needed, distribution losses are significantly reduced and larger spacing between electrical infrastructures compared to AC distribution is permitted which leads to reduced investment.

Today, this technology is still in its infancy, mainly lacking adequate standardisation of operating parameters and the availability of a set of equipment from different vendors as a result of it not having been widely adopted. Viable designs of electrical components for DC current and voltage switching, short-circuit current switching, fault detection and protection system selectivity remain to be developed and still represent technical challenges.

\section{Objectives}

The objective is to raise the readiness level of medium-voltage DC distribution network technology by demonstrating its merits at CERN in close-cooperation with an industry-driven demonstrator project. This case serves as a platform for a consortium of industrial partners to develop standards for this grid technology and to trigger the development of market-ready equipment. Technical challenges that still remain to be addressed mainly concern electrical components for current and voltage switching and protection elements such as short-circuit current switching, the detection of faults and an appropriate approach to implement selective protection for the network (e.g. a fast fault-detection and protection communication system to isolate the faulty element, maintaining overall high system reliability). This project aims at facilitating the acceptance of the technology among potential end-users in the commodity market. Eventually, if this technology becomes wide-spread it will be available to large-scale research infrastructure customers at the time when a future particle accelerator is to be installed.

\section{Description of the work}

The first phase to be carried out during a particle collider preparatory phase is to establish a stable consortium of companies who are active in standardisation bodies and who actively study the market opportunities of commodity sectors, companies who have an interest in the development of key components and integrating them in a demonstrator at one of CERN's accelerators. Universities and research centres that develop solutions for the technical challenges and who have the necessary experience should also be part of this consortium. The first phase of the work consists of analysing a specific use case to provide power for one of CERN's accelerators, the capture of the requirements and a detailed technical gap analysis in cooperation with academic and industrial partners. In a second phase, consortium members draw up an architecture and propose a design for a demonstrator that includes the development of novel components to address the most critical technical challenges. In a third phase, prototypes of the technical components are developed by different contributors, fostering the co-development of academia and industry. Finally, a demonstrator that can also serve as a showcase for industries to raise the acceptance of the technology at large scale should be installed at CERN. This work programme needs to be accompanied by the conceptual and technical design of a DC network for a specific particle collider scenario in order to correctly scale the designs according to the specific needs. 


\section{Collaboration with universities and research institutes}

MVDC distribution systems are an area of active academic research that spans various sectors including electrical engineering, electronics, information and computing technologies, reliability engineering, functional safety, economics and business analysis. Consequently, this is an ideal application to bring academic partners with complementary competencies from different geographical regions together at CERN in a concrete technological research project with tangible impact potential at academic levels, with high-educational value and with opportunities to work in a close-tomarket environment. Specifically, EPFL (Lausanne, Switzerland), a technical university, has an active programme on the development of MVDC power distribution networks with support of the Swiss Federal Office of Energy (SFOE).

\section{Collaboration with industrial partners}

With the increasing availability of modern power electronics technologies such as switch-mode converters with higher power ratings, DC networks are being increasingly considered for high voltage transmission lines (HVDC). DC networks start to be deployed for specific applications such as the supply of power for trains in underground metro transport facilities.

In addition to point to point and residential collection grids, MVDC is an interesting solution for industrial applications due to its efficient operation, the small footprint and the low installation and operating costs. Companies like Hyundai, ABB, Siemens and Rolls-Royce are investigating the suitability of this technology for offshore and maritime vessel applications as well as for heavy industries (e.g. aluminium manufacturing) and DC microgrids like those found in shopping centres, office blocks and in particular ever power hungry data centres, smart residential areas and also rural electrification. Since the technology is also ideal for integrating environmentally friendly power sources such as photovoltaic panels (PV) and to a lesser extent fuel cells, companies that are active in the power generation market are also starting to have an interest in this technology. The "EMerge Alliance" ${ }^{13}$ built around this elusive technology is a mirror of who-is-who in the electrical industry and also the world's most important standardisation organisations IEEE and ISO are involved in the ongoing activities. Hence, this is an ideal opportunity for an international community with a vision for a large-scale research infrastructure to provide a test-bed for academia and industry world-wide with the potential of large benefits for society and at the same time preparing the path for large-scale science infrastructures to be operated in a sustainable fashion.

Initially, the focus can be on designing and standardising components, such as DC current breakers, rectifiers, rectifier-transformer solutions and protection systems. The co-developments should leverage existing application development efforts, such as DC railway systems.

\section{Milestones and deliverables}

A preliminary set of milestones and deliverables for this research activity is outlined in Table 12.9 .

13 See also http://www. emergealliance.org 
Table 12.9. Milestones and deliverables for MVDC demonstrator project.

\begin{tabular}{|l|l|}
\hline Title and description & Year \\
\hline Definition of demonstration case for an existing particle accelerator & 2019 \\
\hline Creation of a consortium for showcase project & 2020 \\
\hline Definition of requirements and corresponding sub-projects complete & 2021 \\
\hline Testbed design and key challenges documented & 2022 \\
\hline Component prototyping complete & 2024 \\
\hline Development of key components for test bed complete & 2026 \\
\hline Testing of key components performed & 2026 \\
\hline Design iteration of test bed complete & 2027 \\
\hline Demonstrator operational & 2028 \\
\hline
\end{tabular}

\subsection{Efficient use of excavation materials}

\section{Motivation}

A $100 \mathrm{~km}$ long underground infrastructure for a circular collider will generate around 10 million $\mathrm{m}^{3}$ of excavation material. Both, the French and Swiss host state authorities require a plan for the treatment and management of this material. The recent evolution of Europe's society towards a circular economy calls for novel approaches, viewing the excavated material as a resource, rather than waste for which final repository scenarios need to be developed. A consensus exists among leading civil engineering experts in France, Switzerland and Austria that the development of novel approaches for the processing of excavation material not only leads to an increased acceptance of new large-scale projects, but also has a potential significant beneficial economic impact which scales with the size of the endeavour.

\section{Objectives}

This R\&D programme has three objectives:

1. The legal frameworks in France and Switzerland have to be explored, since the majority of countries currently treat excavation material as waste, preventing further processing or supply of the material to third parties for commercial use. The first step is therefore to identify the legal instruments that can be engaged to permit using excavation materials and to identify the administrative processes to obtain such permission.

2. A transnational plan for the processing and use of the excavation material needs to be developed in cooperation with experienced partners from the host states.

3. An innovative processing technology, that can lead to an end-user product as an alternative to existing treatment and final storage, needs to be developed for the molasse material, the prevalent soil type in the Geneva area.

\section{Description of the work}

The project consists of the following steps:

- An in depth study of the regulations and guidelines applicable in France and Switzerland will lead to an understanding of the current regulatory situation. It will on one hand permit the identification of those areas where the use of excavation materials is possible within the legal framework and on the other 
hand, will provide input for the process of changing the legal framework either in the host states or at European level. Such efforts have already been initiated by leading organisations, which also happen to be collaborators of CERN in these matters.

- The material expected to be excavated during the tunnelling project has to be sampled and analysed. The civil engineering activities in the frame of the HLLHC project started in July 2018 at CERN. This presents an ideal opportunity to obtain a representative set of samples that covers many of the different molassetype soils that are expected to be found in a new, large-scale tunnel for a future particle collider in the region around CERN. Further analysis requires exploratory drillings down to about 300 metres at selected sites. The analysis includes mineralogical, geotechnical, geophysical and petrophysical parameters and will lead to a first qualitative indication of uses for the materials.

- Development of a novel on-line analysis and pre-treatment method for the molasse material that permits the production of different products based on the excavated materials.

- Development of a novel product for molasse material that can form a case for the use of excavation material in the specific accelerator tunnel project.

- Development of a territorial plan for the pre-processing, dispatching and use of the excavation material that takes into account the trans-national aspects of the projects and in particular the different regulatory frameworks in the European Union and in Switzerland. The study needs to include the socio-economic optimisation including cost of pre-processing, logistics and transport, usability for industries, project risks and the development of scenarios for the deposition of non-usable materials.

- Development of input for the legal "procedure unique" for a project of public interest in France and a "plan sectoriel" in Switzerland which create the legal boundary conditions under which the use of the material can take place.

\section{Cooperation with universities and research institutes}

Academic institutes, which are active in research in the domains of mining, metallurgy and materials including novel construction materials and economic studies are highly motivated to collaborate in this R\&D programme. In particular the European Union incentives to speed up the transformation towards a circular economy are encouraging the commitment of resources to this highly-attractive topic which has potential impact beyond the research and accelerator community. The Montanuniversität Leoben (Austria), the Centre d'Etudes des Tunnels (France) and the Ecole Normale Supérieure de Lyon (France) and the Service de géologie, sols et déchets (GESDEC) of the canton of Geneva (Switzerland) are committed to form a core of the research activities mentioned. Further universities in Switzerland such as the University of Geneva, the Vienna University of Economy (Austria), the Politecnico Milano (Italy), who are already engaged in similar projects (e.g. Lyon-Turin) could be additional partners. Eventually, detailed technical developments such as the fast identification of soil characteristics during the excavation process, permitting efficient material separation and pre-processing, are key topics for mechatronic and mechanical engineering schools and dedicated research institutes.

\section{Cooperation with industrial partners}

The development of advanced technologies and a new legal framework for use of excavation material creates a highly attractive case for industrial cooperation 
partnerships. Civil engineering companies such as PORR (Austria), Tunnelling machinery developers such as Herrenknecht (Germany) and material system analysis integrators such as Anton Paar (Austria) and Microtec (Italy) are interested in participating in such an activity.

\section{Impact potential}

The economic potentials for this engineering domain are high. A new tunnel infrastructure designed and built in the frame of a scientific project by an international organisation features legal, contractual and organisational frameworks which differ from ordinary public infrastructure projects such as railway or road tunnels. This makes the project attractive for industrial partners as a showcase for advanced technologies and as a training environment for professionals in different fields for many years, far beyond the scope of the current project. Identifying if molasse material can be processed in such a way that it can be used for an innovative product would permit an entirely new market to be developed. In any case, the development of advanced mechanisms to pre-process and separate the material on-line during the excavation process will generate benefits beyond a pure margin-oriented business. The accompanying development of a legal framework in at least two European countries (France and Switzerland) is an ideal starting point for a Europe-wide development to make civil engineering enterprises more competitive through an alternative approach. European industry has been searching for a long time for such a demonstration project and is eagerly awaiting a tangible possibility to develop the administrative framework that permits tapping into this, so far, largely undervalued raw-material.

\section{Milestones and deliverables}

The milestones and deliverables are presented in Table 12.10.

Table 12.10. Milestones and deliverables concerning excavation materials.

\begin{tabular}{|l|l|}
\hline Title and description & Year \\
\hline Applicable legal and administrative frameworks documented & 2018 \\
\hline Initial set of soil samples analysed & 2019 \\
\hline Study of potential economic impact available & 2020 \\
\hline First proposal for material processing available & 2021 \\
\hline $\begin{array}{l}\text { National plan for management of excavation material in France estab- } \\
\text { lished }\end{array}$ & 2021 \\
\hline Input for law of unique project procedure in France available & 2021 \\
\hline $\begin{array}{l}\text { National plan for management of excavation material in France estab- } \\
\text { lished }\end{array}$ & 2022 \\
\hline Soil samples from exploratory drilling analysed & 2023 \\
\hline Excavation material processing demonstrated & 2024 \\
\hline European guidelines for use of excavation material published & 2024 \\
\hline Input for contractual documents for underground works available & 2025 \\
\hline Excavation-material based product showcased & 2026 \\
\hline Machinery for material pre-processing commercially available & 2027 \\
\hline
\end{tabular}


We would like to thank the International Advisory Committee members:

\author{
R. Assmann, DESY, Germany \\ C. Biscari, CELLS-ALBA, Spain \\ M. Diemoz, INFN, Italy \\ G. Dissertori (Chair), ETH Zürich, \\ Switzerland \\ V. Egorychev, ITEP, Russia \\ W. Fischer, BNL, USA \\ G. Herten, University Freiburg, Germany \\ P. Lebrun, JUAS, France \\ J. Minervini, MIT, USA
}

\author{
A. Mosnier, CEA, France \\ A. Parker, University of Cambridge, \\ $\mathrm{UK}$ \\ C. Quigg, Fermilab, USA \\ M. Ross, SLAC, USA \\ M. Seidel, PSI, Switzerland \\ V. Shiltsev, Fermilab, USA \\ T. Watson, ITER, IEIO \\ A. Yamamoto, KEK, Japan
}

and the International Steering Committee members:

\author{
S. Asai, University of Tokyo, Japan \\ F. Bordry, CERN, IEIO \\ P. Campana (ECFA \& Chair from 2016), INFN, \\ Italy \\ P. Chomaz, CEA, France \\ E. Colby, DOE, USA \\ G. Dissertori, ETH Zürich, Switzerland
}

\author{
E. Elsen, CERN, IEIO \\ M. Krammer (Chair 2014-2016), \\ HEPHY/CERN, Vienna, Austria \\ A. Lankford, UCI, USA \\ S. Peggs, DOE/BNL, USA \\ L. Rivkin, PSI, Switzerland \\ J. Womersley, ESS, Sweden
}

for the continued and careful reviewing that helped to successfully complete this report.

The editors wish to thank all the scientific, engineering and technical personnel, the students and early stage researchers and all members of personnel involved in the investigations, designs and prototyping for their invaluable contributions that made this work possible.

We also want to express our thanks to the administration officers who prepared the ground and created a framework in which this work could be carried out efficiently. The FCC study management team thanks in particular John Poole for his enthusiastic dedication during the editing phase, contributing significantly to deliver a coherent, consistent and readable set of report volumes. Finally, we wish to thank the CERN management for their strong support and encouragement.

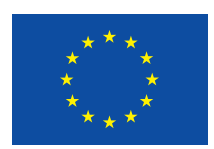

The research, which led to this publication has received funding from the European Union's Horizon 2020 research and innovation programme under the grant numbers 654305 (EuroCirCol), 764879 (EASITrain), 730871 (ARIES), 777563 (RI-Paths) and from FP7 under grant number 312453 (EuCARD-2). The information herein only reflects the views of its authors. The European Commission is not responsible for any use that may be made of the information.

Trademark notice: All trademarks appearing in this report are acknowledged as such.

Open Access This is an open access article distributed under the terms of the Creative Commons Attribution License (http://creativecommons.org/licenses/by/4.0/), which permits unrestricted use, distribution, and reproduction in any medium, provided the original work is properly cited. 


\section{Appendix A: Collider parameters}

Table A.1. FCC-hh baseline parameters compared to LHC, HE-LHC and HL-LHC parameters.

\begin{tabular}{|c|c|c|c|c|}
\hline & \multirow[t]{2}{*}{ LHC } & \multirow[t]{2}{*}{ HL-LHC } & \multicolumn{2}{|c|}{ FCC-hh } \\
\hline & & & Initial & Nominal \\
\hline \multicolumn{5}{|c|}{ Main parameters and geometrical aspects } \\
\hline c.m. Energy $(\mathrm{TeV})$ & \multicolumn{2}{|c|}{14} & \multicolumn{2}{|c|}{100} \\
\hline Circumference C (km) & \multicolumn{2}{|c|}{26.7} & \multicolumn{2}{|c|}{97.75} \\
\hline Dipole field (T) & \multicolumn{2}{|c|}{8.33} & \multicolumn{2}{|c|}{$<16$} \\
\hline Arc filling factor & \multicolumn{2}{|c|}{0.79} & \multicolumn{2}{|c|}{0.8} \\
\hline Straight sections & \multicolumn{2}{|c|}{$8528 \mathrm{~m}$} & \multicolumn{2}{|c|}{$6 \times 1400 \mathrm{~m}+2 \times 2800 \mathrm{~m}$} \\
\hline Number of IPs & \multicolumn{2}{|c|}{$2+2$} & \multicolumn{2}{|c|}{$2+2$} \\
\hline Injection energy $(\mathrm{TeV})$ & \multicolumn{2}{|c|}{0.45} & \multicolumn{2}{|c|}{3.3} \\
\hline \multicolumn{5}{|c|}{ Physics performance and beam parameters } \\
\hline Peak luminosity ${ }^{*}\left(10^{34} \mathrm{~cm}^{-2} \mathrm{~s}^{-1}\right)$ & 1.0 & 5.0 & 5.0 & $<30.0$ \\
\hline Optimum average integrated lumi/day* & \multirow{4}{*}{0.47} & \multirow{4}{*}{2.8} & & \\
\hline$\left(\mathrm{fb}^{-1}\right)$ & & & 2.2 & 8 \\
\hline Assumed turnaround time $(\mathrm{h})$ & & & 5 & 4 \\
\hline Target turnaround time $(\mathrm{h})$ & & & 2 & 2 \\
\hline Peak no. of inelastic events/crossing & 27 & 135 (lev) & 171 & 1026 \\
\hline \multirow{3}{*}{$\begin{array}{l}\text { Total/inelastic cross section } \sigma \text { proton } \\
(\text { mbarn) } \\
\text { Luminous region RMS length }(\mathrm{cm}) \\
\text { Distance IP to first quadrupole, } \mathrm{L}^{*}(\mathrm{~m})\end{array}$} & \multirow{2}{*}{\multicolumn{2}{|c|}{$111 / 85$}} & \multicolumn{2}{|c|}{$153 / 108$} \\
\hline & & & 5.7 & 5.7 \\
\hline & & & 40 & 40 \\
\hline Beam parameters & & & & \\
\hline Number of bunches $n$ & & & & 400 \\
\hline Bunch spacing (ns) & 25 & 25 & & \\
\hline Bunch population $N\left(10^{11}\right)$ & 1.15 & 2.2 & & \\
\hline Nominal transverse normalised emittance & & & 2.2 & \\
\hline$(\mu \mathrm{m})$ & 3.75 & 2.5 & & 2.2 \\
\hline Number of IPs contributing to $\Delta \mathrm{Q}$ & 3 & 2 & $2+2$ & 2 \\
\hline Maximum total b-b tune shift $\Delta \mathrm{Q}$ & 0.01 & 0.015 & 0.011 & 0.03 \\
\hline Beam current (A) & 0.584 & 1.12 & & \\
\hline RMS bunch length (cm) & & & & \\
\hline IP beta function $(\mathrm{m})$ & 0.55 & $0.15(\min )$ & 1.1 & 0.3 \\
\hline RMS IP spot size $(\mu \mathrm{m})$ & 16.7 & $7.1(\min )$ & 6.8 & 3.5 \\
\hline Full crossing angle $(\mu \mathrm{rad})$ & 285 & 590 & 91 & $200^{* *}$ \\
\hline Other beam and machine parameter & & & & \\
\hline Stored energy per beam (GJ) & 0.392 & 0.694 & & \\
\hline SR power per ring ( $\mathrm{MW}$ ) & 0.0036 & 0.0073 & & \\
\hline Arc SR heat load (W/m/aperture) & 0.17 & 0.33 & & \\
\hline Energy loss per turn $(\mathrm{MeV})$ & & & & 67 \\
\hline Critical photon energy (keV) & & & & \\
\hline Longitudinal emittance damping time $(\mathrm{h})$ & & & & \\
\hline Transverse emittance damping time $(\mathrm{h})$ & & & & \\
\hline Dipole coil aperture $(\mathrm{mm})$ & & & & \\
\hline Minimum arc beam half aperture $(\mathrm{mm}$ ( & & & & \\
\hline Installed $\mathrm{RF}$ voltage $(400.79 \mathrm{MHz})(\mathrm{MV})$ & & & & \\
\hline Harmonic number & & & & 680 \\
\hline
\end{tabular}

\section{Appendix B: Uncertainties}

This chapter summarises those uncertainties, which have the highest potential of leading to adverse impacts on the project. They are grouped by technological and implementation-related elements, depending on their origin. The preliminary information provided in this volume is a non-exhaustive, simplified assembly of risks, risk 
origins, impacts and mitigation measures. It merely serves to give a glimpse of the comprehensive risk management process, which has been set up and which is currently ongoing in the Future Circular Collider study. A risk assessment database is maintained by the study group in order to establish an early warning and mitigation process that is expected to grow into a comprehensive project risk management scheme during the project preparatory phase.

\section{B.1 Accelerator and technologies}

Table B.1. Technological uncertainties with decisive impact potentials on the project.

\begin{tabular}{|c|c|c|}
\hline Uncertainty & Impacts & Mitigation measures \\
\hline $\begin{array}{l}\mathrm{Nb}_{3} \mathrm{Sn} \text { wire } \\
\text { performance not } \\
\text { achievable within the } \\
\text { available time window. }\end{array}$ & $\begin{array}{l}\text { Target field level and field } \\
\text { quality of dipole magnets } \\
\text { cannot be reached. Collision } \\
\text { energy will be lower than } \\
\text { designed and consequently } \\
\text { the collider performance is } \\
\text { lower than expected. }\end{array}$ & $\begin{array}{l}\text { Increase the intensity of the } \\
\text { low-temperature superconducting wire } \\
\text { R\&D programme. On a longer time } \\
\text { scale, reinforce an R\&D programme } \\
\text { including high temperature } \\
\text { superconductors aiming at a } \\
\text { performance/cost optimised energy } \\
\text { frontier collider. }\end{array}$ \\
\hline $\begin{array}{l}\text { Cost target of } \mathrm{Nb}_{3} \mathrm{Sn} \\
\text { wire not reachable or } \\
\text { not reachable within } \\
\text { the available time } \\
\text { window. }\end{array}$ & $\begin{array}{l}\text { Project not affordable or } \\
\text { particle collider } \\
\text { performance targets need to } \\
\text { be adjusted. }\end{array}$ & $\begin{array}{l}\text { Increase the intensity of the } \\
\text { low-temperature superconducting wire } \\
\text { R\&D programme. Invest in building up } \\
\text { a co-development with industries } \\
\text { worldwide in an R\&D programme to } \\
\text { avoid a vendor-locked-in situation and } \\
\text { to prevent price limitation. On a longer } \\
\text { time scale, reinforce an R\&D } \\
\text { programme including high temperature } \\
\text { superconductors aiming at a } \\
\text { performance/cost optimised energy } \\
\text { frontier collider. }\end{array}$ \\
\hline $\begin{array}{l}\text { Vendor locking for } \\
\text { superconducting wire. }\end{array}$ & $\begin{array}{l}\text { Project not affordable or } \\
\text { project schedule cannot be } \\
\text { met. }\end{array}$ & $\begin{array}{l}\text { Consider an R\&D initiative with } \\
\text { academic partners to ensure in-house } \\
\text { expertise on the technology and open } \\
\text { standards and designs that can be } \\
\text { licensed to different types of companies } \\
\text { including those established in } \\
\text { metallurgical processing and } \\
\text { manufacturing. }\end{array}$ \\
\hline $\begin{array}{l}\text { Inefficient magnet } \\
\text { series manufacturing } \\
\text { and limited availability } \\
\text { of companies with } \\
\text { comparable } \\
\text { manufacturing } \\
\text { throughput and } \\
\text { adequate quality } \\
\text { management. }\end{array}$ & $\begin{array}{l}\text { Project not affordable, } \\
\text { reduced performance and } \\
\text { reliability of the machine, } \\
\text { possible need to lower the } \\
\text { collision energy in order to } \\
\text { adapt to lower field and } \\
\text { field quality, lower overall } \\
\text { collider performance leading } \\
\text { to a longer operation } \\
\text { schedule. Project } \\
\text { implementation delayed. } \\
\text { Excessive growth of project } \\
\text { cost due to the need to } \\
\text { re-manufacture or exchange } \\
\text { magnets. Unsustainable } \\
\text { operation due to lower than } \\
\text { expected reliability that } \\
\text { causes substantial downtime } \\
\text { for repair or maintenance. }\end{array}$ & $\begin{array}{l}\text { Invest in R\&D to develop magnet } \\
\text { designs that are easier to manufacture, } \\
\text { in particular aiming at streamlined } \\
\text { production, testing and installation. } \\
\text { Optimise the internal system interfaces } \\
\text { with respect to number and simplicity } \\
\text { as well as the external system interfaces, } \\
\text { including test points for total quality } \\
\text { management. Launch studies to improve } \\
\text { the efficiency of assembly, considering } \\
\text { interfaces, simplification and speed-up } \\
\text { of individual production and processing } \\
\text { steps. Invest in automation of } \\
\text { production, assembly, testing and the } \\
\text { integration with a total quality } \\
\text { management system which takes into } \\
\text { account a geographically distributed } \\
\text { production process. }\end{array}$ \\
\hline
\end{tabular}


Table B.1. (Continued.)

\begin{tabular}{|c|c|c|}
\hline Uncertainty & Impacts & Mitigation measures \\
\hline $\begin{array}{l}\text { Capacity of cryogenic } \\
\text { refrigeration system } \\
\text { insufficient. }\end{array}$ & $\begin{array}{l}\text { Lower particle collider } \\
\text { performance due to the } \\
\text { need to adjust operating } \\
\text { parameters (e.g. longer } \\
\text { cycles, lower peak field, } \\
\text { lower intensity). }\end{array}$ & $\begin{array}{l}\text { Invest in bringing the more efficient, } \\
\text { nelium-based cryogenic refrigeration } \\
\text { technology to a higher technological } \\
\text { readiness level in cooperation with } \\
\text { industrial partners through dedicated } \\
\text { R\&D. }\end{array}$ \\
\hline $\begin{array}{l}\text { Electrical supply peak } \\
\text { power requirement is } \\
\text { too high. }\end{array}$ & $\begin{array}{l}\text { Cost of classical electrical } \\
\text { infrastructure and } \\
\text { installation becomes } \\
\text { prohibitive. }\end{array}$ & $\begin{array}{l}\text { Invest in bringing more efficient } \\
\text { cryogenic refrigeration with less } \\
\text { electrical energy requirements for the } \\
\text { same cooling performance to higher } \\
\text { technology readiness level in } \\
\text { cooperation with industrial partners } \\
\text { through dedicated R\&D. Invest in R\&D } \\
\text { to develop a system that can recover } \\
\text { energy from the accelerator subsystems, } \\
\text { buffer that energy temporarily and use } \\
\text { it during the subsequent cycle to reduce } \\
\text { the peak power needs during the ramp } \\
\text { phase. Invest in R\&D on more efficient } \\
\text { electricity distribution systems which } \\
\text { exhibit lower equipment costs and } \\
\text { reduced losses. }\end{array}$ \\
\hline $\begin{array}{l}\text { High amplitude } \\
\text { thermal contraction } \\
\text { and expansion of } \\
\text { conventional cryogenic } \\
\text { distribution system } \\
\text { during cool down and } \\
\text { warm up. }\end{array}$ & $\begin{array}{l}\text { Complicated design of a } \\
\text { conventional cryogenic } \\
\text { distribution system to } \\
\text { absorb thermal contraction } \\
\text { and expansion can result in } \\
\text { significant cost increase, } \\
\text { lower reliability and thus } \\
\text { unsustainable operating } \\
\text { costs. In addition, high loss } \\
\text { rates and excessive } \\
\text { maintenance and repair can } \\
\text { cause unacceptable } \\
\text { downtimes and drive the } \\
\text { cost of operation up. }\end{array}$ & $\begin{array}{l}\text { Bring a cryogenic distribution system } \\
\text { based on a low-thermal expansion } \\
\text { material to industrial production grade } \\
\text { through focused R\&D and a } \\
\text { co-innovation approach with industrial } \\
\text { partners. This activity, which will also } \\
\text { reduce the number of equipment } \\
\text { components and lead to a simpler design } \\
\text { will also help to control the total cost of } \\
\text { ownership through reduced losses and } \\
\text { reduced requirements for maintenance } \\
\text { and repair. }\end{array}$ \\
\hline $\begin{array}{l}\text { Conventional systems } \\
\text { may not be adequate } \\
\text { to extract the } \\
\text { high-energy beams } \\
\text { reliably and with } \\
\text { acceptable electrical } \\
\text { consumption. }\end{array}$ & $\begin{array}{l}\text { Unreliable extraction can } \\
\text { lead to equipment damage } \\
\text { and consequently to } \\
\text { substantial downtimes. This } \\
\text { situation results in the loss } \\
\text { of investments and } \\
\text { unsustainable operation of } \\
\text { the infrastructure. } \\
\text { Conventional systems } \\
\text { consume too much electrical } \\
\text { energy leading to high } \\
\text { operating costs. }\end{array}$ & $\begin{array}{l}\text { Through targeted R\&D, develop a } \\
\text { highly distributed, reliable and } \\
\text { high-performance extraction system } \\
\text { that can cope with the high beam } \\
\text { energies. The research has two facets: a } \\
\text { novel superconducting septum, requiring } \\
\text { less energy, space and system } \\
\text { complexity and secondly, scalable, fast } \\
\text { and affordable high-power switching } \\
\text { devices. Accompanying this equipment } \\
\text { R\&D, and in cooperation with external } \\
\text { partners, there should be the } \\
\text { development and validation of fast, } \\
\text { modular and reliable control system } \\
\text { technologies that can meet the safety } \\
\text { and reliability requirements. }\end{array}$ \\
\hline
\end{tabular}


Table B.1. (Continued.)

\begin{tabular}{|c|c|c|}
\hline Uncertainty & Impacts & Mitigation measures \\
\hline $\begin{array}{l}\text { Depending on the } \\
\text { particle collider } \\
\text { scenario and its } \\
\text { research programme, } \\
\text { particle detection } \\
\text { technologies do not } \\
\text { meet the performance, } \\
\text { reliability and cost } \\
\text { needs. }\end{array}$ & $\begin{array}{l}\text { Under-use of the potential } \\
\text { of the particle collider can } \\
\text { lead to the physics } \\
\text { programme goals not being } \\
\text { met or not meeting the } \\
\text { goals within the planned } \\
\text { schedule and cost envelope. } \\
\text { Excessive costs of } \\
\text { experiment detectors. Low } \\
\text { maintenance intervals and } \\
\text { long repair times as well as } \\
\text { equipment replacement } \\
\text { costs that lead to } \\
\text { unsustainable operation of } \\
\text { the research facility. Loss of } \\
\text { interest of the worldwide } \\
\text { science community due to } \\
\text { limited physics research } \\
\text { reach, loss of a science } \\
\text { vision within an achievable } \\
\text { time frame and within } \\
\text { state-of-the-art scientific } \\
\text { research methods and tools. }\end{array}$ & $\begin{array}{l}\text { Definition of a world-wide coordinated } \\
\text { strategic R\&D initiative focusing on } \\
\text { detector technologies that, with the } \\
\text { selection of a preferred particle collider } \\
\text { scenario, becomes more specific as } \\
\text { technical designs advance, research } \\
\text { communities become organised and the } \\
\text { specification of experimental physics } \\
\text { investigations are developed in greater } \\
\text { detail. }\end{array}$ \\
\hline
\end{tabular}

\section{B.2 Implementation}

Table B.2. Project implementation related uncertainties with decisive potential impact.

\begin{tabular}{|c|c|c|}
\hline Uncertainty & Impacts & Mitigation measures \\
\hline $\begin{array}{l}\text { Limited capacity for } \\
\text { handling excavation } \\
\text { materials in the host } \\
\text { states, relevant for } \\
\text { all particle collider } \\
\text { scenarios, including } \\
\text { the HE-LHC due to } \\
\text { the need for new } \\
\text { underground facili- } \\
\text { ties. }\end{array}$ & $\begin{array}{l}\text { Significant delay of construction } \\
\text { phase start or increase of } \\
\text { construction costs due to unplanned } \\
\text { waste disposal needs. } \\
\text { Non-acceptance of project plan by } \\
\text { host-state representatives with or } \\
\text { with- out public opposition. }\end{array}$ & $\begin{array}{l}\text { Early start to develop a territorial } \\
\text { waste management plan that } \\
\text { considers the current legal situation } \\
\text { in the host states and the } \\
\text { transnational context of the project, } \\
\text { contributing through the } \\
\text { involvement of relevant experts at } \\
\text { international level to the evolution } \\
\text { of the current initiatives on moving } \\
\text { towards a circular economy at } \\
\text { European scale. Through the work } \\
\text { at a stage in which a detailed } \\
\text { project design remains yet to be } \\
\text { developed, invest in R\&D, e.g. } \\
\text { through EC funded projects on } \\
\text { circular economy and infrastructure } \\
\text { construction efficiency, to identify } \\
\text { novel separation and processing } \\
\text { techniques, identify existing and so } \\
\text { far not considered use cases and } \\
\text { bring them to credible technology } \\
\text { readiness levels. Develop a } \\
\text { territorial concept for the logistics } \\
\text { of tracing and transporting the } \\
\text { excavation materials with the } \\
\text { support of ICT systems, involving } \\
\text { the host states, potentially relevant } \\
\text { industries and consumers } \\
\text { throughout the entire process. }\end{array}$ \\
\hline
\end{tabular}


Table B.2. (Continued.)

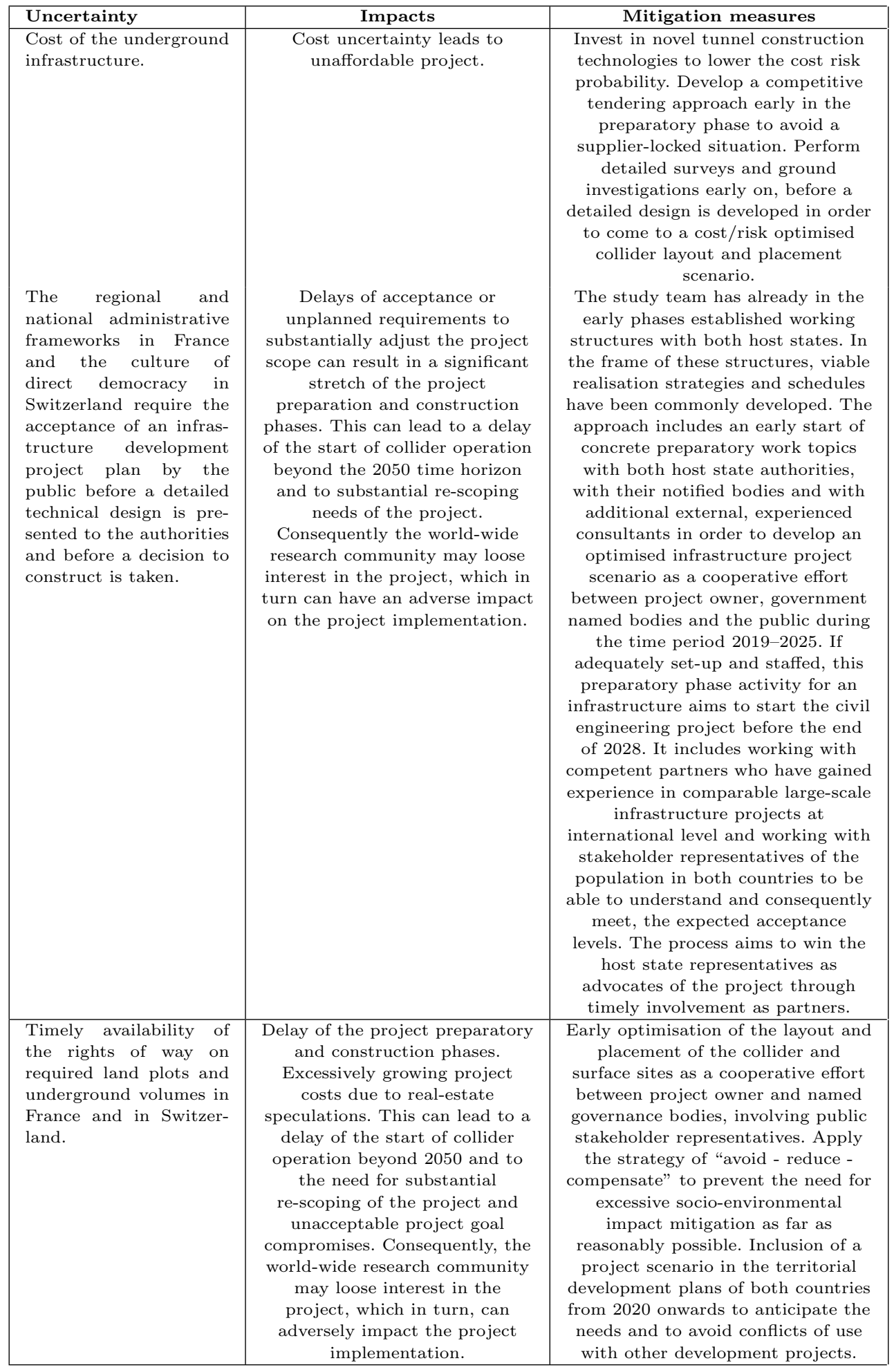


Table B.2. (Continued.)

\begin{tabular}{|c|c|c|}
\hline Uncertainty & Impacts & Mitigation measures \\
\hline $\begin{array}{l}\text { Uncontrolled resource } \\
\text { usage during the oper- } \\
\text { ation phase (examples } \\
\text { include, but are not } \\
\text { limited to, water for } \\
\text { conventional cooling, in } \\
\text { particular if intake and } \\
\text { reject occur in different } \\
\text { countries, electricity } \\
\text { consumption, generation } \\
\text { of waste-heat, cryogens, } \\
\text { potentially harmful } \\
\text { chemicals, introduction } \\
\text { of sealed surfaces on the } \\
\text { land - asphalt, concrete } \\
\text { etc.) }\end{array}$ & $\begin{array}{l}\text { Project-specific regulatory } \\
\text { requirements imposed early } \\
\text { during the preparatory phase } \\
\text { (e.g. in the frame of the } \\
\text { development of the law for the } \\
\text { unique project procedure in } \\
\text { France) can lead to } \\
\text { unsustainable operating costs. } \\
\text { Alternatively, project } \\
\text { acceptance by public } \\
\text { stakeholder representatives may } \\
\text { be delayed, leading to the } \\
\text { project preparation, } \\
\text { construction and commissioning } \\
\text { period being longer than } \\
\text { acceptable, potentially also } \\
\text { requiring technical } \\
\text { modifications at a stage where } \\
\text { such interventions in the design } \\
\text { can be costly. }\end{array}$ & $\begin{array}{l}\text { Timely work with the host-state } \\
\text { representatives on establishing a } \\
\text { communication culture between } \\
\text { administration offices, external } \\
\text { experts and project engineers has } \\
\text { started during the conceptual design } \\
\text { phase. Subsequent early common } \\
\text { work in the form of a partnership } \\
\text { can on one hand help developing a } \\
\text { technical project with reduced } \\
\text { resource needs and on the other } \\
\text { hand trigger targeted investigations } \\
\text { to identify alternative approaches to } \\
\text { cope with the resource needs. } \\
\text { Consequently, a number of design } \\
\text { studies dealing with resource-related } \\
\text { questions during the preparatory } \\
\text { phase, including experience from the } \\
\text { LHC and HL-LHC projects is } \\
\text { indicated. Examples include } \\
\text { applications for making the } \\
\text { waste-heat available, studying } \\
\text { alternative cooling and ventilation } \\
\text { technologies, studying surface site } \\
\text { designs that correspond to the } \\
\text { ecological needs and that can fit } \\
\text { with the cost envelopes, identify } \\
\text { approaches to limit losses and to } \\
\text { recover energy. }\end{array}$ \\
\hline $\begin{array}{l}\text { Sustainable site secu- } \\
\text { rity and effective emer- } \\
\text { gency intervention dur- } \\
\text { ing operation. }\end{array}$ & $\begin{array}{l}\text { Site surveillance, security } \\
\text { enforcement, emergency } \\
\text { intervention and rescue under } \\
\text { the exclusive management } \\
\text { responsibility of the project } \\
\text { owner can lead to unsustainable } \\
\text { operation. Intervention times } \\
\text { which are too long and } \\
\text { inadequate coverage can result } \\
\text { in the need to reduce } \\
\text { operational goals or failure to } \\
\text { meet the operational targets } \\
\text { may result in acceptable costs. }\end{array}$ & $\begin{array}{l}\text { Early identification of the needs as a } \\
\text { collaborative effort between the } \\
\text { project owner, drawing on } \\
\text { experience of the LHC project, and } \\
\text { relevant host-state representatives } \\
\text { will permit the definition of } \\
\text { integrated concepts, making the } \\
\text { best use of existing public security, } \\
\text { emergency and intervention services } \\
\text { combined with joint a continuous } \\
\text { training programme. This approach } \\
\text { also has the advantage of leading to } \\
\text { increased acceptance of the project } \\
\text { by key members of society and to } \\
\text { create a reliable set of project } \\
\text { advocates among the non-scientific } \\
\text { community. }\end{array}$ \\
\hline
\end{tabular}


Table B.2. (Continued.)

\begin{tabular}{|c|c|c|}
\hline Uncertainty & Impacts & Mitigation measures \\
\hline $\begin{array}{l}\text { Low cost-effectiveness } \\
\text { and quality of materials } \\
\text { and services supplied } \\
\text { due to excessive con- } \\
\text { straints on public } \\
\text { tendering processes. } \\
\text { This includes the limi- } \\
\text { tations of a well-defined } \\
\text { cost/performance based } \\
\text { procurement scheme for } \\
\text { the project, ineffective } \\
\text { international networks } \\
\text { with industries and } \\
\text { potential suppliers of all } \\
\text { sizes, too much generic } \\
\text { focus on either in-house } \\
\text { or out-sourced services, } \\
\text { insufficient personnel } \\
\text { (in-house or contracted) } \\
\text { resources for procure- } \\
\text { ment, contract follow up } \\
\text { and inadequate business } \\
\text { processes as well as a } \\
\text { lack of legal advisers } \\
\text { with extensive industrial } \\
\text { experience during peak } \\
\text { periods. }\end{array}$ & $\begin{array}{l}\text { High effort required for } \\
\text { administrative procedures } \\
\text { resulting in lengthy and costly } \\
\text { procurement processes which } \\
\text { have an impact on the duration } \\
\text { of the project preparation and } \\
\text { construction phases. These can } \\
\text { lead to the selection of } \\
\text { under-performing suppliers } \\
\text { which in the long run leads to } \\
\text { potentially unacceptable high } \\
\text { total costs of ownership } \\
\text { (re-construction of subsystems, } \\
\text { high repair or maintenance } \\
\text { costs, high operating costs). }\end{array}$ & $\begin{array}{l}\text { Timely preparation of an adequate } \\
\text { procurement and academia/industry } \\
\text { co-development framework. Timely } \\
\text { investment in preparing the basis of } \\
\text { a well staffed project procurement } \\
\text { system, taking into account the } \\
\text { changing needs during various } \\
\text { phases of the project (preparation, } \\
\text { CE construction, machine } \\
\text { construction, commissioning). } \\
\text { Timely development of an } \\
\text { appropriate concept for in-kind } \\
\text { participation of member- and } \\
\text { non-member states with a } \\
\text { corresponding project governing and } \\
\text { management structure that includes } \\
\text { effective levers to proceed with the } \\
\text { implementation of the project (early } \\
\text { stall warning, re-prioritisation, } \\
\text { re-scheduling, re-assignment). }\end{array}$ \\
\hline $\begin{array}{l}\text { Availability of stable } \\
\text { and effective governing } \\
\text { and management struc- } \\
\text { tures driving forward } \\
\text { the technical design of a } \\
\text { collider, the associated } \\
\text { experiment detectors, } \\
\text { the host state activi- } \\
\text { ties and the financing } \\
\text { strategy. }\end{array}$ & $\begin{array}{l}\text { The lack of a vision of the } \\
\text { priorities, which is propagated } \\
\text { in the framework of a governing } \\
\text { and management structure with } \\
\text { adequate resource assignment, } \\
\text { leads to a stall in the progress } \\
\text { of the technical design and the } \\
\text { development of mitigation } \\
\text { measures for the uncertainties. } \\
\text { This fails to strengthen the } \\
\text { momentum, which is a } \\
\text { pre-requisite of a project of such } \\
\text { scale, of an ever growing } \\
\text { international community. } \\
\text { Consequently, investments made } \\
\text { so far and being made for } \\
\text { limited activities cannot have } \\
\text { lasting impacts. Consequently } \\
\text { the research community may } \\
\text { choose to re-orient towards } \\
\text { alternative projects. }\end{array}$ & $\begin{array}{l}\text { With the next update of the } \\
\text { European strategy for particle } \\
\text { physics, an adequate governing and } \\
\text { management structure for the } \\
\text { preparatory project phase needs to } \\
\text { be established for the accelerator } \\
\text { and infrastructure project and for a } \\
\text { particle detector design project. } \\
\text { This should be well anchored in } \\
\text { CERN's structure and mission } \\
\text { programme, which has the financial } \\
\text { and personnel resources to carry out } \\
\text { the planned tasks before a decision } \\
\text { to build is taken. Consequently, the } \\
\text { successful preparation of a new } \\
\text { research infrastructure for } \\
\text { high-energy particle physics relies } \\
\text { on the unambiguous } \\
\text { recommendation emerging from the } \\
\text { next strategy update, permitting } \\
\text { the available resources to be focused } \\
\text { on the tasks which need to be } \\
\text { accomplished with highest priority. }\end{array}$ \\
\hline
\end{tabular}




\section{Appendix C: Communities}

The impact on the community can be presented in terms of an "onion" type model, starting with the innermost layer comprising the core scientific communities, which need, conceive and use such a facility. Further communities in the European Research Area and beyond, which will benefit throughout the entire lifecycle, starting with the early design phase, include: other sciences, engineering communities, higher education, industrial partners, researchers from non-technical domains, and ultimately all members of society.

\section{Particle physics}

The FCC-hh has broad physics discovery potential with an opportunity to attract a world-wide community of more than 20000 physicists (see arXiv:1707.03711). It addresses the energy frontier, electroweak, Higgs, Dark Matter and heavy flavour physics communities as well as the heavy ion and lepton-hadron communities, presently working on the LHC, flavour factories, Dark Matter experiments and other particle collider experiments worldwide. The theory community is needed to develop scenarios that can be tested at this collider. Together with the experimental physics community they will define a comprehensive physics programme.

\section{Experimental physics}

The detectors for this machine will have to be highly versatile. Requirements include the measurement of multi-TeV jets, leptons and photons with masses up to $50 \mathrm{TeV}$. At the same time, detectors must be highly sensitive to known SM processes. Precision tracking and calorimetry are further fields of activity. The high occupancy and pileup call for unprecedented time resolution and advances in data reduction. The need for high spatial resolution due to boosted objects needs ingenious approaches for particle identifica-tion techniques and precision tracking. Additional experimental physics communities will be attracted by this research facility through the concurrent fixed target and heavy ion operation programmes.

\section{Accelerator physics}

The FCC-hh with its unprecedented collision energy and luminosity will attract the world-wide community of accelerator physicists. Fully automated operation procedures ensuring the concurrent operation of CERN's injector complex and the future high-energy collider, integrating luminosity optimisation, are topics that call for the integration of diverse domains of competence.

\section{Other physics communities}

The research at the FCC-hh will have implications for astrophysics and cosmology, offering an unprecedented opportunity to federate these scientific fields.

\section{Technology, engineering, computing}

The project will drive the development of superconductors for high-field magnet applications including large series production and precision machinery. The collider requires a novel approach to cryogenic refrigeration on a large-scale. The project 
also involves the development of systems for higher efficiency electrical to radiofrequency power conversion. The development of cost-effective, high-performance thin-film coated, superconducting cavities needs material scientists and requires expertise from manufacturing experts. Specific engineering areas include precision mechanics, surface treatment, superconductivity, novel materials, electronic and reliability engineering to improve the particle accelerator efficiency.

Electrical engineering communities will be involved in bringing medium voltage DC technology to the market, to conceive lower-loss electricity distribution systems which are more reliable and develop environmentally friendly and sustainable energy recovery and buffering systems. Designers will be needed to develop waste-heat recovery and reuse systems.

To design and construct the underground infrastructure in a cost-effective way, the civil engineering community needs to make advances in tunnelling technologies and to develop ways for the recovery and reuse of excavation materials. This work will be carried out as a joint endeavour with material scientists, geologists and chemists.

Information and communication technology communities will be involved everywhere. Their activities include simulation algorithms and software infrastructure; parallel and high-performance computing; distributed computing; real-time and embedded systems; mechatronics to conceive new standards and technologies for low-maintenance and easy-to-repair systems in the areas of protection, access, remote handling and autonomous interventions; data acquisition, data visualisation, modelling and operation optimisation,; the introduction of artificial intelligence in machine and detector operation; radiation and fault tolerant systems; environmental information systems; data mining technologies; wireless communications including safety-related functions; data and document management facilities; world-wide computing infrastructures; long-term data stewardship; open access data models and infrastructures and much more.

\section{Higher education}

The design and construction of the accelerator and the detectors will offer many opportunities for science teachers and students at master, doctorate and post-doc levels. Eventually the findings from all the scientific activities will enrich the academic curricula: state-of-science today will become state-of-the art tomorrow. This project will enlarge the impact potential of higher education on highly qualified personnel and apprentices.

\section{Industry}

A project of such scale must be designed, constructed, operated and maintained with strong involvement of industrial partners from all of the participating nations. Where reasonably possible, a shift towards co-development will lead to a research infrastructure which on one hand is sustainable in the long-term and which has greater impact for industry on the other. A specific initiative during the detailed design phase will focus on identifying the fields of cooperation, also elucidating where companies can best profit from enhanced learning to increase their competitiveness and improve the quality of their product and internal processes.

One particular area of interest is to develop ways to increase the technology level in the field of civil engineering: novel methods for on-line excavation material analysis and separation, pathways for reuse of the materials by other industries such as chemical and construction are important levers to increase the economic utility in this domain. 


\section{Non-technical sciences}

This project will engage a variety of scientific communities, beyond the physics, technology and engineering domains. Examples include, but are not limited to research in logistics and systems engineering around the world-wide production chain for the accelerator and detectors (logistics, operations, sales, HR, procurement, accounting, management and organisation, business administration). Architecture and arts will be involved in surface site development. Media and visual arts as well as museums and marketing experts are needed to efficiently engage the public and to communicate with institutional stakeholders.

Radiation protection, technical risk management and waste management experts will facilitate the control of hazards and risks in all areas throughout the entire life cycle. Environmental and urban sciences will help avoiding, reducing and mitigating impacts.

Economics, innovation management and political sciences form another group of non-technical sciences, which have already shown during the FCC study phase that they are essential for the successful preparation of the project.

\section{Members of the global society}

The continued deep exploration of our universe tackles fundamental questions that intrigue everyone: What is the origin of the universe? What is the nature of the matter, that we are all made of? Where do we come from? Why is there something and not nothing?

This project addresses these questions directly and creates opportunities to engage everyone who is interested. During the preparatory phase, an effort will be made to intensify such involvement through community science and a modern communication plan. The conceptual study phase has revealed that the greatest challenge is, however, to create interest among the majority of people who are unaware. FCC-hh is an opportunity to raise awareness on a global scale and to strengthen the support for continued investment in this research by policy makers, funding agencies and ultimately, by every member of society.

\section{Appensix D: Timeline}

\section{D.1 Timeline for FCC-hh without prior implementation of FCC-ee}

The overall project duration of FCC-hh as a "stand-alone" project is 23 years, composed of two major parts: the preparation phase spanning 8 years and the construction phase spanning 15 years. In addition to key technology $R \& D$, the preparation phase includes:

- all administrative procedures with the host states, ultimately leading to the construction permits and delivery of the surface and underground rights of way;

- the consultation process with authorities and public stake holders;

- the development of project financing, organisation and governing structures;

- the site investigations, civil engineering design, tendering for consultant and construction contracts.

The construction phase includes:

- all underground and surface structures;

- technical infrastructure; 


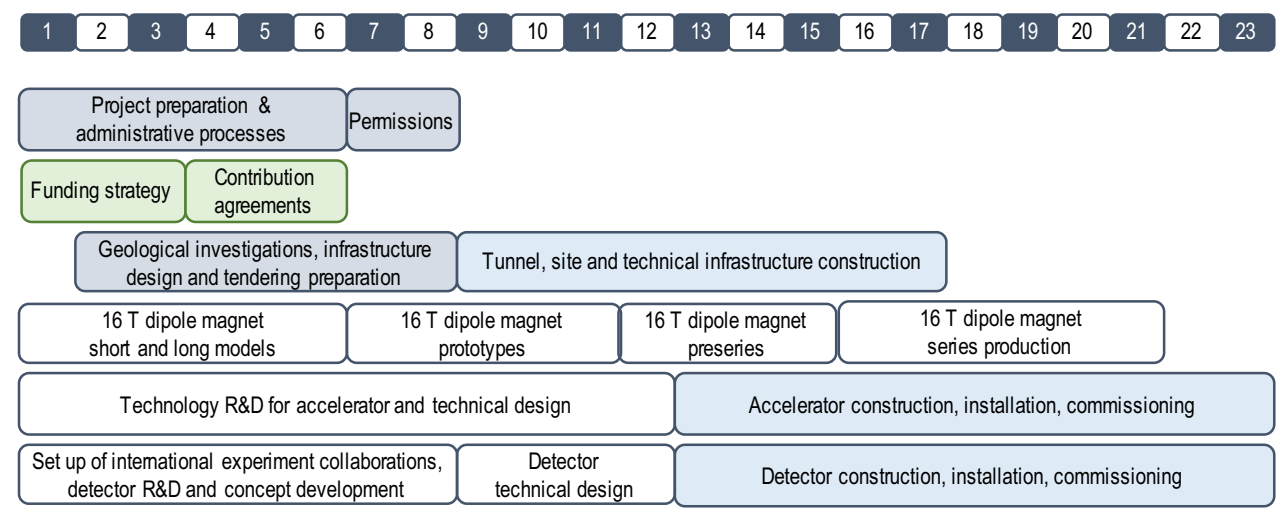

Fig. D.1. Overview of the FCC-hh implementation timeline without prior implementation of FCC-ee starting in 2020. Non technical tasks marked in grey and green are compulsory for any new particle collider project and precede the actual construction. Numbers in the top row indicate the year. Physics operation would start in the mid 2040ies according to this schedule.

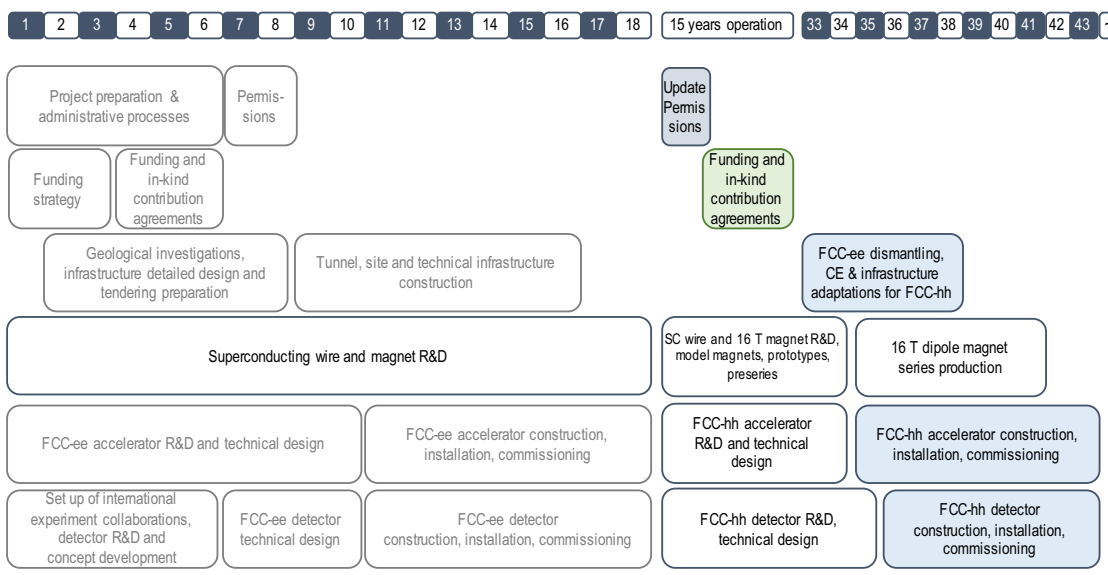

Fig. D.2. Overview of the FCC-hh implementation timeline with prior implementation of FCC-ee starting in 2020. Non technical tasks marked in grey and green are compulsory for any new particle collider project and precede the actual construction. Numbers in the top row indicate the year. Physics operation would start in the mid 2060ies on this schedule.

- accelerators and detectors, including hardware and beam commissioning.

The implementation timeline for FCC-hh without prior implementation of FCCee is shown in Figure D.1. The underground and surface civil engineering construction can be completed in less than 7 years. The first sectors would be ready for technical infrastructure installation about 4.5 years after the start of construction.

\section{D.2 Timeline for FCC-hh with prior implementation of FCC-ee}

If the FCC-hh is constructed as a "combined project", i.e. following the prior implementation of the FCC-ee lepton collider, the entire existing civil structures and technical infrastructures can be reused. The construction of two experiment caverns for 
the lower luminosity experiments, the two beam dump tunnels and the two hadron transfer lines from LHC will be needed in addition. Technical infrastructure installation will be dominated by the construction of the cryogenics plants and distribution lines for the main magnet cooling. Accelerator construction, installation and commissioning will be similar to the FCC-hh "stand-alone" project.

The overall project duration for construction of FCC-hh following a FCC-ee lepton collider is around 10 years from the end of FCC-ee operation. It is composed of three major parts: dismantling of FCC-ee, civil engineering construction and technical infrastructure adaptation and FCC-hh machine installation. This 10 year period is preceded by a preparatory phase similar to that for the FCC-ee project covering construction and operation related agreements with host states, funding and in-kind agreements and technical design and prototypes. The implementation time line for FCC-hh after prior implementation of FCC-ee is shown in Figure D.2.

\section{Appendix E: Costs}

\section{E.1 Construction costs}

A cost study was performed for the FCC-hh based on the conceptual design. The cost estimates for the accelerators (collider and injector complex) and the technical infrastructure are based on machine and system inventories. The cost estimate for civil engineering is based on an analysis of construction methods for underground and surface structures, the associated material quantities and unit prices, derived from several recent large-scale tunnel and civil engineering projects in Central Europe. The resulting precision of the overall cost estimate is at $\pm 30 \%$ level.

\section{E.1.1 Construction cost for FCC-hh without prior implementation of FCC-ee}

The capital cost for construction of the project is summarised in Table E.1. Cost items are indicated in millions of Swiss francs (MCHF) at 2018 values. This cost includes all equipment for operation at the $100 \mathrm{TeV}$ design performance.

The total construction cost amounts to $24000 \mathrm{MCHF}$ as shown in Figure E.1. It is dominated by the by the accelerator and injector, amounting to $57 \%$ or $13600 \mathrm{MCHF}$. The major part of the accelerator cost corresponds to the $4700 \mathrm{Nb}_{3} \mathrm{Sn} 16 \mathrm{~T}$ main dipole magnets, totalling $9400 \mathrm{MCHF}$, at a cost target of $2 \mathrm{MCHF} / \mathrm{magnet}$. The construction cost for surface and underground civil engineering is $6000 \mathrm{MCHF}$ or $25 \%$ of the total construction cost. The capital cost for the technical infrastructures is $4400 \mathrm{MCHF}$ corresponding to $18 \%$ of the total construction cost.

\section{E.1.2 Construction cost for FCC-hh with prior implementation of FCC-ee}

The capital cost for construction of the FCC-hh as a "combined project", i.e. following the prior implementation of the FCC-ee lepton collider with its civil engineering and technical infrastructure, is summarised in Table E.2. The total construction cost amounts to $17000 \mathrm{MCHF}$. In this case the capital cost is reduced by $7000 \mathrm{MCHF}$ compared to the stand-alone variant, since the civil engineering and technical infrastructures from FCC-ee can be fully reused (see also Fig. E.2). The main additional civil engineering structures required for FCC-hh are two experiment caverns with four shafts for the lower luminosity experiments, the beam dump tunnels and the two transfer lines from LHC including an access shaft and injection cavern each, 
Table E.1. Summary of capital cost for the implementation of the FCC-hh project in stand-alone mode.

\begin{tabular}{|l|r|}
\hline Domain & Cost (MCHF) \\
\hline Collider and injector complex & 13600 \\
\hline Technical infrastructure & 4400 \\
\hline Civil engineering & 6000 \\
\hline Total cost & 24000 \\
\hline
\end{tabular}

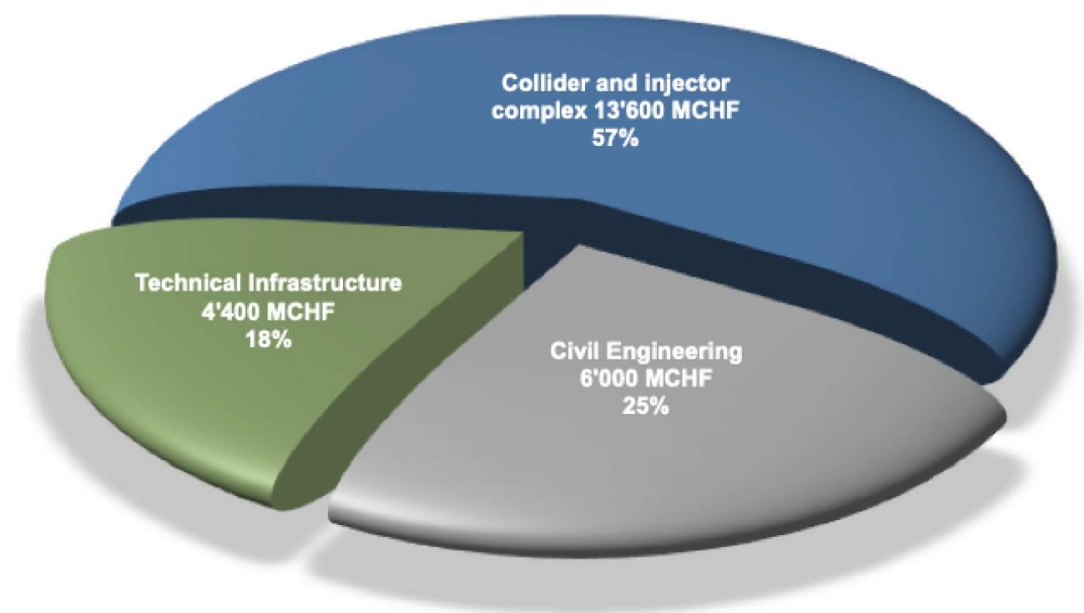

Fig. E.1. FCC-hh capital cost per project domain if implemented as a stand-alone project.

Table E.2. Summary of capital cost for the implementation of the FCC-hh project as a combined project after implementation of FCC-ee.

\begin{tabular}{|l|r|}
\hline Domain & Cost (MCHF) \\
\hline Collider and injector complex & 13600 \\
\hline Technical infrastructure & 2800 \\
\hline Civil engineering & 600 \\
\hline Total cost & 17000 \\
\hline
\end{tabular}

with a total construction cost of $600 \mathrm{MCHF}$. For technical infrastructure, all electrical, cooling and ventilation installations are reused. The cryogenics infrastructure for the main magnet cooling therefore drives the capital cost that amounts to a total of $2800 \mathrm{MCHF}$, including any adaption of the other technical infrastructure systems. The accelerator construction cost is quasi unchanged, apart from a small saving from reuse of the RF system.

This scenario of constructing FCC-hh after FCC-ee operation opens the opportunity for a much longer R\&D period (additional 15-20 years) for the design of FCC-hh key technologies. Consequently, alternative technologies can be considered, e.g. high temperature super-conducting magnets, that in case of a successful R\&D phase could lead to improved parameters (e.g. increase of collision energy) and reduced infrastructure requirements (cryogenics) and/or higher energy efficiency. 


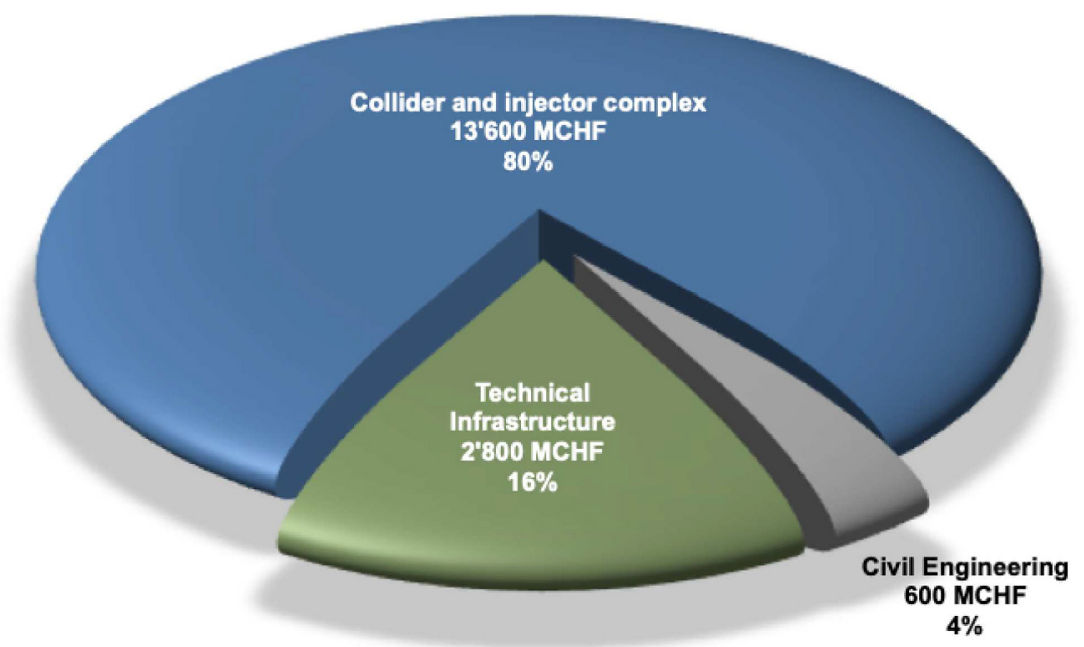

Fig. E.2. FCC-hh capital cost per project domain if FCC-hh is implemented after FCC-ee.

\section{E.2 Operation costs}

Operating costs are a major factor for any research facility and design efforts need to be made from the early concept stage to enable sustainable operation. History of large-scale technical infrastructures reveals a trend of steadily decreasing normalised operating costs: While at the peak of LEP operation CERN had 3300 staff members, in the LHC era the laboratory's staff complement has shrunk to 2300 employees, even though the LHC together with its injectors is a much more complex machine. This decreasing number of personnel is a manifestation of progress in technology, operation and maintenance concepts.

This optimisation trend is expected to continue for the FCC-hh. Looking for example at the basic design unit of the collider, namely the arc FODO cell, reveals that the number of cells is only 1.8 times that of the LHC, although the FCC-hh is nearly four times larger. Consequently, the multiplicity of many components does not scale with the size of the machine and the associated operation and maintenance costs will not do so either. An increase in the absolute number of components goes along with a growth in the maintenance, repair and restore effort required. However, this fact does not necessarily imply higher complexity, i.e. the emergence of dynamic system behaviour that leads to significantly higher operation and mitigation costs. The training and experience requirements for maintenance personnel are further optimised by the use of industry-based, standardised, modular designs for accelerator components. The detailed technical design phase will focus on ensuring that the operation of such a machine is sustainable in the long term.

Sustainable maintenance. The machine design will place emphasis on conceiving the individual systems and subsystems such that they can be monitored, maintained and repaired by service suppliers as much as reasonably possible. Experience with this approach for particle accelerators and imaging devices for healthcare applications has been gained over more than 15 years. Examples include the remote maintenance of power converters and the servicing of superconducting devices including cryogenic refrigeration infrastructure. The effects of this approach are (1) the possibility to renegotiate operation and maintenance during the operation phase and, thus, to profit from an ever improving understanding of the infrastructure's operational behaviour, (2) the possibility to engage financial resources only when needed and with the pos- 
sibility to reduce them when no longer needed, (3) the creation of "local economic benefits" in each of the contributing nations and regions resulting from the financial revenues over sustained time periods for companies and a long-term education effect for highly qualified personnel.

Modular design. Investing early-on in modular designs of basic components and equipment to be installed will enable streamlined operation, service and repair. The successfully demonstrated concept of a vertically integrated "column" that will be replicated many times is the underlying principle of this approach, leading to a scalable system. Thorough analysis with potential industrial partners and the consequent application of best practices will be one of the requirements in the preparatory work plan. One key topic in this approach is the reduction of, and facility-wide agreement on, standard interfaces at all levels (e.g. mechanical, electrical, fluids and their parameters, communication and software). A dedicated activity for interface management is the key to cost-effective production and testing, installation and long-term sustainable operation.

In-kind, collaborative operation. The LHC experiments have already indicated the way by which long-term operability of an experiment can be achieved through a committed involvement of the international collaborating institutes. Intensifying and extending this concept to the entire accelerator and experiment infrastructure is an essential lever to fully engage the entire community in this project. Particle accelerator experts and equipment specialists exist in numerous academic institutes around the globe. Operating a world class particle collider creates a unique learning experience for scientists and engineers at all levels. It is also an essential way to reduce the operating budget through the assignment of in-kind contributions to the operation, maintenance and repair. Information and communication technologies in twenty years from now will permit the distributed monitoring and root-cause analysis of numerous systems. Through unified supervisory control infrastructures, it should be straightforward to operate the technical infrastructure of all experiments with a single set of trained personnel and to share the task across the globe. The CMS "remote operations center" pioneered by FNAL in the US is a first step in this direction.

The electrical energy consumption is an important operating cost, but the analysis of the conceptual design indicates that it is not a major driver of the operation cost. In order to arrive at a long-term, sustainable highest-luminosity particle collider, the FCC-hh conceptual design already integrates a number of energy reduction measures:

- Use of power-saving superconducting magnets and circuit layout optimisation.

- A novel beamscreen design with an optimised temperature working point that permits efficient removal of the synchrotron radiation heat and minimises the load on the cryogenic refrigeration system.

- Use of an innovative cryogenic refrigeration system based on a neon-helium (nelium) light gas mixture, which reduces the electricity consumption and waste heat generation by $20 \%$ with respect to traditional plants.

- Recovery and buffering of the energy stored in the superconducting magnets at the end of the cycle for reuse during the subsequent ramp in order to save energy and to control the peak electricity demand.

- Use of superconducting radiofrequency cavities based on thin-film coating technology at $4.5 \mathrm{~K}$ which have a higher energy efficiency than bulk superconducting materials at $2 \mathrm{~K}$.

- Development of high-efficiency klystrons to increase the effectiveness of electrical to $\mathrm{RF}$ power conversion. 
- Using medium-voltage DC electricity distribution to optimise the size of the powering infrastructure, enabling the introduction of renewable energy and storage systems and supressing the need for a power quality system.

- Waste heat recovery and reuse inside the facility and for storage and provision to district services (heating and air conditioning).

The total electrical energy consumption of the FCC-hh over the 25 years operation period is estimated to be $100 \mathrm{TWh}$, based on an average electricity consumption of $4 \mathrm{TWh} /$ year over the entire physics programme. (Today's electricity consumption at CERN is 1.2 TWh/year. 1.4 TWh/year is expected in the HL-LHC era). For LEP2 the energy consumption ranged between 0.9 and $1.1 \mathrm{TWh} /$ year. Based on the 2014/15 CERN electricity prices, the electricity cost for FCC-hh collider operation would be about 180 Meuro per year. Considering the total luminosity production of $30 \mathrm{ab}^{-1}$, about $150 \mathrm{kEuro}$ for electricity would need to be invested to produce $1 \mathrm{fb}^{-1}$ of integrated luminosity. With more than $10^{10}$ Higgs bosons and $10^{12}$ top-pairs produced in total, this translates into an electricity cost of about 45 cents per Higgs boson and per 100 top quark pair.

\section{Glossary}

$\boldsymbol{\Omega}$ The ohm is the SI derived unit of electrical resistance.

4DCHM 4-dimensional composite Higgs model.

A The ampere (symbol: A) is the base unit of electric current in the International System of Units (SI).

A15 A15 is the Strukturbericht notation for the crystal structure of $\mathrm{Cr}_{3} \mathrm{Si}$, originally reported for $\beta$-tungsten. This structure is shared by a family of intermetallic compounds with the formula $\mathrm{A}_{3} \mathrm{~B}$, where $\mathrm{A}$ is a transition metal. Several of these compounds, with $\mathrm{A}=\mathrm{Nb}$ or $\mathrm{V}$, are superconducting with a critical temperature $T_{\mathrm{c}}$ of around $20 \mathrm{~K}$ and an upper critical magnetic field $\mathrm{H}_{\mathrm{c} 2}$ exceeding $20 \mathrm{~T}$. The most commonly applied example is $\mathrm{Nb}_{3} \mathrm{Sn}$.

ab An attobarn corresponds to an area equal to $10^{-46} \mathrm{~m}^{2}$.

ABCI The azimuthal beam cavity interaction is software which solves the Maxwell equations directly in the time domain when a bunched beam goes through an axi-symmetric structure on or off axis. An arbitrary charge distribution can be defined by the user.

AC Alternating current.

a-C Amorphous carbon.

ADC Analogue-to-digital converter.

ADT The ADT is a transverse damping system deployed in the LHC to reduce the oscillations of the injected proton beam, to reduce emittance blow-up due to ground motion and magnetic noise or ripple and to stabilise the beam against the resistive wall instability and other possible instabilities caused by narrow band parasitic transverse impedances in the ring.

Alcove An alcove is a recessed area open from a larger room but enclosed by walls, pillars, or other architectural elements.

AMD Adiabatic matching device.

APC Artificial pinning centre: a particle, defect or other feature intentionally introduced to act as a site for flux pinning.

Ar Argon is a chemical element with symbol Ar and atomic number 18.

Arc A circular collider is composed of curved cells called arcs that are separated by straight sections (see LSS). An arc half-cell forms the periodic part of the arc lattice (see lattice). 
ARIES A H2020 EC funded research and development project in the area of particle accelerator technologies.

ARMCO American Rolling Mill Company pure iron became synonymous with the purest steel mill produced iron with a purity of more than $99.85 \% \mathrm{Fe}$.

ASIC An application-specific integrated circuit is an integrated circuit (IC) customised for a particular use, rather than intended for general-purpose use.

ATLAS A toroidal LHC apparatus is one of the seven particle detector experiments constructed at the LHC.

B The byte is a unit of digital information that most commonly consists of eight bits, representing a binary number.

b The bit, a binary digit, is a basic unit of information used in computing and digital communications. A binary digit can have only one of two values, and may be physically represented with a two-state device.

$\boldsymbol{B}_{\boldsymbol{i}}$ The dispersion function of a beam line is determined by the strength and placement of dipole magnets. As a consequence, dipole field errors also contribute to the dispersion function. Multipole components corresponding to a magnetic flux distribution, which can be added up are denoted by $B_{i}$ and $A_{i} . B_{1}$ corresponds to a normal dipole component, $B_{2}$ to a normal quadrupole component, $B_{3}$ to a normal sextupole component, $A_{1}$ to a skew dipole, $A_{2}$ to a skew quadrupole and $A_{3}$ to a skew sectupole.

barn A barn (symbol: b) is a unit of area equal to $10^{-28} \mathrm{~m}^{2}$. It is best understood as a measure of the probability of interaction between small particles. A barn is approximately the cross-sectional area of a uranium nucleus.

BCS Theory named after John Bardeen, Leon Cooper, and John Robert Schrieffer. It is the first microscopic theory of superconductivity since Heike Kamerlingh Onnes's 1911 discovery. The theory describes superconductivity as a microscopic effect caused by a condensation of Cooper pairs into a boson-like state.

Be Beryllium is a rare chemical element with symbol Be and atomic number 4 .

Beam pipe Volumes of different shape (e.g. cylindrical, conical, flanges and bellows) and material (e.g. metallic, ceramic) used to transport the beam. The ultrahighvacuum within it reduces beam-gas interactions to a level at which the beam lifetime is acceptable.

Beamline A series of functional elements, such as magnets and vacuum pipe, which carry the beam from one portion of the accelerator to another.

Beamscreen Perforated tube inserted into the cold bore of the superconducting magnets in order to protect the cold bore from synchrotron radiation and ion bombardment.

Beta function An optical function proportional to the square of the local transverse beam size. The beta function describes how the beam width changes around the accelerator. There are separate $\beta$-functions for the $x$ and $y$ planes.

B-factory A B-factory, or sometimes a beauty factory, is a particle collider experiment designed to produce and detect a large number of $\mathrm{B}$ mesons so that their properties and behaviour can be measured with small statistical uncertainty. Tauons and D mesons are also copiously produced at B-factories.

BFPP Bound-free pair production is one of the new types of processes that occur in relativistic collisions of atoms and ions. It is the production of an electronpositron pair with the electron not produced as a free state but as a bound state of one of the ions.

Bhabha scattering The electron-positron scattering process: $\mathrm{e}^{+} \mathrm{e}^{-} \rightarrow \mathrm{e}^{+} \mathrm{e}^{-}$There are two leading-order Feynman diagrams contributing to this interaction: an annihilation process and a scattering process. 
BHWIDE A Monte Carlo event generator software simulating Bhabha-scattering, developed for linear collider detector luminosity studies.

BIM Building information modeling is a process involving the generation and management of digital representations of physical and functional characteristics of places.

BLM Beam loss monitor.

BPM Beam position monitor.

$\mathbf{B q}$ The becquerel is the SI derived unit of radioactivity. One becquerel is defined as the activity of a quantity of radioactive material in which one nucleus decays per second. The becquerel is therefore equivalent to an inverse second, $\mathrm{s}^{1}$.

BR In particle physics and nuclear physics, the branching ratio for a decay is the fraction of particles which decay by an individual decay mode with respect to the total number of particles which decay.

BR (machine) The booster ring is an accelerator in the collider tunnel, which provides the particle collider continuously with particles at collision energy.

BRGM Bureau de Recherches Géologiques et Minières, France.

BS Beamstrahlung.

BSCCO Bismuth strontium calcium copper oxide (pronounced "bisko"), is a family of high-temperature superconductors. Specific types of BSCCO are usually referred to using the sequence of the numbers of the metallic ions. Thus Bi-2201 is the $\mathrm{n}=1$ compound (Bi2Sr2CuO6+x), Bi-2212 is the $n=$ 2 compound (Bi2Sr2CaCu2O8+x), and $\mathrm{Bi}-2223$ is the $n=3$ compound (Bi2Sr2Ca2Cu3O10+x).

BSM Beyond Standard Model.

Bunch A group of particles captured inside a longitudinal phase space bucket.

BX Bunch crossing.

C The coulomb (symbol: C) is the SI (international system of units) unit of electric charge. It is the charge (symbol: Q or q) transported by a constant current of one ampere in one second.

$\mathbf{C}$ band The $\mathrm{C}$ band is a designation by the IEEE (institute of electrical and electronics engineers) for a part of the microwave band of the electromagnetic spectrum covering frequencies from 4 to 8 gigahertz.

$\mathbf{C}++$ An object-oriented programming language.

CAD Computer-aided design is the use of computer systems to aid in the creation, modification, analysis, or optimisation of a design.

CAF A compressed air foam system is used in firefighting to deliver fire retardant foam for the purpose of extinguishing a fire or protecting unburned areas.

CAPEX Capital expenditures are the funds used to acquire or upgrade fixed assets, such as expenditures towards property, plant, or equipment.

Carnot Efficiency Carnot efficiency describes the maximum thermal efficiency that a heat engine can achieve as permitted by the second law of thermodynamics.

CBA Cost-benefit analysis is a systematic process for estimating and comparing benefits and costs of a project. The purpose of CBA is to facilitate a more efficient allocation of resources, demonstrating the convenience for society of a particular intervention rather than possible alternatives.

CC60 The CC60 facility at CERN uses a ${ }^{60}$ Co source for the qualification of components against TID effects.

CCT A canted-cosine-theta magnet is an accelerator magnet that superposes fields of nested and tilted solenoids that are oppositely canted.

CDR A conceptual design report completes the first stage of the ESFRI roadmap methodology, permitting concept screening, consortium formation, access policy and funding concept preparation, scientific and project leadership definition. 
CEA The French alternative energies and atomic energy commission (Commissariat à' l'énergie atomique et aux énergies alternatives).

CepC The circular electron positron collider is an electron-positron collider proposed by the Chinese high energy physics community, acting as a Higgs-factory in a new tunnel $80-100 \mathrm{~km}$ in length.

CEREMA The French "Centre d'Etude et d'Expertise sur les Risques, l'Environnement, la Mobilité et l'Aménagement" is a public administration organisation, governed by the "ministre chargé de l'écologie, du développement durable et de l'énergie" and the "ministre du transport, de l'égalité des territoires et de la ruralité". The organisation works with the territorial state services during all development projects, notably with respect to implement sustainable development goals (urbanism, environment, infrastructures and transport, risk management).

CERN European Organisation for Nuclear Research.

CETU The "centre d'études des tunnels" is a technical service of the French "ministère de l'Écologie, du Développement et de la Mer", a notified body for the safety of tunnels in France.

CFC Carbon fibre composite.

CFD Computational fluid dynamics uses numerical analysis to solve and analyse problems that involve fluid flows.

$\mathbf{C H}_{4}$ Methane is a chemical compound.

CHARM CERN high energy accelerator mixed field facility, located in the CERN east area, supplies a wide spectrum of radiation types and energies.

CHF ISO code of the Swiss franc currency.

Chilled Water Chilled water is a commodity used to cool a building's air and equipment. It's temperature is between $4^{\circ} \mathrm{C}$ and $7^{\circ} \mathrm{C}$.

CIR Circuit.

CIXP CERN internet exchange point.

CL Confidence level.

CLD The CLic detector is a conceptual design for an experiment detector at CLic, which also serves as a design model for an experiment detector at the FCC-ee.

CLIC The compact linear collider (CLIC) is a concept for a future linear electronpositron collider in a new tunnel, ranging from 11 to $50 \mathrm{~km}$, depending on the collision energy.

CLIC-dp The CLIC detector and physics study is an international collaboration currently composed of 30 institutions.

CLIQ The coupling-loss-induced quench method is a superconducting magnet protection approach.

CM Cold mass.

CMM Coordinated measuring machines.

CMOS Complementary metal oxide semiconductor is a technology for constructing integrated circuits patented in 1963 by Frank Wanlass while working for Fairchild Semiconductor.

CMS The compact muon solenoid is one of the seven particle detector experiments constructed at the LHC.

CNDP The French "Commission nationale du débat public" was created in 1995 and is since 2002 an independent administration authority. The organisation ensures that the public participates in the process of construction projects of national interest.

CNES The National Centre for Space Studies is the French government's space agency.

CNRS The French national center for scientific research (Centre National de la Recherche Scientifique).

COL Collimation. 
Collimator A device that removes beam particles at large amplitudes. They are used to keep beam-losses low and to protect critical elements of the accelerator.

Collision A close encounter of particles during which dynamic quantities such as energy, momentum, and charge may be exchanged.

Comsol A cross-platform, finite element analysis, solver and multiphysics simulation software developed by private company COMSOL Inc. (Sweden). It allows conventional physics-based user interfaces and coupled systems of partial differential equations.

Cooper pair Two electrons that appear to "team up" in accordance with theory, BCS or other, despite the fact that they both have a negative charge and normally repel each other. Below the superconducting transition temperature, paired electrons form a condensate, a macroscopically occupied single quantum state, which flows without resistance. However, since only a small fraction of the electrons are paired, the bulk does not qualify as being a "Bose-Einstein condensate".

COTS Commercial-off-the-shelf.

CP Charge conjugation parity symmetry is the product of two symmetries: C for charge conjugation, which transforms a particle into its antiparticle, and $\mathrm{P}$ for parity, which creates the mirror image of a physical system. The strong interaction and electromagnetic interaction seem to be invariant under the combined CP transformation operation, but this symmetry is slightly violated during certain types of weak decay. Historically, CP-symmetry was proposed to restore order after the discovery of parity violation in the 1950s.

$\mathrm{Cr}_{2} \mathrm{O}_{3}$ Chromium(III) oxide is an inorganic compound. It is one of the principal oxides of chromium and is used as a pigment. In nature, it occurs as the rare mineral eskolaite.

Critical temperature Temperature $T_{\mathrm{c}}$ below which characteristics of superconductivity appear. The value varies from material to material and depends on the magnetic field.

Cryo magnet Complete magnet system integrated in a cryostat, including main magnet coils, collars and cryostat, correction magnets and powering circuits.

Cryogenic system A system that operates below a temperature set by convention at $150 \mathrm{~K}\left(-123.15^{\circ} \mathrm{C}\right)$.

CST Computer simulation technology is a computational tool from Dassault Systems for 3D electromagnetic design and analysis.

Cu Copper is a chemical element with symbol $\mathrm{Cu}$ and atomic number 29.

CW A continuous wave is an electromagnetic wave of constant amplitude and frequency, almost always a sine wave, that for mathematical analysis is considered to be of infinite duration.

Cybersecurity Cybersecurity is the protection of computer systems from theft or damage to their hardware, software or electronic data, as well as from disruption or misdirection of the services they provide.

DA Dynamic Aperture.

DA $\Phi$ NE The double annular $\Phi$ factory for nice experiments is an electron-positron collider at the INFN Frascati National Laboratory in Frascati, Italy. It has been colliding electrons and positrons at a centre of mass energy of $1.02 \mathrm{GeV}$ to create $\varphi$ mesons Since 1999.

DAQ Data acquisition.

Dark matter Invisible matter that makes up $26 \%$ of the universe and which can only be detected from its gravitational effects. Only $4 \%$ of the matter in the Universe are visible. The remaining $70 \%$ are accounted to dark energy.

$\mathbf{d B}$ The decibel (symbol: $\mathrm{dB}$ ) is a unit of measurement used to express the ratio of one value of a physical property to another on a logarithmic scale. When 
expressing power quantities, the number of decibels is ten times the logarithm to base 10 of the ratio of two power quantities. That is, a change in power by a factor of 10 corresponds to a $10 \mathrm{~dB}$ change in level.

DC Direct current.

DCAL Digital hadron calorimeter.

DCCT A direct current-current transformer is a current-to-voltage transducer, employed when measurement of very high current is required.

DCH Drift CHamber.

DD4hep A detector description toolkit for high energy physics.

DELPHES Delphes is a C++ framework, performing a fast multipurpose detector response simulation. The simulation includes a tracking system, embedded into a magnetic field, calorimeters and a muon system.

DELPHI The detector with lepton, photon and hadron identification was one of the four main detectors of the large electron-positron collider (LEP) at CERN.

DESY The German electron synchrotron (Deutsches Elektronen-Synchrotron).

DFAE The Département Fédéral des Affaires Étrangères is the Swiss foreign ministry.

Dipole A magnet with two poles, like the north and south poles of a horseshoe magnet. Dipoles are used in particle accelerators to keep particles moving in a circular orbit.

DIS Dispersion suppressor.

DM Dark matter.

DN Diametre nominal, the European equivalent of NPS (nominal pipe size) defines a set of nominal pipe sizes in standard ISO 6708. The dimensionless number after the letters DN indicate the physical size in millimetres of the bore.

DOE The United States Department of Energy.

DPA Displacement per atom.

DR A damping ring reduces the emittances produced by the particle source to the small values required for the collider. Emittance reduction is achieved via the process of radiation damping, i.e. the combination of synchrotron radiation in bending fields with energy gain in RF cavities.

DRAM Dynamic random-access memory.

DVR Dynamic voltage restorer.

Dynamic aperture Maximum transverse oscillation amplitude that guarantees stable particle motion over a given number of turns. If the motion amplitude of a particle exceeds this threshold, the betatron oscillation of the particle will not have any bounds, and the motion will become unstable, leading to loss of the particle. It is expressed in multiples of the beam size together with the associated number of turns. Unlike the physical aperture, dynamic aperture separating stable and unstable trajectories is not a hard boundary.

Dynistor A dynistor is an unidirectional thyristor breakover diode. It can be used as switches in micro- and nanosecond power pulse generators.

EASITrain A H2020 EC funded Marie Słodovska Curie innovative training network project to advance superconducting wire and thin film technologies as well as cryogenic refrigeration systems for particle accelerators.

EBITDA A company's earnings before interest, taxes, depreciation, and amortisation is an accounting measure calculated using a company's net earnings, before interest expenses, taxes, depreciation, and amortisation are subtracted, as a proxy for a company's current operating profitability (i.e. how much profit it makes with its present assets and its operations on the products it produces and sells, as well as providing a proxy for cash flow.

EC Electron cloud. 
ECal Electromagnetic calorimeter (also ECAL).

ECFA European Committee for Future Accelerators.

Eddy current Eddy currents are loops of electrical current induced within conductors by a changing magnetic field in the conductor due to Faraday's law of induction. Eddy currents flow in closed loops within conductors, in planes perpendicular to the magnetic field.

EDLC Electrical double-layer capacitors.

EES Energy extraction system.

EFT An effective field theory is a type of approximation, or effective theory, for an underlying physical theory, such as a quantum field theory or a statistical mechanics model.

EHF Electrohydraulic forming is a type of metal forming in which an electric arc discharge in liquid is used to convert electrical energy to mechanical energy and change the shape of the workpiece.

EIA Environmental impact assessment.

EIE Étude de l'impact sur l'environnement is the Swiss name for the environmental impact assessment in the scope of the OEIE.

EIR Experimental insertion region.

Electron cloud A cloud of electrons generated inside an accelerator beam pipe due to gas ionisation, photoemission from synchrotron radiation, or "beam-induced multipacting" via electron acceleration in the field of the beam and secondary emission. Electron clouds may cause single- and multi-bunch beam instabilities as well as additional heat load on the beamscreen inside the cold magnets.

electronvolt In physics, the electronvolt (symbol eV, also written electron-volt and electron volt) is a unit of energy equal to approximately $1.6 \times 10^{-19}$ joules (symbol $\mathrm{J})$ in SI units. By definition, it is the amount of energy gained (or lost) by the charge of a single electron moving across an electric potential difference of one volt. The electronvolt is not an SI unit, and its definition is empirical. By massenergy equivalence, the electronvolt is also a unit of mass, expressed in units of $\mathrm{eV} / c^{2}$, where $c$ is the speed of light in vacuum.

Electroweak symmetry breaking Although electromagnetism and the weak force have the same strength at high energies, electromagnetism is much stronger than the weak force in our everyday experience. The mechanism by which, at low energies, a single unified electroweak force appears as two separate forces is called electroweak symmetry breaking.

EM Electro magnetic.

EMB Electromagnetic barrel calorimeter.

EMEC Endcap electromagnetic calorimeter.

EMF Forward electromagnetic calorimeter.

Emittance The area in phase space occupied by a particle beam. The units are mm-milliradians for transverse emittance and $\mathrm{eV} \cdot \mathrm{s}$ for longitudinal emittance.

EN European norms are documents that have been ratified by one of the three ESOs (European Standardisation Organisations), CEN, CENELEC or ETSI; recognised as competent in the area of voluntary technical standardisation as for the EU Regulation 1025/2012.

EOT The most common type of overhead crane, found in many factories. These cranes are electrically operated by a control or remote control pendant, or from an operator cabin attached to the crane.

EPFL The École polytechnique fédérale de Lausanne is one of the two Swiss Federal Institutes of Technology.

EPPSU European Particle Physics Strategy Update. See also https:// europeanstrategy. cern.

ERL Energy recovery Linac. 
ERMC Enhanced racetrack model coil.

ES Electro static.

ESD Electron-stimulated desorption.

ESFRI European strategy forum for research infrastructures.

Espoo Convention The Espoo Convention sets out the obligations of parties to assess the environmental impact of certain activities at an early stage of planning. It also lays down the general obligation of states to notify and consult each other on all major projects under consideration that are likely to have a significant adverse environmental impact across boundaries.

ESPP European Strategy for Particle Physics. See also https:// europeanstrategy . cern.

ESRF The European Synchrotron Radiation Facility is a joint research facility situated in Grenoble, France, and supported by 22 countries (13 member countries: France, Germany, Italy, UK, Spain, Switzerland, Belgium, The Netherlands, Denmark, Finland, Norway, Sweden, Russia and 9 associate countries: Austria, Portugal, Israel, Poland, Czech Republic, Hungary, Slovakia, India and South Africa).

ESS Energy storage system.

ETHZ The Eidgenössische Technische Hochschule Zürich is one of the two Swiss Federal Institutes of Technology.

EU European Union.

EuCARD2 A FP7 EC funded research and development project in the area of particle accelerator technologies.

EuroCirCol A H2020 EC funded research and development project to develop the foundations of a future circular hadron collider.

EW Electroweak.

EWPO Electroweak precision observables.

EWPT Electroweak phase transition.

EWSB Electroweak symmetry breaking.

EXP Experiment.

Experiment insertion region Place in the particle collider hosting the interaction region in which the two beams are brought to collision and the surrounding particle physics experiments.

EXT Extraction.

fb A femtobarn corresponds to an area equal to $10-43 \mathrm{~m}^{2}$.

FCC Future circular collider is a feasibility study aiming at the development of conceptual designs for future particle colliders with energies and intensities at the frontier, based on a technically feasible and affordable circular layout permitting staged implementation.

FCC-ee Future circular intensity-frontier electron-positron collider with multiple centre-of-mass collision energies ranging from the $\mathrm{Z}$ peak to $\mathrm{t} \overline{\mathrm{t}}$ collision energies at luminosities up to almost $5 \times 10^{36} \mathrm{~cm}^{-2} \mathrm{~s}^{-1}$ in a new circular tunnel of about $100 \mathrm{~km}$ length.

FCC-eh Future circular energy-frontier electron-hadron collider interaction point scenario. The scenario foresees an energy recovery linac (ERL) to generate electron beams and to collide them with high-energetic proton or ion beams of a hadron collider in a new circular tunnel of about $100 \mathrm{~km}$ length.

FCC-hh Future circular energy-frontier hadron-hadron collider reaching up to $100 \mathrm{TeV}$ centre-of-mass collision energies at luminosities of $5-10 \times 10^{34} \mathrm{~cm}^{-2} \mathrm{~s}^{-1}$ in a new circular tunnel of about $100 \mathrm{~km}$ length. Operation with protons and ions is envisaged. 
FDS Fire dynamics simulator is a software developed and maintained by the U.S. Department of Commerce National Institute of Standards and Technology for the simulation of smoke and heat transport from fires.

FED Fractional effective dose.

FEM European federation of material handling.

FF Final focus.

FIB An FIB setup is a scientific instrument that resembles a scanning electron microscope (SEM). However, while the SEM uses a focused beam of electrons to image the sample in the chamber, a FIB setup uses a focused beam of ions instead.

FinFET A type of non-planar or "3D" field effect transistor used in the design of modern processors. The transistor architecture uses raised channels called "fins", from source to drain.

FLUKA A particle physics Monte Carlo simulation package for high energy experimental physics and engineering, shielding, detector and telescope design, cosmic ray studies, dosimetry, medical physics and radio-biology.

Flux pinning In practical applications, a type II superconductor in a magnetic field is usually in the mixed state, in which the superconducting material is penetrated by magnetic flux. When carrying a current, a force (the Lorentz force) acts on these flux lines. If this was not opposed, the resulting movement of flux would result in energy dissipation, rendering the material useless for current-carrying applications. Flux pinning is the phenomenon by which defects in a superconducting material immobilise flux lines, producing a pinning force to oppose the Lorentz force. The volumetric flux pinning force effectively defines the $\mathrm{J}_{\mathrm{c}}$ of a type II superconductor. The type of defects providing effective flux pinning depend on the material (e.g. grain boundaries in $\mathrm{Nb}_{3} \mathrm{Sn}$.

FMA Radioactive waste of "faible et moyenne activité" (low and intermediate activity), the classification depends on the level of activity and on the radionuclides.

FMECA The failure mode, effects, and criticality analysis is a bottom-up, inductive analytical method which may be performed at either the functional or piece/part level. It is used to chart the probability of failure modes against the severity of their consequences. The result highlights failure modes with relatively high probability and severity of consequences, allowing remedial effort to be directed where it will produce the greatest value.

FNAL Fermi National Accelerator Laboratory.

FODO The focusing and defocusing cell is a widespread lattice concept for designing a particle accelerator based on a magnet structure consisting alternately of focusing and defocusing quadrupole lenses.

FPC Fixed power coupler.

FPGA A field-programmable gate array is an integrated circuit designed to be configured by a customer or a designer after manufacturing.

Free (air) cooling Free cooling is an economical method of using low external air temperatures to assist in chilling water, which can then be used for industrial processes, or air conditioning systems.

FSI Frequency scanning interferometry.

GB A gigabyte is by definition of the IEC, $10^{9} \mathrm{~B}$.

Gd Gadolinium is a chemical element with symbol Gd and atomic number 64 .

$\mathbf{G d}_{2} \mathbf{O}_{3}$ Gadolinium(III) oxide is an inorganic compound. It is one of the most commonly available forms of the rare-earth element gadolinium, derivatives of which are potential contrast agents for magnetic resonance imaging. 
GEANT4 GEANT4 is a platform for the simulation of the passage of particles through matter using Monte Carlo methods. It is the successor of the GEANT series of software toolkits developed by CERN.

GeniLac A project planned in Geneva to use the water of lake Geneva for cooling and heating of public buildings and international organisations.

GESDEC The "service de géologie, sols et déchets" is the office of the canton and state of Geneva in charge of questions related to excavation materials, ground acquisition and classification, protection of underground volumes and underground water.

GeV $10^{9}$ electronvolt.

Gfitter A generic fitter software for for the statistical analysis of parameter estimation problems in high-energy physics.

$\mathbf{G H z} 10^{9} \mathrm{~Hz}$.

Gif ++ The gamma irradiation facility is located in the CERN north area. It combines a 137Cs source with a high-energy particle beam from the SPS H4 beam line.

GIM In quantum field theory, the GIM mechanism (or Glashow-Iliopoulos-Maiani mechanism) is the mechanism through which flavour-changing neutral currents (FCNCs) are suppressed in loop diagrams. It also explains why weak interactions that change strangeness by 2 are suppressed, while those that change strangeness by 1 are allowed, but only in charged current interactions.

GIS Geographical information system.

GNSS The global navigation satellite system is the standard generic term for satellite navigation systems that provide autonomous geo-spatial positioning with global coverage. This term includes e.g. the GPS, GLONASS, Galileo, Beidou and other regional systems.

GRI A global research infrastructure is mandated by the G8+5 and addresses worldwide science and technology challenges, following the "GSO Framework for Global Research Infrastructures". The GSO maintains a list of potential GRIs.

GRN Geodetic reference network.

GSO Group of senior officials mandated by G8+5 to develop GRI concepts.

GTO A gate turn-off thyristor (GTO) is a special type of thyristor, which is a highpower semiconductor device. It acts as a bistable switch, conducting when the gate receives a current trigger, and continuing to conduct until the voltage across the device is reversed biased, or until the voltage is removed. GTOs, as opposed to normal thyristors, are fully controllable switches which can be turned on and off by their third lead, the gate lead.

Guinea-Pig ++ An electron-positron beam-beam simulation software developed at CERN.

GV $10^{9}$ volt.

GV (vacuum) Gate valve.

Gy The gray (symbol: Gy) is a derived unit of ionising radiation dose in the International System of Units (SI). It is defined as the absorption of one joule of radiation energy per kilogram of matter.

$\mathbf{H}$ The Higgs boson (symbol: H) is an elementary particle in the Standard Model of particle physics, produced by the quantum excitation of the Higgs field. The particle has a spin of 0 .

H2020 Horizon 2020 is an EU Research and Innovation funding programme over 7 years (2014-2020). It is the financial instrument implementing the "Innovation Union", a Europe 2020 flagship initiative aimed at securing Europe's global competitiveness. 
$\mathbf{H}_{c}$ The critical magnetic field of a superconductor. For current-carrying and magnet applications of a type II superconductor, in practice the upper critical field $\mathrm{H}_{\mathrm{c} 2}$ must be considered instead.

$\mathbf{H}_{c 2}$ The upper critical magnetic field of a type II superconductor, above which the material enters the normal state and does not show superconducting behaviour.

ha The hectare is an SI accepted metric system unit of area equal to a square with $100 \mathrm{~m}$ sides, or 1 ha $=10000 \mathrm{~m}^{2}$.

Hadron A subatomic particle that contains quarks, antiquarks, and gluons, and so experiences the strong force. The proton is the most common hadron.

HB Barrel hadron calorimeter.

HCAL Hadron calorimeter.

HCal Hadron calorimeter.

HEB High energy booster.

HEC Endcap hadron calorimeter.

HEH High energy hadron fluence, i.e. hadrons with energies greater than $20 \mathrm{MeV}$.

HEIKA The high efficiency international Klystron activity was initiated at CERN in 2014 to evaluate and develop new bunching technologies for high-efficiency Klystrons.

HEL Hollow electron lens.

HE-LHC High-energy large hadron collider. A new particle collider with about twice the LHC collision energy in the existing LHC tunnel, using technologies conceived for the FCC-hh.

HERA The hadron-elektron-ringanlage (English: hadron-electron ring accelerator) was a particle accelerator at DESY in Hamburg. It began operating in 1992. At HERA, electrons or positrons were collided with protons at a center of mass energy of $318 \mathrm{GeV}$. It was the only lepton-proton collider in the world while operating. HERA was closed down on 30 June 2007.

HF Hadron forward calorimeter.

HFSS A 3D electromagnetic simulation software for designing and simulating highfrequency electronic products from ANSYS.

HIE-ISOLDE The high-intensity and energy upgrade of ISOLDE project incorporates a new linear accelerator (linac) into CERN's ISOLDE facility (isotope mass separator on-line device).

Higgs boson An elementary particle linked with a mechanism to model, how particles acquire mass.

HiRadMat The high-radiation to materials facility at CERN provides highintensity pulsed beams to an irradiation area where material samples as well as accelerator component assemblies can be tested.

HL-LHC High Luminosity upgrade of the LHC to a levelled constant luminosity of $5 \times 10^{34} \mathrm{~cm}^{2} \mathrm{~s}^{-1}$. A dedicated FP7 design study (HiLumi LHC DS) precedes the implementation of the upgrade.

HLS Hydrostatic levelling system.

HLT High level trigger.

HOM Higher order modes are undesired Eigenmodes parasitically excited in a resonant (accelerating) radiofrequency cavity.

HTML Hypertext markup language.

HTS High temperature superconductors have critical temperatures above $77 \mathrm{~K}$.

HTTP Hypertext transfer protocol.

HV High voltage.

HVDC High voltage direct current power distribution.

HX Heat exchanger. 
$\mathbf{H z}$ The hertz (symbol: Hz) is the derived unit of frequency in the International System of Units (SI) and is defined as one cycle per second.

IBS Iron-based superconductors (IBS) are iron-containing chemical compounds whose superconducting properties were discovered in 2006.

IC Integrated circuit.

ICS Inverse compton scattering.

ICT Information and communications technology.

IDEA The international detector for electron accelerator is a conceptual design for an experiment detector at the FCC-ee.

IEEE The Institute of Electrical and Electronics Engineers is is the world's largest association of technical professionals. Its objectives are the educational and technical advancement of electrical and electronic engineering, telecommunications, computer engineering and allied disciplines.

ILC The international linear collider (ILC) is a proposed linear electron-positron particle collider aiming at collision energies of $500 \mathrm{GeV}$ and an upgrade to $1000 \mathrm{GeV}(1 \mathrm{TeV})$.

Impedance A quantity that quantifies the self-interaction of a charged particle beam, mediated by the beam environment, such as the vacuum chamber, RF cavities, and other elements encountered along the accelerator or storage ring.

INFN Istituto Nazionale di Fisica Nucleare.

INJ Injection.

Innovation New ideas that respond to societal or economic needs and generate new products, services, business and organisational models that are successfully introduced into an existing market and are able to create new markets and that contribute value to society.

Invar Invar, also known generically as $\mathrm{FeNi} 36$ (64FeNi in the US), is a nickel-iron alloy notable for its uniquely low coefficient of thermal expansion (CTE). The name Invar comes from the word invariable, referring to its relative lack of expansion or contraction with temperature changes. It was invented in 1896 by Swiss physicist Charles Édouard Guillaume. He received the Nobel Prize in Physics in 1920 for this discovery, which enabled improvements in scientific instruments.

Ion An atom or molecule that is not electrically neutral but that carries a positive or negative charge (electrons removed or added).

IP Interaction point.

IPC Incoherent pair creation.

IR Interaction region.

IrCe Iridium-cerium is an alloy used as a photocathode.

ISD Ion-stimulated desorption.

ISO The International Organisation for Standardisation is an international standard-setting body composed of representatives from various national standards organisations.

IT Information technology.

ITER ITER is an international nuclear fusion research and engineering project. It is an experimental Tokamak fusion reactor that is being built next to the Cadarache facility in Saint-Paul-lèz-Durance, in Provence, southern France.

ITS Inner tracking system.

$\mathbf{J}$ The joule (symbol: J) is a derived unit of energy in the International System of Units. It is equal to the energy transferred to or work done on an object when a force of one newton acts on that object in the direction of its motion through a distance of one metre. It is also the energy dissipated as heat when an electric current of one ampere passes through a resistance of one ohm for one second. 
$\mathbf{J}_{\mathrm{c}}$ The scientific notation representing the "critical current density" or maximum current that a superconductor can carry. As the current flowing through a superconductor increases, the $T_{\mathrm{c}}$ will usually decrease.

JLab Thomas Jefferson National Accelerator Facility (TJNAF), commonly called Jefferson Lab or JLab.

K See Kelvin.

Karst Karst is a topography formed from the dissolution of soluble rocks such as limestone, dolomite, and gypsum. It is characterized by underground drainage systems with sinkholes and caves.

kB A kilobyte refers traditionally to 1024 bytes. In december 1998, the IEC defined $2^{10}=1024$ bytes as 1 kibiyte $(\mathrm{KiB})$.

KEKB KEKB is a particle accelerator used in the Belle experiment to study CP violation. KEKB is located at the KEK (High Energy Accelerator Research Organisation) in Tsukuba, Ibaraki Prefecture, Japan.

Kelvin Unit of measurement for temperature $(\mathrm{K})$ using as null point the absolute zero, the temperature at which all thermal motion ceases. $0 \mathrm{~K}=-273.15^{\circ} \mathrm{C}$.

keV $10^{3}$ electronvolt.

kg The kilogram or kilogramme (symbol: $\mathrm{kg}$ ) is the base unit of mass in the International System of Units (SI), and is defined as being equal to the mass of the International Prototype of the Kilogram (IPK, also known as "Le Grand K" or "Big K"), a cylinder of platinum-iridium alloy stored by the International Bureau of Weights and Measures at Saint-Cloud, France.

KLOE A particle physics experiment at the INFN Frascati National Laboratory, Italy

KlyC Software developed at CERN for the optimisation and design of high efficiency Klystrons based on new bunching mechanisms.

Klystron A specialised linear-beam vacuum tube, which is used as an amplifier for high radio frequencies.

$\mathbf{K r}$ Krypton is a chemical element with symbol $\mathrm{Kr}$ and atomic number 36.

kW $10^{3}$ Watt.

$\mathbf{l}$ The litre (SI spelling) or liter (American spelling) (symbol 1 in this document) is an SI accepted metric system unit of volume equal to 1000 cubic centimetres or $1 / 1000$ cubic metre.

$\mathbf{L}^{*}$ The distance from the IP to the start of the magnetic field of the first magnet closest to the IP. The physical equipment is larger and is closer to the IP.

LAL Laboratoire de l'Accélérateur Linéaire (LAL) of CNRS in Orsay in France.

LAr Liquid argon.

LASE Laser-ablated surface engineering.

Lattice In accelerator physics, a magnetic lattice is a composition of electromagnets at given longitudinal positions around the vacuum tube of a particle accelerator, and thus along the path of the enclosed charged particle beam. Many lattices are composed of identical substructures or cells, which denote a special magnet arrangement that may reoccur at several positions along the path.

LCB Lower cold box.

LCBI Longitudinal coupled bunch instability.

LCCS Local Chromatic Correction System.

LEP The large electron-positron collider, which was operated at CERN until 2000.

LEP3 A concept for an electron-positron collider in the existing LHC tunnel with a centre-of-mass of $240 \mathrm{GeV}$ and a peak luminosity of $1 \times 10^{34} \mathrm{~cm}^{-2} \mathrm{~s}^{-1}$ at each of two experiments. 
Lepton A class of elementary particles that do not experience the strong force. The electron is the most common lepton.

LF Low field.

LFV Lepton flavour violation.

LGAD Low gain avalanche detectors.

LHC The large hadron collider is a circular particle collider for protons and heavy ions with a design centre-of-mass energy of $14 \mathrm{TeV}$ for proton-proton collisions at a peak luminosity of $1 \times 10^{34} \mathrm{~cm}^{2} \mathrm{~s}^{-1}$ at CERN in Geneva, Switzerland.

$\mathbf{L H C b}$ The large hadron collider beauty experiment is one of the seven particle detector experiments constructed at the LHC.

LHeC A study to extend the current LHC collider with an energy recovery linac (ERL) to generate electron beams and to collide them with high-energetic proton or ion beams of the LHC.

LIL LEP injector linac.

Limestone Limestone is a sedimentary rock, composed mainly of skeletal fragments of marine organisms such as coral, forams and molluscs. Its major materials are the minerals calcite and aragonite, which are different crystal forms of calcium carbonate $\left(\mathrm{CaCO}_{3}\right)$.

Linac A linear accelerator for charged particles in which a number of successive radiofrequency cavities that are powered and phased such that the particles passing through them receive successive increments of energy.

Linac4 The linear accelerator 4 at CERN accelerates negative hydrogen ions $\left(\mathrm{H}^{-}\right.$, consisting of a hydrogen atom with an additional electron) to $160 \mathrm{MeV}$ to prepare them to enter the PSB (proton synchrotron booster), which is part of the LHC injection chain.

LIU LHC injector upgrade.

LLRF Low Level RF.

$\mathbf{L N}_{2}$ Liquid nitrogen is nitrogen in a liquid state at an extremely low temperature.

LSP Lightest supersymmetric particle.

LSS Long straight section: quasi-straight segments of a circular collider, which are available for beam interactions or utility insertions (e.g. injection, extraction, collimation, RF).

LTO Lithium titanium oxide.

LTS Low temperature superconductors have critical temperatures below $77 \mathrm{~K}$.

Luminometer A calorimeter inside the detector to precisely measure the luminosity.

Luminosity Luminosity is the rate of collision events normalised to the cross section. It is expressed as inverse square centimetre and inverse second $\left(\mathrm{cm}^{-2} \mathrm{~s}^{-1}\right)$ or barn $\left(1\right.$ barn $\left.=10^{-24} \mathrm{~cm}^{2}\right)$.

LV Low voltage.

MAC The medium access control sublayer and the logical link control (LLC) sublayer together make up the data link layer (DLL). Within that data link layer, the LLC provides flow control and multiplexing for the logical link (i.e. EtherType, 802.1Q VLAN tag), while the MAC provides flow control and multiplexing for the transmission medium. MAC is responsible for the transmission of data packets to and from the network-interface card, and to and from another remotely shared channel.

MAD-X MAD-X is a project and computational physics software to aid particle accelerator design and simulation.

MADX-PTC MAD X polymorphic tracking code.

MAPS Monolithic active pixel sensor. A silicon detector technology for high-energy physics particle detectors. 
MB A megabyte are by definition of the IEC, 1000000 bytes $\left(10^{6} \mathrm{~B}\right)$.

MC Main corrector magnet.

MCHF $10^{6}$ CHF.

MD (magnet) Main dipole.

MDI The machine detector interface refers to the topics and regions where the beamlines of the accelerator overlap with the physics experiment's detector. Key elements include mechanical support of final beamline elements, luminosity monitoring, feedback, background suppression and radiation shielding.

MDISim A tool set developed in the frame of the FCC study for Machine Detector Interface SIMulations using and combining the standard tools MAD-X, ROOT and GEANT4.

MDT Monitored drift tube.

MEG A particle physics experiment at the Paul Scherrer Institute, Switzerland.

MeV $10^{6}$ electronvolt.

$\mathbf{M g B}_{2}$ Magnesium diboride is the inorganic compound. It is a dark gray, waterinsoluble solid. The compound becomes superconducting at $39 \mathrm{~K}$.

MGy $10^{6}$ gray.

$\mathrm{MHz} 10^{6} \mathrm{~Hz}$

MI Microwave instability.

MIIT Millions of amp squared seconds. A performance indicator of a superconducting cable that determines two primary factors of the adequate quench protection system: how quickly the current in the magnet must be reduced once a quench is detected and how quickly a quench must be detected, once the initiating spot quenches.

MJ $10^{6} \mathrm{~J}$.

MLI Multi layer insulation.

mm $10^{-3} \mathrm{~m}$

MO Main octupole corrector magnet.

Molasse Variable sedimentary deposits comprising sandstones, shales and conglomerates that form as terrestrial or shallow marine deposits in front of rising mountain chains. It is the typical soil type found in the Franco-Geneva basin.

MolfFlow + Software developed at CERN to calculate the pressure in an arbitrarily complex geometry when ultra-high vacuum condition is met.

MOSFET Metal-oxide-semiconductor field-effect transistor.

MoU Memorandum of understanding.

MP Medium pressure, around 20 bar.

MPGD Micro pattern gas detector.

MQ Main quadrupole.

MQDA Main quadrupole dispersion suppressor magnet.

MQS Main quadrupole skew magnet.

MQT Main quadrupole tuning magnet.

MQTL Main quadrupole trim magnet.

MRI Magnetic resonance imaging.

MRN Metrologial reference network.

MS Main sextupole.

MTE Multi turn extraction.

MTTF Mean time to failure.

MTTR Mean time to repair.

MVDC Medium voltage direct current power distribution.

MW $10^{6}$ Watt 
$\mathbf{N}$ The newton (symbol: N) is the SI unit of force. A newton is how much force is required to make a mass of one kilogram accelerate at a rate of one metre per second squared.

NASA National Aeronautics and Space Administration.

$\mathrm{Nb}$ Niobium, formerly known as columbium, is a chemical element with symbol $\mathrm{Nb}$ (formerly $\mathrm{Cb}$ ) and atomic number 41. It is a soft, grey, crystalline, ductile transition metal, often found in the minerals pyrochlore and columbite, hence the former name "columbium". Niobium is used in various superconducting materials.

$\mathrm{Nb}_{3} \mathbf{S n}$ An intermetallic compound of niobium $(\mathrm{Nb})$ and tin ( $\mathrm{Sn}$ ) with the A15 structure, and a type II LTS with with $T_{\mathrm{c}}=18.3 \mathrm{~K}$ and $\mathrm{H}_{\mathrm{c} 2}=25 \mathrm{~T}$.

NbTi Niobium-titanium is an alloy of niobium $(\mathrm{Nb})$ and titanium $(\mathrm{Ti})$ and a type II LTS with $T_{\mathrm{c}}=9.5 \mathrm{~K}$. Nb-Ti wires, containing $\mathrm{Nb}-\mathrm{Ti}$ filaments in an aluminium or copper matrix, are produced industrially and are used in the majority of superconducting magnets.

NEG Non-evaporable getter materials are mostly porous alloys or powder mixtures of $\mathrm{Al}, \mathrm{Zr}, \mathrm{Ti}, \mathrm{V}$ and iron (Fe). They help to establish and maintain vacuums by soaking up or bonding to gas molecules that remain within a partial vacuum.

Nelium A light gas mixture made of neon and helium.

NEXTorr A patented flanged vacuum pumping solution, combining NEG and ion pumping technologies by the saes group, Milan, Italy.

NIE The notice d'impact sur l'environnement is an assembly of the results of environmental impact assessments for large projects in Switzerland.

NMR Nuclear magnetic resonance.

NN Neural network.

NPV Net Present Value is a measurement of profit calculated by subtracting the present values (PV) of cash outflows (including initial cost) from the present values of cash inflows over a period of time. It is determined by calculating the costs (negative cash flows) and benefits (positive cash flows) for each period of an investment. After the cash flow for each period is calculated, the PV of each one is obtained by discounting its future value at a periodic rate of return.

NTU The number of transfer units is defined as a ratio of the overall thermal conductance to the smaller heat capacity rate. It may also be interpreted as the relative magnitude of the heat transfer rate compared to the rate of enthalpy change of the smaller heat capacity rate fluid.

NuPECC Nuclear physics European Collaboration Committee.

$\mathcal{O}$ Big $\mathrm{O}$ notation is a mathematical notation that describes the limiting behavior of a function when the argument tends towards a particular value or infinity.

OBS Organisation breakdown structure.

ODH Oxygen deficiency hazard.

OEIE The ordonnance relative à' l'étude de l'impact sur l'environnement is the Swiss environmental impact assessment regulation.

OFHC Oxygen-free copper (OFC) or oxygen-free high thermal conductivity (OFHC) copper is a group of wrought high conductivity copper alloys that have been electrolytically refined to reduce the level of oxygen to $0.001 \%$ or below.

OPEX An operating expense, operating expenditure, operational expense, operational expenditure or opex is an ongoing cost for running a product, business, or system.

Optics An optical configuration refers to a powering scheme of the magnets. There can be several different optics for a single lattice configuration. Different optics exist for instance, for injection and for luminosity operation corresponding to different $\beta^{*}$ values in the experiment insertions. 
ORC The organic rankine cycle is named for its use of an organic, high molecular mass fluid with a liquid-vapor phase change, or boiling point, occurring at a lower temperature than the water-steam phase change. The fluid allows Rankine cycle heat recovery from lower temperature sources. The low-temperature heat is converted into useful work, that can itself be converted into electricity.

OSI The open systems interconnection model is a conceptual model that characterises and standardises the communication functions of a telecommunication or computing system without regard to its underlying internal structure and technology.

OSI Model The open systems interconnection model is a conceptual and logical layout that defines network communication used by systems open to interconnection and communication with other systems.

Overburden Material (rock, soil) that lies above an underground structure, e.g. a tunnel or cavern.

Pa The pascal is the SI derived unit of pressure. A common multiple unit of the pascal us the hectopascal $(1 \mathrm{hPa}=100 \mathrm{~Pa})$ which is equal to one millibar.

Pandora PFA The pandora particle flow algorithm is an event reconstruction software developed for future linear collider studies ILC and CLIC.

$\mathbf{P b}$ Lead is a chemical element with symbol $\mathrm{Pb}$ (from the Latin plumbum) and atomic number 82 .

PBR The pre booster ring is an injector to the booster top-up ring (BR), which continuously provides the particle collider with particles at collision energy.

PBS Product breakdown structure.

PCB Printed circuit board.

PCO Power COnverter.

PDF The parton name was proposed by Richard Feynman in 1969 as a generic description for any particle constituent within the proton, neutron and other hadrons. These particles are referred today as quarks and gluons. A parton distribution function is defined as the probability density for finding a particle with a certain longitudinal momentum fraction at a certain resolution scale.

PEDD Peak energy deposition density.

PEP-II The PEP-II facility consists of two independent storage rings, one located atop the other in the at the Stanford Linear Accelerator Center (SLAC). The highenergy ring, which stores a $9 \mathrm{GeV}$ electron beam, was an upgrade of the existing Positron-Electron Project (PEP) collider; it reused all of the PEP magnets and incorporated a state-of-the-art copper vacuum chamber and a new radio-frequency system capable of supporting a stored beam of high current. The low-energy ring, which stores $3.1 \mathrm{GeV}$ positrons, was newly constructed. Injection is achieved by extracting electrons and positrons at collision energies from the SLC and transporting them each in a dedicated bypass line. The low-emittance Stanford Linear Collider (SLC) beams are used for the injection process. The collider was completed in July 1998.

PERLE Powerful energy recovery Linac for experiments, an envisaged test facility for an energy recovery linac (ERL) at Laboratoire de l'Accélérateur Linéaire (LAL) of CNRS in Orsay in France.

PFL Pulse forming line.

PFN Pulse forming network.

pH A logarithmic scale used to specify the acidity or basicity of an aqueous solution. It is approximately the negative of the base 10 logarithm of the molar concentration, measured in units of moles per liter, of hydrogen ions.

Phase Space A six-dimensional space consisting of a particle's position (x, y, z) and divergence $\left(\mathrm{x}^{\prime}, \mathrm{y}^{\prime}, \mathrm{z}^{\prime}\right)$. Phase space is represented in two dimensions by plotting 
position on the horizontal axis and the corresponding divergence on the vertical axis.

PHY In the seven-layer OSI model of computer networking, the physical layer or layer 1 is the first and lowest layer. This layer may be implemented by a dedicated PHY chip. The physical layer consists of the electronic circuit transmission technologies of a network.

Pile-up The situation where a particle detector is affected by several events at the same time.

PIT The powder-in-tube process is often used for making electrical conductors from brittle superconducting materials such as niobium-tin or magnesium diboride and ceramic cuprate superconductors such as BSCCO.

PLC Programmable logic controller.

PMNS The Pontecorvo-Maki-Nakagawa-Sakata matrix, Maki-Nakagawa-Sakata matrix (MNS matrix), lepton mixing matrix, or neutrino mixing matrix is a unitary mixing matrix which contains information on the mismatch of quantum states of neutrinos when they propagate freely and when they take part in the weak interactions. It is a model of neutrino oscillation. This matrix was introduced in 1962 by Ziro Maki, Masami Nakagawa and Shoichi Sakata, to explain the neutrino oscillations predicted by Bruno Pontecorvo.

PMT Photomultiplier tube.

pNGB Pseudo Nambu-Goldstone boson.

PoT Protons on target.

PPLP Parabolic parabolic linear parabolic.

ppm Parts per million $\left(10^{-6}\right)$.

PS The proton synchrotron is a particle accelerator at CERN. It was CERN's first synchrotron, beginning operation in 1959. It has since served as a pre-accelerator for the ISR (intersecting storage rings) and the SPS (super proton synchrotron), and is currently part of the LHC (large hadron collider) accelerator complex. In addition to protons, PS has accelerated alpha particles, oxygen and sulphur nuclei, electrons, positrons and antiprotons.

PSB The proton synchrotron booster at CERN is an accelerator made up of four superimposed synchrotron rings that receive beams of protons from the linear accelerator Linac 2 or 4 at $50 \mathrm{MeV}$ and accelerate them to $1.4 \mathrm{GeV}$ for injection into the PS (proton synchrotron).

PSD Photon stimulation desorption is a phenomenon whereby a substance is released from or through a surface.

PSO In computational science, particle swarm optimisation is a computational method that optimises a problem by iteratively trying to improve a candidate solution with regard to a given measure of quality. It solves a problem by having a population of candidate solutions, here dubbed particles, and moving these particles around in the search-space according to simple mathematical formulae over the particle's position and velocity. Each particle's movement is influenced by its local best known position, but is also guided toward the best known positions in the search-space, which are updated as better positions are found by other particles. This is expected to move the swarm toward the best solutions.

PU Pile-up.

PV Photovoltaics.

PVSS Prozessvisualisierungs- und steuerungssoftware. A SCADA product from SIEMENS company ETM professional control now called WinCC OA.

Px One of the 12 FCC access points. $x$ can be one of A, B, C, D, E, F, G, H, I, J, $\mathrm{K}, \mathrm{L}$, e.g. PA is the access point A close to the CERN Meyrin site.

PyHEADTAIL A macroparticle tracking software designed specifically to simulate collective effects in circular accelerators. 
Pythia A Monte Carlo event generator software.

Q The quality factor or $\mathrm{Q}$ factor is a dimensionless parameter that describes how underdamped an oscillator or resonator is, and characterises a resonator's bandwidth relative to its centre frequency. Higher $\mathrm{Q}$ indicates a lower rate of energy loss relative to the stored energy of the resonator. For an RF cavity it characterises RF losses in the cavity: an RF cavity having a higher Q factor is a more efficient user of RF power.

QC1 First final focus quadrupole next to the IP.

QC2 Second final focus quadrupole, behind QC1.

QCD In theoretical physics, quantum chromodynamics is the theory of the strong interaction between quarks and gluons, the fundamental particles that make up composite hadrons such as the proton, neutron and pion.

QD Defocusing quadrupole.

QED In particle physics, quantum electrodynamics is the relativistic quantum field theory of electrodynamics.

QF Focusing quadrupole.

QFT Quantum field theory.

QGP Quark gluon plasma.

QPS Quench protection system.

QPU Quench processing unit.

QRL Cryogenic distribution line.

QS Quench instrumentation units in the tunnel.

Quench The change of state in a material from superconducting to resistive. If uncontrolled, this process damages equipment due to thermal stress induced by the extremely high-currents passing through the material.

R\&D Research and development refers to activities to develop new services and products, or to improve existing services and products. Research and development constitutes the first stage of development of a potential new service or the production process.

R2E Radiation to electronics.

rad The radian (SI symbol rad) is the SI unit for measuring angles. The length of an arc of a unit circle is numerically equal to the measurement in radians of the angle that it subtends; $360^{\circ}$ correspond to $2 \pi \mathrm{rad}$.

RAMS Reliability, availability, maintainability and safety. Four non-functional key characteristics that determine the performance and total cost of technical systems.

RD52 A research and development project at CERN, carried out by an international collaboration, to develop a detector technology for a future electron-positron particle collider using the simultaneous measurement of scintillation light and Cerenkov light generated in the shower development process.

RDC Radiation dependent capacitor.

RDP Resonant depolarisation.

RDR Radiation dependent resistor.

ReBCO Rare-earth barium copper oxide is a family of chemical compounds known for exhibiting high temperature superconductivity.

RF Radiofrequency.

RF cavity An electromagnetically resonant cavity used to convey energy (accelerate) to charged particles as they pass through by virtue of the electric field gradient across the cavity gap(s). Radio Frequency is a rate of oscillation in the range of around $3 \mathrm{kHz}-300 \mathrm{GHz}$.

RH Relative humidity is the ratio of the partial pressure of water vapor to the equilibrium vapor pressure of water at a given temperature. Relative humidity 
depends on temperature and the pressure of the system of interest. The same amount of water vapor results in higher relative humidity in cool air than warm air.

RHA Radiation hardness assurance.

RI A research infrastructure is a facility, a set of resources and services that are used by research communities to conduct research and foster innovation in their fields. This includes major scientific equipment or sets of instruments, knowledge-based resources such as collections, archives and scientific data, e-infrastructures, such as data and computing systems and communication networks and any other tools that are essential to achieve excellence in research and innovation.

RMC Racetrack model coil.

RMM Racetrack model magnet.

RMS The root mean square is square root of the arithmetic mean of the squares of a set of numbers.

Roadheader Excavation equipment consisting of a boom mounted cutting head with a hydraulic mechanism.

Rockbreaker A hydraulically powered tool used to break up rock during the excavation process.

RoHS The restriction of hazardous substances directive 2002/95/EC, (RoHS 1), short for directive on the restriction of the use of certain hazardous substances in electrical and electronic equipment, was adopted in February 2003 by the European Union.

ROOT ROOT is a modular scientific software toolkit. It provides functionalities needed to deal with big data processing, statistical analysis, visualisation and storage.

ROXIE Software developed at CERN for the electromagnetic simulation and optimisation of accelerator magnets.

RPC Resistive plate chamber.

RRP The restacked-rod restack process is a manufacturing process for $\mathrm{Nb}_{3} \mathrm{Sn}$ wires developed by the OST company (Oxford instruments technologies), which has been subsequently acquired by BEST Inc. (Bruker Energy and Supercon. Technologies).

RRR The residual resistivity ratio is defined as the ratio of the electrical resistivity of a material at room temperature and at a chosen cryogenic temperature. For superconducting materials, a cryogenic temperature above $T_{\mathrm{c}}$ must be chosen. RRR serves as a measure of the purity and overall quality of a sample: as electrical resistivity usually increases as defect prevalence increases, a large RRR is associated with a pure sample.

RTE Réseau de Transport d'Électricité is the French electricity transmission system operator. It is responsible for the operation, maintenance and development of the French high-voltage transmission system.

RW The resistive wall impedance is one of the main sources for beam instabilities in synchrotrons and storage rings.

S The siemens (symbol: S) is the derived unit of electric conductance, electric susceptance and electric admittance in the International System of Units (SI). Conductance, susceptance, and admittance are the reciprocals of resistance, reactance, and impedance respectively; hence one siemens is redundantly equal to the reciprocal of one ohm, and is also referred to as the mho.

$\sqrt{s}$ The total centre of mass energy of the colliding particles.

$\mathbf{S}$ band The $\mathrm{S}$ band is a designation by the IEEE (institute of electrical and electronics engineers) for a part of the microwave band of the electromagnetic spectrum covering frequencies from 2 to 4 gigahertz. 
S275JR A structural steel grade according to EN 10025: part 2: 2004.

SAD Strategic accelerator design is a software tool for particle accelerator design developed at KEK since 1986.

SC coating A very thin layer of superconducting material on normal-conducting material (e.g. copper). Used for various purposes such as quench avoidance of a neighbouring superconductor, reduction of production costs due to use of cheaper support material and impedance reduction.

SCADA Supervisory control and data acquisition.

SCP The "Structure de Concertation Permanente" federates representatives of CERN, the Swiss federal and cantonal governments as well as the Swiss permanent mission at the international organisations in order to work in common on adequate administrative frameworks for CERN's operation and future developments on Swiss territory.

scSPS The superconducting SPS is a superconducting synchrotron, replacing the current SPS accelerator at CERN.

SDA The "surfaces d'assolement" are land plots in Switzerland, which are reserved for potential agricultural purposes in the event of crisis. They are not constructible.

SEE Single event effect is a general class of radiation effects in electronic devices. There are two types of effects: those which cause permanent damage to the equipment's functionality and those, which cause a transient fault.

SEM A scanning electron microscope (SEM) is a type of electron microscope that produces images of a sample by scanning the surface with a focused beam of electrons.

SESAME The synchrotron-light for experimental science and applications in the middle east is a third-generation synchrotron light source. SESAME is located in Allan, Jordan $(30 \mathrm{~km}$ from Amman and $30 \mathrm{~km}$ from the King Hussein/Allenby Bridge crossing of the Jordan River).

SEU A single event upset is a change of state caused by one single ionising particle (ions, electrons, photons) striking a sensitive node in a micro-electronic device, such as in a microprocessor, semiconductor memory, or power transistor. It is not considered to permanently damage the equipment's functionality. It is an example of a general class of radiation effects in electronic devices called single event effects (SEE).

SEY Secondary electron yield.

SFOE The Swiss Federal Office of Energy.

SFOPT Strongly first order phase transition.

SGAR The Secretariat Générale de la région Auvergne-Rhône-Alpes assists the prefect of the region in the implementation of the government's policies in the region.

Shotcrete A sprayed concrete lining that is projected at high velocity via a hose onto a surface.

SI The international system of units (SI, abbreviated from the French système international (d'unités)) is the modern form of the metric system, and is the most widely used system of measurement. It comprises a coherent system of units of measurement built on seven base units that are ampere, kelvin, second, metre, kilogram, candela, mole, and a set of twenty prefixes to the unit names and unit symbols that may be used when specifying multiples and fractions of the units. The system also specifies names for 22 derived units, such as lumen and watt, for other common physical quantities.

SiC Silicon carbide (SiC) devices belong to the so-called wide band gap semiconductor group. They offer a number of attractive characteristics for high voltage power semiconductors when compared to commonly used silicon ( $\mathrm{Si}$ ). In particular, the much higher breakdown field strength and thermal conductivity of $\mathrm{SiC}$ 
allow devices to be created which by far outperform the corresponding Si ones. This way previously unattainable efficiency levels can be achieved.

SiD The silicon detector is a conceptual design for an experiment detector at the ILC.

SiPM Silicon photomultiplier.

SIRIUS Sirius is a synchrotron light source facility based on a 4th generation low emittance storage ring that is under construction in Campinas, Brazil.

SITROS A tracking program developed at DESY in 1983 for the simulation of polarising and depolarising effects in electron-positron storage rings.

Sixtrack A single particle 6D symplectic tracking code optimised for long term tracking in high energy particle accelerators.

SLC Stanford Linear Collider.

Slurry shield TBM A TBM fitted with a full face cutterhead which provides face support by pressurizing boring fluid inside the cutterhead chamber. These machines are most suited for tunnels through unstable material subjected to high groundwater pressure or water inflow that must be stopped by supporting the face with a boring fluid subjected to pressure.

SM Standard Model.

SMC Short model coil.

sMDT Small diameter muon drift tube.

SME Small and medium-sized enterprises.

SMEFT The Standard Model effective field theory is a model independent framework for parameterising deviations from the Standard Model in the absence of light states.

SMS A safety management system integrates autonomously working safety-related subsystems for higher-level operation, such as intrusion alarms, entrance and access control systems, fire and smoke detection, communication with public emergency services, public address and evacuation systems and many more.

SPN Support pre-alignment network.

SPS The super proton synchrotron is a particle accelerator at CERN. It is housed in a circular tunnel, $6.9 \mathrm{~km}$ in circumference and delivers beams to fixed target experiments and the LHC.

SPT The standard penetration test is an in-situ dynamic penetration test designed to provide information on the geotechnical engineering properties of soil. The test procedure is described in ISO 22476-3, ASTM D1586 and Australian Standards AS 1289.6.3.1.

SR Synchrotron radiation.

SRF Superconducting radiofrequency.

SSM Sequential Standard Model.

SSS Short straight section.

Standard Model The Standard Model explains how the basic building blocks of matter interact, governed by four fundamental forces.

STATCOM Static synchronous compensator.

STFC Science and Technology Facilities Council in the UK.

Storz Storz is a type of hose coupling invented by Carl August Guido Storz in 1882 and patented in Switzerland in 1890, and patented in the U.S. in 1893 that connects using interlocking hooks and flanges. It is widely used on fire hoses in firefighting applications.

Strand A superconducting strand is a composite wire containing several thousand superconducting filaments (e.g. $\mathrm{Nb}_{3} \mathrm{Sn}$ ) dispersed in a matrix with suitably small electrical resistivity properties (e.g. copper).

Strong force One of four known fundamental forces (the others are the weak force, electromagnetism and gravity). The strong force is felt only by quarks and gluons, 
and is responsible for binding quarks together to make hadrons. For example, two up quarks and a down quark are bound together to make a proton. The strong interaction is also responsible for holding protons and neutrons together in atomic nuclei.

Superconducting cable Superconducting cables are formed from several superconducting strands in parallel, geometrically arranged in the cabling process to achieve well-controlled cable geometry and dimensions, while limiting the strand deformation in the process. Cabling several strands in parallel results in an increase of the current carrying capability and a decrease of the inductance of the magnet, easing protection.

Superconductivity A property of some materials, usually at very low temperatures that allows them to carry electricity without resistance.

Superferric magnet An iron-dominated magnet based on a magnetic steel structure with a minimal amount of superconductor. The structure is the same as a normal conducting magnet, but the coil is built from superconducting material. The yole is cooled to cryogenic temperature.

SuperKEKB SuperKEKB is a particle accelerator located at KEK (High Energy Accelerator Research Organisation) in Tsukuba, Ibaraki Prefecture, Japan. SuperKEKB will collide electrons at $7 \mathrm{GeV}$ with positrons at $4 \mathrm{GeV}$. The accelerator is an upgrade of KEKB, providing approximately 40 times higher luminosity, due mostly to superconducting quadrupole focusing magnets.

SUSY Supersymmetry.

Synchrotron A circular machine that accelerates subatomic particles by the repeated action of electric forces generated by $\mathrm{RF}$ fields at each revolution. The particles are maintained on constant circular orbits by synchronously increasing the magnetic fields.

Synchrotron Radiation Electromagnetic radiation generated by acceleration of relativistic charged particles in a magnetic or electric field. Synchrotron radiation is the major mechanism of energy loss in synchrotron accelerators and contributes to electron-cloud build-up.

SynRad+ A modified MolFlow + software developed at CERN to trace photons in order to calculate flux and power distribution caused by synchrotron radiation on a surface.

t The "metric ton" is a unit of measure. It corresponds to $1000 \mathrm{~kg}$ in this document.

$\mathbf{T}_{c}$ The critical temperature of a superconducting material, above which material enters the normal state and does not show superconducting behaviour.

Tantalum Tantalum is a chemical element with symbol Ta and atomic number 73. It is a rare, hard, blue-gray, lustrous transition metal that is highly corrosionresistant. It is part of the refractory metals group, which are widely used as minor components in alloys. The chemical inertness of tantalum makes it a valuable substance for laboratory equipment and a substitute for platinum.

TBA Triple bend achromat.

TBM A tunnel boring machine is a machine used to excavate tunnels with a circular cross section through a variety of soil and rock strata. They can bore through anything from hard rock to sand. Tunnel diameters can range from one metre to more than $17 \mathrm{~m}$ to date.

TCLA Active tungsten absorber.

TCLD Dispersion suppression collimator.

TCO Total cost of ownership.

TCP Primary collimator.

TCSG Secondary collimator. 
Technology Spillover Technology spillover refers to the unintentional technological benefits to firms coming from the research and development efforts of other organisations without the costs being shared.

TEM Transmission electron microscopy (TEM) is a microscopy technique in which a beam of electrons is transmitted through a specimen to form an image.

Tesla Unit of magnetic field strength. $1 \mathrm{~T}$ is the field intensity generating one newton $(\mathrm{N})$ of force per ampere (A) of current per metre of conductor.

TETRA Terrestrial trunked radio, a European standard for a trunked radio system, is a professional mobile radio and two-way transceiver specification.

TeV Tera electron Volts $\left(10^{12} \mathrm{eV}\right)$. Unit of energy. $1 \mathrm{eV}$ is the energy given to an electron by accelerating it through 1 Volt of electric potential difference.

Tevatron A $2 \mathrm{TeV}$ proton on anti-proton collider that was operated at Fermilab in Batavia, Illinois (USA) until 2011. The top quark was discovered using this collider.

TFA Radioactive waste of "très faible activité" (very low level activity), the classification depends on the level of activity and on the radionuclides.

Thyratron A type of gas-filled tube used as a high-power electrical switch and controlled rectifier. Thyratrons can handle much greater currents than similar hard-vacuum tubes. Electron multiplication occurs when the gas becomes ionised, producing a phenomenon known as Townsend discharge.

TID Total ionising dose.

TileCal TileCal is a hadronic calorimeter covering the most central region of the ATLAS experiment at the LHC.

TLEP A concept for a circular electron-positron collier in a new $80-100 \mathrm{~km}$ long tunnel acting as a Tera-Z factory

TM Transverse magnetic modes have no magnetic field in the direction of propagation.

$\mathbf{T M}_{210}$ In rectangular waveguides, rectangular mode numbers are designated by two suffix numbers attached to the mode type, such as $\mathrm{TM}_{m n}$, where $m$ is the number of half-wave patterns across the width of the waveguide and $n$ is the number of half-wave patterns across the height of the waveguide. In circular waveguides, circular modes exist and here $m$ is the number of full-wave patterns along the circumference and $n$ is the number of half-wave patterns along the diameter.

TMCI Transverse model coupling instability.

TOT The tunnel optimisation tool is software that has been developed under a cooperation contract for CERN by the company ARUP (UK).

TRL Technology readiness levels (TRLs) are indicators of the maturity level of particular technologies. This measurement system provides a common understanding of technology status and addresses the entire innovation chain. There are nine technology readiness levels; TRL 1 being the lowest and TRL 9 the highest.

TTB Turbo Brayton box.

Tungsten Tungsten, or wolfram is a chemical element with symbol $\mathrm{W}$ and atomic number 74 .

UFO Unidentified falling object.

UNESCO The United Nations Educational, Scientific and Cultural Organization is a specialised agency of the United Nations based in Paris.

UPS Uninterruptible power supply.

V The volt (symbol: V) is the derived unit for electric potential, electric potential difference (voltage), and electromotive force.

VA A volt-ampere is the unit used for the apparent power in an electrical circuit, equal to the product of root-mean-square (RMS) voltage and RMS current. In 
direct current (DC) circuits, this product is equal to the real power (active power) in watts. Volt-amperes are useful only in the context of alternating current (AC) circuits.

Vacuum Pressures much below atmospheric pressure.

Variant A variant of a product has a specific set of characteristics that distinguish it from other products in the same product line. All variants are derived from a common base and share common design features. The development of different variants is managed by distinct processes and different variants co-exist at the same time.

VBF Vector boson fusion.

Version A version of a product represents that same product at a different time. It may or may not have undergone some change (revision).

VFET Vacuum field-effect transistor.

VLP Very low pressure.

VSM An established compact shaft sinking technology for all ground conditions for soft and stable soils, originally developed by Herrenknecht AG.

VXD CLD vertex detector.

W (particle) The $\mathrm{W}$ and $\mathrm{Z}$ bosons are together known as the weak or more generally as the intermediate vector bosons. They mediate the weak interaction. The $\mathrm{W}$ bosons have either a positive or negative electric charge of 1 elementary charge and are each other's antiparticles. The particles have a spin of 1 .

W (Watt) The watt (symbol W) is a unit of power. In the international system of units (SI) it is defined as a derived unit of 1 joule per second, and is used to quantify the rate of energy transfer.

WBS Work breakdown structure.

WCS Warm compressor station.

Weak force A force carried by heavy particles known as the $\mathrm{W}$ and $\mathrm{Z}$ bosons. The most common manifestation of this force is beta decay, in which a neutron in a nucleus is transformed into a proton, by emitting an electron and a neutrino. Weak neutral current is a very weak interaction mediated by the $\mathrm{Z}$ boson that is independent of the electric charge of a particle. Particles can exchange energy through this mechanism, but other characteristics of the particles remain unchanged.

Willingness To Pay An indicator of how much a person values a product or device, measured by the maximum amount she or he would pay to acquire one.

WIMP Weakly interacting massive particles are hypothetical particles that are thought to constitute dark matter.

WinCC OA WinCC Open Architecture is a SCADA system for visualising and operating processes, production flows, machines and plants in all lines of business. It was formerly called PVSS.

WLS Wave length shifting.

WPS Wire positioning sensors.

Xe Xenon is a chemical element with symbol Xe and atomic number 54 .

XFEL A free-electron laser generating high-intensity electromagnetic radiation by accelerating electrons to relativistic speeds and directing them through special magnetic structures.

XRD X-ray powder diffraction (XRD) is a rapid analytical technique primarily used for phase identification of a crystalline material and can provide information on unit cell dimensions. The analysed material is finely ground, homogenised, and average bulk composition is determined. 
YBCO Yttrium barium copper oxide is a family of crystalline chemical compounds, displaying high-temperature superconductivity. YBCO is often categorised as a rare-earth barium copper oxide (REBCO).

$\mathbf{Z}$ The $\mathrm{W}$ and $\mathrm{Z}$ bosons are together known as the weak or more generally as the intermediate vector bosons. They mediate the weak interaction. The $\mathrm{Z}$ boson is electrically neutral and is its own antiparticle. The particles has a spin of 1 .

ZrTiV A zirconium-titanium-vanadium alloy that is used as a coating for a large surface getter pump.

\section{References}

1. CERN Council, European Strategy Session of Council, CERN-Council-S/106 (May 30, 2013)

2. Future Circular Collider Study Kickoff Meeting, University of Geneva (February 1215, 2014), http://indico.cern.ch/e/fcc-kickoff

3. E. Todesco, F. Zimmermann (eds.), in Proceedings of EuCARD-AccNet-EuroLumi workshop: The High-Energy Large Hadron Collider - HE-LHC10, Malta, CERN-2011003 (October 14-16, 2010), arXiv:1111.7188 [physics.acc-ph]

4. J. Osborne, C. Waaijer, Pre-feasibility Assessment for an $80 \mathrm{~km}$ Tunnel Project at $C E R N$, Contribution to the update of the European strategy for particle physics, 165 (July 27, 2012), http://indico.cern.ch/event/175067/call-for-abstracts/165/ file/1.pdf

5. Joint Snowmass-EuCARD/AccNet-HiLumi meeting "Frontier Capabilities for Hadron Colliders 2013" a.k.a. EuCARD VHE-LHC Day, CERN (February 21-22, 2013), http://indico.cern.ch/event/223094

6. A. Blondel, F. Zimmermann, A High Luminosity $\mathrm{e}^{+} \mathrm{e}^{-}$collider in the LHC tunnel to study the Higgs boson (2011), arXiv:1112.2518 [hep-ex]

7. EuCARD LEP3 workshop (June 18, 2012); 2nd EuCARD LEP3 workshop (October 23, 2012); 3rd EuCARD TLEP3 workshop (January 10, 2013); 4th EuCARD TLEP workshop (April 4-5, 2013)

8. The TLEP Design Study Working Group, JHEP 01, 164 (2014)

9. ATLAS Collaboration, Technical Design Report for the Phase-II Upgrade of the ATLAS muon spectrometer CERN-LHCC-2017-017, ATLAS-TDR-026 (CERN, Geneva, Switzerland, September, 2017), https://cds.cern.ch/record/2285580

10. CMS Collaboration, The Phase-2 Upgrade of the CMS Muon Detectors CERN-LHCC2017-012, CMS-TDR-016 (CERN, Geneva, Switzerland, September, 2017), https:// cds.cern.ch/record/2283189, This is the final version, approved by the LHCC

11. LHCb Collaboration, Framework TDR for the LHCb Upgrade: Technical Design Report (April, 2012), https://cds.cern.ch/record/1443882, CERN-LHCC-2012007, LHCb-TDR-12

12. M.L. Mangano, Physics at the FCC-hh, a $100 \mathrm{TeV}$ pp Collider, CERN Yellow Reports: Monographs (CERN, Geneva, Switzerland, 2017), https://cds.cern.ch/ record/2270978

13. O.S. Brüning, P. Collier, P. Lebrun, S. Myers, R. Ostojic, J. Poole, P. Proudlock, $L H C$ Design Report, CERN Yellow Reports: Monographs (CERN, Geneva, Switzerland, 2004), http://cds.cern.ch/record/782076

14. I. Hinchliffe, A. Kotwal, M.L. Mangano, C. Quigg, L.-T. Wang, Int. J. Mod. Phys. A30, 1544002 (2015)

15. C. Helsens, D. Jamin, M. Selvaggi, Search for High-Mass Resonances at FCC-hh (CERN Document Server, October, 2018), https://cds.cern.ch/record/2642473

16. L. Gouskos, J. Incandela, A. Sung, Search for Stop Scalar Quarks at FCC-hh (October, 2018), https://cds.cern.ch/record/2642475

17. M. Selvaggi, Higgs Measurements at FCC-hh (October, 2018), https://cds.cern.ch/ record/2642471 
18. V. Barger, P. Langacker, M. McCaskey, M.J. Ramsey-Musolf, G. Shaughnessy, Phys. Rev. D77, 035005 (2008)

19. A.V. Kotwal, J.M. No, M.J. Ramsey-Musolf, P. Winslow, Phys. Rev. D94, 035022 (2016)

20. D. Curtin, P. Meade, C.-T. Yu, JHEP 11, 127 (2014)

21. M. Low, L.-T. Wang, JHEP 08, 161 (2014)

22. M. Cirelli, F. Sala, M. Taoso, JHEP 10, 033 (2014)

23. S. Asai, M. Saito, R. Sawada, K. Terashi, Search for WIMPs with Disappearing Track Signatures at the FCC-hh (October, 2018), https://cds.cern.ch/record/2642474

24. P. Harris, V.V. Khoze, M. Spannowsky, C. Williams, Phys. Rev. D93, 054030 (2016)

25. T. du Pree, K. Hahn, P. Harris, C. Roskas, Cosmological constraints on Dark Matter models for collider searches, arXiv: 1603.08525 [hep-ph]

26. R. Mahbubani, P. Schwaller, J. Zurita, JHEP 06, 119 (2017)

27. J. Bramante, N. Desai, P. Fox, A. Martin, B. Ostdiek, T. Plehn, Phys. Rev. D93, 063525 (2016)

28. LHeC Study Group Collaboration, J.L. Abelleira Fernandez et al., J. Phys. G39, $075001(2012)$

29. U. Schneekloth, The HERA Luminosity Upgrade (1998), DESY-HERA-98-05

30. M. Kumar, X. Ruan, R. Islam, A.S. Cornell, M. Klein, U. Klein, B. Mellado, Phys. Lett. B764, 247 (2017)

31. J. Jowett, HL-LHC Physics workshop (CERN, October 2017), Indico presentation, https://indico.cern.ch/event/647676/contributions/2721134/

32. A. Dainese et al., Heavy ions at the future circular collider, CERN Yellow Report (2017), pp. 635-692, arXiv:1605.01389 [hep-ph], Preprint CERN-TH-2016-107

33. L. Apolinário, J.G. Milhano, G.P. Salam, C.A. Salgado, Probing the time structure of the quark-gluon plasma with top quarks, arXiv:1711.03105 [hep-ph], Preprint CERN-TH-2017-237

34. D. d'Enterria, K. Krajczár, H. Paukkunen, Phys. Lett. B746, 64 (2015)

35. D. d'Enterria, Nucl. Part. Phys. Proc. 289-290, 237 (2017)

36. D. d'Enterria, A.M. Snigirev, Eur. Phys. J. C78, 359 (2018)

37. C.-M. Ko, Y. Liu, J. Phys. G43, 125108 (2016)

38. Z. Chen, C. Greiner, K. Zhou, P. Zhuang, Phys. Lett. B758, 434 (2016)

39. D. d'Enterria, D.E. Martins, P. Rebello Teles, Prospects for $\gamma \gamma \rightarrow$ Higgs observation in ultraperipheral ion collisions at the Future Circular Collider, arXiv: 1712.10104 [hep-ph]

40. S. Furuseth, Head-On Beam-Beam Interactions in High-Energy Hadron Colliders. GPU-Powered Modelling of Nonlinear Effects, Master thesis, CERN-THESIS-2017279, 2017, https://cds.cern.ch/record/2299580

41. X. Buffat, D. Schulte, Broadband impedance of pumping holes and interconnects in the FCC-hh beams creen, in Proceedings of the IPAC'18 (Vancouver, BC, Canada, 2018)

42. M. Benedikt, D. Schulte, F. Zimmermann, Phys. Rev. ST Accel. Beams 18, 101002 (October, 2015)

43. X. Buffat, D. Schulte, Evolution of the beam parameters during luminosity production in the future circular hadron collider, in Proceedings of the IPAC'16 (Busan, South Korea, 2016)

44. F. Antoniou et al., Can we predict luminosity?, in 7th Evian Workshop on LHC Beam Operation (Evian-les-Bains, France, December 13-15, 2016), pp. 125-132

45. R. Alemany Fernández, A. Apollonio, W. Bartmann, X. Buffat, A. Niemi, D. Schulte, M. Solfaroli Camillocci, L. Stoel, FCC-hh turn-around cycle, Geneva, Switzerland (December, 2016), Preprint CERN-ACC-2016-0341, https://cds.cern.ch/record/ 2239138

46. M. Benedikt, M. Hofer, and R. Tomas, Optics Design for the Low Luminosity Experiments in the FCC-hh, November 28, 2017, https://cds.cern.ch/record/2640686, CERN-THESIS-2017-418.

47. R. Martin, M.I. Besana, F. Cerutti, A. Langner, R. Tomás, E. Cruz-Alaniz, B. Dalena, Phys. Rev. Accel. Beams 20, 081005 (2017) 
48. D. Tommasini, Radiation limits of normal- and superconducting magnets, FCC Other Magnet Design Meeting (2017), https://indico.cern.ch/event/647002/ contributions/2628503/attachments/1481854/2298287/Tommasini_Radiation.pdf

49. W. Bartmann et al., Beam transfer to the FCC-hh collider from a $3.3 \mathrm{TeV}$ Booster in the LHC Tunnel, in Conference proceedings (June, 2015), http://jacow.org/ ipac2015/papers/thpf089.pdf

50. T. Kramer, D. Barna, M. Barnes, W. Bartmann, F. Burkart, L. Ducimetière, B. Goddard, V. Senaj, T. Stadlbauer, D. Woog, Considerations for the injection and extraction kicker systems of a $100 \mathrm{TeV}$ Centre-of-Mass FCC-hh Collider, in Conference Proceedings (May 8-13, 2016), DOI: 10.18429/JACoW-IPAC2016-THPOR049

51. J.A. Uythoven et al., Injection protection upgrade for the HL-LHC, in Conference Proceedings (June, 2015), http://jacow.org/ipac2015/papers/tupty051.pdf

52. W. Bartmann, M. Atanasov, M.J. Barnes, J. Borburgh, F. Burkart, B. Goddard, T. Kramer, A. Lechner, A. Sanz Ull, R. Schmidt, L.S. Stoel, R. Ostojic, J. Rodziewicz, P. van Trappen, D. Barna, Phys. Rev. Accel. Beams 20, 031001 (March, 2017)

53. F. Burkart et al., Conceptual design considerations for the $50 \mathrm{TeV}$ FCC beam dump insertion, in Conference Proceedings (June, 2016), DOI: 10.18429/JACoW-IPAC2016-TUPMR047

54. O.S. Brüning et al. (eds.), LHC Design Report v.1 : The LHC Main Ring, CERN2004-003-V1 (2004)

55. R. Bruce, M. Fiascaris, D. Mirarchi, and S. Redaelli, First design of a proton collimation system for $50 \mathrm{TeV}$ FCC-hh, in Proceedings of the IPAC'16, Busan, Korea (2016), p. 2425

56. M. Fiascaris et al., Conceptual solution for a beam halo collimation system of the Future Circular hadron-hadroCollider (FCC-hh), submitted to Nucl. Instr. Meth. Phys. Res. A (2018)

57. R.W. Aßmann, Collimators and beam absorbers for cleaning and machine protection, in Proceedings of the LHC Project Workshop - Chamonix XIV, Chamonix, France (2005), p. 261

58. G. Robert-Demolaize, Design and performance optimization of the lhc collimation system, Ph.D. thesis, Universite Joseph Fourier, Grenoble, 2006

59. R.W. Aßmann et al., The final collimation system for the LHC, in Proceedings of the EPAC'06, Edinburgh, Scotland (2006), p. 986

60. C. Bracco, Commissioning scenarios and tests for the LHC collimation system, Ph.D. thesis, EPFL Lausanne, 2008

61. R. Bruce, R.W. Assmann, V. Boccone, C. Bracco, M. Brugger, M. Cauchi, F. Cerutti, D. Deboy, A. Ferrari, L. Lari, A. Marsili, A. Mereghetti, D. Mirarchi, E. Quaranta, S. Redaelli, G. Robert-Demolaize, A. Rossi, B. Salvachua, E. Skordis, C. Tambasco, G. Valentino, T. Weiler, V. Vlachoudis, D. Wollmann, Phys. Rev. ST Accel. Beams 17, 081004 (August, 2014)

62. R. Bruce, C. Bracco, R. De Maria, M. Giovannozzi, A. Mereghetti, D. Mirarchi, S. Redaelli, E. Quaranta, B. Salvachua, Nucl. Instr. Meth. Phys. Res. A 848, 19 (2017)

63. G. Apollinari et al. (eds.), High-Luminosity Large Hadron Collider (HL-LHC) : Technical Design Report, CERN-2017-007-M (2017)

64. R. Bruce, A. Marsili, S. Redaelli, Cleaning performance with $11 \mathrm{~T}$ dipoles and local dispersion suppressor collimation at the lhc, in Proceedings of the IPAC'14, Dresden, Germany (2014), p. 170, http://jacow.org/IPAC2014/papers/mopro042.pdf

65. B. Auchmann et al., Power deposition in lhc magnets with and without dispersion suppressor collimators downstream of the betatron cleaning insertion, in Proceedings of the IPAC'14, Dresden, Germany (2014), p. 112

66. R. Bruce et al., Status of the FCC-hh collimation system, in Proceedings of the IPAC'17, Copenhagen, Denmark (2017), p. 64

67. C. Bahamonde Castro et al., Energy deposition in the betatron collimation insertion of the $100 \mathrm{TeV}$ future circular collider, in Proceedings of the IPAC'17, Copenhagen, Denmark (2017), p. 68 
68. A.M. Krainer, Design \& Simulation of new protection devices in the dispersion suppressor regions for the Future Circular Collider Project Master's thesis, TU Graz, 2018

69. A. Fassò, A. Ferrari, J. Ranft, P.R. Sala, FLUKA: a multi-particle transport code Report CERN-2005-10 (CERN, Geneva, Switzerland, 2005), Also available as INFN/TC_05/11 and SLAC-R-773

70. T.T. Böhlen, F. Cerutti, M.P.W. Chin, A. Fassò, A. Ferrari, P.G. Ortega, A. Mairani, P.R. Sala, G. Smirnov, V. Vlachoudis, Nucl. Data Sheets 120, 211 (2014)

71. M.I. Besana et al., Update on energy deposition in betatron cleaning insertion, Presentation at the FCC Collimation Design Meeting (July 17, 2017), https://indico. cern.ch/event/653079/

72. M. Varasteh et al., Energy deposition studies with $30 \mathrm{~cm}$ TCP, Presentation at the $F C C$ Collimation Design Meeting (October 20, 2017), https://indico.cern.ch/event/ 674036/

73. M. Varasteh et al., Energy deposition update with thicker TCP and no skew, Presentation at the FCC Collimation Design Meeting (November 20, 2017), https: //indico.cern.ch/event/674036/

74. M. Varasteh et al., Beam loss in collimators, in Fourth Annual Meeting of the Future Circular Collider study, Amsterdam, The Netherlands (April, 2018), https://indico. cern. ch/event/656491/contributions/2930758/

75. G. Gobbi et al., Thermo-mechanical studies of collimators for slow losses in FCC-hh, Presentation at the FCC Collimation Design Meeting (December 8, 2017), https: //indico.cern.ch/event/685664/

76. F. Cara, G. Gobbi, M. Pasquali, Updates on thermomechanical studies of FCC collimators, in Presentation at the FCC Collimation Design Meeting (June 25, 2018), https://indico.cern. ch/event/738429/

77. R. Bruce et al., Status of collimation system studies, in Fourth Annual Meeting of the Future Circular Collider study, Amsterdam, The Netherlands (April, 2018), https: //indico.cern.ch/event/656491/contributions/2930755/

78. D. Boutinet al., Updates on the optics of the future Hadron-Hadron collider FCChh in Proceedings of the 8th IPAC'17, Copenhagen, Denmark (May 14-19, 2017), p. TUPVA002, http://inspirehep.net/record/1626340/files/tupva002.pdf

79. R. Alemany Fernández et al., First results for a FCC-hh ring optics design CERNACC-2015-0035, (CERN, Geneva, Switzerland, April, 2015), Preprint CERN-ACC2015-0035

80. R. Bruce et al., Status of the FCC-hh Collimation System in Proceedings of 8th the IPAC'17, Copenhagen, Denmark (May 14-19, 2017) p. MOPAB001

81. A. Seryi et al., Overview of design development of FCC-hh experimental interaction regions in Proceedings of the 8th IPAC'17, Copenhagen, Denmark, (May 14-19, 2017), p. TUPVA040, http://inspirehep.net/record/1626415/files/tupva040.pdf

82. M. Boege and F. Schmidt, Tracking studies for the LHC optics 4 at injection energy, (June, 1997), CERN-LHC-Project-Report-103, http://cds.cern.ch/record/327303

83. E. Cruz-Alainz et al., Methods to increase the dynamic aperture of the FCC-hh lattice, in Proceedings of the 9th IPAC'18 (2018), http://jacow.org/ipac2018/papers/ thpak145.pdf

84. O.S. Brüning, S.D. Fartoukh, Field Quality Specification for the LHC Main Dipole Magnets LHC-Project-Report-501. CERN-LHC-Project-Report-501, (CERN, Geneva, Switzerland, October, 2001), Preprint LHC-Project-Report-501, https://cds.cern. $\mathrm{ch} / \mathrm{record} / 522049$

85. B. Dalena et al., Dipole field quality and dynamic aperture for FCC-hh, in Proceedings of the 9th IPAC'18 (2018), http://jacow.org/ipac2018/papers/mopmf024.pdf

86. D. Boutin et al., Updates on the optic correction of FCC-hh, in Proceedings of the 9th IPAC'19 (2018), http://jacow.org/ipac2018/papers/mopmf023.pdf

87. Y. Nosochkov and D.M. Ritson, The provision of IP crossing angles for the SSC, in Proceedings of the PAC'93 (May, 1993), pp. 125-127 
88. M. Cropper et al., Low secondary electron yield of laser treated surfaces of copper, aluminium and stainless steel, in Proceedings of the 7th IPAC'16, Busan, Korea (May 8-13, 2016), pp. 1089-1092

89. R. Valizadeh, O.B. Malyshev, S. Wang, T. Sian, M.D. Cropper, N. Sykes, Appl. Surf. Sci. 404, 370 (2017)

90. R. Bartolini, E. Koukovini-Platia, A. Morgan, G. Rehm, Study of single bunch instabilities with transverse feedback at diamond in Proceedings of the 8th IPAC'17, Copenhagen, Denmark (May 14-19, 2017), pp. 4489-4492

91. N. Klinkenberg, Computational studies of collective effects in particle beams in the Future Circular Collider, Master's thesis, Westfälische Hochschule in cooperation with CERN, 2018, Bachelor thesis

92. X. Buffat, W. Herr, N. Mounet, T. Pieloni, S. White, Phys. Rev. ST Accel. Beams 17, 111002 (November, 2014)

93. C. Tambaso et al., Beam-beam effects, octupoles and Landau damping, Presentation at the EuroCirCol Meeting(October 9-10, 2017), https://indico.cern.ch/event/ 669849/contributions/2740676/attachments/1537807/2410440/FFC_eurocircol_ landau_beam_beam.pdf

94. C. Tambasco et al., Landau damping studies for the FCC: octupole magnets, electron lens and beam-beam effects, in Proceedings of the IPAC'18, THPAF074 (2018)

95. T. Argyropoulos, H. Bartosik, N. Biancacci, X. Buffat, J.E. Muller, W. Herr, G. Iadarola, A. Lasheen, K. Li, A. Oeftiger, IEEE Trans. Nucl. Sci. 63, 1001 (April, 2016)

96. A.A. Gorzawski, Luminosity control and beam orbit stability with beta star leveling at LHC and HL-LHC, Ph.D. thesis, EPFL Lausanne, 2016

97. X. Buffat et al., Colliding during the squeeze and beta star levelling in the LHC, in Proceedings of the IPAC'13, Shanghai, China (May, 2013), http://jacow.org/IPAC2013/ papers/tupfi033.pdf

98. Y. Alexahin, A. Burov, V. Shiltsev, A. Valishev, Phys. Rev. Lett. 119, 134802 (September, 2017)

99. W. Fischer, X. Gu, Z. Altinbas, M. Costanzo, J. Hock, C. Liu, Y. Luo, A. Marusic, R. Michnoff, T.A. Miller, A.I. Pikin, V. Schoefer, P. Thieberger, S.M. White, Phys. Rev. Lett. 115, 264801 (December, 2015)

100. A. Grudiev, Phys. Rev. ST Accel. Beams 17, 011001 (January, 2014)

101. S. De Santis et al., Wideband vertical intra-bunch feedback at the SPS - 2015 results and path forward in Proceedings of the IPAC'15, Richmond, VA, USA (May 3-8, 2015), pp. $1353-1355$

102. M.A. Furman, Electron Cloud Effects in Accelerators in Proceedings of the 5th Workshop on Electron-Cloud Effects (ECLOUD'12), La Biodola, Isola d'Elba, Italy (June 5-9, 2013)

103. C. Yin Vallgren, G. Arduini, J. Bauche, S. Calatroni, P. Chiggiato, K. Cornelis, P. Costa Pinto, B. Henrist, E. Métral, H. Neupert, G. Rumolo, E. Shaposhnikova, M. Taborelli, Phys. Rev. ST Accel. Beams 14 (July, 2011)

104. X. Buffat et al., Long range beam-beam effects in the lhc (CERN, Geneva, Switzerland, 2014), arXiv: 1409.4942 [physics.acc-ph]

105. T. Pieloni et al., Two beam effects, in Proceedings of the 2014 Evian Workshop on LHC beam operation (2014), pp. 69-79, http://cds.cern.ch/record/2289585, CERN-ACC-2014-0319

106. G. Arduini et al., Long-range and head-on beam-beam: what are the limits?, in Proceedings of the 7th Evian Workshop on LHC Beam Operation: Evian-les-Bains, France, 2016 (CERN, Geneva, Switzerland, 2017), pp. 133-140, http://cds.cern.ch/record/ 2289585

107. SixTrack Code, Website, http://sixtrack.web.cern.ch/SixTrack/

108. T. Pieloni, A study of beam-beam effects in hadron colliders with a large number of bunches, Ph.D. thesis, EPFL (2008)

109. M. Giovannozzi, Dynamic Aperture: Simulations vs. Experiments and first DA results in the LHC, in 14th HiLumi WP2 Task Leader Meeting (August, 2013), https://indico.cern. ch/getFile. py/accesscontribId=2\&resId=0\&materialId= slides\&conf $I d=267840$ 
110. T. Pieloni et al., Beam-beam long-range studies and possible reduction of the crossing angle in the LHC, Presentation at the LHC Machine Committee (August, 2016)

111. M. Crouch et al., Dynamic aperture studies of the long-range beam-beam interaction at the LHC, in Proceedings of the 8th IPAC'17, Copenhagen, Denmark (May 14-19, 2017), pp. 3840-3843, http://jacow.org/ipac2017/papers/thpab056.pdf

112. M. Crouch, Luminosity performance limitations due to the beam-beam interaction in the Large Hadron Collider, Ph.D. thesis, Manchester University, 2017

113. M. Giovannozzi, Phys. Rev. ST Accel. Beams 15, 024001 (February, 2012)

114. X. Buffat, Transverse beams stability studies at the Large Hadron Collider, Ph.D. thesis, EPFL, 2015

115. H. Grote, L.H.A. Leunissen, F. Schmidt, LHC Dynamic Aperture at Collision LHCPROJECT-NOTE-197 (CERN, Geneva, Switzerland, August, 1999), Preprint LHCPROJECT-NOTE-197, https://cds.cern.ch/record/691988

116. Y. Luo and F. Schmidt, Dynamic Aperture Studies for LHC Optics Version 6.2 at Collision (CERN, Geneva, Switzerland, January, 2003), Preprint LHC-PROJECTNOTE-310, http://cds. cern.ch/record/692074

117. T. Pieloni et al., Beam-Beam Studies for FCC-hh, in $3^{\text {rd }}$ Annual Meeting of the Future Circular Collider Study, Berlin, Germany (May 29-June 2, 2017)

118. J. Barranco García et al., Beam-Beam Studies for FCC-hh, in Proceedings of the IPAC'17, Copenhagen, Denmark (JACoW, Geneva, Switzerland, May 14-19, 2017), pp. 2109-2112, http://jacow.org/ipac2017/papers/tupva026.pdf

119. J. Barranco García, EuroCirCol Meeting Oct 2017, Presentation at the LHC Machine Committee (October 31, 2017)

120. W. Herr, Features and implications of different LHC crossing schemes LHC-ProjectReport-628. CERN-LHC-Project-Report-628 (CERN, Geneva, Switzerland, February, 2003) Preprint LHC-Project-Report-628, https://cds.cern.ch/record/604005

121. T. Pieloni et al., Colliding High Brightness Beams in the LHC, in Proceedings of the HB'12: Beijing, China (2012), p. MOP250

122. J. Barranco García et al., Probing the behaviour of high brightness bunches in collision at $6.5 \mathrm{TeV}$ and the interplay with an external source of noise (MD1433) (April, 2017), https://cds . cern. ch/record/2261037, CERN-ACC-NOTE-2017-0030

123. S.V. Furuseth, Head-on beam-beam interactions in high-energy hadron colliders, GPUPowered Modelling of Nonlinear Effects Master's thesis, NTNU, 2017, CERN-THESIS2017-279

124. V.A. Lebedev, AIP Conf. Proc. 326, 396 (1995)

125. M. Albert et al., MD 400: LHC emittance growth in presence of an external source of noise during collision (January, 2016), https://cds.cern.ch/record/2125228, CERN-ACC-NOTE-2016-0020

126. X. Buffat et al., Beam-Beam Studies for FCC-hh, Presentation at the $H L$ LHC Collaboration Meeting (Madrid, Spain, November 15, 2017), https: //indico.cern.ch/event/647714/contributions/2646114/attachments/1558511/ 2452144/2017-11-15_BeamBeamInstability-expanded.pdf

127. L. Jin, O. Kheawpum, J. Shi, Proc. AIP'04 693, 265 (2004)

128. J. Barranco García and T. Pieloni, Global compensation of long-range beam-beam effects with octupole magnets: dynamic aperture simulations for the HL-LHC case and possible usage in LHC and FCC (May, 2017), https://cds . cern.ch/record/2263347, CERN-ACC-NOTE-2017-0036

129. D. Schulte, Preliminary collider baseline parameters (CERN, Geneva, Switzerland, 2015), Preprint CERN-ACC-2015-0132, https://cds.cern.ch/record/2059230

130. A. Apollonio, K. Foraz, A. Niemi, Considerations on Operation Schedule and Maintenance Aspects of FCC-hh (CERN, Geneva, Switzerland, May, 2018), Preprint CERNACC-2018-0013, https://cds.cern.ch/record/2317097

131. W. Bartmann et al., High Energy Booster Options for a Future Circular Collider at CERN, no. 7 in International Particle Accelerator Conference. JaCoW, Geneva, Switzerland, June, 2016, Proceedings of the IPAC'16, Busan, Korea (May 8-13, 2016) 
132. D. Jacquet, Injection in 6th Evian Workshop on LHC beam operation, edited by B. Goddard and S. Dubourg (CERN, Geneve, October, 2016) pp. 49-52

133. R. Alemany Fernández et al., FCC-hh turn-around cycle (CERN, 2016)

134. O. S. Brüning et al., ed., The LHC main ring, Vol. 1 of LHC Design report (CERN, Geneva, Switzerland, June, 2004)

135. M. Pojer, LHC operation, in 8th LHC Operations Evian Workshop (CERN, 2017)

136. A. Apollonio, L. Ponce, and B. Todd, LHC Availability 2016: Proton Run, in 7th Evian Workshop on LHC beam operation (CERN, Geneva, Switzerland, December, 2016), pp. 53-58, https://cds.cern.ch/record/2293511

137. A. Apollonio, L. Ponce, C. Roderick, B. Todd, D. Walsh, LHC Availability 2017: Proton Run, in 8th Evian Workshop on LHC beam operation, CERN-ACC-2019-012 (CERN, Geneva, Switzerland, December, 2017), pp. 35-46, https://cds.cern.ch/ record/2654224

138. W. Bartmann et al., Conceptional Design Considerations for a $1.3 \mathrm{TeV}$ superconducting SPS (sc SPS), in Proceedings of the IPAC'17, Copenhagen, Denmark, no. 8 in International Particle Accelerator Conference (JaCoW, Geneva, Switzerland, May 1419, 2017), pp. 3323-3326

139. A. Apollonio et al., Phys. Rev. Accelerators Beams 19, 121003 (December, 2016)

140. J. Gutleber, A. Niemi, J.P. Penttinen, An Open Modelling Approach for Availability and Reliability of Systems - OpenMARS CERN-ACC-2018-0006 (CERN, Geneva, Switzerland, January, 2018), Preprint CERN-ACC-2018-0006, https://cds.cern.ch/ record/2302387

141. J.P. Penttinen, A. Niemi, J. Gutleber, K.T. Koskinen, E. Coatanea, J. Laitinen, Reliab. Eng. Syst. Saf. 183, 387 (2019)

142. J.M. Jowett et al., The 2016 proton-nucleus run of the LHC, in Proceedings of the IPAC'17, Copenhagen, Denmark (2017)

143. M. Schaumann, Phys. Rev. ST Accel. Beams 18, 091002 (2015)

144. M.L. Mangano, Heavy ions at the future circular collider, chapter 4 in Physics at the FCC-hh, a $100 \mathrm{TeV}$ pp collider, Vol. 3 of CERN Yellow Reports: Monographs (CERN, CERN, Geneva, Switzerland, 2017), pp. 635-691, CERN-2017-003-M

145. J.M. Jowett, M. Schaumann, A first look at the performance for $\mathrm{Pb}-\mathrm{Pb}$ and $\mathrm{p}-\mathrm{Pb}$ collisions in FCC-hh, Presentation at FCC Study Kickoff Meeting, Geneva, Switzerland (2014)

146. J.M. Jowett et al., FCC-hh as a heavy-ion collider, Presentation at FCC Week, Washington DC, USA (2015)

147. A. Dainese et al., Heavy-ion physics studies for the Future Circular Collider, Presentation at FCC Week, Rome, Italy (2016)

148. M. Schaumann et al., Heavy-ions at the FCC-hh, Presentation at the FCC Week, Berlin, Germany (2017), https://indico.cern.ch/event/556692/contributions/ 2484258/

149. K. Fuchsberger, Turnaround - analysis and possible Improvements, in Proceedings of the 7th Evian Workshop on LHC beam operation (2017)

150. Indico presentation (March, 2016) http://indico.cern.ch/event/505557/ contribution/1/

151. U. Klein, Higgs Physics at the LHeC, Contribution to ICHEP, Valencia (2014)

152. M. Klein, Ann. Phys. 528, 138 (2016)

153. PERLE Collaboration, G. Arduini et al., Powerful Energy Recovery Linac Experiments, Conceptual Design Report, accepted for publication in Journal of Physics G. (2018), arXiv:1705.08783 [physics.acc-ph]

154. O. Brüning, M. Klein, F. Zimmermann, The LHeC as a Higgs Boson Factory, in Proceedings of the 4th IPAC'13, Shanghai, China (May 12-17, 2013), p. MOPWO054, http://JACoW.org/IPAC2013/papers/mopwo054.pdf, CITATION = INSPIRE-1338636

155. O.S. Brüning, M. Klein, Mod. Phys. Lett. A28, 1330011 (2013) 
156. G. Apollinari et al., High-Luminosity Large Hadron Collider (HL-LHC): Preliminary Design Report, Yellow Report CERN-2015-005 (2015)

157. D. Schulte et al., FCC-he parameters, Talk presented at the FCC Workshop, Rome (2016)

158. F. Zimmermann, FCC accelerator parameters, Talk presented at the FCC Physics Week, Geneva, CERN (2017)

159. D. Schulte, Study of electromagnetic and hadronic background in the interaction region of the TESLA collider, DESY-TESLA-97-08 (1997)

160. H. Artosik and J. Jowett, LHC Performance Workshop (Chamonix, France, January, 2017), https://indico.cern.ch/event/580313/

161. R. Martin, R. Tomas Garcia, Length Optimization of the Detector Region Dipoles in LHeC and FCC-eh, CERN-ACC-2018-0042 (CERN, Geneva, Switzerland, October, 2018), https://cds.cern.ch/record/2644892

162. R. Valente et al., Baseline design of a $16 \mathrm{~T}$ costheta bending dipole for the future circular collider, Presented at the $A S C$, Seattle, USA (2018)

163. R. Wolf, Field error naming conventions for the LHC, LHC-M-ES-0001, EDMS90250 (2001), Engineering Specification

164. S. Bermudez, Persistent Currents Magnetization Effects in The 16 T Main Dipoles for the Future Circular Collider, CERN EDMS 2036614, (2018)

165. T. Salmi et al., Quench protection of the $16 \mathrm{~T} \mathrm{Nb}_{3} \mathrm{Sn}$ dipole magnets designed for the Future Circular Collider, Presented at the $A S C$, Seattle, USA (2018)

166. C. Pes et al., Magnetic and Mechanical Design of the Block-coil Dipole Option for the Future Circular Collider, Presented at the ASC, Seattle, USA (2018)

167. F. Toral, Magnetic and mechanical design of a $16 \mathrm{~T}$ common coil dipole for FCC, FCC Week (2018) https://indico.cern.ch/event/656491/contributions/ 2920132/attachments/1629665/2597178/FCCweek2018_common_coil_ftoral.pdf

168. B. Auchmann, L. Brouwer, S. Caspi, J. Gao, G. Montenero, M. Negrazus, G. Rolando, S. Sanfilippo, IEEE Trans. Appl. SC 28, 1 (April, 2018)

169. A. Ballarino et al., The CERN FCC conductor development program: a world-wide effort for the future generation of high-field magnets, submitted to IEEE Trans. Appl. Supercond., forthcoming on IEEE XPlore

170. C. Lorin et al., Exploration of two layer designs of the Future Circular Collider Main Quadrupoles, Presented at the ASC, Seattle, USA (2018)

171. L. Tavian, Cryogenics, FCC Collider Kickoff Meeting, Univ. Geneva, Switzerland (2014), https://indico.cern.ch/event/282344/contributions/1630775/

172. O. Boine-Frankenheim, FCC-hh Impedances, FCC Week, Rome, Italy (2016), https: //indico.cern.ch/event/438866/contributions/1085013/

173. R. Kersevan, Synchrotron radiation $\&$ vacuum concepts, FCC Collider Kickoff Meeting, Univ. Geneva, Switzerland (2014), https://indico.cern.ch/event/282344/ contributions/1630665/

174. O. Malyshev et al., Laser Treatment at STFC, FCC-hh impedance and beam screen Workshop (March, 2017), https://indico.cern.ch/event/619380/contributions/ 2527422/

175. L.A.G. Gomez, Results on the FCC-hh Beam Screen Prototype at the KIT Electron Storage Ring (FCC Week, Amsterdam, The Netherlands, April, 2018), https: //indico.cern.ch/event/656491/contributions/2938816/

176. C. Garion, FCC-hh Beam Screen Studies and Beam Screen Cooling Scenarios (FCC Week, Rome, Italy, 2016), https://indico.cern.ch/event/438866/contributions/ 1084911/

177. S. Calatroni, IEEE Trans. Appl. Supercond. 26, 1 (April, 2016)

178. V. Baglin, Cryogenic Beam Vacuum Specificities Applicable to FCC hh, (FCC Week, Washington, DC, 2015), https://indico.cern.ch/event/340703/contributions/ 802149/

179. J.F. Topham, FCC-hh Beam Screen Design, WP4 EuroCirCol Coordination Meeting 05, ALBA light source (November 7-9, 2016), https://indico.cern.ch/event/ 579868/ 
180. C. Kotnig, Cold mass cooling with supercritical helium, in 2nd FCC Cryogenics Day (Dresden, Germany, 2016)

181. I. Bellafont, Photon Tracing and Gas-density Profile in the FCC-hh (FCC Week, Berlin, Germany, 2017), https://indico.cern.ch/event/556692/contributions/ 2487660/

182. I. Bellafont et al., Study of the Beam Induced Effects in the FCC-hh Vacuum Chamber, submitted to PR-AB (2018)

183. L. Mether, CERN-EPFL, Personal Communication, and FCC-hh Electron Cloud (FCC Week, Berlin, Germany, 2017), https://indico.cern.ch/event/556692/ contributions/2567985/

184. E. Shaposhnikova, Longitudinal beam dynamics and RF requirements, in Third Annual Meeting of the Future Circular Collider Study, Berlin, Germany, 2017 (CERN, 2017), https://indico.cern.ch/event/556692/contributions/2484257/ attachments/1467380/2269248/FCChh_RF_Berlin.pdf

185. S. Aull, O. Brunner, A. Butterworth, N. Schwerg, Material Options for the Superconducting RF System of the Future Circular Collider (CERN, Geneva, Switzerland, 2018), Preprint CERN-ACC-2018-0019, http://cds.cern.ch/record/2625126

186. E. Palmieri, C. Pira, Coating studies on $6 \mathrm{GHz}$ seamless cavities, in Fourth Annual Meeting of the Future Circular Collider study (Amsterdam, The Netherlands, 2018)

187. W.V. Delsolaro, Thin Film Research: CERN Experience and Possible Future Applications (TESLA Technology Collaboration (TTC) Meeting, Milano, Italy, 2018), https://agenda.infn.it/contributionDisplay $\cdot$ py? sessionId=12\&contribId= 4\&conf $I d=13791$

188. D.L. Hall, S. Posen, Supercond. Sci. Technol. 30, 033004 (2017)

189. K. Ilyina-Brunner, Magnetron Sputtering of $\mathrm{Nb}_{3} \mathrm{Sn}$ thin films on copper for SRF application, in Fourth Annual Meeting of the Future Circular Collider Study, Amsterdam, The Netherlands (2018), https://indico.cern.ch/event/656491/contributions/ 2918336/attachments/1628654/2595685/Ilyina_Nb3Sn_FCC2018.pdf

190. E. Montesinos, FPC challenges and perspectives for FCC, in Third Annual Meeting of the Future Circular Collider Study, Berlin, Germany, 2017 (CERN, 2017), https://indico.cern.ch/event/556692/contributions/2484366/attachments/ 1466409/2269379/20170530_FCC_week_FPC.pdf

191. G. Apollinari et al., High-Luminosity Large Hadron Collider (HL-LHC), in Preliminary Design Report. CERN Yellow Report (2015)

192. K. Papke, A. Carvalho, C. Zanoni, A. Grudiev, Multiphysics simulations of the Wide Opened Waveguide Crab-Cavity, in Proceedings of the SRF'17, Lanzhou, China (2017)

193. J.R. Delayen, S.U.D. Silva, Phys. Rev. ST Accel. Beams 16, 012004 (2013)

194. I. Syratchev, Introduction to HEIKA. Tentative structure and objectives CLIC Workshop 2015 (CERN, Geneva, Switzerland, CERN, 2015), https://indico.cern.ch/ event/336335/contributions/789041/

195. C. Marrelli, I. Syratchev, A. Yu, IEEE Trans. Electron Devices 62, 3406 (2015)

196. I.A. Guzilov, BAC method of increasing the efficiency in Klystrons, in IEEE Vacuum Electron Sources Conference 2014, St. Petersburg, Russia (2014)

197. G. Burt et al., Particle-in-cell simulation of a core stabilization method klystron, in IEEE International Vacuum Electronics Conference, IVEC 2017, London, UK (2017)

198. I. Syratchev, High efficiency klystron technology, in Third Annual Meeting of the Future Circular Collider Study, Berlin, Germany, 2017 (CERN, 2017), https:// indico.cern.ch/event/556692/timetable/\#20170530.detailed

199. J. Cai, I. Syratchev, KlyC: Large Signal Simulation Code for Klystrons, submitted to IEEE TED, January 2016 (2017)

200. A. Grudiev, K. Li, K. Papke, M. Schenk, RF Quadrupole System for FCC-hh, Technical Report cern-ats-2018-xxx (CERN, 2018)

201. A. Grudiev, K. Papke, Phys. Rev. Accel. Beams 20, 082001 (2017)

202. P. Baudrenghien and T. Mastoridis, Phys. Rev. Accel. Beams 20, 011004 (2017)

203. P. Baudrenghien, R. Calaga, E. Shaposhnikova, H. Timko, The Main RF System and its Implications for HL-LHC (7th HL-LHC Collaboration Meeting, 
CIEMAT, Madrid, Spain, 2017), https://indico.cern.ch/event/647714/ contributions/2646158/attachments/1556707/2453084/HL-LHC_2017_Timko.pdf

204. M.J. Barnes et al., Inductive adders for replacing thyratron based modulators at CERN, in Power Electronics and Applications (EPE'15 ECCE-Europe), 2015 17th European Conference, 8-10 (September, 2015)

205. J. Holma, A pulse power modulator with extremely flat-top output pulses for the compact linear collider at CERN, Doctoral thesis, Aalto University publication series, Doctoral Dissertations 196/2015, Helsinki, Finland, 2015, CERN-THESIS-2014-359

206. D. Woog, M.J. Barnes, L. Ducimetiére, J. Holma, T. Kramer, J. Phys.: Conf. Ser. 874, 012096 (2017)

207. M.J. Barnes, J. Holma, Measurements on a $12.5 \mathrm{kV}$ prototype inductive adder for the CLIC DR extraction kickers, in Proceedings of the 8th IPAC'17 (Copenhagen, Denmark, May 14-19, 2017), pp. 3487-3490, WEPVA098

208. M.J. Barnes, A. Kandratsyeu, L.M. Redondo, Marx generator prototype for kicker magnets based on SiC MOSFETs, Special Issue of Transactions on Plasma Science on Pulsed Power Science and Technology (2018), Submitted

209. Y.H. Chin et al., Impedance and beam instability issues at J-PARC Rings, in Proceedings of the HB'08 (Nashville, Tennessee, USA, 2008), WGA01

210. M.J. Barnes et al., Preliminary estimate of beam induced power deposition in a FCChh injection kicker magnet, in Proceedings of the 8th IPAC'17 (Copenhagen, Denmark, May 14-19, 2017), pp. 3475-3478, WEPVA095

211. M.J. Barnes, A. Adraktas, G. Bregliozzi, B. Goddard, L. Ducimetière, B. Salvant, J. Sestak, L.V. Cid, W. Weterings, C.Y. Vallgren, J. Phys.: Conf. Ser. 874, 012101 (2017)

212. K. Sugita, IEEE Trans. Appl. Supercond. 22, 4902204 (June, 2012)

213. D. Barna, Phys. Rev. Accel. Beams 20, 041002 (April, 2017)

214. E. Carlier, T. Fowler, N. Magnin, J. Rodziewicz, Prospects for an optical re-triggering system for the lhc beam dumping system at CERN, in Proceedings of the 2017 IEEE Pulsed Power Conference (Brighton, UK, June 18-22, 2017), https://ieeexplore. ieee.org/document/8291250

215. D. Barna, First experimental results with the SuShi septum prototypes, in Future Circular Collider Week 2017, presentation (Berlin, Germany, May, 2017), https://indico.cern.ch/event/556692/contributions/2488390/attachments/ 1468982/2272308/barna-sushi.pdf

216. K. Sugita, Design status of a high field superconducting magnet, in Future Circular Collider Week 2017, presentation (Berlin, Germany, May, 2017), https://indico.cern.ch/event/556692/contributions/2488389/attachments/ 1466268/2271498/Sc-Septum-GSI.pdf

217. EUCARD2 Transnational Access Facility, Website, http://eucard2.web.cern.ch/ content/transnational-access

218. I. Efthymiopoulos et al., HiRadMat: A New Irradiation Facility for Material Testing at CERN (November, 2011), http://cds.cern.ch/record/1403043, CERN-ATS-2011232

219. F.X. Nuiry et al., Low-Z material R\&D application for Beam Intercepting Devices (BID) at CERN, in 3rd International Workshop on Spallation Materials Technology (Chattanooga, TN, USA, 2016)

220. F. Carra et al., The "Multimat" experiment at CERN HiRadMat facility: advanced testing of novel materials and instrumentation for HL-LHC collimators, in Proceedings of the 8th IPAC'17 (Copenhagen, Denmark, 2017), pp. 76-097, MOPAB005

221. T.T. Böhlen et al., Nucl. Data Sheets 120, 211 (2014)

222. B. Goddard, Beam dumping system, in LHC Design Report, The LHC Main Ring, edited by O.S. Brüning, P. Collier, P. Lebrun, S. Myers, R. Ostojic, J. Poole, P. Proudlock (CERN, Geneva, Switzerland, 2004) Vol. 1, Chap. 17, CERN-2004-003

223. S. Péraire and P.R. Sala, Beam dumps and beam stoppers for LHC and CNGS transfer lines, LHC Project Report 465 (CERN, Geneva, Switzerland, 2001)

224. J. Kidd, N. Mokhov, T. Murphy, M. Palmer, T. Toohig, F. Turkot, A. VanGinneken, IEEE Trans. Nucl. Sci. 28, 2774 (1981) 
225. C. Crawford, B. Hanna, Construction of a new Tevatron collider beam abort dump, in Conference Record of the 1991 IEEE Particle Accelerator Conference (San Sebastian, Spain, 1991), pp. 970-972

226. M. Schmitz, Overview of the beam abort system for the $820 \mathrm{GeV} / \mathrm{c}$ HERA proton ring, in Proceedings of the 3rd EPAC'92 (Berlin, Germany, 1992), pp. 1455-1456

227. T. Kramer et al., Considerations for the Injection and Extraction Kicker Systems of a $100 \mathrm{TeV}$ Centre-of-Mass FCC-hh Collider in Proceedings of the 7th IPAC'16 (Busan, Korea, 2016), pp. 3901-3904, THPOR049

228. N. Tahir et al., Hydrodynamic tunneling studies for a water beam dump in Future Circular Collider Week 2017, presentation (Berlin, Germany, May, 2017)

229. N. Mokhov, C.T. Murphy, S. Pruss, Extracted Beam Lines and Absorbers for a $50 \times 50$ TeV hadron collider, Fermilab Report FERMILAB-VLHCPUB-047 (CERN, Batavia, IL, USA, 1996)

230. O.R. Jones, First years experience of LHC Beam Instrumentation (September, 2011), https://cds.cern.ch/record/1382452

231. CERN EDMS Specification LHC-BPM-ES-0004, CERN EDMS document, https:// edms. cern. ch/document/327557

232. M. Barros Marin, M. Barros Marin, A. Boccardi, C. Donat Godichal, J.L. Gonzalez, T. Lefevre, T. Levens, B. Szuk, J. Inst. 11, C02062 (2016)

233. M. Bergamaschi et al., Cherenkov Diffraction Radiation From Long Dielectric Material: An Intense Source of Photons in the NIR-THz Range (2017), https://cds.cern.ch/ record/2289125, MOPAB118

234. B. Dehning, E. Effinger, J. Emery, G. Ferioli, G. Guaglio, E.B. Holzer, D. Kramer, L. Ponce, V. Prieto, M. Stockner, C. Zamantzas, The LHC beam loss measurement system, in 2007 IEEE Particle Accelerator Conference (PAC) (June, 2007), pp. 41924194, DOI: 10.1109/PAC.2007.4439980

235. M.F. Fernandes et al., Optimized cryogenic current comparator for CERN's lowenergy antiproton facilities, in Proceedings of the IBIC'16, Barcelona, Spain, September 13-18, 2016, no. 5 in International Beam Instrumentation Conference, (JACoW, Geneva, Switzerland, February, 2017), pp. 161-164, http://jacow.org/ibic2016/ papers/mopg48.pdf

236. D. Belohrad, D.E. Pereira, J. Kral, S.B. Pedersen, Up1grade of the LHC bunch by bunch intensity measurement acquisition system, in Proceedings of the IBIC'16, Barcelona, Spain, Sept. 13-18, 2016, no. 5 in International Beam Instrumentation Conference (JACoW, Geneva, Switzerland, February, 2017), pp. 135-138, http:// jacow.org/ibic2016/papers/mopg39.pdf

237. E. Bravin et al., Performance of the upgraded synchrotron radiation diagnostics at the LHC in Proceedings of the IPAC'16, Busan, Korea, May 8-13, 2016, no. 7 in International Particle Accelerator Conference (JACoW, Geneva, Switzerland, June, 2016), pp. 306-309, http://jacow.org/ipac2016/papers/mopmr030.pdf

238. H.D. Zhang et al., A supersonic gas-jet based beam induced fluorescence prototype monitor for transverse profile determination, in Proceedings of the IPAC'17, Copenhagen, Denmark, no. 8 in International Particle Accelerator Conference, (JACoW, Geneva, Switzerland, May 14-19, 2017), pp. 458-461, http://jacow.org/ipac2017/papers/ mopab139.pdf

239. S. Vlachos et al., The LHC beam gas vertex detector - a non-invasive profile monitor for high energy machines, in Proceedings of the IBIC'17, Grand Rapids, Michigan, USA (August, 2017)

240. Review on the needs for a hollow e-lens for the HL-LHC, CERN (October 6-7, 2016), https://indico.cern.ch/event/567839/

241. O.S. Brüning, F. Willeke, Phys. Rev. Lett. 76, 3719 (1996)

242. R. Bruce et al., MD 1691: Active Halo Control Using Tune Ripple at Injection (April, 2017), http://cds.cern.ch/record/2261236, CERN-ACC-NOTE-2017-0031

243. G. Annala et al., Experimental study of magnetically confined hollow electron beams in the Tevatron as collimators for intense high-energy hadron beams, in Conference 
Proceedings (2011), pp. 370-372, Vol. C110328, Preprint PAC-2011-MOP147 FERMILAB-CONF-11-058-AD-APC

244. G. Apollinari et al., High-Luminosity Large Hadron Collider (HL-LHC). Technical Design Report V.0.1, Vol. 4 of CERN Yellow Reports: Monographs (CERN, Geneva, Switzerland, 2017), CERN-2017-007-M

245. Y. Nie, R. Schmidt, V. Chetvertkova, G. Rosell-Tarragó, F. Burkart, D. Wollmann, Phys. Rev. Accel. Beams 20, 081001 (August, 2017)

246. Q. Bai, B. Jin, D. Wang, Y. Wang, X. Liu, J. Inst. 13, T04004 (2018),

247. T. Wilksen et al., The control system for the linear accelerator at the European XFEL: status and first experiences, in Proceedings of the 16th ICALEPCS'17, Barcelona, Spain, October 8-13, 2017 (2018), p. MOAPL01, http://inspirehep.net/record/ 1656098/files/moapl01.pdf

248. R. Ganter et al., SwissFEL Conceptual Design Report, PSI Bericht 10-14, (Paul Scherrer Institut, July, 2010), https://www.psi.ch/swissfel/SwissFELCDREN/SwissFEL_ CDR_web_small.pdf

249. R. Huhmann et al., The FAIR control system - system architecture and first implementations, in Proceedings of the ICALEPCS'13, San Francisco, CA, USA (2013), p. MOPPC097, http://jacow.org/ICALEPCS2013/papers/moppc097.pdf

250. M. Hankel, B. Rexroth, The Reference Architectural Model Industrie 4.0 (RAMI 4.0) Whitepaper Version 1.0, ZVEI: Die Elektroindustrie (April, 2015), https://www.zvei.org/fileadmin/user_upload/Themen/Industrie_4.0/Das_ Referenzarchitekturmodell_RAMI_4.0_und_die_Industrie_4.0-Komponente/pdf/ ZVEI-Industrie-40-RAMI-40-English.pdf

251. E.P. Boven, White rabbit in radio astronomy, in Proceedings of the ICALEPCS'17, Barcelona, Spain (2017), p. TUCPL03, http://jacow.org/icalepcs2017/

252. N. Moreira, J. Lázaro, U. Bidarte, J. Jimenez, A. Astarloa, IEEE Trans. Smart Grid 8, $1932(2017)$

253. R. Excel, G. Gaderer, P. Loschmidt, Comput. Netw. Commun. 2012, 1 (2012)

254. As-2d2 Deterministic Ethernet And Unified Networking, Time-Triggered Ethernet Standard AS 6802 (SAE International, September 11, 2016)

255. J. Allnut et al., Timing challenges in the smart grid, in Natl. Inst. Stand. Technol. Spec. Publ. 1500-08, (U.S. Department of Commerce, January, 2017), DOI: 10.6028/NIST.SP. 1500-08

256. C. Sydlo et al., Femtosecond timing distribution at the European XFEL, in Proceedings of the FEL'15, Daejeon, Korea (2015), p. WEP047, http://jacow.org/FEL2015/ papers/wep047.pdf

257. A. Aghababyan et al., The large scale European XFEL control system: overview and status of the commissioning, in Proceedings of the ICALEPCS'15, Melbourne, Australia (2015), pp. 5-8, http://jacow.org/icalepcs2015/papers/moa3o02.pdf

258. A. Dinius, Q. King, B. Todd, S. Uznanski, J. Inst. 7, C11012 (2012)

259. F. Abdi et al., Application and system-level software fault tolerance through full system restarts, in ACM/IEEE 8th International Conference on Cyber-Physical Systems (ICCPS), Pittsburgh, PA, USA (April 18-21, 2017), pp. 6505-6519, https: //ieeexplore.ieee.org/document/7945009/

260. W. Benjamin et al., An open architecture for embedded systems: hardware open systems technologies, in IEEE SoutheastCon, Charlotte, NC, USA, (March 30-April 2, 2017), pp. 6505-6519, DOI: 10.1109/SECON.2017.7925388

261. P. Bieth, V. Brindejonc, COTS-AEH - Use of complex COTS in airborne electronic hardware - failure mode and mitigation, Research Project EASA.2012.C15 Report EASA.2012/04 (European Aviation Safety Agency, 2013), https ://www . easa. europa. eu/sites/default/files/dfu/Final20Report20EASA202012-04.pdf

262. National Instruments, Considerations When Navigating Build or Buy Decisions for Industrial Embedded Control Projects White Paper 54072 (National Instruments, August 30, 2017), http://www.ni.com/white-paper/54072/en/ 
263. L. Yubin, L. Yucheng, High continuous availability digital information system based on stratus Fault-Tolerant server, in 2010 International Forum on Information Technology and Applications (July, 2010), Vol. 2, pp. 184-187, DOI: 10.1109/IFITA.2010.79

264. B. Chen, J. Wan, L. Shu, P. Li, M. Mukherjee, B, Yin, IEEE Access 6, 6505 (2017)

265. I. Dai, P. Oleniuk, B. Todd, A. Voto, J. Inst. 11, C01047 (2016)

266. IEEE, Systems and Software Engineering - System Lifecycle Processes Standard ISO 15288:2008 (IEEE/ISO/IEC, 2008), https://en.wikipedia.org/wiki/ISO/IEC_ 15288

267. K. Forsberg, H. Mooz, System engineering for faster, cheaper, better, in INCOSE International Symposium Systems Engineering Past, Present and Future I, Brighton, England, (June 6-11, 1999), Vol. 9, pp. 924-932

268. European Commission, Horizon 2020 Work Programme 2016 - 2017. 5.i. Information and Communication Technologies European Commission Decision C(2017)2468 (European Commission, April 24, 2017), http://ec.europa.eu/research/participants/ data/ref/h2020/wp/2016_2017/main/h2020-wp1617-leit-ict_en.pdf

269. J. Gutleber et al., The MedAustron accelerator control system, in Proceedings of the ICALEPCS'11, Grenoble, France (October 10-14, 2011), pp. 9-12, https://jacow . org/icalepcs2011/papers/mobaust03.pdf

270. J. Gutleber, R. Moser, The MedAustron accelerator control system: design installation and commissioning, in 14th International Conference on Accelerator and Large Experimental Physics Control Systems, San Francisco, CA, USA (March 6-11, 2013), p. TUCOAAB04, https://cds.cern.ch/record/1697002

271. Cosylab, Accelerator Control System for PT, Website, (2018), https://www. cosylab. com/accelerator-control-system-for-pt/

272. D. Ondreka, U. Weinrich, The Heidelberg Ion Therapy (HIT) accelerator coming into operation, in European Physical Society Accelerator Group, Proceedings of EPAC 2008, Genoa, Italy (June 23-27, 2008), pp. 979-981, https://jacow.org/e08/papers/ tuocg01.pdf

273. S. Rossi, S. Eur. Phys. J. Plus 126, 78 (2011)

274. S. Giordanengo, M.A. Garella, F. Marchetto, F. Bourhaleb, M. Ciocca, A. Mirandola, V. Monaco, M.A. Hosseini, C. Peroni, R. Sacchi, R. Cirio, M. Donetti, Med. Phys. 42, $263(2016)$

275. P. Bryant, L. Evans (eds.), J. Inst. 3, 98 (2008)

276. B. Kihei, Automotive doppler sensing: the doppler profile with machine learning in vehicle-to-vehicle networks for road safety, in 18th IEEE International Workshop on Signal Processing Advances in Wireless Communications, Sapporo, Japan (July 3-6, 2017)

277. ICFA, Beam Dynamics Mini-Workshop: Machine Learning Applications for Particle Accelerators. SLAC National Accelerator Laboratory, Menlo Park, CA, USA., Indico presentation (27 February-2 March, 2018), https://indico.fnal.gov/event/16327/ other-view?view=standard

278. R.G. Alia et al., Single event effects in high energy accelerators, in Semiconductor Science and Technology (2017), http://iopscience.iop.org/10.1088/1361-6641/ aa5695

279. R.G. Alia et al., IEEE Trans. Nucl. Sci. 65, 448 (January, 2018)

280. A. Apollonio et al., Roadmap towards high accelerator availability for the CERN HLLHC Era, in Proceedings of the IPAC'15 (May 3-8, 2015)

281. J. Mekki et al., IEEE Trans. Nucl. Sci. 63, 2106 (August, 2016)

282. R. Lauckner, R. Rausch, P. Ribeiro, T. Wijnands, IEEE Conf. Real-Time Comput. Appl. Nucl. Particle Plasma Phys. (1999) 194

283. M. Brugger et al., Irradiation Facilities at CERN (October, 2017), https ://cds . cern. $\mathrm{ch} / \mathrm{record} / 2290629$, AIDA-2020-CONF-2017-007

284. P. Carbonez, R. Macián-Juan, F. Pozzi, CERN Radiation Protection (RP) Calibration Facilities (July 1st, 2016), https://cds.cern.ch/record/2256137, CERN-THESIS2015-394 
285. M.R. Jäkel et al., CERN GIF++: a new irradiation facility to test large-area particle detectors for the high-luminosity LHC program, in PoS (TIPP2014), 102, Proceedings of the TIPP'14, Amsterdam, The Netherlands (2014), https://pos.sissa.it/213/ $102 / \mathrm{pdf}$

286. M. Brugger et al., IEEE Trans. Nucl. Sci PP, 1 (2018), DOI: 10.1109/TNS. 2018.2806450

287. M.L. Alles and et al., IEEE Trans. Nucl. Sci. 64, 285 (2017)

288. D. Bouvet et al., IEEE Trans. Nucl. Sci. 65, 1583 (January, 2018)

289. Geoconsult and Gibb and SGI Ingeniere, LHC Civil Engineering Consultancy Services, Package 2 - Geotechnical Interpretative Report, CERN

290. P. Lebrun, L. Tavian, Phys. Procedia 67, 768 (October, 2014)

291. C. Kotnig, L. Tavian, IOP Conf. Ser. Mater. Sci. Eng. 101, 012043 (2015)

292. M. Chorowski et al., IOP Conf. Ser: Mater. Sci. Eng. 278, 012097 (2017)

293. G. Brenn, C. Kotnig, and L. Tavian, IOP Conf. Ser. Mater. Sci. Eng. 171, 012006 (2017)

294. M.P. Chorowski, P. Duda, J. Polinski, Phys. Rev. Accel. Beams 30, 033202 (March, 2017)

295. C. Haberstroh, F. Holdener, S. Kloeppel, H. Quack, IOP Conf. Ser.: Mater. Sci. Eng. 101, $012042(2015)$

296. H. C. Rodrigues, L. Tavian, IOP Conf. Ser.: Mater. Sci. Eng. 278, 012090 (2017)

297. S. Claudet, G. Ferlin, F. Millet, L. Tavian, $1.8 \mathrm{~K}$ Refrigeration Units for the LHC: Performance Assessment of Pre-series Units (September, 2004), https://cds.cern. $\mathrm{ch} / \mathrm{record} / 795007$, CERN-LHC-Project-Report-797

298. D. Boutin et al., Updates on the optic corrections of FCC-hh in Proceedings of the IPAC'18, Vancouver, Canada (2018), pp. 133-136, DOI: 10.18429/JACoW-IPAC2018-MOPMF023

299. P. Brockill et al., A novel multichannel interferometer system for absolute distance measurements, in Large Volume Metrology Conference (2012)

300. R. Bingham et al., The linear collider alignment and survey (LiCAS) Project, in Proceedings of the 7th International Workshop on Accelerators Alignment, 2002, Spring8, Japan (2002)

301. D. Clark, IEEE Comput. Sci. Eng. 5, 84 (1998)

302. I. Bird, L. Robertson, J. Shiers, Deploying the LHC computing grid - the LCG service challenges, in 2005 IEEE International Symposium on Mass Storage Systems and Technology (June, 2005), pp. 160-165

303. J.C. Webber, The ALMA telescope, in 2013 IEEE MTT-S International Microwave Symposium Digest (MTT) (June, 2013), pp. 1-3

304. D. Ding, D. Wu, F. Yu, An overview on cloud computing platform spark for Human Genome mining, in 2016 IEEE International Conference on Mechatronics and Automation (August, 2016), pp. 2605-2610.

305. G. Antchev et al., IEEE Trans. Nucl. Sci. 47, 293

306. K. Anikeev et al., Comput. Phys. Commun. 140, 110 (2001)

307. DESY, MicroTCA Technology Lab, Website (2018), https://techlab.desy.de

308. CERN, openlab, Website (2018), https://openlab.cern

309. Conseil régional Auvergne-Rhône-Alpes, Numérique. Feuille de route stratégique de la Région Auvergne-Rhône-Alpes 2017-2021, Website of the ARA region (2018), https://www . auvergnerhonealpes.fr/95-infrastructures-economieusages-numeriques.htm

310. Grand Genève Agglomération Franco-Valdo-Genevoise, Charte d'engagement du projet de territoire Grand Genève 2016-2030, Website, December (2016), http://www.grand-geneve.org/sites/default/files/fichiers/projetagglomeration3/charte_2016-projet-territoire.pdf

311. Sepura, Case Study: Advancing Safety and Communications at CERN, Website (January, 2014), https://www. sepura.com/media/166841/cern-case-study.pdf

312. Euro-IX, Information on Carrier Neutrality, Website (2018), https://www.euro-ix. net/en/forixps/set-ixp/ixp-models/neutrality 
313. CERN, Documents and Maps of the Worldwide LHC Computing Grid (WLCG), Website (2018) http://wlcg.web.cern.ch/documents-reference

314. H. Schopper, Rivista del Nuovo Cimento 40, 199 (2017)

315. B. Panzer-Steindel, IT Technology and Markets, Status and Evolution, Indico presentation (March 26, 2018), https://indico.cern.ch/event/658060/contributions/ 2889027/attachments/1622791/2583013/tech_market_BPS_Mar2018_v9pptx.pdf

316. A.D. Meglio, M. Girone, A. Purcell, F. Rademakers, CERN openlab white paper on future ICT challenges in scientific research (January, 2018), http://cds.cern.ch/ record/2301895

317. A. Bastianin, M. Florio, Social Cost Benefit Analysis of HL-LHC, CERN-ACC2018-0014 (CERN, Geneva, Switzerland, May, 2018), Preprint CERN-ACC-2018-0014, https://cds.cern.ch/record/2319300

318. S. Schmeling, IEEE Trans. Nucl. Sci. 53, 970 (2006)

319. D. Collaboration, Data Preservation in High Energy Physics (DPHEP), Website (2018), https://hep-project-dphep-portal.web.cern.ch

320. F. Berghaus et al., CERN Services for Long Term Data Preservation (CERN, Geneva, Switzerland, July, 2016), Preprint CERN-IT-Note-2016-004, https://cds.cern.ch/ record/2195937

321. M.J. Barnes, T. Fowler, A. Kandratsyeu, L. M. Redondo, Design strategies for a SiC Marx generator for a kicker magnet, in 2017 IEEE 21st International Conference on Pulsed Power (PPC) (June, 2017), pp. 1-4

322. R. Cappi, M. Giovannozzi, Phys. Rev. ST Accel. Beams 7, 024001 (February, 2004)

323. E. Shaposhnikova, Implications of $5 \mathrm{~ns}$ bunch spacing for the injector chain, in Future Circular Collider Week 2017, presentation (Berlin, Germany, May, 2017), https://indico.cern.ch/event/556692/contributions/2567943/attachments/ 1468210/2271031/spacing5nsFCC_final.pdf

324. M. Benedikt, Design Optimization of PS2 (May, 2009), http://cds . cern.ch/record/ 1215876, CERN-sLHC-PROJECT-Report-0024

325. W. Bartmann et al., Possible reuse of the LHC as a $3.3 \mathrm{TeV}$ high energy booster for Hadron injection into the FCC-hh, in Proceedings of the 6th IPAC'15, Richmond, Virginia, USA (May 3-8, 2015), p. THPF094, http://jacow.org/IPAC2015/papers/ thpf094.pdf

326. W. Bartmann et al., High Energy Booster Options for a Future Circular Collider at CERN (2016), http://cds.cern.ch/record/2207334, CERN-ACC-2016-291

327. B. Goddard, A. Milanese, M. Solfaroli Camillocci, Faster Ramp of LHC for use as an FCC High Energy Hadron Booster, CERN-ACC-2015-0133 (CERN, Geneva, Switzerland, October, 2015), Preprint CERN-ACC-2015-0133, http://cds . cern.ch/record/ 2057723

328. B. Goddard, A. Milanese, M. Solfaroli Camillocci, ICFA Beam Dyn. Newsl. 72, 113 (2017)

329. A. Milanese, M.S. Camillocci, Faster ramping of LHC in 2017 and prospects for lower energy injection into LHC in 2018, in Presented at FCC Week 2018, Amsterdam (April, 2018), https://indico.cern.ch/event/656491/contributions/ 2930783/attachments/1628670/2594859/FCC_week_2018_faster_ramping_LHC.pdf

330. O. Beltramello, Shutdown constraints and radiation and activation effects, in ECFA High Luminosity LHC Experiments Workshop, Aix-les-Bains (2014) (Aix-les-Bains, 2014), https://indico.cern.ch/event/315626/

331. B. Goddard et al., CERN Yellow Rep.: Monographs, 693, 3 (2017)

332. W. Bartmann et al., Conceptual design considerations for a $1.3 \mathrm{TeV}$ superconducting SPS (scSPS), in Proceedings of the 8th IPAC'17, Copenhagen, Denmark (May 14-19, 2017), pp. 3323-3326, http://jacow.org/ipac2017/papers/wepva033.pdf

333. M. Benedikt, Summary of review of FCC-hh injection energy, Presented at FCC Week 2016 Rome (Rome, 2016), https://indico.cern.ch/event/ 438866/contributions/1084974/attachments/1256301/1854686/FCC_Week_Rome_ Injection-Energy-review-summary.pdf 
334. A.D. Kovalenko, W. Scandale, A.M. Taratin, Nucl. Inst. Methods Phys. Res. Sect. B: Beam Interact. Mater. Atoms 355, 390 (2015)

335. A. Milanese, H. Piekarz, L. Rossi, Concept of a Hybrid (Normal and Superconducting) Bending Magnet based on Iron Magnetization for 80-100km Lepton/Hadron Colliders (November, 2014), http://cds.cern.ch/record/1968562, CERN-ACC-2014-0274

336. The ALICE Collaboration, JINST 3, S08002 (2008)

337. The ATLAS Collaboration, JINST 3, S08003 (2008)

338. The CMS Collaboration, JINST 3, S08004 (2008)

339. The LHCb Collaboration, JINST 3, S08005 (2008)

340. H. Brooks, P.Z. Skands, P. Eur. Phys. J. C 78, 963 (2018)

341. J. Alwall et al., JHEP 07, 079 (2014)

342. ATLAS Collaboration, ATLAS Phase-II Upgrade Scoping Document CERN-LHCC2015-020, LHCC-G-166 (CERN, Geneva, Switzerland, September, 2015), https:// cds.cern.ch/record/2055248

343. CMS Collaboration, J. Butler et al., CMS Phase II Upgrade Scope Document, CERNLHCC-2015-019. LHCC-G-165 (CERN, Geneva, Switzerland, September, 2015), https://cds . cern.ch/record/2055167, CERN-LHCC-2015-019

344. ATLAS Collaboration, Technical Design Report for the ATLAS inner tracker pixel detector, CERN-LHCC-2017-021, ATLAS-TDR-030 (CERN, Geneva, Switzerland, September, 2017), https://cds.cern.ch/record/2285585

345. ATLAS Collaboration, Technical Design Report for the ATLAS Inner Tracker Strip Detector CERN-LHCC-2017-005, ATLAS-TDR-025 (CERN, Geneva, Switzerland, April, 2017), https://cds.cern.ch/record/2257755

346. CMS Collaboration, The Phase-2 Upgrade of the CMS Tracker CERN-LHCC-2017009, CMS-TDR-014 (CERN, Geneva, Switzerland, June, 2017), https://cds.cern. $\mathrm{ch} / \mathrm{record} / 2272264$

347. M. Mentink et al., IEEE Trans. Appl. Supercond. 26, 4003506 (2016)

348. M. Morpurgo, Cryogenics 19, 411 (1979)

349. R.L. Gluckstern, Nucl. Instrum. Meth. 24, 381 (1963)

350. Z. Drasal, W. Riegler, Nucl. Instrum. Meth. A 910, 127 (2018)

351. E. Pere Codina, P. Roloff, Tracking and flavour tagging at FCC-hh LCD-Note-2018-002 (CERN, Geneva, Switzerland, August, 2018), http://cds.cern.ch/record/2635893

352. E. Pere Codina, P. Roloff, Initial Study of the Reconstruction of Boosted B-hadrons and $\tau$-leptons at FCC-hh, LCD-Note-2018-003 (CERN, Geneva, Switzerland, October, 2018), http://cds.cern.ch/record/2643897

353. E. Perez Codina, P. Roloff, Hit Multiplicity Approach to b-tagging in FCC-hh, LCD-Note-2018-001 (CERN, Geneva, Switzerland, July, 2018), http://cds.cern.ch/ record/2631478

354. ATLAS Collaboration, Technical Design Report for the Phase-II Upgrade of the ATLAS LAr calorimeter, CERN-LHCC-2017-018, ATLAS-TDR-027 (CERN, Geneva, Switzerland, September, 2017), https://cds.cern.ch/record/2285582

355. CMS Collaboration, The Phase-2 Upgrade of the CMS Endcap Calorimeter, CERNLHCC-2017-023, CMS-TDR-019 (CERN, Geneva, Switzerland, November, 2017), https://cds.cern.ch/record/2293646, Technical Design Report of the endcap calorimeter for the Phase-2 upgrade of the CMS experiment, in view of the HL-LHC run

356. T. Carli, C. Helsens, A. Henriques Correia, C. Solan Sànchez, JINST 11, P09012 (2016)

357. ATLAS Collaboration, Z. Ajaltouni et al., Nucl. Instrum. Meth. A387, 333 (1997)

358. G. Aad et al., Eur, Phys. J. C 77, 490 (2017)

359. D. Bertolini, P. Harris, M. Low, N. Tran, JHEP 10, 059 (2014)

360. M. Cacciari, G.P. Salam, G. Soyez, J. High Energy Phys. 2008, 063 (2008)

361. M.L. Mangano et al., JHEP 07, 001 (2003)

362. J.M. Campbell, K. Hatakeyama, J. Huston, F. Petriello, J. Andersen, L. Barze, H. Beauchemin, T. Becher, M. Begel, A. Blondel, G. Bodwin, R. Boughezal, S. Carrazza, M. Chiesa, G. Dissertori, S. Dittmaier, G. Ferrera, S. Forte, N. Glover, T. Hapola, A. Huss, X.Garcia i Tormo, M. Grazzini, S. Hoche, P. Janot, T. Kasprzik, M. Klein, U. 
Klein, D. Kosower, Y. Li, X. Liu, P. Mackenzie, D. Maitre, E. Meoni, K. Mishra, G. Montagna, M. Moretti, P. Nadolsky, O. Nicrosini, F. Piccinini, L. Reina, V. Radescu, J. Rojo, J. Russ, S. Sapeta, A. Schwartzman, P. Skands, J. Smillie, I.W. Stewart, F.J. Tackmann, F. Tramontano, R. Van de Water, J.R. Walsh, S. Zuberi, Report of the Snowmass 2013 energy frontier QCD working group, arXiv:1310.5189 [hep-ph]

363. ATLAS Collaboration, Technical Design Report for the Phase-II Upgrade of the ATLAS TDAQ system, CERN-LHCC-2017-020, ATLAS-TDR-029 (CERN, Geneva, Switzerland, September, 2017), https://cds.cern.ch/record/2285584

364. CMS Collaboration, The Phase-2 Upgrade of the CMS DAQ Interim Technical Design Report, CERN-LHCC-2017-014, CMS-TDR-018 (CERN, Geneva, Switzerland, September, 2017), https://cds.cern.ch/record/2283193, this is the CMS Interim TDR devoted to the upgrade of the CMS DAQ in view of the HL-LHC running, as approved by the LHCC

365. W.J. Fawcett, A. Sfyrla, G. Iacobucci, A Self-Seeded Track Trigger for the FCC-hh, CERN-ACC-2018-0046 (CERN, Geneva, Switzerland, October, 2018) https://cds. cern.ch/record/2645273

366. S. Mrenna, T. Sjöstrand, P.Z. Skands, Comput. Phys. Commun. 178, 852 (2008)

367. B.C. Allanach, Comput. Phys. Commun. 143, 305 (2002)

368. A. Djouadi, M.M. Muhlleitner, M. Spira, Acta Phys. Polon. B38, 635 (2007)

369. DELPHES 3 Collaboration, C. Delaere et al., JHEP 02, 057 (2014)

370. J. Baglio et al., JHEP 04, 151 (2013)

371. R. Contino et al., CERN Yellow Rep. 255 (2017)

372. D. London, J.L. Rosner, Extra Gauge Bosons in E(6), in Proceedings of the 23rd ICHEP'86, Berkeley, CA, USA (July 16-23, 1986)

373. A. Joglekar, J.L. Rosner, Phys. Rev. D96, 015026 (2017)

374. P. Langacker, Rev. Mod. Phys. 81, 1199 (2009)

375. E. Salvioni, G. Villadoro, F. Zwirner, JHEP 11, 068 (2009)

376. M. Ibe, S. Matsumoto, R. Sato, Phys. Lett. B721, 252 (2013)

377. S.D. Thomas, J.D. Wells, Phys. Rev. Lett. 81, 34 (1998)

378. B.C. Allanach et al., JHEP 03, 045 (2003)

379. C.H. Chen, M. Drees, J.F. Gunion, Phys. Rev. D55, 330 (1997)

380. C.H. Chen, M. Drees, J.F. Gunion, Addendum/erratum for "searching for invisible and almost invisible particles at $\mathrm{e}^{+} \mathrm{e}^{-}$colliders" [hep-ph/9512230] and "a nonstandard string/SUSY scenario and its phenomenological implications" [hep-ph/9607421], arXiv: hep-ph/9902309 [hep-ph], Preprint UCD-99-2

381. S. Asai, T. Moroi, and T. T. Yanagida, Phys. Lett. B664, 185 (2008)

382. J. Blumlein, M. Klein, Nucl. Instrum. Meth. A329, 112 (1993)

383. LHeC Collaboration, Electrons for the LHC, Update of the LHeC Design, Geneva, CERN, in progress (2019)

384. Gouvernement de la République française and CERN, Accord entre le Gouvernement de la République française et l'Organisation Européenne pour la Recherche Nucléaire relatif au statut juridique de ladite Organisation en France, CERN institutional documents (CERN, Geneva, Switzerland, August, 1973), https://cds.cern.ch/record/ 436804/files/CM-B00042459.pdf

385. U. Forsblom-Pärli et al., Connaissez-vous le potentiel des phénomènes dangereux dans votre entreprise? instruction, système selon commission fédérale de coordination pour la sécurité au travail, SUVA - Protection de la santé (June, 2011), Preprint 66105.1, https://www . suva.ch/materiel/documentation/connaissez-vous-le-potentieldes-phenomenes-dangereux-dans-votre-entreprise--le--66105.f-25527-25526

386. S.L. Mendola, FCC performance-based safety design - A proposal for a methodology founded on the SFPE guideline internal presentation (CERN, June, 2017), Preprint EDMS 1770088, https://edms.cern.ch/document/1770088

387. International Electrotechnical Commission, IEC 60812:2018 - Failure modes and effects analysis (FMEA and FMECA), (International Standard. IEC, Geneva, Switzerland, 3.0 ed., August 10, 2018), https ://cds.cern.ch/record/436804/files/ CM-B00042459.pdf 
388. O. Rios and A. Arnalich, Quantitative Assessment of Fire Hazard for FCC-hh (and FCC-ee) internal report (CERN, June, 2018), Preprint EDMS 1975602, https://edms . cern.ch/document/1975602

389. A. Henriques, Private communication (CERN, Geneva, Switzerland, April, 2018)

390. O.R. Rubiras, A. Arnalich, FCC-hh Fire Safety Analysis: Executive Summary EDMS 1982356 (CERN, Geneva, Switzerland, 2018), Preprint FCC-INF-RPT-0060 v.1, https://edms.cern.ch/document/1982356/

391. A. Henriques, FCC Performance-Based Design for Safety Aspects: Qualitative Analysis on Cryogenic Hazards internal report (CERN, December, 2017), Preprint EDMS 1818330, https://edms. cern.ch/document/1818330

392. STUVA e.V., Security and Workplace Safety Concepts for the Construction, Installation and Operation of the XFEL Research Facility European XFEL project documentation (European XFEL GmbH, August, 2005), Project internal, not public

393. K. McGrattan et al., Fire Dynamics Simulator User's Guide, special publication (National Institute of Standards and Technology, June, 2018), Preprint 1019, https://pages.nist.gov/fds-smv/; https://github.com/firemodels/fds/ releases/download/FDS6.7.0/FDS_User_Guide.pdf

394. B. Delille et al., Task Force Report, Safety of Personnel in LHC Underground Areas Following the Accident of 19th September 2008 report, CERN, June 16, 2009, Preprint CERN-ATS-2009-002, https://cds . cern.ch/record/1184225

395. A.J. Henriques, FCC performance-based Design for Safety Aspects - Cryogenic hazards, EDMS 1818330 v.1.1 (CERN, Geneva, Switzerland, December, 20170, Preprint EDMS 1818330, https://edms.cern.ch/document/1818330/1.1

396. La République française, Article L1333-1 to L1333-25, Code de la santé publique (2018), https://www.legifrance.gouv.fr/telecharger_pdf.do?cidTexte= LEGITEXT000006072665

397. L'Assemblée fédérale de la Confédération suisse, Loi sur la radioprotection (LRaP), Recueil officiel du droit fédéral (RO) (2017), https://www.admin.ch/opc/fr/ classified-compilation/19910045/index.html

398. R. Rata, M. Widorski, Radiological studies for the FCC-hh collimation regions, EDMS 1975526 (2018), https://edms.cern.ch/file/1976620/1/TN_FCChh_EXP.pdf

399. L. Bruno, M. Magistris, Radioactive Waste Management at CERN, Technical Note, EDMS 1453489 (2017), https://edms.cern.ch/document/1453489/3.1

400. Energy for Sustainable Science at Research Infrastructures, 4th Workshop in Măgurele, Bucharest, Romania (November 23-24, 2017)

401. EuCard2 (Enhanced European Coordination for Accelerator Research and Development), Website http://eucard2.web.cern.ch/

402. EuCard2 Work Package 3, Website https://www.psi.ch/enefficient/

403. ARIES (Accelerator Research and Innovation for European Science and Society), Website https://aries.web.cern.ch/

404. M. Seidel, Energy efficiency of accelerators in the European programs Eucard2 and ARIES, https://indico.eli-np.ro/event/1/contributions/6/attachments/ 70/106/4_enefficient_seidel.pdf

405. A. Grosjean, Tout un quartier chauffé grâce au CERN, Tribune de Genève (2018), https://www.tdg.ch/geneve/actu-genevoise/Tout-un-quartier-chauffegrce-au-CERN/story/28974479

406. K. Biesheuvel, R. de Boer, S. Smeding, Waste Heat recovery in industrial batch processes: analysis of combines heat storage and heat pump application, in Proceedings of the 12th IEA Heat Pump Conference, no. 12 in HPC (IEA, Rotterdam, The Netherlands, June, 2017), http://hpc2017.org/wp-content/uploads/2017/05/0.3.8.1-Waste-Heat-recoveryin-industrial-batch-processes-analysis-of-combined-heat-storage.pdf

407. F. Campana et al., Energ. Convers. Manage. 76, 244 (2013)

408. P. Colonna et al., J. Eng. Gas Turbines Power 137, 100801-1 (2015)

409. Accord entre le Gouvernement de la République française et l'Organisation européenne pour la Recherche nucléaire relatif au statut juridique de ladite Organisation en France 
(CERN, Geneva, Switzerland, 1973), https://cds.cern.ch/record/436804

410. Convention entre le Conseil fédéral de la Confédération suisse et le Gouvernement de la République française relative à l'extension en territoire français du domaine de l'Organisation européenne pour la recherche nucléaire, Le Conseil fédéral, Chancellerie fédérale, Palais fédéral ouest (3003 Berne, Switzerland, September 13, 1965), https: //www.admin.ch/opc/fr/classified-compilation/19650161

411. A. Poiron, M. Zahnd, FCC Layout Review in Switzerland deliverable report contract ca 3797383 (Ecotec, December, 2017), Preprint EDMS 1838912, https://edms.cern. $\mathrm{ch} /$ document/1838912

412. Le Conseil fédéral suisse, Ordonnance relative à l'étude de l'impact sur l'environnement 814.011 (UVPV), Recueil officiel du droit fédéral (RO) (2016), https://www.admin. $\mathrm{ch} / \mathrm{opc} / \mathrm{fr} / \mathrm{classified-compilation/19880226/index.html}$

413. Office fédéral de l'environnement OFEV, Directive de la Confédération sur l'étude de l'impact sur l'environnement (Manuel EIE), Serie L'environnement pratique (2009), https://www.bafu.admin.ch/bafu/fr/home/themes/eie/publications/ manuel-eie.html

414. Le Président de la République, Code de l'environnement, Journal officiel de la République française (2018), https://www.legifrance.gouv.fr/affichCode.do? cidTexte=LEGITEXT000006074220\&dateTexte $=20180924$

415. UNECE, Convention on environmental impact assessment in a transboundary context Convention ECE/MP.EIA/21/Amend.1, United Nations, Avenue de la Paix 8, 1202 Genève, Suisse (October, 2017), http://www.unece.org/env/eia/about/eia_text. html

416. Office Fédéral de la santé publique, Radioactivité de l'environnement et doses de rayonnements en Suisse, Rapport 2017, Project website (2018), http://www.bag.admin. $\mathrm{ch} / \mathrm{ura}-\mathrm{jb}$

417. R. Rata, M. Widorski, Radiological studies for the FCC-hh arc sections, EDMS 1905211 (2018), https://edms.cern.ch/file/1905211/1/TN_FCChh_ARC.pdf

418. R. Rata, M. Widorski, Radiological studies for the FCC-hh experimental caverns, EDMS 1976620, (2018)

419. A. Bibet-Chevalier, D. Chanal, Étude de sensibilité du scenario d'implantation du project FCC en France et de ses opportunités Rapport d'étude du Cerema FCC-INFRPT-040 and EDMS 1853668 (CEREMA, April 26, 2018), https://edms.cern.ch/ document/1853668

420. Le Conseil fédéral suisse, Ordonnance sur l'aménagement du territoire (OAT) 700.1, Recueil officiel du droit fédéral (RO) (2016) , https://www.admin.ch/opc/fr/ classified-compilation/national.html

421. GeniLac, Website http://www.citedelenergie.ch/fileadmin/user_upload/ Energiestadt/de/Dateien/Instrumente/6_Kommunikation/6_5_2_GeniLa.pdf

422. M. Magistris, H. Vincke, M. Widorski, Radioactive Waste Estimates for the FCC project EDMS 1992036 (CERN, Geneva, Switzerland, 2018), Preprint EDMS 1992036, https://cds.cern.ch/record/1992036

423. Départment du Rhône-Métropole de Lyon, Plan de prévention et de gestion des déchets non dangereux du Rhône et de la Métropole de Lyon version définitive, Départment du Rhône-Métropole de Lyon (June, 2015), http://www . sindra.org/wp-content/uploads/2017/01/Plan-dC3A9chets-nondangereux-RhC3B4ne-MC3A9tropole-de-Lyon.pdf

424. Le Président de la République, Loi n. 2015-992 du 17 août 2015 relative à la transition énergétique pour la croissance verte, Journal officiel de la République française (2018) 14263, https://www.legifrance.gouv.fr/affichTexte. do?cidTexte=JORFTEXT000031044385

425. République et Canton de Genève, Genève - Plan directeur cantonal 2030 permière mise à jour, Direction de la planification directrice cantonale et régionale, service du plan directeur cantonal (February, 2017), https://www.ge.ch/consulter-plans-amenagement-adoptes/plan-directeurcantonal 
426. P. Joxe, J. Officiel République Fr. 33, 2064 (2018)

427. Le Président de la République, J. Officiel République Fr. 75, 5568 (2003)

428. UNECE, Treaty Ser. 2161, 447 (1998)

429. CEREMA, Rapport d'études sur les impacts pour l'État en matière de procédures et d'engagements financiers report, Secrétariat Gégnéral pour les Affaires Régionales d'Auvergne-Rhône-Alpes (April, 2018), This report is for government-internal purposes only.

430. Le Président de la République, J. Officiel République Fr. (JORF) 248, 17545 (2002)

431. Le Président de la République, J. Officiel République Fr. (JORF) 0181, 10 (2016)

432. Commission nationale du débat public, Comment ça marche ? site internet de l'institution, Commission nationale du débat public, 244 boulevard SaintGermain 75007 Paris France (October, 2002), https://www.debatpublic.fr/ comment-ca-marche

433. Journal officiel de la République française (JORF), J. Officiel République Fr. 0261, 19003 (2014)

434. Le Président de la République, J. Officiel République Fr. (2018), https://www. legifrance.gouv.fr/affichTexte.do?cidTexte=JORFTEXT000033926976

435. Le Président de la République, J. Officiel République Fr. 0128, 10339 (2018)

436. Direction de l'ARE, Surfaces d'assolement selon le plan sectoriel SA Modèle de géodonnées minimal. Documentation sur le modèle. 68, Office fédéral du développement territorial ARE, Worblenstrasse 66, CH-3063 Ittingen, Schweiz (November, 2015), https://www.are.admin.ch/are/fr/home/developpementet-amenagement-du-territoire/strategie-et-planification/conceptions-etplans-sectoriels/plans-sectoriels-de-la-confederation/sda.html

437. Conseil d'Etat de la République et canton de Genève, Surfaces d'assolement selon le plan sectoriel SA Législation K 1 70.13, État de Genève, 2, rue Henri-Fazy, case postale 3964, 1211 Genève 3 (January, 2008), https://www.ge.ch/legislation/rsg/ f/s/rsg_k1_70p13.html

438. G. Catalano et al., Guide to Cost-Benefit Analysis of Investment Projects (Regional and Urban Policy, European Union, December, 2014), pp. 321-333

439. Questionnaire for submission of proposals for roadmap 2018, Website (September, 2016), https://ec.europa.eu/research/infrastructures/pdf/esfri/esfri_ roadmap/esfri_rd2018_questionnaire.pdf

440. RI impact pathways H2020 INFRASUPP project 777563, January 2018 to June 2020, Website (September, 2018), https://cordis . europa. eu/project/rcn/212964_ en.html

441. M. Florio, E. Sirtori, Technol. Forecasting Soc. Change 112, 65 (2016)

442. M. Florio, S. Forte, E. Sirtori, Techn. For. and Soc. Change 112, 38 (2016)

443. M. Florio et al., Exploring Cost-Benefit Analysis of Research, Development and Innovation Infrastructures: An Evaluation Framework working paper 01/2016 (CSIL Centre for Industrial Studies, Corso Monforte, 15, 20122 Milano MI, Italy, March, 2016), arXiv:1603.03654 [physics.soc-ph]

444. United Nations Statistical Commission, System of National Accounts 2008, Website (2008), https://unstats.un.org/unsd/nationalaccount/sna2008.asp

445. P. Johansson, B. Kriström, Cost-Benefit Analysis for Project Appraisal (Cambridge University Press, October, 2015), ISBN 9781107548220

446. P. Herson, L. McNeil, Phys. Today 70, 39 (2017)

447. T. Camporesi, G. Catalano, M. Florio, F. Giffoni, European Journal of Physics 38, $025703(2017)$

448. G. Catalano, M. Florio, V. Morretta, T. Portaluri, The Value of Human Capital Formation at CERN CERN-ACC-2018-0025 (CERN, Geneva, Switzerland, August 22, 2018), Preprint CERN-ACC-2018-0025, https://cds.cern.ch/record/2635864

449. Economisti Associati, Marie Curie Researchers and their Long-term Career Development: A Comparative Study, final report, (European Union, Publications Office of the European Union, March, 2014), http://ec.europa.eu/research/fp7/pdf/mca/ marie_curie_researchers_and_their_long-term_career_development.pdf 
450. A. Bibet-Chevalier, D. Chanal, Étude des impacts pour l'État du projet de future collosionneur circulaire du CERN en matière de procédures et d'engagements financiers Rapport d'étude du Cerema pour le SGAR Auvergne-Rhône-Alpes EDMS 1959547 V1.0, CEREMA (March, 2018), https://edms.cern.ch/document/1959547/ 1.0, Access to the report is subject to an NDA

451. European Advances Superconductor Innovation and Training Network, Website http: //easitrain.web.cern.ch

452. CEBR, The importance of physics to the economics of Europe executive summary report of the centre for economics and business research (European Physical Society, 68200 Mulhouse, France, January, 2013), http://www .eps.org/?page=policy_economy

453. M. Bianchi-Streit et al., Economic utility resulting from CERN contracts (second study), CERN Yellow Reports: Monographs (CERN, Geneva, Switzerland, 1984), https://cds.cern.ch/record/156911

454. P. Castelnovo et al., Res. Policy 47, 1853 (2018)

455. M. Florio, F. Giffoni, A. Giunta, E. Sirtori, Ind. Corporate Change 27, 915 (2018)

456. A. Bastianin, M. Florio, Industrial Spillovers from the LHC/HL-LHC Programme at CERN, CERN-ACC-2018-0026 (CERN, Geneva, Switzerland, August 23, 2018), Preprint CERN-ACC-2018-0026, https ://cds . cern. ch/record/2635876

457. P. Seidel, Applied superconductivity: handbook on devices and applications, in The Art of Computer Programming (Wiley, February, 2015), p. 1238, ISBN 978-3-527-41209-9

458. P. Lebrun, Mater. Sci. Eng. 171, 012001 (2017)

459. E. Autio, M. Bianchi-Streit, A.P. Hameri, Technology Transfer and Technological Learning Through CERN's Procurement Activity, CERN Yellow Reports: Monographs (CERN, Geneva, Switzerland, 2003), https://cds.cern.ch/record/680242

460. Le Grand Paris des déblais dévoile ses lauréats, Website (2008), http://www.batiweb.com/actualites/vie-des-societes/le-grand-paris-desdeblais-devoile-ses-laureats-30-03-2017-30184.html

461. C. Benna, Telt-Politecnico, master e dottorandi nel cantiere della TorinoLione, Corriere della Sera (2017), http://torino.corriere.it/cronaca/ 17_dicembre_15/accordo-telt-politecnico-master-dottorandi-cantiere-torinolione-ba0f 2874-e1e7-11e7-980c-f1b8f0b331b7.shtml

462. Sept projets innovants pour les déblais du Grand Paris, Website (2017), https://www .usinenouvelle.com/article/sept-projets-innovants-pour-lesdeblais-du-grand-paris.N520469

463. T.B. Lee, M. Fischetti, Weaving the web: the original design and ultimate destiny of the world wide web by its inventor, Harper Business, 1st edition (November, 2000), ISBN 978-0062515872

464. L. Mascetti et al., J. Phys.: Conf. Ser. 644, 062037 (2015)

465. A. Alberini, A. Longo, J. Cult. Econ. 30, 287 (2006)

466. R.C. Bishop, N.W. Bouwes, P.P. Caulkins, Am. J. Agricult. Econ. 68, 291 (1986)

467. J.P. Poor, J.M. Smith, J. Cult. Econ. 28, 217 (2004)

468. M. Florio, S. Forte, E. Sirtori, Technol. Forecast. Soc. Change 112, 38 (2016)

469. C. Risen, J. Am. Inst. Archit. (2018), https://www.architectmagazine.com/ awards/studio-prize/studio-prize-the-new-grand-tour-a-new-visitors-centerfor-cern_o

470. G. Catalano, I.C. Garido, Cultural Effects at CERN CERN-ACC-2018-0048 (CERN, Geneva, Switzerland, November, 2018), https://cds.cern.ch/record/2649022

471. R.D. Cabana, J.E. Petro, Kennedy Space Center. Future Development Concept, KSC center master plan 2012-2031 (NASA, John F. Kennedy Space Center, FL 32899, USA, 2012), https://www.nasa.gov/centers/kennedy/pdf/634026main_future-concept. pdf

472. A.J. Barr, A. Haas, C. Kalderon, 'That looks weird' = evaluating citizen scientists' ability to detect unusual features in ATLAS images of collisions at the Large Hadron Collider, report, (University of Oxford, UK and New York University, USA and University of Lund, Sweden, 2017), arXiv:1610.02214 [physics.soc-ph], https: //arxiv.org/pdf/1610.02214.pdf 
473. The Zooniverse project: publications, Website https://www.zooniverse.org/about/ publications

474. EXTREME - alla ricerca delle particelle. Museo Nazionale Scienza e Tecnologia Leonardo Da Vinci, Milano, Italia, Website (2016), http://www.museoscienza.org/ extreme

475. Anfang - Wie alles begann. Von Galaxien, Quarks und Kollisionen. Naturhistorisches Museum Wien, Austria, Website (2016), http://www.nhm-wien.ac.at/anfang

476. F. Giffoni, F. Massimo, Scientific Research at CERN as a Public Good: A Survey to French citizens, CERN-ACC-2018-0024, (CERN, Geneva, Switzerland, August, 2018), Preprint CERN-ACC-2018-0024, https ://cds. cern.ch/record/2635861

477. M. Cabriol, La France booste le budget du CNES en 2017, La Tribune newspaper website https://www.latribune.fr/entreprises-finance/industrie/ aeronautique-defense/la-france-booste-le-budget-du-cnes-en-2017-629755. html

478. A. Augier, B. Gindre, Préparation de l'accompagnement stratégie en concertation socioterritoriale dans le cadre de l'étude FCC Final project report EDMS 1745888, iddest - Institut durable de développement économique, social \& territorial (January, 2017), Access to the report is subject to a NDA

479. M. Sauvain, Rapport d'étude relatif aux procédures administratives sur le territoire suisse Rapport de la Structure de Concertation Permanente EDMS 20258995 V1.0, Latitude Durable (November, 2018), https://edms.cern.ch/document/2025895, Access to the report is subject to an NDA

480. European Strategy Forum on Research Infrastructures, Strategy Report on Research Infrstructures, Roadmap 2018 Roadmap 2018 (ESFRI, August, 2018), http:// roadmap2018.esfri.eu/media/1066/esfri-roadmap-2018.pdf

481. European Strategy Forum on Research Infrastructures, Public Roadmap 2018 Guide Guide 2018 (ESFRI, December, 2016), https://ec.europa.eu/ research/infrastructures/pdf/esfri/esfri_roadmap/esfri_rd2018_guide_for_ appplicants.pdf

482. A. Ballarino, L. Bottura, IEEE Trans. Appl. Supercon. 25, 3 (2015)

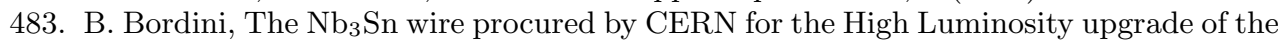
Large Hadron Collider, in Proceedings of the ASC'18, IEEE Conference Proceedings (IEEE, Seattle, USA, October, 2018), forthcoming in 2019 on IEEE XPlore

484. CERN EP department, RESD on experimental technologies, Project website, https: //ep-dep.web.cern.ch/rd-experimental-technologies

485. CERN EP department, First workshop on R\&D on experimental technologies, Indico website, https://indico.cern.ch/event/696066/

486. CERN EP department, Second workshop on R\&D on experimental technologies, Indico website, https://indico.cern.ch/event/743661/ 